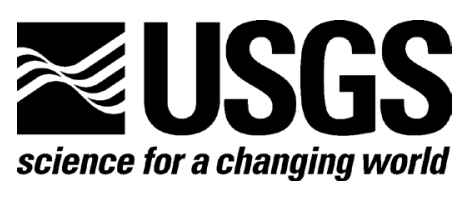

\title{
HAWAIIAN VOLCANO OBSERVATORY 1979 Annual Administrative Report
}

INTRODUCTORY NOTE BY THOMAS L. WRIGHT AND JENNIFER S. NAKATA

COMPILED BY JENNIFER S. NAKATA

\author{
SUMMARY 79, PART 1 \\ SEISMIC DATA, JANUARY TO DECEMBER 1979 \\ By Jennifer S. Nakata, Wilfred R. Tanigawa, and Fred W. Klein \\ CHRONOLOGICAL SUMMARY \\ BY DANIEL DZURISIN
}

OPEN-FILE REPORT 2007-1339

U.S. DEPARTMENT OF THE INTERIOR

U.S. GEOLOGICAL SURVEY 


\section{U.S. Department of the Interior DIRK KEMPTHORNE, Secretary \\ U.S. Geological Survey Mark D. Myers, Director}

\section{U.S. Geological Survey, Reston, Virginia 2007}

For product and ordering information:

World Wide Web: http://www.usgs.gov/pubprod

Telephone: 1-888-ASK-USGS

For more information on the USGS - the Federal source for science about the Earth, its natural and living resources, natural hazards, and the environment: World Wide Web: http://www.usgs.gov

Telephone: 1-888-ASK-USGS

Any use of trade, product, or firm names is for descriptive purposes only and does not imply endorsement by the U.S. Government.

Although this report is in the public domain, permission must be secured from the individual copyright owners to reproduce any copyrighted material contained within this report. 


\section{INTRODUCTORY NOTE}

The Hawaiian Volcano Observatory Summaries have been published in the current format since 1956. The Quarterly Summaries (1956 through 1973) and the Annual Summaries (1974 through 1985) were originally published as Administrative Reports. These reports have been compiled and published as U.S. Geological Survey Open-File Reports. The quarterly reports have been combined and published as one annual summary. All the summaries from 1956 to the present are now available as .pdf files at http://www.usgs.gov/pubprod.

The earthquake summary data are presented as a listing of origin time, depth, magnitude, and other location parameters. Network instrumentation, field station sites, and location algorithms are described. Tilt and other deformation data are included until Summary 77, January to December 1977. From 1978, the seismic and deformation data are published separately, due to differing schedules of data reduction.

There are eight quarters - from the fourth quarter of 1959 to the third quarter of 1961 — that were never published. Two of these ( $4^{\text {th }}$ quarter $1959,1^{\text {st }}$ quarter 1960) have now been published, using handwritten notes of Jerry Eaton (HVO seismologist at the time) and his colleagues. The seismic records for the remaining six summaries went back to California in 1961 with Jerry Eaton. Other responsibilities intervened, and the seismic summaries were never prepared.

\section{Chronology}

The following Kìlauea eruption chronology covers the two recent reports and the six missing quarters:

\begin{tabular}{|l|l|l|l|}
\hline Location & Beginning Date & Ending Date & Comment \\
\hline Kìlauea Iki crater (Kîlauea's summit) & $11 / 14 / 1959$ & $12 / 20 / 1959$ & 19 eruptive episodes \\
\hline Kapoho (lower east rift zone) & $1 / 13 / 1960$ & $2 / 18 / 1960$ & 4 eruption stages \\
\hline Halemaumau (Kīlauea's summit) & $2 / 24 / 1961$ & $2 / 24 / 1961$ & $\begin{array}{l}\text { Intermittent activity during } \\
\text { uninterrupted inflation fol- } \\
\text { lowing the 1960 eruption }\end{array}$ \\
\hline Halemaumau (Kīlauea's summit) & $3 / 22 / 1961$ & $3 / 25 / 1961$ & Same as above. \\
\hline Halemaumau (Kīlauea's summit) & $7 / 10 / 1961$ & $7 / 17 / 1961$ & Same as above. \\
\hline Heiheiahulu (middle east rift zone) & $9 / 22 / 1961$ & $9 / 25 / 1961$ & $\begin{array}{l}\text { First historical east rift erup- } \\
\text { tion at this location }\end{array}$ \\
\hline
\end{tabular}

The 1959-1960 eruptions were among two of the most spectacular Kilauea eruptions. The HVO staff was kept busy with acquisition of unusually high quantities of instrumental data and observations of the two sequences, which were separated by less than one month. Even with a year's interval before the beginning of the summit-east rift sequence in 1961, the staff never caught up, and the seismic records were set aside for later study.

A total of 1,672 earthquakes-1,106 for 1960 and 566 for 1961-are part of HVO's cataloged database. The annual listings have been appended to the $1^{\text {st }}$ Quarter Report of 1960 and to the $4^{\text {th }}$ Quarter Report for 1961 . The number of earthquakes is probably low, biased toward the larger magnitudes. The entire HVO catalog, including 1960 and 1961, is accessible from the ANSS CATALOG SEARCH site at http://www.ncedc.org/anss/catalog-search. 
UNITED STATES DEPARTMENT OF THE INTERIOR GEOLOGICAL SURVEY

HAWAIIAN VOLCANO OBSERVATORY

SUMMARY 79, PART 1

SEISMIC DATA, JANUARY TO DECEMBER 1979

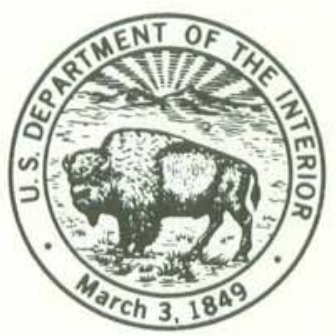

This report is preliminary and has not been edited or reviewed for conformity with Geological Survey standards and nomenclature

Menlo Park, California 


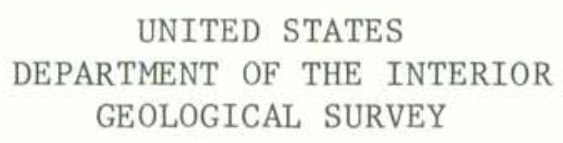

HAWAIIAN VOLCANO OBSERVATORY

SUMMARY 79, PART 1

SEISMIC DATA, JANUARY TO DECEMBER 1979

BY

JENNIFER S. NAKATA, WILFRED R. TANIGAWA, FRED W. KLEIN

CHRONOLOGICAL SUMMARY

BY

DANIEL DZURISIN

OBSERVATORY STAFF

GEOLOGY

JANE M. BUCHANAN-BANKS

NORMAN G. BANKS

ROBERT W. DECKER (SCIENTIST-IN-CHARGE)

DANIEL DZURISIN

JOHN P. LOCKWOOD

RICHARD B. MOORE

\section{GEOPHYSICS}

ROBERT K. CESSARO

KENNETH T. HONMA

DALLAS B. JACKSON

FRED W. KLE IN

GEORGE KOJIMA

ROBERT Y. KOYANAGI

JENNIFER S. NAKATA

ARNOLD T. OKAMURA

IRENE S. TAKAYESU

WILFRED R. TANIGAWA

ALVIN H. TOMORI
GEOCHEMISTRY

THOMAS J. CASADEVALL

BRUCE T. FURUKAWA

L. PAUL GREENLAND

REGINALD T. OKAMURA

(CHIEF OF OPERATIONS)

\section{SUPPORT}

ALEX N. BITTENBINDER

THOMAS T. ENGLISH

DOROTHY C. FOOTER

JOHN C. FORBES

WILLIAM E. FRANCIS

J.D. GRIGGS

JACK E. HARRIS

GAIL E. HILL

MARIAN M. KAGIMOTO

MARIE S. ONOUYE

MAURICE K. SAKO

ELLEN SCANDALIS

AKIRA YAMAMOTO

KENNETH M. YAMASHITA 
Introduction.$\ldots \ldots \ldots \ldots \ldots \ldots \ldots \ldots \ldots \ldots \ldots \ldots \ldots \ldots \ldots \ldots \ldots \ldots \ldots \ldots$

Chronological Summary..................................... 2

Seismic Instrumentation.................................

Figure 1 Map of Hawaii showing geographic and geologic features................................... 6

Figure 2 Map of Hawaii showing active seismic stations and geographic regions......................... 7

Table 1 Seismic stations on Hawaii operated by the USGS........ 8

Table 2 Seismic instrumentation types in use by HVo.......... 9

Figure 3 System response curve of the four basic seismograph types in use by HVo.....................

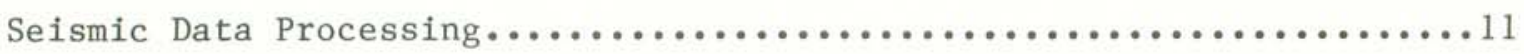

Figure 4 Relationship between duration time and local magnitude...................................

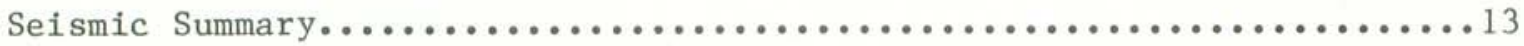

Table 3 Number of earthquakes and minutes of tremor recorded on seismographs around Kilauea.............14

Figure 5 Epicenter map of Hawaii showing only magnitudes

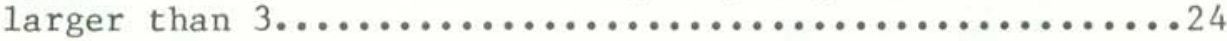

Figure 6 Epicenter map of Hawaii showing all earthquakes.........25

Figure 7 Epicenter map of the Kilauea/Mauna Loa region showing only magnitudes larger than $3 \ldots \ldots \ldots \ldots \ldots \ldots \ldots$

Figure 8 Epicenter map of the Kilauea/Mauna Loa region showing all earthquakes..........................

Figure 9 Epicenter map of Kilauea caldera...................

Table 4 Coordinates of named regions used for classifying

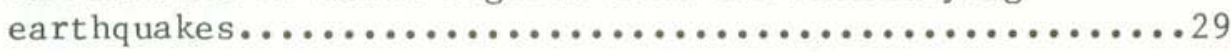

Table 5 List of all located earthquakes.......................

Table 6 List of located earthquakes of magnitude 3.0 or

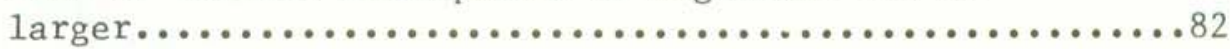




\section{INTRODUCTION}

The Hawaiian Volcano Observatory (HVO) summaries present data gathered during the year together with a chronological narrative intended to describe in geologic terms the volcanic activity associated with the seismic events and tilt data included. The summaries are issued in two parts. Seismic data appears in Part 1, and water tube tilt and other deformation data appears in Part 2. The seismic and chronological summaries are offered without interpretation as a source of preliminary data. The seismic summary is complete in the sense that all data routinely gathered by the observatory are included. The emphasis in collection of tilt and deformation data has recently shifted from quarterly measurements at a few water-tube tilt stations ("wet" tilt) to a larger number of continuously recording borehole tiltmeters, repeated measurements at numerous spirit-level tilt stations ("dry" tilt), and surveying of level and trilateration networks. Because of the large quantity of deformation data now gathered and differing schedules of data reduction, the seismic and deformation summaries will be published separately.

The HVO summaries have been published in various formats since 1956. Summaries prior to 1974 were issued quarterly, but cost, convenience of preparation and distribution, and the large quantities of data dictated an annual format beginning with summary 74 for the year 1974. Summary 74 includes an extensive description of the seismic instrumentation, calibration and processing used in recent years. The present summary includes enough background information on the seismic network and processing to use the data and understand the essentials of how it was gathered.

A report tabulating the instrumentation, calibration and recording history of each seismic station in the network by Klein and Koyanagi is available as a USGS open file report ("Hawaiian Volcano Observatory Seismic Network History 1950-79," 1980). It is designed as a reference for users of seismograms and phase data, and so includes and goes a bit beyond the information in the station table in this summary. 
CHRONOLOGICAL SUMMARY - 1979

by

Daniel Dzurisin

The decade which included the longest-lived rift zone eruption in recorded Hawaiian history (Mauna Ulu, 1969-1974), the first Mauna Loa eruption in 25 years (5-6 July 1975), and the largest Hawaiian earthquake in more than a century (29 November 1975, M = 7.2) ended this year on a relatively tranquil note. Pele's only appearance of the year came on November 16-17 during a small eruption in and near Pauahi crater along Kilauea's upper east rift zone. The eruption briefly closed a section of Chain of Craters Road, which had been reopened in 1979 following its destruction ten years earlier by flows from Mauna Ulu. By year's end, traffic was again flowing smoothly, but Kilauea summit tilt had surpassed its pre-November eruption level and reached the level attained just prior to the major east rift zone eruption of September 1977.

Mauna Loa continued its repose following the July 1975 eruption, and seismicity there remained at background levels throughout most of 1979 . A week-long flurry of shallow nighttime earthquakes near or within Mokuaweoweo caused some concern in March, but these were soon recognized to be a near-surface, temperature-related phenomenon unrelated to volcanic activity. Borehole tiltmeters near the summit and along the northeast rift zone recorded a modest aseismic inflation during June-July, but tilting was negligible throughout the remainder of the year. Summit dry tilt and horizontal distance measurements in August were generally consistent with weak inflation south of the caldera since June 1978 , but at a substantially lower rate than during the previous three years.

As Mauna Loa slumbered, Kilauea continued its struggle to recover from the effects of the November 1975 earthquake. During 1978, summit inflation had overcome roughly $70 \%$ of the deflation associated with the 1977 eruption, but the net summit tilt change during January-August 1979 was essentially zero. This period was punctuated by two shallow intrusions into the upper east rift zone, which in many respects were reminiscent of three east rift zone intrusions which followed the November 1975 earthquake and preceded the September 1977 eruption.

There was a marked increase in the frequency of felt earthquakes at Kilauea during March, and shallow summit quake counts increased to nearly 500 per day during April-May. This activity culminated in an east rift zone intrusion on May 29-30. The event was heralded by several felt earthquakes which roughly coincided with the onset of summit deflation at 0.3 microradians/hour. This modest rate was maintained for roughly 5 hours, after which subsidence slowed before levelling off early on May 30 after a net deflation of 2.7 microradians. A simultaneous swarm of shallow earthquakes which began near buried Aloi crater migrated slightly uprift at an average rate of $1.3 \mathrm{~km} / \mathrm{hr}$ during the first 1.5 hours of intense activity, then moved downrift to Makaopuhi crater at roughly $0.6 \mathrm{~km} / \mathrm{hr}$. Nearly 2,000 earthquakes ranging upward in magnitude to $M=3.3$ were recorded by the HVO seismic network during the first 15 hours of anomalous activity. 
Attention was again focused on Kilauea's upper east rift zone during JuneJuly, when shallow earthquakes became concentrated between Keanakakoi and Pauahi craters. A M $=4.0$ earthquake along the middle east rift zone on July 15 was followed by a brief seismic flurry, but no discernible harmonic tremor or summit deflation. On August 12, a swarm of shallow earthquakes and rapid summit deflation totalling 1 microradian marked a second intrusion into the upper east rift zone. During the first few hours of activity, earthquakes migrated downrift from Keanakakoi to Kokoolau at a mean rate of $1.4 \mathrm{~km} / \mathrm{hr}$. No measureable ground deformation within the epicentral zone accompanied this relatively minor intrusive event.

The period September-November was characterized by resumed summit inflation at roughly $10 \mathrm{microradians/month}$ and generally increasing seismicity at the summit and along the upper east rift zone. Hawaii's largest seismic event since November 1975 occurred on September 21, when a $M=5.4$ quake struck the south flank of Kilauea roughly $7 \mathrm{~km}$ south of Kalalua. Minor damage was caused in Hilo and Puna, but no injuries were reported. The quake followed a substantial inflation of the east rift zone between Makaopuhi and Heiheiahulu since the September 1977 eruption, and was presumably caused in part by accumulated magma pressure. Dry tilt measurements along the rift zone in early September revealed as much as 125 microradians inflation since October 1977, but another survey after the $\mathrm{M}=5.4$ earthquake indicated a distinct reversal of this trend. Presumably, ground movements associated with the quake at least partially relieved accumulated stress caused by shallow magma storage within the rift zone.

An HVO press release in late October noted that high rates of ground deformation and seismic activity had significantly increased the likelihood of eruption at Kilauea. This forecast was borne out only three weeks later when magma returned to the surface of Kilauea after a 25-month absence. Alerted by local harmonic tremor and relatively rapid summit deflation, HVO was on the scene when intense fuming began in the forest east of Pauahi crater at $0805 \mathrm{hrs}$ on November 16. Lava fountains from this eastern vent became audible from Pauahi overlook at roughly 0815, and lava welled up from a new fissure on the northwest wall of Pauahi at 0821. Vents east of the crater migrated a few hundred meters eastward before eruptive activity there ceased at roughly 0915, after closing a short section of Escape Road. Starting at 1130, three new vents opened progressively westward within Pauahi, followed by five more along a westward-migrating fissure which cut Chain of Craters Road at the Pauahi parking area. Apparently, downrift migration of the eruptive fissure stopped as magma gained access to the Koae fault system west of Pauahi. The western vents declined noticeably shortly before 1600, and by 1700 lava was reaching the surface only from vents within Pauahi. Lava production there remained roughly constant until 0100 November 17, when activity slowed before ceasing completely at 0630. After a net summit deflation of only 7 microradians, re-inflation began at 0400 November 17. Roughly $600,000 \mathrm{~m}^{3}$ of differentiated basalt had been erupted in 22.5 hours, presumably from a storage area within the rift zone which was rapidly resupplied with magma from the summit reservoir. At year's end, summit tilt stood slightly above its pre-eruption level.

Pele's brief reappearance was definitely not the only noteworthy happening at HVO during 1979, as staff members took advantage of the 1 ull in eruptive activity to pursue data analysis and field projects. Detailed geologic mapping and radiocarbon dating continued at Kilauea (Banks, Moore), Mauna Loa (Lockwood), 
and Hualalai (Moore), and a stratigraphic/petrologic study of Kilauea caldera was initiated (Casadeval1, Dzurisin). The gas geochemistry program at HVO was expanded with the addition of chemist Paul Greenland and technician Bruce Furukawa, and by the acquisition of a correlation spectrometer (COSPEC) for daily measurements of $\mathrm{SO}_{2}$ flux at Kilauea (Casadeva11). An electrical selfpotential survey of Hualalai's rift zones revealed high-amplitude, short wavelength anomalies suggestive of shallow heat sources, and a program of induction resistivity monitoring was implemented in the Kilauea summit region (Jackson). The Kilauea/Mauna Loa gravity network was expanded in 1979; delivery of HVO's new LaCoste-Romberg gravity meter is expected in late 1980 (Dzurisin). A NASAsponsored study of tephra erosion at Kilauea was completed and readied for publication (Dzurisin). The observatory's Data General Eclipse computer became fully operational, and assumed the task of routine seismic and deformation data processing (English, Klein, Koyanagi, A. Okamura). Sandia personnel drilled six new holes into Kilauea Iki lava lake, one of which successfully penetrated pre-1959 basement. Finally, HVO received additional international exposure when Dzurisin, Koyanagi, and Yamashita spent four weeks in Indonesia as part of a cooperative USGS/USAID geologic assistance program, and Casadevall conducted a geothermal energy assessment in Argentina with DOE support. This dizzying array of activities was coordinated in 1979 by HVO's new scientist-in-charge, Bob Decker, and by Chief of Operations, Reggie Okamura, who will both be on board for HVO's launch into the new decade. 


\section{SEISMIC INSTRUMENTATION}

The network. The Hawaiian Volcano Observatory has installed and maintains an extensive telemetering seismometer network on the island of Hawaii. In December 1979 the seismometer network consisted of 44 stations spread over an area with a diameter of 125 kilometers on the island of Hawaii (Figs. 1 and 2). Of these 44 stations, two are low-gain multicomponent stations (optical), six are two-component, four are three-component, and thirty-two are vertical only. The coverage is most complete on and around the main center of seismic and volcanic activity, Kilauea Volcano. Other stations in the network are part of a larger net located on other volcanoes of the island of Hawaii. With the exception of HIL, all seismometer signals from the short period network are telemetered to the observatory for recording.

Figure 1 is a map of selected geographic and geologic features, and Figure 2 shows the seismic stations which were operated during the year. Table 1 lists all seismic stations operated by the U.S. Geological Survey in Hawaii during 1979. Listed are station name, three letter code, coordinates in degrees and minutes, elevation in meters, and other data described below.

Instrumentation and recording. Each telemetering station has a voltage controlled oscillator (VCO) for FM multiplex transmission to HVO via either hardwire or VHF radio. These telemetering stations are now all of Type 1, the NCER standard system used in USGS seismic networks (see Table 2 for details). After discrimination, the analog signals from thirty-two stations are recorded on two Develocorders using $16 \mathrm{~mm}$ microfilm. FM signals from the telemetering network are also recorded directly on one inch magnetic tape. Selected larger events are copied onto condensed FM library tapes which are currently archived in Menlo Park.

Develocorder records are scanned on a film viewer with $20 \mathrm{x}$ magnification. Individual events are digitized from FM magnetic tape and timed to .01 second using an Eclipse computer. The arrival times, amplitudes (where readable), and other key data are routinely processed by Eclipse computer, which also produced the tables and epicenter maps in this summary.

In addition to the standard stations, optical seismographs are maintained at Uwekahuna (HVO), Hilo, Maui, and on Oahu (Kipapa station operated by the Pacific Tsunami Warning Center). The less sensitive short period records are used primarily for amplitude measurements for magnitude calculations to supplement readings from the high-gain stations. Optical seismographs listed in Table 1 are of four types. Types numbered three and four are electro-mechanical systems of high and low gain respectively. Hilo and Haleakala are each equipped with two low-gain Wood-Anderson torsion seismographs. Long period Press-Ewing seismographs record in three components in the Uwekahuna vault. The paper (optical) records as well as the $16 \mathrm{~mm}$ develocorder microfilm are archived at HVO.

Seismograph response and calibration. Displacement response curves for the four short-period seismograph types in use are given in Figure 3. Types three and four are electro-mechanical systems recorded on paper records. The Type 1 curve gives the displacement magnification of the standard NCER system from ground motion at the seismometer to the seismic trace as seen on a $20 \mathrm{x}$ Develocorder film viewer. The curves plot the unit response which should be multiplied by a constant but known factor (CAL) to get the response for an individual station. Individual CAL factors for Type 1 seismographs are equal 
to the peak-to-peak amplitude measured in $\mathrm{mm}$ on the $20 \mathrm{x}$ Develocorder viewer of a 10 microvolt $5 \mathrm{~Hz}$ signal introduced to the preamp/VCO in place of the geophone. Calibration is normally done each time a station is visited. CAL factors range from about 1 to 8, averaging about 4. A detailed history of Cal factors and other data is given in F.W. Klein and R.Y. Koyanagi, Hawaiian Volcano Observatory Seismic Network History 1950-79, U.S. Geological Survey Open File Report 80-302, 1980.

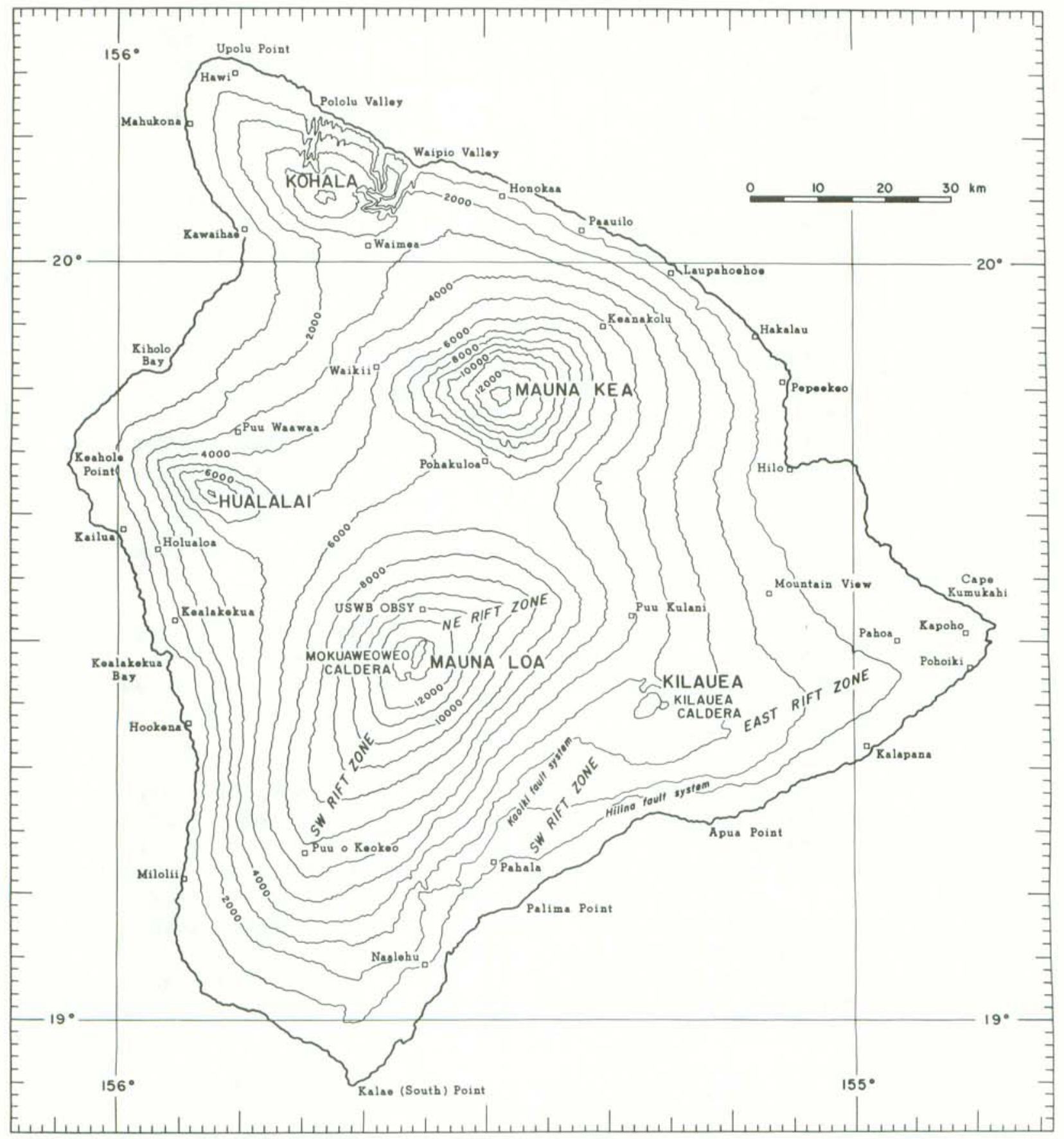

Figure 1. Map of the island of Hawaii showing principal settlements and selected geographic and geologic features. 


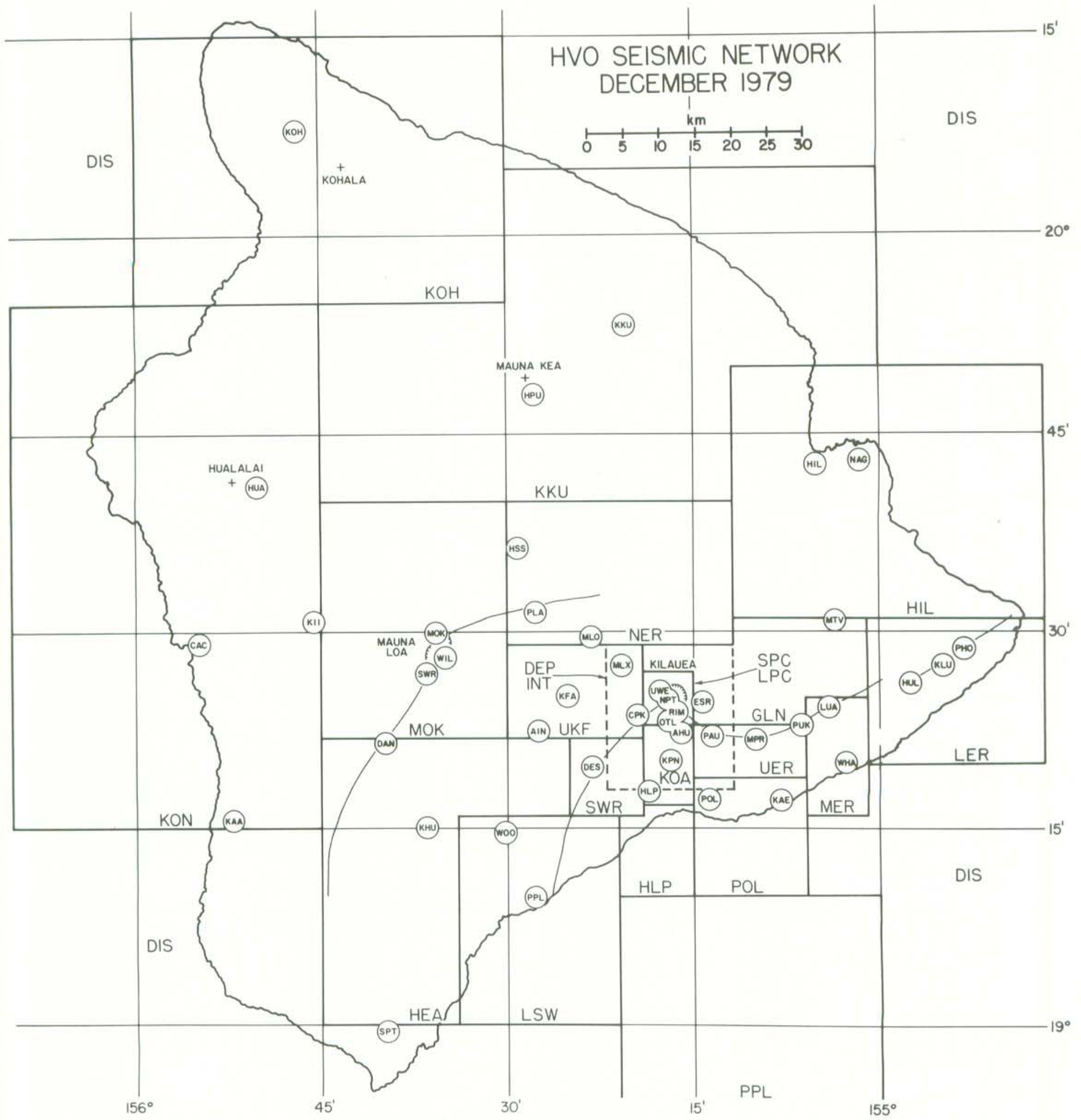

Figure 2. Map of the island of Hawaii showing seismic stations (three letter codes inside circles) and geographic regions (three letter codes inside heavy line boxes). 
Table 1. Seismometer stations in Hawaii operated by the U.S. Geological Survey, 1979.

Telemetered type 1 seismographs.

\begin{tabular}{|c|c|c|c|c|c|c|}
\hline Station Name 1 & Code & LAT-N & LON-W & Elev & $\begin{array}{c}\text { Delay } \\
1\end{array}$ & $\begin{array}{c}\text { Delay } \\
2\end{array}$ \\
\hline AHUA (3) & $\mathrm{AHU}$ & 1922.40 & $155 \quad 15.90$ & 1070 & -.10 & -.13 \\
\hline AINAPO (3) & AIN & 1922.50 & $155 \quad 27.62$ & 1524 & .13 & .17 \\
\hline CAPTAIN COOK & $\mathrm{CAC}$ & 1929.29 & $155 \quad 55.09$ & 323 & .00 & -.16 \\
\hline CONE PEAK (2) & CPK & 1923.70 & $155 \quad 19.70$ & 1030 & -.26 & -.07 \\
\hline DANDELION & DAN & 1921.42 & $155 \quad 40.04$ & 3003 & -.27 & .03 \\
\hline DESERT & DES & 1920.20 & $\begin{array}{ll}155 \quad 23.30\end{array}$ & 815 & -.29 & -.13 \\
\hline ESCAPE ROAD & ESR & 1924.68 & 15514.33 & 1177 & -.17 & -.19 \\
\hline HALE POHAKU & HPU & 2946.85 & $155 \quad 27.50$ & 3396 & .31 & .17 \\
\hline HE IHE IAHULU & HUL & 1925.13 & $155 \quad 58.72$ & 369 & -.17 & -.16 \\
\hline HILINA PALI & HLP & 1917.96 & $155 \quad 18.63$ & 707 & .02 & .07 \\
\hline HUALALAI & HUA & 1941.25 & $155 \quad 50.32$ & 2189 & .67 & .38 \\
\hline HUMUULA（3） & HSS & 1936.31 & $155 \quad 29.13$ & 2445 & .20 & .35 \\
\hline KAAPUNA & KAA & $19 \quad 15.98$ & $155 \quad 52.28$ & 524 & -.12 & -.01 \\
\hline KAENA & $\mathrm{KAE}$ & 1917.35 & $155 \quad 7.95$ & 37 & -.01 & .06 \\
\hline KAHUKU & $\mathrm{KHU}$ & 1914.90 & 15537.10 & 1939 & .03 & -.03 \\
\hline KALALUA & LUA & 1924.55 & 15504.25 & 622 & -.25 & -.30 \\
\hline KALIU & $\mathrm{KLU}$ & 1927.48 & $154 \quad 55.26$ & 271 & -.17 & -.30 \\
\hline KANEKII (3) & KII & 1930.56 & $155 \quad 45.90$ & 1841 & .15 & .37 \\
\hline KAOIKI FAULTS & $\mathrm{KFA}$ & 1925.26 & $155 \quad 25.14$ & 1579 & .13 & .17 \\
\hline KEANAKOLU & $\mathrm{KKU}$ & 1953.39 & $155 \quad 20.58$ & 1863 & .68 & .24 \\
\hline KIPUKA NENE & KPN & 1920.10 & 15517.40 & 924 & -.11 & -.08 \\
\hline KOHALA & $\mathrm{KOH}$ & 207.69 & 15546.77 & 1166 & -.03 & -.17 \\
\hline MAUNA LOA (2) & MLO & 1929.80 & $155 \quad 23.30$ & 2010 & .03 & .08 \\
\hline MAUNA LOA X (2) & MLX & 1927.60 & 15520.70 & 1474 & .06 & .15 \\
\hline MAKAOPUH I & MPR & 1922.07 & $155 \quad 9.85$ & 881 & -.17 & -.20 \\
\hline MOKUAWEOWEO & MOK & 1929.28 & $155 \quad 35.98$ & 4104 & .15 & .16 \\
\hline MOUNTAIN VIEW & MTV & 1930.25 & $155 \quad 3.75$ & 409 & -.02 & .01 \\
\hline NATIONAL GUARD & NAG & $19 \quad 42.12$ & $\begin{array}{ll}155 & 1.72\end{array}$ & 18 & .54 & .30 \\
\hline NORTH PIT & NPT & 1924.90 & $155 \quad 17.00$ & 1115 & -.30 & -.18 \\
\hline OUTLET (2) & OTL & 1923.40 & 15516.80 & 1084 & -.19 & -.18 \\
\hline PAUAHI (2) & PAU & 1922.62 & $\begin{array}{ll}155 \quad 13.10\end{array}$ & 994 & -.21 & -.24 \\
\hline POLIOKEAWE PALI & POL & 1917.02 & $\begin{array}{ll}155 & 13.47\end{array}$ & 169 & -.02 & .03 \\
\hline PUU HOHUAULA & $\mathrm{PHO}$ & 1928.90 & $154 \quad 53.40$ & 215 & -.09 & -.24 \\
\hline PUU KAMOAMOA & PUK & 1923.00 & $155 \quad 6.25$ & 704 & -.25 & -.30 \\
\hline PUU PILI & PPL & 199.50 & $\begin{array}{ll}155 \quad 27.87\end{array}$ & 35 & -.15 & -.15 \\
\hline PUU ULAULA & PLA & 1932.00 & $155 \quad 27.67$ & 2992 & -.03 & .13 \\
\hline RIM (2) & RIM & $19 \quad 23.90$ & $155 \quad 16.60$ & 1128 & -.21 & -.13 \\
\hline SOUTH POINT & SPT & $18 \quad 58.91$ & $155 \quad 39.92$ & 244 & -.17 & -.22 \\
\hline SOUTHWEST RIFT & SWR & 1927.26 & 15536.30 & 4048 & .01 & .04 \\
\hline WAHAULA & WHA & $19 \quad 19.90$ & $155 \quad 2.92$ & 29 & -.10 & -.04 \\
\hline WILKES CAMP & WIL & 1928.15 & $155 \quad 35.02$ & 4037 & .22 & .17 \\
\hline WOOD VALLEY & WOO & $19 \quad 15.08$ & $155 \quad 30.12$ & 909 & -.15 & -.06 \\
\hline
\end{tabular}

$1_{\text {Numbers }}$ in parentheses indicate the total number of seismometers operating if more than a vertical component. 
Table 1. (continued)

Optical Seismographs

\begin{tabular}{|c|c|c|c|c|c|c|}
\hline Station Name & Code & LAT-N & LON-W & Elev & Type & $\mathrm{Ca} 1$ \\
\hline HALEAKALA Z & HAL & 2046.00 & $156 \quad 15.00$ & 2090 & 3 & 0.71 \\
\hline HALEAKALA EW & $\mathrm{HAE}$ & 2046.00 & 15615.00 & 2090 & WA & 1.0 \\
\hline HALEAKALA NS & HAN & 2046.00 & 15615.00 & 2090 & WA & 1.0 \\
\hline HILO Z & HIL & 1943.20 & $155 \quad 5.30$ & 20 & 3 & 1.0 \\
\hline HILO EW & HIE & 1943.20 & 5.30 & 20 & WA & 1.0 \\
\hline HILO NS & HIN & 1943.20 & 5.30 & 20 & WA & 1.0 \\
\hline KIPAPA & KIP & 2125.40 & $158 \quad .90$ & 76 & 3 & 0.56 \\
\hline UWEKAHUNA EW & UWH & 1925.40 & $155 \quad 17.60$ & 1240 & 3 & 0.7 \\
\hline UWEKAHUNA Z & USZ & 1925.40 & $155 \quad 17.60$ & 1240 & 4 & 1.0 \\
\hline UWEKAHUNA EW & USE & 1925.40 & $155 \quad 17.60$ & 1240 & 4 & 1.0 \\
\hline UWEKAHUNA & PEZ & 1925.40 & $155 \quad 17.60$ & 1240 & $\mathrm{PE}$ & \\
\hline UWEKAHUNA & PEE & 1925.40 & $155 \quad 17.60$ & 1240 & $\mathrm{PE}$ & \\
\hline UWEKAHUNA & PEN & 1925.40 & $155 \quad 17.60$ & 1240 & $\mathrm{PE}$ & \\
\hline
\end{tabular}

Table 2. -- Seismic Instrumentation Types

Type 1. Consists of:

a) Geophone - Electrotech EV-17 or Mark Products L4C $1.0 \mathrm{sec}$. period moving magnet vertical component seismometer or horizontal component adjusted for an output of $0.5 \mathrm{volts} / \mathrm{cm} / \mathrm{sec}$. and 0.8 critically damped.

b) Preamp/VCo - Develco Model 6202 voltage controlled oscillator or a USGS/NCER Mode1 J202, J302, or J402. 3 db points for bandpass filter at $0.1 \mathrm{~Hz}$ and $30 \mathrm{~Hz}$. Signals are transmitted on audio FM carrier over cable or FM radio link to HVO.

Type 3. Consists of:

Electrotech EV-17 or observatory-built $0.8 \mathrm{sec}$. period moving coil seismometer with HVO-built solid state seismic preamplifier, galvanometer driver, and $2 \mathrm{~Hz}$ galvanometer. Peak magnification approximately 40,000 at $4 \mathrm{~Hz}$.

Type 4. Consists of:

Sprengnether short period vertical and horizontal seismometers (E-W) with $1.5 \mathrm{sec}$. galvanometers, coupling factor $=0.25,2 \mathrm{X}$ critically damped. Peak magnification approximately $1500 \mathrm{x}$ at $2 \mathrm{~Hz}$.

Type 2 instruments have been discontinued. 


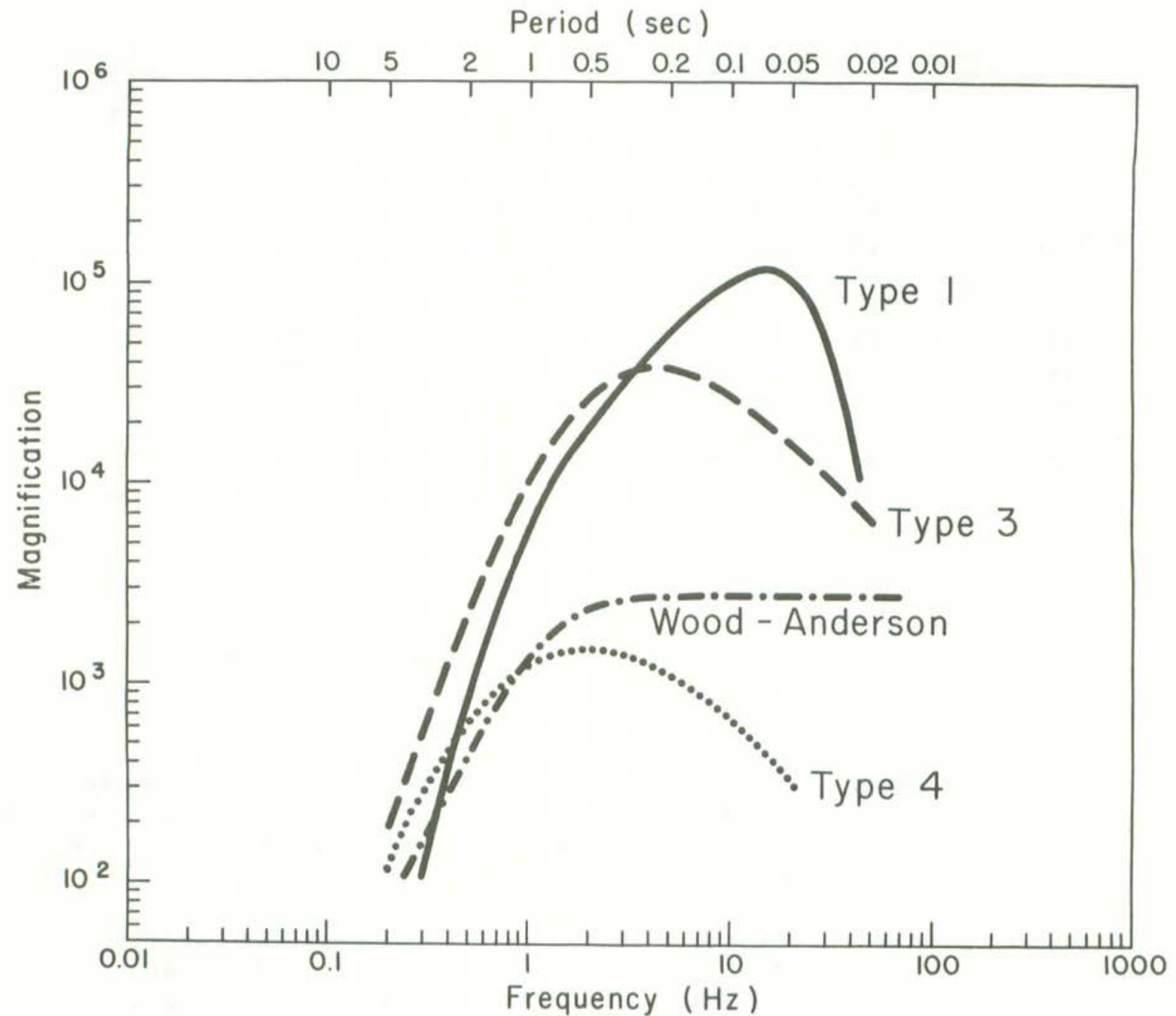

Figure 3. System response curves for the Wood-Anderson torsion seismograph and for the 3 different types of seismometers in use by the Hawaiian Volcano Observatory. Types 3 and 4 are electro-mechanical seismographs recorded optically on photographic paper. Type 1 is the standard NCER seismometer recorded on Develocorder film and magnetic tape. The curve for Type 1 includes response of the geophone, all electronics including telemetry, Develocorder galvanometer, and projection of film by a $20 \mathrm{X}$ viewer. The curves plot the unit response which should be multiplied by a constant but known factor (CAL), to get the response for an individual station. 


\section{SEISMIC DATA PROCESSING}

Develocorder films are scanned for earthquakes, and coda durations are measured for magnitude determination. Events are digitized, timed, and located on the Eclipse computer at HVO. Computer locations are made using the program HYPOINVERSE (Klein, 1978), and problem events are reread and rerun. Magnetic tape copies of all arrival time and output summary data are kept in Menlo Park and HVO.

The crustal model used is specified by velocities at 4 depth points. Velocity at any depth is given by linear interpolation between points and uses a homogeneous half-space below.

\begin{tabular}{cr}
$\begin{array}{c}\text { VELOCITY } \\
(\mathrm{km} / \mathrm{sec})\end{array}$ & DEPTH $(\mathrm{km})$ \\
\cline { 1 - 1 } 1.9 & 0.0 \\
6.5 & 4.6 \\
6.9 & 15.0 \\
8.3 & 16.5
\end{tabular}

Two empirical sets of station delays or corrections were used in the locations, and are given in Table 1. Delay model 1 is used for events on Kilauea and its south flank, and delay model 2 applies to the rest of the island and offshore earthquakes. The delay models are in fact separated by a circle of radius $34 \mathrm{~km}$ centered at $19^{\circ} 22^{\prime} \mathrm{N}$ and $155^{\circ} 10^{\prime} \mathrm{W}$.

Magnitudes for most events were computed using both recorded amplitudes on low gain or Wood-Anderson stations and signal or coda duration on short-period vertical stations. Amplitudes read from other than Wood-Anderson instruments are corrected to an equivalent Wood-Anderson amplitude using the curves of Figure 3 and CAL factors. Amplitude magnitudes larger than 2.5 are generally based on the Wood-Anderson instruments in Hilo or Type 4 seismographs at Uwekahuna. Smaller events may occasionally include amplitude readings from stations AHU, KAA, OTL, PPL, KHU, PHO, or WIL.

Duration magnitudes are determined from the length of signal in seconds read from the Develocorder viewer. This time, also called the "F-P time" is measured from the first $P$ arrival to the point where the earthquake signal has decayed nearly to the noise level. A plot of $10 \mathrm{~g}$ (F-P time) versus local (amplitude) magnitude appears in Figure 4. The bilinear relation shown in the figure is an appropriate fit to the data sample and is used to compute all duration magnitudes. Duration times are only read from Type 1 seismographs. Because duration magnitudes are relatively insensitive to station response and can be determined using the high-gain short-period stations, it is felt that duration magnitudes are more accurate and complete at the lower magnitudes (below 2). 


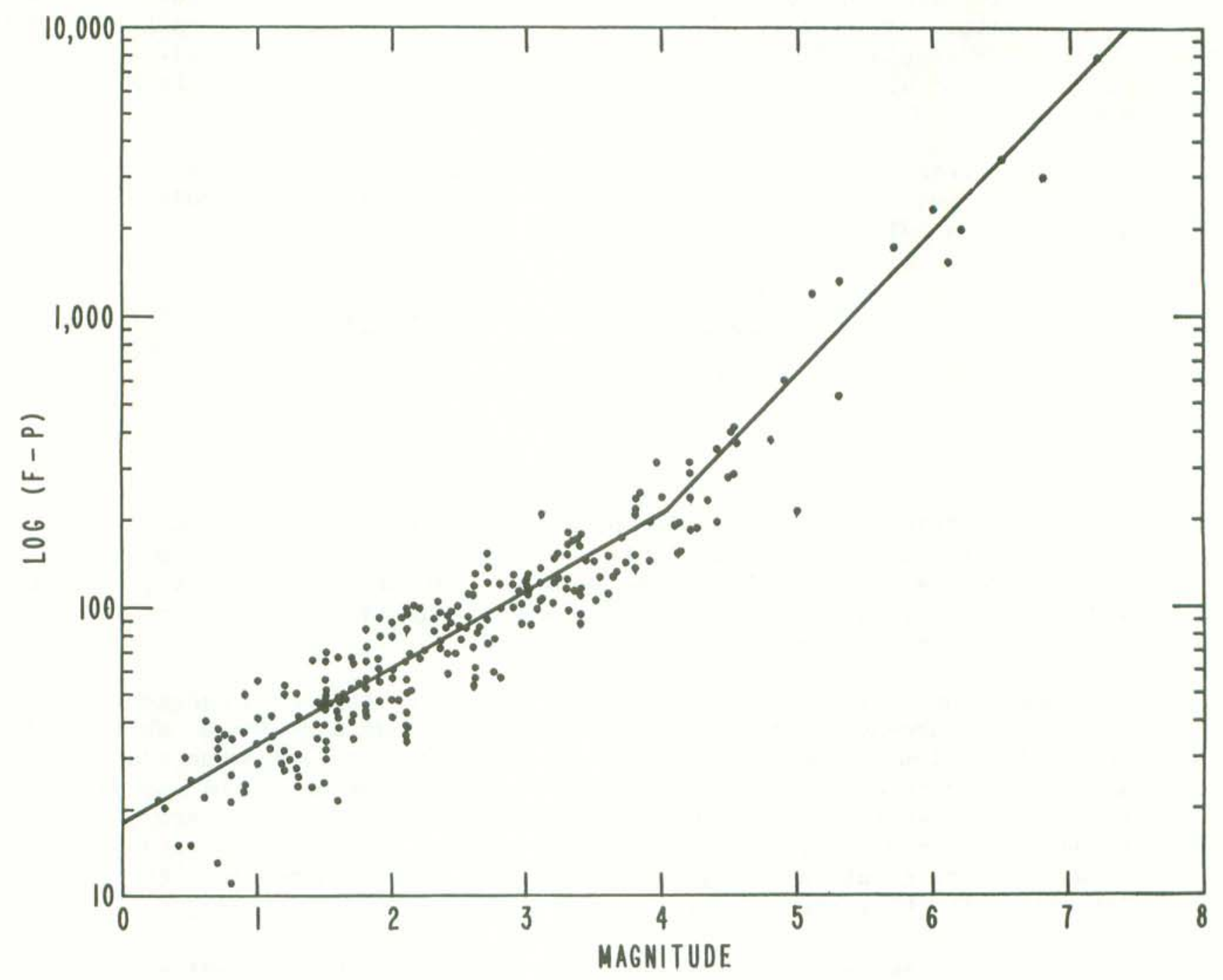

Figure 4. Relationship between signal duration (F-P time) and local magnitude for a large number of earthquakes which occurred during 1975 and 1976. Local magnitude is determined from amplitudes read on Wood-Anderson and other calibrated seismographs. The dual linear relationship between magnitude and $\log (\mathrm{F}-\mathrm{P})$ appears to hold over a magnitude range of 7 units. The equations used in magnitude determination are:

duration $<210 \mathrm{sec} \quad M=-5.2+3.89 \log (\mathrm{F}-\mathrm{P})+.013 \mathrm{z}+.0037 \mathrm{D}$ duration $>210 \mathrm{sec} \quad M=-.905+2.026 \log (\mathrm{F}-\mathrm{P})+.013 \mathrm{z}+.0037 \mathrm{D}$ where $z$ and $D$ are the depth and epicentral distance in $\mathrm{km}$. 


\section{SEISMIC SUMMARY}

The emphasis in both station coverage and detailed data analysis is on the highly active south flank of the island of Hawaii. Hundreds of earthquakes too small to locate are counted daily, and the set of located earthquakes in the Kilauea region is nearly complete above magnitude about 2.0. Many smaller events are located also. Substantial effort is made to locate earthquakes elsewhere on the island and within about $150 \mathrm{~km}$ of the island. Such coverage cannot be as complete as on the south flank, but nearly all events above magnitude 3.0 to 3.5 are located.

Data presented in the seismic summary is in four parts. Table 3 gives duration of harmonic tremor and numbers of earthquakes (most too small to locate) from several source regions around Kilauea. The source region is determined visually from signal character and pattern of arrival times at key stations. Maps showing computer located epicenters of all depths are given in Figures 5, 6, 7, 8 and 9. The epicenter maps are on different scales, and show both all located earthquakes and large events only.

The list of computer locations constitutes the bulk of this summary, and is given in Table 5. Each earthquake in the list is assigned a three-letter code based on its location and in some cases on its depth. Figure 2 is a map of the regions used to assign the location codes. The latitude and longitude limits of rectangular regions are listed in Table 4. When the listed coordinates imply an overlap, precedence is given according to Figure 2. Table 6 relists the events in Table 5 for which either duration or amplitude magnitude is 3.0 or larger. It is felt that this list is a more objective measure of large earthquakes than a list of felt earthquakes. 
Table 3. Number of earthquakes and minutes of tremor recorded on seismographs around Kilauea.

Earthquake categories are:

1) Kilauea summit, short period caldera: shallow earthquakes beneath the caldera.

2) Kilauea summit, long period caldera: earthquakes characterized by low frequency signatures, of ten originating $5-10 \mathrm{~km}$ below the caldera.

3) Kilauea summit $30 \mathrm{~km}$ : from about $30 \mathrm{~km}$ depth.

4) Kaoiki and southwest rift: southwest rift of Kilauea, western parts of the Koae faults and adjacent Kaoiki fault system.

5) Upper east rift zone of Kilauea including the eastern parts of the Koae faults.

6) Lower east rift zone of Kilauea.

7) Offshore PPL: earthquakes from offshore areas south of the Puu Pili station, including Loihị seamount.

8) Mauna Loa long period: low frequency events near Mauna Loa summit.

9) Mauna Loa short period: shallow earthquakes in the Mauna Loa caldera region.

Tremor is separated into four categories: shallow, intermediate and deep Kilauea, and Mauna Loa. Depth is inferred on the basis of relative amplitudes on seismographs. 
Table 3

KILAUEA FLANK

MAIUNA LOA TREMOR (MINUTES)

I ISHORT LONG I KAO. UP. LOW. UFF- I KILAUEA MAIINAI

I DATE I PER. PER. 30 I \& SW EAST EAST SHOREILONG SHORTT INT. LOA I

I 1979 I CALDERA KM I RIFT RIFT RIFT PPL IPER. DFR. T SHAL. DEEP I

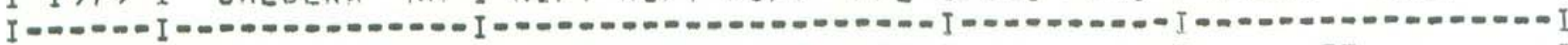

IJAN 1 I $221 \quad 12$ C I $27 \quad 159 \quad 39$

$\begin{array}{lllllllll}I & 2 & I & 225 & 9 & I & 26 & 153 & 27\end{array}$

I 3 I 125

I 4 I 200

$\begin{array}{lllll}11 & 1 & 18 & 85 & 28\end{array}$

5 I 312

$\begin{array}{lllll}21 & I & 29 & 118 & 40\end{array}$

I

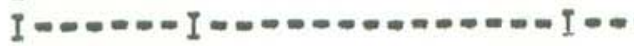

$13 \quad 64 \quad 34$

$\begin{array}{llll}I & 1 & 1 & 1\end{array}$

I

$\begin{array}{rrrrrrrrrrrrrrr}I & 6 & I & 246 & 1 & 3 & I & 27 & 61 & 28 & I & & T & 2 & \text { I } \\ I & 7 & I & 200 & 4 & I & 54 & 112 & 37 & I & 1 & T & I \\ I & 8 & I & 210 & 8 & I & 32 & 139 & 30 & I & 1 & T & & I \\ I & 9 & I & 196 & 6 & I & 45 & 124 & 25 & I & & T & 5 & I \\ I & 10 & I & 243 & 1 & I & 57 & 209 & 36 & I & 1 & 1 & T & & I\end{array}$

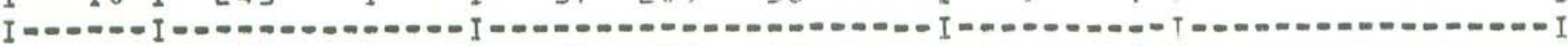

$\begin{array}{llllllllllllll}I & 11 & I & 177 & 4 & 1 & I & 39 & 95 & 20 & I & 1 & 1 & \text { I }\end{array}$

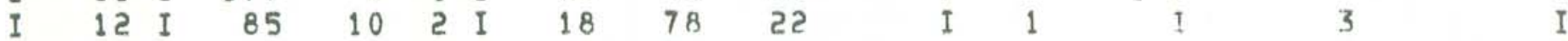

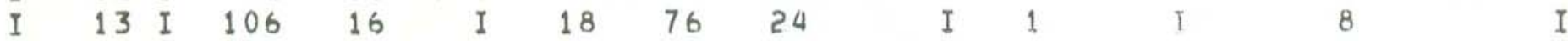

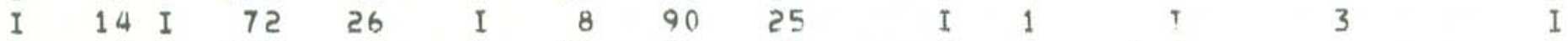

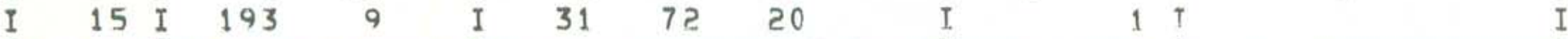

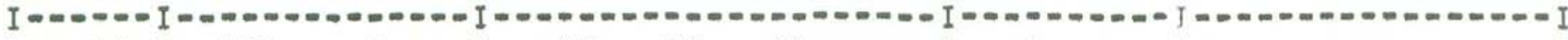

\begin{tabular}{|c|c|c|c|c|c|c|c|c|c|c|c|c|c|c|c|c|c|}
\hline I & 16 & I & 196 & 1 & & I & 45 & 90 & 27 & & I & 1 & & $T$ & & & \\
\hline I & 17 & I & 202 & 1 & & I & 32 & 124 & 27 & & I & & 1 & I & & & \\
\hline I & 18 & I & 143 & & & I & 33 & 101 & 30 & & I & 3 & & $T$ & & & \\
\hline I & 19 & I & 93 & & 2 & I & 23 & 70 & 12 & & I & $?$ & 1 & T & & & \\
\hline I & 20 & I & 149 & 2 & 1 & I & 47 & 86 & 14 & & I & & 1 & Y & & 5 & \\
\hline \multicolumn{18}{|c|}{$I=-\infty=-I=-\infty-\infty=\infty$} \\
\hline I & 21 & I & 182 & & 3 & I & 13 & 61 & 10 & 2 & I & & & I & & 2 & \\
\hline & 22 & I & 261 & 10 & & I & 22 & 94 & 4 & & I & 1 & & J. & & & \\
\hline & 23 & I & 308 & 5 & & I & 27 & 97 & 9 & & I & & & $T$ & 3 & & \\
\hline & 24 & I & 402 & 26 & & I & 57 & 137 & 20 & & I & 1 & & T & 15 & & \\
\hline & 25 & I & 384 & 10 & & I & 30 & 98 & 6 & & I & & ? & $T$ & 3 & 5 & \\
\hline \multicolumn{18}{|c|}{ 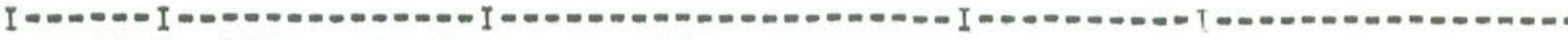 } \\
\hline & 26 & I & 383 & 10 & 3 & I & 26 & 102 & 18 & 1 & I & & & $T$ & 10 & & \\
\hline & 27 & I & 330 & 4 & 3 & I & 31 & 92 & B & 2 & I & & & 1 & 3 & & \\
\hline & 28 & I & 354 & 32 & & I & 36 & 142 & 16 & & I & & 1 & T & & & \\
\hline & 29 & I & 261 & 15 & & I & 52 & 95 & 27 & & I & 1 & 1 & 丁 & 7 & & \\
\hline 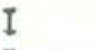 & 30 & I & 150 & 152 & & I & 39 & 71 & 4 & & I & & & I & & 2 & \\
\hline \multirow{2}{*}{\multicolumn{18}{|c|}{ 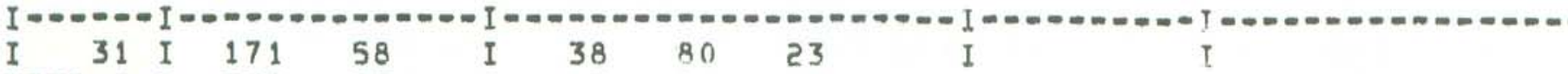 }} \\
\hline & & & & & & & & & & & & & & & & & \\
\hline IFEB & 31 & I & 220 & 23 & & I & 13 & 84 & 6 & & $I$ & & 1 & I & 3 & 20 & \\
\hline & 2 & I & 237 & 40 & & I & 37 & 153 & 30 & & I & & & T & 23 & 34 & \\
\hline & 3 & I & 222 & 25 & & I & 29 & 114 & 19 & & I & & & I & 5 & & \\
\hline 1 & 4 & I & 240 & 35 & & I & 25 & 84 & 6 & & I & & & T & 10 & & \\
\hline \multicolumn{18}{|c|}{ 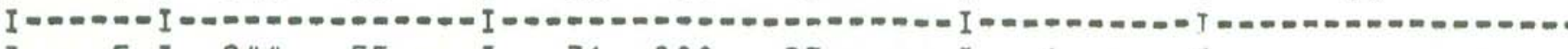 } \\
\hline & 5 & I & 944 & 55 & & I & 31 & 800 & 23 & & I & 1 & & I & & & \\
\hline & 6 & I & 503 & 44 & & I & 35 & 115 & 25 & & $I$ & & 1 & $T$ & 2. & & \\
\hline & 7 & I & 349 & 91 & & I & 52 & 118 & 46 & & I & & 1 & $T$ & 31 & & \\
\hline & 8 & I & 226 & 8 & & I & 30 & 114 & 37 & & I & & & $T$ & & & \\
\hline & 9 & I & 201 & 1 & & I & 43 & 96 & 10 & & I & & 1 & I & & & ᄅ \\
\hline \multicolumn{18}{|c|}{ 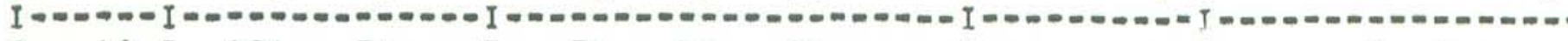 } \\
\hline & 10 & I & 191 & 34 & & I & 36 & 78 & 2.5 & & I & & & $\mathrm{T}$ & 2 & 46 & \\
\hline & 11 & I & 231 & 52 & & I & 42 & 88 & 40 & & I & 1 & & $T$ & 4 & & \\
\hline & 12 & I & 162 & 45 & & I & 29 & 86 & 23 & & I & & & I & & & \\
\hline & 13 & I & 298 & 39 & 1 & I & 39 & 128 & 33 & & $I$ & & $?$ & T & & & \\
\hline & 14 & I & 410 & 76 & & I & 47 & 82 & 33 & & I & & & I & & & \\
\hline
\end{tabular}


I ISHORT LONG I KAO. UP. LOW. OFF- T

I DATE I PER. PER. 30 I \& SW EAST EAST SHOREILOM SHORT

I 1979 I CALDERA KM I RIFT RIFT RIFT

KILAUIFA MAUNAI

INT. LOA I

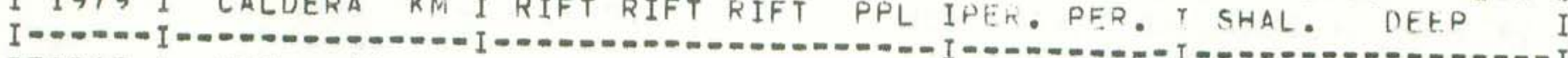

IFEB15 I 420 62 I $55 \quad 130 \quad 19 \quad$ I 19

$\begin{array}{lllllllllllll}\text { I } & 16 & I & 361 & 67 & I & 22 & 83 & 13 & I & 1 & Y & 4\end{array}$

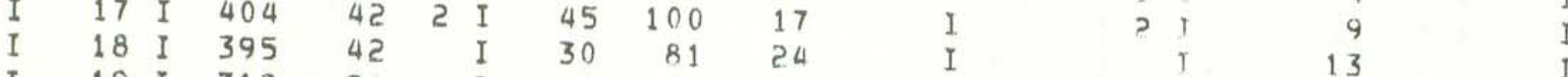

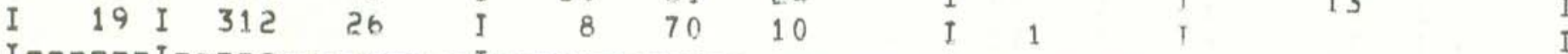

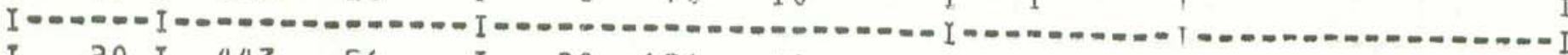

$\begin{array}{lllllllllllll}I & 20 & I & 443 & 56 & I & 28 & 121 & 10 & I & 1 & \text { I }\end{array}$

I 22 I $439 \quad 14 \quad$ I $33 \quad 112 \quad 7$

I 23 I $274 \quad 10$ I $40 \quad 109 \quad 7$

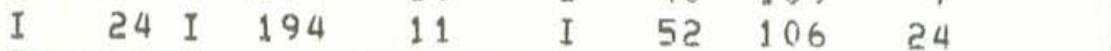

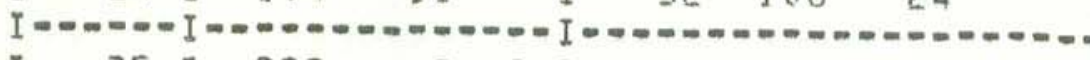

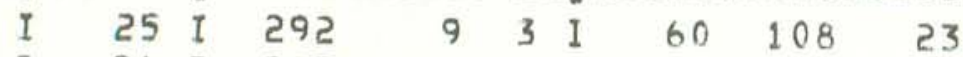

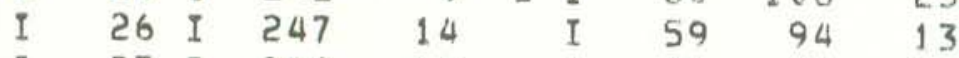

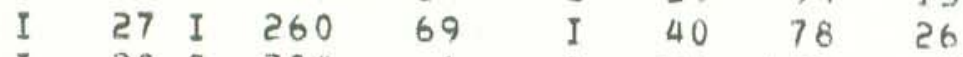

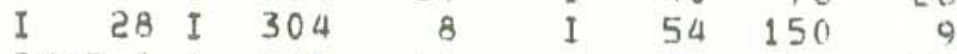

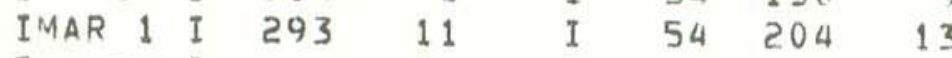

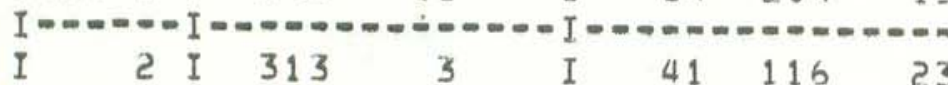

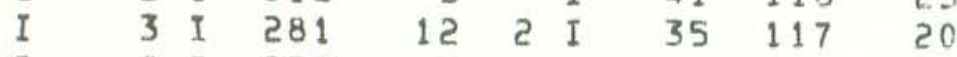

I 4 I $253 \quad 5 \quad 1$ I $64 \quad 140 \quad 10$

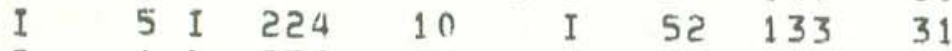

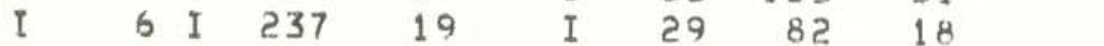

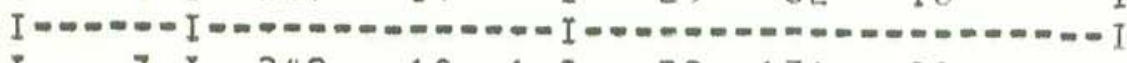

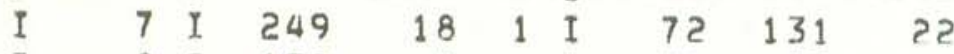

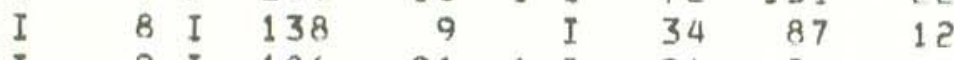

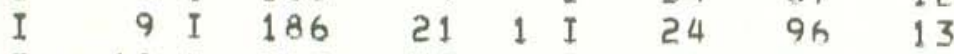

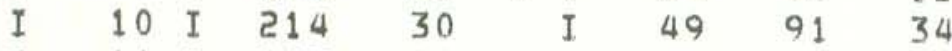

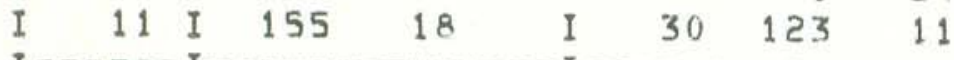

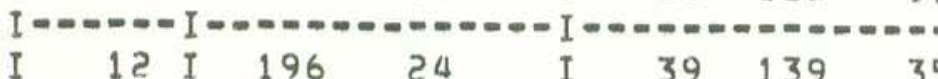

$\begin{array}{lllllllll}13 & 1 & 219 & 39 & 1 & I & 23 & 68 & 20\end{array}$

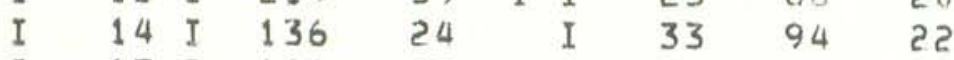

I $\quad \begin{array}{llllllll}15 & 1 & 101 & 15 & \text { I } & 35 & 116 & 20\end{array}$

I 16 I $141 \quad 30$ I $33 \quad 122 \quad 11$

I $\quad \begin{array}{llllllll}17 & 1 & 149 & 40 & \text { I } & 33 & 142 & 19\end{array}$

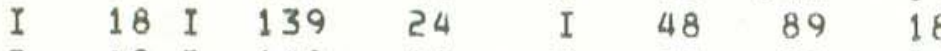

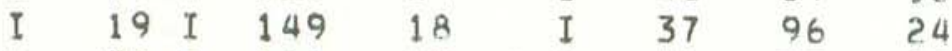

$\begin{array}{llllllllll}\text { I } & 20 & \text { I } & 168 & 17 & 1 & \text { I } & 34 & 90 & 27\end{array}$

I 21 I $105 \quad 16 \quad$ I $38 \quad 98 \quad 15$

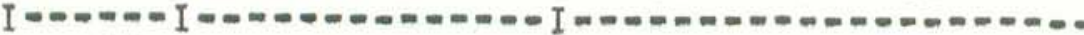

$\begin{array}{lllllllll}I & 22 & I & 126 & 53 & I & 27 & 115 & 25\end{array}$

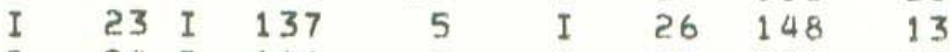

I $\begin{array}{llllllll}24 & \text { I } & 146 & 5 & \text { I } & 37 & 190 & 10\end{array}$

$\begin{array}{llllllll}I & 25 I & 132 & 15 & \text { I } & 29 & 148 & 26\end{array}$

$\begin{array}{llllllllll}I & 26 & I & 134 & 2 & I & 34 & 244 & 17\end{array}$

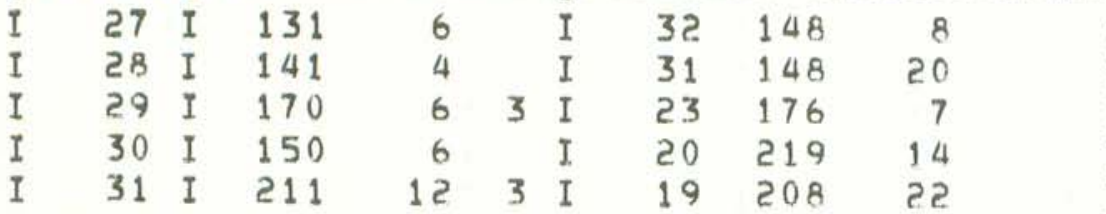

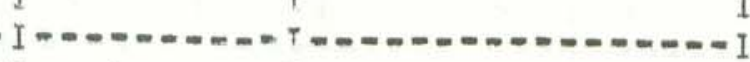

3

I

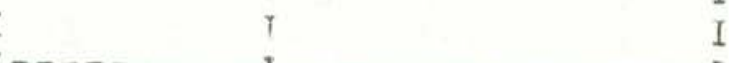


I ISHORT LONG I KAO, UP. LOW. OFF- I

I DATE I PER. PER. 30 I \& SW EAST EAST SHOREILONG SHORTI

KILAUEA MAUNAI

I 1979 I CALDERA KM I RIFT RIFT RIFT PPL IPER. PFR, I SHAL. DEEP I

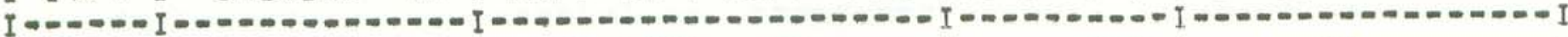

\begin{tabular}{|c|c|c|c|c|c|c|c|c|c|c|c|c|c|c|}
\hline I APR & I & 210 & 7 & 3 & I & 22 & 240 & 17 & I & & 159 & 1 & 29 & \\
\hline I & I & 166 & 19 & & I & 21 & 171 & 20 & I & 1 & 105 & $T$ & \multirow{4}{*}{\multicolumn{2}{|c|}{$\begin{array}{r}7 \\
22\end{array}$}} \\
\hline 3 & 3 & 235 & 12 & 2 & I & 32 & 192 & 6 & I & & 19 & $T$ & & \\
\hline 4 & 4 & 231 & 6 & & I & 25 & 207 & 26 & I & & 27 & $\tau$ & & \\
\hline 5 & 5 & 250 & 12 & & I & 32 & 183 & 23 & I & & 69 & r & & \\
\hline 6 & 6 & 263 & 12 & 1 & I & 20 & 191 & 11 & I & 4 & 70 & T & \multirow{4}{*}{\multicolumn{2}{|c|}{20}} \\
\hline 7 & 7 & 231 & 23 & & I & 9 & 2.55 & 7 & I & 3 & 19 & I & & \\
\hline 8 & 8 & 213 & 4 & & I & 27 & 206 & 14 & I & & 35 & Y & & \\
\hline 9 & 9 & 214 & 10 & & I & 37 & 146 & 34 & I & & 27 & $T$ & & \\
\hline 10 & I & 229 & 2 & & I & 31 & 155 & 2 & I & & $1 n$ & $T$ & \multicolumn{2}{|r|}{3} \\
\hline$=-0$ & $=-I$ & $=--0-0$ & 10 & $\infty$ & $I-$ & $=-$ & 154 & $=-1$ & I. & $=-$ & $=-5$ & $T^{T}$ & & \\
\hline 12 & 1 & 219 & 5 & 1 & $I$ & 10 & 151 & 19 & I & & 8 & T & \multirow{3}{*}{\multicolumn{2}{|c|}{7}} \\
\hline 13 & 31 & 309 & 5 & 2 & I & 22 & 164 & & I & & 4 & $T$ & & \\
\hline 14 & 4 I & 310 & 2 & 1 & I & 23 & 134 & 3 & I & & & T & & \\
\hline 15 & 51 & 317 & 9 & & I & 20 & 177 & 12 & I & & 11 & $T$ & & \\
\hline
\end{tabular}

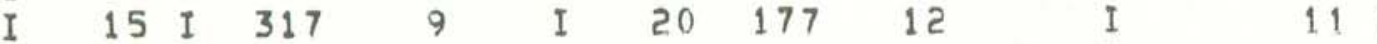

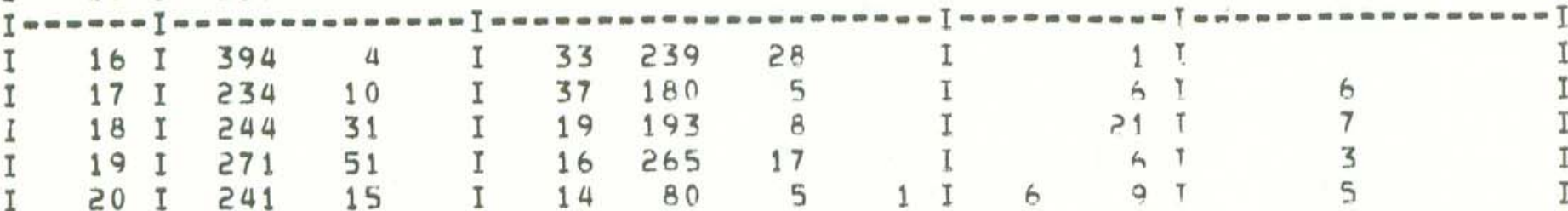

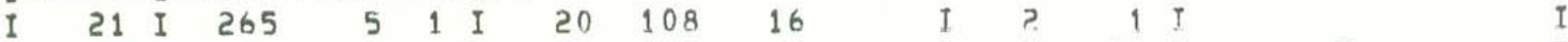

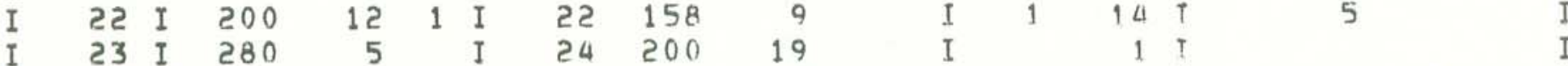

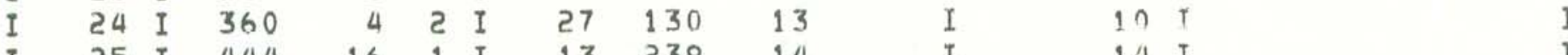

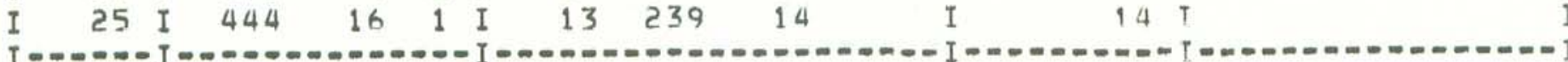

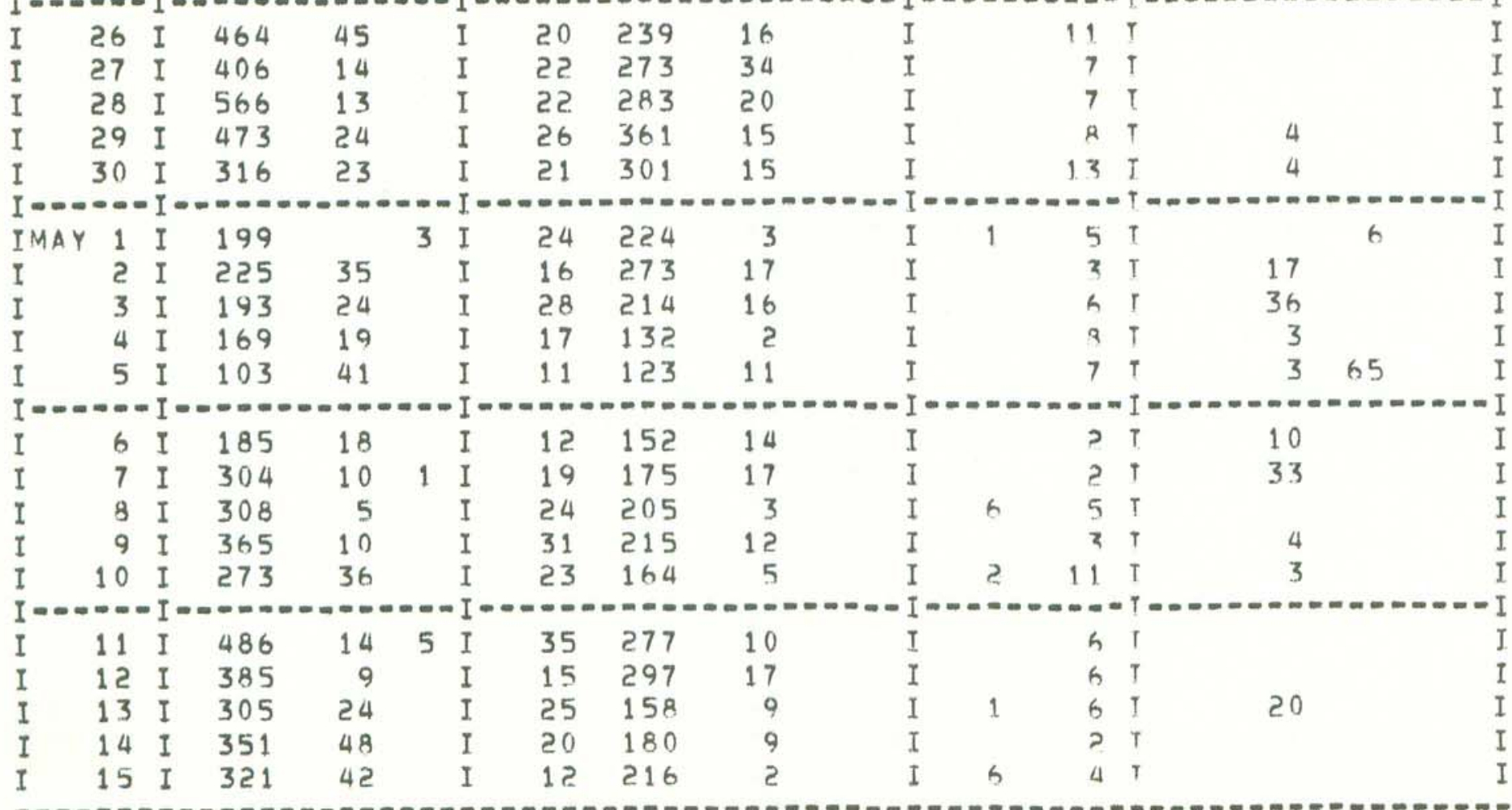


I ISHORT LONG
I DATE I PER. PER. 30 I \& SW UPST LOW. OFF- I EAST SHOREILONG SHORTT TILAUEA MAUNAI

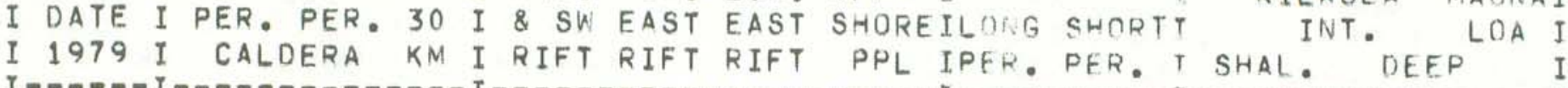

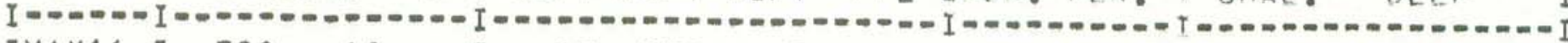

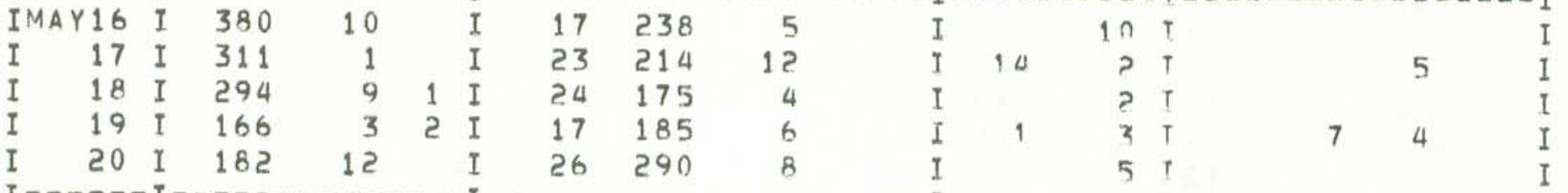

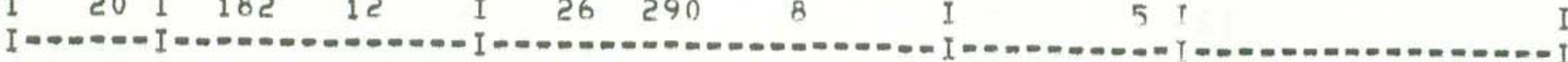

$\begin{array}{llllllllllll}I & 21 & I & 158 & 19 & \text { I } & 15 & 220 & 2 & I & 3 & \text { I }\end{array}$

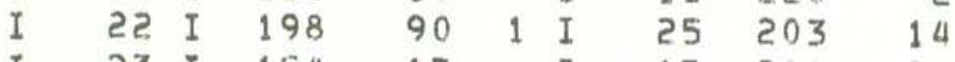

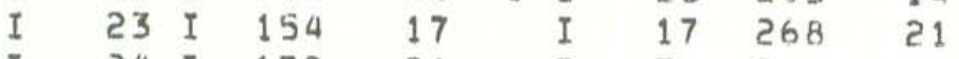

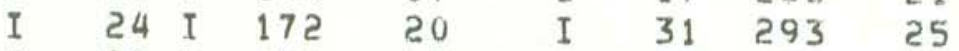

I $\quad 25$ I $181 \quad 13 \quad$ I $39 \quad 238 \quad 16$

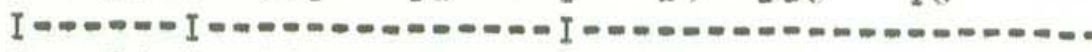

$\begin{array}{llllllllll}I & 26 & I & 198 & 44 & 1 & I & 21 & 263 & 50\end{array}$

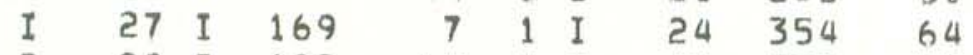

$\begin{array}{lllllllllll}I & 28 & I & 183 & 15 & 1 & I & 46 & 357 & 50\end{array}$

I 29 I $149 \quad 2 \quad$ I $82166 \quad 2$

$\begin{array}{llllllllll}I & 30 & I & 74 & 10 & I & 17 & 188 & 8\end{array}$

I 31 I 101 I

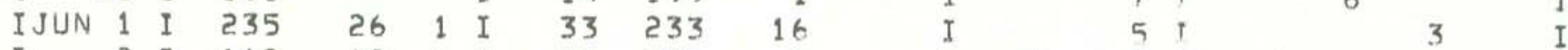

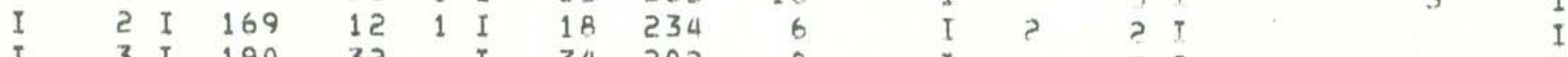

I $\quad \begin{array}{lllllllllll} & 3 & I & 190 & 32 & I & 34 & 202 & 8 & I & 4\end{array}$

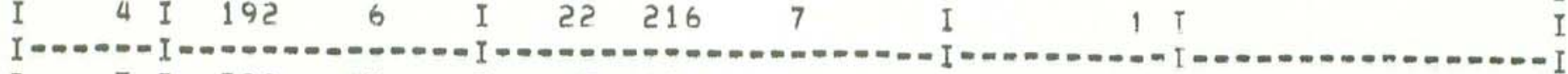

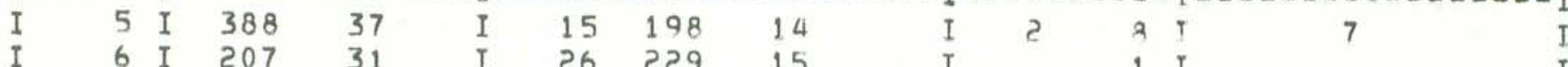

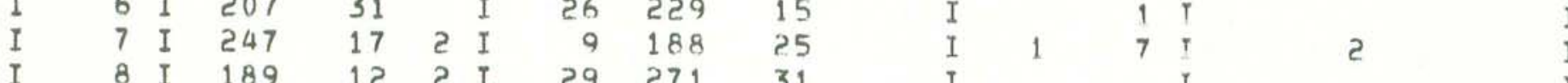

$\begin{array}{lrrrrrrrrrrrl}I & 8 & I & 189 & 12 & 2 & I & 29 & 271 & 31 & I & I & I\end{array}$

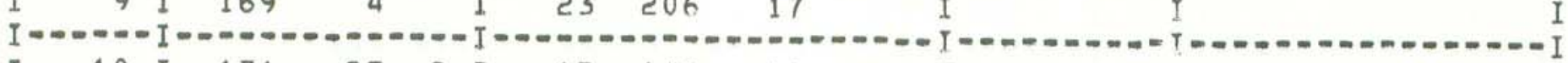

I $\quad \begin{array}{llllllllllll} & 10 & I & 131 & 25 & 2 & \mathrm{I} & 17 & 162 & 16 & \mathrm{I} & 3\end{array}$

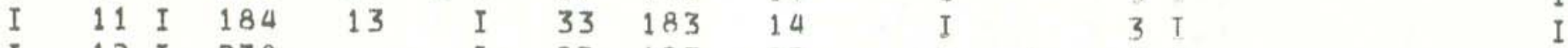

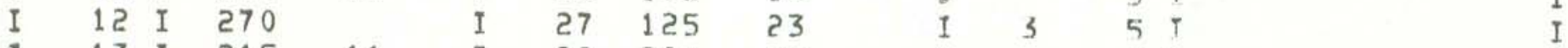

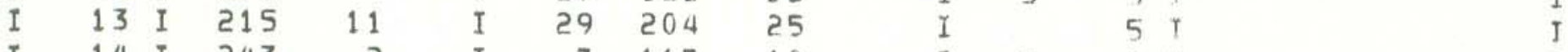

$\begin{array}{llllllllllll}I & 14 & I & 243 & 2 & I & 7 & 167 & 10 & I & 1 & 4\end{array}$

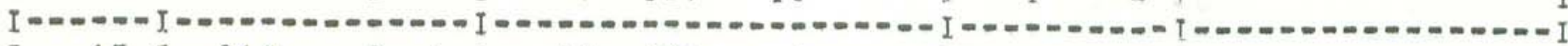

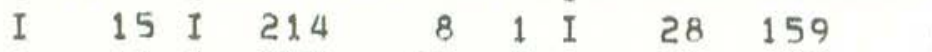

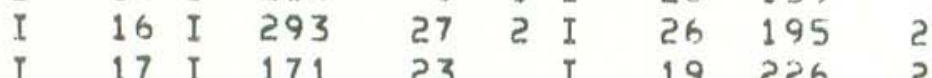

$\begin{array}{lllllllll}\text { I } & 18 & \text { I } & 172 & 37 & \text { I } & 17 & 208 & 26\end{array}$

I 19 I 201 9 I $16 \quad 163$ ?

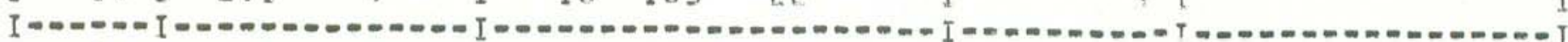

I 20 I $165 \quad 5 \quad 2$ I $30 \quad 219 \quad 14$

$\begin{array}{lllllllll}I & 21 & \text { I } & 342 & 8 & \text { I } & 13 & 184 & 10\end{array}$

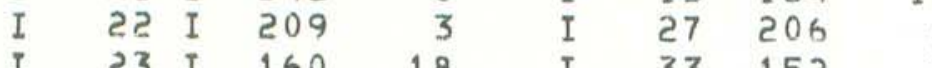

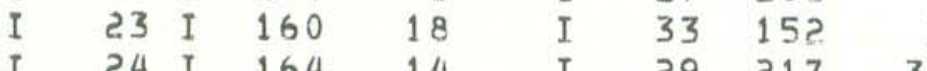

I 24 I $164 \quad 14 \quad$ I $29217 \quad 33$

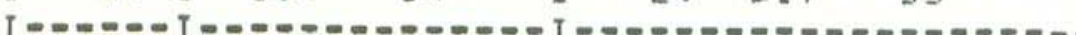

I 25 I $224 \quad 41 \quad 1$ I 33 234 $24 \quad$ I $\quad$ ? I

$\begin{array}{lllllllll}I & 26 & I & 340 & 13 & \text { I } & 21 & 175 & 1\end{array}$

I $\quad \begin{array}{llllllll}27 & \text { I } & 191 & 87 & \text { I } & 16 & 176 & 20\end{array}$

I $\begin{array}{llllllll}28 & \text { I } & 310 & 42 & \text { I } & 39 & 208 & 19\end{array}$

I $29 \begin{array}{lllllll}1 & 302 & 7 & \text { I } & 45 & 156 & 13\end{array}$

$111 / 4$

16

? 104 
I ISHORT LONG I KAO. UP. LOW. OFF - I

I DATE I PER. PER. 30 I \& SW EAST FAST SHOREILODG I

I 1979 I CALDERA KM I RIFT RIFT RIFT PPL IPFR. DFH. I SHAL INT DEFPOA I I =-0.-0-I I -

I.JUN30 I 255

IJUL 1 I 117$$
\text { I }
$$

I

? I 102

3 I 149

$\begin{array}{rrrrrrr}2 & 1 & \text { I } & 33 & 118 & 6 & 1 \\ 9 & 1 & \text { I } & 25 & 175 & 14 & I \\ 13 & 1 & \text { I } & 23 & 145 & 14 & I \\ 3 & & \text { I } & 27 & 237 & 14 & I \\ & & \text { I } & -52 & 18 & I\end{array}$

I
I
I
I
I

$\begin{array}{ll}7 & T \\ 4 & T\end{array}$

$\begin{array}{ll}4 & T \\ 1 & T\end{array}$

4 I 286

5 I

6 I 209

7 I 310

50 I

I $33 \quad 169$

3. I 206

33

11 I

$2.5 \quad 175$

$41 \quad 181$

12

$43 \quad 214$

9 I $35 \quad 173$

4

20

35

13

26

(6) I

10

6

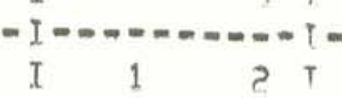

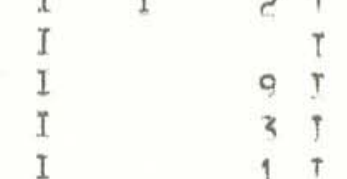

$\begin{array}{ll} & T \\ 9 & r \\ 3 & 1 \\ 1 & T\end{array}$

10

6

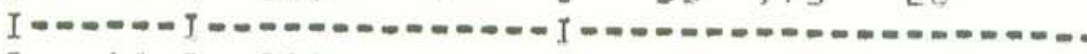

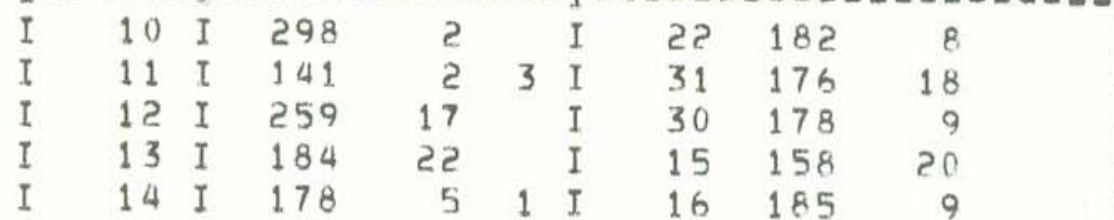

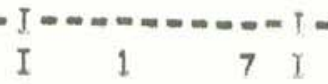

39

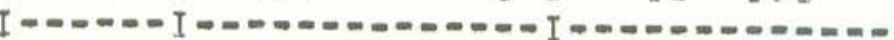

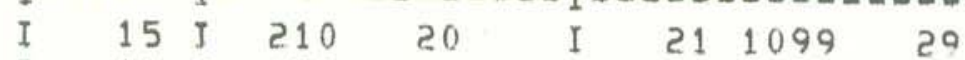

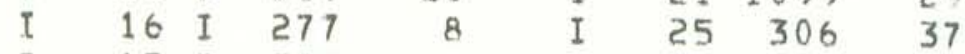

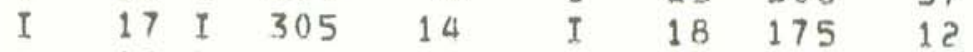

$\begin{array}{lllllllll}I & 18 & I & 176 & 17 & I & 18 & 215 & 24\end{array}$

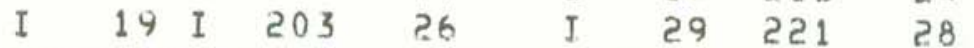

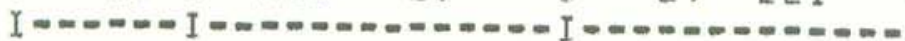

I 20 I $157 \quad 20$

21 I 17

20 I $40 \quad 165$

22 I 111

23 I 128

10 I 28

177

11

$18 \quad 108$

I

24 I 313

$\begin{array}{lll}1 & 1 \\ 8 & 1\end{array}$

$11 \quad 110$

$1=-$

I

I

25 I 329

15

$32 \quad 145$

17

8

I 5 I 1 I

I 1

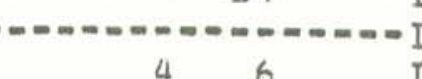

$\begin{array}{lllll}7 & \text { I } & 48 & 176 & 24\end{array}$

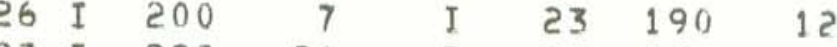

27 I 298

28 I. 289

$27 \quad 193$

$\begin{array}{lll}33 & 172 & 13\end{array}$

29 I 179

$51-1$

$\begin{array}{lllll}3 & \text { I } & 32 & 138 & 6\end{array}$

13

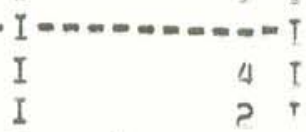

I 2

1

1

$-1$

I

$+1$

19

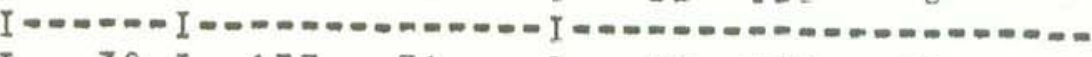

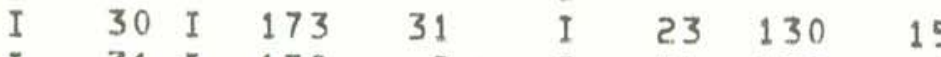

I 31 I 178

IAUG 1 I 448

I $\quad$ I 560

$\begin{array}{rl}5 & I \\ 11 & I\end{array}$

$21 \quad 155$

15
4

$28 \quad 211 \quad 21$

I $\quad 3$ I 268

22. I $29 \quad 148$

21

$\begin{array}{lll}19 & 198 \quad 16\end{array}$

I

I

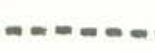

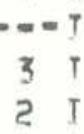

314

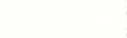

$$
4 \text { I }
$$

38 ?

5

244

17

$20 \quad 167 \quad 16$

6 I 216

7

10

10

27

12

$\begin{array}{ll}35 & 157\end{array}$

$29 \quad 193$

20

8 I 121

I

$17 \quad 133$

9
13

-.....-

4

5

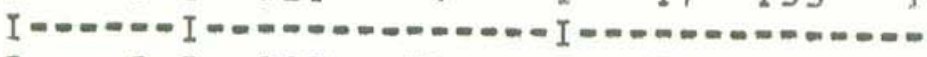

I $\quad 9$ I $205 \quad 31 \quad$ I $33 \quad 135$

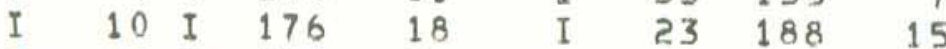

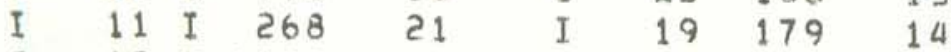

I

12 I 1101

7 I

23

679

13 I $559 \quad 14$ I $14 \quad 174 \quad 1$ ?

18

-1
1

5 I

\section{I}

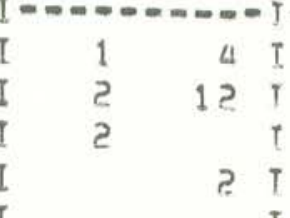

3

5010

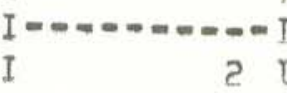

$2 \mathrm{I}$

I

$3 \mathrm{~T}$

2

9

30

2 I

$-1$

56 I

$\begin{array}{ll}2 & I \\ 3 & 1\end{array}$

$5 \quad 1 \frac{T}{T}$

67

I

12 I

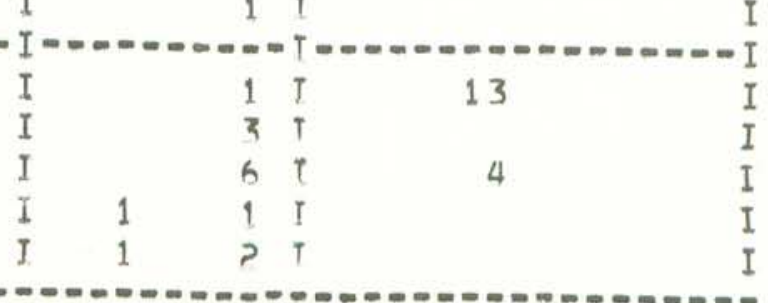




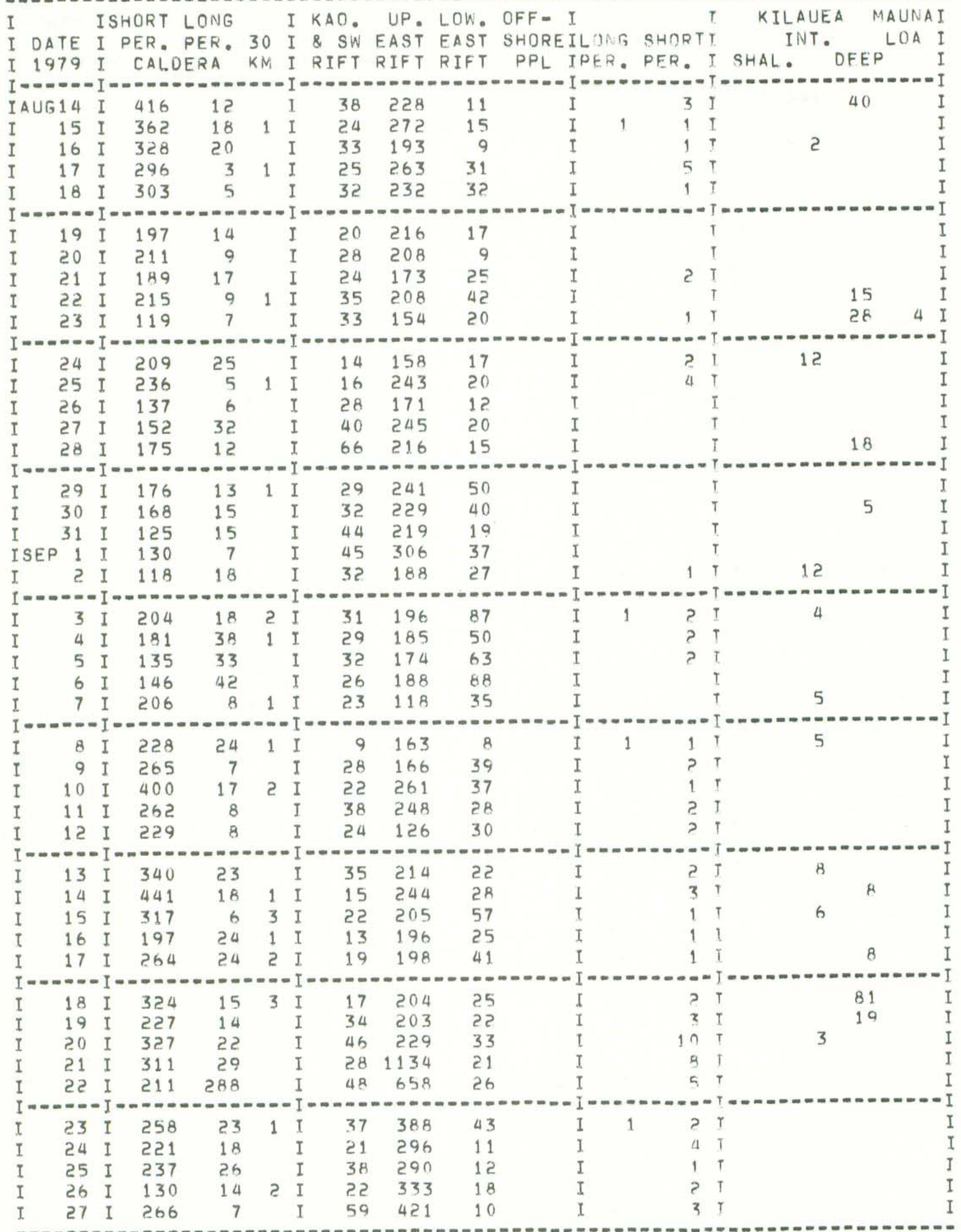




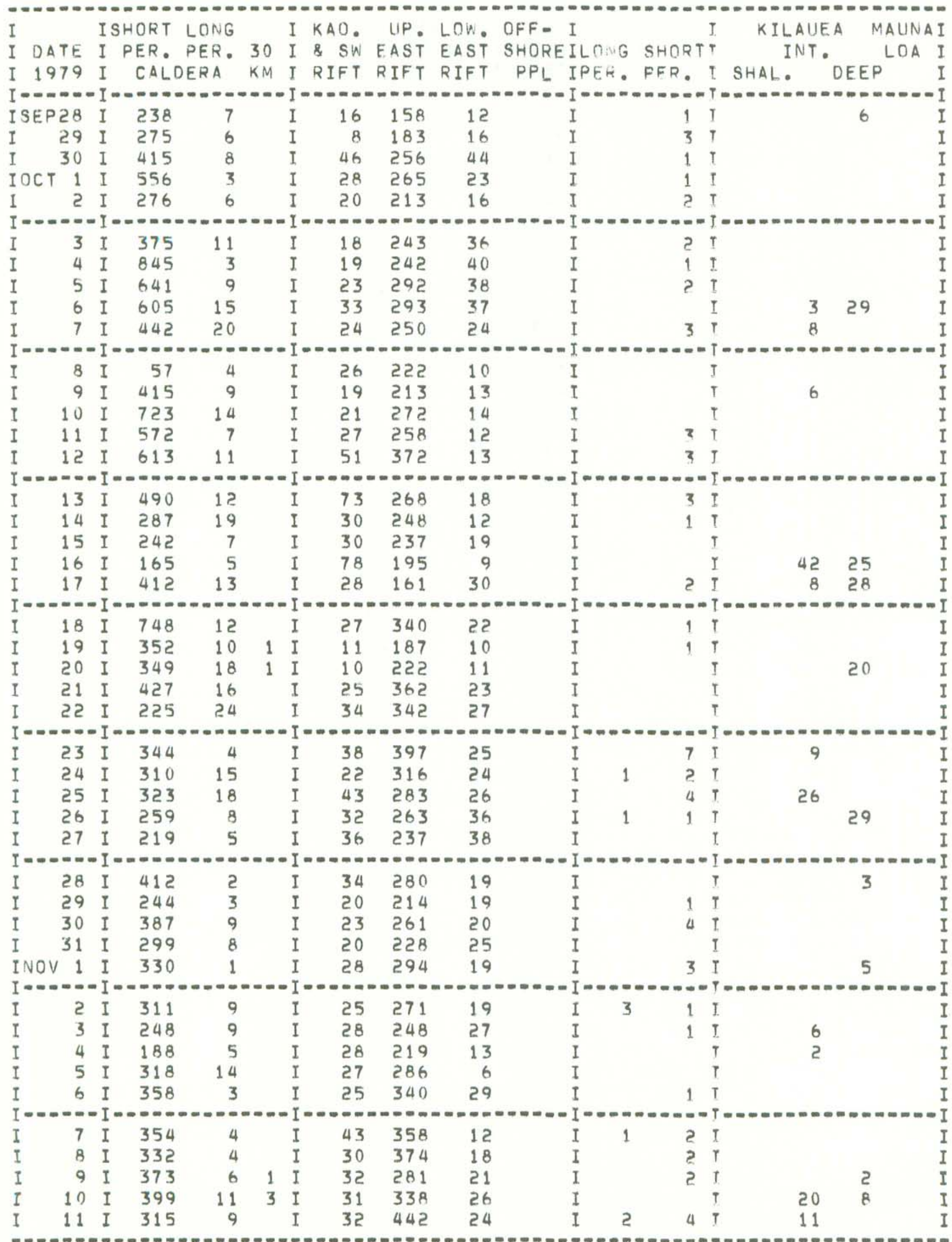


KILAUEA SUMMIT KILAUEA FLANK

MAUNA LOA TREMOR (MINUTES)

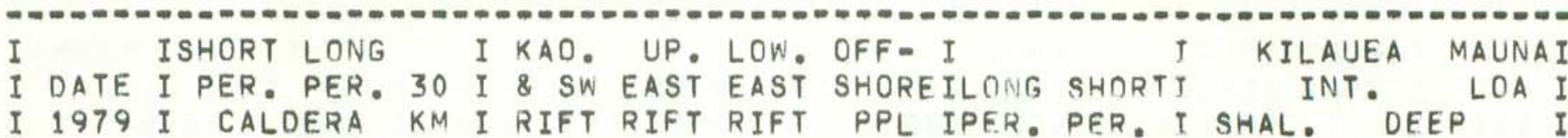

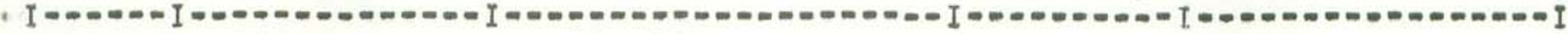

$\begin{array}{llllllllll}\text { INOV12 } & \text { I } & 399 & 29 & \text { I } & 33 & 521 & 10 & I & \text { I }\end{array}$

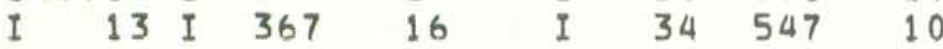

I $\quad 14$ I $66 \quad 50 \quad$ I $25 \quad 611 \quad 12$

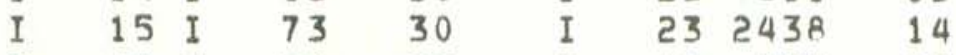

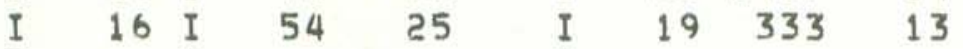

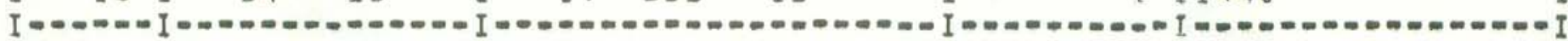

$\begin{array}{lllllllllll}I & 17 & I & 220 & 94 & I & 21 & 402 & I & \text { ? } T\end{array}$

$\begin{array}{lllllllll}I & 18 & I & 43 & 16 & I & 16 & 203 & 16\end{array}$

$\begin{array}{llllllll}1 & 19 & I & 83 & 79 & \text { I } & 10 & 460\end{array}$

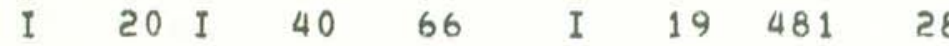

I 21 I $64 \quad 84$ I $22 \quad 349 \quad 19 \quad$ I 1 ?

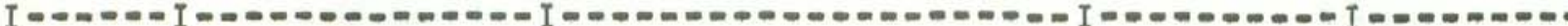

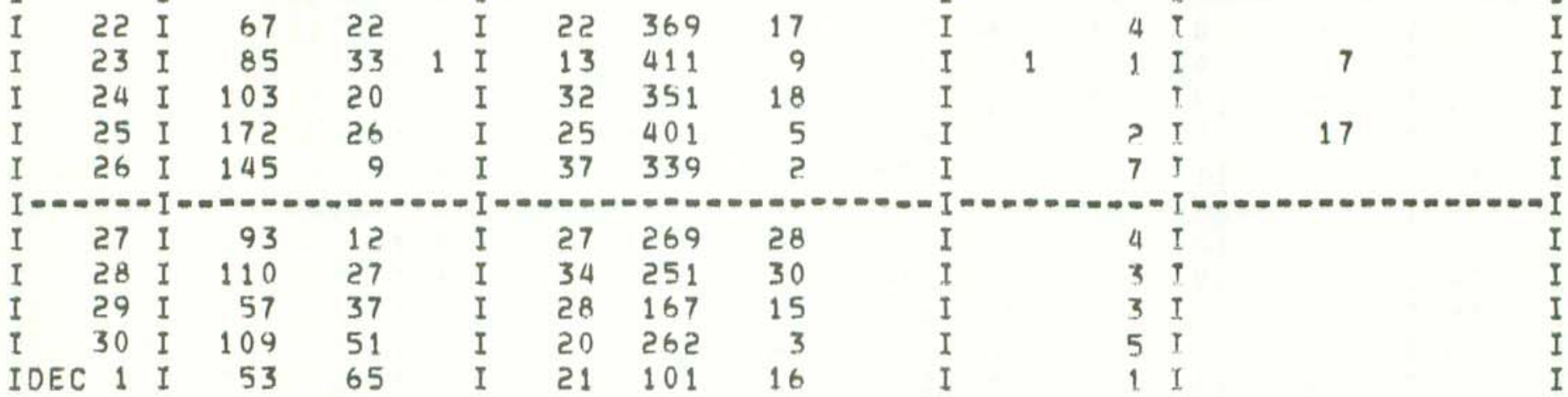

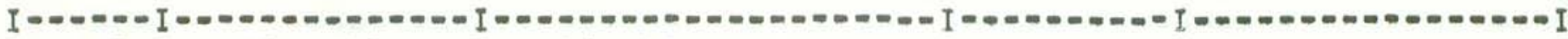

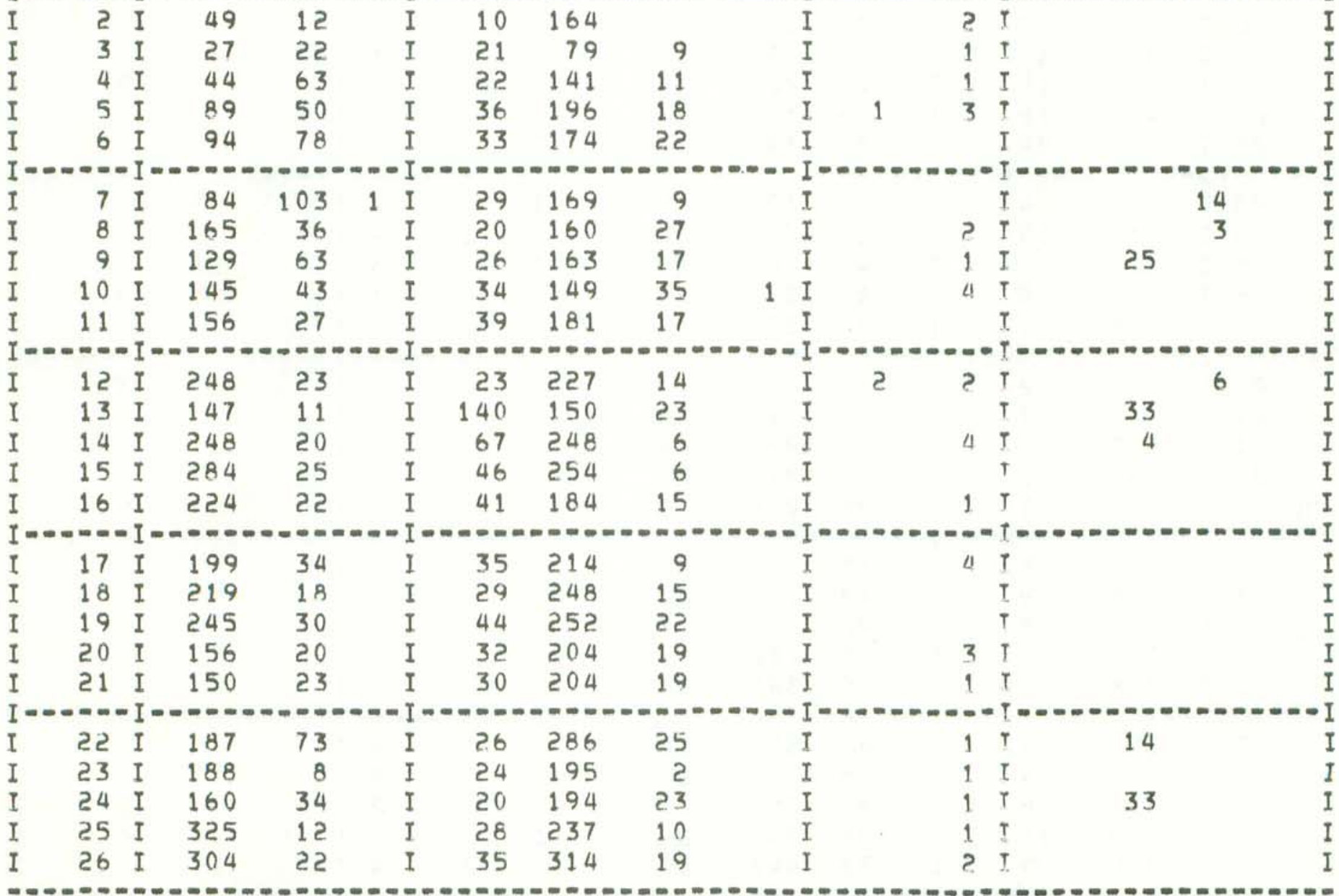


KILAUEA SUMMIT KILAUEA FLANK

MAIJNA LOA TREMOR (MINUTES)

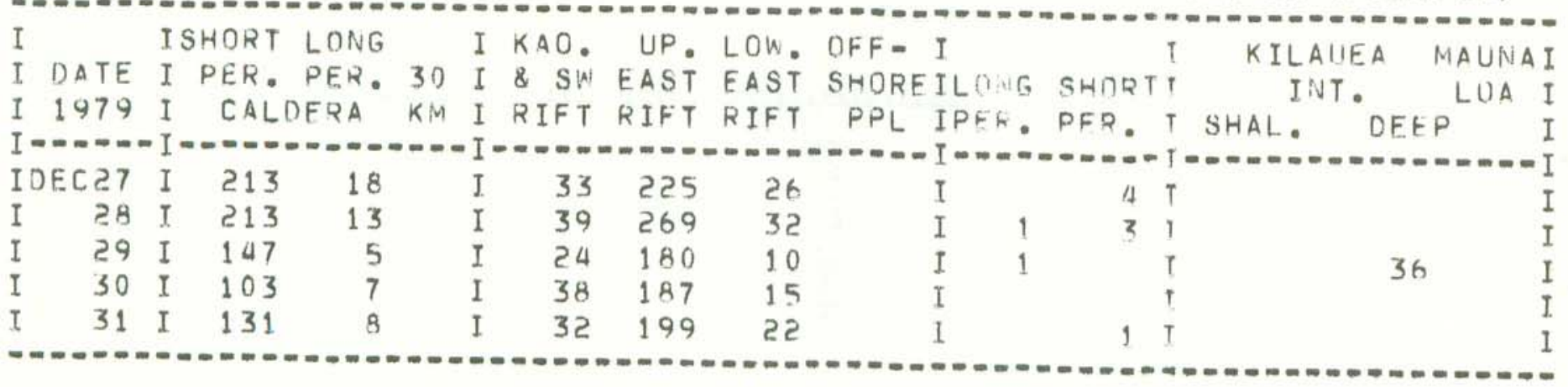




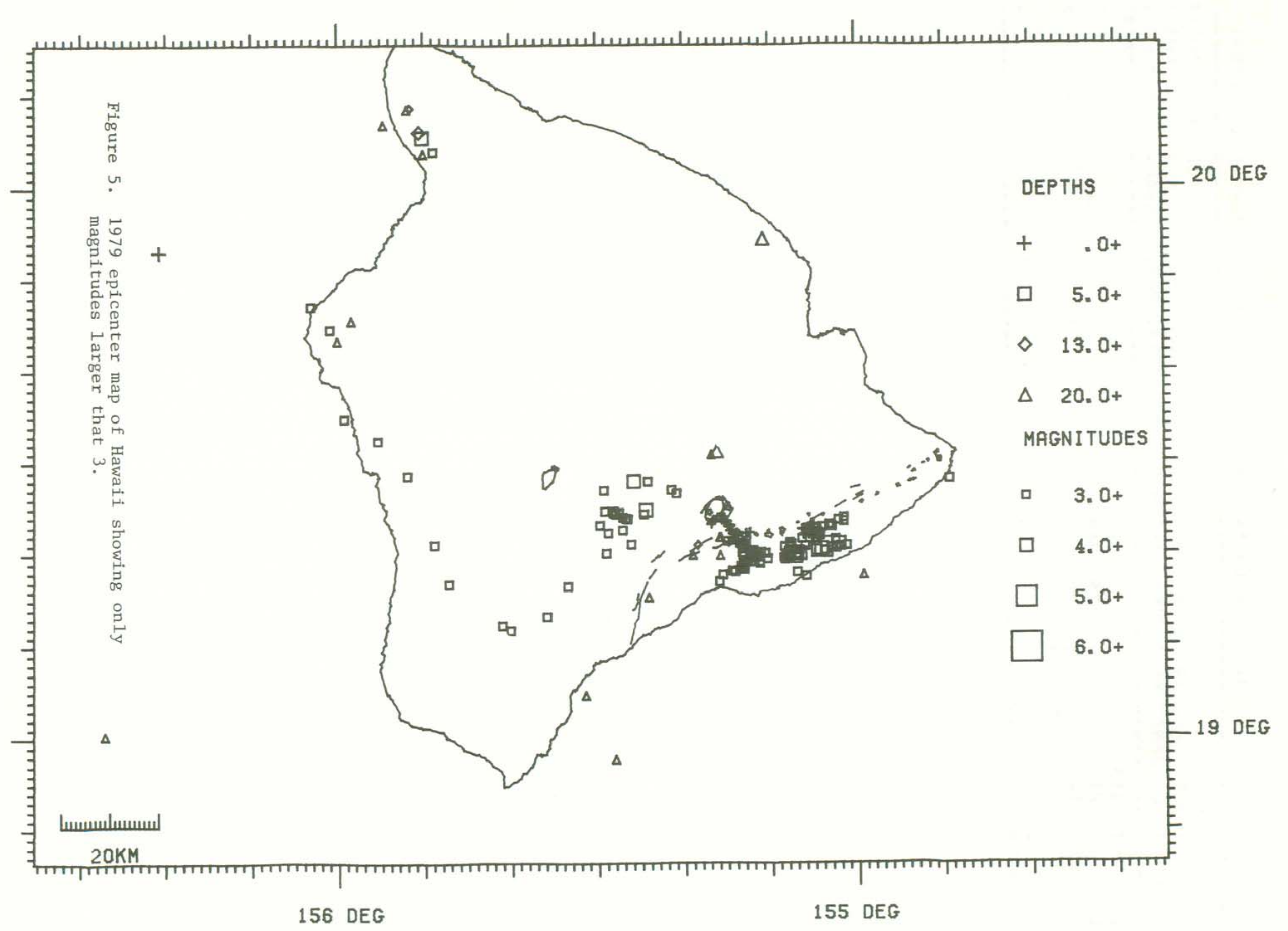




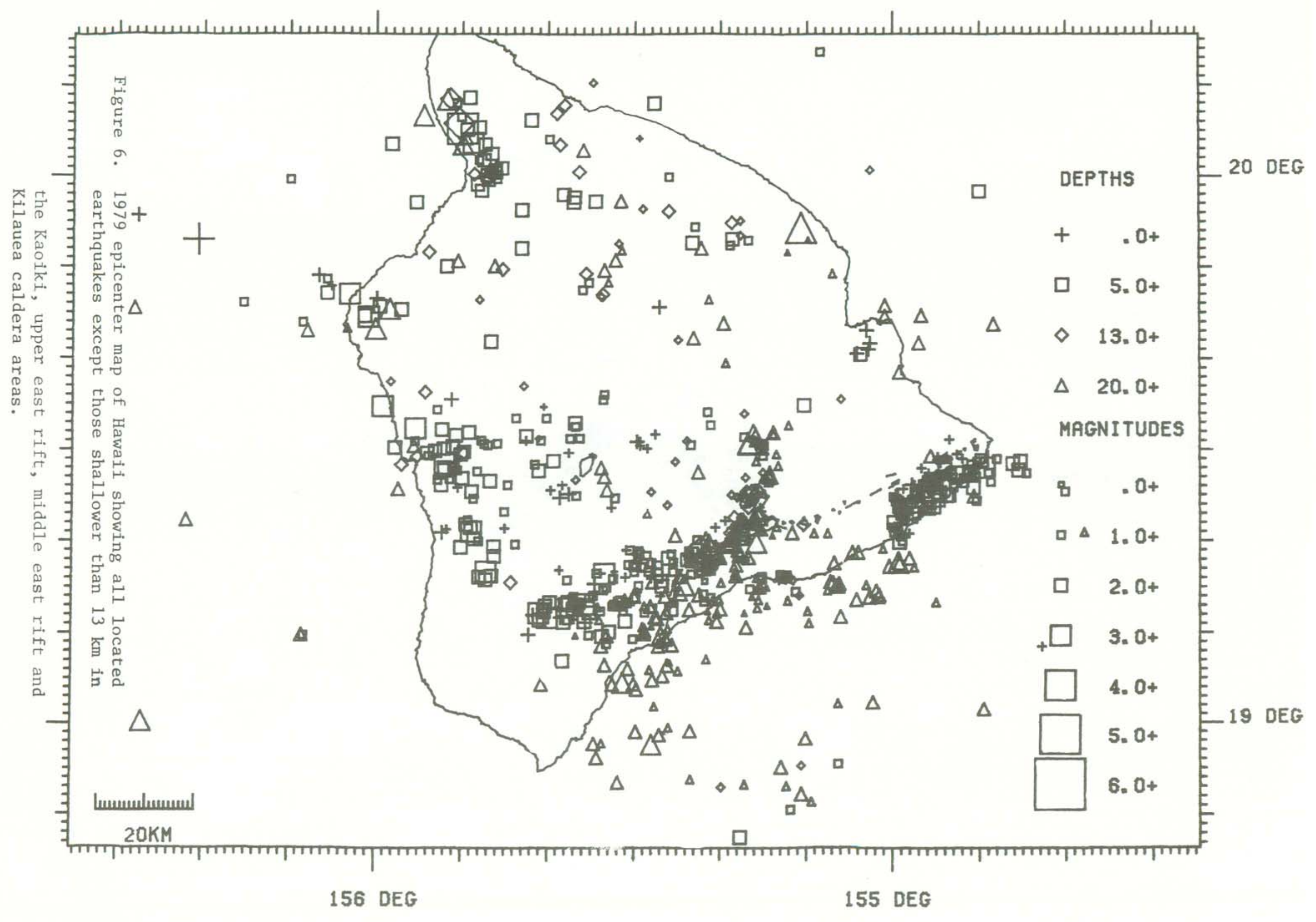




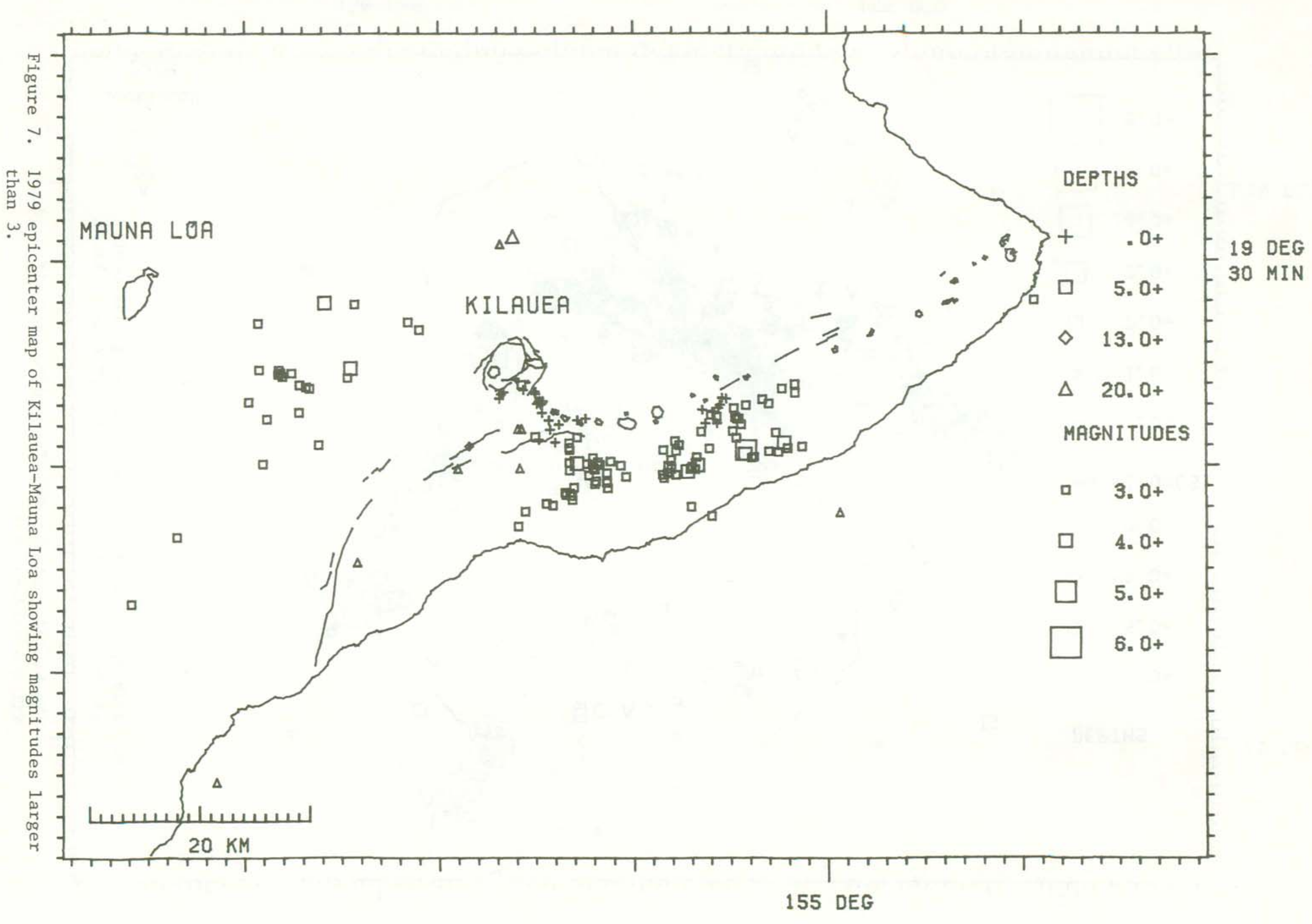




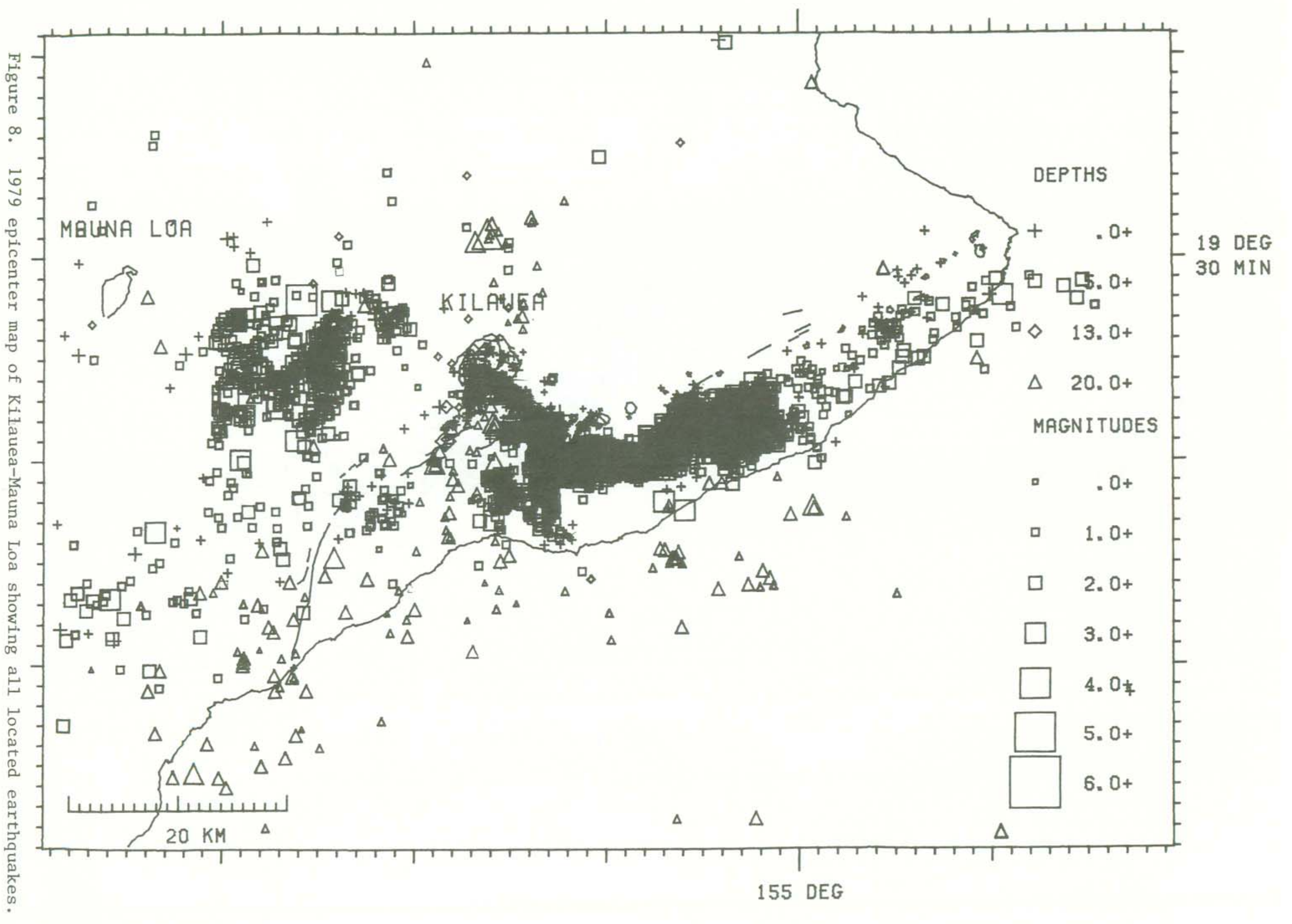




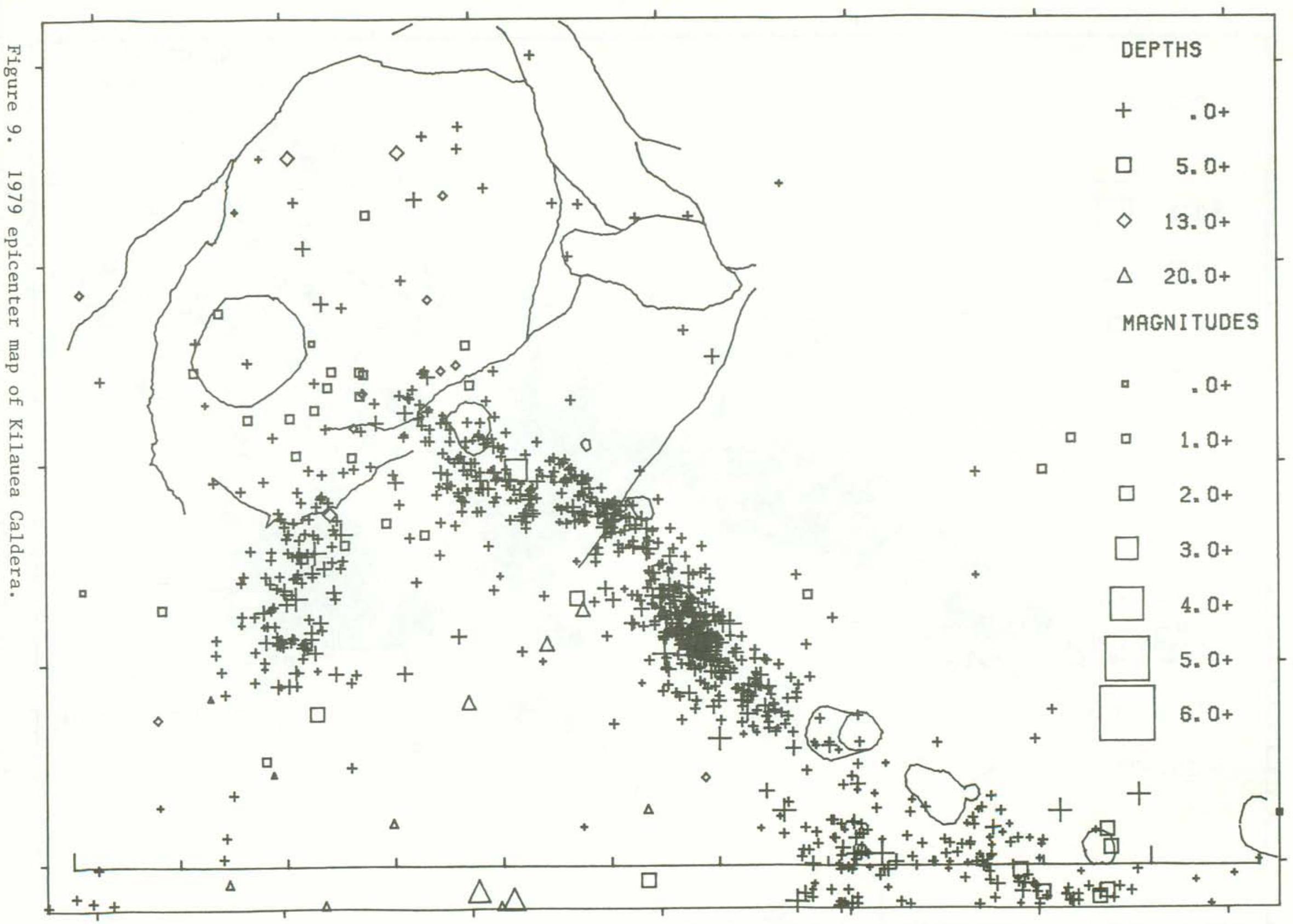


Table 4. Coordinates of named regions.

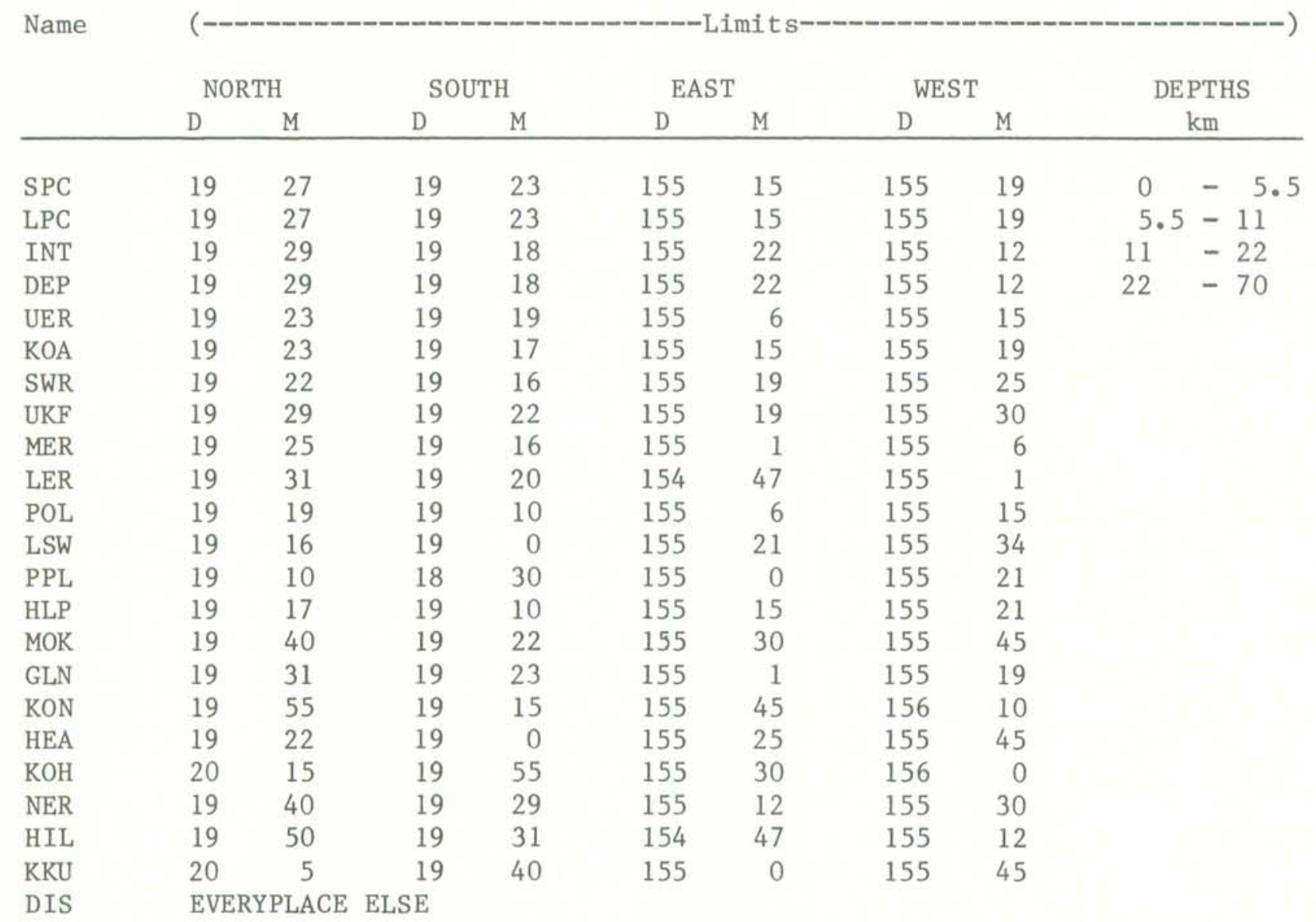

When coordinates imply an overlap, precedence is given as shown in Figure 2. 
Table 5 is a chronological listing of successfully located earthquakes. For each event the following data are presented:

Origin time in Hawaiian Standard Time: date, hour (HR), minute (MN), and second (SEC).

Epicenter in degrees and minutes of north latitude (LAT N) and west longitude (LON W).

DEPTH - Depth of focus in $\mathrm{km}$.

AMP MAG - Amplitude magnitude, if determined.

DUR MAG - Duration magnitude, if determined.

NR - Number of arrivals ( $P$ or $S$ ) used for solution.

NS - Number of S arrivals used for solution.

GAP DEG - Largest azimuthal separation in degrees between stations.

RMS SEC - Root mean square error of time residuals in sec.

$$
\mathrm{RMS}=\left(\sum \mathrm{R}_{1}^{2} / \mathrm{NR}\right)^{1 / 2}
$$

MIN DIS - Epicentral distance in $\mathrm{km}$ to the third nearest station.

ERH $\mathrm{km}$ - Standard error of the epicenter in $\mathrm{km}$.

ERZ km - Standard error of depth of focus in $\mathrm{km}$.

REMK - Remarks, three letter code for geographic location of event. See Figure 2 for location of mnemonic code. Additional one letter codes have the following meanings:

$\mathrm{F}-\mathrm{felt}$

L - long period character

T - associated with harmonic tremor

B - quarry or other blast

* - the location program had a convergence problem, which usually means that the depth may be unreliable.

Table 5 lists all events located during 1979. Table 6 lists only events of magnitude 3.0 or larger. 
Table S HVO EARTHQUAKE SUMMARY LIST ORIGIN TIME LAT N LON $N$ OEPTH ANP OUR G GAP RMS MIN ERH ERZ NO
DA HRMN SEC DEG MIN DEG MIN 1979 JAN $1 \quad 3 \quad 6 \quad 15.751916 .05 \quad 15528.07$

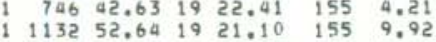

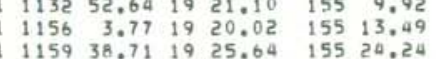

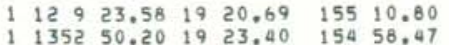
135250.201923 .4019550 .47

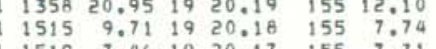
$\begin{array}{llllll}1519 & 7,46 & 19 & 20,17 & 155 & 7,71\end{array}$

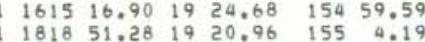
181851.261920 .96 154 59.19

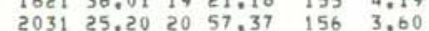
$\begin{array}{lllll} & & & \end{array}$ $\begin{array}{lllllll}21 & 2 & 34.60 & 19 & 19.70 & 155 & 13.15\end{array}$ 1
2 $213753.751919 .56 \quad 155 \quad 14.82$

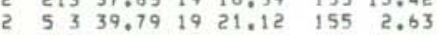

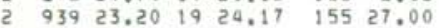

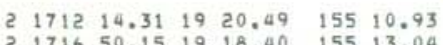

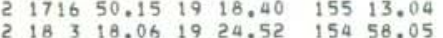
184045.301926 .62

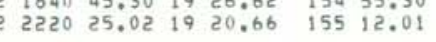
$\begin{array}{llllll}2 & 2222 & 8.19 & 19 & 20.38 \quad 155 & 11.80\end{array}$

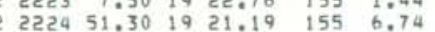

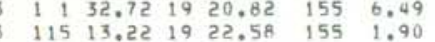
$\begin{array}{lllllll}3 & 351 & 29.74 & 19 & 18.76 & 155 & 13.34\end{array}$

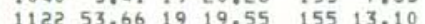
312554.711919 .58 155 9.46 $\begin{array}{lllllll}3 & 1256 & 46.74 & 19 & 19.50 & 155 & 9.35\end{array}$

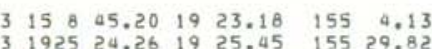

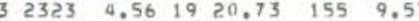

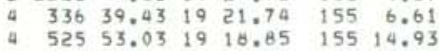
$\begin{array}{lllllll}1130 & 46.11 & 19 & 21.00 & 155 & 2.47\end{array}$

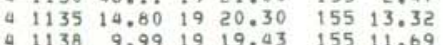

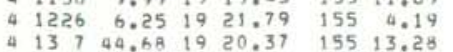
$4026 \quad 49.19 \quad 19 \quad 20.36 \quad 155 \quad 13.46$ 4 $231520.04 \quad 19 \quad 19.85 \quad 155 \quad 10.09$

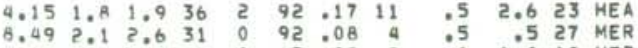

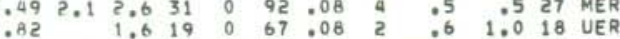

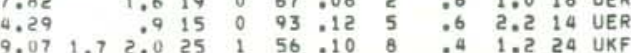
$\begin{array}{rrrrrrrrrrrr}6.81 & .9 & .9 & 11 & 0 & 83 & 010 & 3 & .7 & 1.5 & 11 & \text { UER } \\ 7.71 & 1.9 & 1.4 & 21 & 2 & 195 & 018 & 3 & 1.3 & 1.2 & 18 & \text { LER }\end{array}$

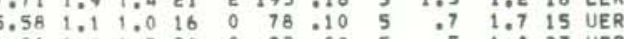
$\begin{array}{llllllllllll}6.91 & 1.4 & 1.7 & 24 & 0 & 92 & .08 & 5 & .5 & 1.0 & 23 & \text { UER } \\ 6.53 & 1.2 & 1.6 & 23 & 0 & 92 & .11 & 5 & .6 & 1.5 & 23 & \text { UER }\end{array}$ $\begin{array}{llllllllllll}7.18 & 1.8 & 1.5 & 22 & 1 & 153 & 14 & 2 & .9 & 1.0 & 22 & \text { LER }\end{array}$

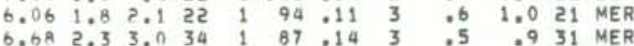

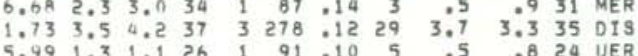
$\begin{array}{llllllllllll}6.79 & 1.1 & 1.4 & 25 & 0 & 72.12 & 5 & .6 & 1.1 & 21 & \text { UER }\end{array}$

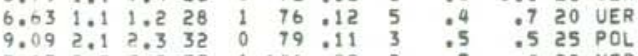

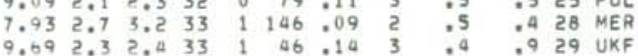

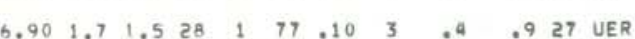
$\begin{array}{llllllllllll}5.81 & & 7.1 & 16 & 0 & 191 & 11 & 2 & 1.4 & 1.6 & 14 & \text { LER } \\ 7.45 & 1.6 & 7.9 & 28 & 0 & 71 & : 13 & 4 & .6 & .8 & 25 & \text { UER }\end{array}$

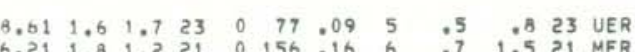

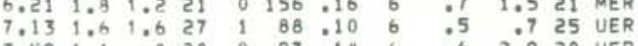

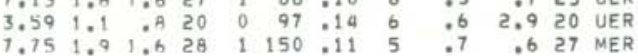
$\begin{array}{llllllllllll}0.64 & 1.5 & 1.4 & 23 & 0 & 79 & .11 & 3 & .5 & 1.1 & 22 & \text { POL }\end{array}$

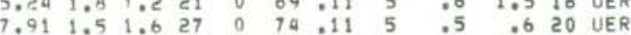

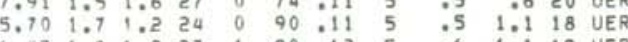

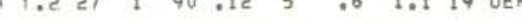
$\begin{array}{llllllllllll}6.39 & 1.6 & 1.0 & 13 & 1 & 91 & .11 & 3 & .7 & 1.4 & 7 & \text { MER } \\ 7.91 & .7 & 0.0 & 24 & 1 & 59 & 11 & 7 & 5 & 1.5 & 12 & \text { UKF }\end{array}$

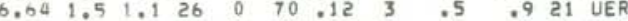
$\begin{array}{rllllllllllll}7.59 & 1.8 & 1.5 & 31 & 0 & 80 & .12 & 6 & .5 & .8 & 27 & \text { UER } \\ 7.63 & 1.8 & 1.1 & 14 & 1 & 118 & .13 & 4 & .6 & 1.1 & 13 & \text { POL }\end{array}$ $\begin{array}{llllllllllll}6.52 & 2.1 & 1.8 & 23 & 0 & 157 & .12 & 2 & .9 & .9 & 21 & \text { MER }\end{array}$

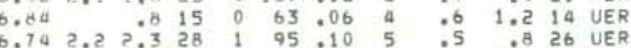

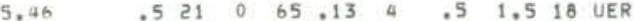

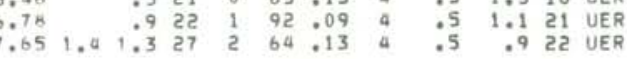

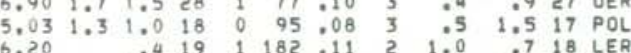

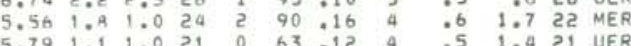

HVO EARTHQUAKE SUMMARY LIST

PAGE a

DRIGIN TIME LATN TON W DEPTH AMP DUR GAP RMS MIN ERH ERZ NO TEAR MON DA HRMN SEC DEG MIN DEG MIN KM MAG MAG NR NS OEG SEC OIS KM KM FM REMK 1979 JAN 5 S $515 \quad 5.63 \quad 1923.97 \quad 15515.63$ $52941.031921 .31 \quad 155 \quad 4.62$

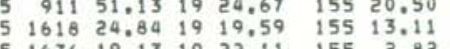
$\begin{array}{llll}1712 & 8.21 & 19 & 14.58 \quad 155 \quad 29.69\end{array}$ $\begin{array}{llllll}1726 & 53.21 & 19 & 15.22 & 155 \quad 6.36\end{array}$ $\begin{array}{llllll}1738 & 59.39 & 19 & 20.61 & 155 & 12.72\end{array}$

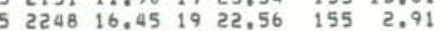

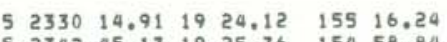
$\begin{array}{lllllll}5 & 2342 & 45.17 & 19 & 25.76 & 154 & 58.84\end{array}$ $6 \quad 14456,5119 \quad 18,46 \quad 15514,10$

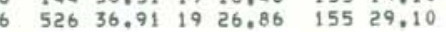

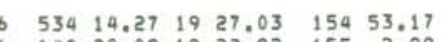

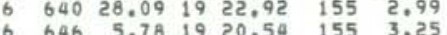

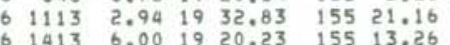
$\begin{array}{lllllll}6 \quad 15 & 1 & 21.36 & 19 & 23.73 & 155 & 28.73\end{array}$

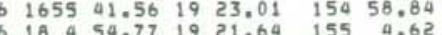

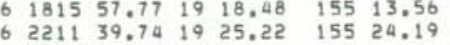

\begin{tabular}{l}
$6252 \quad 37.02 \quad 19 \quad 14.77 \quad 155 \quad 7.60$ \\
\hline
\end{tabular}

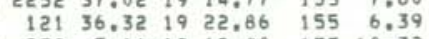
$\begin{array}{llllll}238 & 5.66 & 19 & 19.40 & 155 & 10.32\end{array}$

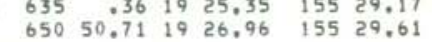

$7 \quad 73341.74 \quad 19 \quad 19.62 \quad 155 \quad 11.89$ $\begin{array}{llllll}840 & 22.14 & 19 & 19.74 & 155 & 7.32\end{array}$ \begin{tabular}{l}
$1024 \quad 8.191923 .35 \quad 15458.15$ \\
\hline
\end{tabular}

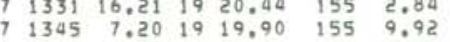
$\begin{array}{lllllll}7 & 1758 & 32.38 & 19 & 18.59 & 155 & 13.76\end{array}$ $\begin{array}{lllll}71830 & .38 & 19 & 20.32 \quad 155 & 11.18\end{array}$

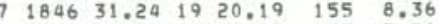
$71914 \quad 5.91 \quad 1921.13 \quad 155 \quad 13.30$

$\begin{array}{lllllll}7 & 2045 & 24.72 & 19 & 19.54 & 155 & 11.62\end{array}$

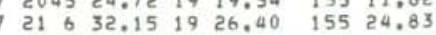

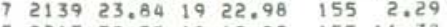

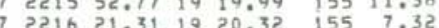
$\begin{array}{llllll}2239 & 52.63 & 19 & 20.46 & 155 & 6.49\end{array}$ $\begin{array}{lllllll}7 & 2316 & 36.30 & 19 & 21.84 & 155 & 6.31 \\ 8 & 427 & 32.62 & 19 & 20.72 & 155 & 12.85\end{array}$ $\begin{array}{lllllllllll}2.55 & 1.0 & .9 & 9 & 0 & 113 & .06 & 3 & .4 & .5 & 9\end{array}$

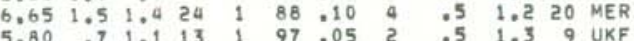

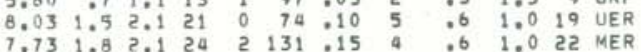
$\begin{array}{llllllllllll}3.51 & 1.3 & 1.4 & 19 & 0 & 79 & .14 & 10 & .6 & 23.4 & 8 & \text { LSW }\end{array}$

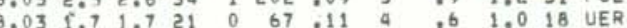

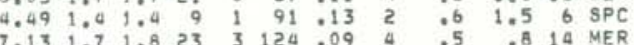
$\begin{array}{lllllllllllll}.78 & 1.1 & 1.3 & 12 & 1 & 114 & .12 & 2 & .3 & .6 & 5 & \mathrm{SPC}\end{array}$

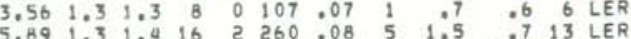

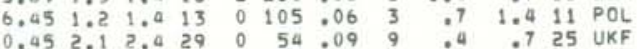
$\begin{array}{llllllllllll}5.86 & 1.2 & 1.1 & 15 & 2 & 250 & .13 & 4 & 1.7 & .8 & 11 & \text { LER }\end{array}$

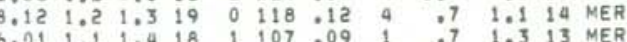
$\begin{array}{lllllllllllll}1.97 & 2.0 & 1.8 & 39 & 3 & 62 & .11 & 7 & .4 & 1.1 & 23 & \text { NER } \\ 6.00 & 1.0 & -3 & 23 & 0 & 65 & 12 & 4 & 5 & 1.3 & 18 & \text { UER }\end{array}$ $9,30 \quad 1,1 \quad 1,323 \quad 2 \quad 112.11 \quad 3 \quad .4 \quad 1.112$ UKF

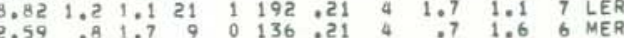

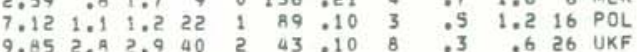
$\begin{array}{llllllllllll}1.57 & 1.7 & 1.6 & 30 & 8 & 203 & .07 & 5 & .9 & .9 & 19 & \text { POL }\end{array}$

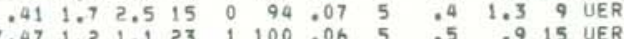

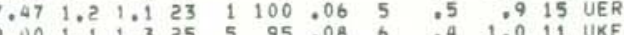

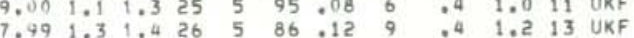

$\begin{array}{llllllllllll}7.43 & 1.1 & 1.0 & 19 & 0 & 89.05 & 6 & .5 & 1.3 & 10 & \text { UER }\end{array}$

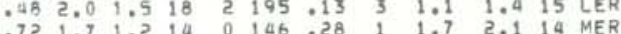

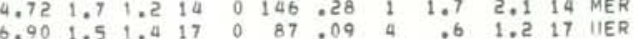

$\begin{array}{llllllllllll}6.19 & 1.6 & 1.7 & 23 & 0 & 92 & .09 & 3 & .6 & 1.2 & 20 & \mathrm{POL}\end{array}$

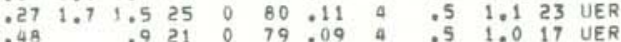

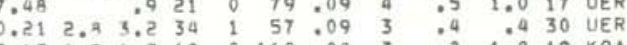

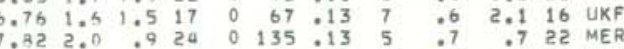

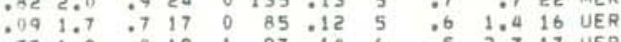

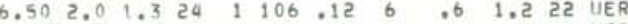
$\begin{array}{llllllllllll}7.29 & 1.9 & .8 & 18 & 2 & 86 & .10 & 6 & .6 & 9 & 16 & \text { UER } \\ 7.67 & 1.6 & 0 & 25 & 1 & 63 & .11 & 4 & .5 & 9 & 22 & \text { UERR }\end{array}$

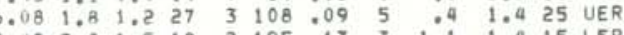




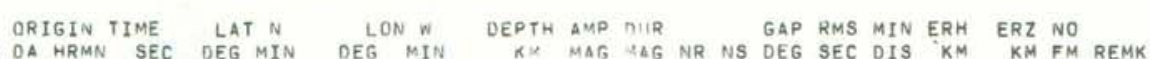
YEAR MON OA HRMN SEC DEG MIN DEg MIN KX MAG HAG NR NS DEg SEC DIS KM KM FM REM

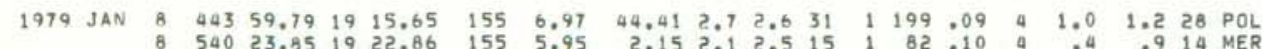

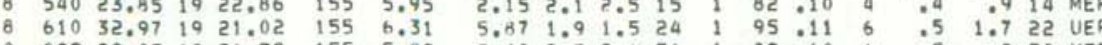

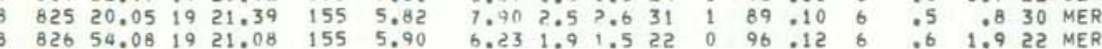
$\begin{array}{llllllll}8 & 1011 & 49.73 & 19 & 19.57 & 155 & 14.16 \\ 8 & 1057 & 9.25 & 19 & 20.20 & 155 & 13.19\end{array}$

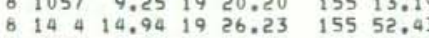

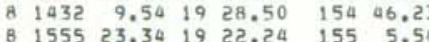

$\begin{array}{lllllll}8 & 1726 & 15.61 & 19 & 23.06 & 155 & 5.71\end{array}$ $\begin{array}{lllllll}8 & 1816 & 6.35 & 19 & 20.27 & 155 & 13.78 \\ 8 & 1937 & 19.44 & 19 & 20.02 & 155 & 11.97\end{array}$

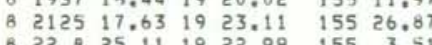

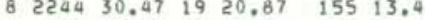

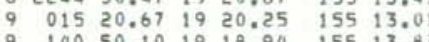

$921356.411922 .45 \quad 15517.05$

$9 \quad 646 \quad 40.64 \quad 1921.32 \quad 155 \quad 4.01$

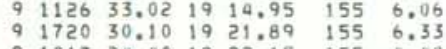

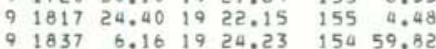
$91859 \quad 10.70 \quad 19 \quad 21.15 \quad 155 \quad 8.03$
9

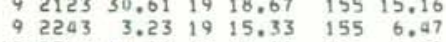

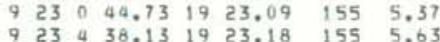

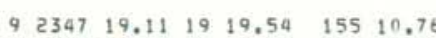

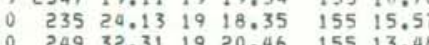

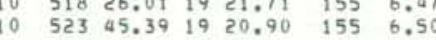

$\begin{array}{lllllll}10 & 617 & 38.95 & 19 & 20.61 & 155 & 11.15\end{array}$

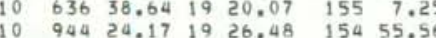

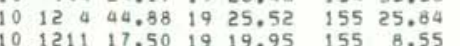

$\begin{array}{lllllll}10 & 1235 & 55.18 & 19 & 23.09 & 155 & 6.33\end{array}$

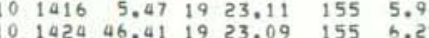
$\begin{array}{llrllll}10 & 1752 & 13.03 & 19 & 17.28 & 155 & 18.38 \\ 10 & 1753 & 7.59 & 19 & 17.47 & 155 & 18.16\end{array}$

$\begin{array}{lllllllll}10 & 18 & 8 & 14.92 & 19 & 16.30 & 155 & 18.21\end{array}$ $101822 \quad 49.66 \quad 1920.44 \quad 155 \quad 2.42$ $\begin{array}{llllllllllll}8.00 & 1.6 & 1.1 & 13 & 0 & 85 & .07 & 5 & .6 & 1.6 & 12 & \text { UER }\end{array}$

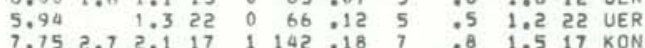

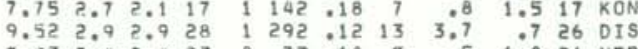

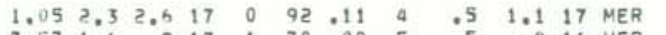
$\begin{array}{llllllllllll}7.57 & 1.6 & .9 & 17 & 1 & 70 & .08 & 5 & .5 & .8 & 16 & \text { UER } \\ 6.36 & 0 & 1.0 & 17 & 0 & 81 & .11 & 5 & .6 & .5 & 17 & \text { UER }\end{array}$

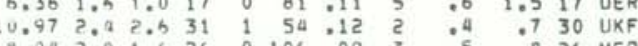
$\begin{array}{llllllllllll}7.04 & 1.5 & 1.3 & 24 & 0 & 59.14 & 3 & .6 & 1.0 & 24 \\ 5.25 & 1.5 & .02 & 10 & 0 & 67 & .12 & 4 & .6 & .9 & \text { UER }\end{array}$

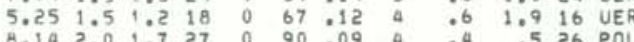

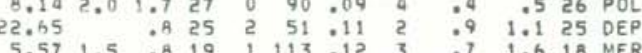

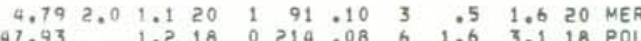

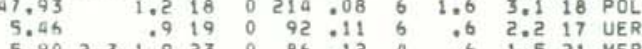

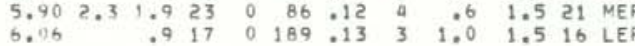
$\begin{array}{llllllllllll}7.40 & 1.2 & 19 & 0 & 74 & .10 & 4 & .5 & 1.3 & 19 & \text { UER }\end{array}$

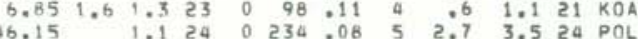
$\begin{array}{llllllllllll}1.42 & 2.7 & 3.1 & 23 & 0 & 78 & .16 & 3 & .5 & 1.1 & 23 & \text { MER } \\ 2.48 & 1.9 & 1.9 & 16 & 0 & 82 & .11 & 3 & .6 & 1.0 & 16 & \text { MER }\end{array}$ $\begin{array}{llllllllllll}7.16 & 1.7 & 1.2 & 22 & 0 & 97 & .08 & 5 & .5 & 1.0 & 22 & \text { UER }\end{array}$

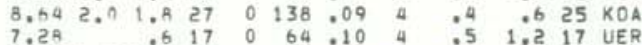

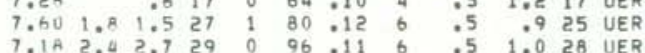

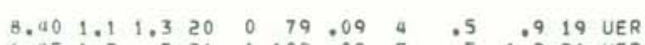

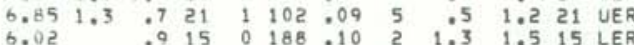

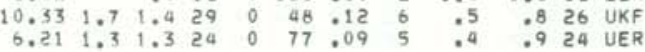
$\begin{array}{rrrrrrrrrrrr}.02 & 1.8 & 1.9 & 10 & 0 & 96 & .10 & 6 & .6 & 3.5 & 10 & \mathrm{GLN}\end{array}$

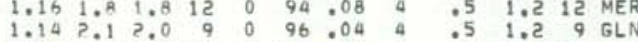
$\begin{array}{llllllllllllll}32.57 & 1.4 & 1.2 & 24 & 0 & 136 & 07 & 1 & 09 & 1.6 & 24 & \mathrm{KOA} \\ 34.58 & 2.4 & 7.7 & 36 & 1 & 133 & .10 & 1 & .7 & 1.1 & 34 & \mathrm{KOA}\end{array}$

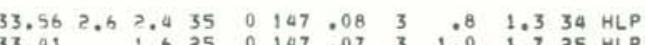
$\begin{array}{lllllllllll}6.61 & 8 & 20 & 1 & 179 & 14 & 1 & .8 & 1.2 & 19 & \text { MER }\end{array}$
ORIGIN TIME LAT N LON N DEPTH AMP RIR GAP RMS MIN ERH ERZ NO NEMK

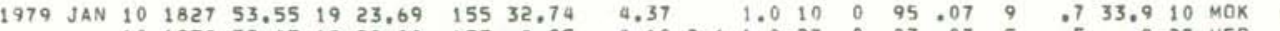
$101938 \quad 32.45 \quad 1920.08 \quad 155 \quad 8.25$ $102136 \quad .791919 .71 \quad 155 \quad 8.03$

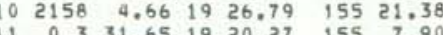

$11 \quad 024 \quad 1.66 \quad 1911.84 \quad 155 \quad 6.11$

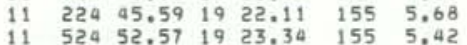

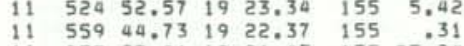

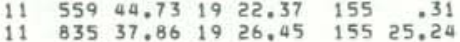

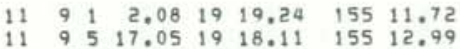

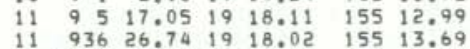
$111028 \quad 56.92 \quad 1923.07 \quad 155 \quad 5.69$ 11 $1113 \quad 53.82 \quad 1921.90 \quad 155 \quad 25.25$

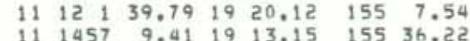
$11 \quad 1457 \quad 9.41 \quad 19 \quad 13.15 \quad 155 \quad 36.22$ 11151038.96 19 21.67 155 7.10 $1844 \quad 2.191921 .46 \quad 15459.85$ $\begin{array}{rrrrrrr}11 & 1914 & 13.73 & 19 & 19.20 & 155 & 12.43 \\ 11 & 1919 & 5.49 & 19 & 24.67 & 155 & 14.87\end{array}$ 111919.5 .4919 19.67 15519.87

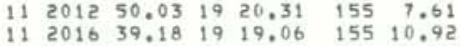
$\begin{array}{lllllll}11 & 2351 & .39 & 19 & 19.48 & 155 & 46,32\end{array}$ $\begin{array}{lllllll}12 & 058 & 11.11 & 19 & 19.29 & 155 & 8.61\end{array}$

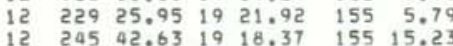

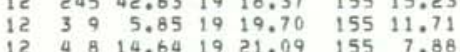
$\begin{array}{lllllll}12 & 959 & 34.58 & 19 & 19.28 & 155 & 11.77\end{array}$

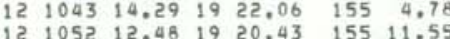

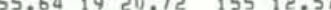
$12171935.5219 \quad 17.24 \quad 15530.18$ $\begin{array}{lllllll}12 & 1842 & 40.86 & 19 & 19.55 & 155 & 10.84\end{array}$

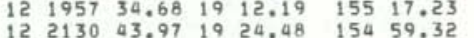

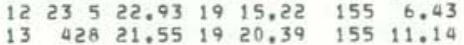

$\begin{array}{llllllll}13 & 637 & 13.73 & 19 & 19.88 & 155 & 13.61\end{array}$ $\begin{array}{rrrrrrr}13 & 637 & 13.73 & 19 & 19.88 & 155 & 13.61 \\ 13 & 78 & 8.92 & 19 & 12.74 & 155 & 15.72\end{array}$ $\begin{array}{lllllll}13 & 755 & 26.99 & 19 & 20.06 & 155 & 7.98\end{array}$ $\begin{array}{llllllll}13 & 8 & 4 & 1.84 & 19 & 20.52 & 155 & 13.21 \\ 13 & 10 & 9 & 40.80 & 19 & 25.84 & 155 & 28.29\end{array}$ $\begin{array}{lllllll}13 & 1023 & 33.40 & 18 & 57.79 & 155 & 33.83\end{array}$

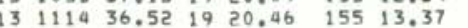

$\begin{array}{llllllllllll}4.37 & 1.0 & 10 & 0 & 95 & 07 & 9 & .7 & 33.9 & 10 & \text { MOK } \\ 8.18 & 2.1 & 1: 0 & 27 & 0 & 83 & 07 & 5 & .5 & .8 & 27 & \text { UER }\end{array}$

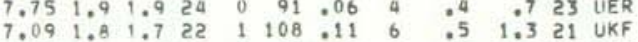

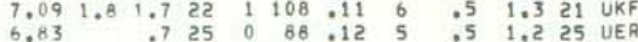
$\begin{array}{lllllllllllllll}45.30 & 2.6 & 2.7 & 35 & 0 & 213 & .08 & 11 & 1.2 & 1.8 & 34 & \text { POL } \\ 2.53 & 3.0 & 3.4 & 27 & 0 & 75 & .15 & 6 & .5 & 2.6 & 27 & \text { MER }\end{array}$

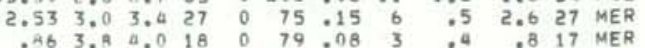

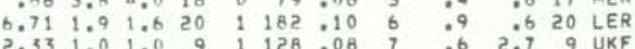
$\begin{array}{llllllllllllll}7.81 & 1.7 & 2.5 & 23 & 0 & 100 & .09 & 5 & .5 & 1.0 & 21 & \text { UER } & 0.0\end{array}$

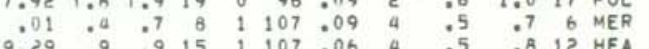
$\begin{array}{lllllllllllll}7.80 & 1.1 & 1.8 & 18 & 0 & 97 & .07 & 5 & .6 & .9 & 16 & \text { UER } \\ 8.60 & 1.0 & & 18 & 0 & 07 & .17 & 4 & .0 & 9 & 9 & \text { HEA }\end{array}$

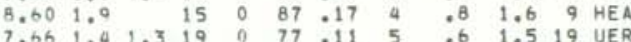

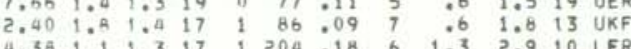
$\begin{array}{rrrrrrrrrrrr}8.16 & 1.5 & 1.3 & 14 & 0 & 92 & 05 & 4 & .6 & 1.6 & 12 & \text { UER } \\ 4.31 & 1.8 & 1.9 & 12 & 1 & 119 & .10 & 5 & .6 & 1.5 & 6 & \text { GLN }\end{array}$

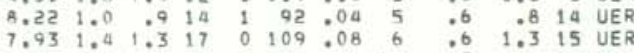
$\begin{array}{lllllllllll}14 & 2.6 & 2.3 & 28 & 2 & 81 & .10 & 12 & .5 & .6 & 25 \\ \mathrm{kON}\end{array}$ $\begin{array}{llllllllllll}8.58 & 1.5 & 1.1 & 13 & 0 & 82 & .06 & 4 & .7 & 1.7 & 11 & \text { UER } \\ 7.87 & 2.2 & 1.9 & 21 & 0 & 79 & .11 & 6 & .8 & 1.1 & 20 & \text { MER }\end{array}$

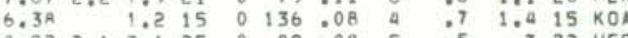
$\begin{array}{lllllllllllll}8.92 & 2.1 & 2.1 & 25 & 0 & 89 & 08 & 5 & .5 & .7 & 22 & \text { UER } \\ 7.47 & .8 & 17 & 0 & 82 & .08 & 4 & .5 & 1.1 & 17 & \text { UER }\end{array}$ $\begin{array}{lllllllllll}6.33 & .9 & 19 & 0 & 98 & .10 & 5 & .5 & 1.1 & 17 & \text { UER } \\ 5.95 & .8 & 19 & 0 & 79 & .14 & 5 & .6 & 1.9 & 10 & \text { MER }\end{array}$

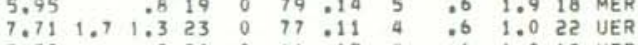

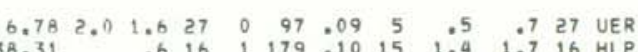

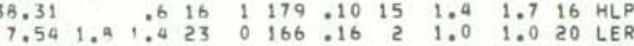

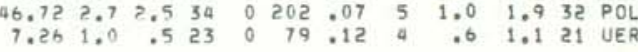

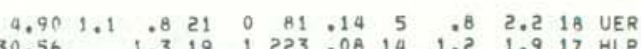
$\begin{array}{llllllllllll}8.60 & 2.8 & 3.1 & 33 & 0 & 88 & .10 & 5 & .5 & .6 & 28 & \text { UER }\end{array}$

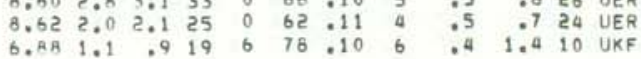
$\begin{array}{llllllllllll}41.26 & 1.9 & 1.4 & 22 & 5 & 248 & 0.07 & 11 & 1.1 & 1.5 & 10 & \text { OIS }\end{array}$

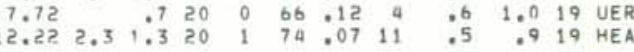




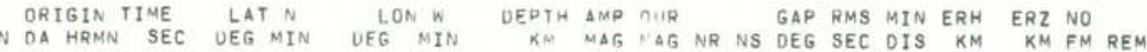
1979 JAN $13 \quad 1610 \quad 15.96 \quad 1921.61 \quad 155 \quad .64$

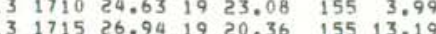

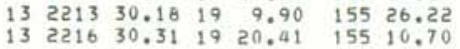

$\begin{array}{lllllll}13 & 2223 & 16.06 & 19 & 20.15 & 155 & 7.77\end{array}$

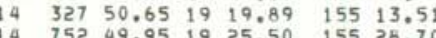

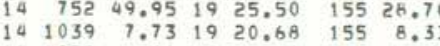

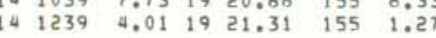
$\begin{array}{lllllll}14 & 1241 & 14.31 & 19 & 21.27 & 155 & 2.29\end{array}$

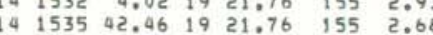

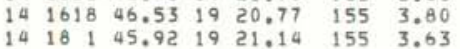
$\begin{array}{lllllll}14 & 2046 & .08 & 19 & 22.52 & 155 & 4.74\end{array}$

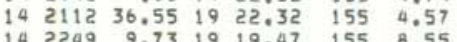

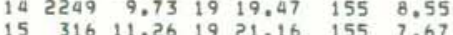
is $411 \quad 53.74 \quad 1920.83 \quad 155 \quad 13.10$

$\begin{array}{lllllll}15 & 523 & 46.96 & 19 & 19.34 & 155 & 11.35\end{array}$

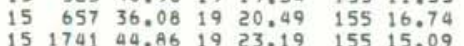
$15193319.421924: 99 \quad 15524.32$

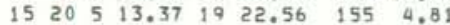

$\begin{array}{lllllll}16 & 154 & 38.81 & 19 & 22.56 & 155 & 24.37 \\ 16 & 927 & 48.34 & 19 & 22.48 & 155 & 5.313\end{array}$

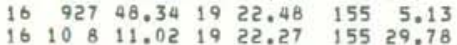

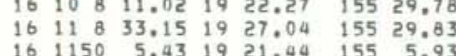

$\begin{array}{lllllll}16 & 1412 & 18.65 & 19 & 21.00 & 155 & 12.94\end{array}$ $\begin{array}{lllllll}16 & 1439 & 29.47 & 19 & 41.76 & 154 & 57,06 \\ 16 & 1745 & 59,38 & 19 & 20.13 & 155 & 11,29\end{array}$ $16175359.381920 .13 \quad 15511.29$ $16 \quad 1843 \quad 33.25 \quad 19 \quad 17.52 \quad 15514.41$

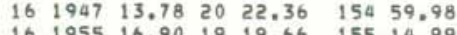
$16 \quad 195516.901902 .36 \quad 154 \quad 14.98$

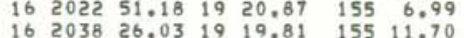

$\begin{array}{lllllll}17 & 236 & 45,58 & 19 & 18,10 & 155 & 14.14\end{array}$

$\begin{array}{rrrrrrr}17 & 943 & 46.89 & 19 & 35.03 & 155 & 10.38 \\ 17 & 1020 & 35.31 & 19 & 17.25 & 155 & 14.07\end{array}$

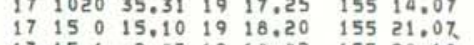

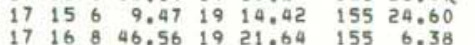
$\begin{array}{lllllll}17 & 1756 & 44.91 & 19 & 19.04 & 155 & 12.47\end{array}$

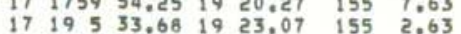

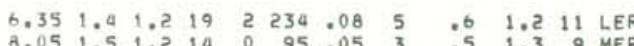

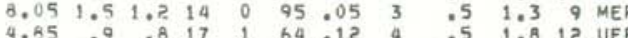
$\begin{array}{rrrrrrrrrrrr}15.50 & 1.0 & 0.4 & 12 & 1 & 171 & .25 & 3 & 2.9 & 1.1 & 7 & \text { LSW } \\ 8.56 & 1.5 & 1.4 & 27 & 2 & 79 & .05 & 3 & .4 & .6 & 17 & \text { UER }\end{array}$

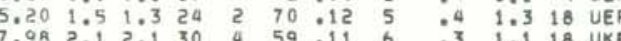

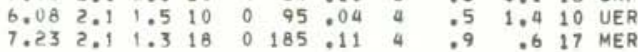
$\begin{array}{lllllllllllll}6.00 & 2.1 & 1.2 & 17 & 0 & 159 & .14 & 3 & 1.1 & 1.6 & 16 & \text { MEP }\end{array}$

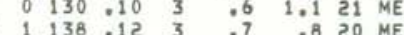

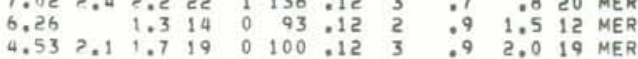
$\begin{array}{llllllllllll}8.82 & 2.8 & 2.6 & 31 & 1 & 81 & .08 & 4 & .5 & .6 & 29 & \text { MER }\end{array}$

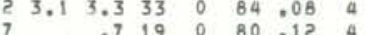

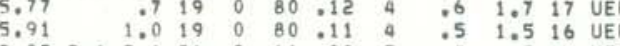

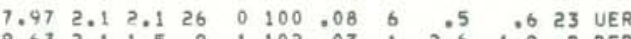

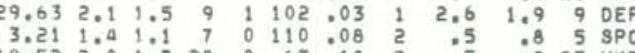

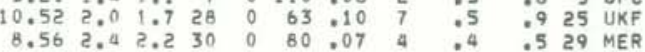

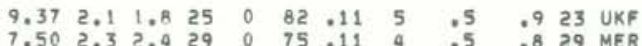

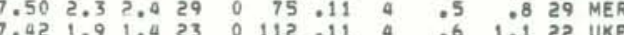

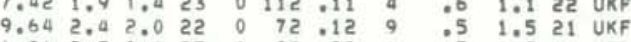
$\begin{array}{rrrrrrrrrrrr}6.77 & 1.8 & 1.5 & 23 & 0 & 61 & .12 & 3 & .5 & .8 & 23 & \text { UER }\end{array}$

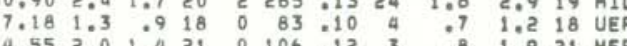
$\begin{array}{llllllllllll}4.55 & 2.0 & 1.4 & 21 & 0 & 106 & .12 & 3 & .8 & 1.9 & 21 & \text { MER } \\ 7.19 & 1.2 & 1.1 & 22 & 0 & 137 & .09 & 1 & .7 & 1.1 & 22 & \text { POL }\end{array}$ $\begin{array}{rrrrrrrrrrrrr}.09 & 3.4 & 3.1 & 32 & 0 & 308 & .14 & 65 & 12.1 & 6.7 & 32 & \text { OIS } \\ 4.83 & 1.3 & 9 & 19 & 1 & 87 & 15 & 4 & .6 & 1.8 & 19 & \text { UER }\end{array}$

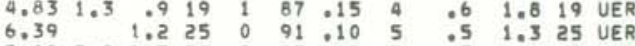

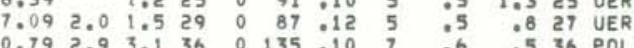
$\begin{array}{rrrrrrrrrrrr}1.44 & 2.3 & 2.1 & 23 & 0 & 79 & 10 & 15 & .5 & 1.0 & 23 & \mathrm{HIL} \\ 7.33 & 1.7 & 1.6 & 22 & 0 & 155 & : 10 & 1 & .7 & .9 & 21 & \text { POL }\end{array}$

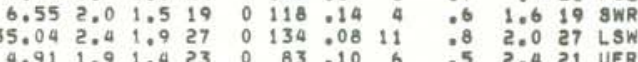
$\begin{array}{llllllllllll}5.17 & 1.3 & 1.1 & 18 & 0 & 95 & .09 & 4 & .6 & 2.0 & 17 & \text { UER }\end{array}$

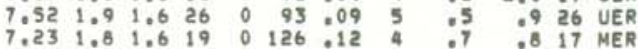
$\begin{array}{llllllllllll}6.58 & 1.1 & 1.1 & 17 & 4 & 92 & .09 & 5 & .4 & 1.1 & 4 & \\ 5 & \text { UER }\end{array}$
ORIGIN TIME LAT N LON W DEPTH AMP OUR GAP RMS MIN ERH ERZ NO YEAR MON DA HRMN SEC DEG MIN DEG MIN KM MAG MAG NR NS DEG SEC DIS KM KM FM REMK

1979 JAN $17 \quad 2126 \quad 42.44 \quad 19 \quad 19.32 \quad 155 \quad 12.97$

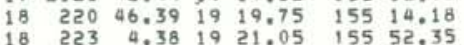

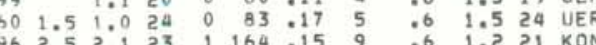

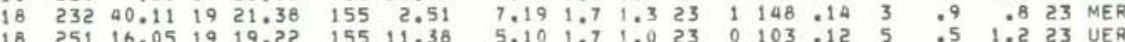

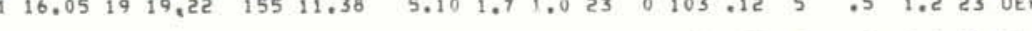
$\begin{array}{lllllllllllllllllllll}18 & 10 & 1 & 52.89 & 19 & 25.74 & 155 & 24.30 & 11.16 & 2.1 & 2.2 & 25 & 1 & 60 & .07 & 8 & .4 & 1.1 & 18 & \text { UKF } \\ 18 & 1855 & 5.08 & 19 & 19.74 & 155 & 1156 & 8.07 & 2.1 & 2.0 & 30 & 9 & 90 & .06 & 5 & .4 & .9 & 16 & \text { UER }\end{array}$

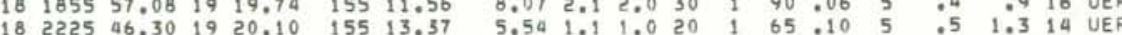

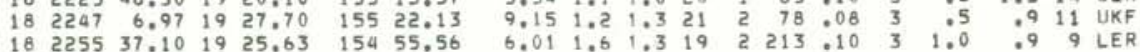

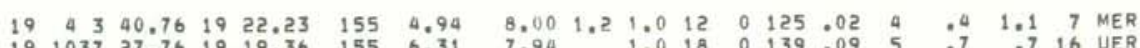

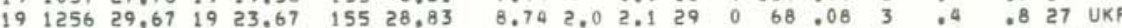

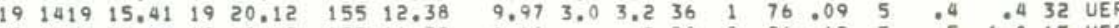

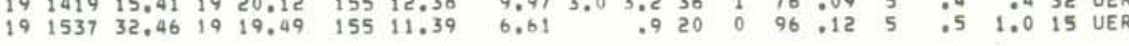

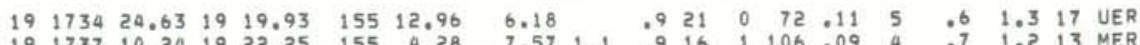

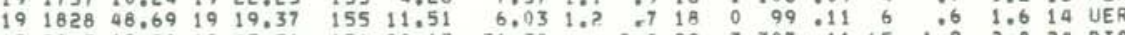

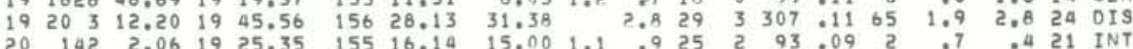

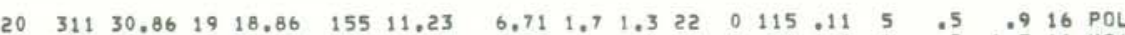

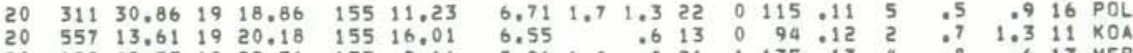

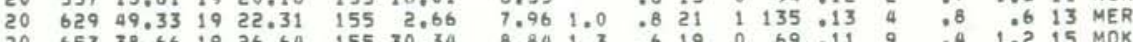

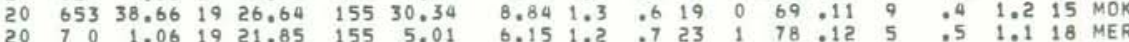

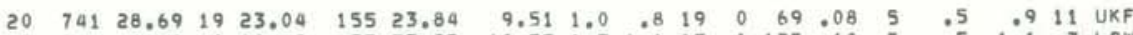

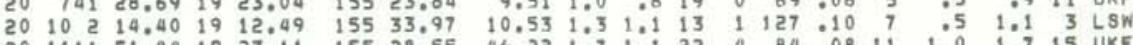
20111151.841923 .11 155 28.55 46. 251.71 .1222 4 84.08 11 1.0 1.7 15 UKF

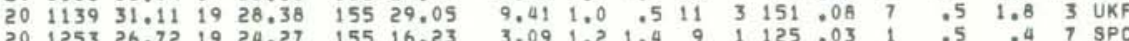

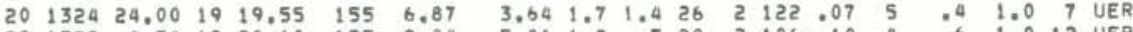

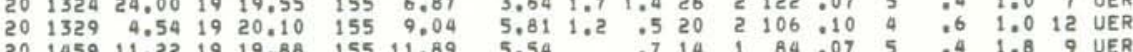

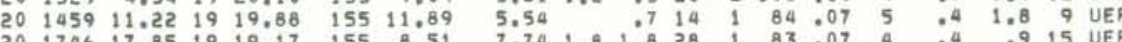

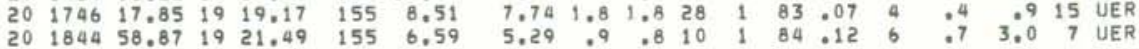

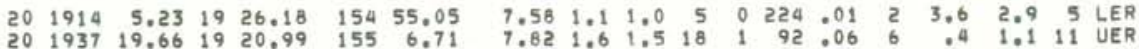

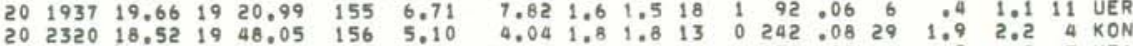

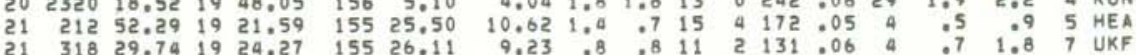

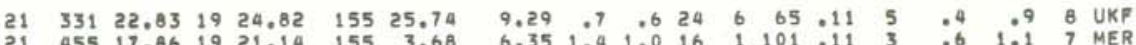

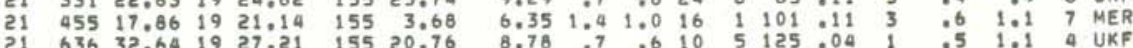

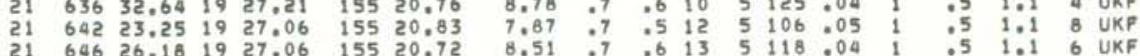

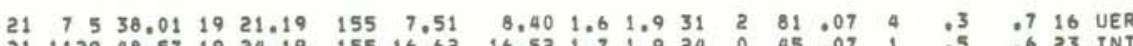

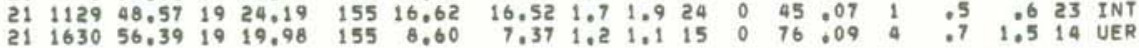


HVO EARTHQUAKE SUMMARY LIST

ORIGIN TIME LATN LON W OEPTH AMP DUR GAP RMS MIN ERH ERZ NO YEAR MON DA HRMN SEC DEG MIN DEG MIN KM MAG MAG NR NS DEG SEC OIS KM KM FM REMK $\begin{array}{rllllll}1979 \text { JAN } 21 & 1746 & 17.46 & 19 & 20.57 & 155 & 2.74 \\ 21 & 1931 & 55.29 & 19 & 11.26 & 155 & 38.17\end{array}$ $21201352.2319 \quad 7.01 \quad 15538,33$ $\begin{array}{lllllll}21 & 2125 & 11.27 & 19 & 18.85 & 155 & 20.24 \\ 22 & 040 & 14.85 & 19 & 28.16 & 155 & 28.32\end{array}$

$\begin{array}{lllllll}22 & 225 & 2.32 & 19 & 22.30 & 155 & 4.54\end{array}$

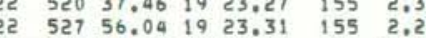

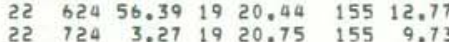

$\begin{array}{lllllll}22 & 728 & 8.07 & 19 & 22.44 & 155 & 29.01\end{array}$ $\begin{array}{lllllll}22 & 1229 & 40.35 & 19 & 21.92 & 155 & 6.45 \\ 22 & 1245 & 58.12 & 19 & 22.00 & 155 & 6.09\end{array}$

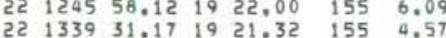
$\begin{array}{llllllr}22 & 1339 & 31.17 & 19 & 21.32 & 155 & 4.57 \\ 22 & 2025 & 14.09 & 19 & 20.06 & 155 & 13,26\end{array}$

$\begin{array}{rrrrrrr}22 & 2152 & 21.60 & 19 & 21.66 & 155 & 6.99 \\ 23 & 051 & 57.54 & 19 & 15.21 & 155 & 26.80\end{array}$ $23 \quad 449 \quad 41.301920,79 \quad 155 \quad 4.38$ $\begin{array}{lllllll}23 & 713 & 37.57 & 19 & 20.67 & 155 & 10.97\end{array}$

$\begin{array}{lllllll}23 & 852 & 29.25 & 19 & 22.35 & 155 & 5.75\end{array}$

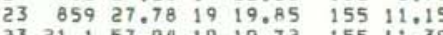
$\begin{array}{llllllll}23 & 21 & 1 & 57.94 & 19 & 19.72 & 155 & 11.39 \\ 23 & 22 & 3 & 38.86 & 19 & 23.72 & 155 & 15.73\end{array}$ $\begin{array}{lllll}24 & 140 & 29.85 & 19 & 18.06 \quad 155 \quad 13.13\end{array}$ $\begin{array}{lllllll}24 & 140 & 29.85 & 19 & 18.06 & 155 & 13.13 \\ 24 & 236 & 20.09 & 19 & 23.32 & 155 & 24.30\end{array}$ $\begin{array}{lllllll}24 & 745 & 40.82 & 19 & 19.53 & 155 & 8.24 \\ 24 & 843 & 45.03 & 19 & 20.39 & 155 & 12.49\end{array}$ $24 \quad 846 \quad 47.90 \quad 1921.04 \quad 155 \quad 4.71$

$\begin{array}{lllllll}24 & 1322 & 45.87 & 19 & 15.74 & 155 & 26.99\end{array}$

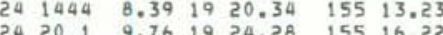
$242056 \quad 41.201924 .05 \quad 155 \quad 6.67$ $24 \quad 227 \quad 5,91 \quad 19 \quad 19,82 \quad 155 \quad 8,16$ $\begin{array}{lllllll}24 & 2228 & 24.97 & 19 & 19.48 & 155 & 10.07\end{array}$ $25020 \quad 45.09 \quad 1920.64 \quad 155 \quad 7.12$

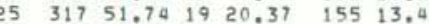
$25 \quad 812 \quad 53.691920 .68 \quad 155 \quad 6.09$ $\begin{array}{lllllll}25 & 1029 & 43.74 & 19 & 20.52 & 155 & 10.50\end{array}$ $\begin{array}{lllllll}25 & 1244 & 15.47 & 19 & 18.00 & 155 & 13.15\end{array}$

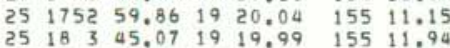

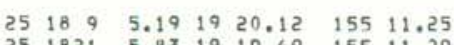

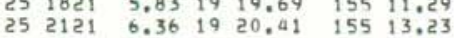

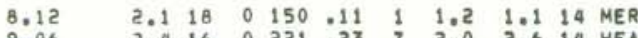

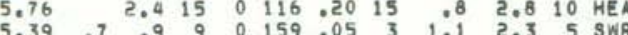

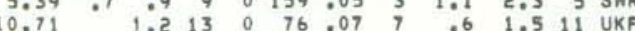
$\begin{array}{rrrrrrrrrrrr}7.66 & 1.8 & 1.8 & 16 & 0 & 85 & 011 & 4 & .6 & 1.1 & 16 & \text { MER } \\ 7.38 & & 1.2 & 13 & 1 & 129 & : 11 & 4 & 1.0 & 1.7 & 11 & \text { MER }\end{array}$

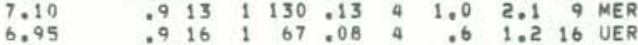
$\begin{array}{llllllllllll}6.95 & & 9 & 16 & 1 & 67 & 08 & 4 & .6 & 1.2 & 16 & \text { UER } \\ 7.30 & 1.4 & 1.0 & 18 & 0 & 71 & .08 & 2 & .6 & 1.2 & 16 & \text { UER }\end{array}$ $\begin{array}{cccccccccccc}8.84 & 1.1 & 1.2 & 19 & 0 & 114 & 10 & 2 & .5 & 1.9 & 16 & \text { UKF } \\ 6.86 & 1.4 & . & 31 & 0 & 77 & 10 & 6 & 97 & 1.4 & 20 & \text { UER }\end{array}$

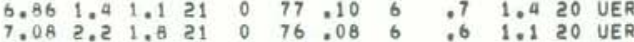

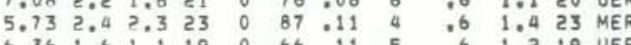

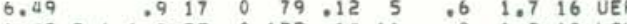

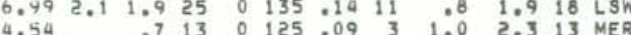

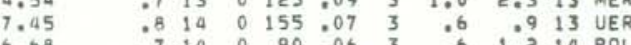

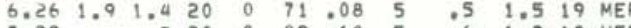

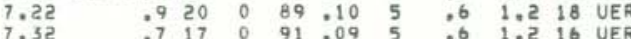

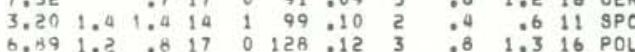
$\begin{array}{lllllllllllll}8.24 & 1.7 & 1.5 & 25 & 0 & 100 & 10 & 2 & .5 & .8 & 24 & P O L\end{array}$

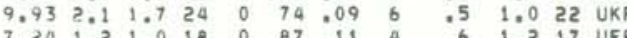

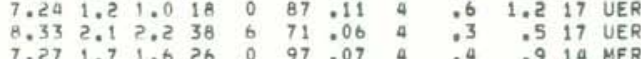
$\begin{array}{lllllllllllll}9.00 & 2.8 & 3.0 & 35 & 4 & 104 & .12 & 11 & .3 & .9 & 20 & \text { LSW }\end{array}$

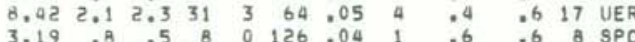

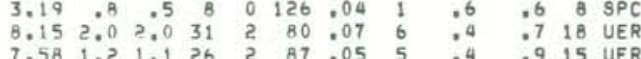
$\begin{array}{llllllllllll}7.80 & 1.0 & .7 & 17 & 0 & 108 & .03 & 5 & .6 & 1.5 & 13 & \text { UER }\end{array}$

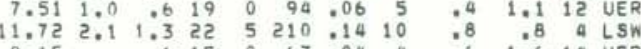

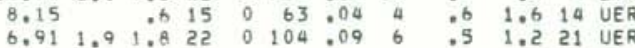
$\begin{array}{llllllllllll}9.06 & 1.3 & .9 & 13 & 0 & 77 & .04 & 3 & .7 & 1.4 & 12 & \text { UER }\end{array}$

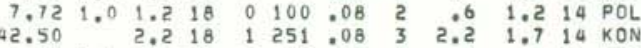
$\begin{array}{llllllllllll}8.28 & 2.4 & 2.2 & 26 & 0 & 85 & 09 & 4 & .4 & .7 & 26 & \text { UER } \\ 7.57 & & 1.0 & 19 & 0 & 82 & .07 & 5 & .6 & 1.0 & 17 & \text { UER }\end{array}$

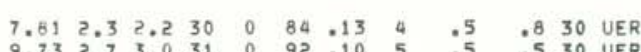

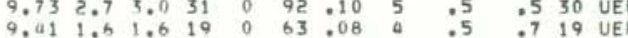

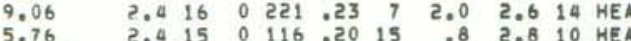

HVO EARTHQUAKE SUMMARY LIST

PAGE 8

ORIGIN TIME LAT N LON W DEPTH AMP OUR GAP RMS MIN ERH ERZ NO YEAR MON DA HRMN SEC DEG MIN DEG MIN DEPTH AMP DUR NR NS GAP RMS MIN ERH ERT NAM REMK

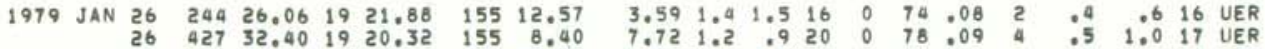

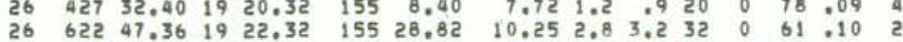

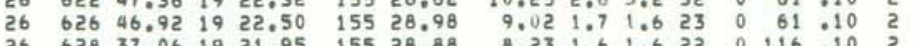

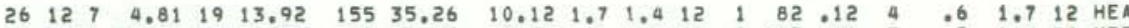

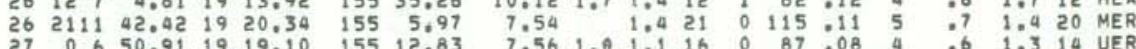

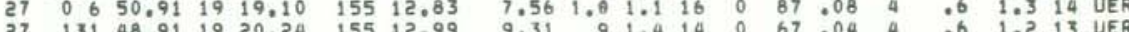

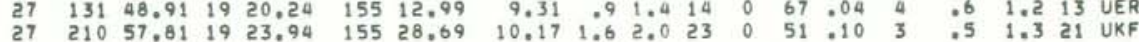

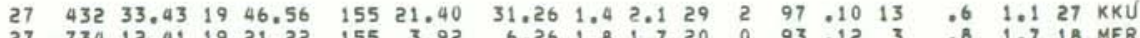

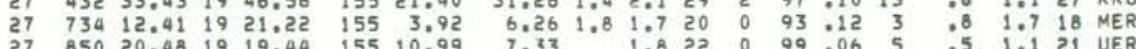

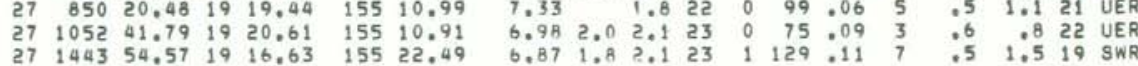

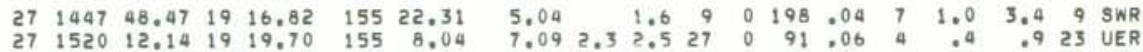

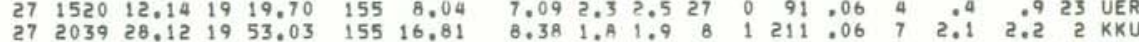

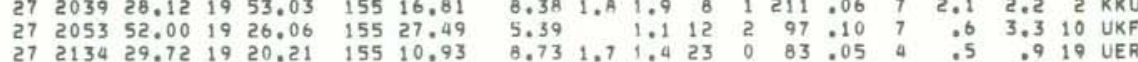

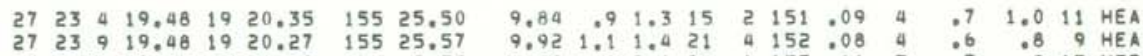

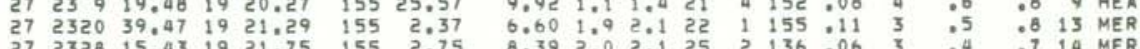

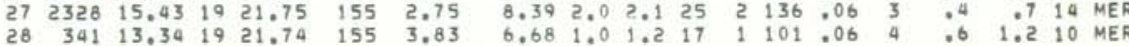
$\begin{array}{lllllllllllllllllll}28 & 347 & 25.73 & 19 & 19.76 & 155 & 12.90 & 5.81 & 1.6 & 1.6 & 22 & 3 & 74 & .05 & 5 & .3 & 1.1 & 12 & \text { UER }\end{array}$

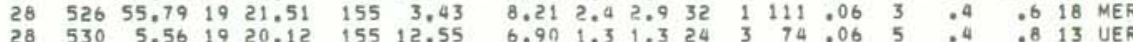

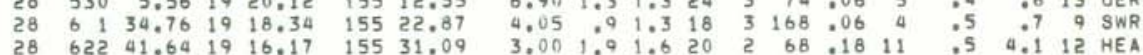
\begin{tabular}{lllllllllllllllllll}
28 & 1145 & 46.87 & 19 & 19.37 & 155 & 11.92 & 9.57 & 2.4 & 2.6 & 24 & 0 & 94 & .09 & 5 & .5 & .8 & 19 & 0 \\
\hline
\end{tabular}

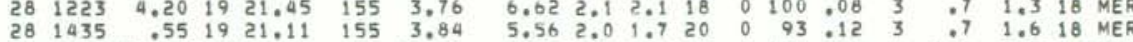

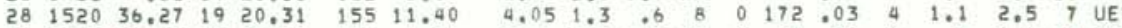

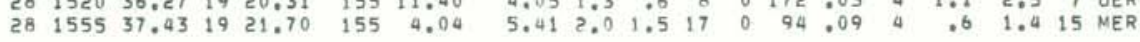

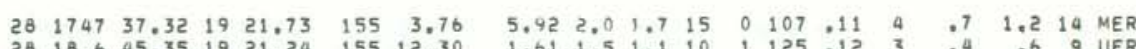

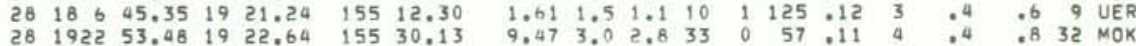

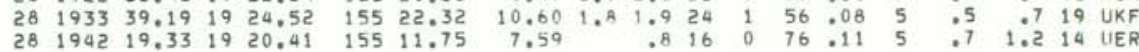

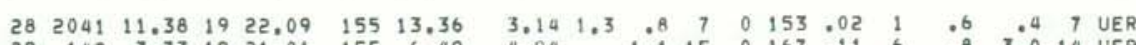

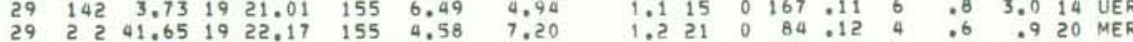

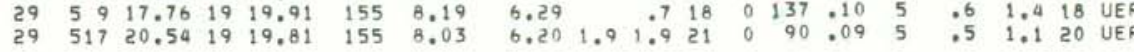
$\begin{array}{llllllllllllllllll}29 & 716 & 9.30 & 19 & 21.12 & 155 & 8.84 & 7.0 R & 1.6 & 18 & 0 & 72 & .12 & 3 & .7 & 1.4 & 14 & \text { UER }\end{array}$

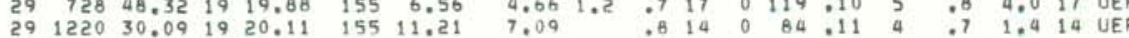


ORIGIN TIME LAT N LON W DEPTH AMP DUR GAP RMS MIN ERH ERZ NO YEAR MON DA HRMN SEC DEG MIN DEG MIN XM MAG HAG NR NS DEG SEC DIS KM KM FM REMK $\begin{array}{lllllll}29 & 1250 & 24.69 & 19 & 24.09 & 155 & 15.85\end{array}$ $29 \begin{array}{llllll}1342 & 35.70 & 19 & 23.87 & 155 & 17.2\end{array}$

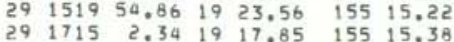

$29 \begin{array}{lllll}1843 & 44.67 \quad 19 & 19.57 \quad 155 \quad 11.72\end{array}$

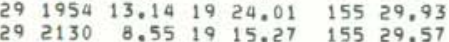

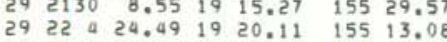

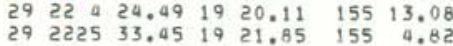

$\begin{array}{lllllll}30 & 050 & 51.36 & 19 & 20.18 & 155 & 13.04 \\ 30 & 252 & 26.48 & 19 & 20.17 & 155 & 11.44\end{array}$

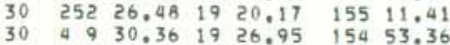
$\begin{array}{lllllll}30 & 633 & 21,15 & 19 & 22.80 & 155 & 2.01\end{array}$

$\begin{array}{lllllll}30 & 912 & 41.47 & 19 & 23.46 & 155 & 2.66\end{array}$ $\begin{array}{lllllll}30 & 931 & 1.58 & 19 & 18.70 & 155 & 13.66\end{array}$ $30104625.8319 \quad 9.73 \quad 15533.80$

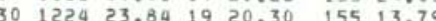
$\begin{array}{lllllll}30 & 1328 & 10.23 & 19 & 26.90 & 155 & 51.48 \\ 30 & 1717 & 50.89 & 19 & 26.94 & 155 & 30.11\end{array}$ $\begin{array}{llllllll}30 & 1816 & 7.11 & 19 & 25.59 & 155 & 24.66 \\ 30 & 2257 & 6 & 33 & 19 & 20.77 & 155 & 7.43\end{array}$ $\begin{array}{llllll}30 & 2312 \quad 35.72 & 19 & 19.46 \quad 155 \quad 7.85\end{array}$ $\begin{array}{lllllll}30 & 2332 & .45 & 19 & 20.10 & 155 & 8.85\end{array}$

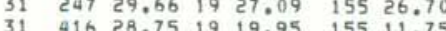
$\begin{array}{llllllll}31 & 525 & 8.86 & 19 & 22.38 & 155 & 11.75\end{array}$ $\begin{array}{llllllll}31 & 9 & 0 & 51.70 & 19 & 21.70 & 155 & 18.45\end{array}$ $\begin{array}{llllllll}31 & 99 & 34.33 & 19 & 19.76 & 155 & 7.98\end{array}$

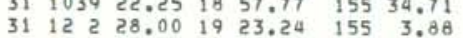

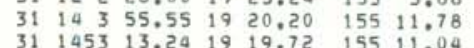
$\begin{array}{lllllll}31 & 150 & 39.75 & 19 & 46.22 & 156 & 15.38\end{array}$

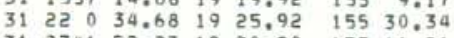
$\begin{array}{lllllll}31 & 2341 & 52.27 & 19 & 20.20 & 155 & 11.21\end{array}$

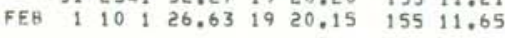
1
1 $1043 \quad 15.07 \quad 19 \quad 20.76 \quad 155 \quad 11.83$

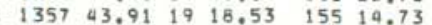
$\begin{array}{lllllll}1 & 1425 & 32.21 & 20 & 30.58 & 155 & 35.75 \\ 1 & 1830 & 26.02 & 20 & 23.09 & 155 & 3.26\end{array}$

$\begin{array}{lllllll}1 & 1935 & 46.49 & 19 & 20.84 & 155 & 2.31\end{array}$

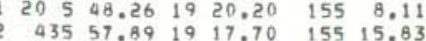

$\begin{array}{lllllllllllllll}3.06 & 1.6 & 1.6 & 14 & 1 & 117 & .07 & 3 & .3 & .5 & 12 & \mathrm{SPC}\end{array}$

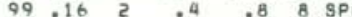

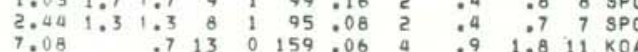

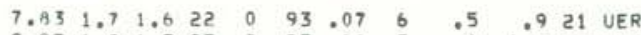

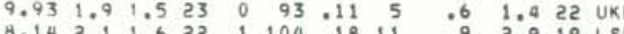

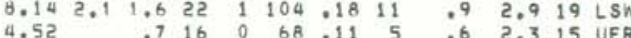

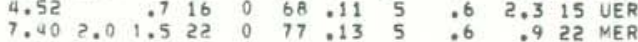
$\begin{array}{llllllllll}7.21 & 1.4 & 19 & 0 & 68 & .12 & 5 & .6 & 1.2 & 17\end{array}$

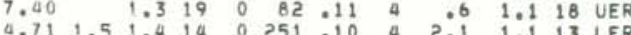

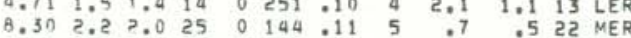

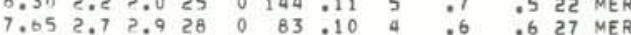

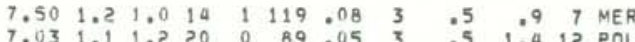

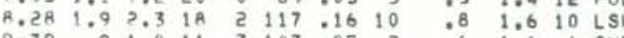

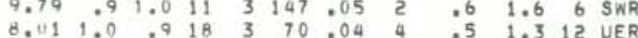
$\begin{array}{rrrrrrrrrrrr}7.71 & 1.4 & 1.1 & 11 & 2 & 122 & 06 & 8 & .8 & 1.6 & 7 & \text { KON } \\ 9.48 & 1.0 & 1.3 & 18 & 4 & 91 & .05 & 9 & .5 & 1.1 & 13 & \text { MOK }\end{array}$

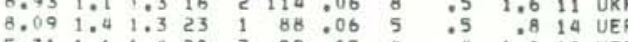

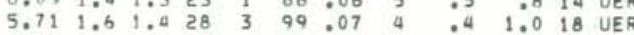
$\begin{array}{lllllllllllll}7.53 & 1.3 & .9 & 19 & 1 & 72 & 05 & 4 & .4 & 1.0 & 11 & \text { UER } \\ 7.42 & 19 & 0.9 & 18 & 4 & 62 & 10 & 8 & 04 & 1.3 & 12 & \text { UKF }\end{array}$

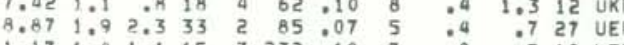

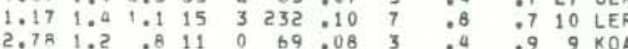
$\begin{array}{lllllllllll}5.62 & .8 & 18 & 0 & 91 & .09 & 4 & .6 & 1.8 & 16 & \text { UER }\end{array}$

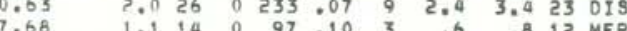

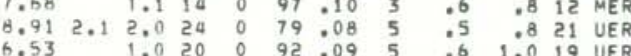
$\begin{array}{llllllllllll}12 . & 47 & 1.7 & 13 & 0 & 317 & .08 & 45 & 15.9 & 52.5 & 11 & 019\end{array}$

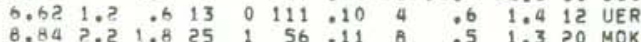

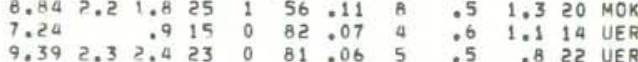
$\begin{array}{lllllllllllll}7.64 & 1.4 & 1.6 & 16 & 0 & 71 & 08 & 4 & .0 & 1.1 & 14 & \text { UER }\end{array}$

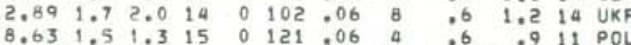

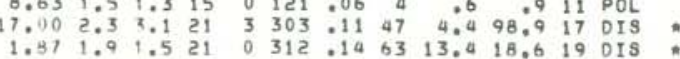
$\begin{array}{llllllllllll}8.60 & 1.2 & 1.3 & 12 & 0 & 169 & .06 & 2 & 1.1 & 1.4 & 10 & \text { MER } \\ 7.38 & 1.5 & 2.4 & 17 & 0 & 84 & .07 & 5 & 0 & 1.4 & 17 & \text { MER }\end{array}$

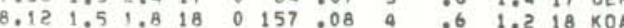

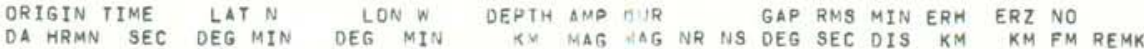

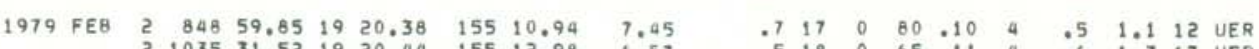

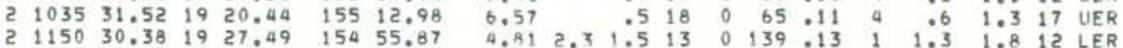

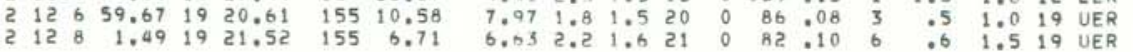

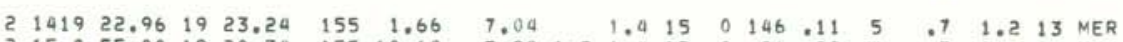

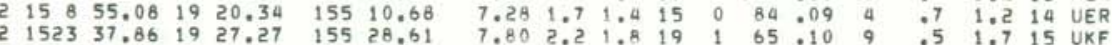
$\begin{array}{llllll}1631 & 36.00 & 18 & 53.86 & 155 & 23.43 \quad 28.09\end{array}$

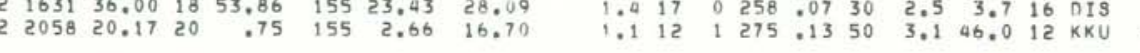

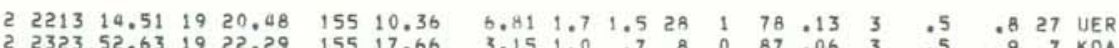
$\begin{array}{llllllllllll}5.81 & 1.5 & 13 & 0 & 109 & .15 & 4 & .8 & 20 & 11 & 0 & 0\end{array}$

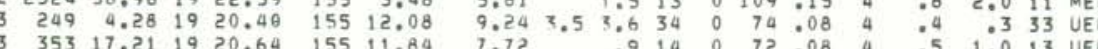

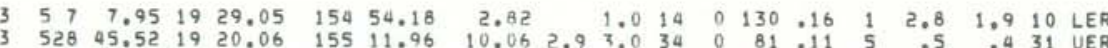

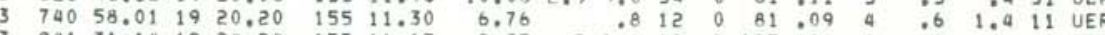

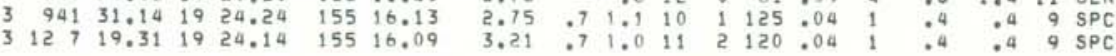

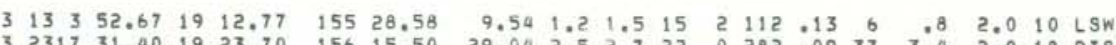

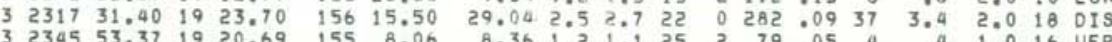

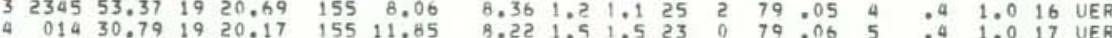

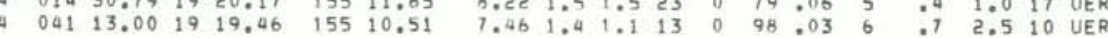

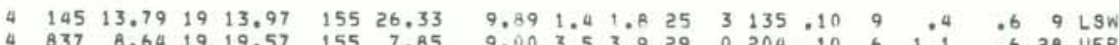

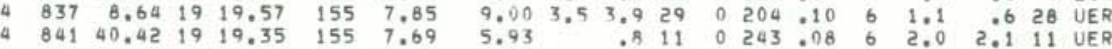

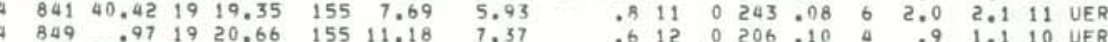

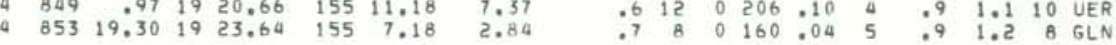

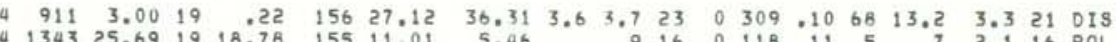

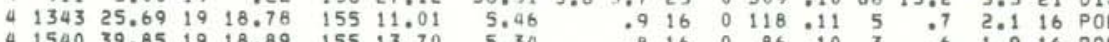

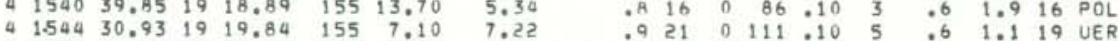

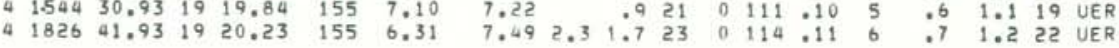

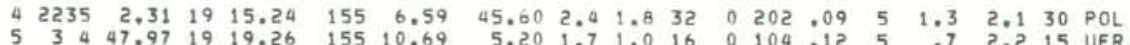

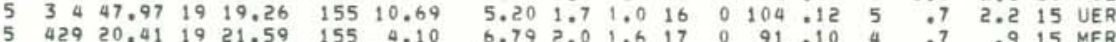

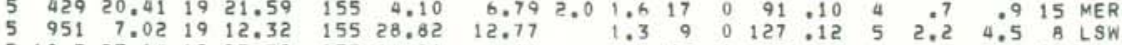

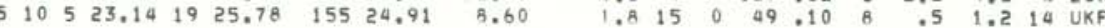

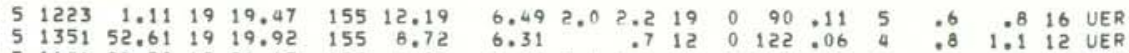

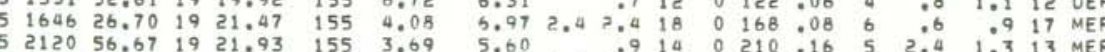

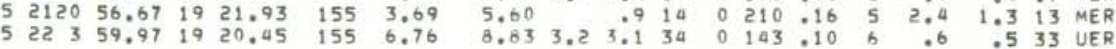

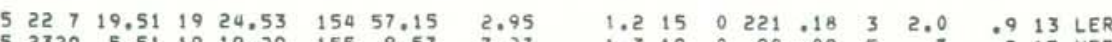

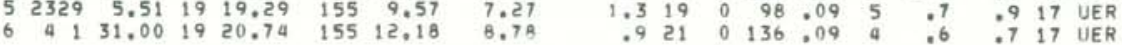




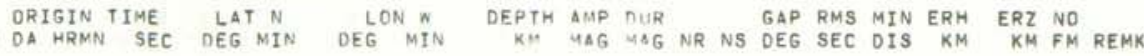

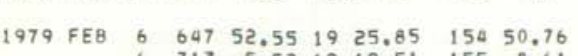
$\begin{array}{lrrrrrr}6 & 713 & 5.52 & 19 & 19.51 & 155 & 8.61 \\ 6 & 9 & 0 & 17.98 & 19 & 27.22 & 155 \\ 6 & 1 & 179 & 52\end{array}$

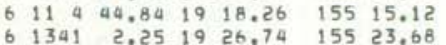
$\begin{array}{lllllll}6 & 1651 & 18.16 & 19 & 22.76 \quad 155 \quad 6.46\end{array}$

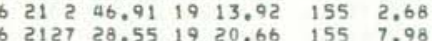
$62144 \quad 38.35 \quad 19 \quad 19.68$ - 155 15.

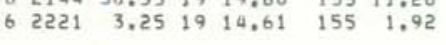

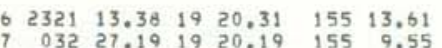

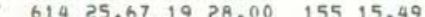
$\begin{array}{lllllll}7 & 640 & 24.33 & 19 & 34.50 & 155 & 52.88 \\ 7 & 743 & 28.58 & 19 & 19.46 & 155 & 28.73\end{array}$

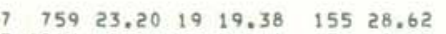

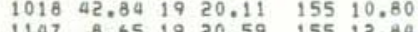

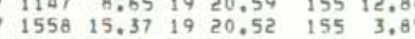

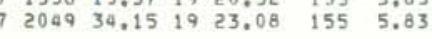

\begin{tabular}{l}
$72224 \quad 29.07 \quad 1920.93 \quad 155 \quad 1.84$ \\
\hline
\end{tabular}

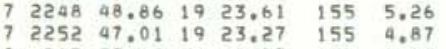
$\begin{array}{lllllll}8 & 217 & 35.69 & 19 & 19.37 & 155 & 7.07 \\ 8 & 410 & 37.51 & 19 & 21.01 & 155 & 10,78\end{array}$

$\begin{array}{lllllll}618 & 39.02 & 19 & 19.71 & 155 & 8.35\end{array}$

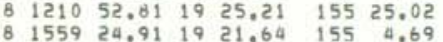

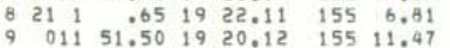
$\begin{array}{rrrrrrr}9 & 232 & 5.02 & 19 & 21.43 & 155 & 2.90 \\ 9 & 4 & 599.63 & 19 & 20.79 & 155 & 11.45\end{array}$

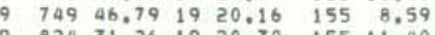
4.82431 .261920 .130 155 11.40

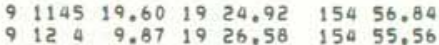
$131613.121918 .45 \quad 15546.26$ Q $184758.261919 .79 \quad 155 \quad 7.54$

$92016 \quad 31.34 \quad 19$
$90.21 \quad 155 \quad 8.57$ $205944.481924 .04 \quad 15524,38$
9 $92224 \quad 45.46 \quad 1919.59 \quad 155 \quad 11.46$ $10 \quad 09 \quad 7,47 \quad 19 \quad 19,49 \quad 155 \quad 30,26$ $\begin{array}{lllllll}10 & 017 & 35.28 & 19 & 20.85 & 155 & 30.16 \\ 10 & 021 & 22.63 & 19 & 22,30 & 155 & 5.03\end{array}$

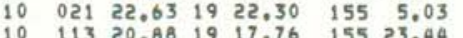

$\begin{array}{lllllllllllll}8.98 & 2.6 & 2.5 & 25 & 0 & 286 & .16 & 7 & 3.8 & .7 & 22 & \text { LER }\end{array}$

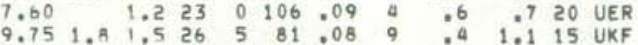

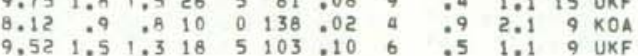
$\begin{array}{rrrrrrrrrrrr}.81 & 2.7 & 3.3 & 21 & 1 & 84 & .07 & 5 & .3 & .8 & 11 & \text { UER }\end{array}$

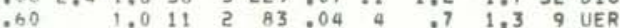

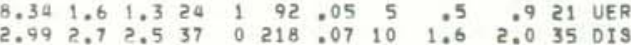
$\begin{array}{llllllllllll}8.25 & 1.2 & .9 & 19 & 3 & 67 & .04 & 4 & .5 & 1.2 & 15 & \text { UER }\end{array}$

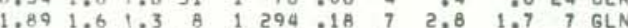

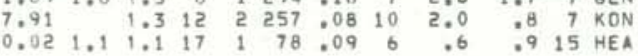
$\begin{array}{lllllllllllll}1.26 & 1.1 & .8 & 14 & 1 & 142 & .06 & 6 & .6 & .8 & 12 & \text { HEA }\end{array}$

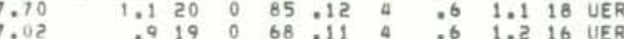

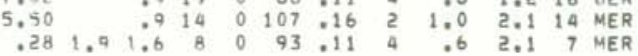
$\begin{array}{lllllllllll}4.85 & 1.0 & 13 & 0 & 182 & .10 & 3 & 1.2 & 1.9 & 13 & \text { MER }\end{array}$

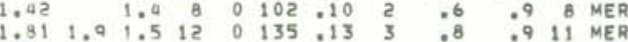

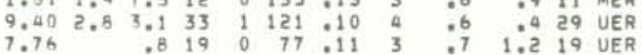

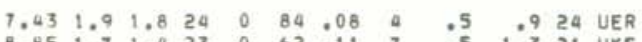

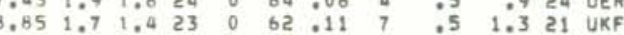
$\begin{array}{llllllllllll}5.85 & 2.3 & 2.5 & 28 & 0 & 80 & 11 & 4 & .5 & 1.4 & 27 & \text { MER } \\ 6.78 & 1.9 & 1.9 & 28 & 0 & 73 & 11 & 5 & : 5 & 1.3 & 25 & \text { UER }\end{array}$ $\begin{array}{llllllllllll}6.78 & 1.9 & 1.9 & 28 & 0 & 73 & .11 & 5 & .5 & 1.3 & 25 & \text { UER } \\ 10.42 & 2.6 & 2.9 & 31 & 1 & 83 & .09 & 5 & .9 & .6 & 28 & \text { UER }\end{array}$ $\begin{array}{lllllllllllll}6.35 & 1.9 & 2.2 & 26 & 1 & 132 & .13 & 3 & .7 & 1.0 & 23 & \text { MER } \\ 7.25 & 1.6 & 1.6 & 37 & 0 & 75 & .13 & 4 & .6 & .9 & 26 & \text { UER }\end{array}$

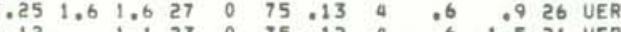

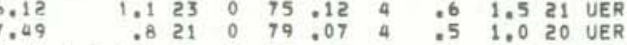

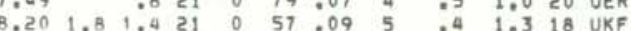

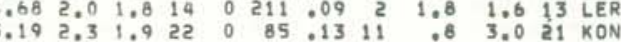

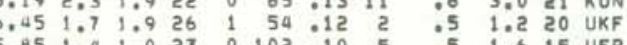

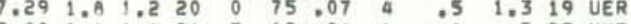

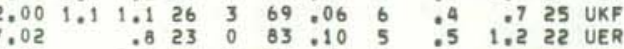

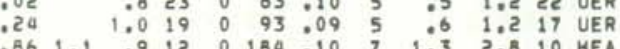
$\begin{array}{lllllllllllll}8.10 & 1.3 & 1.0 & 13 & 0 & 141 & 05 & 5 & 09 & 1.4 & 10 & \text { MEA } \\ 8.49 & 1.3 & 1.1 & 16 & 0 & 76 & .07 & 4 & \text {.5 } & 1.2 & 12 & \text { MER }\end{array}$

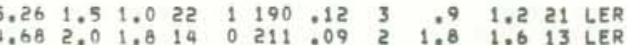

YEAR MON ORIGIN TIME LAT SEC OEG MIN DEG MIN 1979 FEB 10

10 3.6 $9.691923 .71 \quad 155 \quad 15.53$

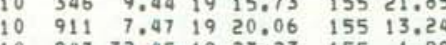
$\begin{array}{rrrrrrr}10 & 947 & 32.45 & 19 & 23.23 & 155 & 1.99 \\ 10 & 1050 & 39.03 & 19 & 20.38 & 155 & 6.46\end{array}$ $\begin{array}{lllllll}10 & 1720 & .28 & 19 & 21.68 & 155 & 12.70\end{array}$ $10202056.57 \quad 1920.61 \quad 15510.37$ $\begin{array}{lllllll}10 & 2121 & 48.69 & 19 & 20.63 & 155 & 13.15 \\ 10 & 2359 & 41.39 & 19 & 19.83 & 155 & 10.27\end{array}$

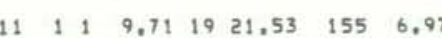

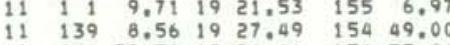
$11 \quad 958 \quad 58.71 \quad 1926.66 \quad 154 \quad 55.04$

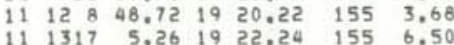
$\begin{array}{lllllll}11 & 1447 & 3.99 & 19 & 22.02 \quad 155 & 4.19\end{array}$ $\begin{array}{llllllll}1 & 15 & 9 & 50.30 & 19 & 16.09 & 155 & 15.77\end{array}$ $1119721.6419 \quad 15.38 \quad 155 \quad 15.05$

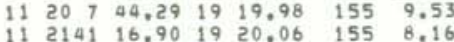
$\begin{array}{lllllll}12 & 218 & 25.44 & 19 & 23.62 & 154 & 55.53\end{array}$ $12 \quad 232 \quad 19.591921 .41$ 155 3.46 $12 \quad 4$ a $32.9019 \quad 18.63 \quad 155 \quad 13.17$

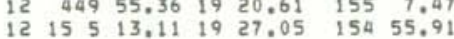

$12 \quad 1533 \quad 49.971921 .73 \quad 155 \quad 5.81$ $12 \quad 1922 \quad 39.27 \quad 1920.29 \quad 155 \quad 3.19$ $121933 \quad 8.931919 .63 \quad 155$ 13.4 $121938 \quad 55.6919 \quad 19.86 \quad 155 \quad 10.41$

$\begin{array}{rrrrrrr}12 & 1946 & .34 & 19 & 21.99 & 155 & 5.01 \\ 12 & 1956 & 22.92 & 19 & 23.54 & 154 & 56.33\end{array}$ $122251 \quad 42.591919 .85$ 155 8.00

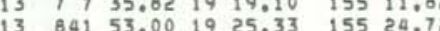

$\begin{array}{lllllll}13 & 917 & 31.69 & 19 & 14.13 & 155 & 26,47\end{array}$

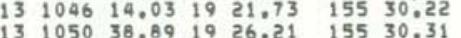

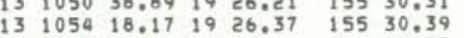
$13 \quad 105533.031920 .48 \quad 15512.75$

$\begin{array}{lllllll}13 & 1628 & 31.37 & 19 & 25.96 & 155 & 25.23 \\ 13 & 1652 & 51.05 & 19 & 20.60 & 155 & 4.25\end{array}$ $13 \quad 165251.051920 .60 \quad 155 \quad 4.25$ $13193429.41 \quad 1929.43 \quad 15526.21$

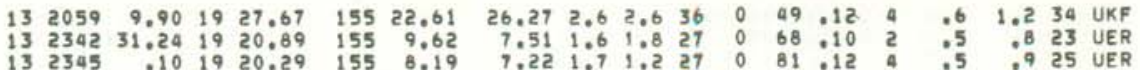

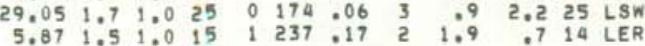

KM MAG WAG NR NS OEG SEC OIS KM KM FM REMK $\begin{array}{lllllllllllll}2.99 & 1.2 & .8 & 8 & 0 & 98 & .12 & 3 & 1.1 & 1.7 & 6 & \text { SPC } & \end{array}$ $\begin{array}{lllllllllll}52 & 2.0 & 1.9 & 10 & 0 & 87 & .03 & 5 & .8 & 1.5 & 10 \\ 0\end{array}$

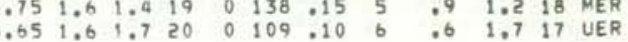

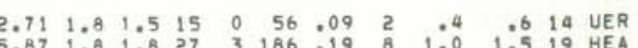
$\begin{array}{llllllllllll}.46 & 1.6 & 1.0 & 22 & 0 & 75 & .12 & 3 & .6 & 1.1 & 17 & \text { UER }\end{array}$ $\begin{array}{llllllllllll}7.80 & 1.5 & 1.1 & 18 & 0 & 62 & .12 & 4 & .6 & 1.0 & 17 & \text { UER } \\ 8.39 & .9 & .8 & 17 & 0 & 90 & .07 & 4 & .6 & 1.3 & 17 & \text { UER }\end{array}$ $\begin{array}{lllllllllll}.13 & 1.6 & 1.5 & 22 & 0 & 80 & .10 & 5 & .5 & 1.3 & 21 \\ \text { UER }\end{array}$ $\begin{array}{lllllllllll}5.92 & .9 & 14 & 0 & 208 & 012 & 2 & 2.2 & 1.7 & 14 & \text { LER }\end{array}$

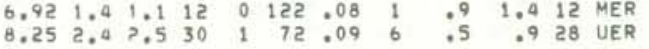

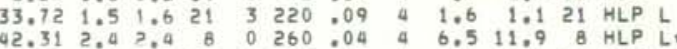

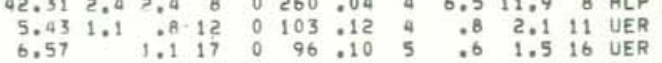

$\begin{array}{llllllllll}.12 & 1.0 & 9 & 0 & 241 & .12 & 6 & 1.5 & 3.6 & 9 \text { LER }\end{array}$

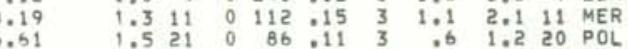

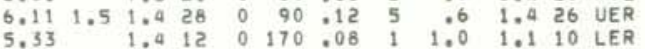

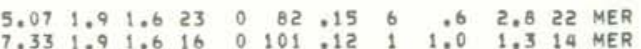

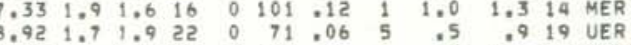

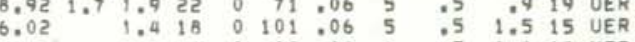

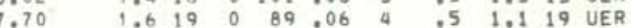
$\begin{array}{lllllllllllll}7.25 & 2.0 & 1.8 & 20 & 0 & 75 & .11 & 5 & .6 & 1.2 & 20 & \text { MER } \\ 5.73 & .5 & .5 & 1.6 & 0 & 213 & .15 & 5 & 1.5 & 1.2 & 15 & \text { LER }\end{array}$

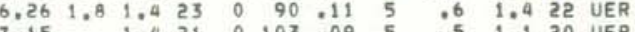

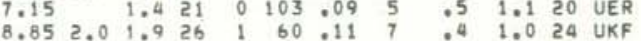

$\begin{array}{rrrrrrrrrrrr}36.51 & 2.4 & 2.1 & 29 & 0 & 117 & .08 & 9 & .9 & 2.0 & 28 & \text { LSW } \\ 7.49 & 2.5 & 2.5 & 29 & 0 & 61 & .11 & 5 & 04 & 1.0 & 25 & \text { HEA }\end{array}$

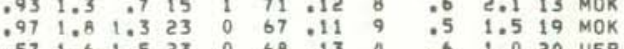

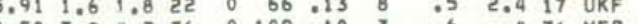

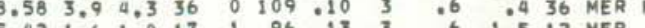

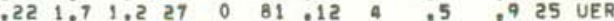
$\begin{array}{llllllllllllll}5.34 & 1.6 & 1.3 & 19 & 0 & 92 & .16 & 4 & .7 & 2.4 & 16 & \text { MER }\end{array}$ 
ORIGIN TIME
OA HRMN SAT N
DEG MIN 1979 FEB $14 \quad 040 \quad 46.64 \quad 19 \quad 15.05 \quad 155 \quad 33.29$ 1432354.96 19 20.42 15511.38

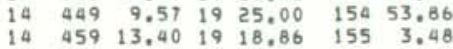
$\begin{array}{lllllll}14 & 511 & 41.87 & 19 & 21.59 & 155 & 2.69\end{array}$ $1465324,19 \quad 1928,04 \quad 155 \quad 23,76$

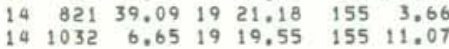

$\begin{array}{llllllll}14 & 13 & 0 & 31.53 & 20 & 19.03 & 154 & 53.77\end{array}$

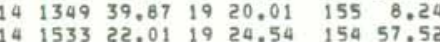

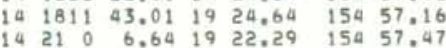

$\begin{array}{lllllll}14 & 2212 & 48.30 & 19 & 19.02 & 155 & 11.15\end{array}$

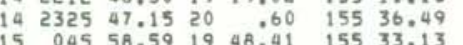

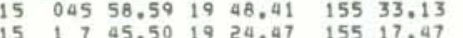

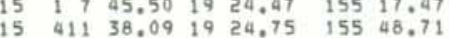

$\begin{array}{lllllll}15 & 421 & 12.28 & 19 & 23.91 & 155 & 15.49\end{array}$

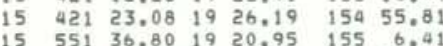
$\begin{array}{rrrrrrr}15 & 551 & 36.80 & 19 & 20.95 & 155 & 6.41 \\ 15 & 856 & 35.63 & 19 & 19.56 & 155 & 11.61\end{array}$ $15 \quad 9116,96 \quad 1920.11 \quad 155 \quad 4,21$

$\begin{array}{lllllll}15 & 1011 & 29.74 & 19 & 20.65 & 155 & 10.87 \\ 15 & 1635 & 34.48 & 19 & 19.86 & 155 & 8.48\end{array}$

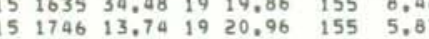

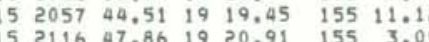

$\begin{array}{lllllll}15 & 2117 & 48.54 & 19 & 20.79 & 155 \quad 3.00\end{array}$

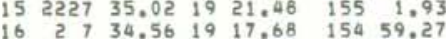

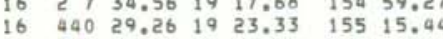

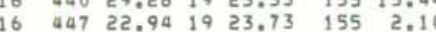

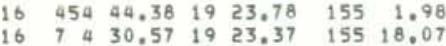

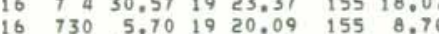

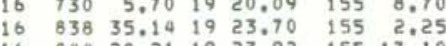
$\begin{array}{lllllll}16 & 1125 & 40.22 & 19 & 20.28 & 155 & 12.93\end{array}$

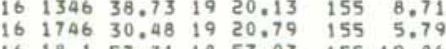
$\begin{array}{llllllll}16 & 18 & 1 & 57.71 & 18 & 53.03 & 155 & 19.86 \\ 16 & 21 & 9 & 20.25 & 19 & 22.52 & 155 & 17.09\end{array}$ $\begin{array}{lllllll}16 & 2151 & 24.44 & 19 & 24.24 & 155 & 16.96\end{array}$

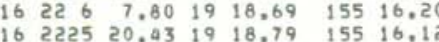

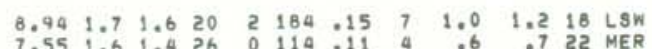

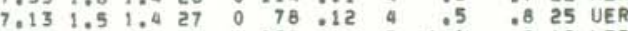

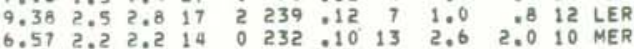
$\begin{array}{llllllllllll}5.11 & 1.2 & .8 & 12 & 1 & 218 & .20 & 10 & 2.2 & 9.7 & 11 & \text { MER }\end{array}$

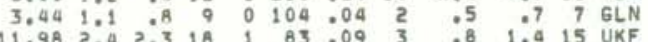

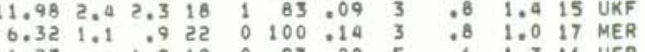

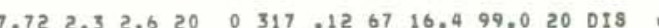

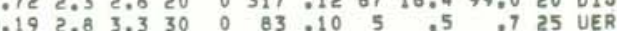

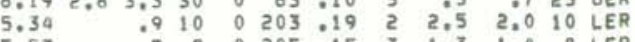
$\begin{array}{llllllllllll}5.53 & 5 & 9 & 0 & 205 & 15 & 3 & 1.3 & 1.4 & 8 & \text { LER } \\ 7.60 & 9 & 7 & 0 & 216 & 06 & 6 & 1.3 & 2.1 & 7 \text { LER }\end{array}$

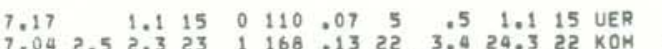

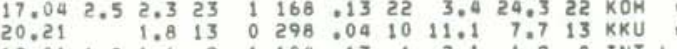
$\begin{array}{lllllllllllll}12,01 & 1.8 & 1.6 & 9 & 1 & 104 & 13 & 1 & 2.1 & 1.9 & 8 & \text { INT } \\ 10,31 & 1.8 & 1.6 & 16 & 1 & 177 & .12 & 12 & .9 & 9 & 16 & \text { KON }\end{array}$

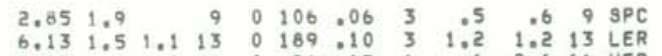
$\begin{array}{lllllllllll}5.83 & 1.0 & 16 & 0 & 96 & .12 & 6 & .6 & 2.1 & 16 & \text { UER } \\ 7.56 & 2.2 & 2.0 & 26 & 0 & 93 & 010 & 6 & .6 & .9 & 25 \\ 5 & \text { UER }\end{array}$

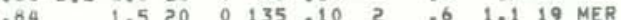

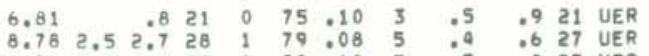

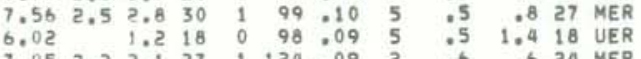

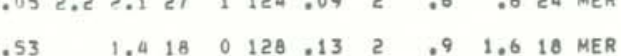

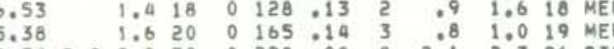
$\begin{array}{llllllllllll}39.38 & 2.9 & 2.9 & 30 & 0 & 228 & 00 & 8 & 2.1 & 2.3 & 26 & \text { OIS } \\ 11.42 & 2.1 & 2.5 & 10 & 1 & 168 & 0.07 & 2 & 1.2 & .9 & 9 & \text { INT }\end{array}$ $\begin{array}{lllllllllllll}6.46 & 1.1 & 17 & 0 & 128 & 0.10 & 4 & .8 & 1.0 & 17 & \text { MER }\end{array}$

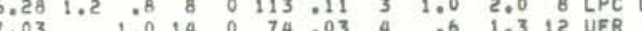

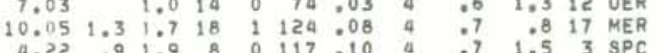
$\begin{array}{llllllllllll}9.16 & 1.3 & 14 & 0 & 68 & .03 & 4 & .7 & 1.3 & 14 & \text { UER }\end{array}$

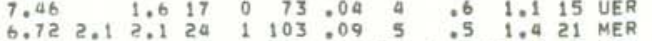

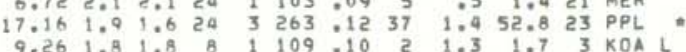
$\begin{array}{rrrrrrrrrrrr}6.62 & 1.1 & 1.9 & 9 & 0 & 82 & .12 & 1 & .8 & 1.6 & 1 & \text { LPC L }\end{array}$

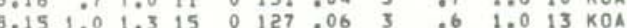

YEAR MON OAR HRMN SEC DEG MIN DEG MIN

1979 FEB $17 \quad 031 \quad 30.6919 \quad 19.51 \quad 155 \quad 10.57$ $\begin{array}{lllllll}17 & 033 & 53.10 & 19 & 20.63 & 155 & 7.43\end{array}$ $17 \quad 64919.351923 .60 \quad 155 \quad 16.6$

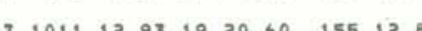

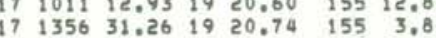

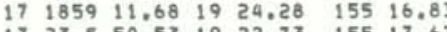
$1723 \quad 5 \quad 50.531922 .73 \quad 155 \quad 17.67$

$\begin{array}{lllllll}18 & 039 & 39.22 & 19 & 18.09 & 155 & 13.12\end{array}$

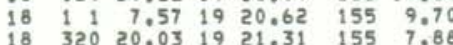
$\begin{array}{lllllll}18 & 320 & 20.03 & 19 & 21.31 & 155 & 7.88 \\ 18 & 423 & 7.25 & 19 & 23.71 & 155 & 16.45\end{array}$ $\begin{array}{llrllll}18 & 423 & 7.25 & 19 & 23.71 & 155 & 16.45 \\ 18 & 443 & 52.93 & 19 & 27.20 & 155 & 29.13\end{array}$

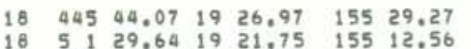
$\begin{array}{lllllll}18 & 522 \quad 031 & 19 & 26.76 & 155 & 29.62\end{array}$

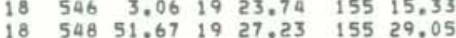

$\begin{array}{lllllll}18 & 552 & 53.96 & 19 & 27.27 & 155 & 29.21 \\ 18 & 630 & 32.16 & 19 & 27.210 & 155 & 29.19\end{array}$

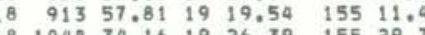
$\begin{array}{llllll}18 & 1158 & 49.21 & 19 & 19.88 & 155\end{array}$

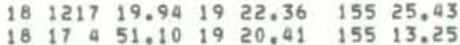
18 17 $491.101920 .46155 \quad 13.25$ $18 \quad 1950 \quad 55.62 \quad 19 \quad 30.63 \quad 155 \quad 47.03$

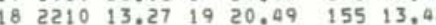

$\begin{array}{llllllll}19 & 320 & 2.90 & 19 & 30.78 & 155 & 47.46\end{array}$ $19 \quad 448 \quad 23.801921 .55 \quad 15459.98$ $1964358.4619 \quad 18.92 \quad 15512,90$ $19 \quad 65421.26 \quad 1923.85 \quad 155 \quad 16,10$

$19 \quad 759 \quad 6.74 \quad 1920.44 \quad 155 \quad 13.19$ $19112935.592051 .51 \quad 15458.96$ $19 \quad 1715 \quad 30.00 \quad 1926.41 \quad 15524.96$

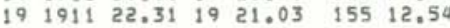
$\begin{array}{llllll}19 & 1942 \quad 31.12 \quad 19 & 20.68 \quad 155 & 10.74\end{array}$ $20 \quad 023 \quad 27.24$ 19 23,34 15515.04

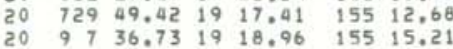
$\begin{array}{llllllll}20 & 1016 & 6.87 & 19 & 18.11 & 155 & 12.78\end{array}$ $20 \quad 1136 \quad 16,70 \quad 19 \quad 19,48 \quad 155 \quad 11,71$
DEPTH AMP DUR G GAP RMS MIN ERH ERZ NO

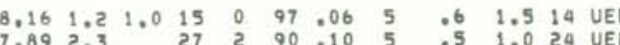
$7.771_{3} 1.910$ 1 $89: 10$ 2 :8 $1,33_{3}$ LPC

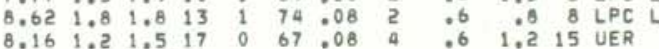

$9.48 \quad 1.51 .917 \quad 0 \quad 65.04 \quad 4 \quad .5 \quad 1.017$ UER

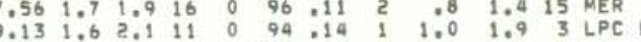
13.57 1.8 2.188 \& $106: 10 \quad 3 \quad 1.6 \quad 1.8$ O INT

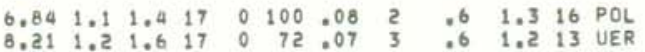

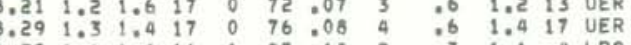

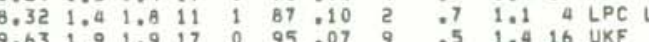
$\begin{array}{llllllllllll}10.58 & 3.2 & 3.5 & 37 & 1 & 46 & .11 & 9 & .4 & .6 & 36 & \text { UKF }\end{array}$

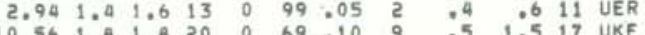

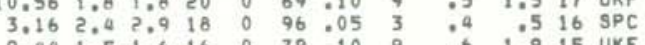
$\begin{array}{cccccccccccc}10.00 & 2.1 & 2.4 & 22 & 1 & 72 & .12 & 9 & .5 & 1.4 & 20 & \text { UKF } \\ 9.76 & 2.2 & 2.4 & 23 & 1 & 71 & .09 & 9 & .5 & 1.3 & 20 & \text { UKF } \\ 0.30 & .9 & .0 & 9 & 0 & 96 & .09 & 5 & .6 & 9.6 & 10 & \text { UKF }\end{array}$ $\begin{array}{llllllllllll}6.30 & .9 & 1.0 & 17 & 0 & 94 & .09 & 5 & .6 & 1.6 & 16 & \text { UER } \\ 9.72 & 9.4 & 0.7 & 13 & 0 & 67 & .00 & 5 & .6 & 3.3 & 10 & \text { UKF }\end{array}$ $6.782 .12 .121 \quad 1 \quad 97: 07 \quad 5 \quad .5 \quad 1.419$ UER $\begin{array}{llllllllllllll}10.40 & .7 & 1.3 & 9 & 0 & 138 & .04 & 4 & .9 & 1.9 & 8 & \text { UKF }\end{array}$

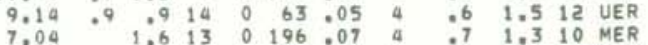

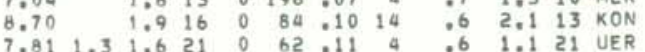

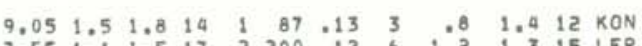

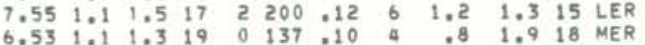

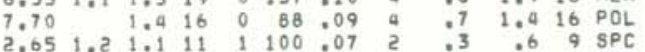
$8.96 \quad 1.2 \quad 1.315 \quad 0 \quad 64.05 \quad 4 \quad 0.6 \quad 1.2 \quad 13$ UER

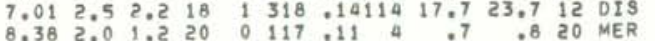
$\begin{array}{rrrrrrrrrrrr}2.08 & 1.8 & 1.1 & 16 & 0 & 123 & : 17 & 7 & .6 & 3.5 & 14 & \text { UKF } \\ 7.88 & 1.6 & 1.1 & 23 & 0 & 63 & : 12 & 3 & .5 & .6 & 20 & \text { UER }\end{array}$ $\begin{array}{lllllllllllll}8.19 & 1.7 & 1.6 & 23 & 0 & 75 & .09 & 3 & .4 & .7 & 22 & \text { UER }\end{array}$ $\begin{array}{llllllllllllll}3.00 & 2 & 2 & 2.0 & 7 & 0 & 110 & .04 & 2 & .5 & .6 & 6 & \text { SPC }\end{array}$

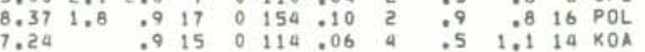

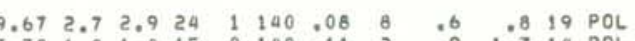

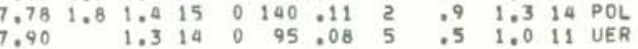




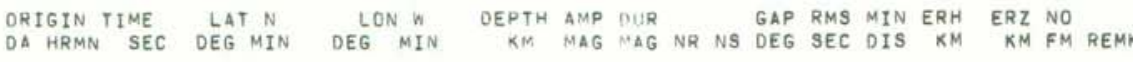
1979 FEB $201144 \quad 47.27 \quad 1931.72 \quad 155 \quad 50.74$

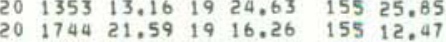

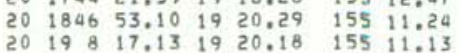

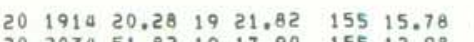

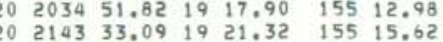

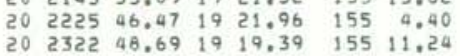
$21 \quad 0 \quad 9 \quad 45.10 \quad 19 \quad 18.31 \quad 155 \quad 15.65$

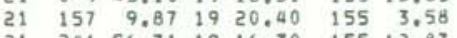
$\begin{array}{lllllll}21 & 241 & 56.71 & 19 & 16.30 & 155 & 12.87\end{array}$

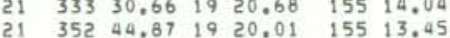

21 $43 \quad .901921 .22 \quad 155 \quad 3.47$ $21 \quad 826 \quad 23.101920 .22 \quad 155 \quad 8.65$ $212235 \quad 43.73 \quad 1920.15 \quad 155 \quad 13.06$

$\begin{array}{lllllll}22 & 635 & 9.57 & 19 & 58.07 & 155 & 38.29\end{array}$

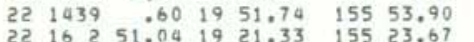

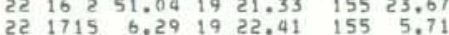

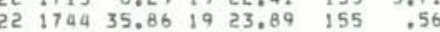
$\begin{array}{rrrrrrr}22 & 1749 & 3.04 & 19 & 23.56 & 155 & .08 \\ 22 & 1811 & 26.65 & 19 & 44.09 & 156 & 8.45\end{array}$ $\begin{array}{lllllll}22 & 1824 & 59.50 & 19 & 20.89 & 155 & 6.23\end{array}$ $22 \quad 215 \quad 16.19$ 19 $19.18 \quad 155 \quad 15.37$

$\begin{array}{rrrrrrr}22 & 2127 & 55.17 & 19 & 16.97 & 155 & 38.67 \\ 22 & 2225 & 12.66 & 19 & 24.06 & 155 & .99\end{array}$

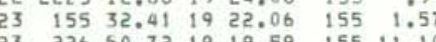

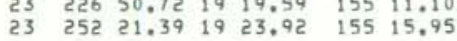

$\begin{array}{llrllll}23 & 320 & 9.84 & 19 & 23.71 & 155 & 16.16 \\ 23 & 330 & 25.97 & 19 & 23.96 & 155 & 15.95\end{array}$

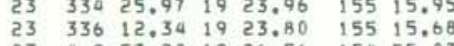

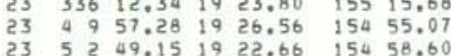
$\begin{array}{lllllll}23 & 821 & 2.43 & 19 & 20.34 & 155 & 12.86\end{array}$

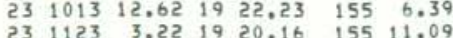

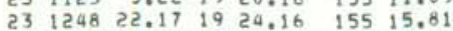
$\begin{array}{llllllll}23 & 13 & 5 & 46.96 & 19 & 26.74 & 154 & 56.14\end{array}$

$\begin{array}{lllllll}23 & 1332 & 57.32 & 19 & 26.81 & 154 & 55.98\end{array}$

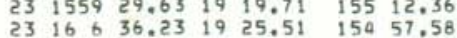

$\begin{array}{lllllllllllll}6.14 & 2.6 & 1.9 & 8 & 0 & 197 & .10 & 9 & 1.8 & 3.9 & 8 & \mathrm{KON}\end{array}$

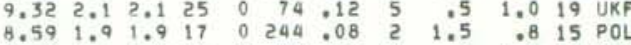
$\begin{array}{rrrrrrrrrrrr}8.12 & 1.7 & 2.0 & 17 & 0 & 80 & 12 & 4 & .7 & 1.2 & 15 & \text { UER } \\ 7.14 & & 1.0 & 16 & 0 & 82 & .08 & 4 & .6 & 1.1 & 16 & \text { UER }\end{array}$ $\begin{array}{llllllllllll}26.89 & 3.1 & 3.1 & 31 & 1 & 60 & .09 & 1 & .7 & .9 & 26 & \text { DEP }\end{array}$

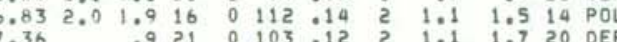

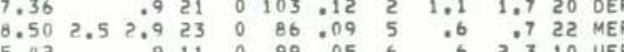

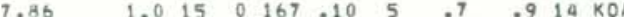

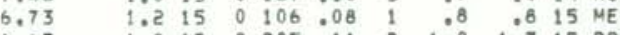

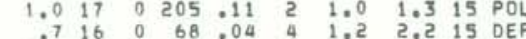

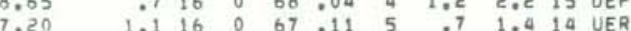

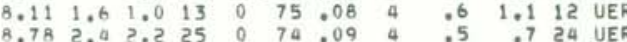
8.631 .92 .026000790850 .40 .0622 UER $\begin{array}{lllllllll}1.08 & 2,4 & 2.8 & 33 & 1 & 251 & 09 & 28 & 1.3\end{array}$

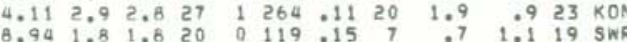

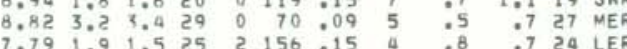

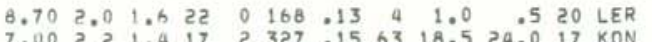

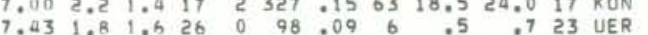

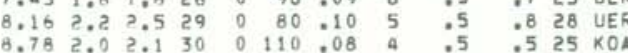
$\begin{array}{llllllllllll}1.41 & 2.0 & 1.4 & 14 & 0 & 165 & .20 & 5 & 2.1 & 4.3 & 13 & \text { HEA }\end{array}$

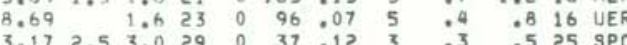
$\begin{array}{lllllllllllll}2.58 & 1.2 & 1.6 & 13 & 1 & 93 & .11 & 3 & .4 & .6 & 11 & \mathrm{SPC}\end{array}$

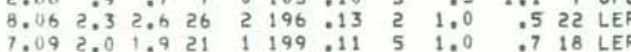

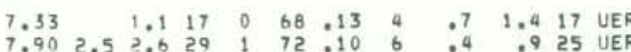

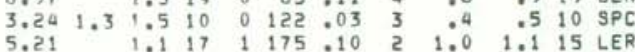
$\begin{array}{lllllllllll}4.99 & 1.3 & 11 & 0 & 188 & .12 & 2 & 2.0 & 1.9 & 10 & \text { LER }\end{array}$

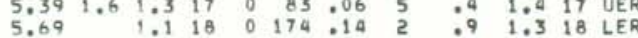

$\begin{array}{lllllllllllll}6.70 & 1.0 & 15 & 0 & 110 & 08 & 3 & .9 & 1.4 & 14 & \text { MER }\end{array}$

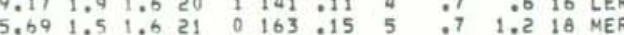

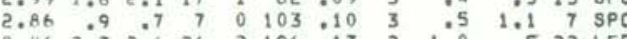

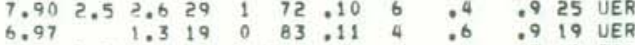

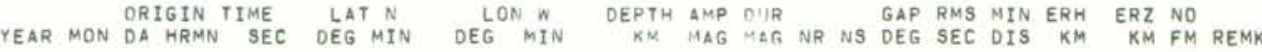
1979 FEB $23 \quad 1941 \quad 9.68 \quad 19 \quad 20.75 \quad 155 \quad 8.7$ $\begin{array}{lllllll}24 & 139 & 53.92 & 19 & 19.73 & 155 & 8.74\end{array}$

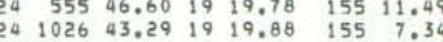
$4 \quad 1127 \quad 37.811920 .31 \quad 155 \quad 13.29$ $24 \quad 1427 \quad 30.8519 \quad 19.35 \quad 155 \quad 11.20$

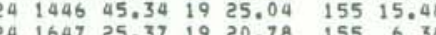

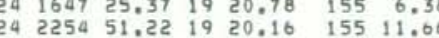
$504619.981920 .75 \quad 155 \quad 9.42$

$\begin{array}{lllllll}25 & 535 & 2.19 & 19 & 52.66 & 155 & 31.96\end{array}$

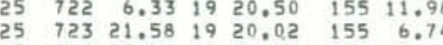
$\begin{array}{lllllll}25 & 831 & 32.80 & 19 & 20.40 & 155 & 7.77 \\ 25 & 94 & 32.22 & 19 & 19.80 & 155 & 7.48\end{array}$

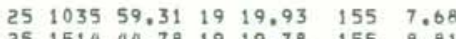
$25 \quad \begin{array}{llllll}1514 & 44.78 & 19 & 19.78 & 155 & 8.81\end{array}$

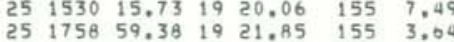

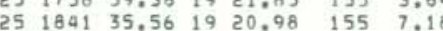
$\begin{array}{lllllll}25 & 2052 & 52.55 & 19 & 25.27 & 155 & 17.24 \\ 25 & 2358 & 28.35 & 19 & 23.91 & 155 & 24.76\end{array}$ $\begin{array}{lllllll}26 & 257 & 14.45 & 19 & 26.17 & 154 & 56.22 \\ 26 & 429 & 43.80 & 19 & 20.85 & 155 & 1.97\end{array}$ $26 \quad 914 \quad 32.09 \quad 19 \quad 19.73 \quad 155 \quad 10.31$ $\begin{array}{llll}26 & 1232 & .71 & 19 \\ 26 & 19.53 & 155 & 10.7\end{array}$

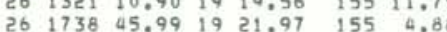
$\begin{array}{llllll}2034 & 6.61 & 19 & 20.44 & 155 & 12.40\end{array}$

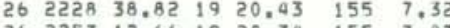
.26 253312.661920 .34 155 7.07

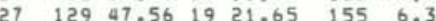
$27456 \quad 49,79 \quad 19 \quad 18,42 \quad 155 \quad 15,51$ $\begin{array}{llllllll}27 & 8 & 4 & 56.11 & 19 & 21.66 & 155 & 2.28\end{array}$ 715513.22 iी 25.96415553 .00 $27172231.45 \quad 1924.21 \quad 15522.98$ $27 \quad 1824 \quad 31.37 \quad 1919.00 \quad 155 \quad 13.40$ $\begin{array}{llllllll}27 & 1840 & 10.48 & 19 & 25.24 & 155 & 14.84\end{array}$ $28 \quad 333 \quad 1.91 \quad 19 \quad 23.84 \quad 15515.69$ $28 \quad 516 \quad 58.47 \quad 19 \quad 19.26 \quad 15513.11$ $\begin{array}{lllllll}28 & 916 & 31.26 & 19 & 23.95 & 155 & 16,41\end{array}$ $28 \quad 10 \quad 4 \quad 46.67 \quad 1920.58 \quad 155 \quad 10.86$ $281213 \quad 25.411919 .80 \quad 155 \%, 20$ $\begin{array}{lllllllllll}6.37 & 1.0 & 24 & 0 & 69.08 & 3 & .5 & 1.1 & 23 & \text { UER }\end{array}$

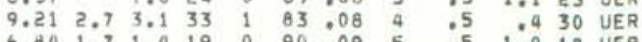
6.841 .71 .4190090 .0950 .51 .018 UER

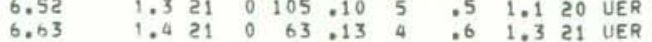
$\begin{array}{lllllllllll}5.50 & 1.3 & 16 & 0 & 100 & .10 & 6 & .6 & 1.8 & 16 & \text { UER }\end{array}$ $\begin{array}{lllllllllllll}1.33 & 1.1 & .9 & 7 & 1 & 187 & .03 & 2 & .9 & .5 & 6 & \mathrm{SPC}\end{array}$

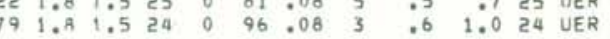

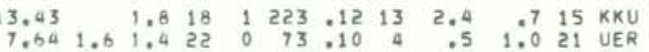

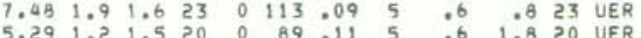

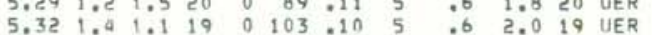
$\begin{array}{lllllllllll}8.35 & 2.4 & 2.6 & 26 & 0 & 96 & .09 & 5 & .5 & .7 & 24 \\ 5 & \text { UER }\end{array}$

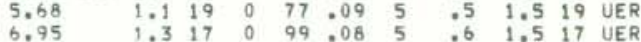

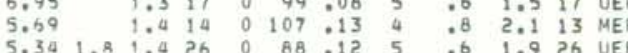

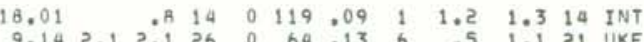

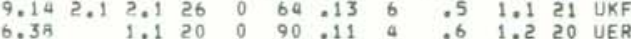

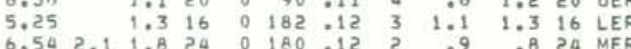

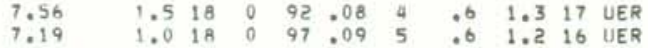

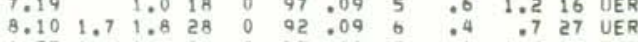

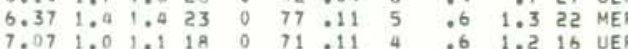

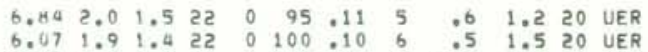

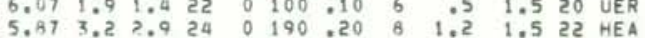

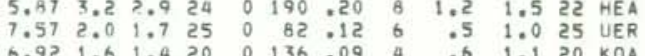

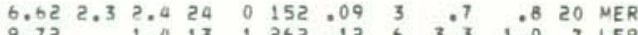

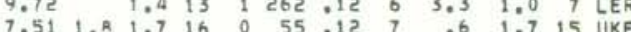

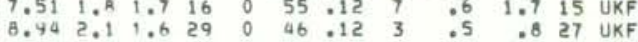

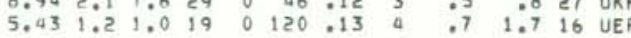

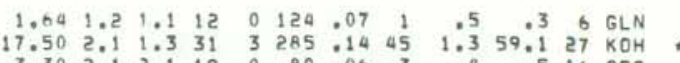

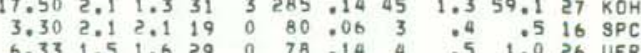

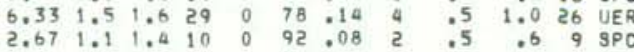
$\begin{array}{llllllllllll}8.26 & 2.0 & 2.2 & 23 & 0 & 76 & .07 & 3 & .5 & .8 & 23 & 23 \\ 8 & 0 E R\end{array}$

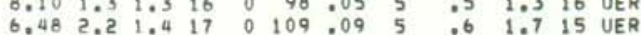

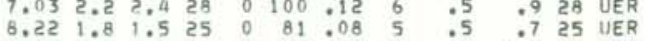




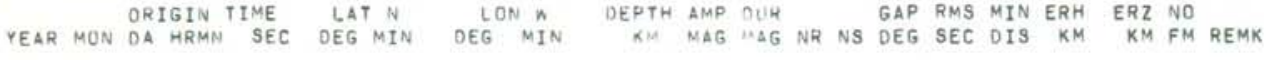
1979 FEB $28 \quad 1322 \quad 19.45 \quad 19 \quad 19.47 \quad 15520.36$

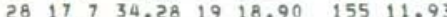

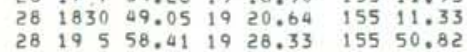

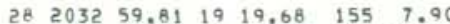

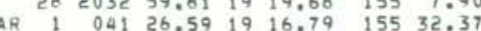
$41758.611920 .21 \quad 155$
13.92

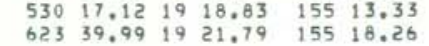
$\begin{array}{llllll}1 & 74211.711920 .20 \quad 155 \quad 10.41\end{array}$

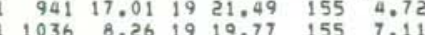
$161914.081920 .70 \quad 15512.96$

$\begin{array}{lllll}11 & 23548.04 \quad 19 & 20.80 \quad 155 \quad 3.09\end{array}$

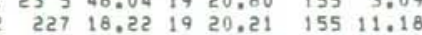

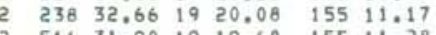

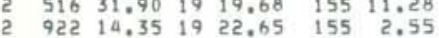

$\begin{array}{lllllll}2 & 1221 & 23.17 & 19 & 23.61 & 155 & 24.63\end{array}$ 2
2
2

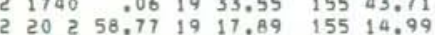

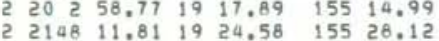

$\begin{array}{lllllll}3 & 159 & 10.72 & 19 & 19.94 & 155 & 10.99 \\ 3 & 537 & 18.82 & 19 & 36.16 & 155 & 33.56\end{array}$ $\begin{array}{lllllll}3 & 537 & 18.82 & 19 & 36.16 & 155 & 33.56 \\ 3 & 932 & 47.50 & 19 & 20,75 & 155 & 1.99\end{array}$

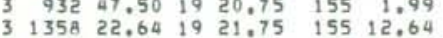
$\begin{array}{llllll}32343 & 27.31 & 19 & 22.81 & 155 & 16.02\end{array}$

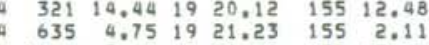

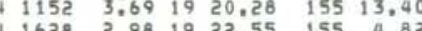
$\begin{array}{llllll}48 & 41.54 \quad 19 & 19,35 \quad 155 \quad 9.66\end{array}$

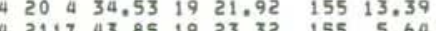
211743.851923 .32
155 $5 \quad 129 \quad 27.87$ 19 $19.27 \quad 155 \quad 13.04$

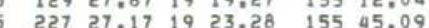

$\begin{array}{lllllll}5 & 347 & 47.19 & 19 & 19.51 & 155 & 8.63 \\ 5 & 546 & 26.94 & 19 & 22.23 & 155 & .24\end{array}$

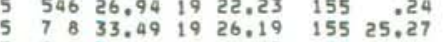

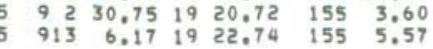

$5 \quad 930 \quad 36.99 \quad 19 \quad 26.18 \quad 155 \quad 25.16$

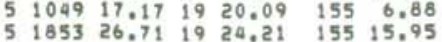

$\begin{array}{rlllllllllllll}.01 & 01 \\ 8.29 & 1.8 & 1.1 & 10 & 0 & 210 & .28 & 5 & 2.0 & 2.7 & 0 & 98 & \text { SWR } \\ 0.07 & 5 & .5 & 1.0 & 21 & \text { UER }\end{array}$

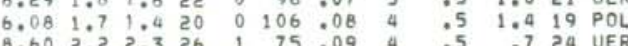

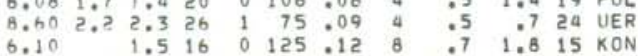
$\begin{array}{llllllllllll}9.31 & 2.4 & 2.6 & 27 & 0 & 95 & .08 & 4 & .6 & .6 & 26 & \text { UER } \\ 3.47 & .5 & .8 & 17 & 0 & 113 & 11 & 9 & 7 & 18.99 & 16 & \text { HEA }\end{array}$ $9.142 .52 .733 \quad 1 \quad 58: 10 \quad 5 \quad .450 .5$ 29 UER

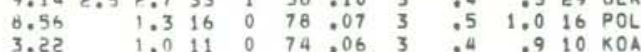

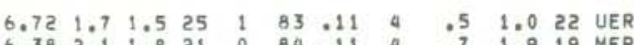

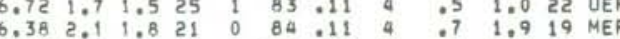

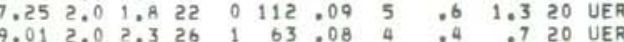

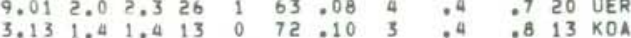

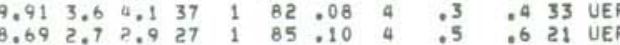

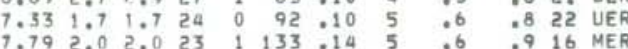
$\begin{array}{lllllllllll}10.43 & 2.1 & 2.0 & 28 & 0 & 75 & .12 & 6 & .5 & .9 & 26\end{array}$

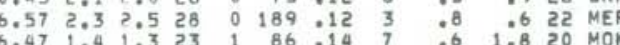

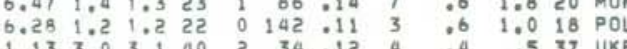
$9.52 \quad 2.62 .835 \quad 0 \quad 87 \quad .12 \quad 4 \quad .5 \quad .430$ UER

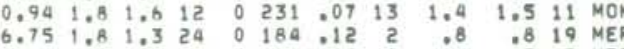

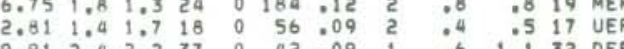

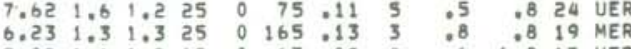

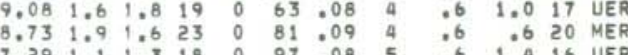

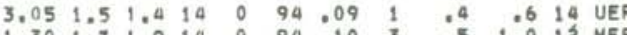

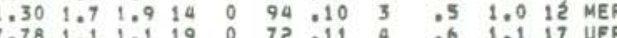
$\begin{array}{llllllllllll}7.41 & 1.2 & 1,1 & 17 & 0 & 95 & 08 & 5 & 06 & 1.2 & 17 & \text { UER } \\ 0.51 & & 1.5 & 15 & 0 & 86 & 08 & 9 & 0 & 1.2 & 1.14 & \text { KON }\end{array}$

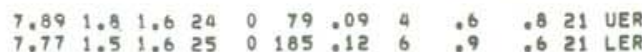

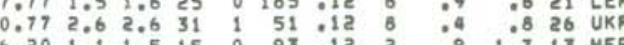

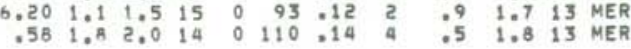
$8.911 .720 .021 \quad 0 \quad 51.09 \quad 7 \quad .51 .416$ UKF

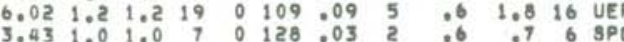

$\begin{array}{lllllllllllll}6.82 & 2.1 & 1.8 & 24 & 0 & 122 & .08 & 2 & .8 & .7 & 22 & \text { MER }\end{array}$
YEAR MON ORIGIN TIME LAMN SEC DEG MIN DEG MIN OEPTH AMP DUR G GAP RMS MIN ERH ERZ NO

1979 MAR $5 \quad 1859 \quad 50.44 \quad 1920.14 \quad 155 \quad 7.50$

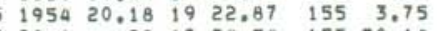
520 2 $\quad .991924 .54 \quad 15530.14$

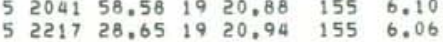

$52250 \quad 40.87 \quad 1925.02 \quad 155 \quad 26.00$ $5225522.75 \quad 1925.02 \quad 15425.00$ $5235924.911923 .75 \quad 15515.32$ - $250 \quad 50.141919 .1515513 .30$

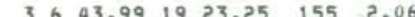
52247.641931 .72 155 15.94 $653257.501932 .01 \quad 15513.90$

$\begin{array}{lllllll}550 & 8.31 & 19 & 31.79 & 155 & 13.89\end{array}$

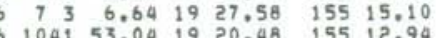

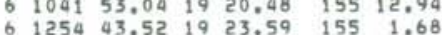

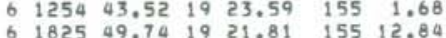
$\begin{array}{llllll}1827 & 55.44 & 19 & 21.81 & 155 & 12.82\end{array}$

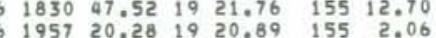

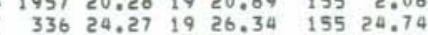
$\begin{array}{llllll}5 & 8 & 7.91 & 19 & 19.97 \quad 155 & 7.45\end{array}$

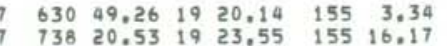
13820.531923 .5515516 .17

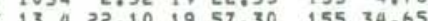
$\begin{array}{lllll}1338 & 3.57 \quad 19 & 20,88 & 155 & 10.75\end{array}$

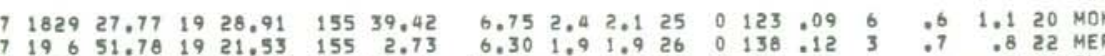

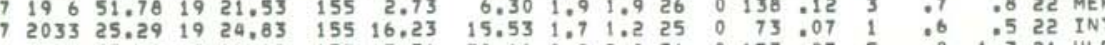

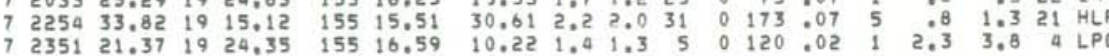
$\begin{array}{llllllllllllllllllllll}8 & 326 & .31 & 19 & 19.46 & 155 & 8.85 & 8.59 & 2.4 & 2.8 & 31 & 1 & 83 & .10 & 4 & .5 & .7 & 24 & \text { UER } \\ 8 & 332 & 45 & 0.71 & 19 & 25.72 & 155 & 33.25 & 29.27 & 2.3 & 2.6 & 11 & 0 & 116 & .23 & 5 & 2.0 & 4.1 & 10 & \text { MOK }\end{array}$

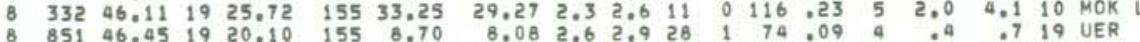

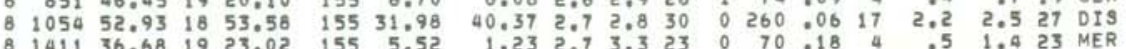

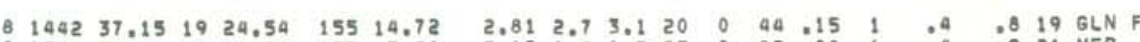

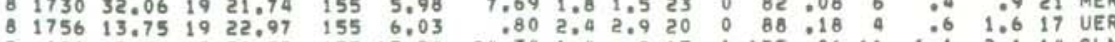

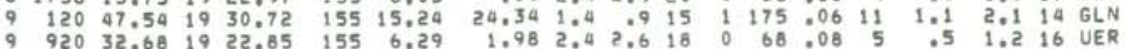

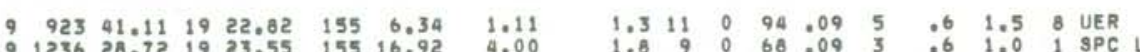

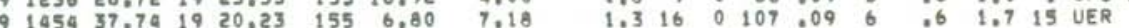


HVO EARTHQUAKE SUMMARY LIST

PAGE 19

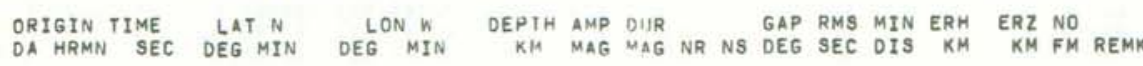
YEAR MON DA HRMN SEC DEG MIN DEG MIN KM MAG MAG NR NS DEG SEC DIS KM KM FM REMK

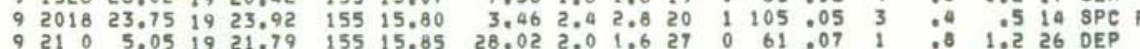

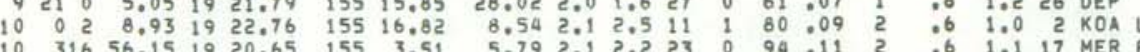

$10 \quad 355 \quad 14.6519,20.05 \quad 155 \quad 6.60$ $10 \quad 454 \quad 49.35 \quad 19 \quad 20.050155 \quad 6.68$

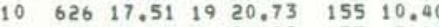

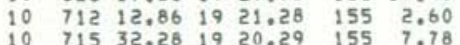

$\begin{array}{lllllll}10 & 949 & 33.31 & 19 & 18.03 & 155 & 7.03\end{array}$ $\begin{array}{lllllll}10 & 1012 & 55.86 & 19 & 20.00 & 155 & 6.59\end{array}$

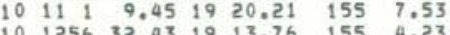

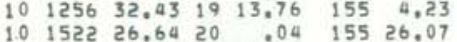

$\begin{array}{lllllll}10 & 2056 & 50.90 & 19 & 18.63 & 155 & 6.88\end{array}$ $\begin{array}{lllllll}10 & 2133 & 26.19 & 19 & 35.74 & 155 & 6.13 \\ 10 & 3313 & 21.71 & 19 & 22.59 & 155 & 2.06\end{array}$

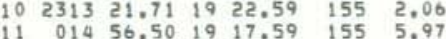

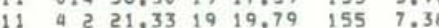
$\begin{array}{rrrrrrr}11 & 744 & 9.32 & 19 & 19.56 & 155 & 11.26 \\ 11 & 1728 & 5.17 & 19 & 30.84 & 155 & 16.84\end{array}$ $11747 \quad 2.351920 .39$ 155 7.02 11 18 $4 \quad 44.411920 .24$ 155 8.05 $11 \quad 1820 \quad .22 \quad 1921.14 \quad 155 \quad 10.72$

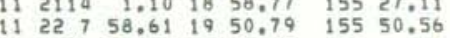

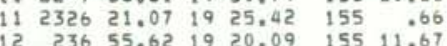
$\begin{array}{lllllll}12 & 249 & 57.99 & 19 & 20.36 & 155 & 12.76\end{array}$ 12 1040 $13.231919 .82 \quad 15510.25$

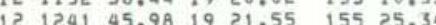
$121512 \quad 58,37 \quad 19 \quad 19,88 \quad 155 \quad 11.11$

$\begin{array}{lllllll}12 & 1640 & 33.04 & 19 & 21.57 & 155 & 2.50\end{array}$ $12511716.44 \quad 1924.55$ 154 54.82

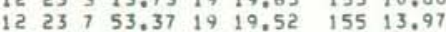
$\begin{array}{lllllll}13 & 341 & 34.39 & 19 & 4,47 & 155 & 40,74\end{array}$

$13 \quad 43453.66 \quad 1920.04 \quad 155 \quad 6.45$ $\begin{array}{llllllll}13 & 8 & 58.59 & 19 & 20.22 & 155 & 12.82\end{array}$ $13 \quad 957 \quad 8.811921 .10 \quad 15526.16$ $\begin{array}{lllllll}13 & 1435 & 34.54 & 19 & 19.97 \quad 155 \quad 10.03\end{array}$

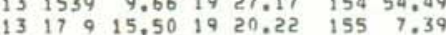

HVO EARTHOUAKE SUMMARY LIST

PAGE 20

ORIGIN TIME LAT N LON W DEPTH AMP DUR GAP RMS MIN ERH ERZ NO

YEAR MON DA HRMN SEC DEG MIN DEg MIN KM MAG MAG NR NS DEG SEC DIS KM KM FM REMK

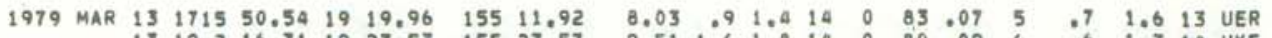

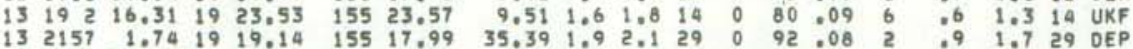

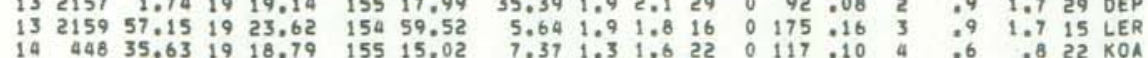

$14 \quad 6 \quad 8 \quad 8.19 \quad 1920.39 \quad 155 \quad 10.50$ $14613 \quad 45.071920,22 \quad 15510,70$ $14101135.131919 .93 \quad 15513.61$

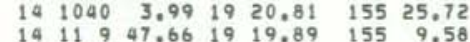

$14 \quad 111425.181920 .18 \quad 155 \quad 7.53$

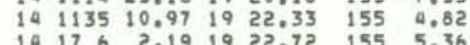
$14.176 \quad 2.191922 .72 \quad 155 \quad 5.36$ $1573854.951918 .64 \quad 15521.58$

$\begin{array}{lllllll}15 & 855 & 1.06 & 19 & 22.12 & 155 & 6.30 \\ 15 & 858 & 26.00 & 19 & 22.88 & 155 & 6.07\end{array}$ $\begin{array}{lllllll}15 & 858 & 26.00 & 19 & 22.88 & 155 & 6.07 \\ 15 & 947 & 47.89 & 19 & 22.84 & 155 & 5.68\end{array}$

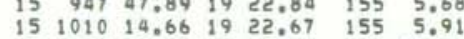
$15 \quad 113 \quad 45.42 \quad 19 \quad 19.64 \quad 155 \quad 10.96$

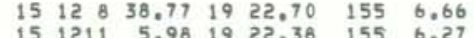

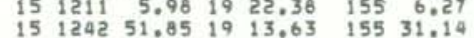

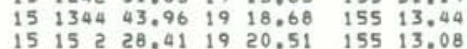
$\begin{array}{lllllll}15 & 1619 & 2.76 & 19 & 29.33 & 154 & 54.88\end{array}$ $15195955.86 \quad 1919.29 \quad 15515.90$ $16 \quad 1635 \quad 02.17 \quad 1929.07 \quad 155 \quad 100.85$ $\begin{array}{lllllll}16 & 1741 & 48.18 & 19 & 22.67 & 155 & 2.18\end{array}$

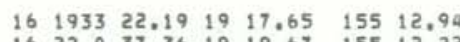

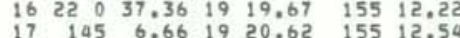
$\begin{array}{lllllll}17 & 537 & 7.91 & 19 & 20.39 & 155 & 8.78\end{array}$ $17 \quad 643 \quad 30.83 \quad 1921.16 \quad 155 \quad 5.79$ $\begin{array}{lllllll}17 & 647 & 56.37 & 19 & 21.43 & 155 & 5.83\end{array}$ $17104657.751919 .93 \quad 15511.86$ $17 \quad 193057.3111921 .32 \quad 155 \quad 40.0060$

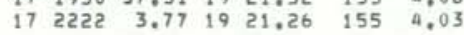

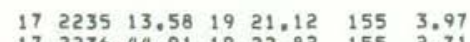
$1723957.891918,60 \quad 15513.82$

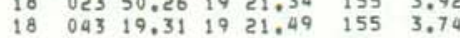
$\begin{array}{lllllll}18 & 637 & 22.21 & 19 & 20.60 & 155 & 8.69\end{array}$

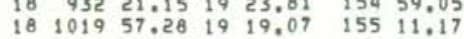

$\begin{array}{llllllll}8.54 & 2.2 & 2.7 & 30 & 0 & 79.09 & 3\end{array}$

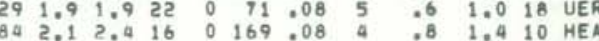

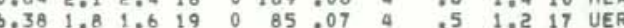

$\begin{array}{llllllllllll}8.12 & 2.3 & 2.3 & 23 & 0 & 96 & 09 & 5 & .5 & .9 & 20 & \text { UER }\end{array}$

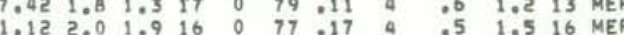

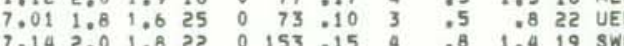

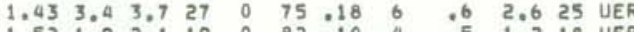
$1.502 .02 .119 \quad 1100: 22 \quad 4 \quad 051.218$ UER

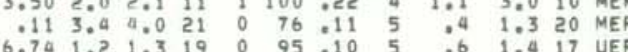

$\begin{array}{lllllllllll}12 & 1.8 & 1.6 & 9 & 0 & 93 & .10 & 5 & .6 & 2.0 & 9 \text { UER }\end{array}$

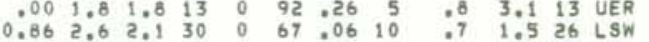

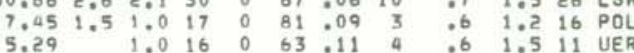
$\begin{array}{rrrrrrrrrrrr}1.40 & 1.5 & 1.1 & 13 & 1 & 133 & .10 & 3 & 1.5 & 2.3 & 12 & \text { LER } \\ 8.92 & 2.4 & 2.4 & 31 & 0 & 110 & .09 & 3 & .9 & .4 & 27 & \text { KOA }\end{array}$

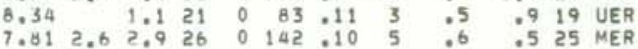
$\begin{array}{rrrrrrrrrrrr}8.15 & 1.8 & 1.1 & 24 & 0 & 126 & .09 & 1 & .6 & .7 & 19 & \text { POL } \\ 5.58 & 1.6 & 1.2 & 19 & 0 & 85 & .12 & 5 & .5 & 1.5 & 17 & \text { UER }\end{array}$

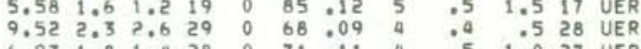

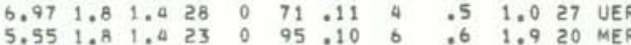
$\begin{array}{llllllllllll}5.90 & 1.8 & 1.5 & 23 & 0 & 89 & .10 & 6 & .5 & 1.7 & 23 & \text { MER }\end{array}$

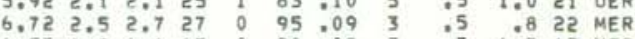

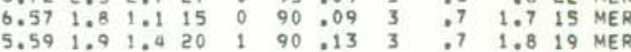
$\begin{array}{llllllllllllll}7.76 & 2.5 & 2.8 & 31 & 1 & 88 & .10 & 3 & .6 & .5 & 29 & \text { MER }\end{array}$ $8.261 .71 .321 \quad 196.08 \quad 3 \quad .6 \quad 0816 \mathrm{POL}$

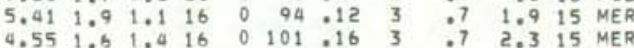
$\begin{array}{lllllllllll}7.55 & 1.5 & 26 & 0 & 70 & .09 & 3 & .5 & .8 & 25 & \text { UER }\end{array}$

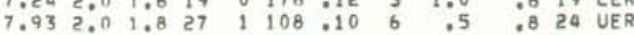

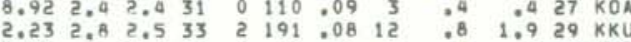




\section{HVO EARTHQUAKE SUMMARY LIST}

PAGE 21

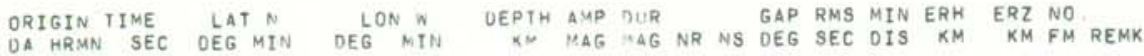
$\begin{array}{lllllllllllllllllll}1979 \text { MAR } 18 & 1139 & 10.03 & 19 & 20.43 & 155 & 6.81 & 7.79 & 2.0 & 1.8 & 28 & 0 & 102 & .08 & 6 & .5 & .925 & 25 & 9 E R\end{array}$ $18 \quad 135456.551919 .50 \quad 15511.44$

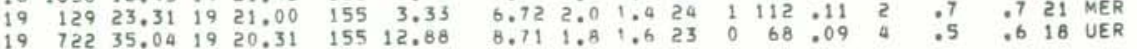

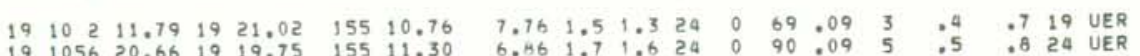
$\begin{array}{lllllll}19 & 12 & 84 & 19 & 20.71 & 155 & 3.46\end{array}$

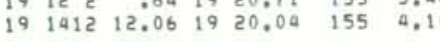

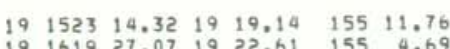
19 टाषी दट.99 1920.71 155

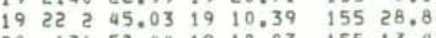

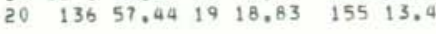

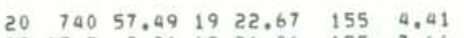

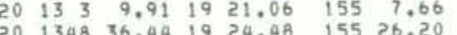
$\begin{array}{lllllll}20 & 1348 & 36.44 & 19 & 24.48 & 155 & 26.20\end{array}$

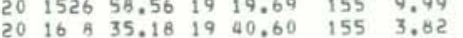

$\begin{array}{lllllll}20 & 1645 & 28.39 & 19 & 19.12 & 155 & 16.31\end{array}$

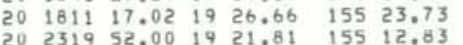

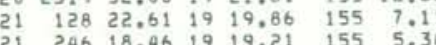

$\begin{array}{lllllll}21 & 738 & 20.57 & 19 & 24.87 & 155 & 28.85\end{array}$ $\begin{array}{lllllll}21 & 836 & 50.09 & 19 & 21.94 & 155 & 6.87 \\ 21 & 839 & 36.66 & 19 & 20.84 & 155 & 6.25\end{array}$

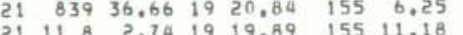
$\begin{array}{lllllll}21 & 11 & 8 & 2.74 & 19 & 19.89 & 155 \\ 21 & 1347 & 53.67 & 19 & 22,34 & 155 & 4,50\end{array}$

$21 \quad 1542 \quad 14.07 \quad 19 \quad 19.92 \quad 155 \quad 8.27$

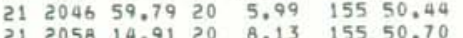
$\begin{array}{lllllll}21 & 2058 & 14.91 & 20 & 8.13 & 155 & 50.70 \\ 21 & 21 & 70.84 & 19 & 59.77 & 155 & 47.20\end{array}$

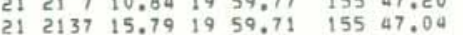

$\begin{array}{lllllll}21 & 2151 & 34.42 & 20 & 59 & 155 & 46.29 \\ 21 & 2218 & 43.67 & 20 & 53 & 155 & 46.62\end{array}$

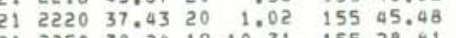

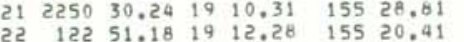

$\begin{array}{lllllll}22 & 232 & 39.12 & 19 & 21.87 & 155 & 6.62 \\ 22 & 3 & 55.28 & 19 & 23.61 & 155 & 2.11\end{array}$

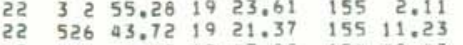

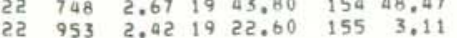
$22 \quad 1018 \quad 57.68 \quad 1920.55 \quad 155 \quad 4.11$

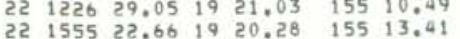

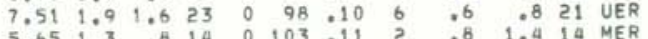

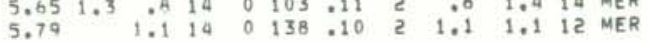

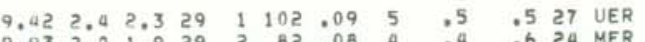

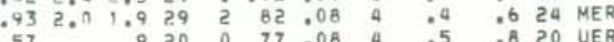

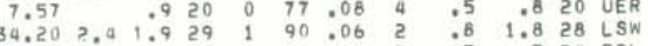
0.120 MER

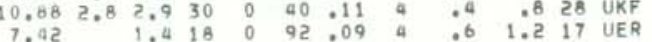

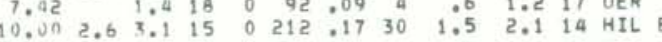
$\begin{array}{rrrrrrrrrrrrr}.93 & 2.0 & 1.9 & 18 & 0 & 116 & 06 & 3 & .6 & 1.0 & 17 & \text { KOA } \\ 0.05 & 09 & 0 & 21 & 0 & 78 & 08 & 6 & : 5 & 1.1 & 16 & \text { UKF }\end{array}$

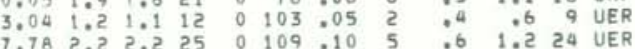
$4.03 \quad 1.41 .323$ o 161.16 a 1.01 2, 19 MER

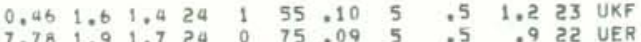

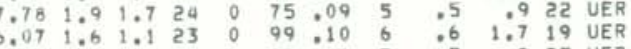

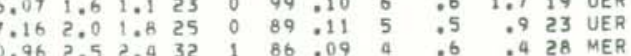
$\begin{array}{rrrrrrrrrrrrr}8.37 & 2.1 & 2.2 & 27 & 0 & 84 & 07 & 5 & .5 & .7 & 27 & \text { UER } \\ 8.22 & 4.5 & 4.5 & 37 & 1 & 144 & 011 & 46 & : 7 & 17.6 & 32 & \text { KOH F. }\end{array}$

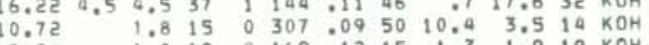
$\begin{array}{lllllllllllll}11.29 & 2.1 & 26 & 1 & 162 & 010 & 13 & .7 & .9 & 25 & \mathrm{KOH} \\ 11.76 & 1.9 & 22 & 1 & 164 & 010 & 13 & 09 & 1.0 & 20 & \mathrm{KOH}\end{array}$

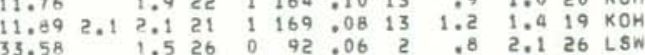
$\begin{array}{lllllllllll}1.5 & 26 & 0 & 92 & 06 & 2 & .8 & 2.1 & 26 & \text { LSW } \\ 46.72 & 1.8 & 26 & 0 & 169 & .09 & 15 & 1.1 & 2.7 & 24 & \text { HLP }\end{array}$ $\begin{array}{rrrrrrrrrrrr}5.62 & 1.3 & 1.1 & 24 & 0 & 77 & .10 & 6 & .5 & 1.5 & 21 & \text { UER } \\ 7.20 & 1.6 & 1.4 & 15 & 0 & 128 & : 11 & 0 & : 6 & : 9 & 15 & \text { MER } \\ 7.62 & 2.1 & 2.1 & 32 & 0 & 64 & : 14 & 3 & .5 & : 8 & 27 & \text { UER }\end{array}$

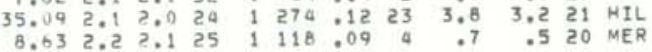

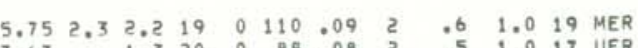

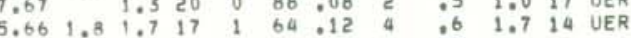

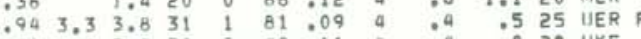

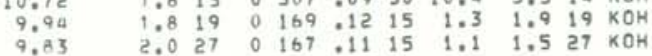

HVO EARTHQUAKE SUMMARY LIST

PAGE 2Z VEAR MON ORIGIN TIME LAM SEC DEG MIN DEG MIN DERTH AMP DUR NR GAP RMS MIN ERH ERZ NO RE

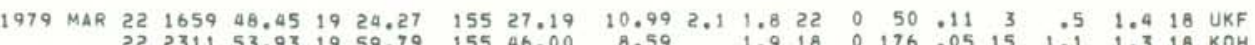

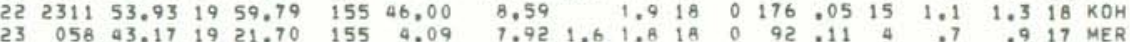

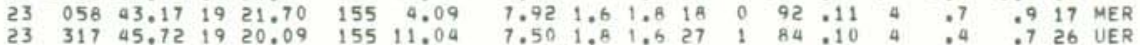

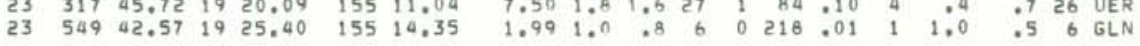

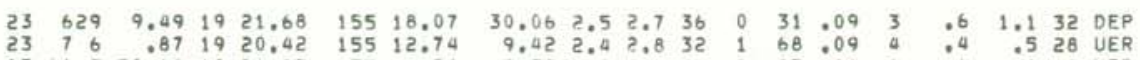

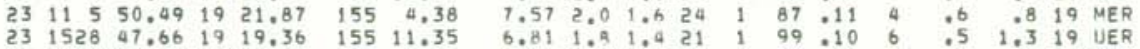

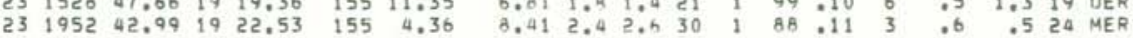

$23 \quad 2136 \quad 30.93 \quad 19 \quad 20.73 \quad 155 \quad 7.50$ $232258 \quad 13.94 \quad 1921.92 \quad 155 \quad 10.40$

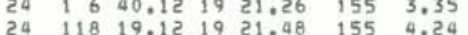
$241021 \quad 9.66 \quad 1921.12 \quad 155 \quad 6.58$

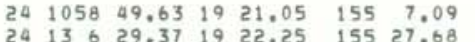
$2413629.371922 .25 \quad 15527.68$ $24 \quad 161624.18 \quad 19 \quad 18.95 \quad 15515.48$

$\begin{array}{lllllll}24 & 1825 & 1.33 & 19 & 20.29 & 155 & 8.47\end{array}$

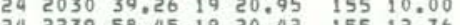
$2533516.01 \quad 1920.01 \quad 15510.25$

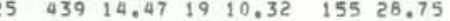
$\begin{array}{rrrrrrr}25 & 650 & 17.87 & 19 & 21.25 & 155 & 7.88 \\ 25 & 1018 & 52.05 & 19 & 23.15 & 155 & 3.10\end{array}$ $25102130.101922 .83 \quad 155 \quad 3.10$ $251053 \quad 8.26 \quad 1920.10 \quad 155 \quad 7.99$ $\begin{array}{lllllll}25 & 1748 & 51.31 & 19 & 2.38 & 155 & 6.37\end{array}$

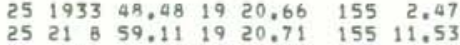

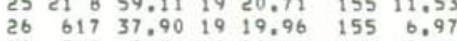
$26 \quad 711 \quad 8.78$ 19 $20.84 \quad 155 \quad 3.97$ $\begin{array}{lllllll}26 & 928 & 47.77 & 19 & 18.15 & 155 & 12,95\end{array}$ $26 \quad 1058 \quad 20.20 \quad 1923.88 \quad 155 \quad 16.02$

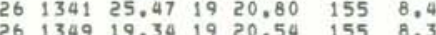

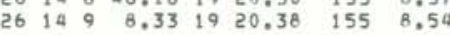

$\begin{array}{lllllll}26 & 2212 & 44.87 & 19 & 18.86 & 155 & 13.48\end{array}$

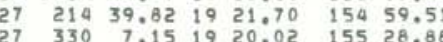
$\begin{array}{rrrrrrr}27 & 829 & 2.92 & 19 & 22.27 & 155 & 5.22 \\ 27 & 1537 & 36.59 & 19 & 21.55 & 155 & 15.31\end{array}$ $\begin{array}{lllllll}27 & 1610 & 45.15 & 19 & 22.01 & 155 & 1.60\end{array}$

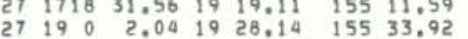

$\begin{array}{llllllllllll}9.08 & 2.7 & 5.1 & 31 & 1 & 88 & .07 & 5 & .5 & .5 & 29 & \text { UER }\end{array}$

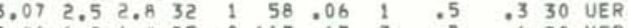

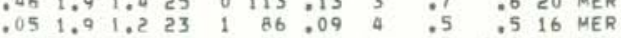

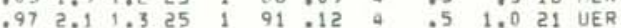

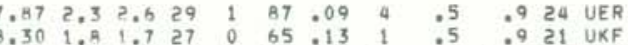

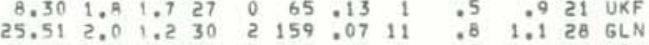

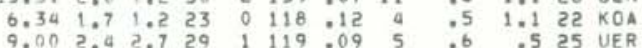

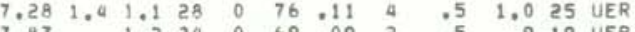

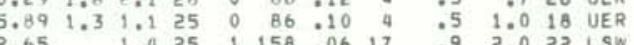

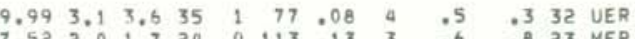

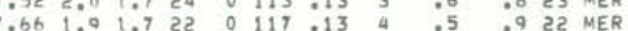

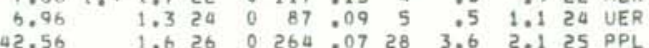
$\begin{array}{rrrrrrrrrrrr}7.58 & 2.3 & 2.6 & 25 & 0 & 165 & .11 & 2 & .7 & .8 & 23 & \text { MER } \\ 6.83 & & 1.2 & 23 & 0 & 73 & .09 & 4 & .5 & .8 & 21 & \text { UER }\end{array}$

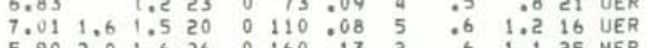

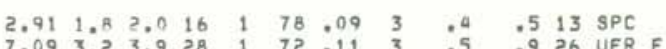

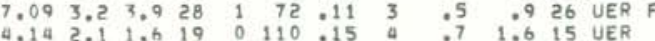
$\begin{array}{lllllllllll}4.45 & 9 & 8 & 0 & 128 & 04 & 4 & .6 & 1.6 & 7 & \text { UER } \\ 6.52 & 0 & 6 & 0 & 130 & 05 & 4 & .0 & 2.0 & 6 & \text { UER }\end{array}$ $\begin{array}{llllllllllll}5.07 & 1.3 & 1.2 & 19 & 0 & 81 & .11 & 3 & .6 & 1.8 & 18 & \text { POL }\end{array}$

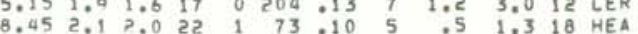
$\begin{array}{rrrrrrrrrrrr}9.03 & 2.1 & 1.7 & 18 & 1 & 116 & .09 & 2 & .7 & 1.0 & 17 & \text { MER } \\ 9.31 & 2.0 & 1.8 & 19 & 0 & 76 & .08 & 2 & .5 & .8 & 16 & \text { KOA }\end{array}$

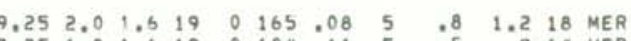

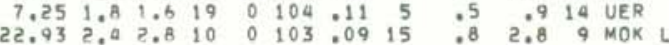

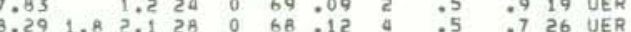

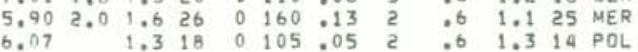


ORIGIN TIME LATN LON W DEPTH AMP OUR N GAP RMS MIN ERH ERZ NO
DA HRMN SEC DEG MIN DEG MIN TKN MAG TAG NR NS DEG SEC DIS KM KM FM REMK

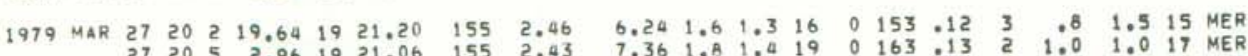

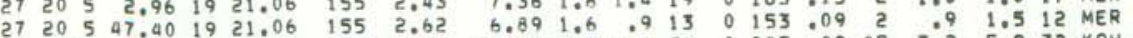

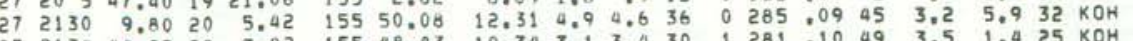

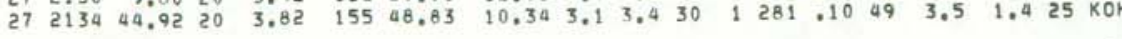

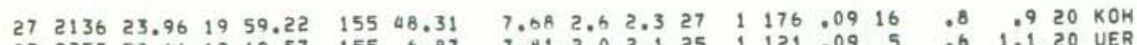

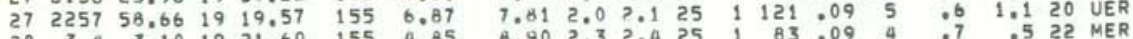

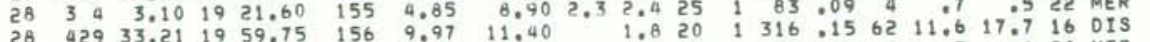

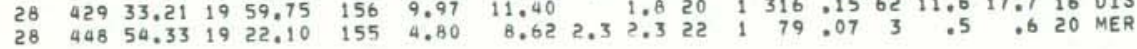

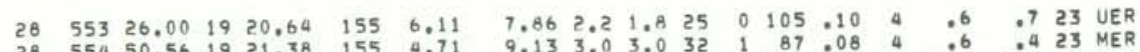

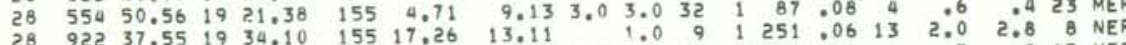

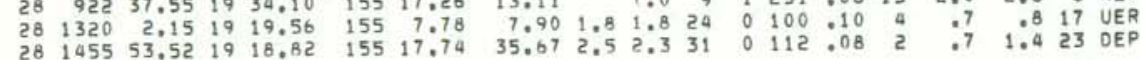

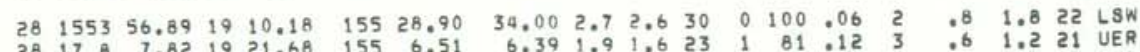

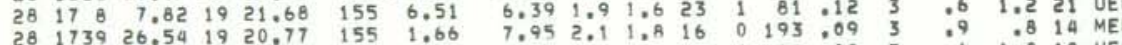

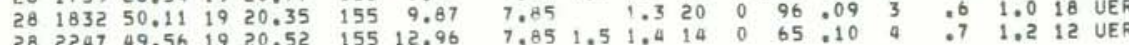
$\begin{array}{llllllllllllllllllllll}28 & 23 & 3 & 31.21 & 19 & 12.08 & 155 & 41.70 & 4.02 & 2.4 & 1.6 & 16 & 0 & 191 & .31 & 10 & 2.0 & 46.9 & 12 & \text { MEA }\end{array}$

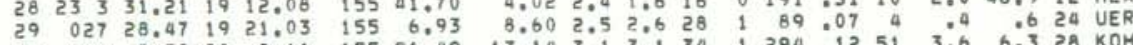

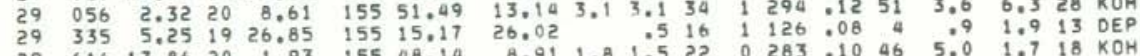

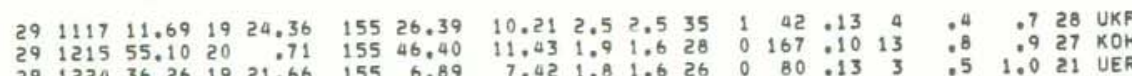

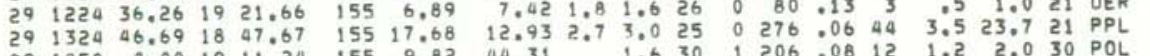
$\begin{array}{lllllllllllllllllllll}29 & 23 & 6 & 44.78 & 20 & 48.35 & 158 & 41.18 & .07 & 5.5 & 5.7 & 37 & 1 & 285 & .18 & 98 & 13.2 & 2.3 & 34 & 013\end{array}$

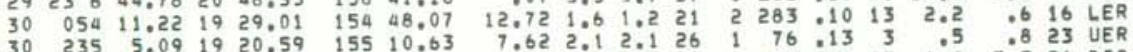

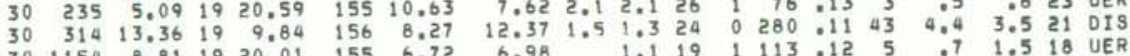
$\begin{array}{llllllllllllllllllll}30 & 1238 & 55.95 & 20 & 1.89 & 155 & 47.56 & 10.31 & 2.6 & 2.6 & 20 & 0 & 306 & .08 & 57 & 8.5 & 29.6 & 13 & \mathrm{KOH} F \mathrm{~F} & \end{array}$

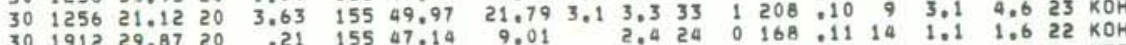

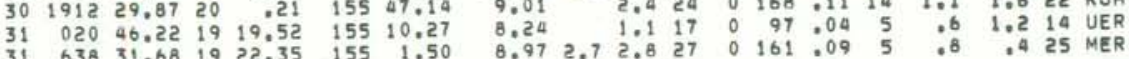

$\begin{array}{llllllllllllllllll}31 & 1018 & 12.32 & 19 & 21.84 & 155 & 12.79 & 2.69 & 1.4 & 1.1 & 11 & 0 & 64 & .06 & 2 & .5 & .6 & 7 \text { UER }\end{array}$

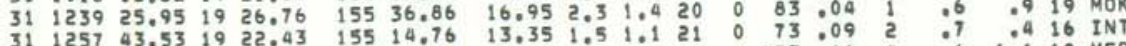

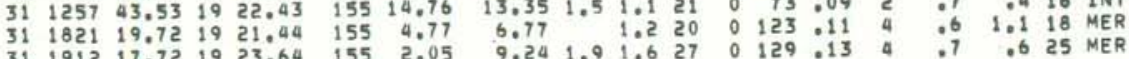
$\begin{array}{lllllllllllllllllll}31 & 2337 & 56.38 & 19 & 24.74 & 155 & 26.35 & 8.98 & 1.8 & 1.8 & 28 & 0 & 51 & .09 & 5 & .4 & .9 & 25 & \text { UKF }\end{array}$

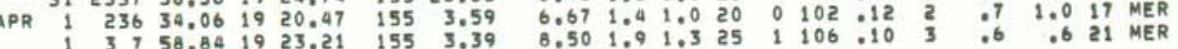

ORIGIN TIME LAT N LON W DEPTH AMP DUR GAP RMS MIN ERH ERZ NO

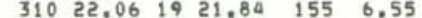
$326 \quad 42.901921 .86 \quad 155 \quad 6.95$

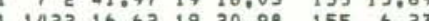
$1536 \quad 18.49$ 19 $20.86 \quad 155 \quad 6.19$ $11948 \quad 44.151926 .57 \quad 15524.85$ $203328.521924 .88 \quad 15459.94$
33353.09 (2)

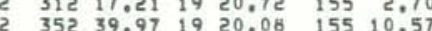

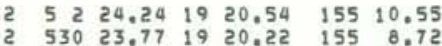
$\begin{array}{lllllll}530 & 23.77 & 19 & 20.22 & 155 & 8.72\end{array}$

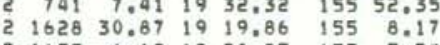

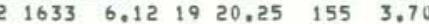

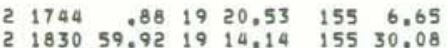
2 $183059.921914 .14 \quad 15530.08$

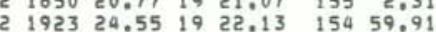
$\begin{array}{llllll}1927 & 5.55 \quad 1920.93 & 155 & 9.96\end{array}$

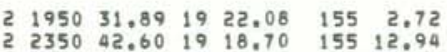
195011.601918 .7015512 .9 3
3
1327

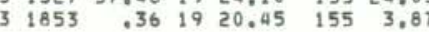

$4047 \quad 46.70 \quad 1924.15 \quad 155 \quad 29.51$ $710 \quad 52.8819 \quad 18.90 \quad 15513.42$
$401645.571925 .63 \quad 15520.36$ $4115 \quad 4.52 \quad 19 \quad 21.97 \quad 155 \quad 4.69$

$\begin{array}{llllll}4 & 1144 & 43.97 \quad 19 & 20.40 \quad 155 \quad 8.84\end{array}$

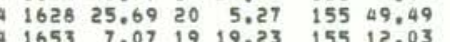
$4 \begin{aligned} & 1916 \quad 14.35 \quad 19 \quad 19.59 \\ & 155 \quad 8.80\end{aligned}$

$\begin{array}{lllllll}4 & 1958 & 49.08 & 19 & 21.55 \quad 155 & 15.15\end{array}$ \begin{tabular}{l}
$401458.71 \quad 1920.94 \quad 155 \quad 1.33$ \\
\hline
\end{tabular} $\begin{array}{llllllll}5 & 1 & 4 & 29.24 & 19 & 20.69 & 155 & 2.57 \\ 5 & 455 & 22.18 & 19 & 20.57 & 155 & 17.15 & 30\end{array}$

$\begin{array}{lllllll}5 & 527 & 59.44 & 19 & 20.73 & 155 & 11.42\end{array}$ $584056.041921 .43 \quad 155 \quad 5.36$

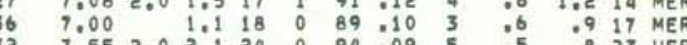

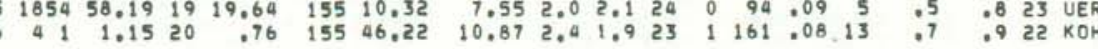

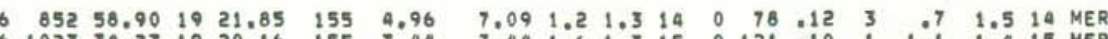

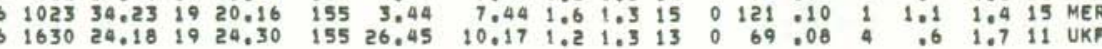


ORIGIN TIME
OA HRMN SEC DEG MIN DE LON N

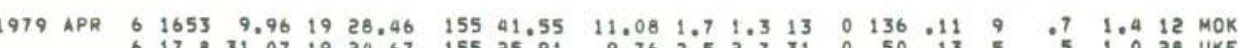

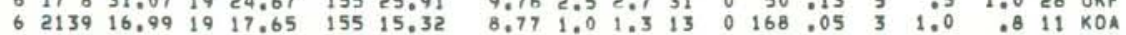

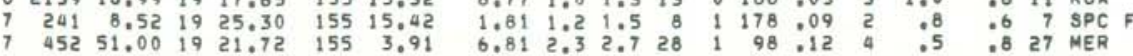
$\begin{array}{llllllll}7 & 5 & 2 & 33.27 & 19 & 21.43 \quad 155 \quad 3.73\end{array}$ $65251.461923 .80 \quad 15528.53$ $\begin{array}{lllllll}7 & 836 & 16.05 & 19 & 19.86 & 155 & 7.13 \\ 7 & 837 & 49.08 & 19 & 23.91 & 155 & 17.37\end{array}$ $\begin{array}{lllllll}7 & 911 & 23.73 & 19 & 22.23 & 155 & 4.68\end{array}$

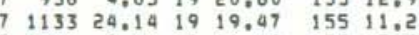

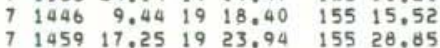
$7 \begin{array}{llllll}7517 & 27.18 & 19 & 19.45 \quad 155 & 7.93\end{array}$

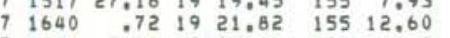
$\begin{array}{llllll}1947 & 2.11 & 19 & 21.14 & 155 & 4.18 \\ 1952 & 49.52 & 19 & 21.26 & 155 & 4.23\end{array}$

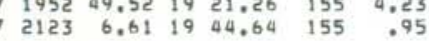

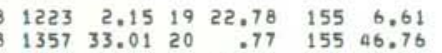
$8145911.951922 .91 \quad 155 \quad 5.6$ $158,14.101922 .19 \quad 15527.23$

$9 \begin{array}{llll}217 & 52.53 \quad 19 & 20.73 \quad 155 \quad 10.60\end{array}$ $9 \begin{array}{llllll}254 & 56.21 & 19 & 25.35 & 155 & 31.96\end{array}$ $9123425.1919 \quad 17.44 \quad 15520.67$

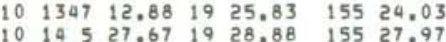

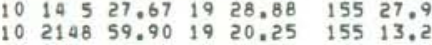
$10 \quad 2251 \quad 13.30 \quad 1924.36 \quad 15523.93$

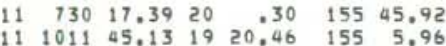
$11519 \quad 12.891920 .59 \quad 155 \quad 5.96$ $\begin{array}{lrrrrrr}11 & 2239 & 28.05 & 19 & 19.75 & 155 & 11.09 \\ 12 & 231 & 35.46 & 19 & 20.00 & 155 & 5.99\end{array}$ $\begin{array}{lllllll}12 & 615 & 42.51 & 19 & 22.45 & 155 & 2.34 \\ 12 & 839 & 46.44 & 19 & 20.15 & 155 & 13.29\end{array}$ $12 \quad 83946.441920 .15 \quad 15513.29$ 12 1457 40.85 19 $23.46 \quad 15529.7$ $121519 \quad 18,48 \quad 19 \quad 18,49 \quad 155 \quad 13.05$ $\begin{array}{lllllll}12 & 1543 & 25.44 & 19 & 21.46 & 155 & 1.74\end{array}$ $\begin{array}{lrrrrrr}12 & 2318 & 13.50 & 19 & 19.90 & 155 & 11.58 \\ 13 & 012 & 42.51 & 19 & 17.38 & 155 & .47\end{array}$ $\begin{array}{lllllllllllllll}7.59 & 1.8 & 2.0 & 26 & 0 & 101 & .11 & 3 & .5 & .7 & 26 & \text { MER }\end{array}$

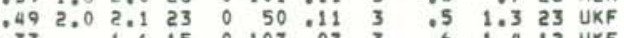
$\begin{array}{llllllllllll}9.33 & 1.6 & 15 & 0 & 107 & 07 & 3 & .6 & 1.4 & 12 & \text { UKF } \\ 7.24 & 9 & 1.0 & 15 & 0 & 109 & .07 & 5 & .5 & 1.0 & 12 & \text { UER }\end{array}$ $\begin{array}{lllllllllllllll}.11 & 9 & 1.5 & 9 & 1 & 80 & 10 & 2 & .4 & .7 & 8 & \text { SPC }\end{array}$ $\begin{array}{lllllllllllll}8.05 & 1.8 & 1.7 & 16 & 0 & 82 & .10 & 3 & .6 & 1.0 & 13 & \text { MER }\end{array}$

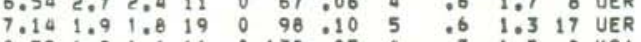

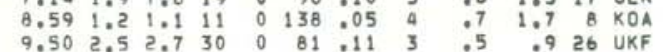
$\begin{array}{lllllllllllll}7.03 & 1.0 & 1.1 & 15 & 0 & 97 & .06 & 4 & .6 & 1.4 & 13 & \text { UER }\end{array}$

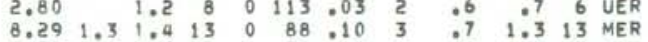
$\begin{array}{rrrrrrrrrrrr}7.90 & 1.8 & 1.8 & 19 & 1 & 86 & 09 & 3 & .5 & 1.0 & 16 & \text { MER } \\ 39.95 & 2.0 & 2.1 & 29 & 2 & 219 & .12 & 5 & 1.4 & 2.4 & 27 & \text { HIL }\end{array}$

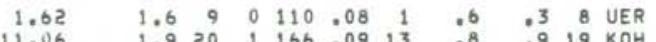

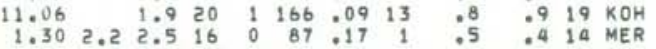

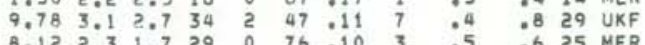
$\begin{array}{llllllllllll}7.78 & 2.1 & 2.1 & 29 & 2 & 74 & .11 & 3 & .4 & .7 & 23 & \text { UER }\end{array}$

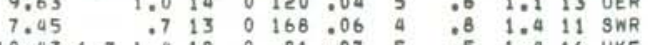

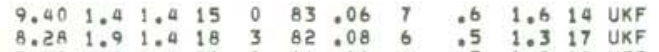

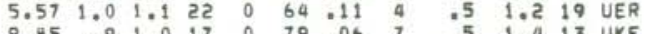
$\begin{array}{llllllllllll}8.97 & 1.5 & 1.6 & 13 & 0 & 161 & .08 & 14 & 1.5 & 1.5 & 12 & \mathrm{KOH}\end{array}$

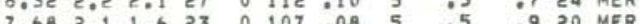

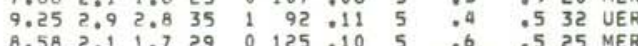

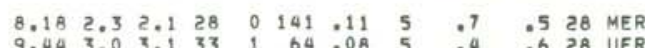

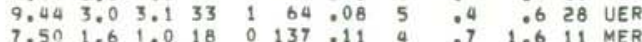

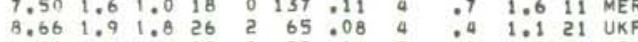

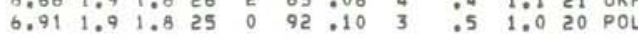
$\begin{array}{lllllllllll}5.16 & 1.6 & 1.2 & 14 & 0 & 172 & .12 & 4 & 1.0 & 1.8 & 9 \text { MER }\end{array}$ $\begin{array}{rrrrrrrrrrrr}8.61 & 1.5 & 1.0 & 16 & 0 & 90 & .05 & 5 & .6 & 1.4 & 14 & \text { UER } \\ 37.23 & 2.2 & 1.9 & 31 & 3 & 236 & .06 & 6 & 1.1 & 1.1 & 27 & 019\end{array}$

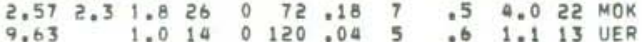

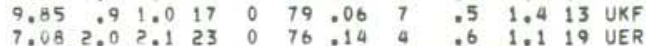

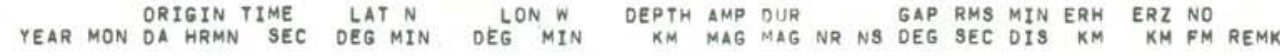
$\begin{array}{rrrrrrr}13 & 039 & 29.95 & 19 & 20.38 & 155 & 13.15 \\ 13 & 422 & 4.79 & 19 & 20.50 & 155 & 13.19 \\ 13 & 641 & .67 & 19 & 31.86 & 155 & 27.66 \\ 13 & 819 & 2.86 & 19 & 15.34 & 155 & 3.17 \\ 13 & 1442 & 19.73 & 19 & 21.01 & 155 & 5.94\end{array}$

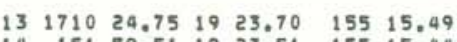
$14 \quad 330 \quad 36.51 \quad 1923.51 \quad 15515.4$

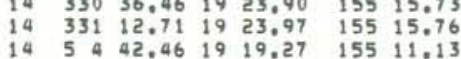
$14 \quad 526 \quad 49.29 \quad 1923.42 \quad 155 \quad 15.03$ $14528 \quad 22,1219 \quad 9.46 \quad 15526,35$

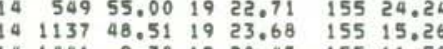

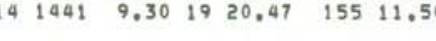
$\begin{array}{lllllll}14 & 1524 & .29 & 19 & 20.94 & 155 & 13.31\end{array}$

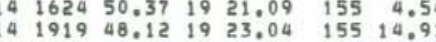
$142152 \quad 8.721923 .72$ 155 15.43 $\begin{array}{lllllll}15 & 115 & 18,55 & 19 & 23,70 & 155 & 15.28\end{array}$

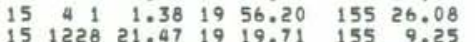

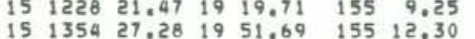
$\begin{array}{lllllll}15 & 1354 & 27.28 & 19 & 51.69 & 155 & 12.30 \\ 15 & 1411 & 24.22 & 19 & 20.27 & 155 & 7.26\end{array}$ $\begin{array}{lllllll}15 & 1722 & 8.46 & 19 & 30.00 & 155 & 53.15\end{array}$ $\begin{array}{lllllll}15 & 1744 & 34.16 & 19 & 21.02 & 154 & 59.86 \\ 16 & 018 & 56.21 & 19 & 21.87 & 155 & 12.85\end{array}$

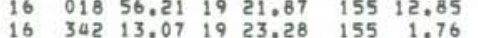

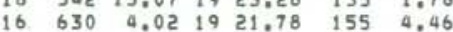
$\begin{array}{llllllll}16 & 9 & 5 & 58.05 & 19 & 19.65 & 155 & 11.20\end{array}$ $\begin{array}{lllllll}16 & 1046 & 53.18 & 19 & 22.16 & 155 & .40 \\ 16 & 1346 & 30.96 & 19 & 21.05 & 155 & 12.87\end{array}$ 16134630.961921 .0515512 .87 $162029 \quad 53.87$ 19 $15.34 \quad 155 \quad 24.17$

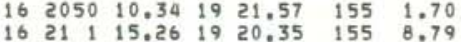

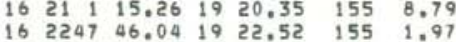

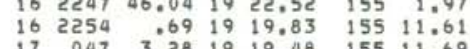

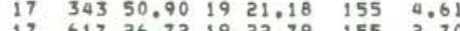

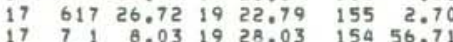

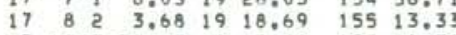

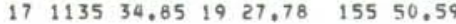
$\begin{array}{lllllll}17 & 1141 & 54.04 & 19 & 21.07 & 155 & 10.30\end{array}$ 17 1250 $32.021920 .51 \quad 15512.93$

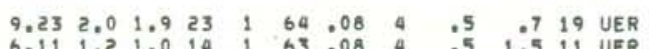

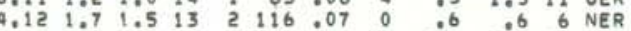

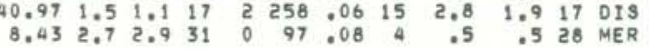
$\begin{array}{lllllllllllll}3.06 & 1.3 & 1.3 & 13 & 1 & 97 & .06 & 3 & .4 & .5 & 12 & 9 P C\end{array}$ $\begin{array}{llllllllllllll}2.51 & .9 & .8 & 12 & 2 & 109 & .25 & 3 & .6 & .6 & 8 & \mathrm{SPC}\end{array}$ $\begin{array}{llllllllllll}5.37 & 3.4 & 3.8 & 36 & 0 & 44 & 013 & 3 & 04 & .7 & 33 & \text { SPC F } \\ 7.70 & 1.7 & 1.4 & 28 & 0 & 103 & .07 & 6 & 04 & 06 & 26 & \text { UER }\end{array}$ $\begin{array}{llllllllllllll}2.77 & 1.4 & 1.2 & 13 & 1 & 78 & .10 & 2 & .4 & .5 & 9 \mathrm{SPC}\end{array}$

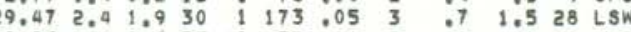

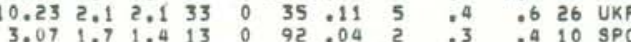

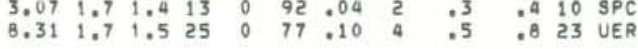
\begin{tabular}{lllllllllll}
9.54 & 2.8 & 3.0 & 33 & 1 & 58 & .09 & 3 & .4 & .5 & 29 \\
\hline & UER
\end{tabular}

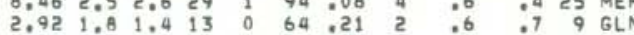

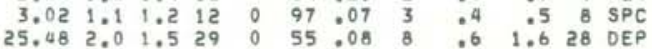
$\begin{array}{llllllllllllll}3.41 & 2.6 & 3.0 & 30 & 1 & 68 & .09 & 2 & .3 & .5 & 20 & \mathrm{SPC}\end{array}$ $\begin{array}{lllllllllllll}14.68 & 2.4 & 1.6 & 28 & 3 & 182 & .13 & 37 & .9 & 3.7 & 26 & K K U & \end{array}$

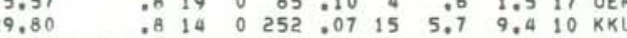

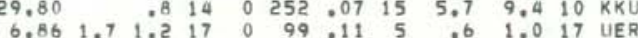

$\begin{array}{lllllllllll}6.30 & 2.0 & 21 & 0 & 120 & 19 & 4 & .8 & .9 & 19 & \text { KON } \\ 5.37 & 1.1 & 13 & 0 & 213 & 020 & 6 & 2.0 & 3.3 & 11 & \text { LER }\end{array}$

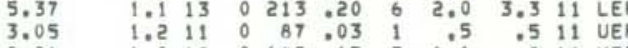
$\begin{array}{llllllllllll}8.21 & & 1.0 & 19 & 0 & 142 & 0.15 & 5 & 1.1 & .8 & 16 & \text { MER } \\ 7,39 & 1.6 & 1.1 & 15 & 0 & 86 & .13 & 4 & .7 & 1.4 & 14 & \text { MER }\end{array}$ $\begin{array}{lllllllllll}8.24 & .9 & 18 & 0 & 94 & 09 & 5 & .7 & 1.2 & 18 & \text { UER }\end{array}$

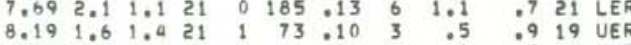

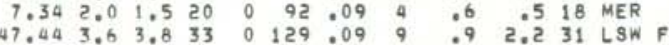
$\begin{array}{rrrrrrrrrrrr}7.10 & 1.6 & 1.2 & 26 & 0 & 170 & 011 & 4 & .8 & .5 & 22 & \text { MER } \\ 6.41 & .8 & .8 & 17 & 0 & 120 & .08 & 4 & : 6 & 1.3 & 17 & \text { UER }\end{array}$

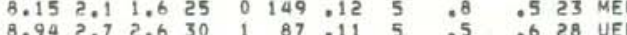

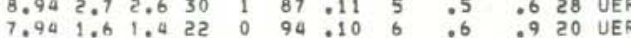
$\begin{array}{rrrrrrrrrrrr}6.58 & 1.3 & .8 & 23 & 0 & 92 & 0.13 & 4 & .6 & 1.1 & 21 & \text { MER } \\ 7.49 & 1.9 & 1.3 & 23 & 1 & 127 & : 13 & 4 & 06 & .6 & 21 & \text { MER }\end{array}$

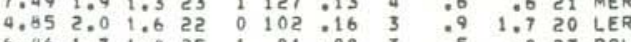
$\begin{array}{llllllllllll}6.46 & 1.7 & 1.4 & 25 & 1 & 81 & 08 & 3 & .5 & .8 & 23 & \text { POL } \\ 6.39 & 1.9 & 1.7 & 22 & 1 & 117 & .16 & 8 & .7 & 1.1 & 18 & \text { KON }\end{array}$ $\begin{array}{lllllllllllll}7.82 & 1.6 & 1.5 & 25 & 0 & 68 & .10 & 2 & .5 & .8 & 23 & \text { UER }\end{array}$

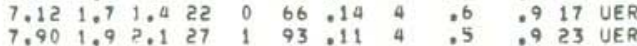




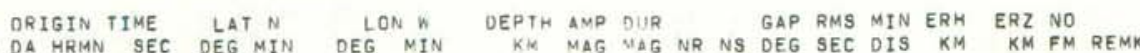

1979 APR $17 \quad 2047 \quad 47.70 \quad 19 \quad 19.64 \quad 155 \quad 10.40$

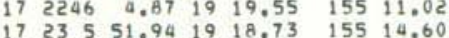

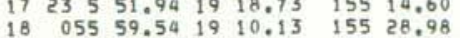

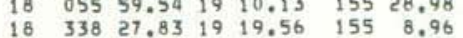
$\begin{array}{lllllll}18 & 427 & 15.62 & 19 & 25.83 & 155 & 27.80 \\ 18 & 446 & 14.39 & 19 & 21.19 & 155 & 2.31\end{array}$

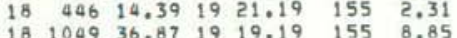

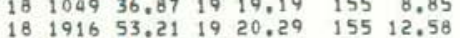

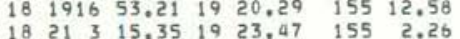

$\begin{array}{lllllll}18 & 2357 & 3.03 & 19 & 18.69 & 155 & 13.59\end{array}$

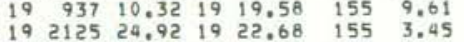
192125 24.92 19 22.68 1553.45 $\begin{array}{ll}10 & 026 \quad 22,27 \quad 19 \quad 20,32 \quad 155 \quad 11.46\end{array}$ $\begin{array}{llllllll}20 & 1 & 7 & 6.04 & 19 & 20.51 & 155 & 6.51 \\ 20 & 357 & 13.39 & 19 & 24.08 & 155 & .67\end{array}$ $20 \quad 445 \quad 10.68 \quad 19 \quad 19.10 \quad 15514.12$ $\begin{array}{lllllll}20 & 1348 & 58.87 & 19 & 15.91 & 155 & 47.35 \\ 20 & 1519 & 30.77 & 19 & 19.09 & 155 & 30.65\end{array}$ $\begin{array}{lllllll}20 & 1554 & 37.98 & 19 & 21.49 & 155 & 1.77\end{array}$

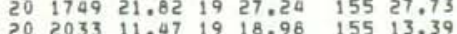
$\begin{array}{lllllllll}21 & 0 & 3 & 33.34 & 19 & 20,31 & 155 & 2.47\end{array}$ $21032 \quad 4.2219 \quad 19.68 \quad 155 \quad 9.96$

$21 \quad 149 \quad 41.91 \quad 1923.88 \quad 155 \quad 27.42$ $\begin{array}{llrrrrr}21 & 213 & 5.58 & 19 & 20.60 & 155 & 5.77 \\ 21 & 756 & 31.67 & 19 & 21.44 & 155 & 12.74\end{array}$ $\begin{array}{lllllll}21 & 814 & 4,55 & 19 & 23,11 & 155 & 2,79\end{array}$ $21 \quad 121452.33 \quad 1920.12 \quad 15529.04$ $\begin{array}{lllllll}21 & 1552 & 46.23 & 19 & 20.79 & 155 & 7.92\end{array}$ $\begin{array}{lllllll}21 & 19 & 34.77 & 19 & 18.56 & 155 & 13.31\end{array}$ $\begin{array}{llllllll}22 & 0 & 6 & 38.09 & 19 & 23.41 & 155 & 1.92 \\ 22 & 2 & 2 & 36.12 & 19 & 23.20 & 155 & 15.14\end{array}$

$\begin{array}{lllllll}22 & 247 & 2.63 & 19 & 20.78 & 155 & 7.10\end{array}$

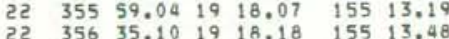
$\begin{array}{lllllll}22 & 523 & 15.90 & 19 & 20.23 & 155 & 12.06 \\ 22 & 622 & 27.10 & 19 & 10.73 & 155 & 29.17\end{array}$

$258.8519,18.08$ 155 13.15

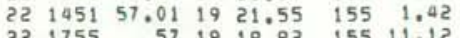
$22 \quad 1755 \quad .57 \quad 19 \quad 19.92 \quad 155 \quad 11.12$

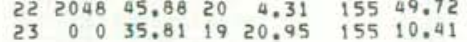

$\begin{array}{lllllll}23 & 128 & 33.12 & 19 & 22.86 & 155 & 5.11\end{array}$ $\begin{array}{lllllll}23 & 258 & 4.17 & 20 & 6.62 & 155 & 50.26 \\ 23 & 551 & 43.61 & 19 & 20,04 & 155 & 6.55\end{array}$
KK. MAG MAG NR NS DEG SEC DIS KM KM FM REMK $\begin{array}{llllllllllll}8.34 & 1.9 & 1.9 & 26 & 1 & 94 & .10 & 5 & .6 & .7 & 21 & \text { UER } \\ 7.15 & 1.9 & 1.3 & 27 & 0 & 96 & .12 & 5 & .5 & .7 & 26 & \text { UER }\end{array}$ $\begin{array}{llllllllllll}7.40 & 1.4 & 1.2 & 24 & 0 & 112 & .13 & 4 & .6 & .9 & 21 & \text { POL } \\ 33.11 & 1.8 & 1.6 & 26 & 1 & 103 & .05 & 2 & .8 & 2.0 & 23 & \text { LSW }\end{array}$

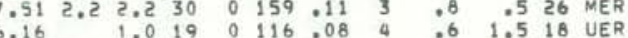

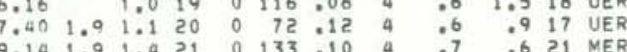
$\begin{array}{llllllllllll}8.93 & 1.9 & 1.7 & 23 & 0 & 87 & .09 & 3 & .6 & .6 & 19 & P O L\end{array}$

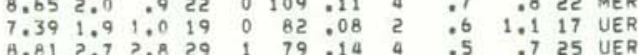
$\begin{array}{rrrrrrrrrrrr}9.18 & 2.6 & 2.6 & 29 & 1 & 105 & 10 & 5 & .6 & .5 & 26 & \text { UER } \\ 6.08 & & .9 & 19 & 0 & 148 & .17 & 4 & .8 & 1.0 & 15 & \text { LER }\end{array}$

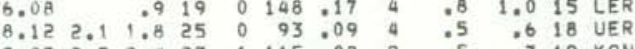

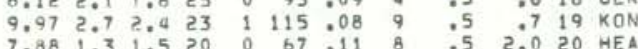
$\begin{array}{llllllllllll}3.25 & 1.2 & 1.3 & 16 & 0 & 170 & .09 & 4 & .9 & 1.1 & 10 & \text { MER }\end{array}$

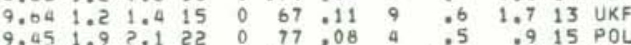

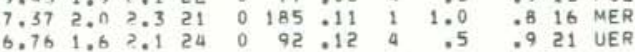
$\begin{array}{llllllllllll}8.60 & 1.0 & 1.3 & 18 & 0 & 64 & .10 & 3 & .5 & 1.2 & 15 & \text { UKF }\end{array}$

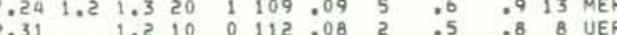
$\begin{array}{llllllllllll}8.52 & 1.9 & 2.0 & 19 & 2 & 125 & .13 & 6 & .8 & 1.1 & 16 & \text { MER }\end{array}$

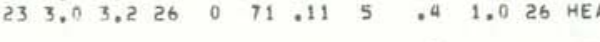

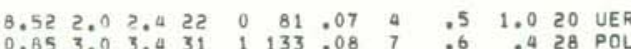

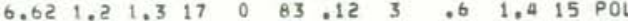

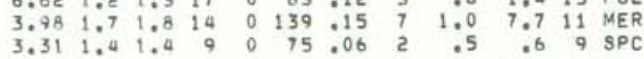
$\begin{array}{llllllllllll}7.61 & 2.1 & 2.4 & 23 & 2 & 92 & .13 & 5 & .5 & 1.2 & 19 & \text { UER }\end{array}$

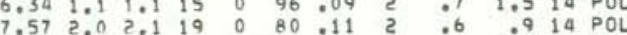

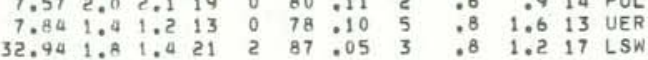

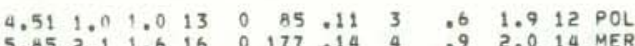
$8.24 \quad 1.4 \quad 1.1 \quad 17 \quad 0 \quad 88 \quad .11 \quad 5 \quad 06 \quad 1.013$ UER

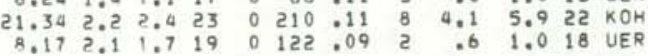
$\begin{array}{lllllllllllll}6.34 & 1.9 & 1.3 & 12 & 0 & 204 & 08 & 7 & 1.0 & 2.5 & 10 & \text { MER }\end{array}$

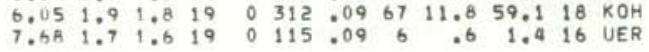

$\begin{array}{llllllllllll}8.84 & 1.9 & 1.2 & 27 & 1 & 42 & .11 & 6 & .4 & .9 & 16 & \text { UKF }\end{array}$

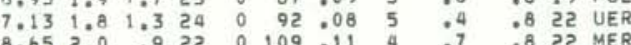

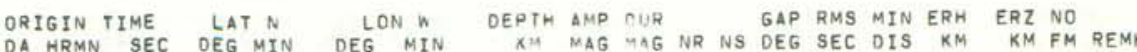

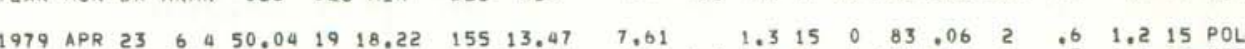
$23 \quad 173 \quad 49.0019 \quad 20,00 \quad 155 \quad 7.25$ 23 2235 93.431919 .89 155 11.20 $24 \quad 112 \quad 38.11 \quad 20 \quad 6.79 \quad 155 \quad 54.62$

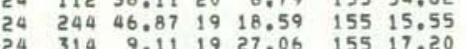
$24 \quad 614 \quad 3.30$ i9 $23,18 \quad 155 \quad 26,18$

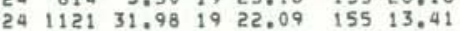
$\begin{array}{lllllll}24 & 1428 & 30.40 & 19 & 22.19 & 155 & 4.88\end{array}$

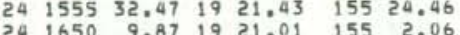
$2417513.801926 .97 \quad 15529,43$

$\begin{array}{lllllll}24 & 2024 & 14.76 \quad 19 & 52.17 \quad 155 & 22.37\end{array}$

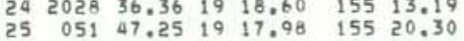
$\begin{array}{lrrrrrr}25 & 548 & 58.78 & 19 & 20.10 & 155 & 48.18 \\ 25 & 95 & 23.10 & 19 & 23.94 & 155 & 15.62\end{array}$ $\begin{array}{lllllll}25 & 949 & 3.89 & 19 & 23.93 & 155 & 15.38\end{array}$

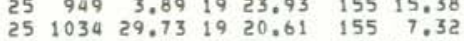
$25112012.891923 .05 \quad 15514.81$ $\begin{array}{lrrrrrr}25 & 1135 & 53.86 & 19 & 23.29 & 155 & 15.03 \\ 25 & 1520 & 33.36 & 19 & 20.98 & 155 & 6.09\end{array}$ $\begin{array}{lllllll}25 & 1624 & 36.11 & 19 & 20.79 & 155 & 6.13\end{array}$

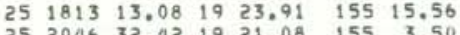

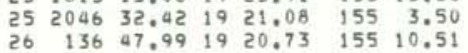

$\begin{array}{lllllll}26 & 216 & 33.31 & 19 & 23.92 & 155 & 15.38\end{array}$ $26 \quad 658 \quad 37.83 \quad 1923,58 \quad 155 \quad 15,20$ $\begin{array}{lllllll}26 & 719 & 4.95 & 19 & 19.44 & 155 & 13.99\end{array}$ $26 \quad 74134.471921 .62 \quad 15549.10$

$26 \quad 827 \quad 21.63 \quad 1924.09 \quad 155 \quad 16.03$ $26 \quad 916 \quad 54.69 \quad 19 \quad 17.45 \quad 155 \quad 14.13$ $\begin{array}{lllllll}26 & 932 & 18.47 & 19 & 20.92 & 155 & 2.86\end{array}$ $26 \quad 105916.98 \quad 1921.96 \quad 155 \quad 6.18$ $\begin{array}{llllllll}26 & 1330 & 47.50 & 19 & 19.21 & 155 & 9.66\end{array}$

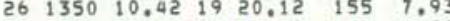
26155225.951923 .82015515 .76 $2621959.941920 .45 \quad 155 \quad 5.56$

$\begin{array}{lllllll}26 & 2129 & 12.60 & 19 & 23.77 & 155 & 15.67\end{array}$

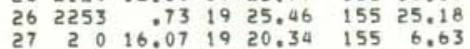

$\begin{array}{lllllllllllll}7.61 & 1.3 & 15 & 0 & 83 & .06 & 2 & .6 & 1.2 & 15 & \text { POL } \\ 6.59 & 2.0 & 1.3 & 24 & 1 & 104 & .10 & 5 & .5 & 1.4 & 22 & \text { UER } \\ 9.55 & 0.0 & 0.5 & 24 & & 98 & .08 & 5 & .4 & .6 & 19 & \text { UER }\end{array}$

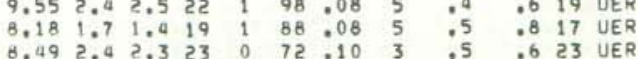
$3.053 .13 .133,262.1014 \quad 2.5 \quad 2.829$
$\mathrm{KOH}$

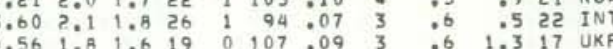

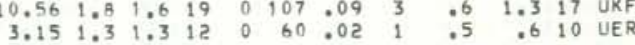
$\begin{array}{llllllllllll}8.54 & 1.1 & 15 & 0 & 127 & .08 & 3 & .6 & 1.2 & 12 & \text { MER }\end{array}$ $\begin{array}{llllllllllll}6.54 & 1.8 & : 4 & 19 & 2 & 114 & 12 & 9 & .5 & 2.4 & 11 & \text { UKF } \\ 3.93 & 1.5 & 1.5 & 19 & 2 & 214 & .20 & 4 & 1.8 & 2.6 & 9 & \text { POL }\end{array}$

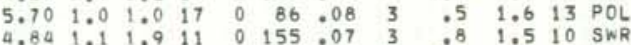
8.931 .91 .817

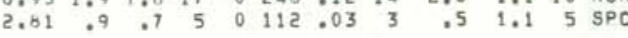

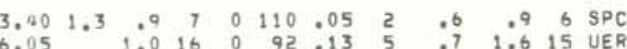
$\begin{array}{lllllllllllll}3.29 & 1.1 & 9 & 6 & 0 & 112 & 04 & 2 & .04 & .9 & 4 & G L N\end{array}$

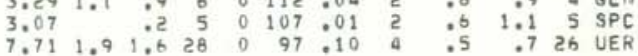
$\begin{array}{lllllllllllll}8.01 & 2.6 & 0.6 & 29 & 0 & 101.07 & 4 & .4 & .6 & 26 & \text { UER }\end{array}$

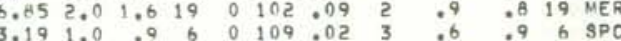

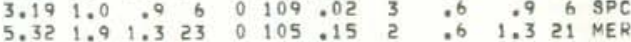

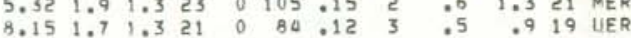
$\begin{array}{lllllllllllllll}3.17 & .9 & .5 & 5 & 0 & 109 & .03 & 2 & .6 & 1.2 & 5 & \mathrm{SPC}\end{array}$

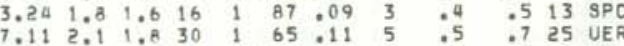

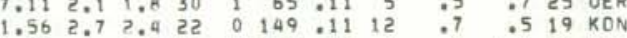

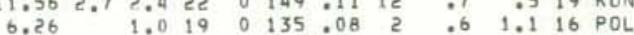

$\begin{array}{lllllllllllll}3.18 & \text { B } & .8 & 5 & 0 & 118 & 01 & 2 & .7 & 9 & 5 & \text { SPC }\end{array}$

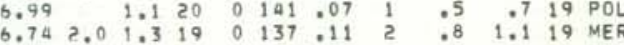

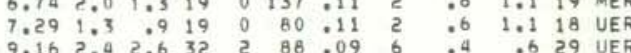

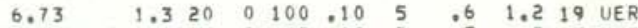

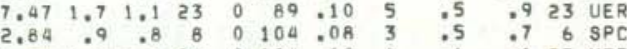
$\begin{array}{llllllllllll}7.99 & 1.9 & 1.8 & 29 & 0 & 104 & 110 & 4 & .6 & .6 & 27 & \text { MER } \\ 1.70 & 2.9 & 3.3 & 29 & 0 & 277 & .14 & 33 & 3.6 & 3.0 & 29 & 019\end{array}$ $\begin{array}{rllllllllllll}2.45 & 1.2 & 1.1 & 12 & 0 & 101 & .07 & 3 & .4 & .5 & 11 & \text { SPC } \\ 8.52 & 1.8 & 1: 3 & 24 & 0 & 54 & : 12 & 7 & .5 & 1.1 & 23 & \text { UKF } \\ 7.30 & 1.7 & 1.4 & 24 & 0 & 107 & .09 & 5 & .5 & .9 & 23 & \text { UER }\end{array}$

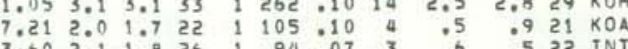

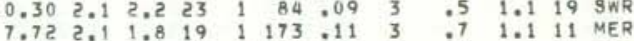
$\begin{array}{llllllllllll}34.27 & 2.0 & 2.2 & 14 & 1 & 188 & .06 & 4 & 1.5 & 2.1 & 9 & \text { KKU }\end{array}$ 
HVO EARTHDUAKE SUMMARY LIST

PAGE 29

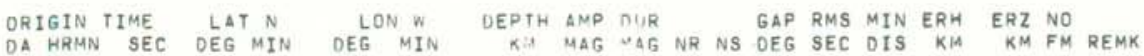

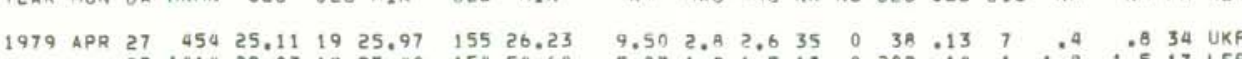

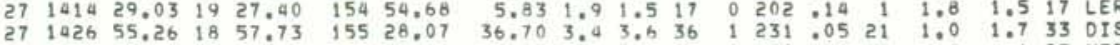

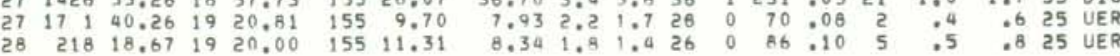
$\begin{array}{lllllllllllllllllllll}28 & 615 & 52.85 & 19 & 18.33 & 155 & 15.34 & 10.16 & 2.7 & 2.6 & 29 & 0 & 136 & .11 & 4 & .7 & .5 & 28 & \text { KOA }\end{array}$

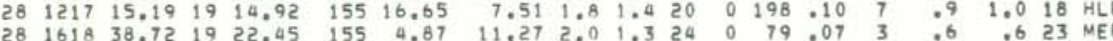

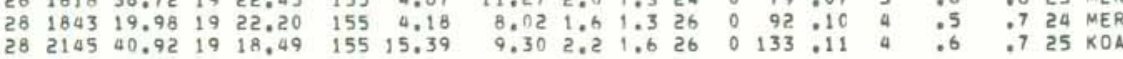
$\begin{array}{llllllllllllllllllll}28 & 2228 & 51.63 & 19 & 26.95 & 155 & 27.71 & 10.01 & 2.1 & 1.6 & 33 & 1 & 52 & .11 & 8 & .4 & .8 & 28 & \text { UKF }\end{array}$

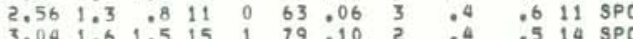

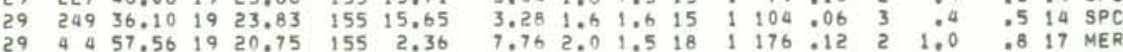

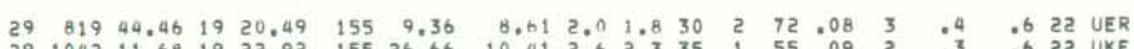
$\begin{array}{lllllllllllllll}9.66 & 10.41 & 2.6 & 2.3 & 35 & 1 & 55 & .09 & 2 & .3 & .6 & 22 & 22 & \text { UKF }\end{array}$

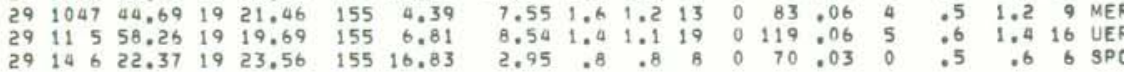
$\begin{array}{llllllll}29 & 16 & 8 & 18.19 & 19 & 18.81 & 155 & 13.61\end{array}$

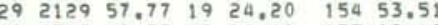
$29231749.751918 .41 \quad 15529.69$

$\begin{array}{lllllll}30 & 111 & 23.35 & 19 & 23.03 & 155 & 14.97\end{array}$

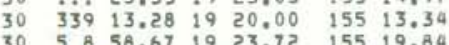

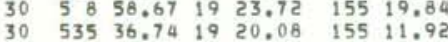

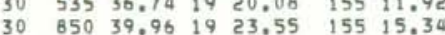
$\begin{array}{lllllll}30 & 1751 & 5.33 & 19 & 22.98 & 155 & 14.79\end{array}$

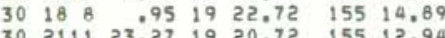
30211123.271920 .72 155 12.90

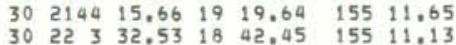

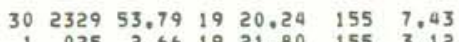

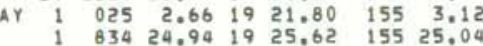
$1 \quad 103 \quad 1.071923 .54 \quad 15527.79$

$\begin{array}{lllllll}1 & 1541 & 53.28 & 19 & 20.84 & 155 & 12.91\end{array}$ $11656 \quad 4.08 \quad 1920.89 \quad 155 \quad 1.52$

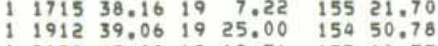

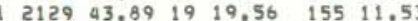

$\begin{array}{lllllll}1 & 2233 & 35.27 & 19 & 32.82 & 155 & 12.19 \\ 1 & 2334 & 41.71 & 19 & 19.91 & 155 & 3.69\end{array}$ $205517.01 \quad 19 \quad 20,32 \quad 155 \quad 13,21$

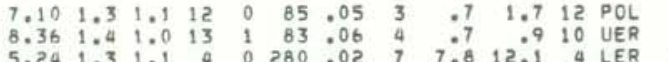

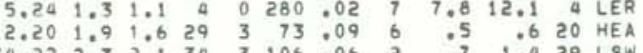
$\begin{array}{llllllllllll}3.54 & 1.5 & 1.3 & 10 & 0 & 82 & .06 & 2 & .5 & .7 & 10 & \text { GLN }\end{array}$

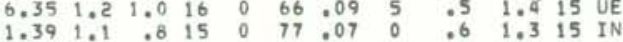
$\begin{array}{llllllllllll}8.65 & 1.5 & 1.0 & 17 & 1 & 80 & 05 & 5 & .5 & 1.3 & 10 & \text { UER } \\ 3.29 & 2.1 & 2.2 & 20 & 1 & 45 & .07 & 2 & .3 & .4 & 17 & \text { SPC }\end{array}$

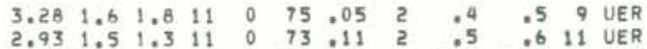

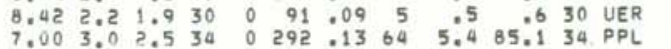
$\begin{array}{rrrrrrrrrrrr}7.77 & 1.4 & 1.3 & 25 & 1 & 96 & : 10 & 5 & .5 & .9 & 23 & \text { UER } \\ 6.05 & 1.9 & 1.3 & 19 & 0 & 122 & : 13 & 4 & : 7 & 1.2 & 18 & \text { MER }\end{array}$

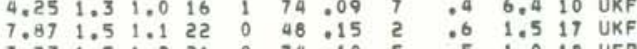
$\begin{array}{lllllllllll}7.11 & 1.5 & 1.4 & 23 & 0 & 62 & 0.14 & 3 & .6 & .920 & 20 \text { UER }\end{array}$

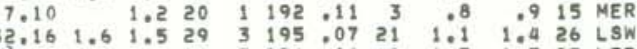

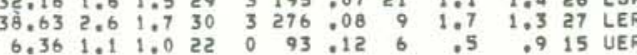
$\begin{array}{rrrrrrrrrrrrr}26.57 & 1.3 & .8 & 25 & 3 & 147 & 08 & 15 & 07 & 1.4 & 17 & \text { NER } \\ 5.27 & 1.2 & 1.2 & 23 & 2 & 149 & 14 & 1 & 06 & 1.1 & 15 & \text { MER }\end{array}$

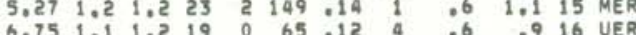
\begin{tabular}{lllllllllll}
8.94 & 2.0 & 1.5 & 7 & 0 & 154 & .02 & 4 & 1.0 & 2.9 & 5 \\
\hline
\end{tabular}
HVO EARTHOUAKE SUMMARY LIST

PAGE 30 YEAR MON ORIGIN TIME LAT NET SEC DEG MIN DEG MIN DE KM MAG UAG NR NS DEG SEC DIS KM KM FM REMK $\begin{array}{lllllllllllllllllll}1979 \text { MAY } 2 & 731 & 19.56 & 19 & 31.33 & 155 & 15.61 & 24.44 & 1.2 & 1.0 & 22 & 4 & 181 & .07 & 12 & 9 & 1.5 & 19 & \text { NER }\end{array}$

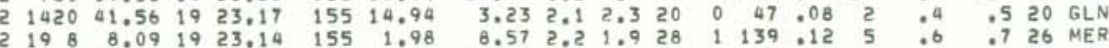

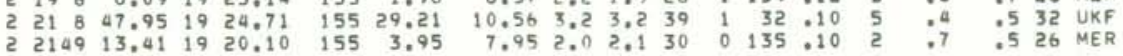
2
$2150 \quad 40.30 \quad 19 \quad 16.72 \quad 155 \quad 13.52$

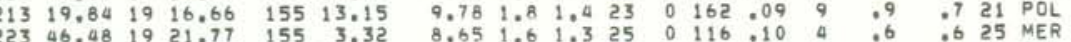

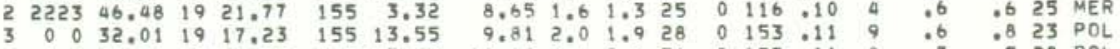

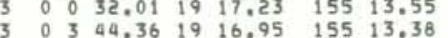

$3 \quad 045 \quad 44,72 \quad 19 \quad 17,13 \quad 155 \quad 13,45$

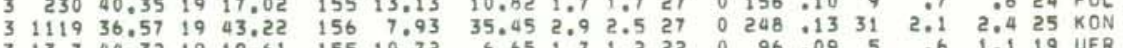

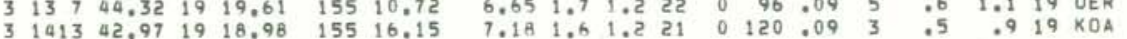

$31549 \quad 23.72 \quad 19 \quad 14.08 \quad 155 \quad 37.09$

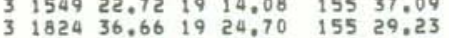
$\begin{array}{lllllll}3 & 1914 & 6.05 & 19 & 19.62 & 155 & 11.25 \\ 3 & 2028 & 21.15 & 19 & 19.79 & 155 & 9.86\end{array}$ $3225625,951926.01 \quad 15527,44$ $8.502 .31 .516 \quad 0201.20 \quad 2 \quad 1.0 \quad 1.516$ HEA

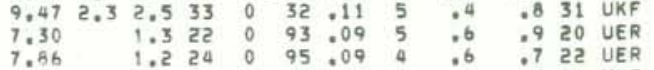

$\begin{array}{lllllllllllllllllll}4 & 829 & 54.45 & 19 & 52.77 & 155 & 23.37 & 12.31 & 2.6 & 2.5 & 32 & 1 & 172 & .11 & 32 & .7 & .8 & 29 & \text { KKU }\end{array}$

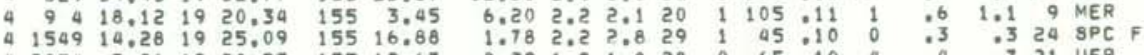

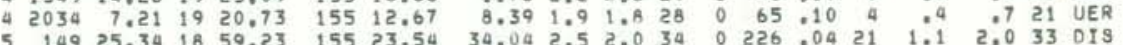
$\begin{array}{lrllllllllllllllllll}5 & 255 & 35.73 & 19 & 19.25 & 155 & 11.75 & 8.22 & 2.6 & 2.9 & 28 & 0 & 100 & .08 & 5 & .4 & .7 & 18 & \text { UER } \\ 5 & 258 & 29.30 & 19 & 23.69 & 155 & 26.95 & 9.75 & 1.6 & 1.2 & 24 & 1 & 72 & .12 & 3 & .5 & 1.0 & 21 & \text { UKF }\end{array}$

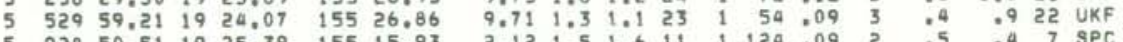

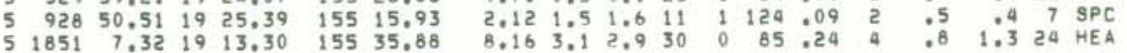

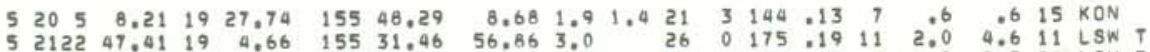

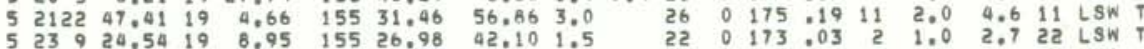

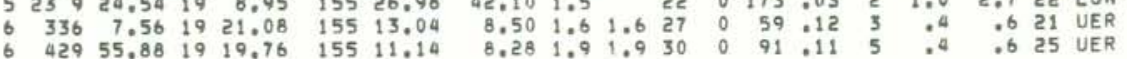

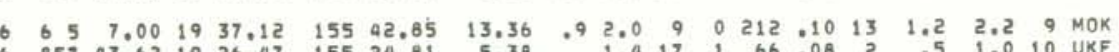

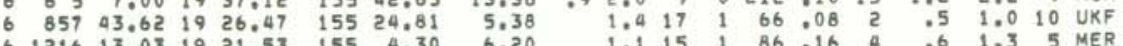

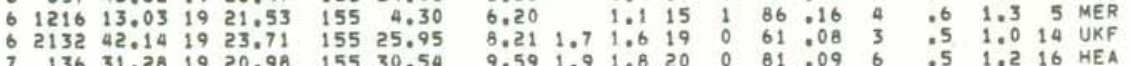
$\begin{array}{lllllllllllllllll}7 & 217 & 6.40 & 19 & 22.61 & 155 & 29.98 & 9.66 & 2.3 & 2.2 & 30 & 1 & 69 & .09 & 4 & .49 & .918 \text { UKF }\end{array}$

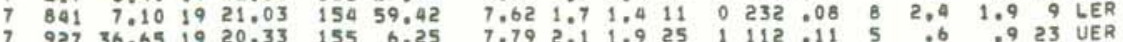

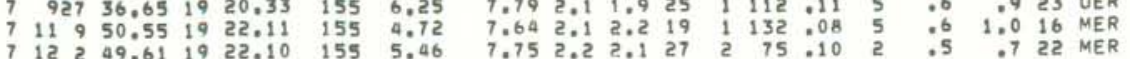

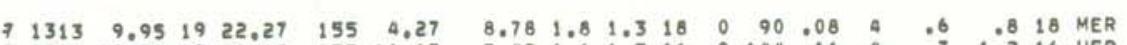

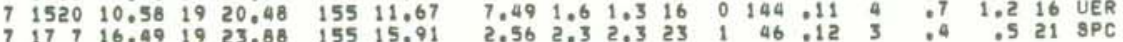


HVO EARTHQUAKE SUMMARY LIST

PAGE 31

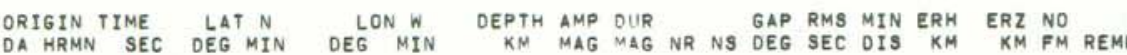

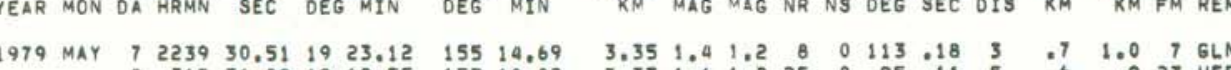

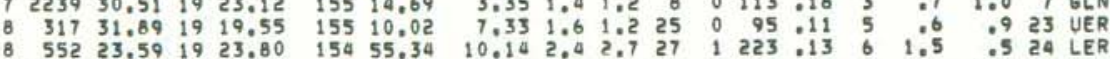

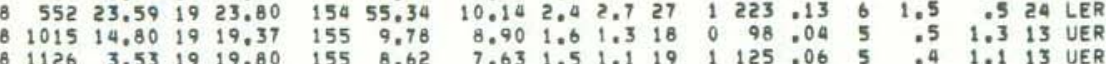

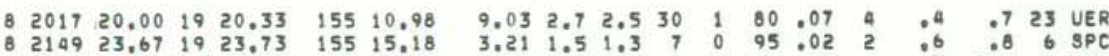

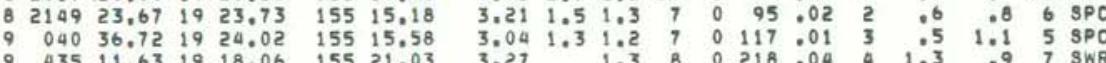

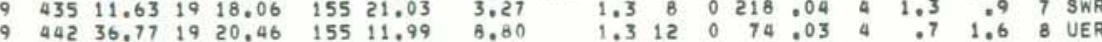
$\begin{array}{lllllllllllllllllll}9 & 627 & 55.87 & 19 & 9.77 & 155 & 33.26 & 32.66 & 2.5 & 1.8 & 26 & 1 & 119 & .06 & 9 & .7 & 1.8 & 20 & \mathrm{LSW}\end{array}$

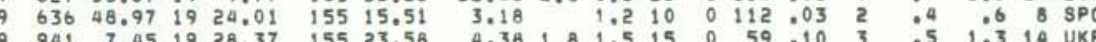

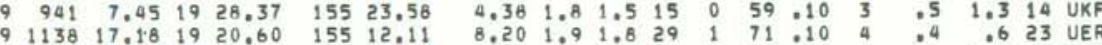

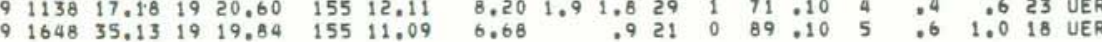

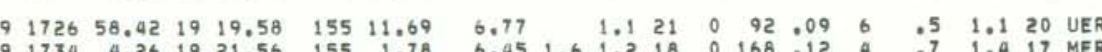

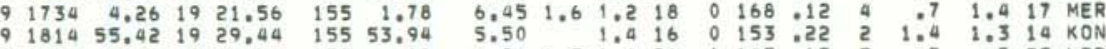

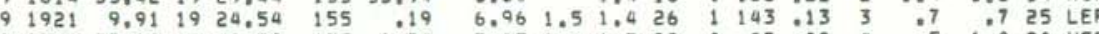

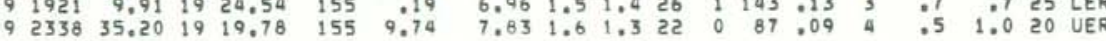
\begin{tabular}{lllllllllllllllllll}
10 & 2 & 6 & 51.19 & 19 & 21.06 & 155 & 2.65 & 7.04 & 2.1 & 1.7 & 27 & 0 & 147 & .12 & 2 & .8 & .5 & 26 \\
\hline
\end{tabular}

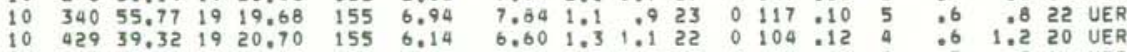

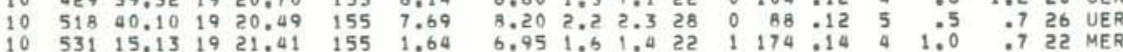

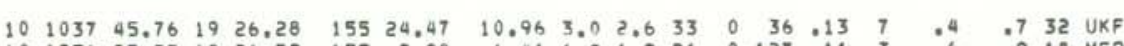

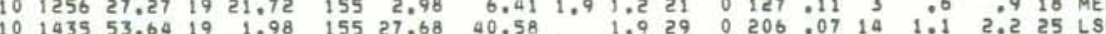

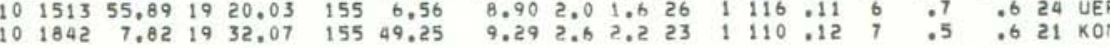

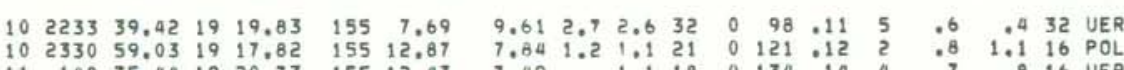

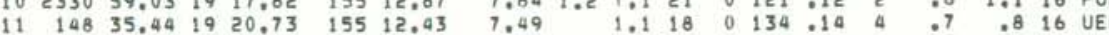

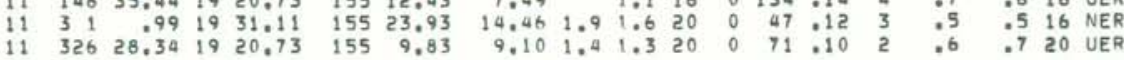

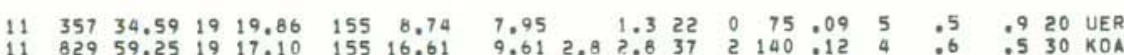

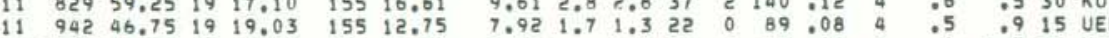

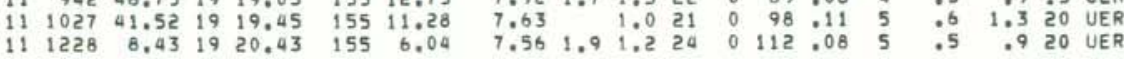

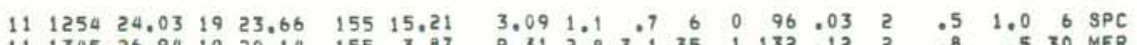

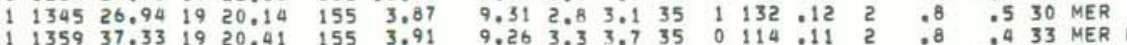

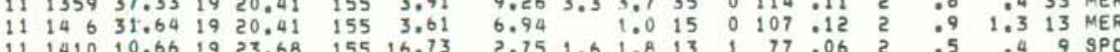

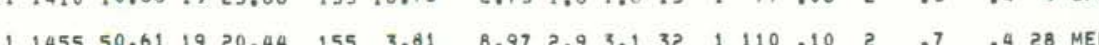

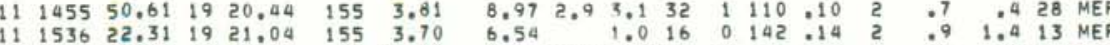

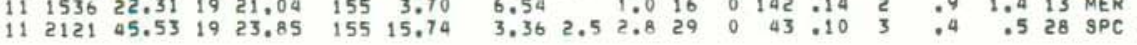

HVO EARTHQUAKE SUMMARY LIST

PAGE 32

ORIGIN TIME LAT N LON W OEPTH AMP OUR GAP KMS MIN ERH ERZ NO YEAR MON ORIGIN TIME LRMN SEC OEG MIN DEG MIN OEPTH AMP OUR MAG GAP KMS MIN ERH ERZ NO REMK

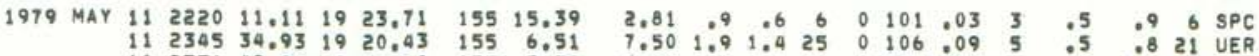

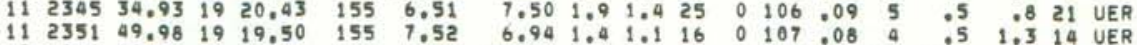

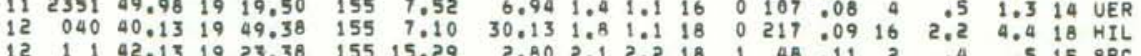
$12 \quad 140 \quad 38.90 \quad 19 \quad 25.31 \quad 155 \quad 15.56$ $214226.81 \quad 1926.05 \quad 15515.67$ $15453.9191920 .27 \quad 155 \quad 10.75$ $12 \quad 617 \quad 37.10 \quad 1919.91 \quad 155 \quad 3.17$ $12 \quad 842 \quad 29.46 \quad 1921.00 \quad 155 \quad 4.43$ $121150 \quad 25.76 \quad 1918.26 \quad 155 \quad 15.36$ $121617 \quad 2.47$ 19 19,33 155 3.36 $12 \quad 1857 \quad 39.16 \quad 1929,91 \quad 15549.83$ $\begin{array}{lllllll}12 & 1951 & 40.69 & 19 & 20.97 \quad 155 \quad 3.30\end{array}$

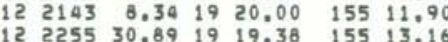
$23328 \quad 10.801919 .76 \quad 155 \quad 0.76$

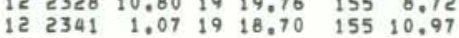
$12234424.37 \quad 1928.07 \quad 15522.42$ 13 13 $02355.941921 .81 \quad 155 \quad 12.79$ $\begin{array}{lllllll}13 & 227 & 5.34 & 19 & 20.40 & 155 & 3.73 \\ 13 & 853 & 7.99 & 19 & 22.72 & 155 & 1.72\end{array}$ $\begin{array}{lllllll}13 & 1138 & 39.85 & 19 & 23.05 & 155 & 3.04\end{array}$ 30.150

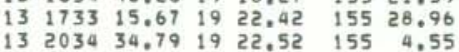
$13 \quad 2049 \quad 8.88 \quad 1923.83 \quad 155 \quad 30.12$ $4 \quad 83124,4819 \quad 18,38 \quad 15515.65$ $\begin{array}{lllllll}14 & 851 & 33.37 & 19 & 24.31 & 155 & 1.69\end{array}$ $1492 \quad 1.16 \quad 1923.94 \quad 155 \quad 15.57$ $14 \quad 96 \quad 20.88 \quad 1925.54 \quad 155 \quad 17.11$ $40057 \quad 31.861923 .00 \quad 15517.85$

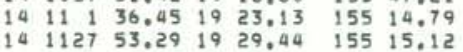
$\begin{array}{llllllll}14 & 12 & 0 & 2.54 & 19 & 20.74 & 155 & 8.72\end{array}$

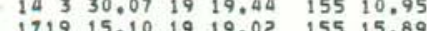
$14 \quad 1838 \quad 45.11$ 19 2.42 $155 \quad 2.28$

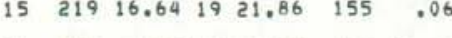

$\begin{array}{lllllll}15 & 317 & 1.77 & 19 & 25.32 & 155 & 16.93\end{array}$

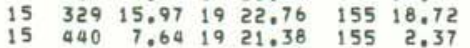

$\begin{array}{llllllllllll}1.71 & 1.3 & 1.4 & 10 & 0 & 153 & 05 & 2 & .6 & .5 & 8 & \mathrm{SPC} \\ 1.72 & 1.0 & 1.1 & 7 & 0 & 240 & 02 & 3 & 1.1 & : 5 & 7 & \mathrm{SPC}\end{array}$

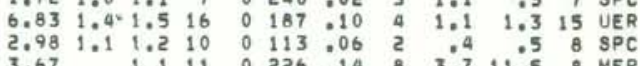

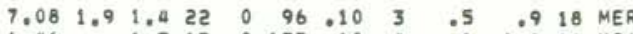
$\begin{array}{lllllllllll}6.46 & 1.3 & 17 & 0 & 136 & .10 & 4 & .6 & 1.1 & 16 & \mathrm{KOA}\end{array}$ 9.282 .92 .935 0 $102: 095$ :.5 509 :4 32 UER $\begin{array}{lllllllllll}7.40 & 1.0 & 19 & 0 & 112 & .09 & 2 & .6 & 1.0 & 16 & \text { MER }\end{array}$

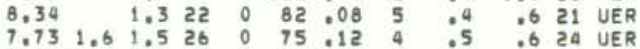

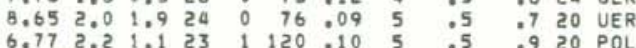
$\begin{array}{llllllllllll}5.61 & 2.0 & 1.6 & 20 & 1 & 79 & .12 & 4 & .5 & 1.6 & 19 & \text { UKF }\end{array}$

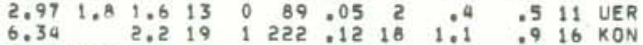
$\begin{array}{lllllllllllll}7.39 & 2.2 & 1.3 & 19 & 0 & 111 & .08 & 2 & .5 & .8 & 17 & \text { MER } \\ 8.56 & 1.6 & 22 & 0 & 152 & 010 & 6 & : 7 & .6 & 17 & \text { MER }\end{array}$ $9.613 .03 .225 \quad 1120.05 \quad 4 \quad .5 \quad .423$ MER

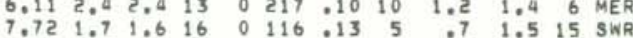
$\begin{array}{llllllllllll}8.91 & 1.9 & 1.6 & 22 & 0 & 61 & .11 & 2 & .5 & .9 & 21 & \text { UKF } \\ 7.98 & 1.6 & 1.4 & 22 & 0 & 85 & .08 & 3 & .4 & .6 & 18 & \text { MER }\end{array}$ $\begin{array}{lllllllllllll}7.89 & 1.9 & 1.3 & 19 & 0 & 60 & .11 & 5 & .5 & 1.3 & 17 & \text { MOK }\end{array}$ $7.56 \quad 1.6 \quad 1.628 \quad 0 \quad 139.12 \quad 9 \quad 0 \% 918$ LER

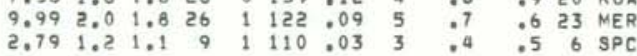
$\begin{array}{llllllllllllllllll}.92 & .7 & .7 & 9 & 1 & 161 & .08 & 1 & .7 & .3 & 8 & \text { SPC }\end{array}$

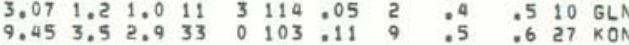

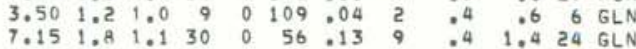
$\begin{array}{llllllllllll}6.59 & 1.4 & 1.1 & 25 & 0 & 70 & .10 & 3 & .5 & .8 & 19 & \text { UER }\end{array}$

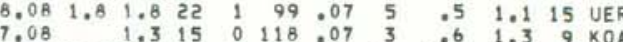

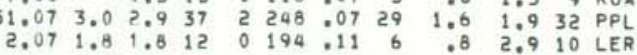
$\begin{array}{llllllllllllll}1.52 & 1.4 & 1.6 & 11 & 1 & 157 & .07 & 1 & .5 & .2 & 3 & \mathrm{SPC}\end{array}$

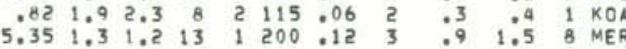


HVO EARTHQUAKE SUMMARY LIST

PAGE 33

ORIGIN TIME LATN LON W OEPTH AMP DUR GAP RMS MIN ERH ERZ NO YEAR MON DA HRMN SEC DEG MIN DEG MIN KY MAG MAG INR NS DEG SEC DIS KM KM FM REMK $\begin{array}{lllllll}15 & 557 & 45.47 & 19 & 26.77 & 155 & 24.55 \\ 15 & 717 & 33.42 & 19 & 23.66 & 155 & 16.68\end{array}$ $15 \quad 1126 \quad 35.77 \quad 1923.02 \quad 155 \quad 15.00$ $15163526.651920 .66 \quad 155 \quad 5.89$

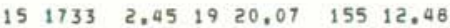

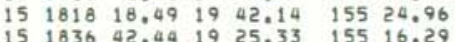

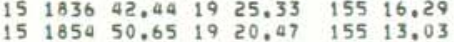

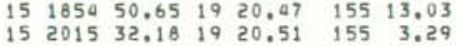

$\begin{array}{lllllll}15 & 2053 & 9.95 & 19 & 23.34 & 155 & 14.98\end{array}$

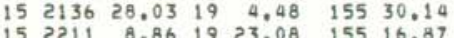
$152239 \quad 44.291920 .56 \quad 155 \quad 3.45$

$\begin{array}{lllllll}16 & 0.46 & 20.41 & 19 & 18.75 & 155 & 29.42\end{array}$ $\begin{array}{lllllll}16 & 130 & 50.27 & 19 & 24.50 & 155 & 16.08 \\ 16 & 330 & 57.21 & 19 & 20.49 & 155 & 9,00\end{array}$ $16 \quad 4 \quad 8 \quad 55.591921 .50 \quad 155 \quad 1.80$ $\begin{array}{lllllll}16 & 740 & 42.97 & 19 & 25.69 & 155 & 16.06\end{array}$ $16 \quad 1317 \quad 57.87 \quad 1920.62 \quad 15513.10$ $\begin{array}{lllllll}16 & 1320 & 48.41 & 19 & 24.06 & 155 & 16.15 \\ 16 & 1721 & 58.92 & 19 & 23.86 & 155 & 15.68\end{array}$

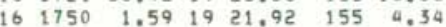

$\begin{array}{llllllll}16 & 18 & 5 & 54.42 & 19 & 23.75 & 155 & 15.29\end{array}$

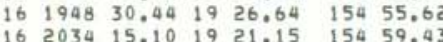

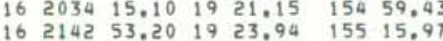

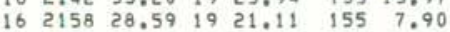
$\begin{array}{lllllll}16 & 2316 & 56.06 & 19 & 20.31 & 155 & 12.88\end{array}$

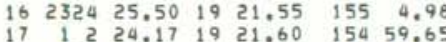
$\begin{array}{llllllll}17 & 225 & 54.31 & 19 & 23.93 & 155 & 15.45\end{array}$ $\begin{array}{lllllll}17 & 232 & 40.53 & 19 & 26.47 & 154 & 48.79\end{array}$

$\begin{array}{lllllll}17 & 530 & 52.27 & 19 & 27.90 & 155 & 24.29\end{array}$

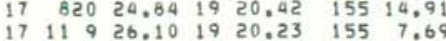
$\begin{array}{lllllll}17 & 1134 & 35.86 & 19 & 25.70 & 155 & 24.29 \\ 17 & 1311 & 37,50 & 19 & 20.18 & 155 & 7,50\end{array}$

$\begin{array}{lllllll}17 & 1721 & 44.88 & 19 & 20.79 & 155 & 10.99\end{array}$

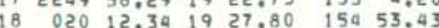
18 ट 638.231923 .36 154 59.00 $\begin{array}{lllllll}18 & 346 & 16.50 & 19 & 25.47 & 155 & 23.78\end{array}$

$\begin{array}{llllllll}18 & 618 & 15.34 & 19 & 20.69 & 155 & 11.75\end{array}$ $\begin{array}{lllllll}18 & 73 & 23.36 & 19 & 21.05 & 155 & 6.14 \\ 18 & 724 & 37.60 & 19 & 23.80 & 155 & 15.27\end{array}$

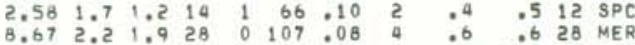

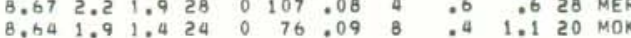

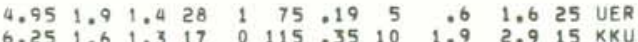

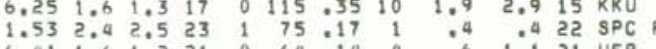

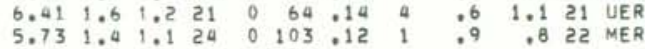
$\begin{array}{rrrrrrrrrrrrr}3.48 & 1.1 & 1.2 & 8 & 0 & 87 & .04 & 2 & .5 & .8 & 8 & 6 L N\end{array}$

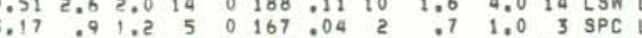

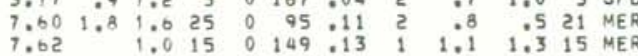

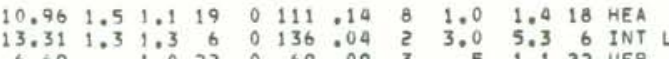
$\begin{array}{lllllrllllll}6.69 & & 1.0 & 22 & 0 & 69 & 09 & 3 & .5 & 1.1 & 22 & \text { UER } \\ 5.36 & 2.0 & 1.6 & 22 & 0 & 169 & .20 & 4 & 1.0 & 1.4 & 21 & \text { MER }\end{array}$ $\begin{array}{rrrrrrrrrrrr}1.51 & 1.0 & 1.0 & 7 & 1 & 179.03 & 2 & .6 & .4 & 6 & \text { SPC } \\ 7.91 & 1.7 & 1.6 & 21 & 0 & 62 & 03 & 4 & .5 & .7 & 20 & \text { UER }\end{array}$

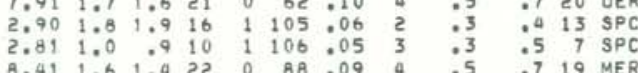

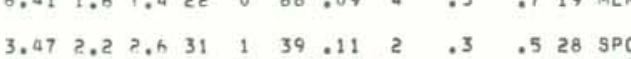

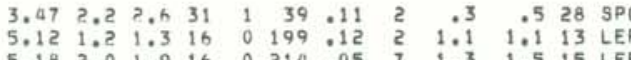

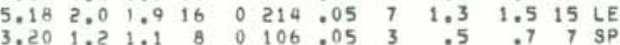

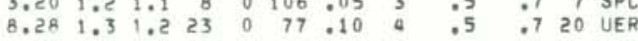
$\begin{array}{llllllllllll}8.79 & 1.5 & 1.4 & 19 & 0 & 68 & .09 & 4 & .5 & .7 & 18 & \text { UER }\end{array}$

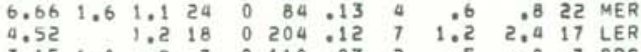
$\begin{array}{lllllllllllll}3.15 & 1.0 & 09 & 7 & 0 & 110 & 03 & 2 & .5 & .8 & 7 & \text { SPC } \\ 7.69 & 2.2 & : .6 & 16 & 0 & 291 & .16 & 9 & 0.4 & 1.1 & 13 & \text { LER }\end{array}$

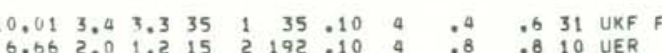

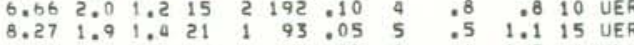

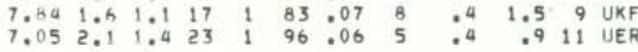
$\begin{array}{lllllllllllll}9.52 & 1.7 & 0.0 & 16 & 0 & 78 & 04 & 3 & .6 & 1.1 & 11 & \text { UER } \\ 9.52 & 2.7 & 0.0 & 27 & 1 & 92 & 08 & 3 & .5 & .7 & 16 & \text { MER }\end{array}$

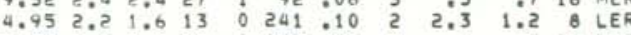

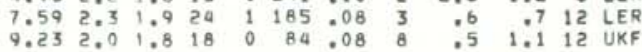
$\begin{array}{llllllllllll}9.12 & 1.9 & 1.4 & 13 & 0 & 72 & 03 & 4 & .5 & .7 & 10 & \text { UER }\end{array}$

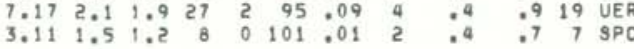

$\begin{array}{lllllllllllll}9.19 & 2.0 & 1.6 & 21 & 0 & 81 & 09 & 6 & .5 & 1.2 & 17 & \text { UKF } \\ 2.93 & 1.8 & 1.3 & 14 & 1 & 71 & .04 & 1 & .3 & .3 & 10 & \text { SPC }\end{array}$
HVO EARTHQUAKE SUMMARY LIST

PAGE 34

ORIGIN TIME LAT N LON W DEPTH AMP DUR GAP RMS MIN ERH ERZ NO

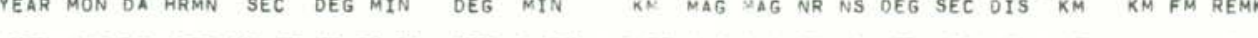

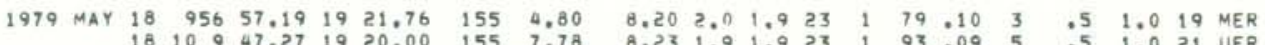

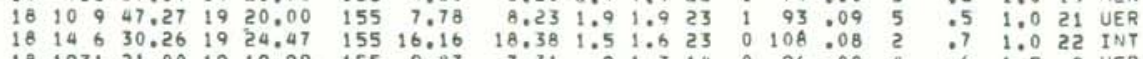
$\begin{array}{lllllllllllllllllll}18 & 1931 & 21.00 & 19 & 19.99 & 155 & 9.87 & 7.31 & 09 & 1.3 & 14 & 0 & 96 & 08 & 4 & .6 & 1.5 & 8 & 8 \\ 18 & 2210 & 46.04 & 19 & 23.04 & 155 & 24.68 & 9.29 & 1.1 & 1.5 & 16 & 0 & 82 & .05 & 5 & .5 & 1.4 & 12 & \text { UKF }\end{array}$

$\begin{array}{lllllll}19 & 327 & 42.29 & 19 & 20.23 & 155 & 12.69\end{array}$ $19 \quad 350 \quad 46.641928 .87 \quad 155 \quad 28.00$ $\begin{array}{lllllll}19 & 410 & 18.81 & 19 & 20.68 & 155 & 2.68 \\ 19 & 550 & 19.69 & 19 & 22.61 & 155 & 3.32\end{array}$

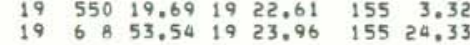
$\begin{array}{lllllll}19 & 944 & 15.47 & 19 & 20.41 & 155 & 3.33\end{array}$

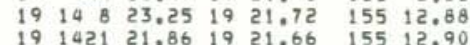

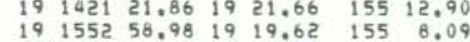
$\begin{array}{lllllll}19 & 1714 & 23 & 19 & 23,70 & 155 & 2,32\end{array}$ $\begin{array}{lllllll}19 & 1756 & 40.50 & 19 & 20.52 & 155 & 3.50 \\ 19 & 1759 & 30.12 & 19 & 42,31 & 155 & 23.17\end{array}$

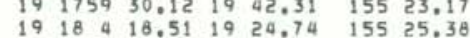

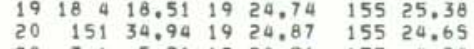
$\begin{array}{lllllll}20 & 36 & 5.31 & 19 & 20.56 & 155 & 4.20\end{array}$ $\begin{array}{lllllll}20 & 457 & 15.01 & 19 & 21.90 & 155 & 13.11 \\ 20 & 559 & 13.45 & 19 & 20.02 & 155 & 9.93\end{array}$ $\begin{array}{lllllll}20 & 627 & 16.27 & 19 & 13.41 & 155 & 25.71 \\ 20 & 931 & 20.56 & 19 & 19.02 & 155 & 11.92\end{array}$

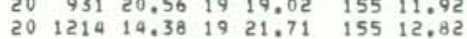
$\begin{array}{lllllll}20 & 1336 & 49.76 & 19 & 18.96 & 155 & 11.42\end{array}$ $20178028.701925 .17 \quad 15457.31$

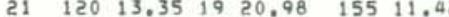
$21 \quad 125 \quad 52.93 \quad 19 \quad 20.93 \quad 155 \quad 11.36$

$\begin{array}{lllllll}21 & 151 & 3.47 & 19 & 27.60 & 154 & 51.25\end{array}$

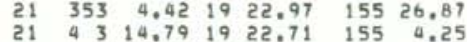

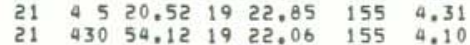
21 $714 \quad 39.591921 .01 \quad 155 \quad 3.16$ $\begin{array}{lllllll}21 & 717 & 31.15 & 19 & 19.09 & 155 & 13.54 \\ 21 & 857 & 9.68 & 19 & 20.31 & 155 & 8.72\end{array}$ $\begin{array}{rrrrrrr}21 & 929 & 37.60 & 19 & 19.89 & 154 & 59.25 \\ 21 & 1349 & 4.11 & 19 & 57.13 & 155 & 37.17\end{array}$ $\begin{array}{lllllll}21 & 1638 & 21.80 & 19 & 23.02 & 155 & 3.07\end{array}$ $\begin{array}{llllllll}22 & 018 & 99 & 19 & 20,74 & 155 & 15.35\end{array}$

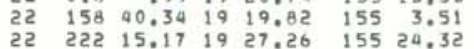

$\begin{array}{lllllll}22 & 1153 & 8.69 & 19 & 20.38 & 155 & 3.30\end{array}$

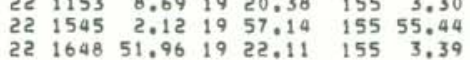

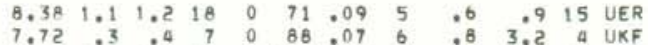

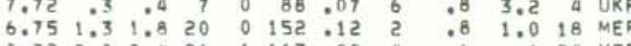

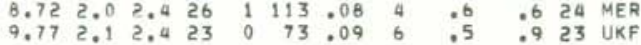

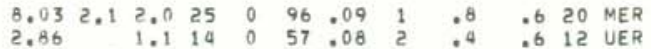

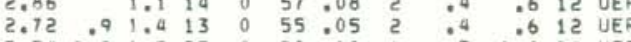

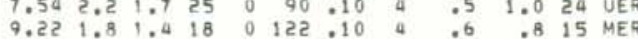
$\begin{array}{rrrrrrrrrrrr}7.18 & 1.1 & 1.3 & 19 & 0 & 96 & .11 & 2 & .8 & 1.2 & 11 & \text { MER } \\ 24.09 & 2.2 & 1.9 & 32 & 3 & 78 & 10 & 11 & .0 & 1.6 & 27 & \text { KKV }\end{array}$

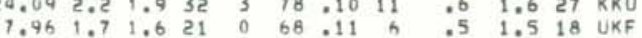

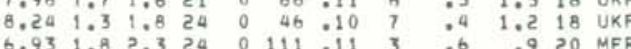
$\begin{array}{lllllllllll}2.94 & 1.1 & 10 & 0 & 97 & 010 & 1 & .6 & .7 & 6 & \text { UER } \\ 7.45 & 1.6 & 17 & 0 & 96 & 09 & 4 & .6 & 1.1 & 15 & \text { UER }\end{array}$

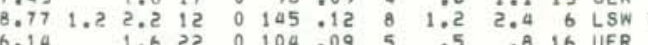

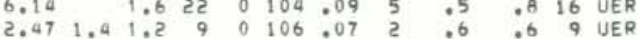
$\begin{array}{llllllllllll}6.56 & 1.7 & 1.2 & 17 & 0 & 110 & .05 & 5 & .5 & 1.1 & 15 & \text { POL }\end{array}$ $\begin{array}{rlllllllllll}5.19 & 1.3 & 14 & 0 & 212 & 0.14 & 2 & 1.3 & 1.1 & 11 & \text { LER } \\ 7.01 & 1.7 & 1.6 & 18 & 0 & 74 & .08 & 3 & .6 & 1.1 & 15 & \text { UER }\end{array}$

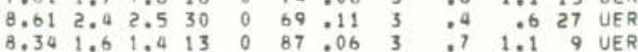
$\begin{array}{lllllllllllll}7.47 & 2.5 & 2.3 & 26 & 1 & 270 & 0.13 & 5 & 1.2 & .7 & 20 & \text { LER }\end{array}$

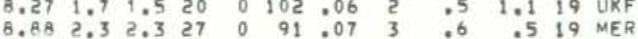
$\begin{array}{llllllllllll}8.68 & 1.9 & 1.5 & 28 & 0 & 90 & : 10 & 3 & .5 & .5 & 20 & \text { MER } \\ 7.75 & 1.3 & 1.3 & 16 & 0 & 94 & : 10 & 4 & .6 & .8 & 14 & \text { MER }\end{array}$ $\begin{array}{lllllllllll}3.98 & 1.0 & 14 & 0 & 120 & .20 & 2 & 1.1 & 1.7 & 13 & \text { MER }\end{array}$

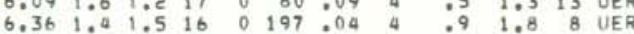
$\begin{array}{llllllllllll}8.19 & 2.5 & 2.0 & 20 & 1 & 222 & .13 & 6 & 1.3 & .6 & 9 & \text { OIS } \\ 0.95 & 2.8 & 2.8 & 22 & 0 & 245 & .06 & 25 & 2.0 & .8 & 19 & \text { KOH }\end{array}$ $\begin{array}{lllllllllllll}8.66 & 2.2 & 1.9 & 21 & 0 & 115 & 08 & 4 & .5 & .7 & 12 & \text { MER } \\ 9.23 & 3.2 & 3.2 & 35 & 1 & 85 & 08 & 6 & .5 & \text {.5 } & 21 & \text { UER }\end{array}$

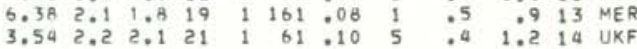
$\begin{array}{llllllllllllll}7.08 & 2.1 & 1.9 & 26 & 2 & 96 & 012 & 1 & .7 & .7 & 22 & \text { MER }\end{array}$

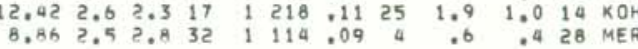

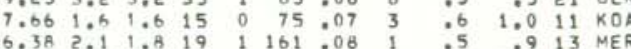


ORIGIN TIME LAT N LON W DEPTH AMP NUR N WAP RMS MIN ERH ERZ NO
DA HRMN SEC DEG MIN OEG MIN

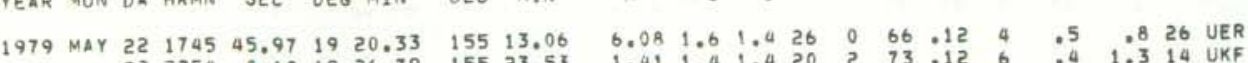

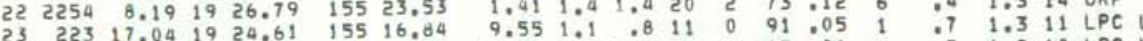

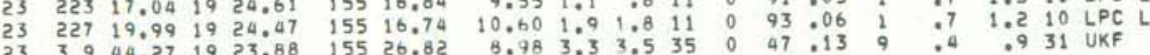

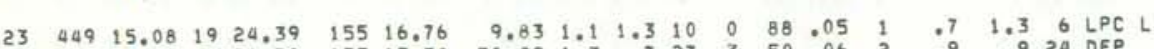

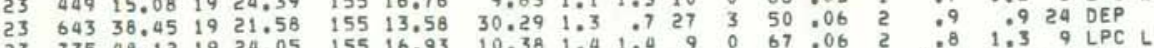

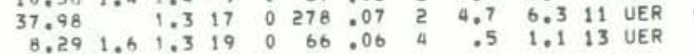
$\begin{array}{lllllll}23 & 956 & 32.86 & 19 & 20.38 & 155 & 12.97\end{array}$ $\begin{array}{lllllll}23 & 1158 & 50.62 & 19 & 18.07 & 155 & 13.14\end{array}$

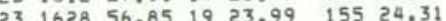
$23 \quad 1651 \quad 14.06 \quad 1957.66 \quad 155 \quad 37.00$ $2320521.491921 .85 \quad 15512.6$

$\begin{array}{lllllll}23 & 2011 & 32.00 & 19 & 25.43 & 155 & 28.14\end{array}$ $23.2327 \quad 40.88 \quad 19 \quad 20.73 \quad 155 \quad 3.23$

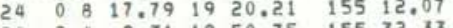
$\begin{array}{llllllll}24 & 236 & 48.72 & 19 & 19.62 & 155 & 8.46\end{array}$ $24 \quad 310 \quad 37.67 \quad 19 \quad 21.53 \quad 155 \quad 1.79$ $24 \quad 430 \quad 22.14 \quad 19 \quad 15,54 \quad 15544.40$

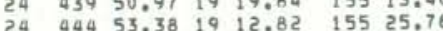

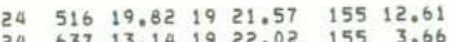
$24 \quad 85658.54 \quad 1921.23 \quad 155 \quad 3.49$

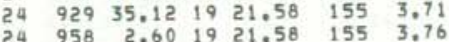
$24 \begin{array}{llllll}1038 & 36.36 & 19 & 23.23 & 155 & 1.51\end{array}$ $24 \quad 126019.74 \quad 1927.11 \quad 15454.80$

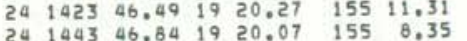
$\begin{array}{lllllll}24 & 1621 & 23.86 & 19 & 20.40 & 155 & 9.03\end{array}$

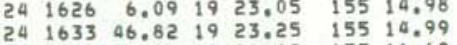
$24214459.92 \quad 1920.02 \quad 15511.69$ $\begin{array}{lllllll}24 & 2247 & 42.96 & 19 & 49.33 \quad 155 \quad 35.7\end{array}$

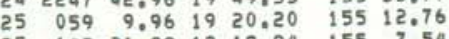
$\begin{array}{lllllll}25 & 147 & 21.29 & 19 & 19.94 & 155 & 7.54 \\ 25 & 759 & 16.77 & 19 & 24.81 & 155 & 16.79 \\ 25 & 843 & 27.23 & 19 & 53.51 & 155 & 17.82\end{array}$ $251356 \quad 36.46 \quad 19 \quad 16.26 \quad 155 \quad 23.90$ $\begin{array}{lrrrrrr}25 & 1356 & 36.46 & 19 & 16.26 & 155 & 23.94 \\ 26 & 052 & 7.23 & 19 & 18.43 & 155 & 23.30 \\ 26 & 114 & 20.01 & 19 & 22.85 & 155 & 27.10\end{array}$

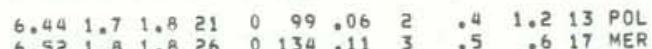
$\begin{array}{lllllllllllllll}10.27 & 1.7 & 1.4 & 14 & 0 & 81 & .06 & 6 & .6 & 1.6 & 13 & \text { UKF }\end{array}$

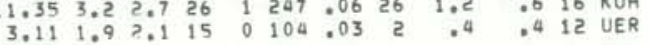
$\begin{array}{rrrrrrrrrrrr}13.94 & 1.9 & 1.5 & 11 & 0 & 193 & 04 & 6 & 3.1 & 5.1 & 8 & \text { UKF } \\ 8.00 & 199 & 1.8 & 26 & 1 & 81 & .09 & 2 & .5 & .9 & 21 & \text { UER }\end{array}$

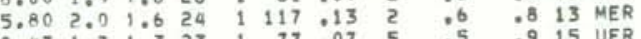

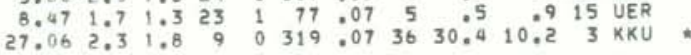
\begin{tabular}{llllllllllll}
7.79 & 1.4 & 1.3 & 23 & 0 & 82 & 05 & 4 & .5 & 1.0 & 15 & UER \\
\hline
\end{tabular}

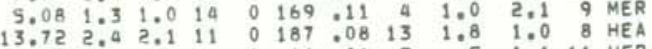

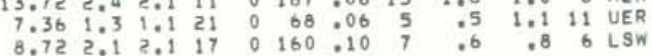
$\begin{array}{lllllllllllll}2.79 & .6 & .9 & 10 & 0 & 112 & .02 & 2 & .4 & .6 & 5 & \text { UER }\end{array}$

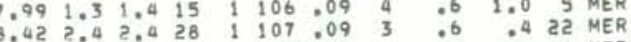
$8.112 .02 .02330103 .0830 .6 \quad 0619$ MER

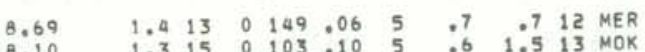

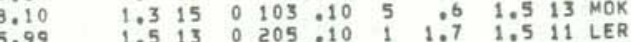

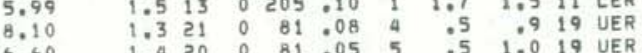
$\begin{array}{llllllllllll}6.50 & 1.8 & 1.7 & 24 & 0 & 71 & .10 & 3 & .5 & .9 & 20 & \text { UER } \\ 3.41 & 3.2 & 3.5 & 36 & 1 & 49 & .09 & 2 & .3 & .5 & 32 & \text { GLN F }\end{array}$

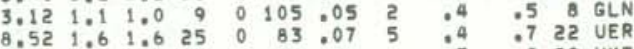

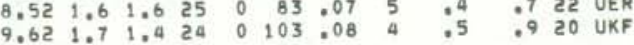

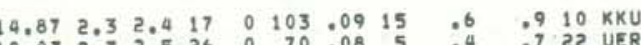

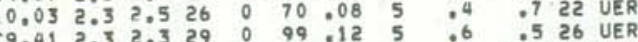

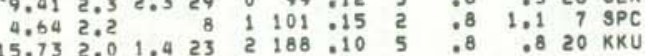
$\begin{array}{lllllllllllll}3.10 & 1.8 & 1.6 & 14 & 1 & 179 & .12 & 7 & .8 & 2.5 & 13 & \text { SWR }\end{array}$

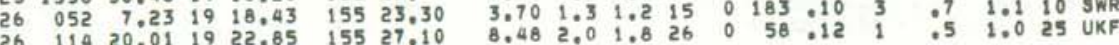
YEAR MON ORIGIN TIME LATMN SEC DEG MIN DEG MIN

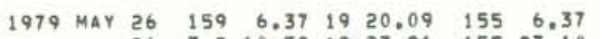

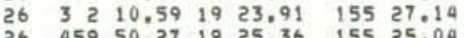

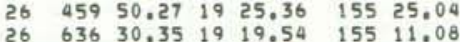

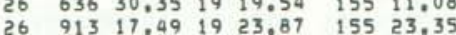

$26 \quad 940 \quad 26.26 \quad 19 \quad 19.40 \quad 155 \quad 11.34$ $\begin{array}{lllllll}26 & 1435 & 46.44 & 19 & 26.30 & 155 & 24.27\end{array}$

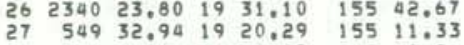
$27 \quad 832 \quad 54.36 \quad 19 \quad 53.03 \quad 155 \quad 10.04$

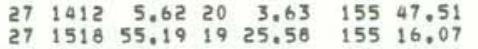
$27 \quad 1711 \quad 8.18 \quad 1926.74 \quad 155 \quad 24.30$ $28 \quad 1942.19 \quad 19 \quad 23.94 \quad 155 \quad 3.10$

$\begin{array}{lllllll}28 & 547 & 22.72 & 19 & 21.24 & 155 & 15.16 \\ 28 & 644 & 58.32 & 18 & 50.07 & 154 & 21.42\end{array}$

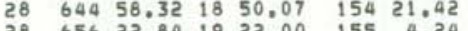
$\begin{array}{lllllll}28 & 656 & 22.84 & 19 & 22.00 & 155 & 4.24 \\ 28 & 8 & 49.49 & 19 & 26.89 & 155 & 24.37\end{array}$ $\begin{array}{lll}1359 & 37.2019 & 193.32 \\ 155 & 6.377\end{array}$ $\begin{array}{lllllll}28 & 1436 & 30.90 & 19 & 20.72 & 155 & 3.98 \\ 28 & 1850 & 33.82 & 19 & 22.39 & 155 & 4.77\end{array}$ ट8 $185033.821922 .39 \quad 155 \quad 4.77$

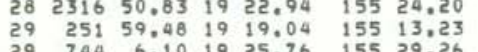
$29 \quad 1157 \quad 4.76 \quad 19 \quad 13.06 \quad 155 \quad 14.72$

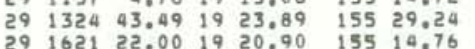

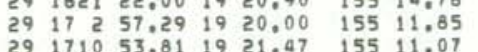
$\begin{array}{lllllll}29 & 1715 & 20.77 & 19 & 21.86 & 155 & 12.51\end{array}$ $17926.721921 .96 \quad 15513.10$ $291722 \quad 3.791921 .64 \quad 155 \quad 12.41$ $291724 \quad 38.52 \quad 1921.63 \quad 15512.27$

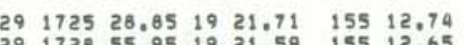
$291729 \quad 59.101921 .56 \quad 15512.74$ $\begin{array}{rrrrrrr}29 & 1731 & 7.39 & 19 & 21.41 & 155 & 12.59 \\ 29 & 1731 & 55,53 & 19 & 21.51 & 155 & 12.33\end{array}$

$\begin{array}{llllll}29 & 1733 \quad 54.35 \quad 19 & 21.66 \quad 155 \quad 12.65\end{array}$

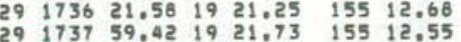

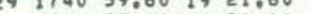
$29174355.401921 .86 \quad 15512.64$

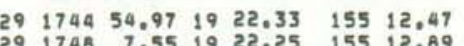
$29174954,11 \quad 1922,16 \quad 15512,64$
PAGE 36

DEPTH AMP NUR GAP RMS MIN ERH ERZ NO

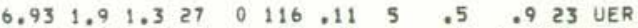

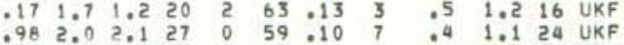

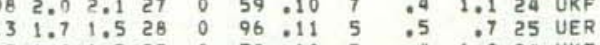
8.691 .91 .7 27 $0058.10 \quad 7 \quad 04 \quad 1.024$ UKF

\begin{tabular}{lllllllllllll}
5.20 & 1.3 & 1.3 & 26 & 0 & 99 & .13 & 6 & .5 & 1.3 & 26 & UER \\
\hline .70 & 2.0 & .9 & 19 & 1 & 49 & 13 & 7 & 4 & 1.9 & 14 & UKF
\end{tabular}

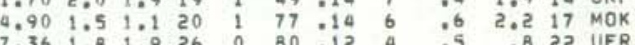

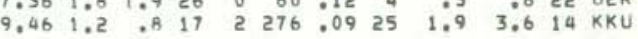

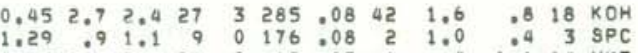

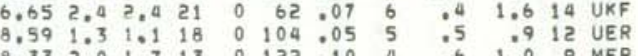

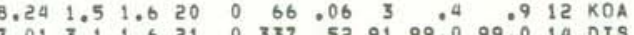

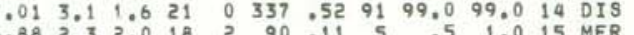

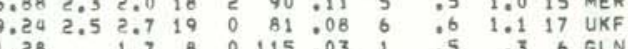
$\begin{array}{lllllllllllllll}6.38 & 2.1 & 1.3 & 18 & 1 & 100 & .07 & 2 & .6 & 1.2 & 15 & \text { MER }\end{array}$

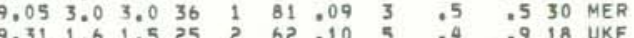

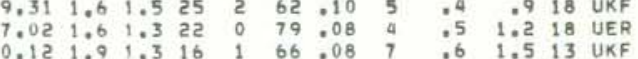

$\begin{array}{llllllllllll}0.94 & 1.2 & .5 & 19 & 2 & 235 & 08 & 8 & 2.1 & 1.7 & 15 & \text { POL }\end{array}$ $\begin{array}{rrrrrrrrrrr}8.48 & 1.2 & 21 & 0 & 119: 10 & 4 & .6 & 1.1 & 20 & \text { UKF } \\ 7.35 & 9.9 & 12 & 0 & 87 & : 13 & 3 & : 7 & 1.6 & 11 & \text { UER }\end{array}$ $\begin{array}{llllllllllll}4.88 & 1.1 & .1 & 13 & 0 & 83 & .09 & 5 & .6 & 2.3 & 12 & \text { UER } \\ 5.66 & & 7 & 13 & 0 & 81 & .14 & 2 & .7 & 1.5 & 11 & \text { UER }\end{array}$ $2.962 .01 .322 \quad 0 \quad 55.0920 .4 \quad .518$ UER

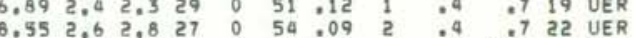

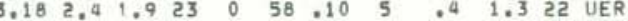
$\begin{array}{llllllllllll}2.19 & 1.5 & 1.0 & 16 & 0 & 75 & .14 & 2 & .4 & .7 & 12 & \text { UER }\end{array}$

$\begin{array}{lllllllllllll}2.46 & 2.3 & 2.3 & 23 & 0 & 56 & .09 & 5 & .4 & 1.1 & 17 & \text { UER }\end{array}$

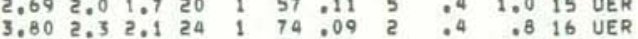
$\begin{array}{lllllllllllll}2.20 & 2.2 & 1.3 & 24 & 0 & 59.17 & 2 & .4 & .7 & 16 & \text { UER } \\ 1.74 & 1.6 & .8 & 13 & 0 & 74 & .14 & 2 & .6 & 1.1 & 10 & \text { UER }\end{array}$

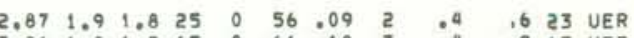

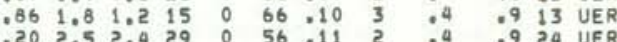

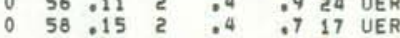
$6.793 .02 .932 \quad 1 \quad 55.11$ 2 44 .8 25 UER $\begin{array}{lllllllllll}4.74 & 3.1 & 3.3 & 16 & 0 & 89.15 & 6 & .6 & 2.2 & 12 & 12 \\ 0 & \text { UER }\end{array}$

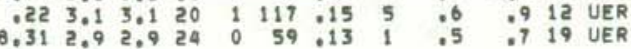



$29180035.461921 .85 \quad 15512,9$

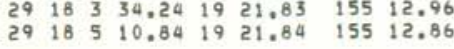

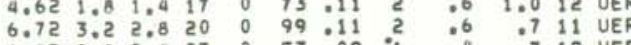
$\begin{array}{llllllllllll}6.07 & 2 . .8 & 2.4 & 27 & 0 & 53 & .09 & 1 & .4 & .7 & 19 & \text { UER } \\ 1.92 & 1.8 & 1.3 & 21 & 0 & 53 & .17 & 2 & 04 & 06 & 13 & \text { UER } \\ 2.66 & 1.4 & 1.1 & 16 & 0 & 59 & .07 & 2 & .3 & .5 & 15 & \text { UER }\end{array}$ $\begin{array}{llllllll}29 & 18 & 6 & 19.71 & 19 & 22.07 & 155 & 12.62 \\ 29 & 18 & 9 & 23.34 & 19 & 21.69 & 155 & 13.19\end{array}$

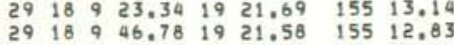
$\begin{array}{lllllll}29 & 18 & 46.78 & 19 & 21.58 & 155 & 12.83 \\ 29 & 1810 & 26.24 & 19 & 21.52 & 155 & 13.07\end{array}$ $\begin{array}{llllll}29 & 1812 & 22.02 \quad 19 & 21.96 \quad 155 & 12.46\end{array}$ $291814 \quad 15.291921 .72 \quad 15513.22$ $291818 \quad 6.591922 .00 \quad 15513,33$

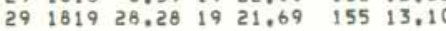
$29 \quad 1819 \quad 57.03 \quad 19 \quad 21.59 \quad 155 \quad 12.93$

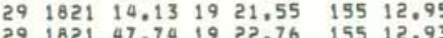
$\begin{array}{lllllll}29 & 1823 & 22.00 & 19 & 21.79 & 155 & 13.00 \\ 29 & 1825 & 18.06 & 19 & 21.72 & 155 & 12,93\end{array}$

$\begin{array}{llllllll}29 & 1828 & 35.34 & 19 & 21.43 & 155 & 12.25\end{array}$

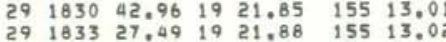

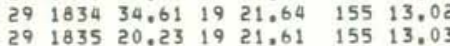

$29 \begin{array}{llllll}1837 & 19.13 & 19 & 21.89 & 155 & 13.22\end{array}$ $29184651.151921 .84 \quad 15512.86$

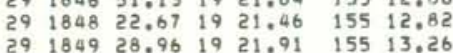
$\begin{array}{lllllll}29 & 1859 & 45.97 & 19 & 22.01 & 155 & 11.84\end{array}$ $291918 \quad 24.951921,31$ 155 12.17 $\begin{array}{rrrrrrr}29 & 1918 & 44.42 & 19 & 20.13 & 155 & 6.35 \\ 29 & 1923 & 4.96 & 19 & 21.87 & 155 & 11.45\end{array}$

$\begin{array}{lllllll}29 & 1924 & 44.76 & 19 & 21.54 & 155 & 11.53\end{array}$ $29193646.971921 .91 \quad 15512.17$

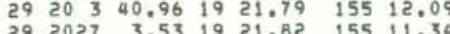
$\begin{array}{lrrrrrr}29 & 2027 & 3.53 & 19 & 21.82 & 155 & 11.34 \\ 29 & 2028 & 16.41 & 19 & 21.94 & 155 & 11.97\end{array}$

$29 \begin{array}{llllll}2030 & 8.63 & 19 & 22.66 & 155 & 11.29\end{array}$

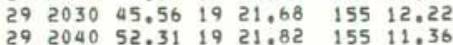

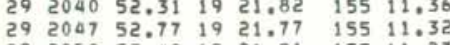
$29205253.421921 .91 \quad 15511.27$ $\begin{array}{llllllllllll}5.26 & 2.8 & 2.7 & 29 & 0 & 53 & -11 & 1 & .4 & 1.0 & 21 & \text { UER }\end{array}$

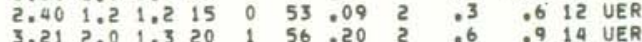

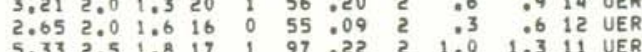

$\begin{array}{llllllllllllll}3.71 & 1.4 & 1.3 & 15 & 0 & 76 & .16 & 2 & .6 & .9 & 11 & \text { UER }\end{array}$

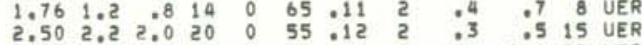

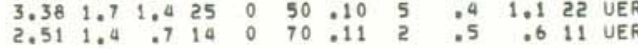
\begin{tabular}{lllllllllll}
2.14 & 1.3 & 1.0 & 20 & 0 & 55 & .18 & 2 & .5 & .9 & 15 \\
\hline & UER
\end{tabular} $\begin{array}{llllllllllll}2.51 & 1.5 & 1.3 & 17 & 0 & 56 & .13 & 2 & .4 & .7 & 14 & \text { UER } \\ 3.91 & .04 & .8 & 7 & 0 & 83 & .09 & 0 & 7 & : 8 & 6 & \text { UER }\end{array}$ $\begin{array}{lllllllllllll}2.68 & 1.9 & 1.3 & 20 & 0 & 54 & 10 & 2 & 03 & .5 & 14 & \text { UER } \\ 2.22 & & 1,0 & 9 & 0 & 87 & .07 & 2 & .5 & .7 & 7 & \text { UER }\end{array}$ \begin{tabular}{llllllllllll}
2.39 & 1.8 & 1.2 & 16 & 0 & 61 & .18 & 3 & .5 & .9 & 9 \\
\hline & UER
\end{tabular} $\begin{array}{llllllllllll}2.78 & 1.2 & .9 & 12 & 0 & 75 & 08 & 1 & .5 & .6 & 11 & \text { UER } \\ 3.01 & 1: 7 & 1.5 & 24 & 1 & 52 & : 13 & 1 & : 4 & : 7 & 17 & \text { UER }\end{array}$ $\begin{array}{lllllllllll}2.67 & 1.2 & 1.0 & 12 & 0 & 64 & 10 & 2 & .4 & .8 & 8 \\ 2.30 & 1.4 & 1.0 & 10 & 0 & 79 & .05 & 2 & .5 & .7 & 9 \text { UER }\end{array}$ $\begin{array}{llllllllllllll}3.24 & 2.0 & 2.1 & 25 & 0 & 51 & 10 & 1 & .4 & .6 & 16 & \text { UER } \\ 6.99 & & .3 & 5 & 0 & 140 & .17 & 3 & 9.1 & 22.3 & 5 & \text { UER }\end{array}$

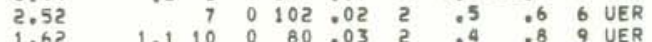

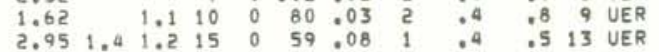

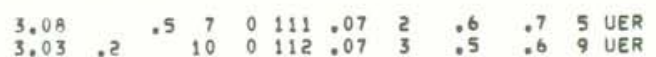

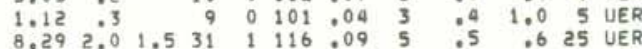

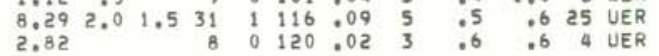
$\begin{array}{llllllllllll}2.91 & .6 & 11 & 0 & 105 & .05 & 3 & .4 & .7 & 9 & \text { UER } \\ 2.61 & .2 & 9 & 0 & 109 & .05 & 2 & .6 & .5 & 6 & \text { UER }\end{array}$

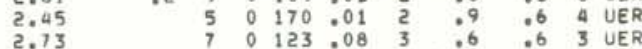

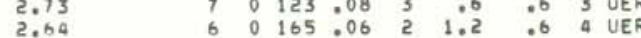

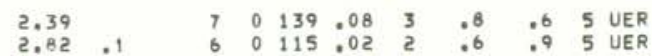

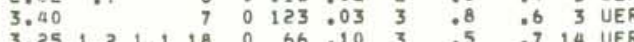

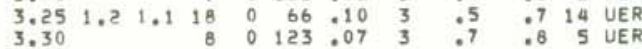

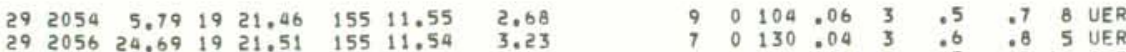

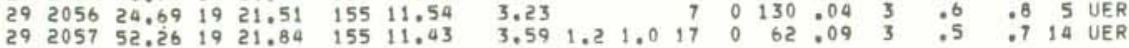

HVO EARTHQUAKE SUMMARY LIST

PAGE 38 ORIGIN TIME LAT N
OA HRMN SEC DEG MIN DEG MIN DEPTH AMP DUR N

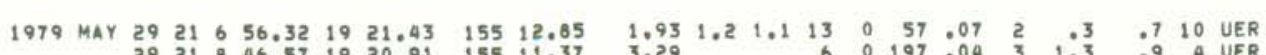

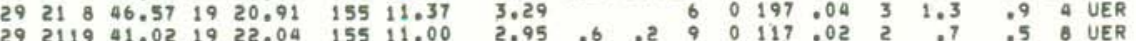

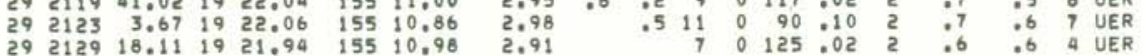
$29220 \quad 31.69 \quad 1922.21 \quad 155 \quad 10.96$ $\begin{array}{llllllll}29 & 22 & 0 & 31.69 & 19 & 22.21 & 155 & 10.96 \\ 29 & 22 & 3 & 1.71 & 19 & 21.96 & 155 & 10.90\end{array}$ $29 \begin{array}{ll}22 & 9\end{array} 48,73$ 19 21,82 15510,53

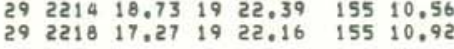
$\begin{array}{llllll}29 & 2219 & 09 & 19 & 22.01 \quad 155 \quad 10.82\end{array}$ $292240 \quad 2.961922 .16 \quad 15510.91$

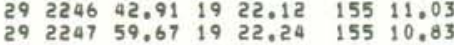

$292251 \quad 16.11 \quad 1921.98 \quad 155 \quad 10.85$ $292316 \quad 8.36 \quad 1921.00 \quad 155 \quad 4.51$

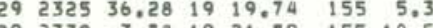

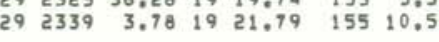

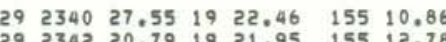
$\begin{array}{lllllll}30 & 034 & 9.76 & 19 & 22.06 & 155 & 10.68 \\ 30 & 040 & 5.77 & 19 & 22.18 & 155 & 4.81\end{array}$ 30 ₹ $839,28 \quad 19$ 21.81 $155 \quad 4,46$

$\begin{array}{llllllll}30 & 3 & 8 & 51.13 & 19 & 21.43 & 155 & 12.71\end{array}$

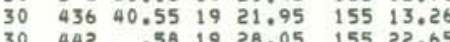
$\begin{array}{lllllll}30 & 442 & .58 & 19 & 28.05 & 155 & 22.65 \\ 30 & 628 & 43.74 & 19 & 19.31 & 155 & 13\end{array}$ $\begin{array}{lllllll}30 & 654 & 37,04 & 19 & 17,94 & 155 & 13.17\end{array}$

$\begin{array}{llllllll}30 & 721 & 31.56 & 19 & 19.58 & 155 & 10.92\end{array}$ $\begin{array}{lllllll}30 & 1011 & 6.48 & 19 & 20.99 & 155 & 13.02\end{array}$ $\begin{array}{lllllll}30 & 1327 & 48.81 & 19 & 20.42 & 155 & 6.79 \\ 30 & 1921 & 51.17 & 19 & 25.23 & 155 & 15.12\end{array}$ $\begin{array}{lllllll}30 & 1921 & 51.17 & 19 & 25.23 & 155 & 15,12 \\ 30 & 1946 & 25.74 & 19 & 16.90 & 155 & 11.79\end{array}$

$\begin{array}{lllllll}30 & 2127 & 2.82 & 19 & 20.22 & 155 & 3.87\end{array}$ $\begin{array}{lllllll}31 & 358 & 8.03 & 19 & 22.82 & 155 & 4.67 \\ 31 & 743 & 10.62 & 10 & 30.52 & 155 & 15.12\end{array}$ $\begin{array}{rrrrrrr}31 & 743 & 14.62 & 19 & 30.52 & 155 & 15.12 \\ 31 & 2340 & 6.06 & 19 & 18.01 & 155 & 12.93\end{array}$

$101611.6419 \quad 20.36 \quad 15513.78$

$\begin{array}{llllllllllllll}.79 & .1 & 6 & 0 & 154 & .03 & 2 & 1.3 & .6 & 5 & \text { UER }\end{array}$

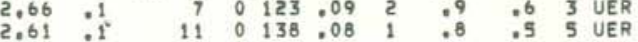
$\begin{array}{lrrrrrrrrrr}3.18 & & 6 & 0 & 135 & 08 & 1 & 1.6 & .6 & 9 & \text { UER } \\ 2,77 & .4 & 10 & 1 & 111 & .09 & 2 & .7 & .5 & 4 & \text { 4 UER }\end{array}$

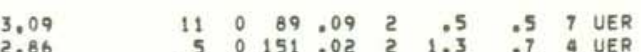

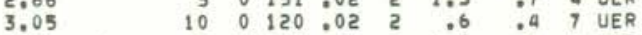

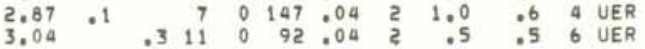
$\begin{array}{lllllllllll}2.85 & .7 & 10 & 0 & 122 & .14 & 2 & .8 & .6 & 5 & \text { UER }\end{array}$

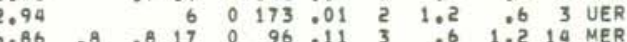
$\begin{array}{llllllllllll}6.86 & .8 & .8 & 17 & 0 & 96 & .11 & 3 & .6 & 1.2 & 14 & \text { MER }\end{array}$

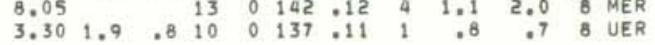
$\begin{array}{llllllllll}2.77 & 7 & 0 & 137 & 09 & 2 & 1.1 & \text {.7 } & \text { a UER } \\ 2.20 & 9 & 0 & 101 & 06 & 1 & 5 & \text { :5 } & 6 & \text { UER }\end{array}$

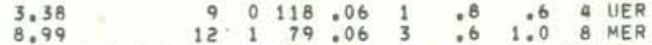
$\begin{array}{llllllllllll}8.99 & & & 12 & 1 & 79 & 06 & 3 & .6 & 1.0 & 8 & \text { MER } \\ 7.83 & 1.8 & 1.6 & 27 & 0 & 85 & .12 & 4 & .6 & .6 & 22 & \text { MER }\end{array}$

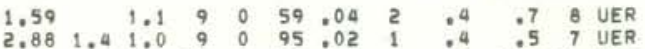

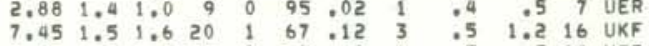

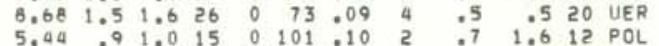

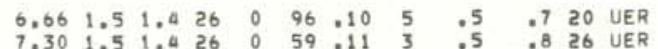

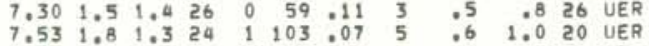
$\begin{array}{llllllllllll}1.79 & 1.3 & 1.2 & 11 & 0 & 149 & .05 & 2 & .6 & .5 & 11 & \mathrm{SPC}\end{array}$ $\begin{array}{lllllllllll}6.60 & 1.2 & 8 & 0 & 126 & .06 & 2 & .7 & 1.6 & 7 \text { MER }\end{array}$

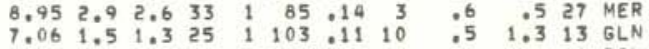

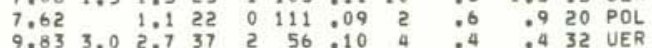

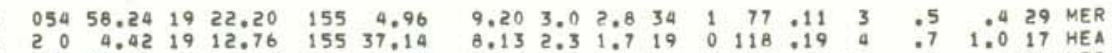

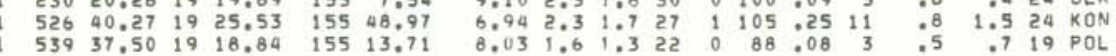
$\begin{array}{lllllllllllllllllll}956 & 10.58 & 19 & 22.38 & 155 & 4.83 & 8.85 & 2.3 & 2.1 & 28 & 0 & 80 & .09 & 3 & .5 & .7 & 23 & \text { MER }\end{array}$

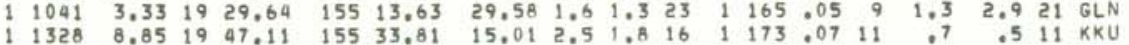


HVO EARTHQUAKE SUMMARY LIST

PAGE 39 ORIGIN TIME LATN
DA HRMN SEC DEG MIN DEG MIN OEPTH AMP OUR KM GAP RMS MIN ERH ERZ NO
MAG WOG NR NS DEG SEC OIS KM KM FM REMK

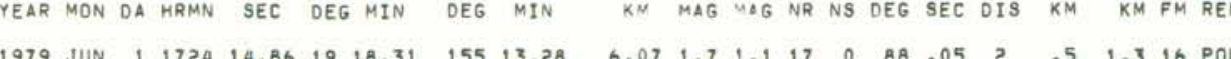

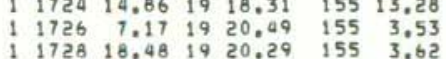

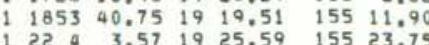
$\begin{array}{llllllll}2 & 0 & 0 & 26.58 & 19 & 37.62 & 155 & 58.31 \\ 2 & 256 & 41.35 & 19 & 23.92 & 155 & 24.21\end{array}$

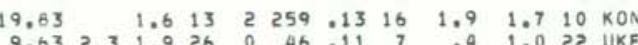

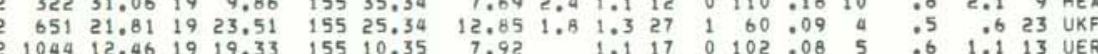

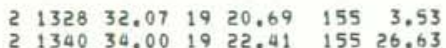

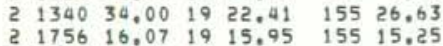

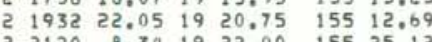

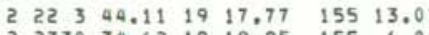
$23330 \quad 34.62 \quad 19 \quad 19.95 \quad 155 \quad 6.84$

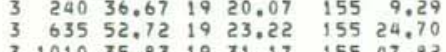
$3 \quad 132028.62 \quad 1924.16 \quad 15525.23$

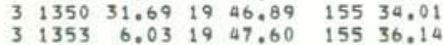
3
3 $168824.1919 .16 .82 \quad 15528.45$

$\begin{array}{lllllll}3 & 2319 & 13.07 \quad 19 & 44.79 \quad 156 \quad 1.33\end{array}$

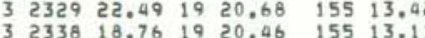
$\begin{array}{lrrrrrr}4 & 147 & 55.64 & 19 & 20.23 & 155 & 7.11 \\ 4 & 7 & 13.06 & 19 & 23.02 & 155 & 3.61\end{array}$ $4 \quad 710 \quad 41.70 \quad 19 \quad 22.99 \quad 155 \quad 3.70$ $4 \quad 1423 \quad 3.401922 .51 \quad 15528.55$ $\begin{array}{lllllll}5 & 019 & 23.56 & 19 & 19.51 & 155 & 3.14 \\ 5 & 310 & 7.44 & 19 & 20.65 & 155 & 6.40\end{array}$

$\begin{array}{lllllll}5 & 429 & 44.32 & 19 & 21.31 & 155 & 6.67\end{array}$ $5 \quad 52102.291919 .26 \quad 15510.7$

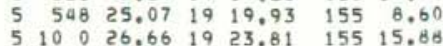

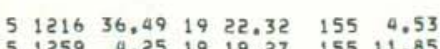

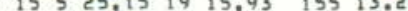

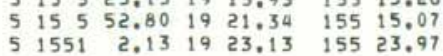

$52148 \quad 6.10 \quad 1919.21 \quad 15511.41$

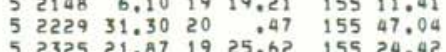

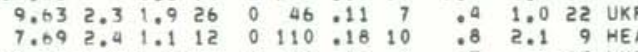

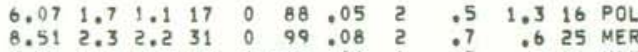

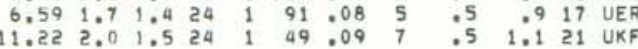
$\begin{array}{rrrrrrrrrrrr}7.21 & 1.8 & 1.6 & 17 & 0 & 96 & 012 & 2 & 07 & 1.3 & 12 & \text { MER } \\ 9.76 & 2.1 & 1.8 & 18 & 0 & 109 & 011 & 2 & : 5 & 1.2 & 17 & \text { UKF }\end{array}$

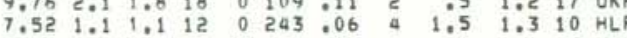

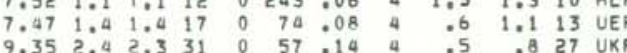
$\begin{array}{lllllllllllll}7.34 & 2.0 & 1.9 & 22 & 0 & 116 & .10 & 2 & .6 & 1.1 & 21 & \text { POL } \\ 7.28 & 1.6 & 1.6 & 20 & 0 & 113 & .09 & 5 & .6 & 1.3 & 17 & \text { UER }\end{array}$

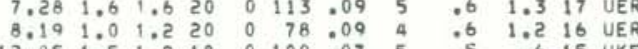

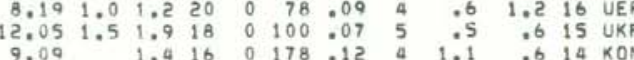
$\begin{array}{rrrrrrrrrrrrr}10.20 & 1.7 & 1.6 & 18 & 0 & 77 & .06 & 5 & .5 & 1.3 & 17 & \text { UKF } \\ 15.07 & 1.8 & : .8 & 18 & 1 & 91 & .11 & 11 & .7 & 9.8 & 17 & \mathrm{KKU}\end{array}$

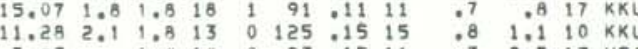
$\begin{array}{llllllllllll}7.43 & 1.4 & 14 & 0 & 93 & .15 & 11 & \text { :7 } & 2.7 & 13 & 13 \\ 8.22 & 1.7 & 1.5 & 22 & 0 & 81 & .09 & 3 & \text { HEA } & 1.0 & 20 & \text { UER }\end{array}$

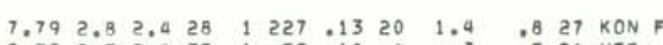

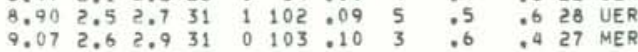
$\begin{array}{lllllllllllll}8.75 & 2.1 & 2.0 & 25 & 1 & 101 & .10 & 3 & .6 & .5 & 21 & \text { MER }\end{array}$ $\begin{array}{lllllllllllll}6.45 & 1.4 & 1.2 & 10 & 0 & 237 & 07 & 1 & 1.5 & 1.2 & 10 & \text { MER } \\ 6.97 & 1.2 & 1.2 & 17 & 0 & 103 & .07 & 6 & .5 & 1.0 & 11 & \text { UER }\end{array}$ $\begin{array}{lllllllllll}8.65 & 2.5 & 2.6 & 33 & 3 & 87 & 06 & 6 & .4 & .5 & 24 \\ 5 & .5 E R\end{array}$

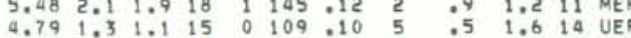

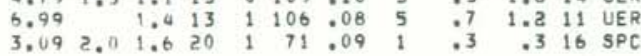
$\begin{array}{llllllllllll}8.82 & 3.1 & 2.8 & 31 & 0 & 86 & 09 & 3 & .5 & .5 & 31 & \text { MER }\end{array}$

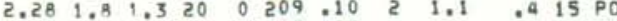

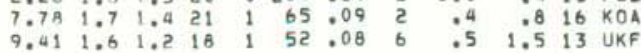
$\begin{array}{llllllllllll}6.75 & 1.4 & 1.1 & 18 & 0 & 103 & 07 & 5 & .5 & 1.6 & 14 & \text { UER }\end{array}$ $\begin{array}{llllllllllll}8.05 & & 1.6 & 9 & 0 & 175 & .05 & 13 & 1.6 & 1.8 & 4 & \text { KOH } \\ 9.38 & 1.2 & 1.3 & 17 & 1 & 77 & .10 & 8 & .6 & 1.5 & 11 & \text { UKF }\end{array}$

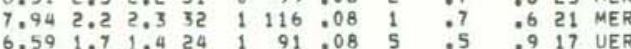

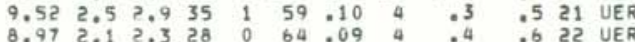

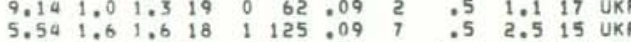

HVO EARTHQUAKE SUMMARY LIST

PAGE 40

ORIGIN TIME GATN LON N DEPTH AMP OUR GAP RMS MIN ERH ERZ NO TEAR MON DA HERN $\begin{array}{lllllllllllllllllllll}1979 \text { JUN } & 6 & 235 & 34.81 & 19 & 20.38 & 155 & 13.04 & 8.31 & 1.8 & 1.2 & 23 & 0 & 65 & .07 & 4 & .5 & 1.0 & 19 \text { UER } \\ & 6 & 236 & 58.37 & 19 & 20.66 & 155 & 12.73 & 9.29 & 1.8 & 1.4 & 22 & 0 & 65 & .08 & 4 & .5 & .8 & 19 \text { UER }\end{array}$

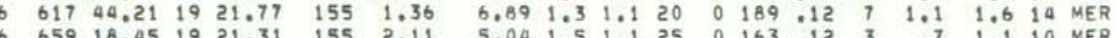

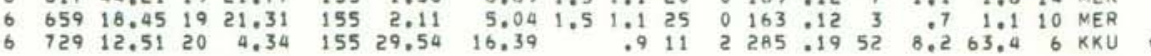

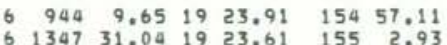

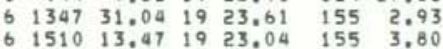

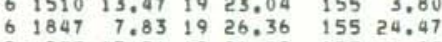

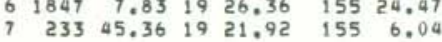

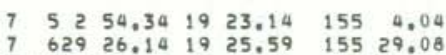

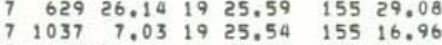
$\begin{array}{lllllll}7 & 1037 & 7.03 & 19 & 25.54 & 155 & 16.96 \\ 7 & 1136 & 31.21 & 19 & 24.00 & 154 & 59.04\end{array}$

$\begin{array}{lllllll}7 & 1225 & 51.88 & 19 & 18.96 & 155 & 8.36\end{array}$

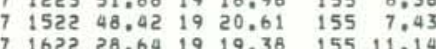
$\begin{array}{lllllll}1622 & 28.64 & 19 & 19.38 & 155 & 11.14 \\ 7 & 1648 & 12.57 & 1922.02 & 155 & 0.43\end{array}$ $\begin{array}{lllllll}7182 & 58.87 & 19 & 19.91 & 155 & 16.98\end{array}$ $\begin{array}{lllllll}7 & 1836 & 16.10 & 19 & 19.37 & 155 & 13.01 \\ 7 & 2017 & 20.61 & 19 & 19.56 & 155 & 3.49\end{array}$ $\begin{array}{lllllll}8 & 246 & 30.49 & 19 & 19.00 & 155 & 10.58 \\ 8 & 3 & 91.22 & 19 & 16.63 & 155 & 34.46\end{array}$ $\begin{array}{lllllll}8 & 730 & 46.62 & 19 & 21.99 & 155 & 4.57\end{array}$

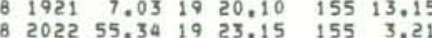
$\begin{array}{lllllll}8 & 2031 & 6.54 & 19 & 22,96 & 155 & 2.92 \\ 8 & 2116 & 20.56 & 19 & 14,81 & 155 & 33,66\end{array}$

$\begin{array}{lllllll}8 & 2224 & 13.35 & 19 & 21.31 & 155 & 2.69\end{array}$ $\begin{array}{lrllllll}8 & 2241 & 43.86 & 19 & 23.66 & 155 & 15.26 \\ 9 & 0 & 17.88 & 19 & 49.82 & 155 & 45.37\end{array}$ $\begin{array}{lllllll}9 & 051 & 11.06 & 19 & 11.84 & 155 & 38.51 \\ 9 & 157 & 38.80 & 19 & 19.31 & 155 & 13.85\end{array}$

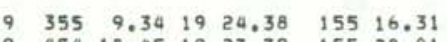
$9,9753.301924 .7915528 .01$

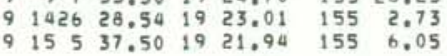

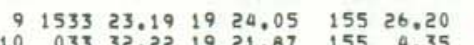

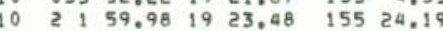
10 4 $553.991925 .11 \quad 154 \quad 54.68$

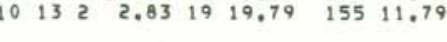
$\begin{array}{lllllll}10 & 1717 & 57.93 & 19 & 20.12 \quad 155 \quad 13.39\end{array}$

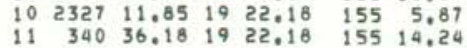

$\begin{array}{rrrrrrrrrrrr}7.87 & 2.0 & 2.1 & 19 & 0 & 201 & 016 & 4 & 1.5 & .6 & 16 & \text { LER } \\ 7.84 & 1.3 & 1.5 & 13 & 0 & 112 & : 12 & 3 & .8 & 1.3 & 12 & \text { MER }\end{array}$ $\begin{array}{llllllllllll}8.28 & 1.3 & 1.4 & 19 & 0 & 99 & 10 & 3 & .6 & .7 & 18 & \text { MER } \\ 7.40 & 2.1 & 1.9 & 24 & 1 & 49 & 010 & 7 & 04 & 1.4 & 21 & \text { UKF }\end{array}$

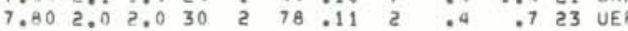
$\begin{array}{lllllllllll}8.31 & 2.8 & 3.1 & 29 & 0 & 94 & .09 & 3 & .5 & .5 & 28 \\ 9.37 & 1.8 & \text { MER }\end{array}$ $\begin{array}{lllllllll} & 0.5 & 1.4 & 18 & \text { UKR }\end{array}$

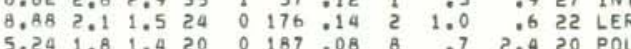
$\begin{array}{llllllllllll}6.88 & 1.3 & 1.2 & 21 & 0 & 87 & 09 & 3 & .6 & 1.3 & 21 & \text { POL } \\ 7.44 & 1.9 & 1.5 & 26 & 1 & 90 & .08 & 5 & .04 & .7 & 24 & \text { UER }\end{array}$

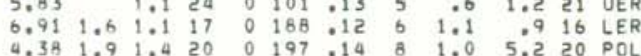
$\begin{array}{llllllllllll}8.37 & 1.9 & 1.2 & 28 & 3 & 96 & -15 & 1 & .6 & .9 & 24 & \mathrm{KOA}\end{array}$

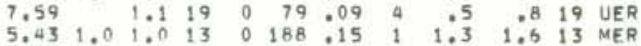
$\begin{array}{llllllllllll}7.31 & 1.4 & 1.1 & 23 & 0 & 112 & 017 & 6 & .7 & 1.1 & 21 & \text { UER } \\ 8.61 & & 1.2 & 24 & 1 & 109 & .12 & 6 & .5 & .9 & 21 & \text { HEA }\end{array}$ $\begin{array}{llllllllllll}8.52 & 2.3 & 2.1 & 29 & 1 & 83 & .12 & 4 & .6 & .5 & 25 & \text { MER }\end{array}$

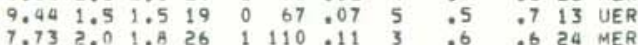

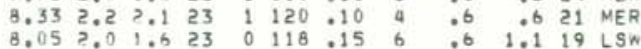
$\begin{array}{llllllllllllll}7.55 & 1.8 & 1.6 & 25 & 0 & 141 & 11 & 3 & .6 & .7 & 20 & \text { MER }\end{array}$

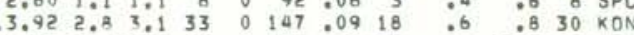

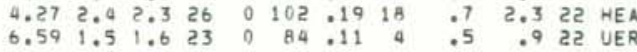
$\begin{array}{llllllllllll}2.98 & 1.3 & 1.3 & 8 & 1 & 130 & .07 & 3 & .5 & .7 & 7 & \text { SPC }\end{array}$

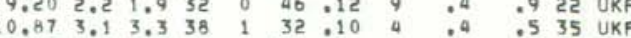

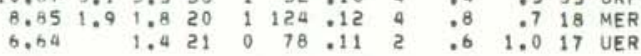
$\begin{array}{llllllllllll}3.25 & 2.1 & 1.8 & 18 & 1 & 77 & .27 & 4 & 1.1 & 1.2 & 14 & \text { UKF } \\ 7.55 & 2.3 & 2.4 & 26 & 0 & 87 & 09 & 4 & .5 & .7 & 24 & \text { MER }\end{array}$ \begin{tabular}{lllllllllll}
8.92 & 2.1 & 2.0 & 30 & 1 & 47 & 0.11 & 6 & .4 & .9 & 27 \\
\hline
\end{tabular}

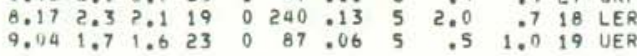
$\begin{array}{llllllllllll}6.77 & 1.6 & 1.3 & 20 & 1 & 65 & .11 & 5 & .5 & 1.3 & 15 & \text { UER }\end{array}$

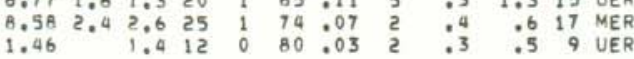
$\begin{array}{rlllllllllll}1.44 & 1.9 & 1.5 & 26 & 1 & 90 & .08 & 5 & .4 & .7 & 24 & \text { UER } \\ 5.83 & & 1.1 & 24 & 0 & 101 & .13 & 5 & .6 & 1.2 & 21 & \text { UER }\end{array}$ 


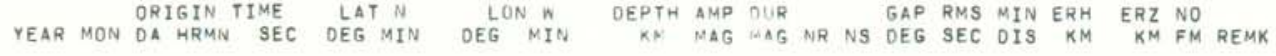

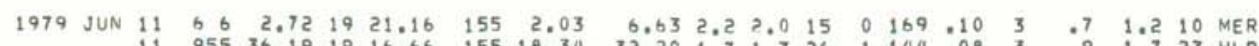

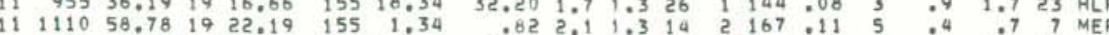

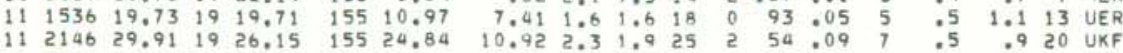
$\begin{array}{lllllllllllllllllllll}11 & 22 & 2 & 46.59 & 19 & 19.13 & 155 & 16.17 & 8.48 & 2.1 & 2.3 & 25 & 2 & 115 & .06 & 3 & .4 & .9 & 16 & \text { KOA }\end{array}$

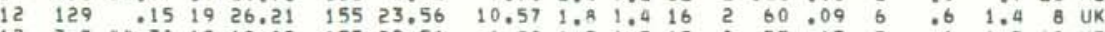

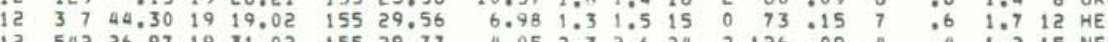

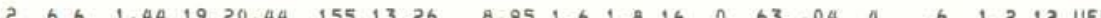

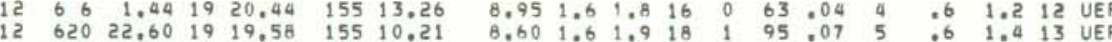

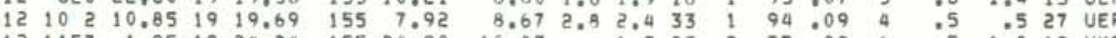

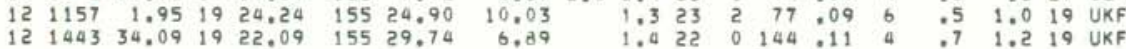

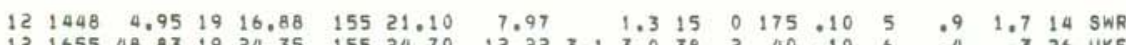

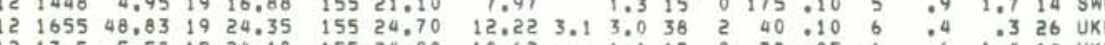

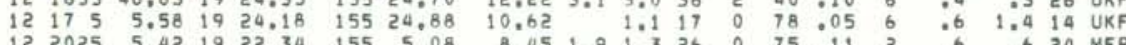

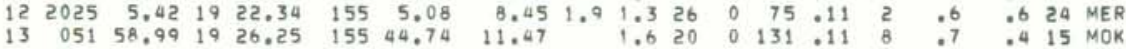

\begin{tabular}{lllllllllllllllllll}
13 & 953 & 11.37 & 19 & 20.52 & 155 & 11.64 & 8.47 & 1.7 & 1.3 & 20 & 1 & 75 & .07 & 4 & .5 & 1.0 & 11 & UER \\
\hline
\end{tabular}

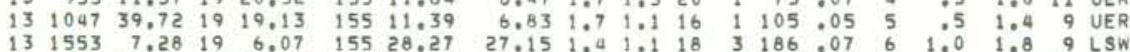

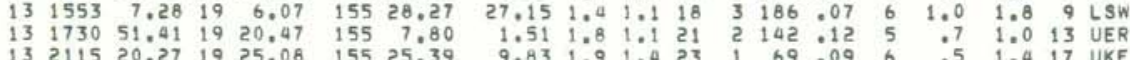

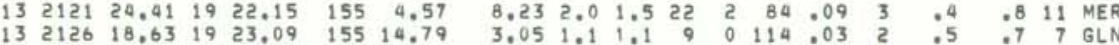

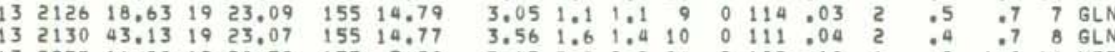

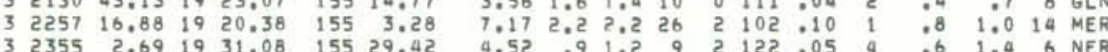

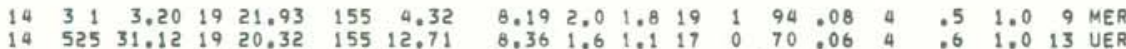

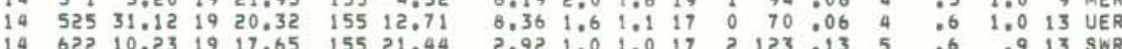

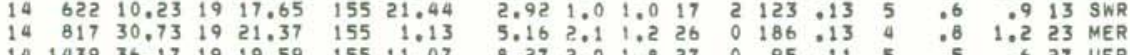
4. 143936.171919 .59 155 11.007

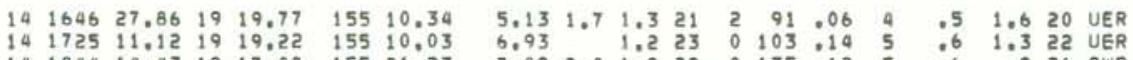

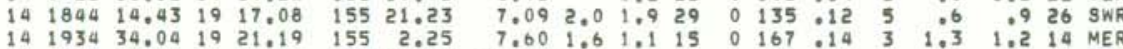

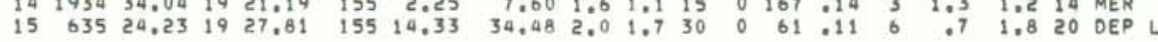

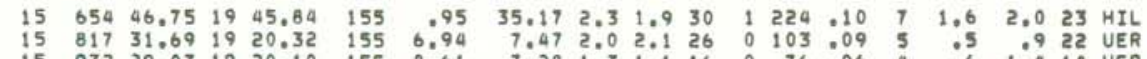

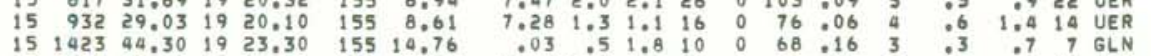

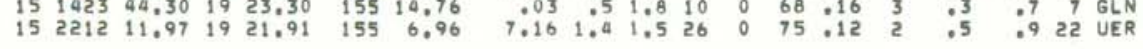
$\begin{array}{llllllllllllllllll}15 & 2347 & 33.29 & 19 & 19.53 & 155 & 11.00 & 4.21 & 2.0 & 2.1 & 12 & 1 & 96 & .14 & 5 & .6 & 2.7 & \text { B UER }\end{array}$

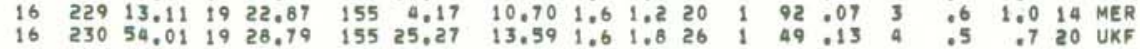

ORIGIN TIME
DA HRMN SAT N
DEG MIN

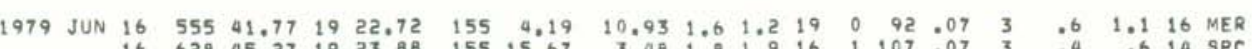

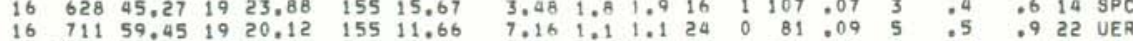

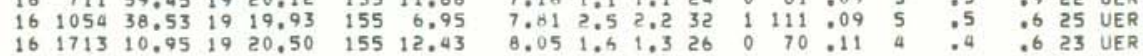
$\begin{array}{lllllllllllllllllll}16 & 1746 & 22.25 & 19 & 23.64 & 155 & 1.61 & 8.42 & 2.1 & 1.2 & 17 & 0 & 138 & .15 & 5 & .9 & 1.2 & 16 & \text { MER } \\ 16 & 1835 & 4.94 & 19 & 22.22 & 155 & 5.00 & 7.57 & 1.9 & .1 & 28 & 0 & 73 & .11 & 2 & .5 & .7 & 24 & \text { MER }\end{array}$

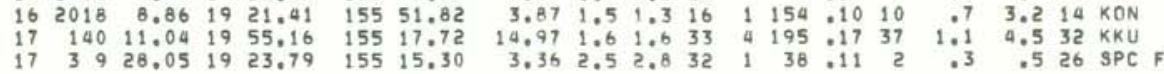
$\begin{array}{lllllllllllllllllll}17 & 420 & 52.25 & 19 & 19.65 & 155 & 12.13 & 9.70 & 2.9 & 2.9 & 36 & 1 & 86 & .09 & 5 & .4 & .4 & 35 & \text { UER }\end{array}$

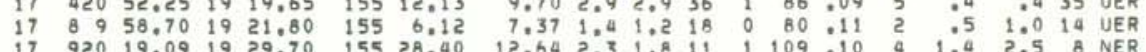

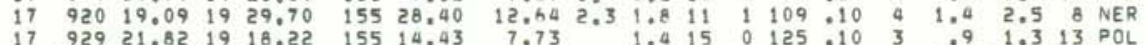

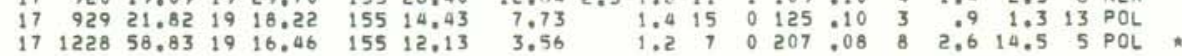

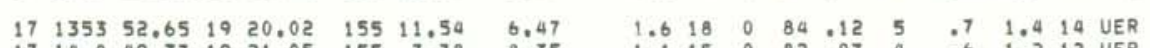

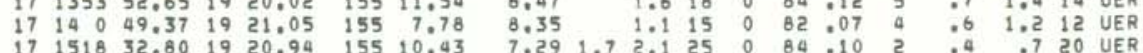

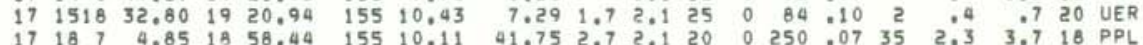

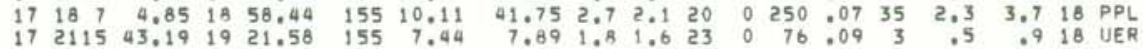

$\begin{array}{lllllllllllllllllll}17 & 2122 & 56.98 & 19 & 19.56 & 155 & 10.65 & 7.08 & 1.7 & 1.7 & 24 & 0 & 97 & 10 & 5 & .5 & .8 & 17 & \text { UER }\end{array}$

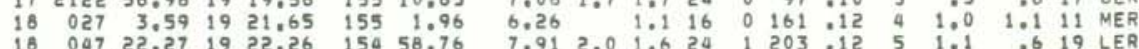

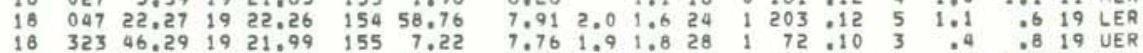

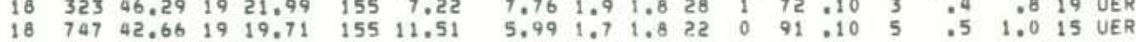

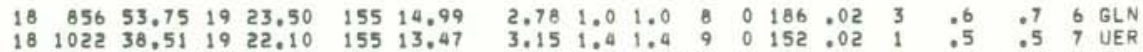

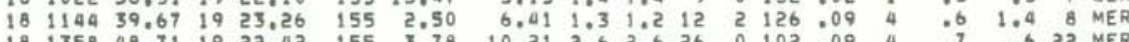

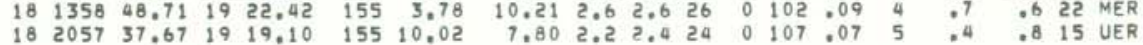
$\begin{array}{rrrrrrrrrrrrrrrrrrr}18 & 2213 & 18.71 & 19 & 18.18 & 155 & 13.03 & 4.88 & .8 & 1.1 & 8 & 0 & 163 & .05 & 2 & 1.5 & 2.5 & 7 & \text { POL . } \\ 19 & 340 & 17.22 & 19 & 48.77 & 156 & 5.62 & 6.43 & & 1.9 & 9 & 0 & 311 & .13 & 30 & 17.4 & 6.4 & 1 & \text { KON . }\end{array}$

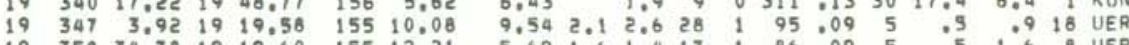

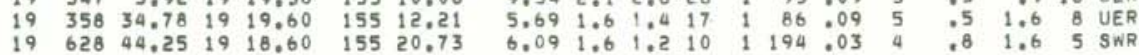

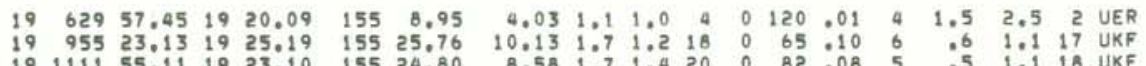

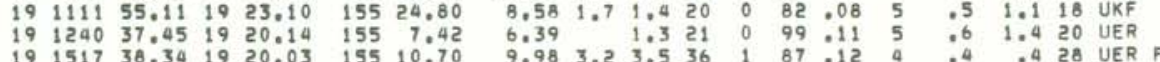
$191524 \quad 10.291921 .82 \quad 155 \quad 1.62 \quad 2.19 \quad \begin{array}{llllllllll}1.1 & 14 & 1 & 168 & 19 & 4 & 1.1 & 2.9 & 11 & \text { MER }\end{array}$

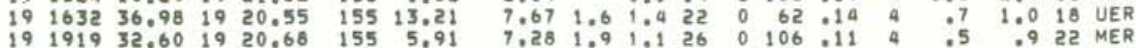

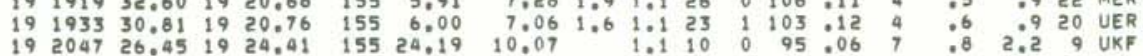

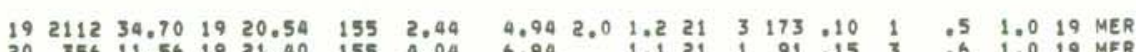

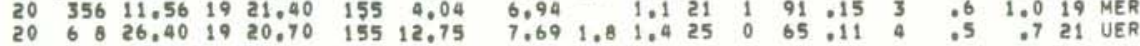


HVO EARTMQUAKE SUMMARY LIST

PAGE 43

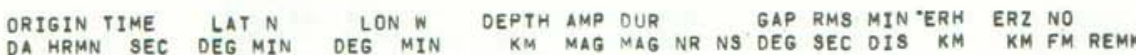
YEAR MON DA HRN

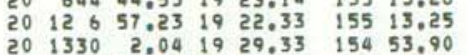

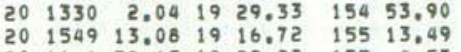

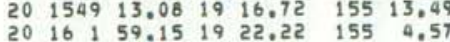

$\begin{array}{lllllll}20 & 1849 & 57.72 & 19 & 24.78 & 155 & 28.81 \\ 20 & 23 & 52.92 & 19 & 17.58 & 155 & 15.37\end{array}$ 21 $61520.501917 .24 \quad 15457.58$ $21 \quad 62231.611919 .58 \quad 155 \quad 8.56$ $\begin{array}{lllllll}21 & 822 & 34.32 & 19 & 9.42 & 155 & 30.21 \\ 21 & 926 & 7.19 & 19 & 19.86 & 155 & 9.93\end{array}$ $\begin{array}{lllllll}21 & 926 & 7.19 & 19 & 19.86 & 155 & 9.93 \\ 21 & 1212 & 3.02 & 19 & 17.01 & 155 & 22.29\end{array}$

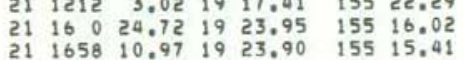

$\begin{array}{lrrrrrr}21 & 1713 & 26.55 & 19 & 22.93 & 155 & 14.21 \\ 21 & 1923 & 6.21 & 19 & 22.75 & 155 & 3.02\end{array}$ $\begin{array}{lllllll}21 & 1923 & 6.21 & 19 & 22.75 & 155 & 3.02 \\ 21 & 1936 & 20.63 & 19 & 20.01 & 155 & 8.86\end{array}$ $21 \quad 194538.74 \quad 19 \quad 14.19 \quad 15534.82$ $21214723.521919 .44 \quad 15$

$\begin{array}{lllllll}21 & 2153 & 18.63 & 19 & 19.53 & 155 & 10.46 \\ 21 & 2347 & 40.41 & 19 & 20.70 & 155 & 1.33\end{array}$

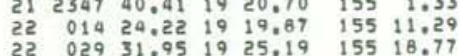
$22 \quad 249 \quad 53.60 \quad 18 \quad 43.58 \quad 155 \quad 13.22$ $\begin{array}{lllllll}22 & 410 & 3.71 & 19 & 27.16 & 155 & 27.74\end{array}$ 25 520 27. 17 19 26.04315456 .73 $22 \quad 534 \quad 27,15 \quad 19 \quad 19,40 \quad 155 \quad 11.02$ $\begin{array}{lllllll}22 & 743 & 21,11 & 19 & 21.84 & 155 & 6.55\end{array}$

$\begin{array}{lllllll}22 & 854 & 1.91 & 19 & 19.89 & 155 & 12.16\end{array}$

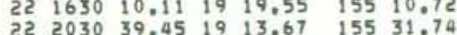

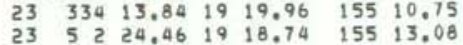

$\begin{array}{lllllll}23 & 834 & 3.79 & 19 & 23.15 & 155 & 14.92\end{array}$ $\begin{array}{lllllll}23 & 936 & 45.74 & 19 & 20.47 & 155 & 4.22\end{array}$ $\begin{array}{lllllll}23 & 2353 & 12.73 & 19 & 20.14 & 155 & 7.52 \\ 24 & 032 & 38.13 & 19 & 22.02 & 155 & 7.16\end{array}$

$\begin{array}{llllllll}24 & 044 & 1.88 & 19 & 30.68 & 155 & 23.49\end{array}$

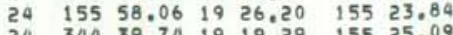
$39,74 \quad 19 \quad 19.29$
15525.09

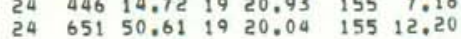

$\begin{array}{llllllll}24 & 8 & 9 & 38.11 & 19 & 21.69 & 155 & 6.37\end{array}$ $\begin{array}{lrlllll}24 & 846 & 57.81 & 19 & 19.49 & 155 & 13.67\end{array}$

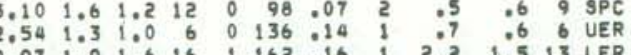
$\begin{array}{rlllllllllll}2.97 & 1.9 & 1.6 & 16 & 1 & 162 & .16 & 1 & 2.2 & 1.5 & 13 & \text { LER } \\ 7.89 & & 1.3 & 15 & 0 & 163 & .09 & 1 & .8 & 1.1 & 15 & \text { POL }\end{array}$ $9.612 .1 \quad 1.828 \quad 0 \quad 49.10 \quad 5 \quad .4 \quad 9920$ UKF . 51 .

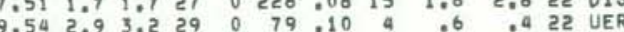
$6,771,311.2220099 .11$ 4 .6 1.220 MER $11.48 \quad 1.32111135 .13 \quad 4 \quad .6 \quad 1.016$ LSW

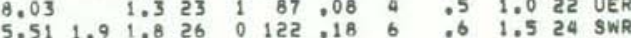

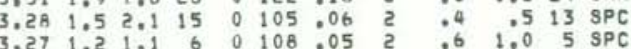
$\begin{array}{llllllllllll}1.55 & 1.1 & 1.7 & 7 & 0 & 115 & .09 & 2 & .5 & .6 & 7 \text { UER }\end{array}$

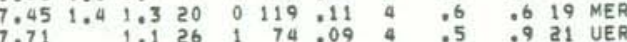

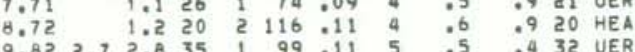
$\begin{array}{lllllllllllll}6.49 & 0 & 0 & 9 & 18 & 0 & 102 & .13 & 5 & .6 & 1.3 & 18 & \text { UER }\end{array}$

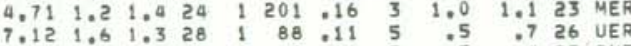

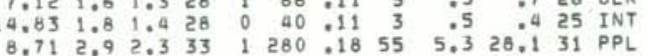
$\begin{array}{llllllllllllll}5.36 & .9 & 1.0 & 15 & 0 & 66 & .10 & 9 & .5 & 3.4 & 12 & \text { UKF }\end{array}$ $8.18 \quad 2.0 \quad 1.729 \quad 0 \quad 87,10 \quad 4 \quad 050.6$ 27 UER

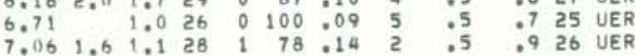
$\begin{array}{llllllllllll}6.71 & 1.2 & 1.3 & 17 & 0 & 82 & .11 & 5 & .6 & 1.4 & 16 & \text { UER }\end{array}$

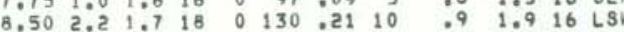
8.161 .92 .025 o 88.08 a .5 .8 25 UER $\begin{array}{llllllllllll}59 & 2.6 & 2.9 & 25 & 0 & 65 & .08 & 2 & .4 & .4 & 24 & G L N\end{array}$

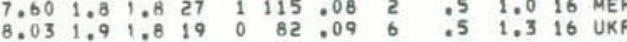
$\begin{array}{lllllll:lllll}7.12 & 1.4 & 1.3 & 20 & 0 & 97 & 06 & 5 & .5 & 1.3 & 15 & \text { UER } \\ 9.13 & 2.7 & 2.9 & 37 & 3 & 73 & .07 & 2 & .3 & .5 & 26 & \text { UER }\end{array}$ $\begin{array}{lllllllllllll}6.84 & .9 & 1.4 & 17 & 2 & 85 & .10 & 2 & .6 & 1.0 & 10 & \text { NER }\end{array}$

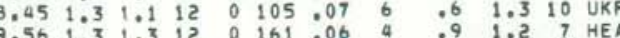

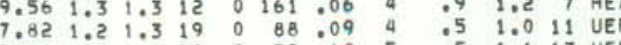
$\begin{array}{lllllllllll}5.60 & 1.3 & .9 & 20 & 0 & 79 & .10 & 5 & .5 & 1.6 & 13 \\ \text { UER }\end{array}$ $\begin{array}{lllllllllll}7.11 & 1.3 & 24 & 0 & 82 & .12 & 2 & .6 & 1.0 & 20 & \text { UER }\end{array}$ $\begin{array}{llllllllllll}6.85 & 1.7 & 24 & 0 & 123 & .12 & 6 & .6 & .9 & 15 & \text { UER } \\ 8.35 & 1.6 & 1.7 & 22 & 0 & 72 & .08 & 5 & .5 & .9 & 16 & \text { UER }\end{array}$
HVO EARTHQUAKE SUMMARY LIST

PAGE 44 ORIGIN TIME LAT N LONW DEPTH AMP OUR GAP RMS MIN ERH ERZ NO YEAR MON DA HRMN TIMEC DEAT NIN DEG MIN D KM MAG MAG NR NG OEEG SEC OIS KM KM FM REMK

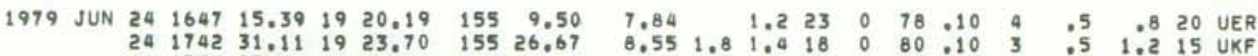

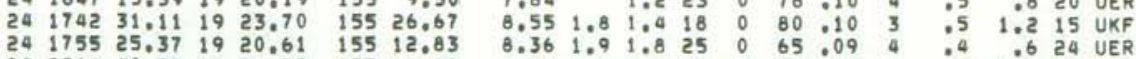

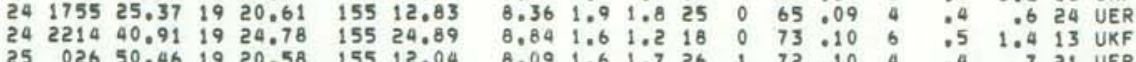

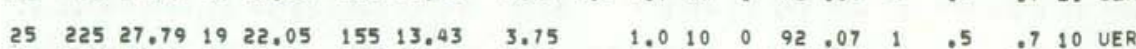

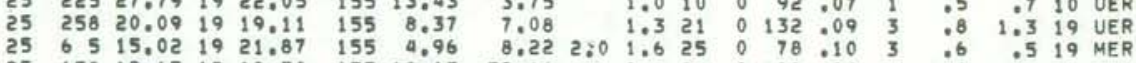

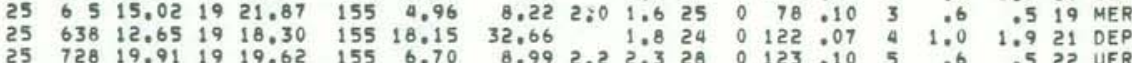
$\begin{array}{llllllllllllllllllll}25 & 1615 & 57.01 & 19 & 48.39 & 155 & 35.40 & 11.84 & 1.7 & 1.8 & 10 & 0 & 187 & .09 & 25 & 1.0 & 2.6 & 8 & \text { KKU }\end{array}$

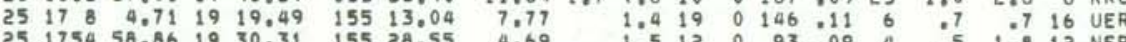

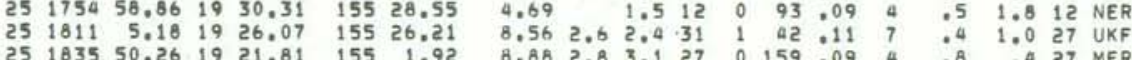

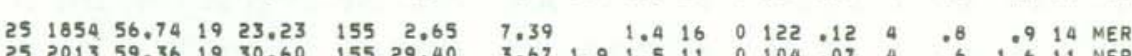

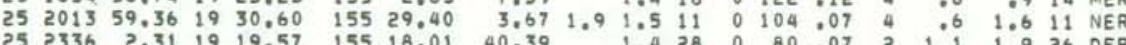

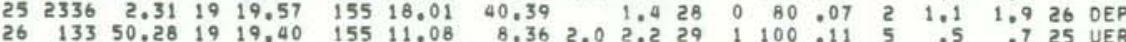

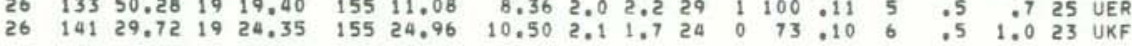

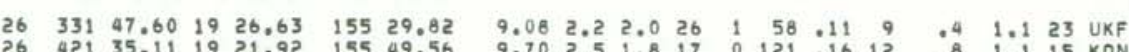

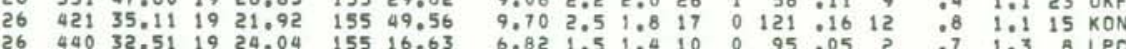

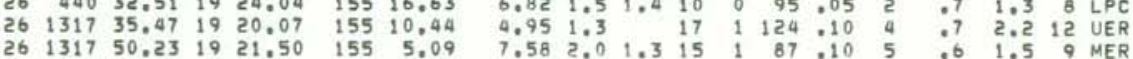
$26.519 \quad 41.52 \quad 19 \quad 20.64 \quad 155 \quad 0.26$

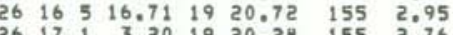

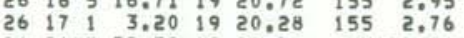

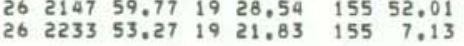

$\begin{array}{lllllll}26 & 2252 & 24.30 & 19 & 20.89 & 155 & 2.05\end{array}$

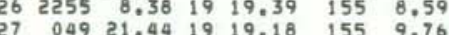

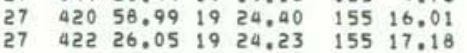
$\begin{array}{llllll}27 & 522 & 46.81 & 19 & 20.68 & 155 \\ 27 & 2.06 & 06\end{array}$

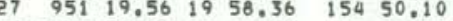
$\begin{array}{lllllll}27 & 1338 & 39.83 & 19 & 32.66 & 155 & 36.87 \\ 27 & 1537 & 34.64 & 19 & 26.57 & 155 & 24.80\end{array}$

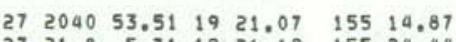

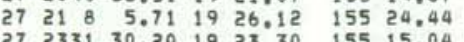

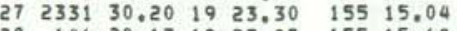

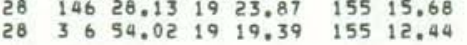
$\begin{array}{lllllll}28 & 318 & 32.99 & 19 & 19.79 & 155 & 8.72\end{array}$ $\begin{array}{lllllll}28 & 442 & 50.79 & 19 & 21.04 & 155 & 2.28 \\ 28 & 648 & 4.10 & 19 & 20.00 & 155 & 12.09\end{array}$
$8.41 \quad 1.91 .6 \quad 25 \quad 1 \quad 100.08 \quad 4 \quad .451 .013$ UER

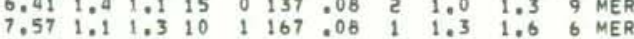

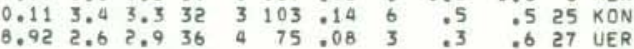
$\begin{array}{llllllllllll}6.37 & 1.3 & 1.3 & 11 & 1 & 185 & .11 & 2 & 1.2 & 1.9 & 4\end{array}$

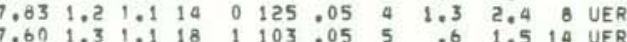

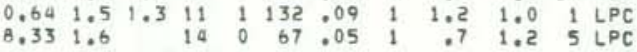
$\begin{array}{lllllllllllllll}4.68 & 1.3 & 1.2 & 7 & 0 & 193 & .06 & 2 & 1.5 & 1.7 & 5 & \text { MER }\end{array}$

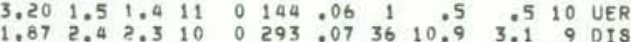
$\begin{array}{lllllllllllll}8.67 & 2.3 & 1.2 & 13 & 0 & 118 & .15 & 6 & .7 & 1.6 & 11 & \text { MOK } \\ 3.26 & 1.8 & 1.2 & 14 & 0 & 79 & : 11 & 7 & .5 & 3.3 & 11 & \text { UKF }\end{array}$ $\begin{array}{llllllllllll}1.67 & 1.3 & 1.1 & 10 & 0 & 181 & .04 & 3 & .4 & .6 & 7 & 7 \text { UER }\end{array}$

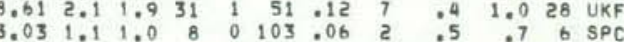

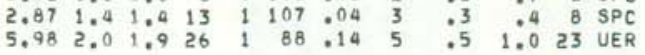

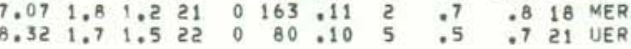
$\begin{array}{llllllllllll}5.26 & 1.2 & 20 & 0 & 122 & .09 & 5 & .5 & 1.2 & 21 & \text { UER }\end{array}$ 


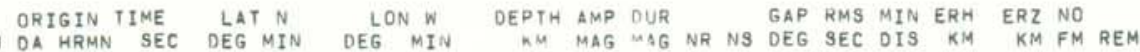

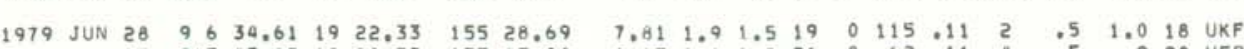

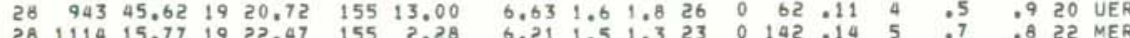

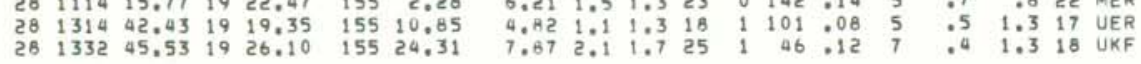
$\begin{array}{lllllllllllllllllllll}28 & 1721 & 21.01 & 19 & 17.90 & 155 & 12.99 & 9.36 & 2.5 & 2.6 & 29 & 0 & 144 & .09 & 9 & .6 & .7 & 29 & \text { POL }\end{array}$

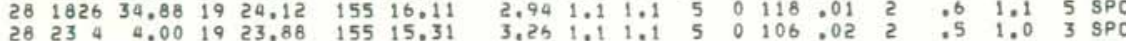

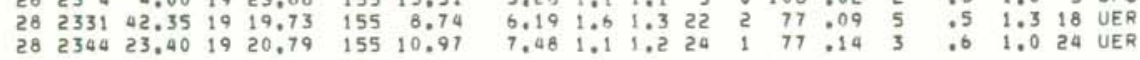
$\begin{array}{lllllllllllllllllll}29 & 514 & 29.16 & 19 & 27.59 & 155 & 27.52 & 8.57 & 1.5 & 1.4 & 11 & 0 & 86 & .07 & 8 & .6 & 2.2 & 11 & \text { UKF }\end{array}$

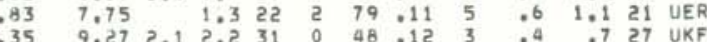

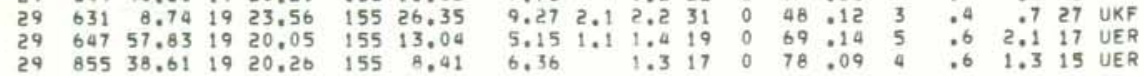

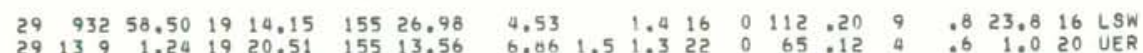

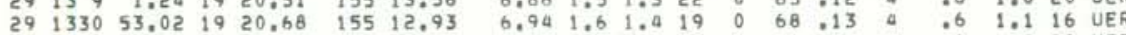

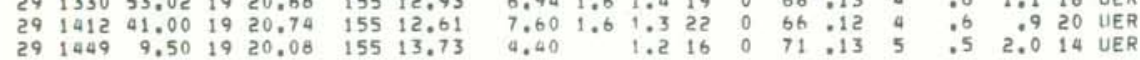
$\begin{array}{lllllllllllllllllll}29 & 1624 & 13.19 & 19 & 23.29 & 155 & 17.05 & 2.41 & 1.4 & 1.4 & 14 & 1 & 69 & .10 & 3 & .4 & .5 & 12 & \mathrm{SPC}\end{array}$

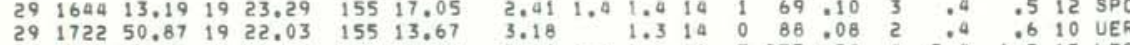

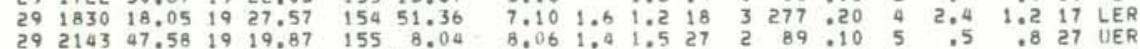

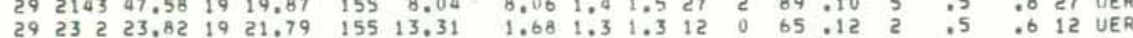

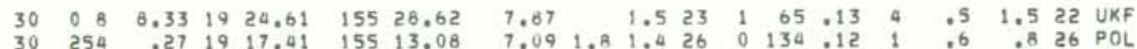

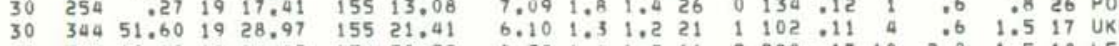

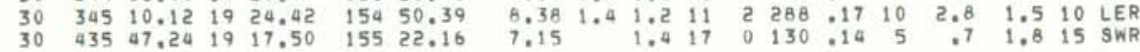

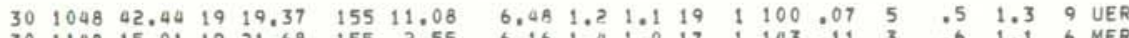

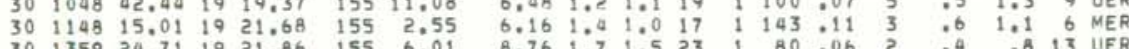

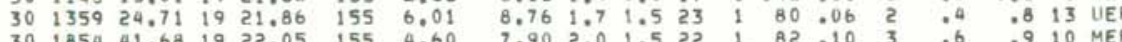

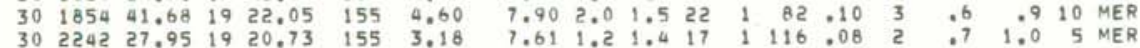

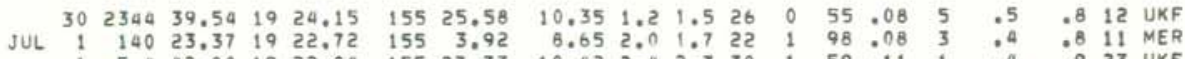

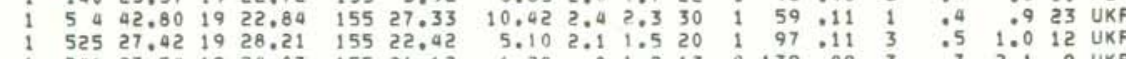

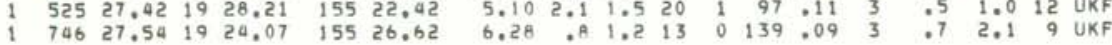
$\begin{array}{lllllllllllllllllll}1 & 1242 & 26.83 & 19 & 26.75 & 155 & 30.01 & 9.12 & 1.9 & 1.4 & 18 & 1 & 78 & .13 & 9 & .5 & 1.7 & 16 & \text { MOK } \\ 1 & 16 & 43.03 & 19 & 20.24 & 155 & 11.76 & 6.98 & 1.6 & 1.3 & 15 & 0 & 79 & : 12 & 5 & : 6 & .99 & 9 \text { UER }\end{array}$

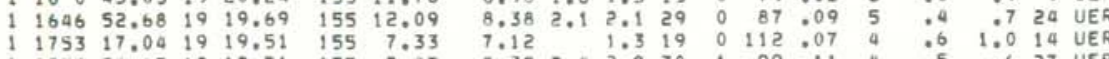

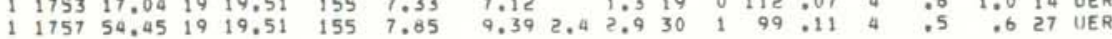

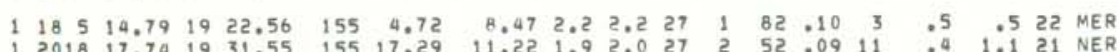

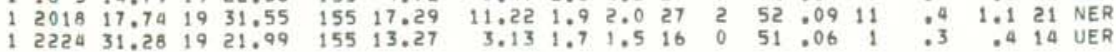

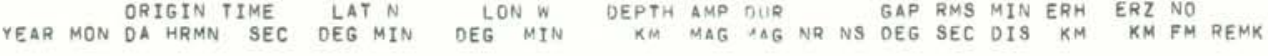

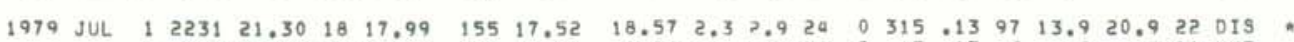

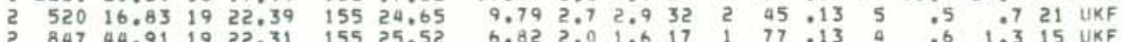

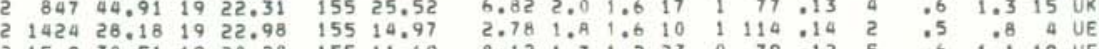
द $15938.511920 .28 \quad 15511.69 \quad 8.121 .71 .223 \quad 0 \quad 79.12 \quad 5 \quad .6 \quad 1.119$ UER

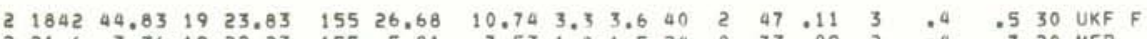

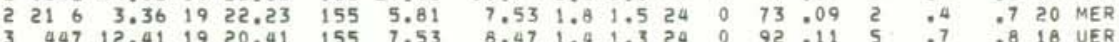
$\begin{array}{llllllllllllllll}3 & 447 & 12.41 & 19 & 20.41 & 155 & 7.53 & 8.47 & 1.4 & 1.3 & 24 & 0 & 92 & .11 & 5 & .7 \\ 3\end{array}$

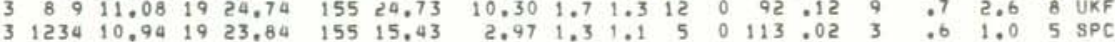

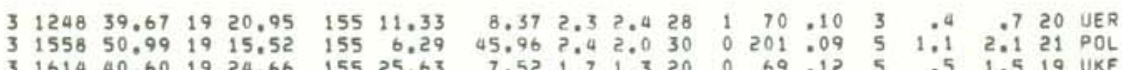

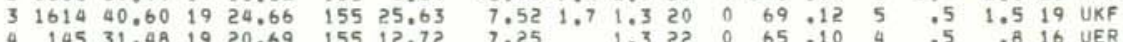

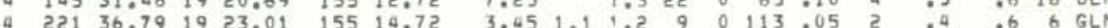

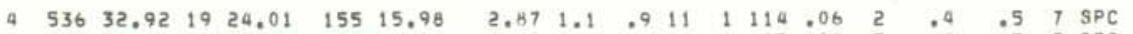

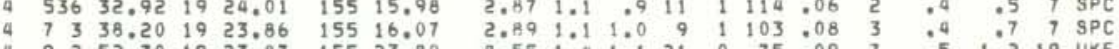

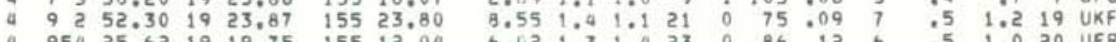
$4 \quad 95425.62$
4 $443928.85 \quad 19$
$43.25 \quad 155 \quad 1.95$ 4
4
4

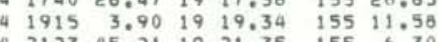
$\begin{array}{lllll}49.21 & 19 & 21,35 & 155 & 6,30\end{array}$

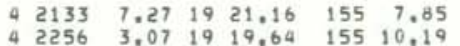

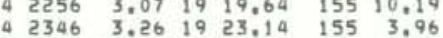
5
5 $233 \quad 46.11 \quad 1928.09 \quad 154 \quad 49.45$

$\begin{array}{lllllll}5 & 720 & 27.44 & 19 & 23.65 & 155 & 15.26 \\ 5 & 722 & 12.33 & 19 & 18.54 & 155 & 23.75\end{array}$ 1
5

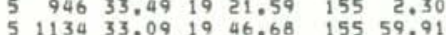
$51452 \quad 3.041920 .22 \quad 155 \quad 12.89$

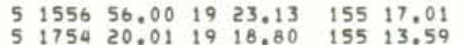
5124.0190 .0315513 .59 52028 41.62 1928.2915523 .09

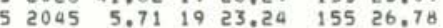

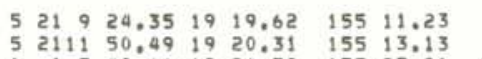

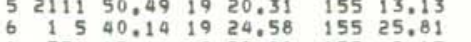

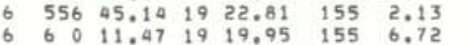
$\begin{array}{llllllll}6 & 6 & 4 & 11.44 & 19 & 18.24 & 155 & 13.28\end{array}$

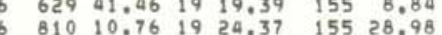

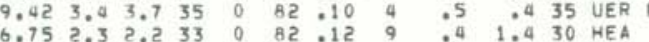

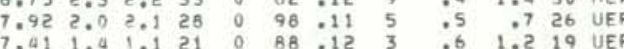

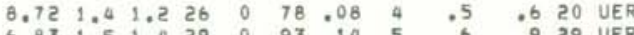

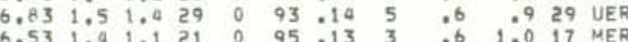

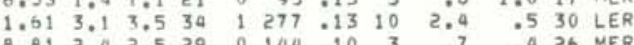
$\begin{array}{lllllllllllll}3.34 & 1.1 & 1.3 & 8 & 0 & 92 & .06 & 3 & .5 & .8 & 8 & \mathrm{SPC}\end{array}$

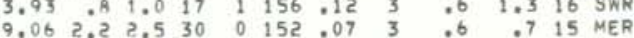

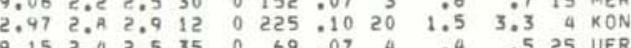

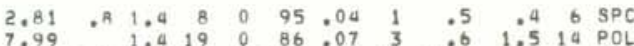

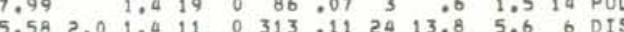

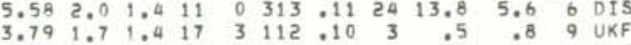

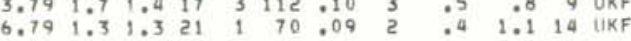
$\begin{array}{lllllllllllll}5.75 & 1.9 & .5 & 16 & 1 & 99 & .05 & 5 & .5 & 2.0 & 10 & \text { UER } \\ 7.37 & 1.6 & .5 & 23 & 1 & 65 & 08 & 4 & .5 & 1.0 & 15 & \text { UER }\end{array}$

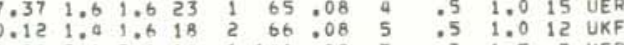

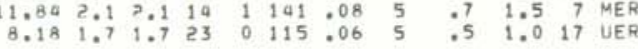
$\begin{array}{lllllllllllll}6.51 & 1.3 & 1.4 & 21 & 0 & 90 & .07 & 2 & .5 & 1.2 & 12 & \text { POL }\end{array}$

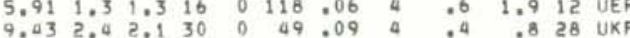

$\begin{array}{lllllllllll}6.63 & 1.4 & 1.2 & 23 & 0 & 138 & .14 & 5 & .6 & .8 & 22 \\ 9.62 & \text { MER }\end{array}$ 
ORIGIN TIME LATN LON N DEPTH AMP DUR N GAP RMS MIN ERH ERZ NO
DA HRMN SEC DEG MIN DEG MIN DKM MAG

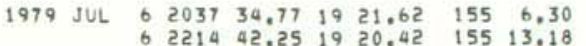

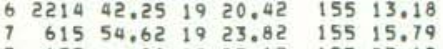

$7 \quad 75234.001920 .81 \quad 155 \quad 3.59$ $\begin{array}{rrrrrrr}7 & 917 & 4.24 & 19 & 21.31 & 155 & 30.25 \\ 7 & 1010 & 8.88 & 19 & 31.61 & 155 & 42.58\end{array}$ $\begin{array}{lllllll}7 & 1015 & 2.60 & 19 & 20.65 & 155 & 9.25\end{array}$ $723532.62 \quad 1927.26 \quad 155 \quad 20.89$ $\begin{array}{lllllll}8 & 053 & 5.17 & 19 & 11.42 & 155 & 31.14 \\ 8 & 412 & 20.13 & 19 & 27.86 & 155 & 52.07\end{array}$

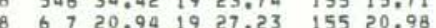
B $9740,00 \quad 1924,12 \quad 155 \quad 16,03$ 8) $914 \quad 39.08 \quad 1923.71 \quad 155 \quad 15.71$ - $111739.04 \quad 1922.70 \quad 15515,25$ 8 $2018 \quad 14.14 \quad 1920,23 \quad 155 \quad 11.07$ 8 $213958.131924 .86 \quad 155 \quad 18,08$

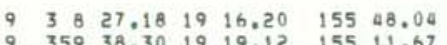

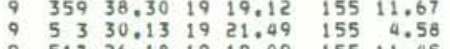
$\begin{array}{rrrrrrr}9 & 517 & 26.18 & 19 & 19.09 & 155 & 11.45 \\ 9 & 1359 & 1.82 & 19 & 20.14 & 155 & 11.69\end{array}$ $9 \begin{array}{llllll}154 & 3.57 \quad 19 & 20.34 & 155 & 13.04\end{array}$

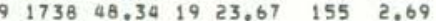
$\begin{array}{lllllll}10 & 411 & 26.72 & 19 & 18.35 & 155 & 13.24 \\ 10 & 711 & 17.07 & 19 & 18.08 & 155 & 13.20\end{array}$ $\begin{array}{llllllll}10 & 728 & 10.98 & 19 & 18.60 & 155 & 13.41\end{array}$ $10 \quad 99 \quad 31.47 \quad 1912.65 \quad 15523.59$ $\begin{array}{rrrrrrr}10 & 917 & 44.92 & 19 & 19.56 & 155 & 8.95 \\ 10 & 1024 & 10.61 & 19 & 22.05 & 155 & 4.00\end{array}$ $\begin{array}{lllllll}10 & 1037 & 21.23 & 19 & 19.04 & 155 & 13.38\end{array}$ $10 \quad 1118 \quad 5.96 \quad 19017.82 \quad 155 \quad 13.13$ $\begin{array}{lllllll}10 & 12 & 88.03 & 19 & 19.66 & 155 & 12.20 \\ 10 & 1346 & 39.99 & 19 & 19.50 & 155 & 11.41\end{array}$ $\begin{array}{llllllll}10 & 14 & 52.78 & 19 & 24.70 \quad 155 & 23.58\end{array}$ $10 \quad 1553 \quad 36.94 \quad 1922.75 \quad 155 \quad 1.84$ $\begin{array}{lllllll}10 & 2211 & 57.11 & 19 & 21.72 & 155 & 11.31\end{array}$

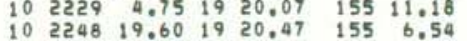

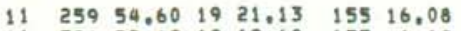

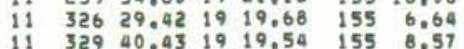

$\begin{array}{llllllllllll}6.82 & 1.5 & 1.2 & 29 & 0 & 83 & .10 & 3 & .5 & .8 & 27 & \text { UER }\end{array}$

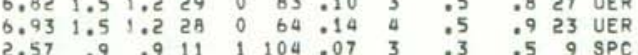

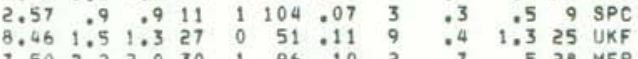

$\begin{array}{llllllllllll}0.21 & 1.2 & 1.2 & 19 & 0 & 78 & .04 & 5 & .7 & 1.2 & 7 \text { HEA }\end{array}$

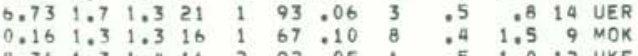

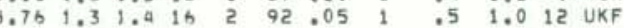
$\begin{array}{rrrrrrrrrrrr}8.19 & 2.3 & 2.0 & 24 & 2 & 162 & : 11 & 7 & .5 & .9 & 9 & \text { LSW } \\ 5.58 & 2.6 & 1.7 & 19 & 2 & 128 & .09 & 6 & .5 & 1.1 & 11 & \text { KON }\end{array}$

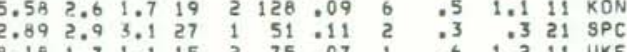

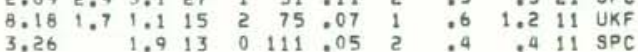
$\begin{array}{llllllllllll}2.75 & 1.3 & 1.3 & 8 & 1 & 105 & .10 & 2 & .5 & .7 & 7 & \mathrm{SPC}\end{array}$

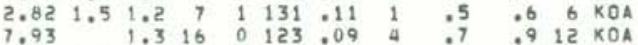

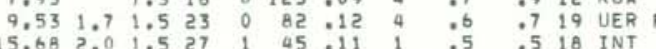
$\begin{array}{llllllllllll}9.29 & 2.5 & 1.6 & 21 & 1 & 159 & .09 & 7 & .8 & .6 & 17 & \text { KON }\end{array}$

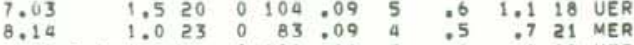

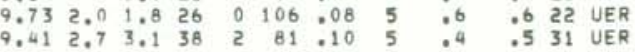
$\begin{array}{llllllllllll}6.89 & 1.3 & 1.7 & 17 & 0 & 80 & .06 & 4 & .5 & 1.3 & 10 & \text { UER }\end{array}$

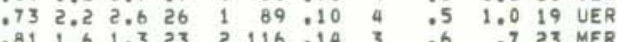

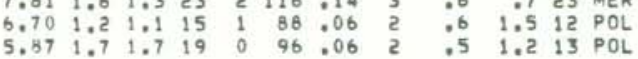
$\begin{array}{rrrrrrrrrrrrr}7.01 & 1.7 & 1.6 & 20 & 1 & 81 & .07 & 3 & .5 & 1.2 & 11 & \text { POL }\end{array}$ $\begin{array}{lllllllllll}2.05 & 2.1 & 14 & 0 & 235 & .09 & 14 & 2.5 & 4.9 & 14 & \text { LSW }\end{array}$

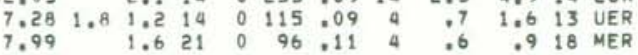
$\begin{array}{lllllllllllll}9.14 & 2.2 & 2.2 & 25 & 0 & 128 & .08 & 7 & .5 & .7 & 22 & \text { UER }\end{array}$

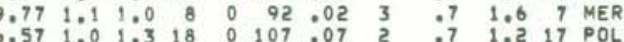

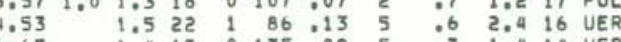

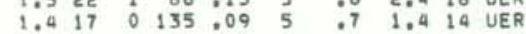
$\begin{array}{llllllllllll}8.36 & 1.5 & 1.5 & 20 & 0 & 64 & .12 & 8 & .6 & 1.6 & 16 & \text { UKF }\end{array}$

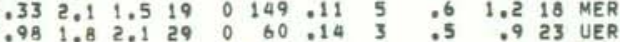

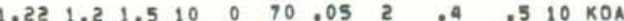

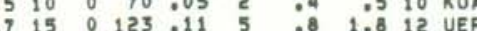

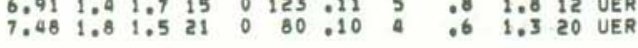

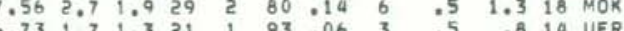

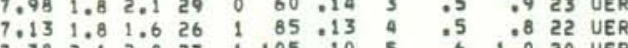

ORIGIN TIME LATN LON N OEPTH AMP NUR GAP RMS MIN ERH ERZ NO

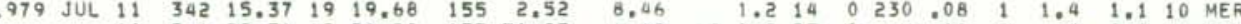
$11 \quad 56 \quad 28.31 \quad 1926.20 \quad 155 \quad 31.25$ $1173 \quad 50.591919 .92 \quad 15511.85$ $11745 \quad 43.75 \quad 19 \quad 19,66 \quad 155 \quad 10,49$

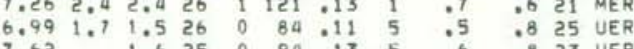
$\begin{array}{llllllllllllllllll}12 & 041 & 22.12 & 19 & 21.76 & 155 & 14.11 & 10.43 & 1.5 & 1.3 & 20 & 0 & 57.08 & 2 & .5 & .6 & 16 & 16 \\ 1 & \text { UER }\end{array}$

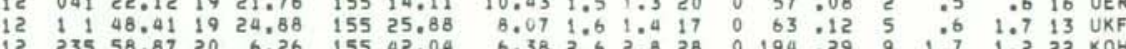

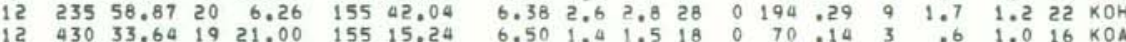

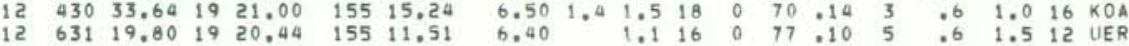

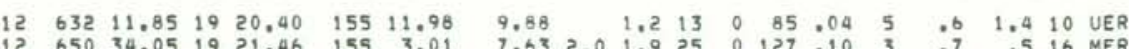

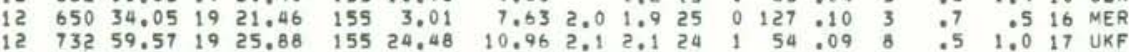

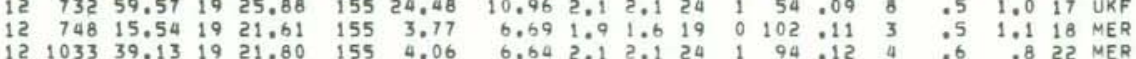
$12 \quad 1416 \quad 43.10 \quad 19 \quad 20.03 \quad 155 \quad 6.56$

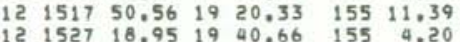
12183844.631921 .70 155 5.04 $12215129.71 \quad 1924.12 \quad 15512.82$ $\begin{array}{lrrrrrr}12 & 2151 & 29.71 & 19 & 24.12 & 155 & 12.82 \\ 12 & 2214 & 3.49 & 19 & 22.12 & 155 & 4.08\end{array}$

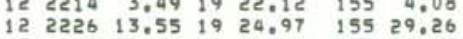
$\begin{array}{llllllll}13 & 3 & 3 & 58.03 & 19 & 20.54 & 155 & 12.89 \\ 13 & 4 & 8 & 59.91 & 19 & 46.50 & 155 & 48.04\end{array}$ $\begin{array}{lllllll}13 & 439 & 25.91 & 19 & 9.81 & 155 & 36.85\end{array}$ $\begin{array}{llllll}13 & 731 & 1.34 & 19 & 20.36 & 155\end{array}$

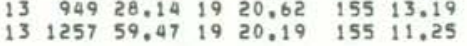
$\begin{array}{lllllll}13 & 1440 & 51.39 & 19 & 22.01 & 155 & 4.53\end{array}$

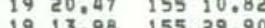

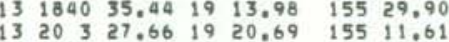
$13 \quad 2117 \quad 13,66 \quad 1920.02 \quad 155 \quad 10,76$

$\begin{array}{llllllll}14 & 1 & 3 & 15.20 & 19 & 23.26 & 155 & 5.59\end{array}$ $14 \quad 255 \quad 37.09 \quad 19 \quad 19.48 \quad 155 \quad 8.76$

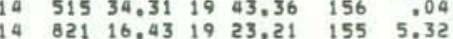
$14122359.96 \quad 19 \quad 20,30 \quad 15511.44$ $14 \quad 1726 \quad 40.09 \quad 19 \quad 16.64 \quad 155 \quad 33.96$

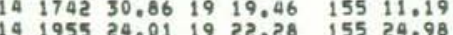

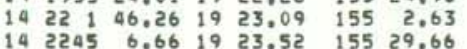
$15 \quad 323 \quad 30.44 \quad 19 \quad 18.35 \quad 155 \quad 15.72$

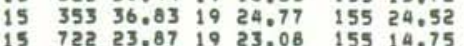

$\begin{array}{llllllllllllll}6.95 & 1.1 & 1.4 & 16 & 0 & 116 & .10 & 6 & .7 & 1.1 & 15 & \text { UER }\end{array}$

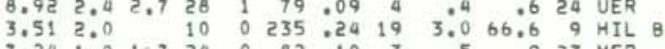

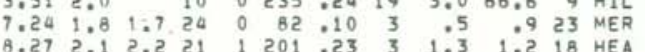

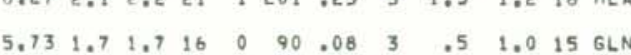

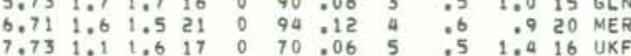

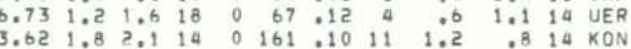
$\begin{array}{llllllllll}6.51 & 12 & 0 & 270 & 11 & 16 & 12.3 & 19.0 & 11 & \text { HEA L }\end{array}$

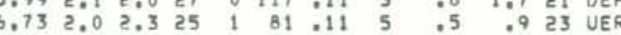

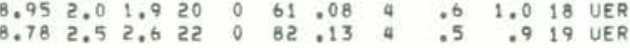
$\begin{array}{llllllllll}8.03 & 2.52 .6 & 24 & 1 & 84 & .09 & 4 & .5 & .8 & 22 \\ 0.3 E R\end{array}$

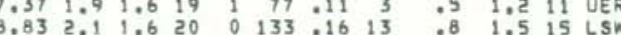
$\begin{array}{llllllllllll}8.68 & 1.7 & 1.9 & 25 & 0 & 73 & .08 & 4 & .4 & .7 & 22 & \text { UER } \\ 6.80 & 1.1 & 1.4 & 25 & 0 & 86 & .08 & 4 & .5 & .8 & 23 & \text { UER }\end{array}$ $\begin{array}{llllllllllll}1.50 & 1.9 & 2.4 & 13 & 0 & 93 & .11 & 1 & .6 & .4 & 10 & \text { MER }\end{array}$

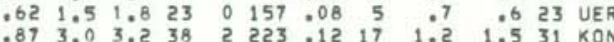

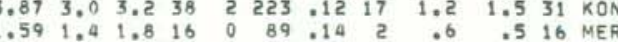

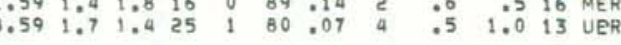
$\begin{array}{llllllllllll}4.56 & 1.7 & 1.7 & 27 & 1 & 110 & .17 & 6 & .5 & 2.8 & 26 & \text { HEA }\end{array}$

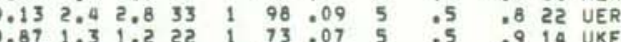

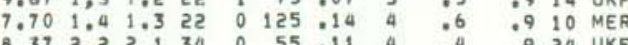

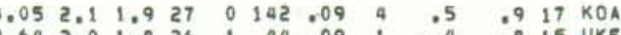

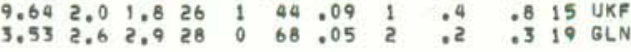




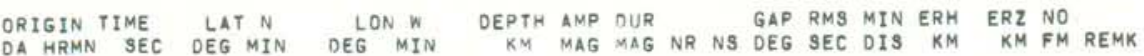
\begin{tabular}{llllllllllllll}
\hline & 83 & 23 & HEA
\end{tabular} 15 12 $559.091924 .36 \quad 15516.57$

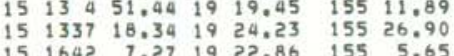
$\begin{array}{llllll}15 & 1936 & 6.42 & 19 & 22.11 \quad 155 \quad 4.85\end{array}$ 15193716.001922 .19 155 4.74 $151991 \quad 8.06$ 19 22.32 155 5.12 $\begin{array}{lllllll}15 & 2011 & .76 & 19 & 22.28 & 155 & 5.09\end{array}$

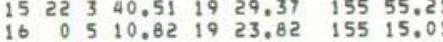
$16 \quad 24757.76 \quad 1923.28$ 155 5.31

$\begin{array}{lllllll}16 & 340 & 18.27 & 19 & 22.96 \quad 155 & 6.29\end{array}$ $\begin{array}{lllllll}16 & 413 & 15.73 & 19 & 24.00 & 155 & 1.73\end{array}$ 16 $534 \quad 15,5719330.32 \quad 15557.80$ i6 $1016 \quad 25.9919 \quad 23.10 \quad 155 \quad 5.89$ $\begin{array}{llllllll}16 & 1017 & 2.64 & 20 & 2.61 & 155 & 46.61\end{array}$ $16 \quad 1239 \quad 10.26 \quad 1924.57 \quad 15528,00$

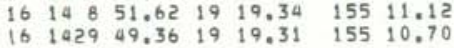

$\begin{array}{lllllll}16 & 1653 \quad 44.69 & 19 & 23.75 \quad 155 & 15.24\end{array}$ $16 \quad 1826 \quad 59.93 \quad 1926.22 \quad 154 \quad 57.83$

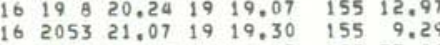

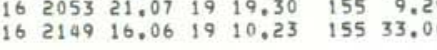

$\begin{array}{lllllllll}16 & 22 & 1 & 12.77 & 19 & 20.02 & 155 & 11.92\end{array}$ $1752458.501923 .96 \quad 155 \quad 16.16$

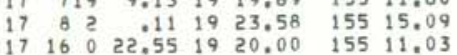

$\begin{array}{llllllll}17 & 19 & 8 & 28.80 & 19 & 24.24 & 155 & 15.87\end{array}$

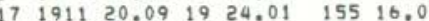
$17 \quad 2252 \quad 38.13 \quad 1920.16 \quad 155 \quad 11.63$

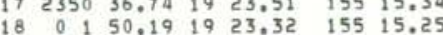
$\begin{array}{lllrllll}18 & 0 & 6 & 38.53 & 19 & 25.69 & 155 & 25.35 \\ 18 & 333 & 8.22 & 19 & 25.10 & 155 & .54\end{array}$ $\begin{array}{lllllll}18 & 1053 & 1.56 & 19 & 19.71 & 155 & 11.15\end{array}$ 1816217.721921 .29 155 4.7

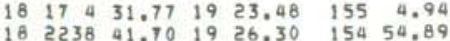

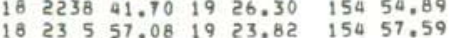

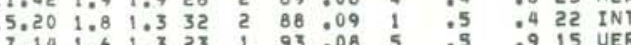
$\begin{array}{lllllllllllll}9.99 & 1.8 & 1.3 & 20 & 1 & 60 & 07 & 3 & .5 & .9 & 11 & \text { UKF } \\ 1.97 & 3.6 & 4.0 & 34 & 0 & 68 & 11 & 1 & .3 & .2 & 27 & \text { MER }\end{array}$ $\begin{array}{lllllllllll}9.33 & 2.4 & 2.3 & 32 & 2 & 78 & .06 & 3 & .4 & .6 & 21 \\ 9 E R\end{array}$

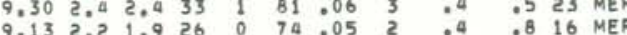

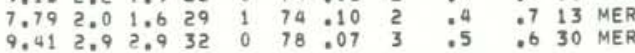
$\begin{array}{llllllllllll}8.89 & 1.4 & 1.3 & 23 & 0 & 75 & .07 & 2 & .5 & 1.0 & 14 & \text { MER }\end{array}$

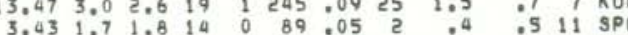
$\begin{array}{llllllllllll}1.87 & 2.1 & 2.3 & 14 & 0 & 91 & .08 & 2 & .5 & .3 & 10 & \text { MER } \\ 1.97 & 3.1 & 3.7 & 28 & 0 & 74 & .12 & 2 & .5 & .3 & 18 & \text { MER }\end{array}$ $\begin{array}{llllllllllll}1.45 & 1.0 & 1.4 & 12 & 0 & 95 & .06 & 0 & .5 & .2 & 6 & \text { UER }\end{array}$

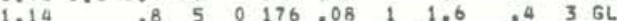

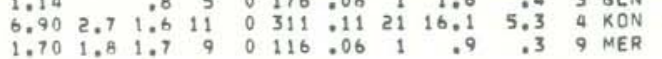
$\begin{array}{lllllllllllll}9.50 & 2.7 & 2.2 & 12 & 0 & 169 & .16 & 9 & 1.9 & 2.1 & 10 & \mathrm{KOH}\end{array}$

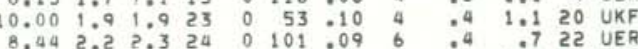

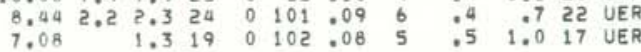
$\begin{array}{llllllllllll}3.23 & 1.1 & 1.1 & 6 & 0 & 98 & .03 & 2 & .5 & 1.0 & 5 & \mathrm{SPC}\end{array}$

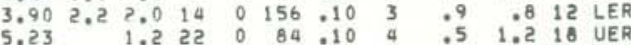

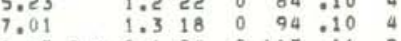

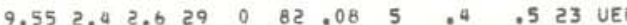

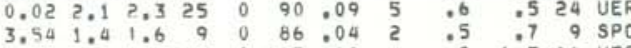
$\begin{array}{llllllllllll}3.36 & 1.6 & 1.5 & 10 & 1 & 116 & 07 & 2 & .4 & .5 & 4 & 9 P C\end{array}$ $\begin{array}{llllllllllll}6.88 & 0 & 1.3 & 17 & 0 & 81 & 11 & 5 & 7 & 1.1 & 16 & \text { UER }\end{array}$

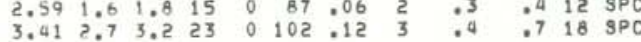
$\begin{array}{rrrrrrrrrrrr}9.31 & .9 & 1.6 & 22 & 1 & 54 & 09 & 7 & .5 & 1.1 & 18 & \text { UKF } \\ 0.12 & -9 & 0.3 & 12 & 0 & 115 & : 13 & 3 & .8 & 1.9 & 10 & \text { LER }\end{array}$

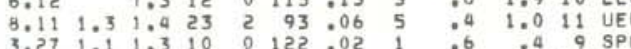

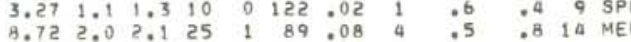
$\begin{array}{rrrrrrrrrrrr}1.81 & & 1.7 & 8 & 0 & 99 & 06 & 2 & .6 & .4 & 8 & \text { MER } \\ 6.70 & 1.3 & 1.4 & 12 & 0 & 224 & .09 & 2 & 2.7 & 1.1 & 7 & \text { LER }\end{array}$ \begin{tabular}{lllllllllll}
6.70 & 1.3 & .4 & 12 & 0 & 224 & 09 & 2 & 2.7 & 1.1 & 7 LER \\
5.20 & .6 & 0 & 19 & 2 & 196 & .19 & 3 & 1.1 & 1.4 & 11 \\
\hline
\end{tabular}

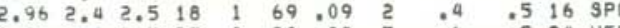

ORIGIN TIME
DA HRT N
LON W

1979 JUL $19 \quad 016 \quad 39.18 \quad 19 \quad 18.51 \quad 155 \quad 23.42$ Q 31022.32 19 $20.44 \quad 15512.05$ $\begin{array}{lllllll}19 & 356 & 41.44 & 19 & 19.37 & 155 & 12.12 \\ 19 & 441 & 56.51 & 19 & 23.46 & 155 & 5.02\end{array}$ $19 \quad 552 \quad 5.66 \quad 19 \quad 20.47 \quad 155 \quad 12.91$ Q $828 \quad 10.83 \quad 19 \quad 18.68 \quad 15523.5$ $\begin{array}{lllllll}19 & 842 & 25.54 & 19 & 22.19 & 155 & 13.34 \\ 19 & 858 & 6.17 & 19 & 18.28 & 155 & 23.59\end{array}$ $19 \begin{array}{llllll}1136 & 6.05 \quad 19 & 18.27 \quad 155 \quad 12.96\end{array}$

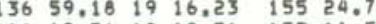
$191411 \quad 19.76 \quad 1919.56 \quad 15511.54$

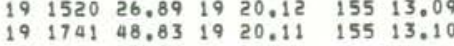

$19 \quad 1748 \quad 59.54 \quad 19 \quad 20.46 \quad 155 \quad 13.03$ $\begin{array}{llllll}920 & 0.87 & 19 & 26.33 & 155 & 27.03\end{array}$ $192034 \quad 13.001921 .04 \quad 15525.46$

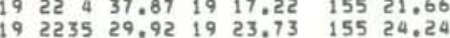

$\begin{array}{lllllll}20 & 033 & 13.92 & 19 & 20.09 & 155 & 7.62\end{array}$ $20052 \quad 55.28$ 19 20.40 15517.44

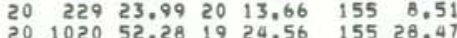
2010 1110 42.21 $1926.06 \quad 15528.44$

$20 \quad 1221 \quad 30.89 \quad 19 \quad 19.60 \quad 155 \quad 11.99$ $\begin{array}{lllllll}20 & 1244 & 15.81 & 19 & 20.25 & 155 & 12.92\end{array}$ $20 \quad 2042 \quad 36.10 \quad 1920.44 \quad 155 \quad 6.00$ $\begin{array}{ll}0.53 & 155 \quad 10.57\end{array}$ $\begin{array}{lllllll}20 & 2322 & 30.25 & 19 & 24.56 & 155 & 27.60 \\ 20 & 2344 & 46.79 & 19 & 27.30 & 155 & 29.51\end{array}$ ह1 $01014.611920 .65 \quad 155 \quad 7.41$ 21 $33618.619020 .18 \quad 15510.5$

$21 \quad 5655.04 \quad 19 \quad 14,32 \quad 155 \quad 21,97$ $21 \quad 546 \quad 24.61 \quad 1923.33 \quad 15516.72$

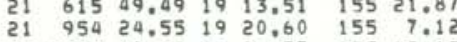
$\begin{array}{lllll}1048 & 48.17 & 19 & 21.53 \quad 155 \quad 18.68\end{array}$ $\begin{array}{lllllll}21 & 1221 & 23.44 & 19 & 20.78 & 155 & 6.96\end{array}$ $\begin{array}{lllllll}21 & 1310 & 45.60 & 19 & 21.47 & 155 & 6.95 \\ 21 & 1551 & 48.78 & 19 & 20.83 & 155 & 2.29\end{array}$ $21 \quad 554 \quad 1.96 \quad 1920.08 \quad 155 \quad 2.29$ $21 \quad 1719 \quad 58,15 \quad 19 \quad 20,78 \quad 155 \quad 6,19$ $21 \quad 1817 \quad 14.40 \quad 1923.51 \quad 155 \quad 16.90$

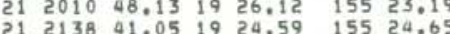

$3.983 .13 .426+107.0833 .4 \quad 8 \quad 19$ SWR $\begin{array}{lllllllllllll}6.73 & 1.5 & 1.6 & 25 & 1 & 68 & .09 & 5 & .4 & .8 & 18 & \text { UER } \\ 9.41 & 2.7 & 2.9 & 31 & 0 & 74 & .07 & 4 & 4 & .5 & 26 & \text { UER }\end{array}$

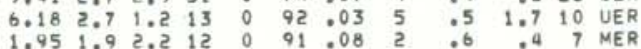
$\begin{array}{llllllllllll}9.21 & 1.5 & 1.5 & 19 & 1 & 66 & .04 & 4 & .5 & .9 & 14 & \text { UER }\end{array}$

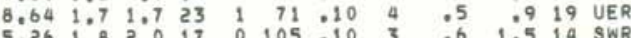

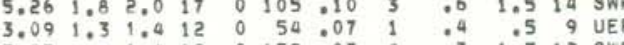
$\begin{array}{llllllllllllll}0.90 & 2.1 & 2.1 & 22 & 0 & 139 & .10 & 8 & .7 & 1.0 & 18 & \text { POL }\end{array}$ $\begin{array}{ccccccccc}33 & 1 & 119 & 111 & 8 & 18 & 1.5 & 20 & \text { SW } \\ 18 & 0 & 93 & 08 & 6 & : 5 & 1.1 & 16 & \text { UEE }\end{array}$

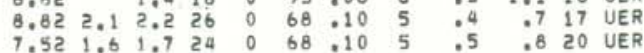

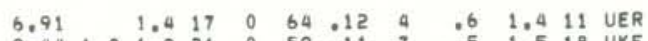
$\begin{array}{llllllllllll}9.44 & 1.9 & 1.9 & 21 & 0 & 59 & 11 & 7 & .5 & 1.5 & 18 & \text { UKF }\end{array}$

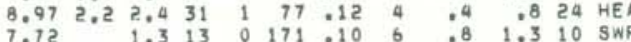

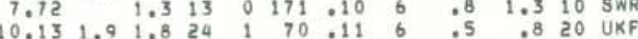
$\begin{array}{llllllllllll}7.43 & 1.3 & 20 & 0 & 95 & .07 & 5 & .5 & 1.1 & 20 & \text { UER }\end{array}$

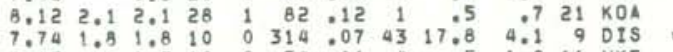

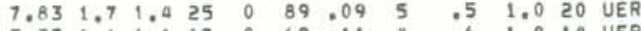
5.331 .61 .117 0 69.11 4 .6 1.8 14 UER

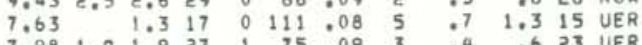
$\begin{array}{lllllllllll}10.83 & 3.6 & 3.9 & 39 & 1 & 45 & .12 & 4 & .4 & .5 & 29 \\ 9 & 0 K F & 0\end{array}$

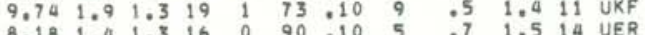

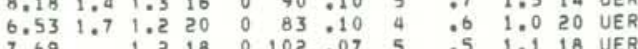
$\begin{array}{llllllllllll}9.15 & 1.7 & 16 & 0 & 159 & 08 & 9 & 09 & 1.6 & 13 & \text { LSW }\end{array}$

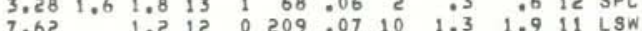

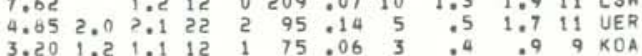

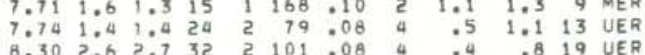
$\begin{array}{lllllllllllll}2.87 & 1.3 & 1.4 & 14 & 1 & 49 & .07 & 0 & .4 & .3 & 8 & \text { SPC }\end{array}$

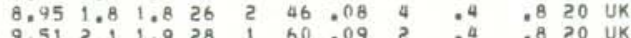

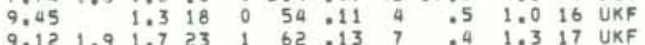
$\begin{array}{lllllllllll}6.92 & 1.7 & 1.4 & 23 & 2 & 94 & .11 & 4 & .5 & 1.2 & 14\end{array}$ UER

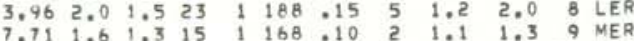


ORIGIN TIME
DA HRMN SAT N

1979 JUL $21 \quad 22 \quad 8 \quad 27.451918 .65 \quad 155 \quad 13.55$

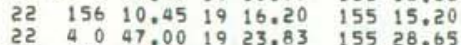

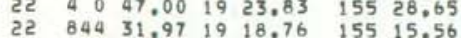
$22 \quad 844 \quad 31.971918 .76 \quad 155 \quad 15.56$ $\begin{array}{rrrrrrr}22 & 1554 & 4.53 & 19 & 20.52 & 155 & 6.10 \\ 22 & 1849 & 56.24 & 19 & 23.95 & 155 & 27.09\end{array}$ ट2 $19852.511919 .72 \quad 15520.98$

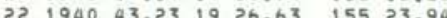

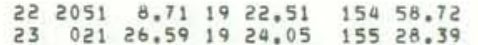

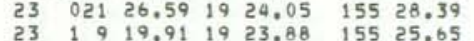
$\begin{array}{lllllll}23 & 1 & 19.91 & 19 & 23.88 & 155 & 25.65 \\ 23 & 231 & 43.13 & 19 & 19.58 & 155 & 8.19\end{array}$ $\begin{array}{lllllll}23 & 246 & 8.79 & 19 & 20.01 & 155 & 8.35\end{array}$ $\begin{array}{lllllll}23 & 1419 & 31.86 & 19 & 25.57 & 155 & 24.63\end{array}$ $\begin{array}{lllllll}23 & 1429 & 17.10 & 19 & 19.79 & 155 & 11.24\end{array}$ $24 \quad 3,14.03$ 19 21.05 155 5.67

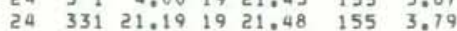
$\begin{array}{lllllll}24 & 629 & 11.33 & 19 & 19.96 & 155 & 8.98\end{array}$ $\begin{array}{lrrrrrr}24 & 936 & 7.61 & 19 & 23.86 & 155 & 15.96 \\ 24 & 1335 & 31.22 & 19 & 21.40 & 155 & 3.96\end{array}$

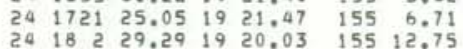

$\begin{array}{llllllll}24 & 18 & 78.33 & 19 & 19.68 & 155 & 8.30\end{array}$ $\begin{array}{lllllll}24 & 1813 & 45.34 & 19 & 20.07 & 155 & 8.16\end{array}$

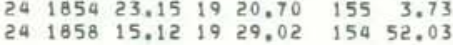
$\begin{array}{lllllll}24 & 1859 & 26.89 & 19 & 20.25 & 155 & 7.39\end{array}$

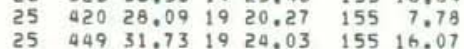

$\begin{array}{llllllll}25 & 5 & 8 & 3.84 & 19 & 20.55 & 155 & 2.92\end{array}$ $\begin{array}{llllllll}25 & 8 & 0 & 14.21 & 19 & 20.66 & 155 & 6.51\end{array}$

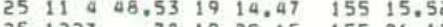
$25 \quad 132017.93 \quad 1924.69 \quad 15526.00$

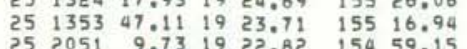
260568.16 19 $19.71 \quad 15511.09$ $26 \quad 127 \quad 25.25 \quad 19+24.23$

$\begin{array}{lllllll}26 & 229 & 48.33 & 19 & 24.05 & 155 & 15.71\end{array}$ $\begin{array}{lllllll}26 & 330 & 1.87 & 19 & 33.57 & 155 & 40.21 \\ 26 & 611 & 9.34 & 19 & 27.89 & 155 & 27.53\end{array}$ $\begin{array}{lllllllllllll}7.60 & 1.2 & 1.3 & 16 & 0 & 86 & .06 & 3 & .6 & 1.5 & 12 & \text { POL }\end{array}$

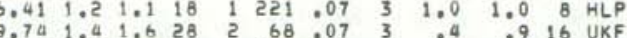

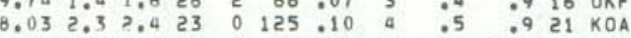

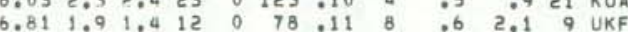

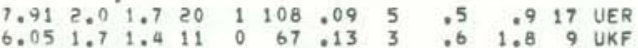

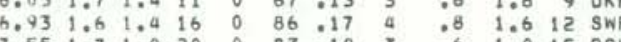

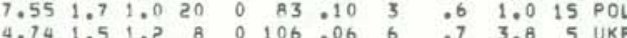

$\begin{array}{rrrrrrrrrrrr}5.73 & 1.3 & 1.3 & 11 & 0 & 199 & .14 & 5 & 1.3 & 2.2 & 6 & \text { LER } \\ 0.14 & 2.2 & 1.6 & 21 & 0 & 68 & .13 & 3 & .5 & 1.0 & 17 & \text { UKF }\end{array}$

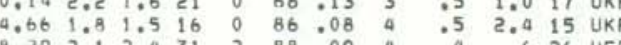

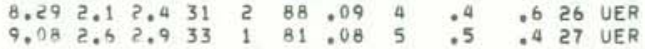
$\begin{array}{llllllllllll}5.05 & 1.6 & 1.1 & 12 & 1 & 99 & .08 & 1 & .5 & 1.4 & 10 & \text { UKF }\end{array}$

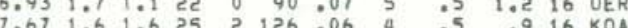

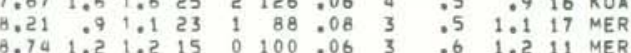
$\begin{array}{lllllllllllll}7.96 & 1.4 & 1.6 & 27 & 1 & 77 & .07 & 4 & .5 & .9 & 20 & \text { UER }\end{array}$

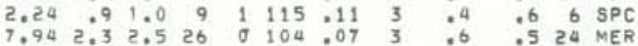

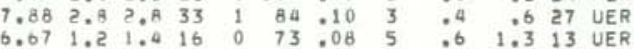
$9.843 .5 \quad 3.734 \quad 0 \quad 85 \quad .10 \quad 4 \quad .6 \quad .528$ UER F

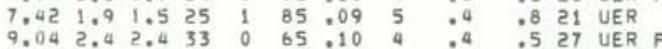
$\begin{array}{llllllllllll}6.62 & 2.1 & 1.0 & 23 & 0 & 95 & .12 & 2 & .6 & .8 & 21 & \text { MER } \\ 4.36 & 2.4 & 1.0 & 11 & 0 & 273 & .10 & 2 & 4.7 & 1.0 & 9 & \text { LER }\end{array}$ $\begin{array}{lllllllllll}6.53 & 1.3 & 16 & 0 & 97 & .10 & 5 & .6 & 1.3 & 16 & \text { UER }\end{array}$ $2.902 .125 .325 \quad 1 \quad 66: 09 \quad 3 \quad .3 \quad .520$ SPC

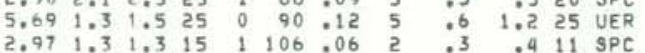

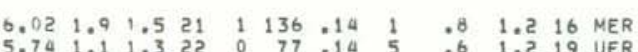
$6.311 .01 .420 \quad 0 \quad 101.11$ 4 60 .6 1.4 18 UER $\begin{array}{llllllllllllll}1.96 & 1.8 & 1.2 & 17 & 1 & 220 & : 09 & 6 & 09 & 1.2 & 9 & \text { HLP } \\ 5.15 & 1.7 & 1.2 & 15 & 1 & 139 & 09 & 2 & 0 & 1.2 & 13 & \text { UKF }\end{array}$

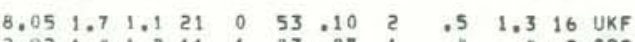

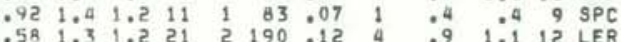

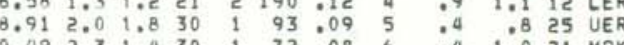

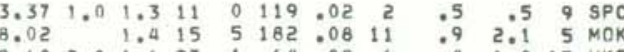

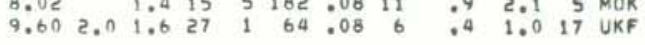

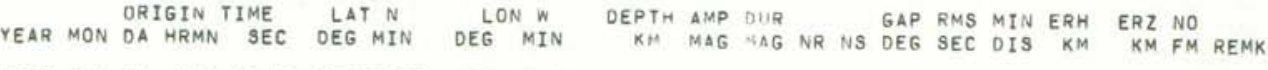

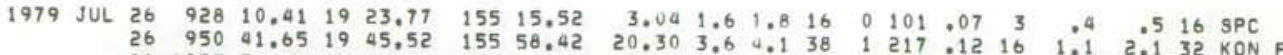

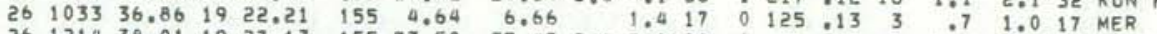

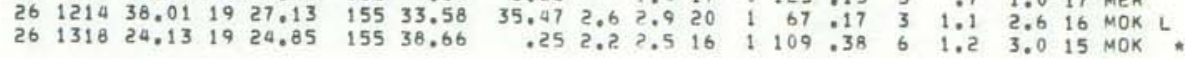

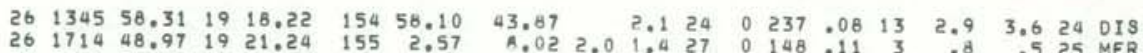

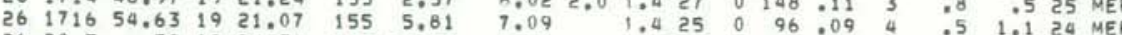

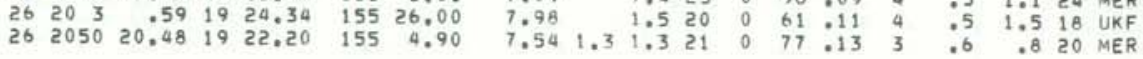

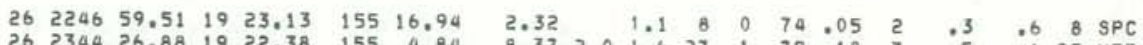

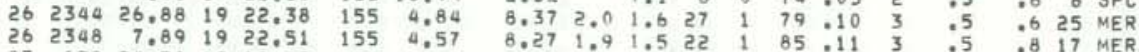

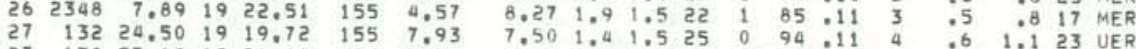

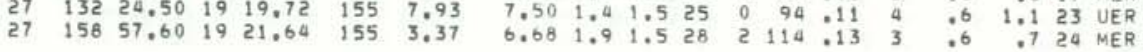

$\begin{array}{lllll}27 & 321 & 57.22 & 19 & 22.55 \quad 155 \quad 4.64\end{array}$

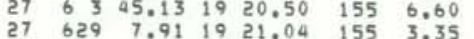

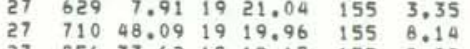
$27 \quad 856 \quad 33.62 \quad 19 \quad 19.65 \quad 155 \quad 8.22$

$\begin{array}{llllllllllll}8.65 & 2.3 & 2.3 & 30 & 1 & 83 & .10 & 3 & .5 & .5 & 28 & \text { MER }\end{array}$

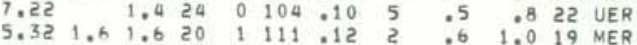

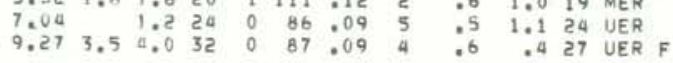

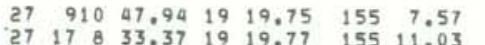

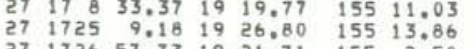

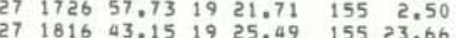
$\begin{array}{llllllllllll}6.06 & 1.3 & 18 & 0 & 102 & .09 & 5 & .6 & 1.5 & 18 & \text { UER }\end{array}$

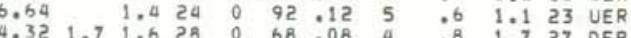

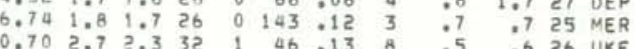

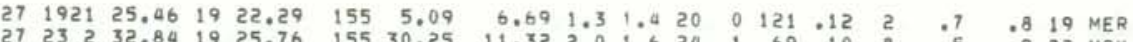

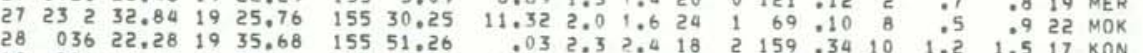

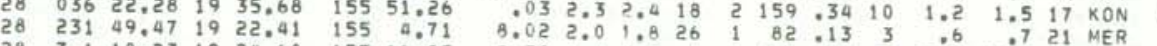

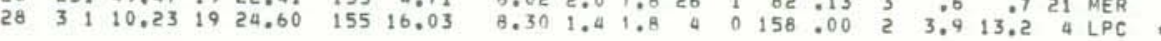

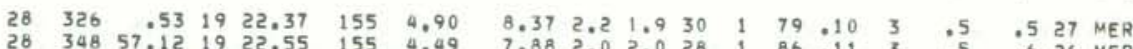

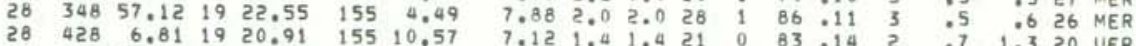

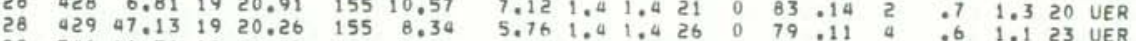
$\begin{array}{lllllllllllllllllll}28 & 741 & 46.51 & 19 & 19.73 & 155 & 8.31 & 6.81 & 1.8 & 1.8 & 25 & 0 & 85 & .09 & 4 & .5 & 1.1 & 23 & \text { UER } \\ \text { UER }\end{array}$ $\begin{array}{llllllllllllllllll}28 & 916 & 38.54 & 19 & 33.07 & 155 & 36.94 & 10.53 & 2.7 & 2.6 & 33 & 0 & 92 & .12 & 7 & .5 & .5 & 29 \mathrm{MOK}\end{array}$

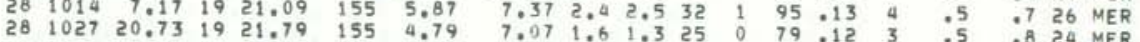

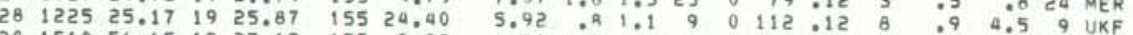

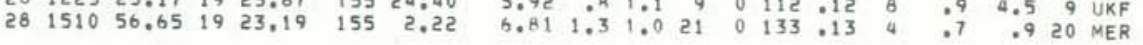

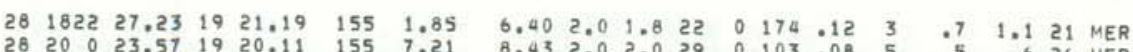

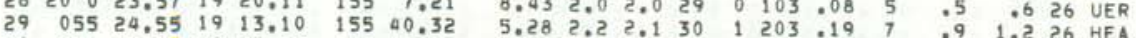

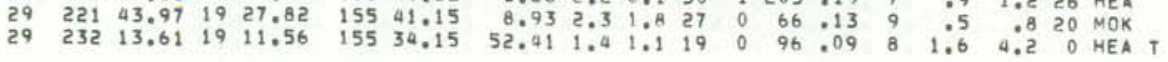

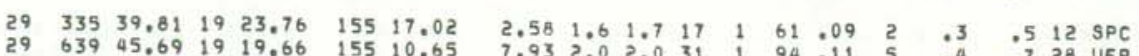

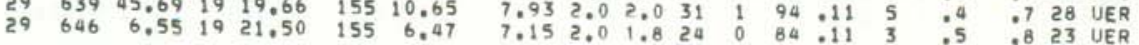


ORIGIN TIME LAT N L LON W DEPTH AMP DIR N GAP RMS MIN ERH ERZ NO
DA HRMN SEC DEG MIN DEG MIN TKM MAG MAG NR NS DEG SEC DIS KM KM FM REMK

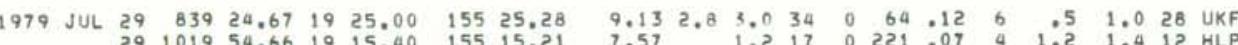

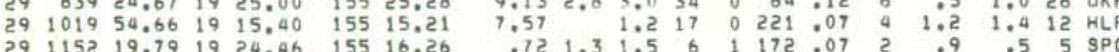

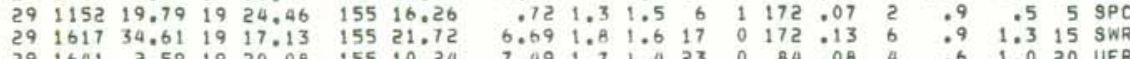

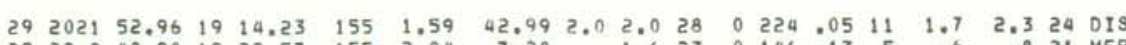

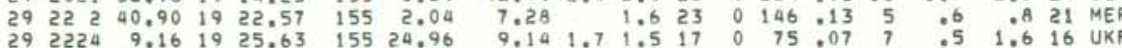

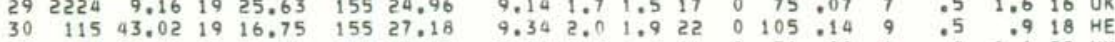

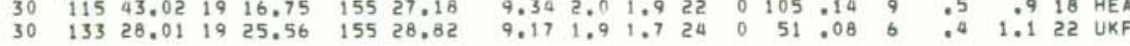

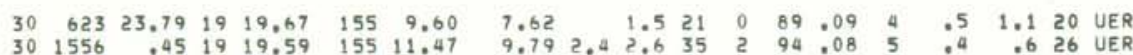

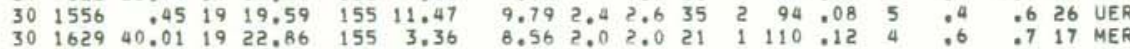

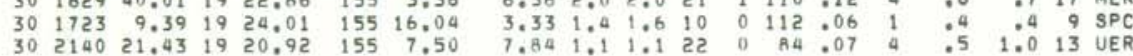

$\begin{array}{lllllllllllllllllll}31 & 330 & 51.28 & 19 & 27.98 & 155 & 25.89 & 11.65 & 4.3 & 4.2 & 42 & 1 & 40 & .11 & 5 & .4 & .4 & 40 & \text { UKF }\end{array}$

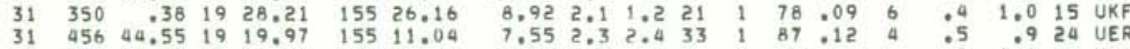

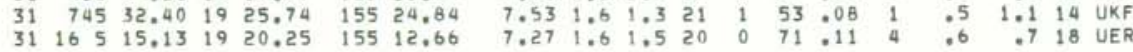
$\begin{array}{lllllllllllllllllll}31 & 1759 & 26.33 & 19 & 22.96 & 155 & 24.78 & 9.93 & 1.8 & 1.6 & 21 & 1 & 84 & .09 & 5 & .5 & 1.2 & 18 & \text { UKF }\end{array}$

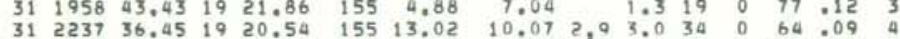
AUG 1 1 038 42.06 $1923.05 \quad 1551.88$

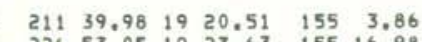
.

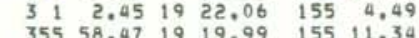

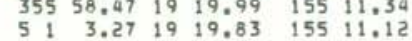
$1614 \quad 11.791923 .33 \quad 155 \quad 16.93$ 630
1 $7.351925 .97 \quad 15550.50$

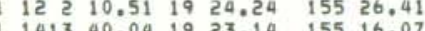
1
1
1 141736.621924 .11 155 15,91

$\begin{array}{lllllll}1 & 1434 & 47.99 & 19 & 24.03 & 155 & 15.56\end{array}$

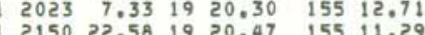

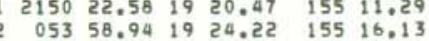
$05459,991922.25 \quad 15526.54$

$\begin{array}{lllllll}124 & 16.29 & 19 & 25.99 & 154 & 54.87\end{array}$

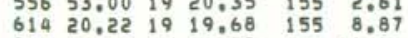
8033.70 19 $21.02 \quad 15511.06$

$\begin{array}{lllllll}2 & 944 & 15.64 & 19 & 23.78 & 155 & 15.49 \\ 2 & 958 & 49.60 & 19 & 20.49 & 155 & 12.87\end{array}$

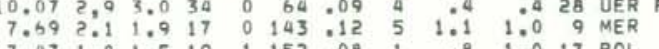

$\begin{array}{lllllllllllllll}7.62 & 2.4 & 2.6 & 24 & 0 & 107 & -10 & 2 & .6 & .6 & 17 & \text { MER } & 0\end{array}$ $9.39 \quad 2,9 \quad 3.1 \quad 33 \quad 1 \quad 85,10 \quad 4 \quad: 5 \quad .429$ MER $\begin{array}{llllllllllll}8.31 & 2.0 & 2.2 & 30 & 1 & 86 & .11 & 5 & .4 & .7 & 17 & \text { UER } \\ 6.89 & 1.2 & 17 & 0 & 90 & .12 & 5 & 06 & 1.0 & 16 & \text { UER }\end{array}$ $\begin{array}{lllllllllllllll}3.43 & 3.0 & 3.5 & 30 & 1 & 36 & .11 & 3 & .3 & .5 & 25 & \mathrm{SPC} F\end{array}$

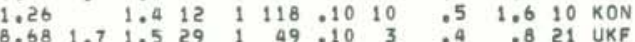

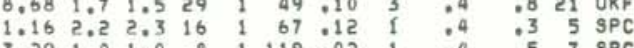

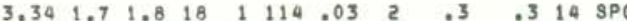

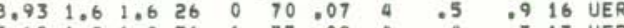

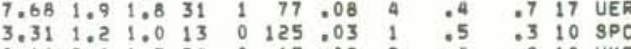

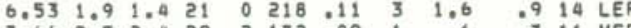

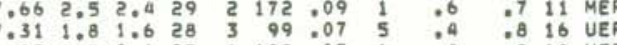

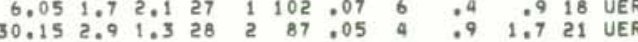
$\begin{array}{llllllllllll}3.09 & 1.5 & 1.5 & 13 & 0 & 102 & .06 & 2 & .3 & .4 & 8 & 9 P C \\ 9.30 & 2.7 & 3.2 & 37 & 1 & 66 & .10 & 4 & .3 & .5 & 26 & \text { UER }\end{array}$
ORIGIN TIME LAT N LON W DEPTH AMP OIR GAP RMS MIN ERH ERZ NO YEAR MON DA HRMN SEC DEG MIN DEG MIN KM MAG AG NR NS DEG SEC DIS KM KM FM REMK

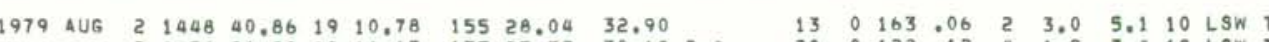

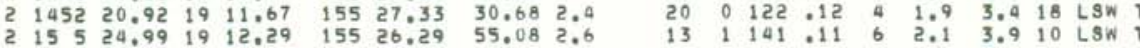

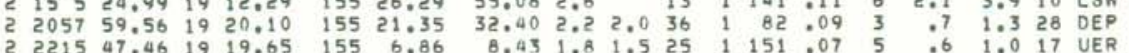

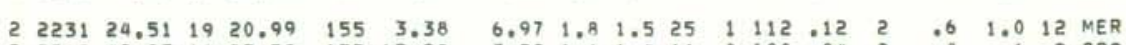

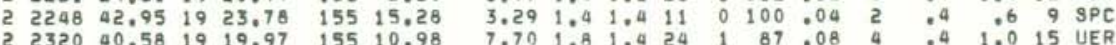

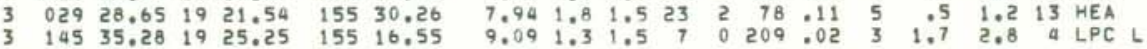

$\begin{array}{llllllllllllllllll}3 & 147 & 39.85 & 19 & 20.09 & 155 & 13.12 & 4.91 & 1.5 & 2.0 & 18 & 0 & 68 & .10 & 5 & .5 & 1.7 & 11 \\ \text { UER }\end{array}$

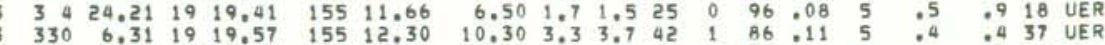

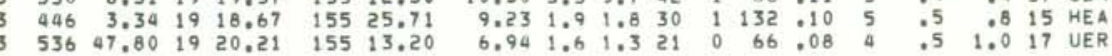

$\begin{array}{llllllllllllllllll}3 & 626 & 57.29 & 19 & 24.21 & 155 & 16.27 & 2.72 & 1.1 & 1.3 & 11 & 0 & 106 & .04 & 1 & .3 & .3 & 9 \text { SPC }\end{array}$

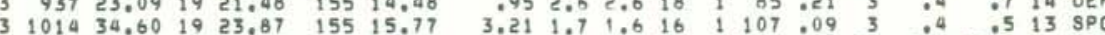

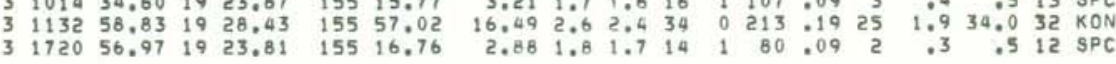

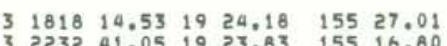
$3232525.291921 .02 \quad 155 \quad 6.14$

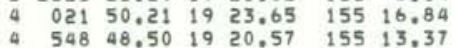
$4 \quad 1159 \quad 24,2019 \quad 19.86 \quad 155 \quad 13.46$ $41237 \quad 36,56 \quad 19 \quad 19,72 \quad 155 \quad 8,15$ $417257.741925 .58 \quad 15524.40$ $41730 \quad 36.301923 .26 \quad 15514.86$

$\begin{array}{lllll}4.1912 \quad 22.74 \quad 19 & 23.28 \quad 155 \quad 3.35\end{array}$ $4202452,161923,80 \quad 155 \quad 15,28$ $421029.121921 .69 \quad 155 \quad 6,90$ $4225545.001919 .89155 \quad 3.95$ $\begin{array}{lllllll}5 & 541 & 4,35 & 19 & 24,31 & 155 & 16,51\end{array}$ $510957.11 \quad 1920.54 \quad 155 \quad 13.02$ $5165855.811921 .23 \quad 15524.81$

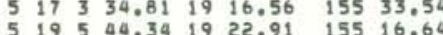

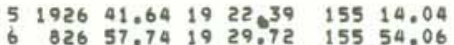
$682657.741929 .72 \quad 15554.06$ 6
6
6 $23557.591922 .61 \quad 15530.20$

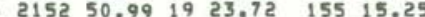
$\begin{array}{llllllll}6 & 2211 & 58.03 & 19 & 23.74 & 155 & 15.21 \\ 6 & 23 & 3 & 19.86 & 19 & 21.39 & 155 & 4.47\end{array}$ 6232238,12 19 23,92 15515,87

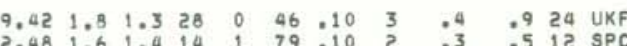

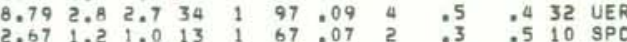

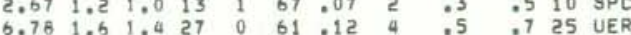

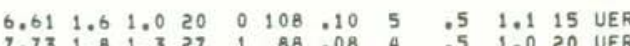

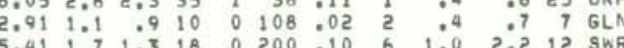
$\begin{array}{llllllllllll}9.10 & 2.6 & 2.4 & 33 & 0 & 106 & .08 & 3 & .5 & .5 & 18 & \text { MER }\end{array}$

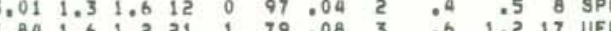

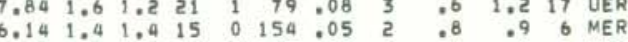

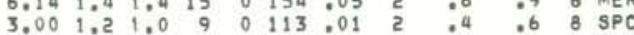

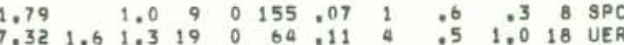

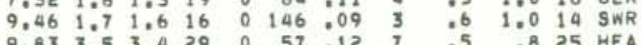

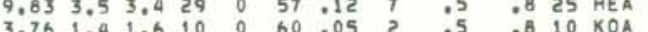

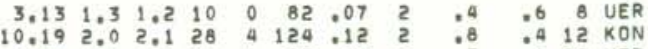

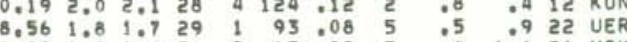

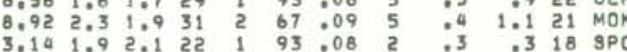
$\begin{array}{rrrrrrrrrrrr}2.93 & 1.3 & 1.4 & 12 & 0 & 96 & 05 & 2 & .3 & .5 & 8 & \text { SPC } \\ 8.37 & 2.3 & 2.4 & 33 & 1 & 85 & .08 & 4 & .4 & .7 & 20 & \text { MER }\end{array}$

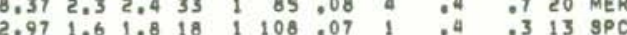

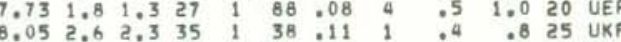


ORIGIN TIME
DA HRMN SAT N

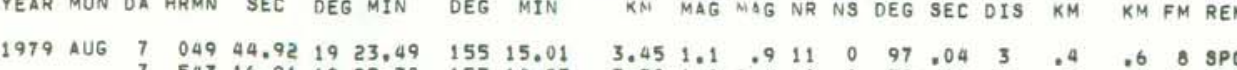

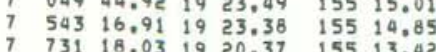

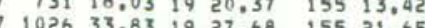
$1038 \quad 53.951920 .52 \quad 155 \quad 13.96$

$1041 \quad 54.15 \quad 1923.25 \quad 155 \quad 14.79$ $1054 \quad 7.14$
1515 $\begin{array}{llllll}7626 & 43.97 \quad 19 & 20.16 & 155 & 13.72\end{array}$

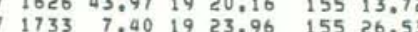

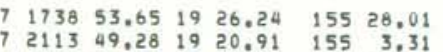

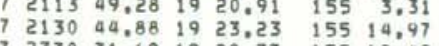
$2330 \quad 31.691920 .37 \quad 15512.49$
0.969

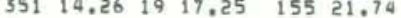

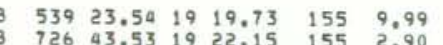
$8 \quad 91859.541922 .75155$ 2.90 8 $1010 \quad 5.93 \quad 19 \quad 20.30 \quad 155 \quad 6.43$ $8 \quad \begin{array}{llllll}826 & 12.15 & 19 & 20,69 & 155 & 8.12\end{array}$

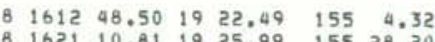

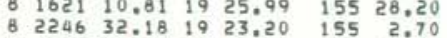

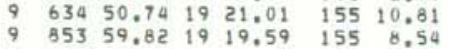

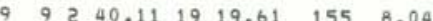
$\begin{array}{llllll}1156 & 41.48 & 19 & 20.13 & 155 & 7.52\end{array}$ 155638.7319 22.44 155 a.09 \begin{tabular}{l}
$164332.321922 .22 \quad 155 \quad 4.53$ \\
\hline
\end{tabular}

$\begin{array}{lllllll}9 & 2157 & 47.76 & 19 & 23.02 & 155 & 2.53\end{array}$

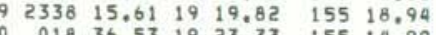
$\begin{array}{lllllll}10 & 018 & 36.53 & 19 & 23.33 & 155 & 14.99 \\ 10 & 437 & 21.08 & 19 & 17.53 & 155 & 30.74\end{array}$ $10 \quad 5841.301924 .20 \quad 15516.26$

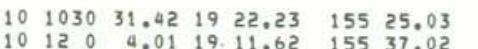

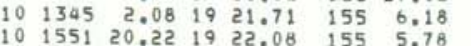

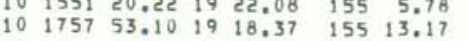

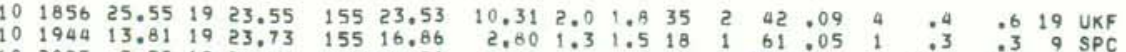

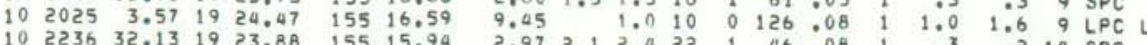

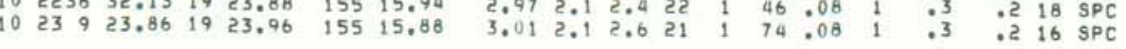

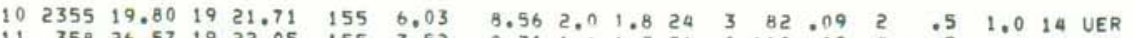

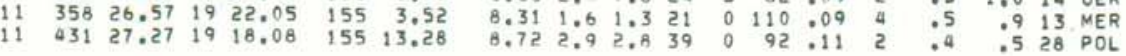

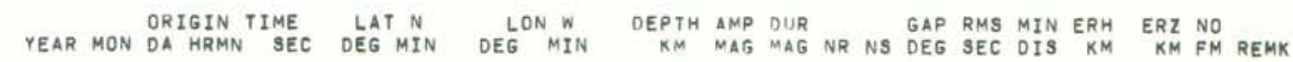

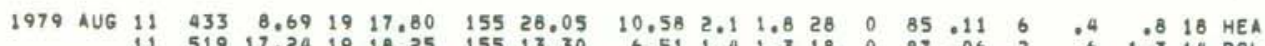

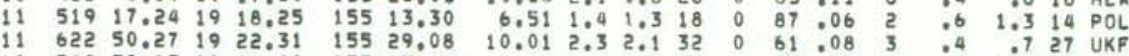

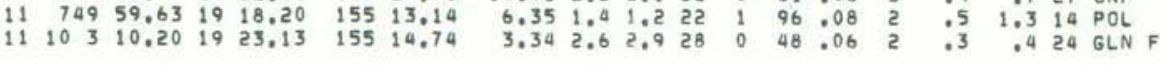

$135913.791919 .82 \quad 155 \quad 6.21$ $11 \quad 1535 \quad 27.601930 .82 \quad 155 \quad 45.21$ $11 \quad 161213,38 \quad 1928,87 \quad 154 \quad 49,82$ $112017 \quad 55.841923,98 \quad 15516,62$ $112257 \quad 20.3019 \quad 19.43 \quad 155 \quad 15.32$ 12 12 $245 \quad 47.96 \quad 19 \quad 16.48 \quad 155 \quad 13.11$ 1. $25515.971924 .27 \quad 15529.62$

$12 \quad 845 \quad 59.02 \quad 19 \quad 23.72 \quad 155 \quad 15.79$

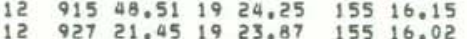
$12 \quad 931 \quad 13.931924 .12 \quad 15516.22$ $12 \quad 932 \quad 27.951923 .97 \quad 15516.04$

$\begin{array}{lllllll}12 & 935 & 4.10 & 19 & 24.03 & 155 & 16.12 \\ 12 & 936 & 13.06 & 19 & 23.89 & 155 & 16.17\end{array}$

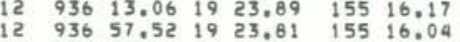

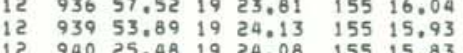

$\begin{array}{lllllll}12 & 943 & 18.91 & 19 & 23.76 & 155 & 15.97\end{array}$ $12 \quad 946 \quad 19.57 \quad 1923.97 \quad 155 \quad 16.00$ $1295220.401924,32 \quad 155$ 15.44 $\begin{array}{lllllll}12 & 958 & 27.51 & 19 & 23.75 & 155 & 15.67\end{array}$

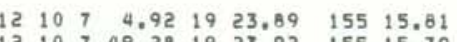
$1210855.071923 .92 \quad 15515.79$ $12101136.38 \quad 1924.09 \quad 155 \quad 15.88$ $121013 \quad 48,341924,30 \quad 155 \quad 16.27$ $\begin{array}{llllllll}12 & 1015 & 7.96 & 19 & 23.97 & 155 & 15.81\end{array}$

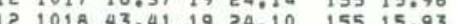
$121024 \quad 12.56 \quad 1923.93 \quad 155 \quad 15,86$

$12 \quad 1027 \quad 31.46 \quad 1923.82 \quad 155 \quad 15.69$ $121034 \quad 4.831923 .68 \quad 15515.30$ $\begin{array}{rrrrrrr}12 & 1038 & 7.50 & 19 & 23.85 & 155 & 15.52 \\ 12 & 1039 & 45.29 & 19 & 23.72 & 155 & 15.10\end{array}$

$\begin{array}{lllllll}12 & 1041 & 54.67 & 19 & 23.84 & 155 & 15.43\end{array}$

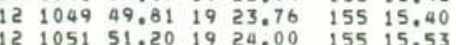

$\begin{array}{lllllllllllll}8.94 & 2.2 & 2.1 & 28 & 0 & 126 & .09 & 6 & .7 & .5 & 24 & 0 & \text { UER }\end{array}$

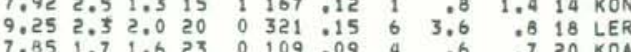

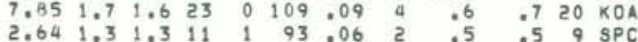

$\begin{array}{llllllllllll}6.52 & 1.4 & 1.2 & 24 & 0 & 88 & .12 & 4 & .5 & .9 & 22 & \text { KOA }\end{array}$ $\begin{array}{lllllllllll}9.32 & 2.0 & 1.8 & 29 & 0 & 50.09 & 3 & .4 & .7 & 20 & \text { UKF }\end{array}$

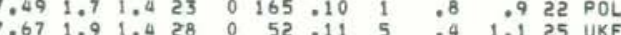

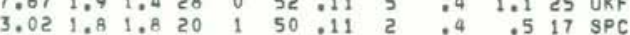
$\begin{array}{lllllllllllll}3.0 & 0 & 9 & 9 & 1 & 127 & .02 & 1 & .7 & .4 & 6 & \text { SPC }\end{array}$

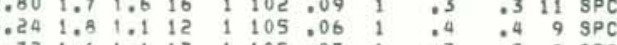
$8.89+3,3 \quad 4 \quad 13$

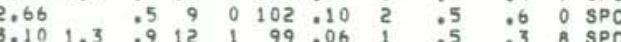

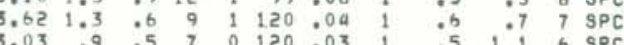
$\begin{array}{llllllllllll}2.99 & 1.3 & 15 & 0 & 99 & .10 & 3 & .4 & .5 & 0 & \mathrm{SPC}\end{array}$

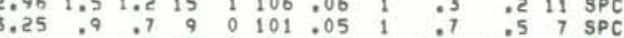

$\begin{array}{lllllllllllllll}3.03 & 1.9 & 2.0 & 23 & 1 & 74 & .09 & 1 & .3 & .2 & 18 & \mathrm{SPC}\end{array}$ 3.301 .52 .015 i $112: 06$ 1 $30.316 \mathrm{SPC}$

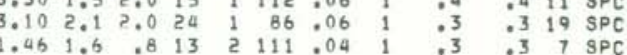

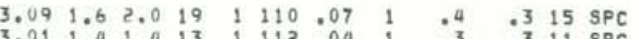

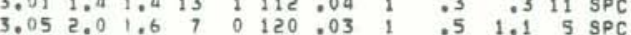

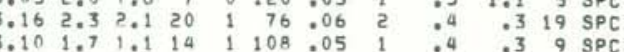
$\begin{array}{llllllllllll}3.37 & 2.2 & 2.0 & 25 & 1 & 104 & .07 & 2 & .3 & .3 & 20 & \text { SPC }\end{array}$

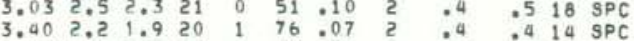
$\begin{array}{lllllllllllllll}3.06 & 1.7 & 1.6 & 15 & 0 & 103 & .04 & 2 & .3 & .3 & 11 & \text { SPC }\end{array}$

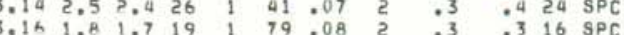

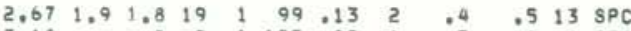

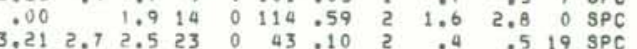
$\begin{array}{llllllllllll}3.12 & 1.6 & 1.3 & 18 & 1 & 76 & .06 & 2 & .3 & .3 & 14 & \mathrm{SPC} \\ 3.34 & 2.2 & 1.9 & 19 & 0 & 90 & .07 & 2 & .3 & .3 & 17 & \mathrm{SPC}\end{array}$ 


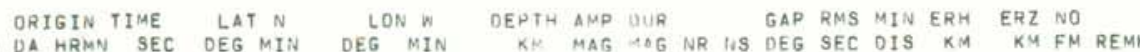

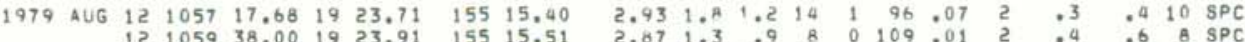
$12105938.001923 .91 \quad 15515.51$ $12 \quad 105955.17 \quad 1923.31 \quad 15514.86$

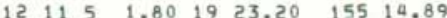
$\begin{array}{rrrrrrr}12 & 1110 & 28.58 & 19 & 24.35 & 155 & 16.38 \\ 12 & 1116 & 3.83 & 19 & 24.08 & 155 & 15.83\end{array}$

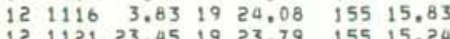
12 12 $112251.961923,38 \quad 15515.07$

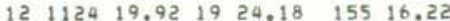
$\begin{array}{llllll}12 & 1125 & 2.63 & 19 & 23.93 \quad 155 & 15.47\end{array}$ $\begin{array}{lllllll}12 & 1127 & 26.99 & 19 & 23.46 & 155 & 14.96\end{array}$ $\begin{array}{lllll}12 & 1133 & 43.22 & 19 & 23.55 \\ 12 & 155 & 15.06\end{array}$ $\begin{array}{llllllll}12 & 1135 & 10.85 & 19 & 23.93 & 155 & 15.38\end{array}$ $12 \quad 1136 \quad 48.51 \quad 1924.79 \quad 155 \quad 16.68$

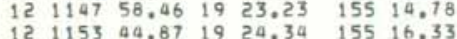
$\begin{array}{lll}12 & 12752,86 \quad 19 \quad 23,97 \quad 155 \quad 15.89\end{array}$ $12129 \quad 1.691923,28 \quad 15514,92$

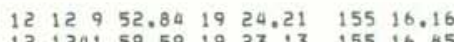
$\begin{array}{lllllll}12 & 1241 & 59.59 & 19 & 23.13 & 155 & 16.85 \\ 12 & 1258 & 50.42 & 19 & 24.04 & 155 & 15.84\end{array}$ $\begin{array}{lllllll}12 & 1531 & 23.85 & 19 & 24.47 & 155 & 15.88 \\ 12 & 1540 & 31.72 & 19 & 23,33 & 155 & 15.00\end{array}$

$\begin{array}{llllllll}12 & 16 & 0 & 30.58 & 19 & 23.30 & 155 & 14.95\end{array}$ $\begin{array}{lllll} & \end{array}$ $\begin{array}{lllllll}12 & 1758 & 17.22 & 19 & 18.53 & 155 & 28.57\end{array}$

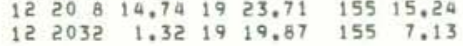
$\begin{array}{lllllll}12 & 2143 & 42.06 & 19 & 23.75 & 155 & 15.41\end{array}$

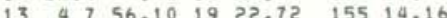
$\begin{array}{llllllll}13 & 6 & 3 & 40.61 & 19 & 17.80 & 155 & 15.54\end{array}$ $\begin{array}{lllllll}13 & 641 & 34.32 & 19 & 16.64 & 155 & 15.49\end{array}$

$\begin{array}{lllllll}13 & 722 & 00.28 & 19 & 16.92 & 155 & 15.64\end{array}$

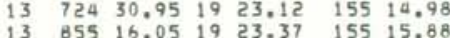

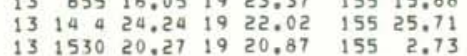
$\begin{array}{llllllll}13 & 1744 & 10.77 & 19 & 23.13 & 155 & 14.79\end{array}$ $\begin{array}{lllllll}13 & 1745 & 3.19 & 19 & 23.81 & 155 & 14.69\end{array}$ $\begin{array}{llllllll}13 & 1755 & 43.31 & 19 & 24.21 & 155 & 16.50\end{array}$ 13 1915 11.03 19 21.82 15513.23

$\begin{array}{lllllllllllllllllll}13 & 2135 & 9.58 & 19 & 21.66 & 155 & 4.18 & 7.02 & 1.6 & 1.2 & 20 & 0 & 90 & .12 & 4 & .6 & .9 & 18 & \text { MER }\end{array}$

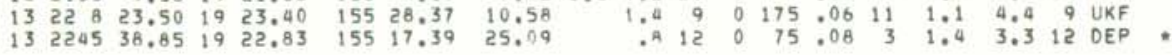

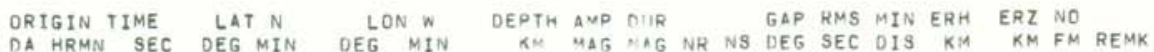

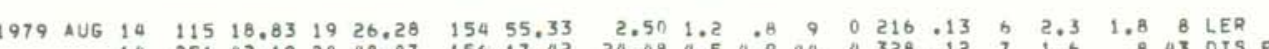

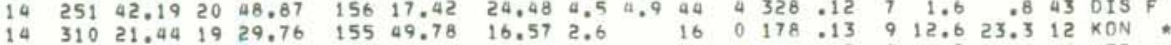

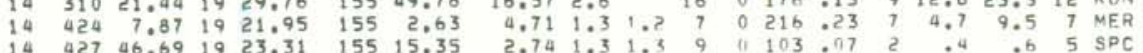

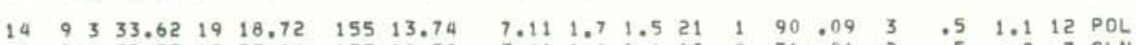

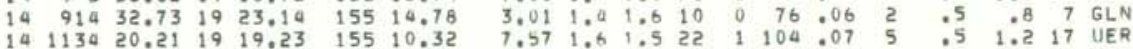

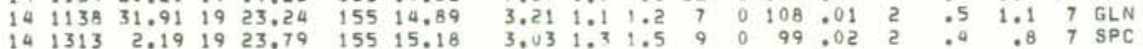

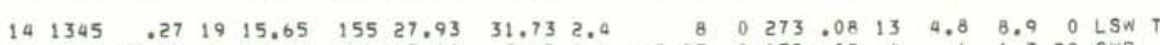

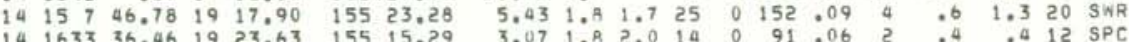

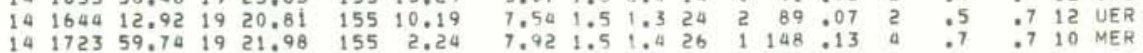

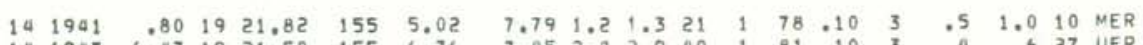

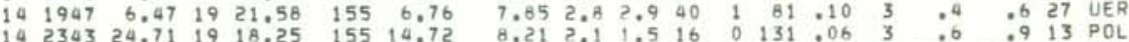

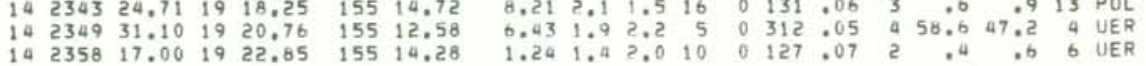

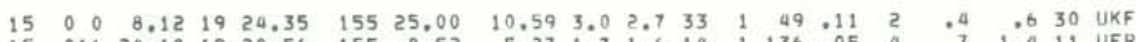

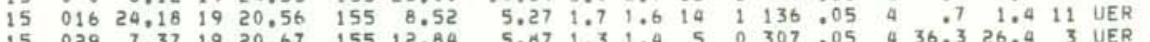

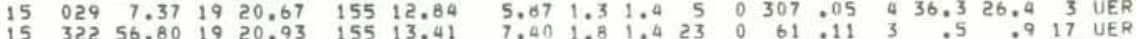

$15 \quad 410 \quad 36,08 \quad 19 \quad 23,39 \quad 155 \quad 14.95$ $15 \quad 554 \quad 05.49 \quad 1920.19 \quad 155 \quad 14.95$ $15 \quad 1038 \quad 58,14 \quad 1923.25 \quad 15514.92$

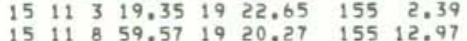

$\begin{array}{lllll}15 & 1124 & .62 & 19 & 23.02 \quad 155 \quad 14.68\end{array}$

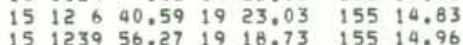

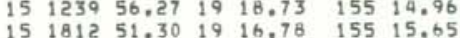

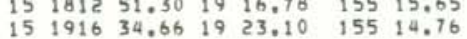

$151916 \quad 59.16 \quad 1920.41 \quad 15510.90$

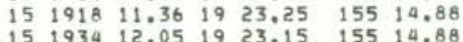

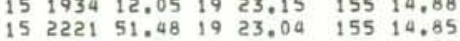
$15231514,931922,96 \quad 15514,73$

$\begin{array}{rrrrrrr}16 & 021 & 2.29 & 19 & 20.25 & 155 & 8.31 \\ 16 & 038 & 40.70 & 19 & 22.99 & 155 & 14.84\end{array}$ 16 2 $09.841925 .23 \quad 15456.24$

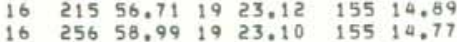

$\begin{array}{lllllll}16 & 330 & 4.74 & 19 & 23.10 & 155 & 14.82\end{array}$

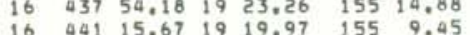

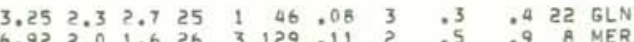

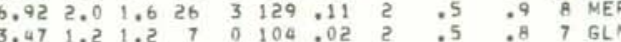

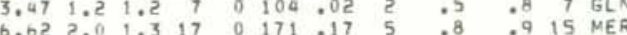

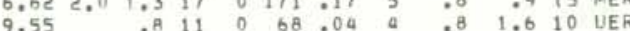

$\begin{array}{lllllllllllll}3.11 & 1.8 & 2.0 & 16 & 0 & 64 & .08 & 2 & .4 & .5 & 14 & 6 L N\end{array}$

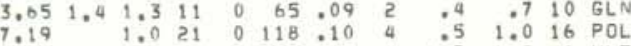

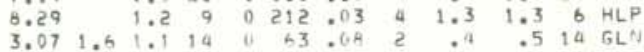

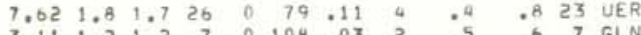

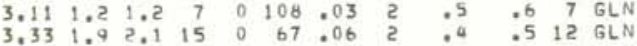

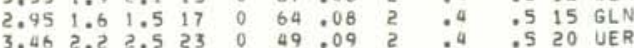

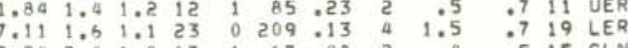

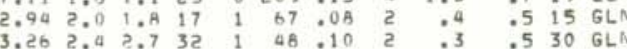

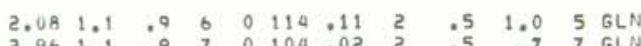
$\begin{array}{llllllllllll}2.96 & 1.1 & .9 & 7 & 0 & 104 & .02 & 2 & .5 & .7 & 7 & 6 L N \\ 7.34 & 1.8 & 1.2 & 24 & 0 & 82 & 08 & 4 & .0 & .8 & 19 & \text { UER }\end{array}$

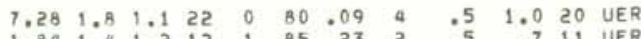


HVO EARTHQUAKE SUMMARY LIST

ORIGIN TIME LAT N LON W DEPTH AMP OIR T GAP RMS MIN ERH ERZ NO YEAR MON DA HAMN SEC DEG MIN DEG MIN TEM MAG MAG NR NS DEG SEC DIS KM KM F FM REMK

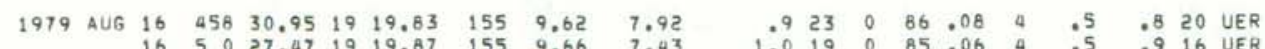

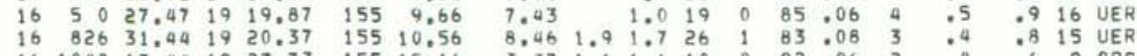

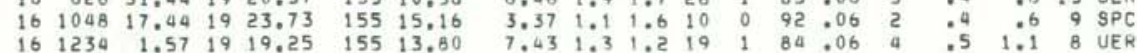

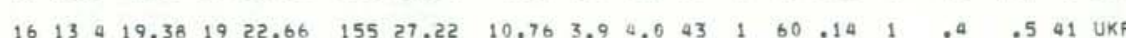

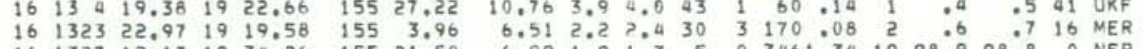

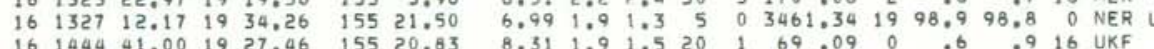

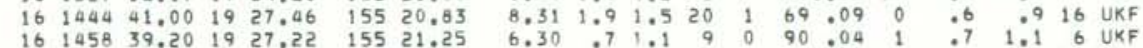

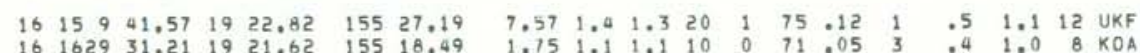

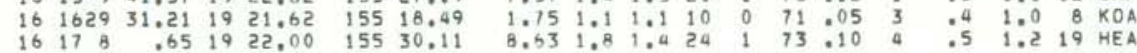

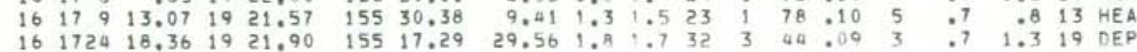

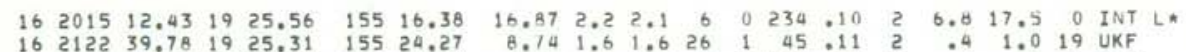

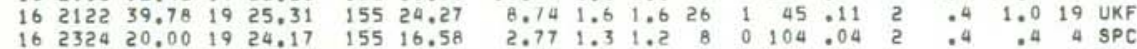

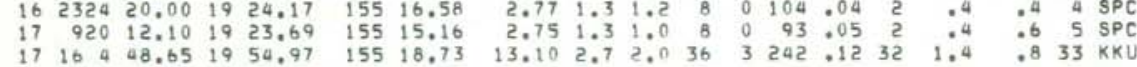

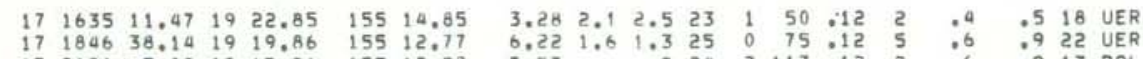

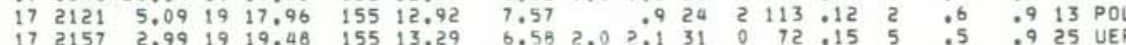

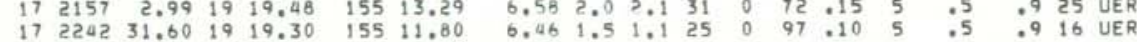

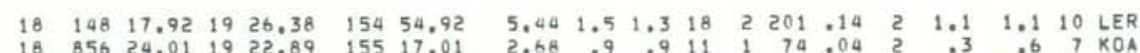

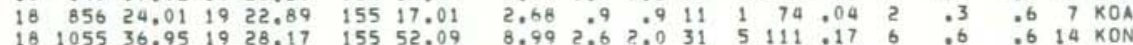

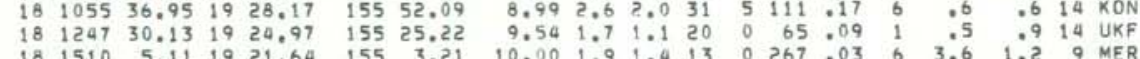

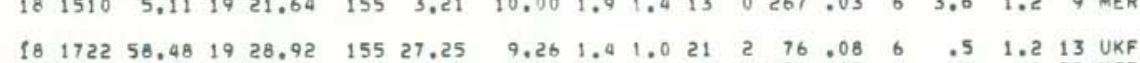

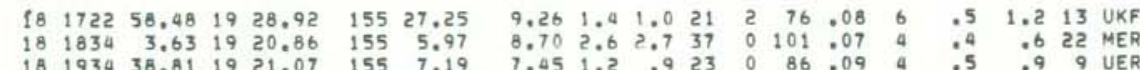

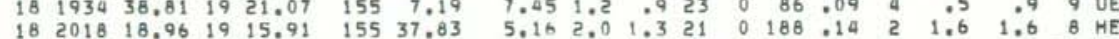

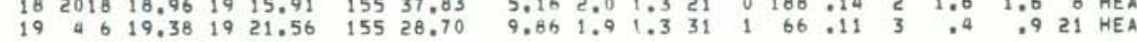

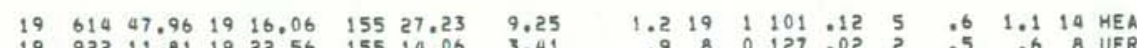

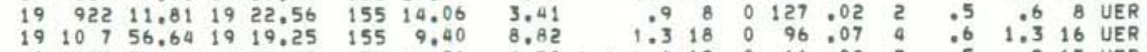

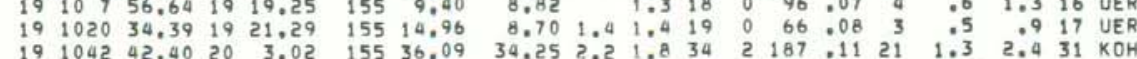

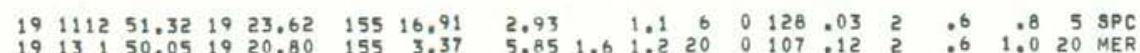

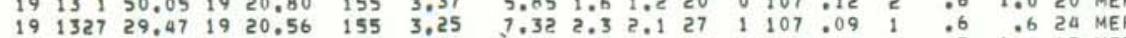

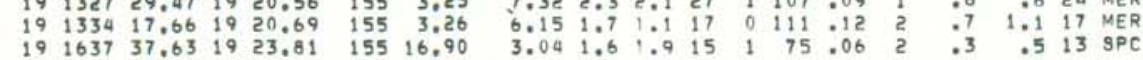

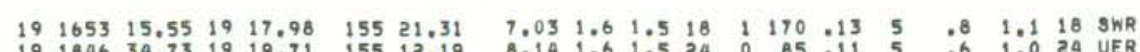

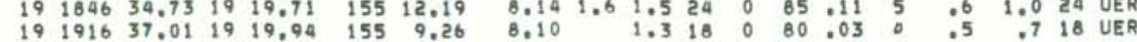

HVO EARTHOUAKE SUMMARY LIST

PAGE 60

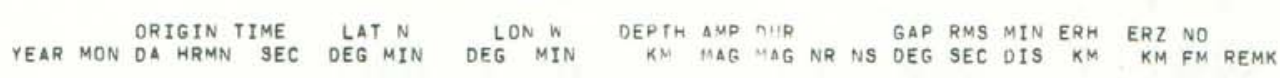

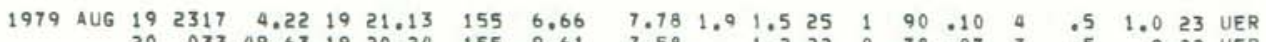

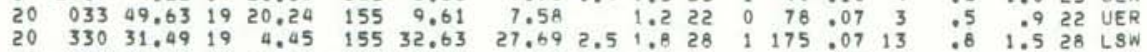

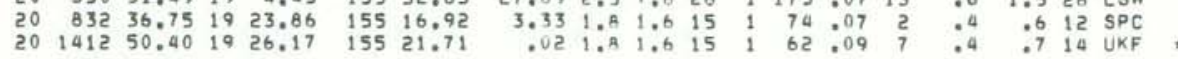

$\begin{array}{lllllll}20 & 1723 & 11.75 & 19 & 22.10 & 155 & 3.67\end{array}$ 20185516.081921 .60 155 3.72

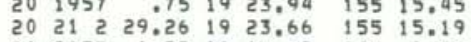

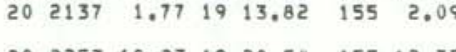
$\begin{array}{lrrrrrr}20 & 2253 & 18.23 & 19 & 20.54 & 155 & 12.75 \\ 21 & 416 & 3.53 & 19 & 22.27 & 155 & 4.52\end{array}$ $21 \quad 43350.05 \quad 1922.00 \quad 155 \quad 2,37$

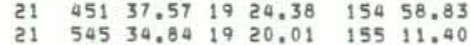

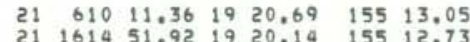
$\begin{array}{lllllll}21 & 1614 & 51.92 & 19 & 20.14 & 155 & 12.73 \\ 21 & 1745 & 28.81 & 19 & 20.25 & 155 & 22.67\end{array}$ $21 \quad 225940.6019 \quad 19,75 \quad 15511.23$ $21234257,57 \quad 19 \quad 18,60 \quad 155 \quad 13,89$ $\begin{array}{lllllll}22 & 0 & 77.26 & 19 & 23.81 & 155 & 15.24 \\ 22 & 138 & 55.12 & 19 & 23.52 & 155 & 16.83\end{array}$

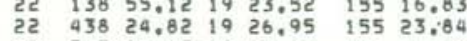
$\begin{array}{lllllll}22 & 513 & 16.43 & 19 & 23.16 & 155 & 14.86 \\ 22 & 521 & 28.37 & 19 & 23.59 & 155 & 16.74\end{array}$ $\begin{array}{lllllll}22 & 745 & 36.36 & 19 & 19.62 & 155 & 10.21\end{array}$ 22 13 . $2216250.36 \quad 1923.45 \quad 15514,79$ $\begin{array}{lllllll}22 & 1628 & 46.56 & 20 & 3.77 & 155 & 58.35\end{array}$ $\begin{array}{lllllll}22 & 1657 & 24.56 & 19 & 20.27 & 155 & 8.55\end{array}$

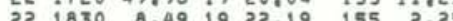

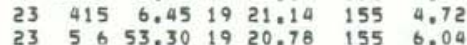
$\begin{array}{lllllll}23 & 814 & 28.39 & 19 & 19.94 & 155 & 7.53\end{array}$ $23113659.321920 .21 \quad 155 \quad 7.20$

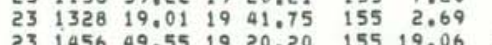
$\begin{array}{llllllll}23 & 21 & 0 & 12.56 & 19 & 23.16 & 155 & 14.91\end{array}$ $\begin{array}{llllllll}23 & 210 & 12.56 & 19 & 23.16 & 155 & 14.91 \\ 23 & 2238 & 8.48 & 19 & 20.48 & 155 & 3.88\end{array}$ $24 \quad 121 \quad 19,50 \quad 1923,70 \quad 155 \quad 1.45$

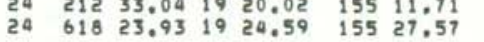
$\begin{array}{rrrrrrr}24 & 824 & 32.51 & 19 & 20.71 & 155 & 6.67 \\ 24 & 1822 & 20.34 & 19 & 19.11 & 155 & 16.19 \\ 24 & 1924 & 36.80 & 19 & 19,94 & 155 & 11.34\end{array}$

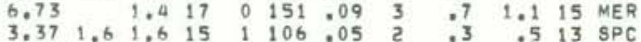

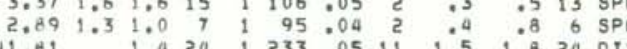

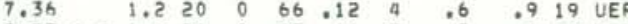

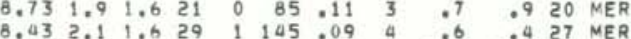

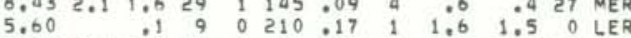

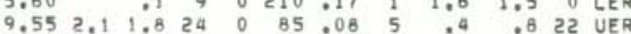

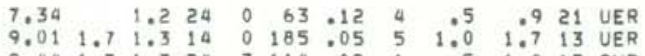
$\begin{array}{lllllllllll}8.71 & 1.7 & 1.5 & 14 & 0 & 205 & .03 & 7 & 1.1 & 1.6 & 12\end{array}$ $\begin{array}{rrrrrrrrrrrr}2.9 A & 1.0 & 1.1 & 8 & 0 & 101 & 02 & 2 & .5 & .7 & 6 & \mathrm{SPC} \\ 2.89 & 1.4 & 1.3 & 17 & 1 & 48 & 07 & 0 & : 5 & .3 & 8 & \mathrm{SPC}\end{array}$

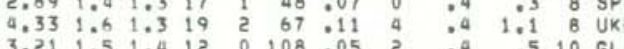

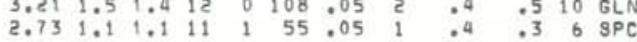

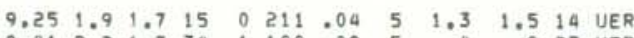

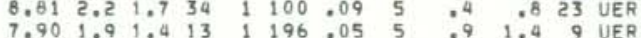

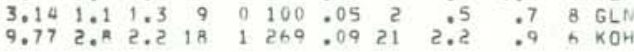
$\begin{array}{llllllllllll}8.40 & 1.8 & 1.5 & 17 & 2 & 214 & 05 & 4 & .9 & 1.1 & 11 & \text { UER } \\ 9.23 & .5 & 1.5 & 17 & 0 & 220 & 03 & 4 & 1.3 & 1.6 & 10 & \text { UER }\end{array}$

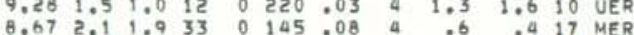

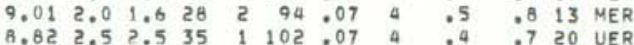
$\begin{array}{lllllllllll}6.38 & 2.2 & 1.3 & 12 & 1 & 202 & 06 & 6 & 1.2 & 1.7 & 9 \text { UER }\end{array}$

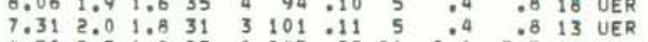

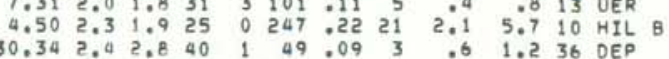
$\begin{array}{llllllllllll}3.70 & 1.4 & 1.2 & 11 & 0 & 108 & .05 & 2 & .4 & .7 & 8 & \text { GLN } \\ 6.85 & 1.8 & 1.4 & 29 & 3 & 110 & .11 & 2 & .5 & 07 & 12 & \text { MER }\end{array}$

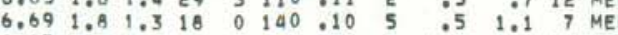
$\begin{array}{llllllllllll}8.67 & 1.7 & 1.6 & 16 & 0 & 194 & 06 & 5 & 1.0 & 1.4 & 19 & \text { UER } \\ 1.49 & 2.1 & 1.4 & 12 & 0 & 251 & .06 & 4 & 4.4 & 5.1 & 10 & \text { UKF }\end{array}$ $\begin{array}{llllllllllllll}7.66 & 1.7 & 1.1 & 24 & 3 & 98 & .10 & 6 & .5 & 1.0 & 12 & \text { UER }\end{array}$ $\begin{array}{lllllllllllll}8.19 & 1.9 & 1.8 & 35 & 2 & 116 & 06 & 3 & 04 & .6 & 18 & \text { KOA } \\ 9.27 & 1.9 & 1.3 & 17 & 0 & 198 & 06 & 5 & 1.1 & 1.4 & 12 & \text { UER }\end{array}$ $\begin{array}{lllllllllllll}0.25 & 1.9 & 1.2 & 10 & 0 & 108 & .13 & 4 & .8 & 1.7 & 10 & \text { MER }\end{array}$

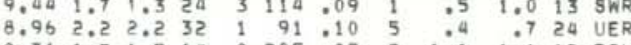


ORIGIN TIME LAT N LON W OEPTH AMP RIR N N GAP RMS MIN ERH ERZ NO
DA HRMN SEC DEG MIN OEG MIN
KM MAG MAG NR NS OEG SEC OIS KM KM FM REMK 1979 AUG $24 \quad 2240 \quad 46.53 \quad 1923.42 \quad 155 \quad 14.74$

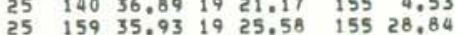

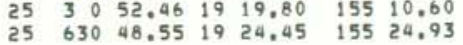
$25 \quad 920 \quad 37.32 \quad 1920.01 \quad 15511.80$ $\begin{array}{lllllll}25 & 1127 & 25.65 & 19 & 23.54 & 155 & 16.95\end{array}$ $2514119.671923 .10 \quad 15457.78$

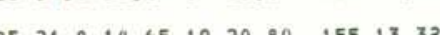

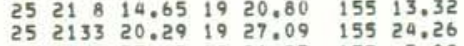
$\begin{array}{lllllll}25 & 2317 & 53.17 & 19 & 21.97 & 155 & 5.02\end{array}$

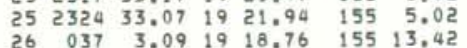

$\begin{array}{lllllll}26 & 626 & 4.85 & 19 & 31.14 & 155 & 40.84\end{array}$ $26 \quad 832 \quad 12.66$ 19 $19.11 \quad 15515.39$

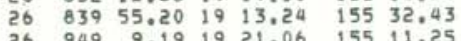

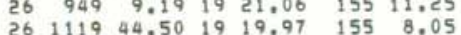

$\begin{array}{lllllll}26 & 1222 & 47.18 & 19 & 22.29 & 155 & 4.20\end{array}$ $\begin{array}{lllllll}26 & 1414 & 36.80 & 19 & 28.11 & 155 & 25.33\end{array}$

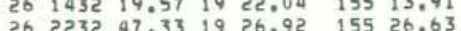
$\begin{array}{lllllll}26 & 2232 & 47.33 & 19 & 26.92 & 155 & 26.63 \\ 26 & 2321 & 37.64 & 19 & 20.64 & 155 & 6.58\end{array}$

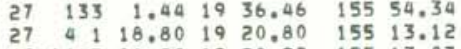

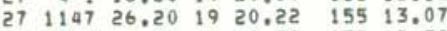
$27 \quad 144223.051919 .59 \quad 15512.38$ $\begin{array}{lllllll}27 & 1650 & 35.02 & 19 & 21.25 & 155 & 3.55 \\ 27 & 21.57 & 1.023 & 19 & 23.50 & 155 & 23.67\end{array}$ $\begin{array}{lllllll}27 & 2117 & 14.23 & 19 & 23.50 & 155 & 23.67 \\ 27 & 22 & 5 & 24.29 & 19 & 21.64 & 155 \\ 13.16\end{array}$

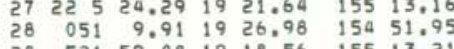
$\begin{array}{lllllll}28 & 521 & 59.08 & 19 & 18.56 & 155 & 13.21\end{array}$

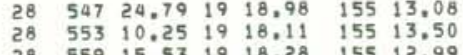

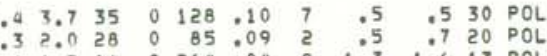

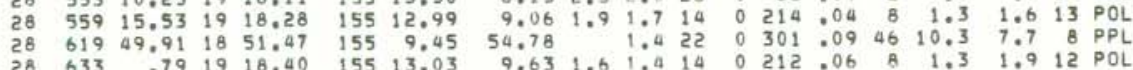
$\begin{array}{llllllllllllllllllll}28 & 654 & 14.06 & 19 & 18.54 & 155 & 13.25 & 8.38 & 1.5 & 1.4 & 13 & 0 & 209 & .02 & 8 & 1.2 & 1.9 & 12 & \mathrm{POL}\end{array}$

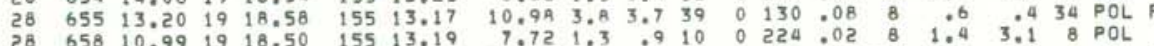

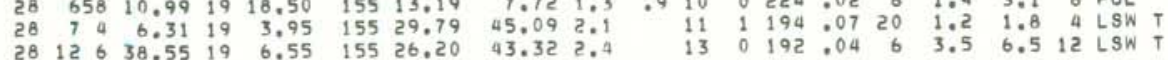

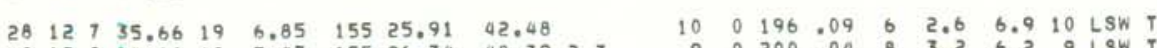

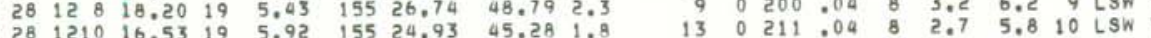

$\begin{array}{lllllllllllllll}3.72 & 1.6 & 1.5 & 15 & 0 & 76 & 07 & 2 & .4 & .5 & 14 & \mathrm{GLN}\end{array}$

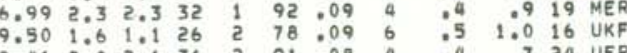
$\begin{array}{lllllllllllll}8.41 & 2.0 & 2.1 & 31 & 2 & 91 & .08 & 4 & .4 & .7 & 24 & \text { UER } \\ 9.60 & 1.4 & 1.1 & 24 & 1 & 92 & 08 & 2 & .5 & .8 & 10 & \text { UKF }\end{array}$

$\begin{array}{lllllllllllll}9.86 & 2.5 & 2.5 & 30 & 1 & 82 & .07 & 5 & .4 & .6 & 22 & \text { UER }\end{array}$

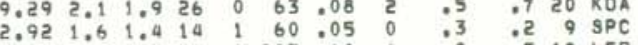

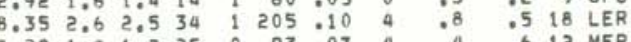
$9.66 \quad 3.3 \quad 3.141 \quad 1 \quad 58,10 \quad 3 \quad .3 \quad .433$ UER F

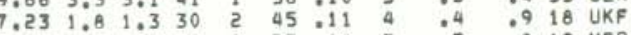
$\begin{array}{llllllllllll}8.15 & 1.7 & 1.3 & 28 & 2 & 75 & 10 & 3 & .5 & .8 & 19 & \text { MEF } \\ 7.46 & 1.6 & 0.0 & 27 & 1 & 77 & 13 & 3 & .5 & 1.0 & 18 & \text { MER }\end{array}$

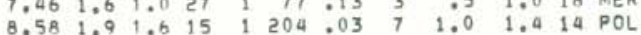

\begin{tabular}{lllllllllll}
9.78 & 1.6 & 1.1 & 24 & 2 & 79 & .09 & 9 & .5 & 1.2 & 16 \\
\hline $0 K$
\end{tabular}

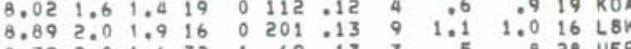

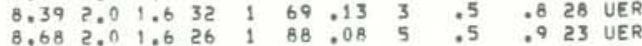
$\begin{array}{llllllllllll}8.22 & 1.9 & 1.4 & 23 & 1 & 92 & .12 & 4 & .5 & .7 & 22 & \text { MER } \\ 7.61 & 2.2 & -5 & 21 & 1 & 54 & .09 & 5 & : 4 & 1.1 & 19 & \text { UKF }\end{array}$

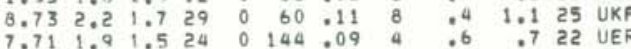

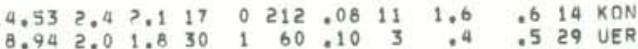

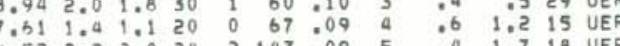

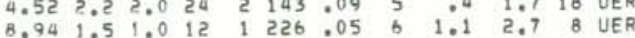

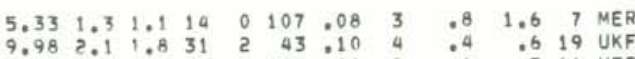

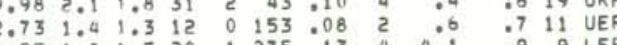

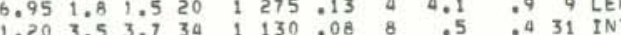
1.551 .61 .9012 0 $86.06 \quad 2 \quad .3 \quad 0.511$ UER GAP RMS MIN ERH ERZ NO YEAR MON DA HRMN SEC DEG MIN DEG MIN KM MAG MAG NR NG DAPG REC MIN ERH ERZ NOM

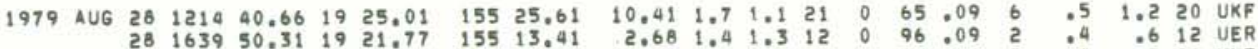

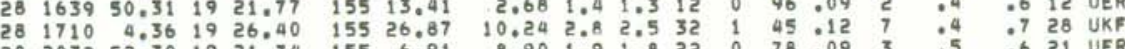

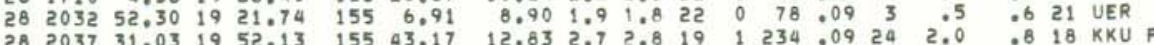

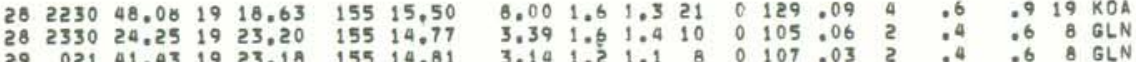

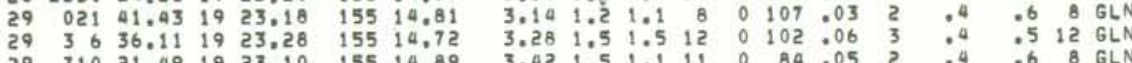

$\begin{array}{llllllllllllllllllll}29 & 1153 & 29.90 & 19 & 44.00 & 155 & 19.73 & 27.20 & 2.7 & 2.1 & 33 & 2 & 97 & 010 & 15 & .7 & 1.8 & 31 & \mathrm{KKU} & \end{array}$

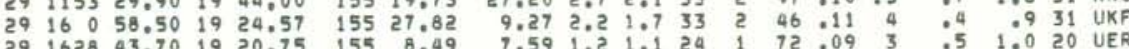

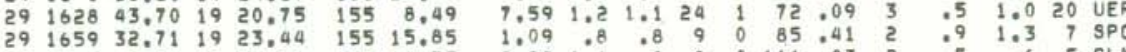

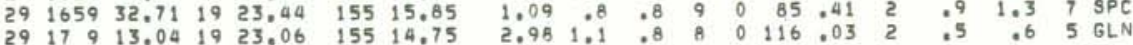

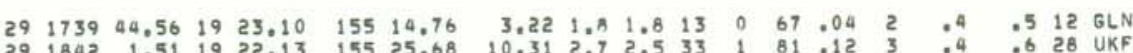

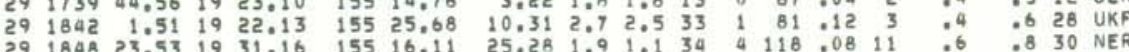

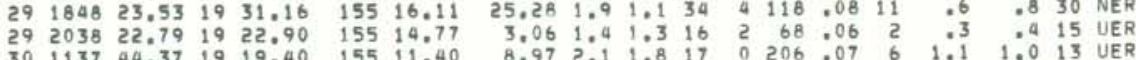

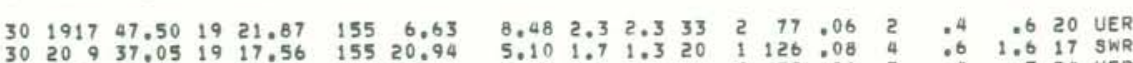

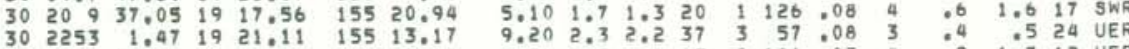

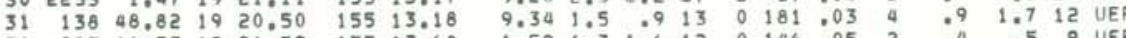

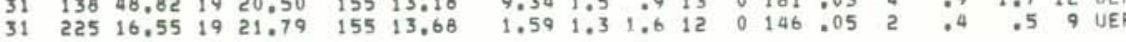

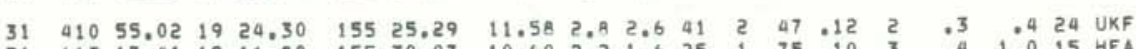

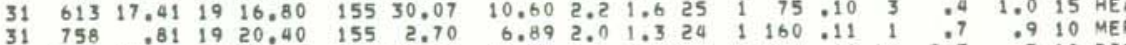

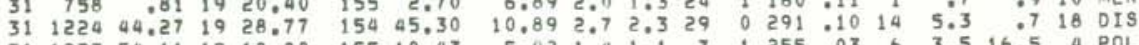

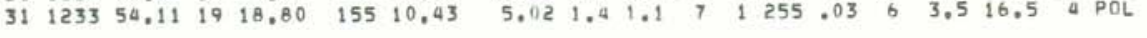
$\begin{array}{lllllllllllllllllll}31 & 1314 & 5.88 & 19 & 20.24 & 155 & 2.55 & 7.83 & 2.6 & 2.6 & 33 & 3 & 185 & 09 & 1 & .6 & .5 & 17 & \text { MER } \\ 31 & 1547 & 53.81 & 19 & 24.92 & 155 & 24.55 & 9.50 & 1.9 & 1.4 & 25 & 0 & 38 & .09 & 1 & .4 & .8 & 18 & \text { UKF }\end{array}$

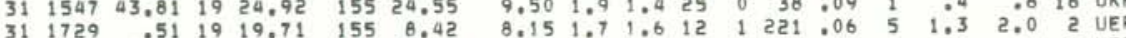

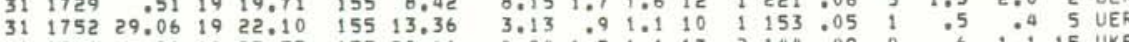

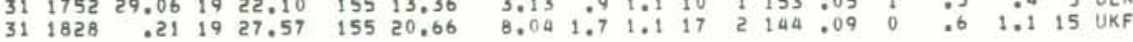
$\begin{array}{lllllllllllllllllll}31 & 2216 & 58.49 & 19 & 27.38 & 155 & 20.56 & 7.79 & 1.9 & 1.4 & 18 & 2 & 132 & .08 & 7 & .6 & 1.7 & 11 & \text { UKF }\end{array}$

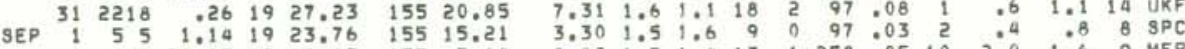

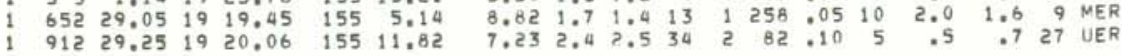

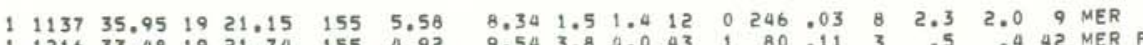

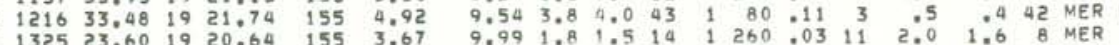

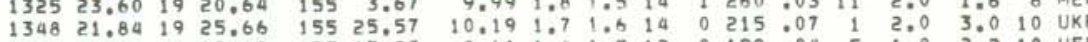

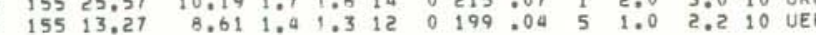

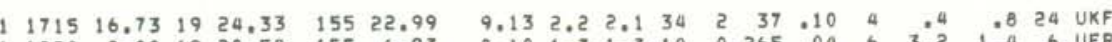

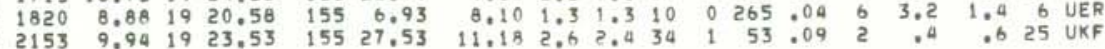


ORIGIN TIME LAT N LON W DEPTH AMP OUR GAP RMS MIN ERH ERZ NO YEAR MON DA HRMN SEC DEG MIN DEG MIN NN MAG ARG NR NS DEG SEC OIS KM KM FM REMK

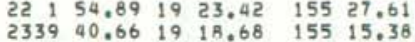

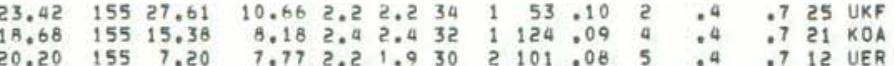
$1123 \quad 29.331920 .34 \quad 15510.55$

$21444 \quad 15.07 \quad 19 \quad 19.39 \quad 155 \quad 10.19$

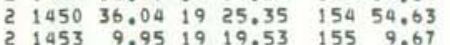

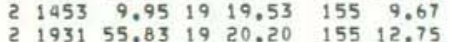
$\begin{array}{lrrrrrr}2 & 1931 & 55.83 & 19 & 20.20 & 155 & 12.75 \\ 3 & 127 & 12.58 & 19 & 19.50 & 155 & 10.43\end{array}$

$\begin{array}{lllllll}3 & 152 & 47.37 & 19 & 17.87 & 155 & 14,10\end{array}$ $\begin{array}{lllllll}3 & 157 & 13.84 & 19 & 26.87 & 155 & 29.54 \\ 3 & 254 & 25.91 & 19 & 21.68 & 155 & 6.13\end{array}$

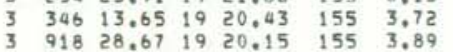

$\begin{array}{llllll}3 & 1343 \quad 38.91 & 19 & 19.37 & 155 & 11.52\end{array}$

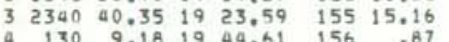

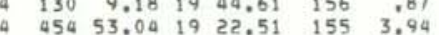
\begin{tabular}{l}
$62212.8918 \quad 37.78 \quad 155 \quad 7.51$ \\
\hline
\end{tabular}

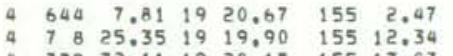

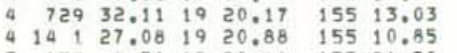

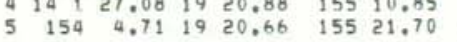

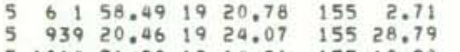
$\begin{array}{lllllll}5 & 1014 & 36.20 & 19 & 14.21 & 155 & 10.90 \\ 5 & 1125 & 50.26 & 19 & 19.66 & 155 & 10.86\end{array}$

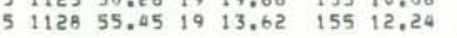
$\begin{array}{lllllll}5 & 14 & 72.28 & 19 & 19.35 & 155 & 11.77 \\ 5 & 1631 & 13.01 & 19 & 26.90 & 155 & 24.71\end{array}$ $51835 \quad 19.81 \quad 19 \quad 29.54 \quad 155 \quad 11.80$ $\begin{array}{lllllll}5 & 2233 & 7.50 & 19 & 19.03 & 155 & 13.44\end{array}$ $\begin{array}{lllllll}5 & 2316 & 56.16 & 19 & 27.19 & 155 & 23.32\end{array}$

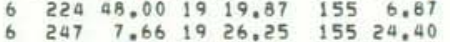

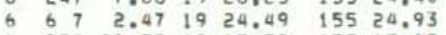

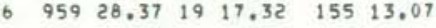
$\begin{array}{llllllll}6 & 1136 & 45.01 & 19 & 19.82 & 155 & 12.89\end{array}$

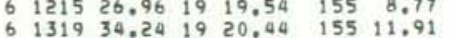
$\begin{array}{lllllll}6 & 1610 & 38.51 & 19 & 20.00 & 155 & 6.90 \\ 6 & 1956 & 26.22 & 19 & 19.46 & 155 & 7.87\end{array}$

$\begin{array}{llllllll}6 & 21 & 2 & 7.07 & 19 & 17.31 & 155 & 22.43\end{array}$ $62256 \quad 3.41 \quad 1920.29 \quad 155 \quad 11.91$

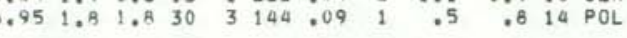
$\begin{array}{llllllllllll}.81 & 2.3 & 2.4 & 31 & 1 & 99 & .09 & 5 & .4 & .6 & 23 & \text { UER }\end{array}$

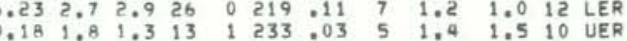

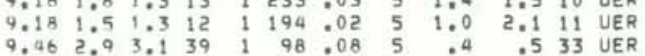
$\begin{array}{lllllllllllll}8.29 & 1.4 & 1.3 & 12 & 0 & 217 & .02 & 7 & 1.3 & 2.1 & 8 & \text { POL }\end{array}$

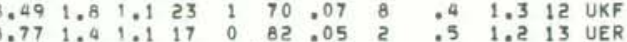

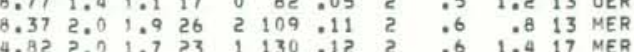

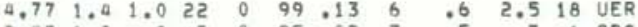

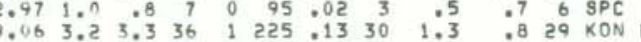

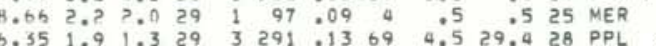

$\begin{array}{rrrrrrrrrrrr}7.73 & 2.7 & 2.8 & 30 & 0 & 165 & .13 & 2 & .8 & .5 & 28 & \text { MER } \\ 6.38 & 1.3 & 1.0 & 26 & 0 & 80 & 010 & 5 & .5 & .8 & 23 & \text { UER }\end{array}$

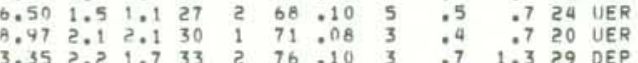

$\begin{array}{llllllllllll}1.23 & 2.0 & 2.2 & 31 & 2 & 148 & .10 & 2 & .7 & .6 & 19 & \text { MER }\end{array}$

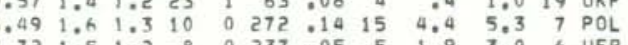

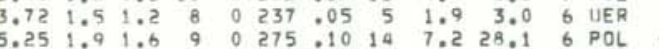

$\begin{array}{llllllllllll}9.27 & 2.7 & 2.9 & 30 & 0 & 97 & .08 & 5 & .4 & .6 & 26 & \text { UER } \\ 5.33 & 1.9 & 1.5 & 30 & 2 & 55 & 12 & 3 & 04 & .19 & 19 & \text { UKF }\end{array}$

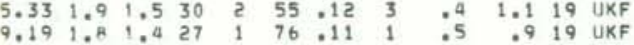

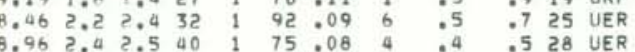
$\begin{array}{lllllllllllll}3.65 & 1.5 & 1.4 & 14 & 2 & 136 & .10 & 5 & .5 & 1.3 & 8 & \text { UKF } \\ 0.55 & 3.5 & 3.5 & 37 & 1 & 114 & .09 & 5 & .5 & & 3 & 30 & \text { UER }\end{array}$

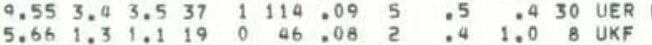

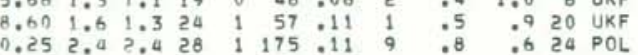
$\begin{array}{llllllllllll}9.67 & 2.0 & 0.0 & 24 & 0 & 75 & .07 & 5 & .5 & .8 & 22 & \\ 0.9 E R\end{array}$ \begin{tabular}{lllllllllll}
6.94 & 1.4 & 22 & 0 & 81.10 & 4 & .5 & 1.0 & 21 & UER \\
8.57 & 1.7 & 1.4 & 20 & 0 & 75 & .09 & 5 & .6 & .8 & 19 \\
\hline
\end{tabular}

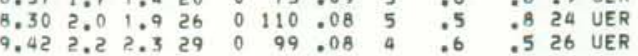
$\begin{array}{rrrrrrrrrrrr}6.97 & 1.8 & 1.6 & 19 & 0 & 123 & .13 & 6 & .7 & 1.1 & 16 & \text { SWR }\end{array}$

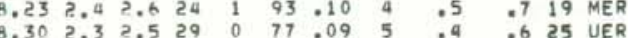
$\begin{array}{llllllllllll}.77 & 2.2 & 1: 9 & 30 & 2 & 101 & 08 & 5 & 04 & 07 & 12 & \text { UER } \\ 6.94 & 1.9 & 1.5 & 13 & 1 & 233 & 04 & 3 & 1: 1 & 1: 9 & 10 & \text { UER }\end{array}$

HVO EARTHQUAKE SUMMARY LIST

PAGE $6 a$

YEAR MON ORIGIN TIME LAMM SEC DEG MIN DEG MIN

1979 SEP $7 \quad 210 \quad 40.94 \quad 19 \quad 19.13 \quad 155 \quad 11.72$

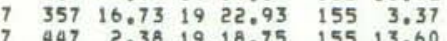

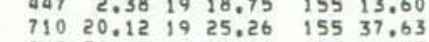
7.89
$710.151920 .08 \quad 155 \quad 4.05$

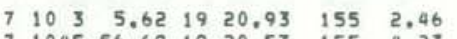

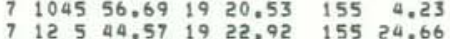

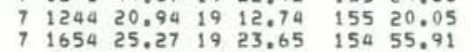

$\begin{array}{lllllll}7 & 1730 & 45.09 & 19 & 19.17 & 155 & 12.04\end{array}$

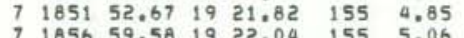

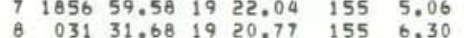
803131.681920 .77 155 6.30

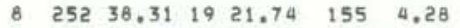

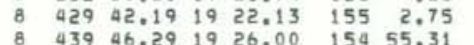
$\begin{array}{lllllll}8 & 439 & 46.29 & 19 & 26.00 & 154 & 55.31 \\ 8 & 918 & 49.78 & 18 & 56.27 & 155 & 34.37\end{array}$

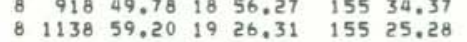

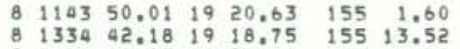
$8 \quad 143214.03 \quad 1926.41 \quad 15524.52$ 8
8 $1951 \quad 13.37 \quad 1920.12 \quad 155 \quad 6.55$

\begin{tabular}{l}
$82034 \quad 37.25 \quad 1921.62 \quad 155 \quad 20.60$ \\
\hline
\end{tabular} 9104233.151924 .24 155 29.72

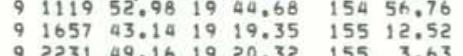
\begin{tabular}{l}
$92242 \quad 52.10 \quad 19 \quad 20.37 \quad 155 \quad 13.73$ \\
\hline
\end{tabular} $\begin{array}{llllllll}10 & 1 & 9 & 48.65 & 19 & 19.89 & 155 & 12.58 \\ 10 & 2 & 0 & 7.39 & 19 & 25.09 & 155 & 25.89\end{array}$

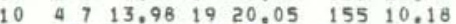
$10 \quad 513 \quad 51.04 \quad 19 \quad 19.38 \quad 155 \quad 15.60$ $10 \quad 6 \quad 0 \quad 18.91 \quad 19 \quad 18.73 \quad 155 \quad 13.51$

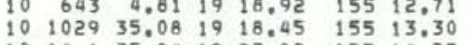
$1014 \quad 135.841923,02 \quad 15514.77$ $\begin{array}{lllllll}10 & 1526 & 17.72 & 19 & 20.90 & 155 & 1.30\end{array}$ $102032 \quad 33.57$ 19 23,09 15516.89 $\begin{array}{lllllll}10 & 2315 & 15.00 & 19 & 23,91 & 155 & 4.00\end{array}$ $\begin{array}{lllllll}10 & 2327 & 54.74 & 19 & 20.87 & 155 & 13.92\end{array}$ $\begin{array}{rrrrrrr}10 & 2356 & 11.27 & 19 & 22.02 & 155 & 3.27 \\ 11 & 150 & 52.87 & 19 & 15.58 & 155 & 19.91\end{array}$ i1 $42453.31 \quad 1922.28 \quad 155 \quad 5.66$
OEPTH AMP NUR GR GAP RMS MIN ERH ERZ NO

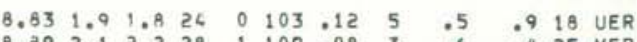

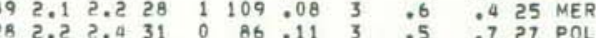

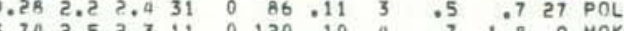
$\begin{array}{llllllllllll}0.97 & 2.5 & 2.3 & 11 & 0 & 120 & .10 & 4 & .7 & 1.8 & 9 & \text { MOK } \\ 2.1 & 1.8 & 21 & 1 & 136 & .10 & 2 & .6 & 1.1 & 16 & \text { MER }\end{array}$ $\begin{array}{llllllllllll}0.47 & 1.7 & 1.2 & 23 & 0 & 159 & .10 & 2 & .6 & .9 & 13 & \text { MER }\end{array}$

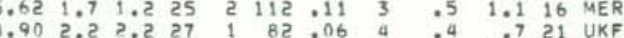
$\begin{array}{llllllllllll}45.90 & 2.0 & 2.0 & 23 & 2 & 180 & 09 & 10 & .9 & 1.8 & 5 & \text { HLP } \\ 7.51 & 2.1 & 1.9 & 17 & 1 & 217 & .25 & 11 & 1.8 & 2.4 & 5\end{array}$ $\begin{array}{lllllllllllll}7.87 & 1.6 & 1.0 & 12 & 0 & 225 & .04 & 7 & 1.3 & 2.1 & 9 & \text { UER }\end{array}$

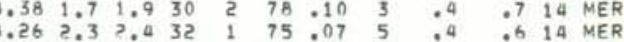

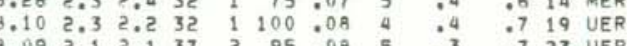

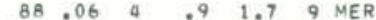

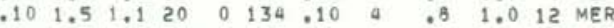

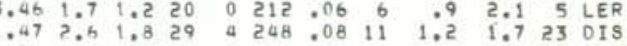

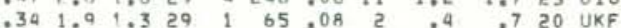

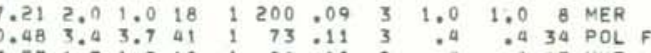

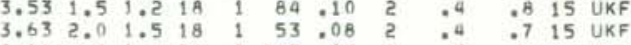

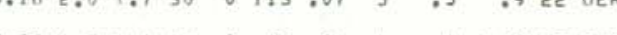

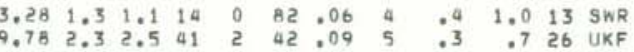
$\begin{array}{rlllllllllll}8.11 & 2.6 & 2.0 & 32 & 1 & 231 & 09 & 10 & 1.2 & 1.8 & 21 & \text { HIL } \\ 72 & 1.3 & 0.4 & 17 & 1 & 88 & 04 & 5 & .5 & 1.3 & 12 & \text { UER }\end{array}$

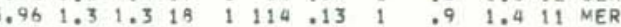

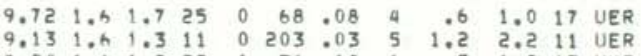

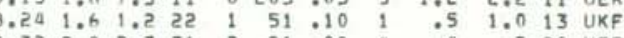

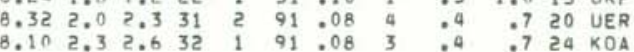
$\begin{array}{lllllllllllll}0.01 & 2.1 & 2.2 & 35 & 2 & 79 & .09 & 3 & .4 & .7 & 20 & \text { POL }\end{array}$

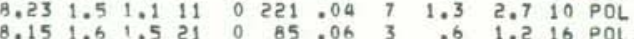

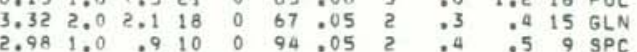
$\begin{array}{llllllllllll}3.61 & 2.1 & 2.3 & 32 & 2 & 196 & .08 & 3 & .4 & .6 & 15 & \text { MER }\end{array}$

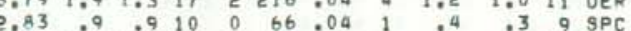
$\begin{array}{lllllllllllll}.18 & 1.8 & 1.2 & 29 & 2 & 95 & .08 & 3 & .5 & .7 & 91 & 9 & \text { MER }\end{array}$

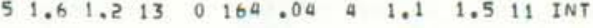

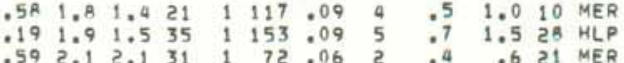


ORIGIN TIME LAT N LON W DEPTH AMP DUR NR GAP RMS MIN ERH ERZ NO
DA HRMN SEC DEG MIN DEG NIN KM MAG AAG NR NS DEG SEC OIS KM KM FM REMK $\begin{array}{lllllllllllllllllllll}1979 \text { SEP } 11 & 7 & 3 & 56.00 & 19 & 21.81 & 155 & 3.37 & 7.15 & 2.2 & 2.2 & 24 & 1 & 114 & .10 & 4 & .5 & .8 & 17 & \text { MER }\end{array}$

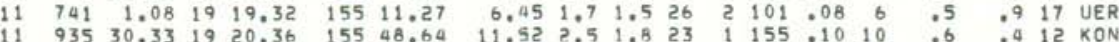

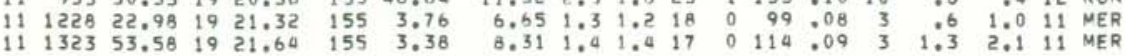
\begin{tabular}{llllllllllllllllll}
11 & 1327 & 58.79 & 19 & 21.62 & 155 & 3.32 & 7.55 & 1.3 & 1.0 & 20 & 0 & 116 & .08 & 3 & .5 & .8 & 12 \\
\hline 1 & MER
\end{tabular}

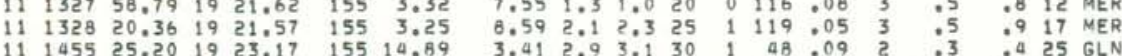

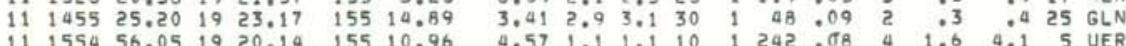

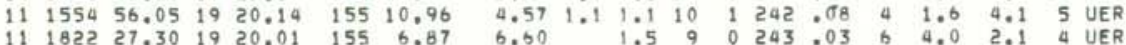

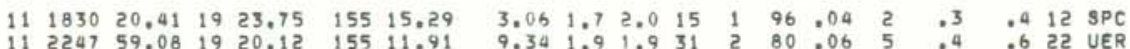

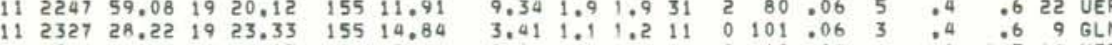

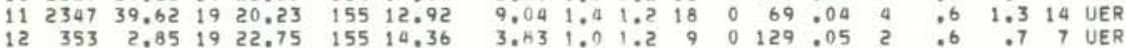

$\begin{array}{llllllllllllllllllll}12 & 438 & 32.93 & 19 & 19.15 & 155 & 10.24 & 5.93 & 1.4 & 1.4 & 12 & 1 & 255 & .05 & 5 & 1.3 & 3.9 & 10 & \text { UER } \\ 12 & 55 & 41.80 & 19 & 20.14 & 155 & 7.39 & 9.03 & 1.6 & 1.6 & 11 & 0 & 309 & .04 & 6 & 7.1 & 4.8 & 10 & \text { UER }\end{array}$

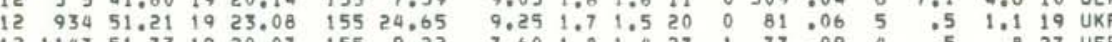

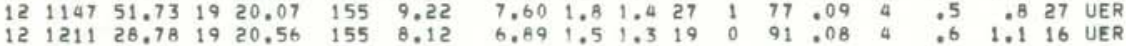

$\begin{array}{lllllll}12 & 1212 & 43.28 & 19 & 19.86 & 155 & 13.45\end{array}$

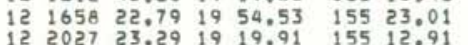
12 2213 $4.241912 .99 \quad 15536.61$

$\begin{array}{lllllll}13 & 522 & 13.30 & 19 & 21.17 & 155 & 3.54\end{array}$

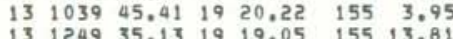
$131333 \quad 1.8919 \quad 18.18 \quad 15523.65$

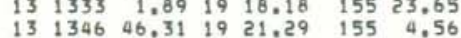

$\begin{array}{lllllll}13 & 1510 & 32.21 & 19 & 23.57 & 154 & 59.13 \\ 13 & 1614 & 40.78 & 19 & 23.10 & 155 & 14.84\end{array}$

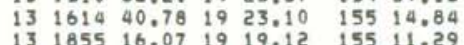
$13201759.62 \quad 1920,32 \quad 155 \quad 4.46$ $\begin{array}{llllll}13 & 2030 & 34.14 & 19 & 20,83 & 155 \quad 2,80\end{array}$ $\begin{array}{lllllll}13 & 2142 & 36.00 & 19 & 23.51 & 154 & 59.23\end{array}$

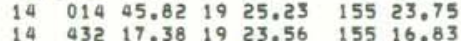
$1454855.821924 .02 \quad 15529.22$ 14 $735 \quad 18.71 \quad 1920,09 \quad 15511.76$

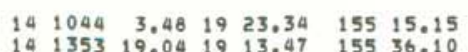
141532 48.01 1921.1015536 .10

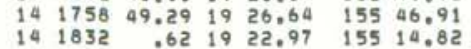

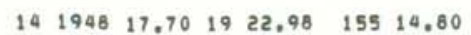

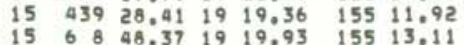

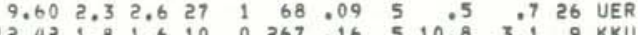

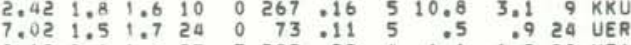

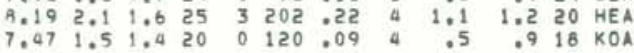
$\begin{array}{llllllllllll}6.44 & 1.9 & 1.5 & 22 & 1 & 104 & 13 & 3 & .7 & 1.0 & 17 & \text { MER } \\ 7.29 & .7 & 1.7 & 20 & 0 & 144 & 09 & 2 & 9 & 1.8 & 11 & \text { MER }\end{array}$

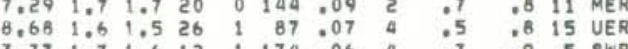

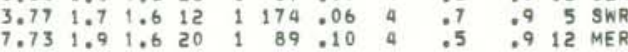

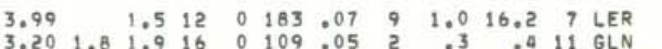
$\begin{array}{lllllllllllll}3.20 & 1.8 & 1: 9 & 16 & 0 & 109 & 05 & 2 & 0.3 & 0.4 & 11 & \text { GLN } \\ 9.13 & 1.4 & 1: 1 & 12 & 0 & 237 & 05 & 6 & 1.7 & 2.7 & 9 \text { UER }\end{array}$

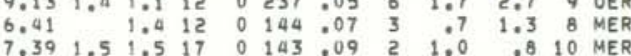
$\begin{array}{llllllllllll}6.25 & 1.7 & 1.4 & 17 & 0 & 180 & .10 & 9 & .7 & 1.1 & 7 & 7 \text { LER }\end{array}$ $\begin{array}{rrrrrrrrrrrr}0.41 & 1.6 & 1.3 & 26 & 1 & 46 & .09 & 2 & .5 & 1.0 & 17 & \text { UKF } \\ 3.00 & 3.0 & 3.5 & 37 & 0 & 35 & .09 & 0 & .5 & .02 & 31 & \text { SPC F }\end{array}$

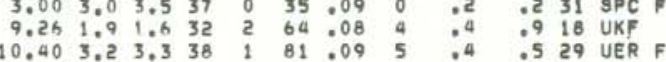
$\begin{array}{llllllllllllll}2.99 & 1.7 & 2.0 & 16 & 1 & 78 & .15 & 2 & .4 & .6 & 12 & \text { SPC } \\ 7.51 & 2.3 & 1.6 & 22 & 1 & 86 & .23 & 3 & 08 & 1.5 & 18 & \text { HEA }\end{array}$

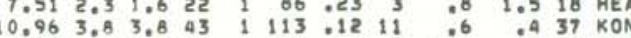

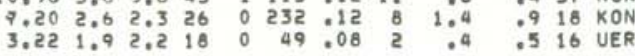
$\begin{array}{llllllllllllll}3.20 & 1.1 & .8 & 6 & 0 & 114 & .05 & 2 & .5 & .7 & 5 & \text { UER }\end{array}$

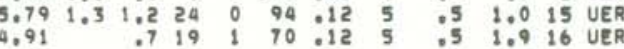

ORIGIN TIME LAT N LON W DEPTH AMP RUR N GAP RMS MIN ERH ERZ NO RE EAR MON DA

1979 SEP $15 \quad 85251.22 \quad 1923.18 \quad 155 \quad 14.80$

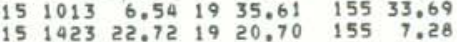
$15 \quad 154516.001925 .06 \quad 15453.58$

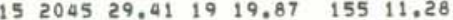
$15 \quad 2143 \quad 4.16 \quad 19 \quad 19.60 \quad 155 \quad 12.10$ 15225556.511920 .2915512 .86 $16,12357.66$ 19 $17.97 \quad 15513.26$

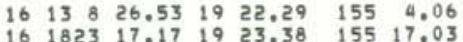

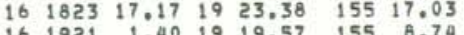
16 16 $194620.971920 .41 \quad 155 \quad 13.15$ $162048 \quad 26.40 \quad 18 \quad 55.62 \quad 155 \quad 6.28$ $\begin{array}{lllllll}16 & 2111 & 54.12 & 19 & 20.07 & 155 & 3.97 \\ 16 & 2116 & 23.47 & 19 & 23.34 & 155 & 14.96\end{array}$ $16211623.471923 .34 \quad 15514.96$ $17 \quad 43625.18$ 19 22.20 15522439 $17 \quad 715 \quad 43.92 \quad 1920.33 \quad 155 \quad 12.61$

$17 \quad 151933.011920 .71 \quad 155 \quad 2.86$ 17194534.013920 .79715529 .57

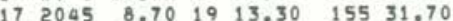

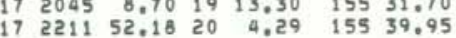
$\begin{array}{ll}17 & 2245 \\ 59.42 \quad 19 & 26.77 \quad 155 \quad 29.14\end{array}$

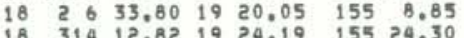

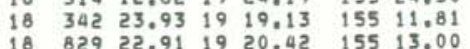
$\begin{array}{lllllll}18 & 846 & 17.59 & 19 & 20.09 \quad 155 \quad 8.96\end{array}$ 18 1144 $7.781919 .41 \quad 15511.00$ $181523 \quad 38.361938 .60 \quad 15459.33$

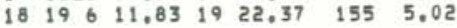
$\begin{array}{lllllllllll}3.27 & 1.7 & 14 & 0 & 106 & .04 & 2 & .3 & .5 & 11 & \text { GLN }\end{array}$

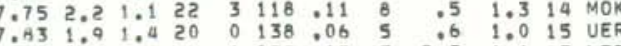

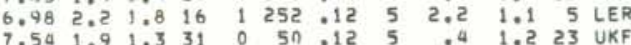

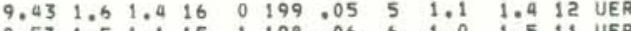
.

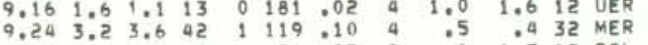
$5.711 .51 .220 \quad 1 \quad 96.07 \quad 2 \quad .6 \quad 1.3 \quad 12$ POL $\begin{array}{llllllllllll}7.16 & 1.3 & 1.3 & 12 & 0 & 149 & .11 & 4 & .7 & 1.2 & 7 & \text { MER }\end{array}$

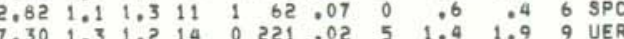

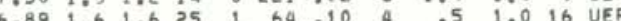
$.98 \quad 2,0 \quad 1.538 \quad 5263 \quad 15 \quad 40 \quad 1,3 \quad 1,028$ PPL $\begin{array}{lllllllllllll}6.48 & 1.5 & 1.5 & 13 & 0 & 154 & .06 & 2 & 1.0 & 1.4 & 6 & \text { MER }\end{array}$

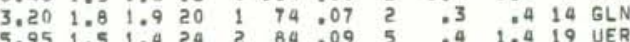

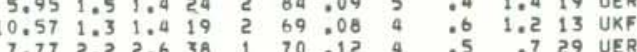
$\begin{array}{llllllll}.77 & 2.2 & 2.6 & 38 & 1 & 70\end{array}$

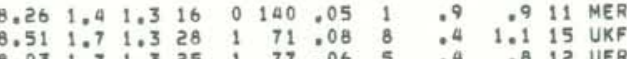

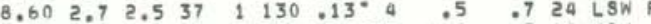

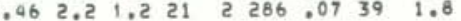
$\begin{array}{llllllllllllllllll}18 & 2053 & 24.26 & 19 & 21.94 & 155 & 4.68 & 8.56 & 1.5 & 1.2 & 21 & 0 & 80 & 0.07 & 3 & .4 & .9 & 21 \\ 1 & \text { MER }\end{array}$

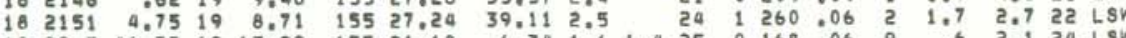

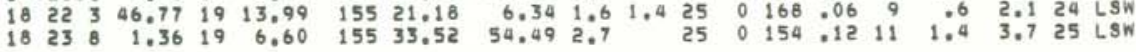

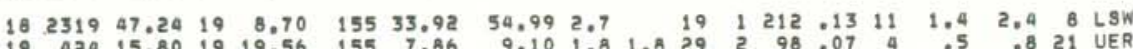

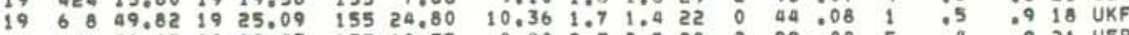

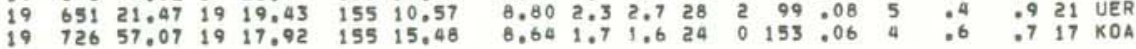

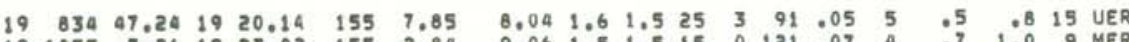

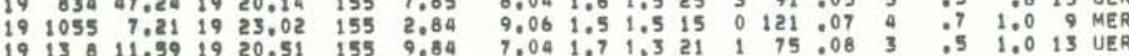


ORIGIN TIME LAT N LON N N DEPTH AMP OUR N GAP RMS MIN ERH ERZ NO
OA HRMN SEC DEG MIN DEG MIN KM MAG MAG NR NS OEG SEC DIS KM KM FM REMK

1979 SEP $19 \quad 1310 \quad 47.12 \quad 19 \quad 17.81 \quad 155 \quad 25,51$

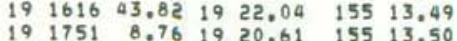

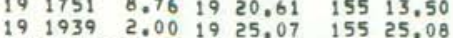
$192128 \quad 50.25 \quad 1920.04 \quad 155 \quad 8.10$

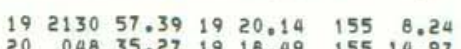
$20,335.86$ 19 $23.32 \quad 155120.97$ $\begin{array}{lllllll}20 & 230 & 19.47 & 19 & 21.62 & 155 & 6.70 \\ 20 & 253 & 43.87 & 19 & 19.01 & 155 & 13.51\end{array}$ $\begin{array}{lllllll}20 & 516 & 36.45 & 19 & 19.79 & 155 & 6.59\end{array}$ $20 \quad 75255,70$ 19 $25,29 \quad 155 \quad 24,48$ $\begin{array}{llrllll}20 & 818 & 6.46 & 19 & 30.28 & 155 & 52.12 \\ 20 & 856 & 57.75 & 19 & 31.21 & 154 & 53.54\end{array}$ $\begin{array}{lllllll}20 & 918 & 32.85 & 19 & 23.57 & 155 & 15.07\end{array}$

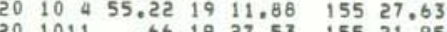
20121714.6619 $20 \begin{array}{llllll}1646 & 13.70 & 19 & 23.80 & 155 & 15.33 \\ 22.41 & 155 & 30.16\end{array}$ $\begin{array}{lllllll}20 & 1647 & 26.16 & 19 & 21.25 & 155 & 14.97\end{array}$

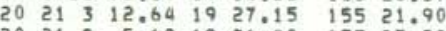
$\begin{array}{llrllll}20 & 21 & 5.62 & 59 & 26.80 & 155 & 23.29 \\ 20 & 2121 & 21.12 & 19 & 20.89 & 155 & 13.17\end{array}$

$\begin{array}{lllllll}20 & 2344 & 12.20 & 19 & 25.71 & 155 & 39.73\end{array}$ $21 \quad 12924.091920 .23 \quad 155 \quad 11.85$ $\begin{array}{lllllll}21 & 255 & 37.98 & 19 & 21.45 & 155 & 10.03 \\ 21 & 25 & 19 & 20.56 & 155 & 10.20\end{array}$ 15511.66 $\begin{array}{llllllll}21 & 7 & 8 & .66 & 19 & 20.89 & 154 & 58.14 \\ 21 & 833 & 27.91 & 19 & 21.73 & 155 & 6.28\end{array}$

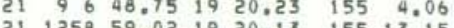
ट1 $125859.021920 .13 \quad 15513.15$

21 $\begin{array}{llllll}1716 & 23.67 & 19 & 21.98 & 155 & 5.07\end{array}$

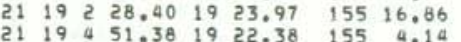
$21211153.16 \quad 1920.11 \quad 15512.07$

$\begin{array}{lllllll}21 & 2119 & 17.37 & 19 & 20.99 & 155 & 3.24\end{array}$

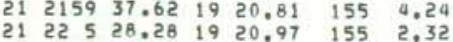
$\begin{array}{lllllll}21 & 22 & 94.04 & 19 & 211.46 & 155 & 6.10\end{array}$ $\begin{array}{lllllll}21 & 2215 & 55.07 & 19 & 21.30 & 155 & 2.15 \\ 21 & 2216 & 23.12 & 19 & 20.60 & 155 & 3.92\end{array}$ $\begin{array}{lllllll}21 & 2216 & 23.12 & 19 & 20.60 & 155 & 2.92 \\ 21 & 2217 & 38.43 & 19 & 21.01 & 155 & 2.88\end{array}$ $\begin{array}{llllllllllll}8.64 & 1.9 & 1.8 & 19 & 0 & 107 & 09 & 6 & .5 & .8 & 14 & \text { HEA }\end{array}$

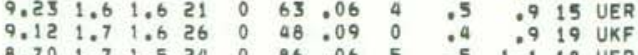
$\begin{array}{rrrrrrrrrrrr}8.76 & 1.8 & 2.0 & 28 & 0 & 82 & 05 & 5 & .5 & .9 & 23 & \text { UER } \\ 6.91 & 1.0 & 1.1 & 19 & 0 & 127 & .08 & 4 & .6 & 1.2 & 18 & \text { POL }\end{array}$

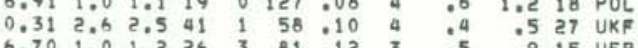
$\begin{array}{llllllllllll}6.70 & 1.0 & 1.2 & 26 & 3 & 81 & 12 & 3 & .5 & 09 & 15 & \text { UER } \\ 8.15 & 1.4 & 1.5 & 24 & 1 & 78 & .07 & 4 & .5 & 99 & 18 & \text { UER }\end{array}$

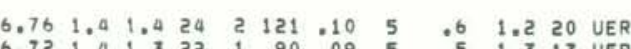

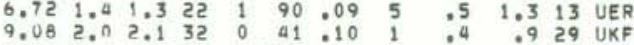

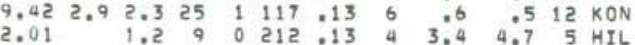
$\begin{array}{lllllllllllll}3.36 & 2.9 & 3.2 & 31 & 1 & 45 & .10 & 2 & .3 & .4 & 27 & \mathrm{SPC} F\end{array}$

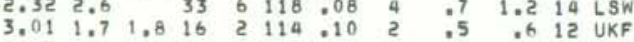

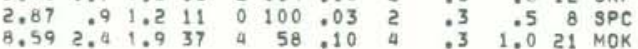
$\begin{array}{lllllllllll}0.21 & 1.5 & 1.3 & 14 & 0 & 147 & .06 & 3 & 1.0 & 1.5 & 9 \text { UER }\end{array}$ $\begin{array}{ccccccccccccc}3.61 & 1.2 & 1.6 & 19 & 0 & 168 & 04 & 3 & .5 & .7 & 10 & \text { SWR } \\ 8.21 & 1.7 & 1.4 & 23 & 2 & 73 & : 12 & 2 & : 5 & 1: 0 & 11 & \text { UKF }\end{array}$

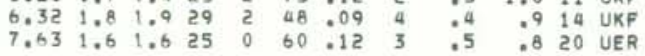
$1.951 .21 .814 \quad 0219.09 \quad 7 \quad 1.3 \quad 2.1 \quad 7$ MOK \begin{tabular}{llllllllllll}
7.59 & 1.4 & 1.1 & 14 & 2 & 199 & 06 & 1 & 1.3 & 9 & 6 & 6 \\
\hline
\end{tabular}

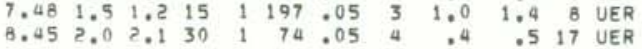

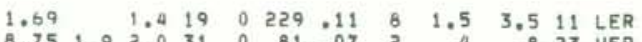

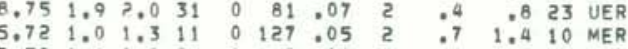

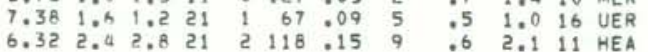
$\begin{array}{llllllllllll}8.78 & 2.3 & 2.5 & 31 & 1 & 76 & .06 & 3 & .4 & .6 & 21 & \text { MER }\end{array}$

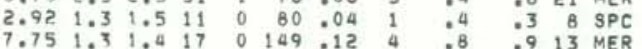

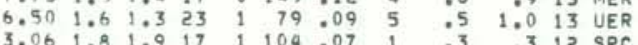

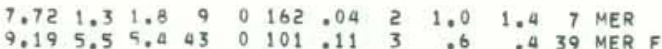

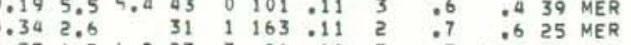
$\begin{array}{rlllllllllll}7.55 & 1.7 & : 9 & 23 & 3 & 86 & 10 & 3 & .5 & 1.2 & 18 & \text { UER } \\ 6.09 & 1.8 & : .8 & 21 & 0 & 160 & : 12 & 3 & : 6 & 1.0 & 15 & \text { MER }\end{array}$

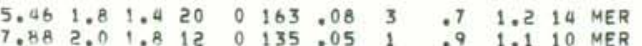

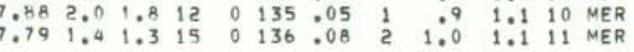

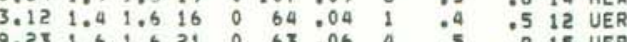

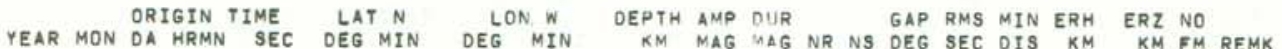

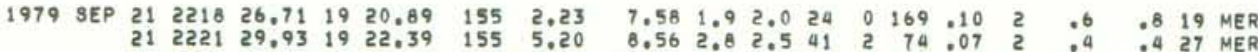

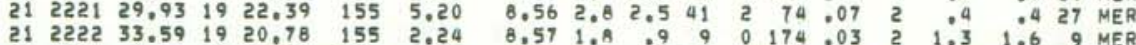

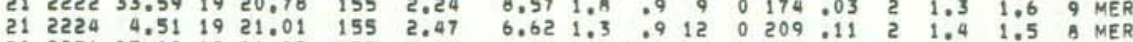

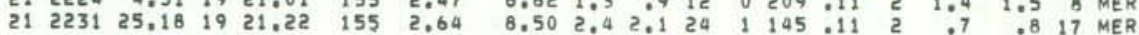
$\begin{array}{lllllllllllllllllllll}21 & 2232 & 32.98 & 19 & 20.45 & 155 & 2.77 & 8.21 & 2.4 & 2.7 & 27 & 1 & 151 & .09 & 1 & .7 & .6 & 21 & \text { MER } \\ 21 & 2236 & 29 & 51 & 19 & 21.89 & 155 & 5.60 & 7.31 & 1.2 & 1.0 & 14 & 2 & 103 & .09 & 2 & .0 & 1.1 & 8 & \text { MER }\end{array}$

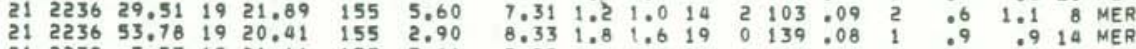

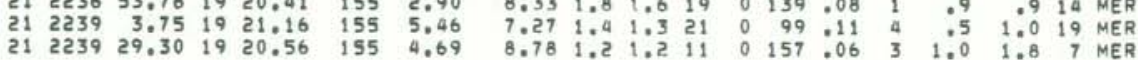
$21223929,301920.56$ is5 4.69

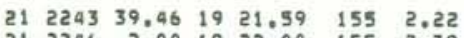
$212246 \quad 2.001922 .00 \quad 155 \quad 2.79$ $21224651.241921 .01 \quad 155 \quad 2.05$ $21 \quad 2255 \quad 50,75 \quad 19 \quad 20,82 \quad 155 \quad 2.78$

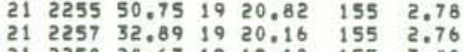
21 $2258 \quad 24.631919 .18 \quad 155 \quad 3,40$

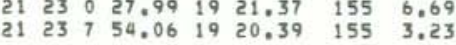

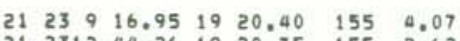
21231647.26 19 20.75155 2.62

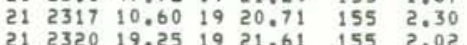

$\begin{array}{lllllll}21 & 2324 & 25.36 & 19 & 21.04 & 155 & 5.99\end{array}$ $.65 \quad 155 \quad 3.35$

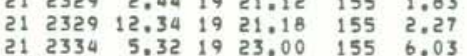

$\begin{array}{lllllll}21 & 2334 & 14.75 & 19 & 21.03 \quad 155 & 2.03\end{array}$

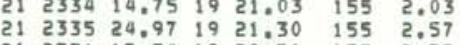
$212336 \quad 17.34 \quad 1920.71 \quad 155 \quad 2.57$ $\begin{array}{lllllll}21 & 2346 & 42.06 & 19 & 20.34 & 155 & 4.83 \\ 21 & 2351 & 34.46 & 19 & 20.44 & 155 & 2.69\end{array}$ $\begin{array}{lllllll}21 & 2353 & 35.23 & 19 & 19.91 & 155 & 3.95\end{array}$ $\begin{array}{llllll}2356 & 36.51 & 19 & 21.87 & 155 & 1.93\end{array}$ $\begin{array}{llllllll}22 & 0 & 0 & 28.27 & 19 & 21.00 & 155 & 1.24\end{array}$

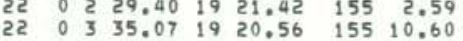
$22 \quad 0 \quad 5 \quad 10.55 \quad 19 \quad 20.94 \quad 155 \quad 2.51$ 22 $07750.741921 .60 \quad 155 \quad 5.75$

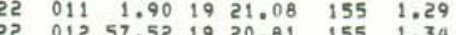

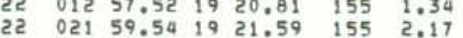

$\begin{array}{lllllll}22 & 022 & 34.36 & 19 & 21.29 & 155 & 2.65\end{array}$ $\begin{array}{lllllll}22 & 029 & 55.83 & 19 & 22.08 & 155 & 1.84 \\ 22 & 039 & 55.54 & 19 & 21.60 & 155 & 1.38\end{array}$ $\begin{array}{llllllllllll}6.20 & 1.6 & 1.6 & 16 & 0 & 155 & 0.10 & 3 & .7 & 1.1 & 12 & \text { MER } \\ 6.59 & 1.4 & 07 & 15 & 0 & 182 & : 13 & 4 & 1.0 & 1.1 & 15 & \text { MER }\end{array}$

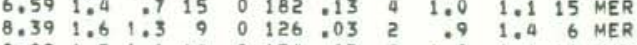

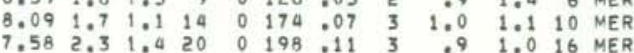

$\begin{array}{llllllllllll}7.56 & 2.8 & 2.9 & 20 & 1 & 143 & .09 & 2 & .5 & 1.0 & 10 & \text { MER }\end{array}$

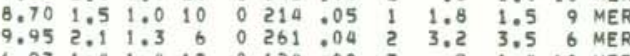

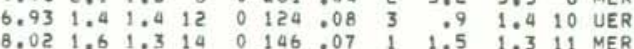
$\begin{array}{lllllllllllll}6.48 & 1.7 & 1.6 & 22 & 0 & 136 & .11 & 2 & .7 & .8 & 19 & \text { MER }\end{array}$

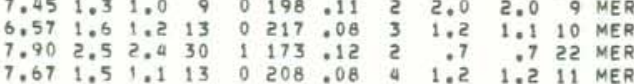
$\begin{array}{llllllllllll}7.03 & 1.6 & 1.6 & 22 & 0 & 96 & .10 & 9 & .5 & 1.0 & 22 & \text { MER }\end{array}$

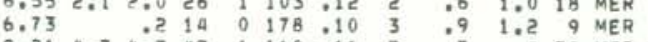
$\begin{array}{lllllllllll}9.21 & 4.3 & 4.3 & 43 & 1 & 160 & .11 & 3 & .7 & .4 & 39 \\ 6.32 & 1.6 & 1.0 & 14 & 0 & 201 & .09 & 0 & 3.1 & 2.2 & 7 \text { MER F }\end{array}$

$\begin{array}{lllllllllllll}7.28 & 2.6 & 2.3 & 27 & 2 & 173 & .13 & 3 & .6 & 1.0 & 16 & \text { MER }\end{array}$

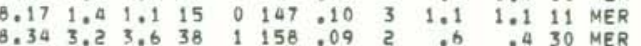
$\begin{array}{llllllllllll}7.17 & 1.4 & 1.3 & 12 & 0 & 145 & .09 & 3 & .7 & 1.3 & 12 & \text { MER } \\ 7.73 & & 1.1 & 11 & 0 & 202 & .09 & 1 & 1.6 & 1.3 & 10 & \text { MER }\end{array}$

$\begin{array}{llllllllllllll}7.27 & 1.5 & 1.4 & 13 & 0 & 164 & .10 & 2 & .8 & 1.1 & 10 & \text { MER }\end{array}$

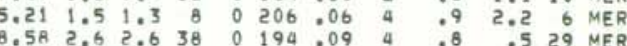

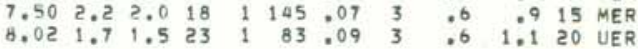
$\begin{array}{llllllllllll}7.79 & 2.4 & 2.5 & 25 & 1 & 155 & .10 & 2 & .6 & .8 & 16 & \text { MER }\end{array}$

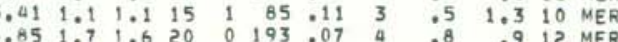
$\begin{array}{rllllllllllll}8.03 & 1.4 & 1.7 & 25 & 0 & 198 & 0 & 10 & 3 & .9 & 09 & 17 & \text { MER } \\ 6.81 & 1.7 & 1.5 & 22 & 1 & 157 & .15 & 3 & .8 & 1.4 & 19 & \text { MER }\end{array}$ $\begin{array}{llllllllllll}0.74 & 2.1 & 2.2 & 26 & 0 & 144 & .12 & 3 & .6 & .7 & 21 & \text { MER }\end{array}$

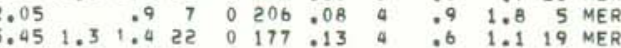




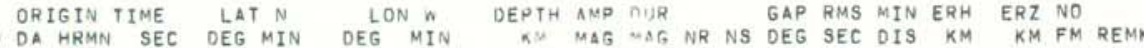

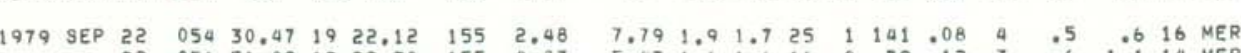
$22 \quad 056 \quad 51.09 \quad 1922.38 \quad 155 \quad 4.87$ $\begin{array}{llllllll}22 & 059 & 34.47 & 19 & 21.38 & 155 & 3.04\end{array}$ $22 \quad 112 \quad 45.68 \quad 1920.45 \quad 155 \quad 3.18$ $\begin{array}{lllllll}22 & 119 & 3.45 & 19 & 21.98 & 155 & 2.99\end{array}$

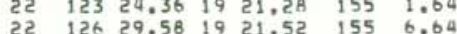

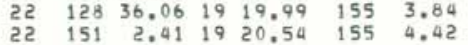

$\begin{array}{lllllll}22 & 214 & 22.86 & 19 & 21.05 & 155 & 2.75\end{array}$ $\begin{array}{llllll}22 & 257 & 25.0619 & 19.97 & 155 & 2.63\end{array}$

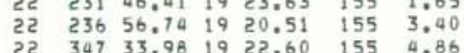

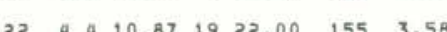

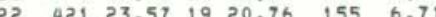

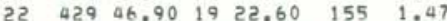
$\begin{array}{lllllll}22 & 438 & 20.84 & 19 & 20.54 & 155 & 2.79 \\ 22 & 513 & 26.09 & 19 & 21.70 & 155 & .94\end{array}$

$\begin{array}{lllllll}22 & 513 & 54.25 & 19 & 21.30 & 155 & .82\end{array}$ $\begin{array}{lllllll}22 & 639 & 56.22 & 19 & 18.86 & 155 & 13.29\end{array}$ $\begin{array}{lllllll}22 & 652 & 18.88 & 19 & 21.52 & 155 & 2.39 \\ 22 & 77 & 10.83 & 19 & 21.39 & 155 & 1.05\end{array}$

$\begin{array}{lllllll}22 & 759 & 6.10 & 19 & 23.25 & 155 & 25.31\end{array}$ $\begin{array}{llllllll}22 & 8 & 3 & 55.35 & 19 & 23.25 & 155 & 25.32\end{array}$ $2281954.6419200 .69155 \quad 2.70$

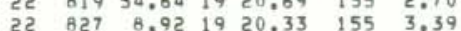

$\begin{array}{lllllll}22 & 836 & 1.27 & 19 & 19.79 & 155 & 6.99\end{array}$ $\begin{array}{lllllll}22 & 915 & 2.62 & 19 & 21.67 & 155 & 6.67\end{array}$

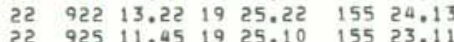

$\begin{array}{lllllll}22 & 932 & 9.96 & 19 & 21.56 & 155 & 1.22 \\ 22 & 934 & 34.79 & 19 & 21.78 & 155 & 1.26\end{array}$

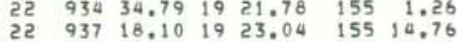
$22 \quad 109 \begin{array}{lllll}46.31 & 19 & 22.00 & 155 & 2.66\end{array}$

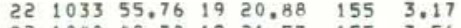

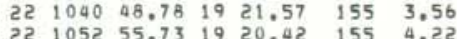
टर $119525.931920 .42 \quad 155$ 4.22

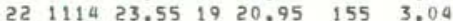

$\begin{array}{lllllll}22 & 1128 & 49.39 & 19 & 20.36 & 155 & 11.75 \\ 22 & 1130 & 52.63 & 19 & 21.38 & 155 & 2.66\end{array}$ $\begin{array}{lllllll}22 & 1130 & 52.63 & 19 & 21.38 & 155 & 2.66 \\ 22 & 1156 & 31.25 & 19 & 20.90 & 155 & 4.96\end{array}$
$5.471 .61 .6 \quad 16 \quad 0 \quad 79.12 \quad 3 \quad .6 \quad 1.1144$ MER

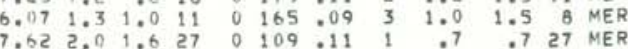
$\begin{array}{lllllllllll}8.91 & 1.2 & 1.1 & 10 & 0 & 183 & .02 & 4 & 1.1 & 1.6 & 9 \text { MER }\end{array}$

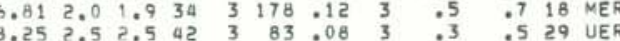
$\begin{array}{llllllllllll}7.61 & 1.1 & 1.1 & 13 & 0 & 159 & 07 & 2 & 1.2 & 1.6 & 10 & \text { MER } \\ 8.47 & 2.8 & 3.1 & 40 & 2 & 114 & : 10 & 3 & .4 & .5 & 29 & \text { MER }\end{array}$ $\begin{array}{lllllllllll}7.70 & 1.1 & 12 & 0 & 142 & .12 & 2 & 1.2 & 1.5 & 9 & \text { MER }\end{array}$

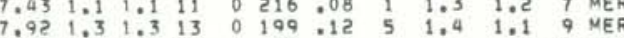
$\begin{array}{llllllllllll}7.45 & 1.6 & .6 & 26 & 0 & 95 & .11 & 1 & .6 & .9 & 18 & \text { MER } \\ 8.13 & 2.0 & 1.8 & 25 & 0 & 79.09 & 3 & .5 & .8 & 18 & \text { MER }\end{array}$ $\begin{array}{llllllllllll}8.32 & 2.0 & 1.9 & 30 & 1 & 108 & .08 & 4 & .5 & .5 & 20 & \text { MER }\end{array}$ $8.781 .919625 \quad 197.07 \quad 4 \quad 551.018$ UER $\begin{array}{llllllllllll}8.78 & 2.1 & 1.7 & 25 & 0 & 158 & .09 & 6 & .7 & .9 & 19 & \text { MER }\end{array}$ $\begin{array}{lllllllllll}8.38 & 2.4 & 2.8 & 29 & 0 & 148 & -11 & 1 & .8 & .8 & 23 \\ 4.41 & \text { MER }\end{array}$

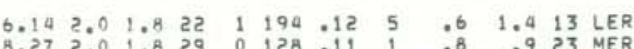

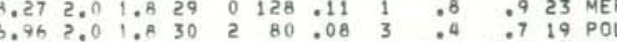

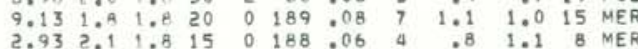

$\begin{array}{lllllllllllll}9.06 & 1.1 & .9 & 21 & 0 & 63 & .10 & 4 & .5 & 1.0 & 13 & \text { UKF }\end{array}$ $\begin{array}{llllllllllll}5.91 & 1.3 & 1.0 & 21 & 0 & 146 & .13 & 4 & .7 & 1.0 & 18 & \text { MEF }\end{array}$

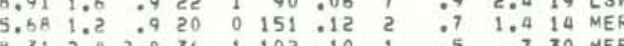

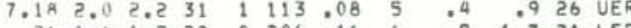

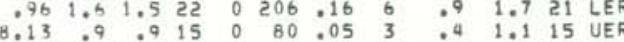

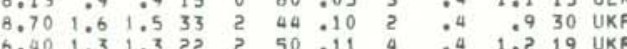
$\begin{array}{lllllllllll}5.45 & 2.0 & 2.1 & 29 & 1 & 180 & .12 & 4 & .6 & 1.2 & 27 \\ 5 & \text { MER }\end{array}$

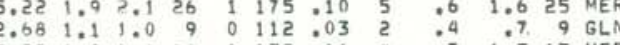

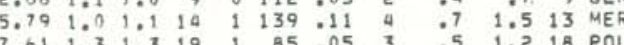

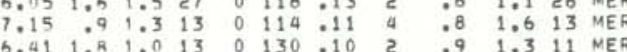
$\begin{array}{llllllllllllll}7.91 & 1.7 & .3 & 23 & 1 & 77 & .06 & 5 & .4 & .9 & 21 & 91 & \text { UER }\end{array}$

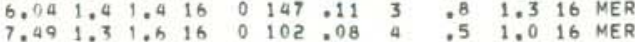

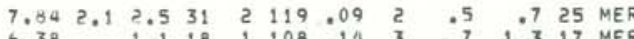

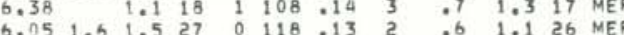

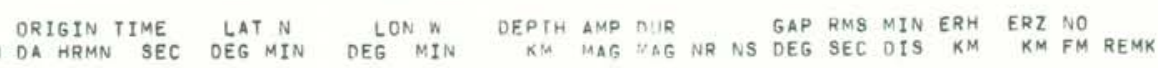

TOT9 SEF $25123024.481920 .96 \quad 155 \quad 605$

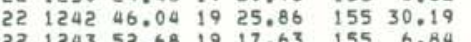

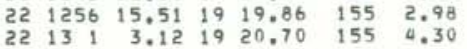
$\begin{array}{llllllll}\text { 22 } & 13 & 3 & 22.35 & 19 & 19.48 & 155 & 5.59\end{array}$ $22 \quad 133218.701924 .30 \quad 15517.41$ $22 \quad 133339.641924 .42 \quad 155 \quad 17.97$

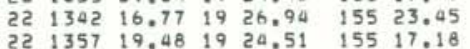

$2214041.82 \quad 19 \quad 12.97 \quad 155 \quad 28.18$ 22 14 658.12 19 $24.52 \quad 155 \quad 16.64$ $\begin{array}{lllllll}22 & 1421 & 9.28 & 19 & 23.74 & 155 & 16.30 \\ 22 & 1421 & 37.37 & 19 & 18.78 & 155 & 13.55\end{array}$

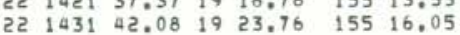

$22 \quad \begin{array}{llllll}445 & 16.20 & 19 & 24.18 & 155 & 16.77\end{array}$

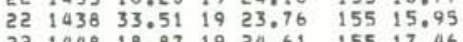

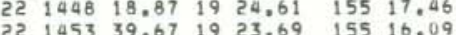
$22.45730 .571923 .81 \quad 15516.04$

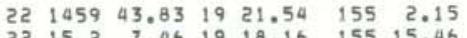

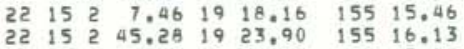

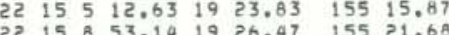

$\begin{array}{lllllll}22 & 1513 & 19.42 & 19 & 24.47 & 155 & 16.25\end{array}$

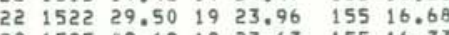
22 152509.691923 .63 155 16.60 .33 22. $152907.75 \quad 1924,04 \quad 155 \quad 16.06$

22 $1532 \quad 24.68 \quad 1923.84 \quad 155 \quad 16.07$

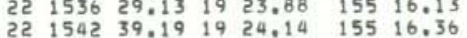

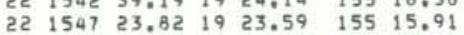
$22 \quad 1550 \quad 30.09 \quad 1925.76 \quad 155 \quad 19.64$

$\begin{array}{lllllll}22 & 1552 & 15.14 & 19 & 24.32 & 155 & 15.92 \\ 22 & 1554 & 49.87 & 19 & 23.99 & 155 & 16.53\end{array}$

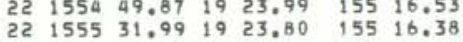

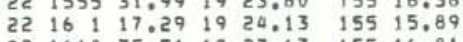
$22 \quad 1610 \quad 35.51 \quad 19 \quad 23.63 \quad 155 \quad 16.81$ $\begin{array}{lllllll}22 & 1622 & 26.55 & 19 & 20.66 & 155 & 2.97\end{array}$ $221713 \quad 41.11$ 19 $21.49 \quad 155 \quad .38$

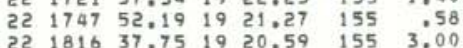
$\begin{array}{llllll}1920 & 31.57 & 19 & 19.82 & 155 \quad 7.99\end{array}$ $\begin{array}{lllllll}22 & 1920 & 31.57 & 19 & 19.82 & 155 & 7.99 \\ 22 & 1930 & 22,98 & 19 & 20003 & 155 & 9.12\end{array}$

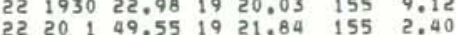

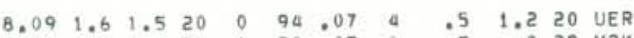

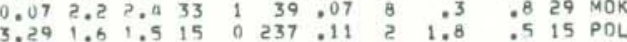
$\begin{array}{llllllllllll}5.40 & 1.2 & 1.1 & 16 & 0 & 205 & 12 & 0 & 1.0 & 1.2 & 16 & \text { MER } \\ 6.49 & 9.9 & 9 & 16 & 0 & 105 & : 13 & 3 & .7 & 1.3 & 16 & \text { MER }\end{array}$ $\begin{array}{lllllllllllll}5.75 & .9 & .6 & 10 & 0 & 148 & .08 & 5 & .9 & 2.6 & 10 & \text { MER }\end{array}$

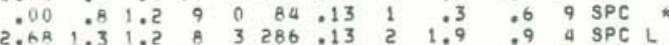

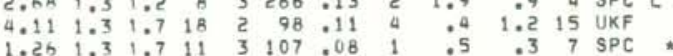

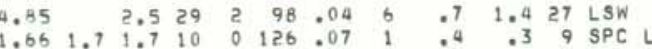

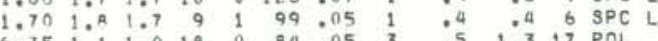

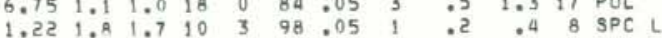

$\begin{array}{lllllllllllll}2.29 & 1.8 & 1.8 & 10 & 3 & 151 & .07 & 1 & .5 & .4 & 7 & \text { SPC L }\end{array}$

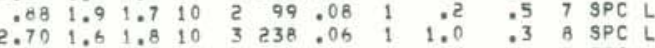

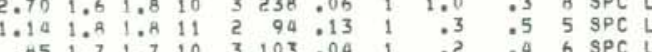

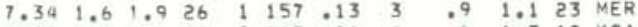

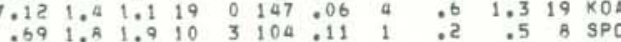

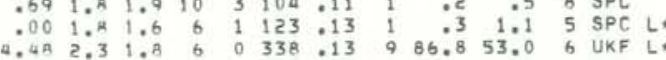

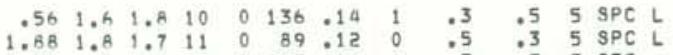

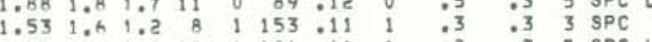

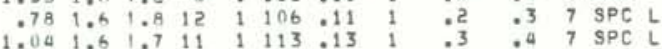
$\begin{array}{llllllllllllll}.94 & 1.6 & 1.7 & 12 & 1 & 105 & .13 & 1 & .2 & .3 & 5 & \mathrm{SPC} L\end{array}$

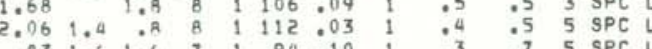

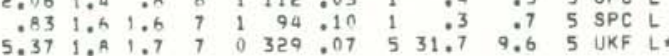

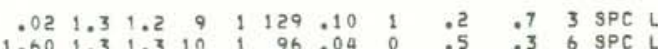

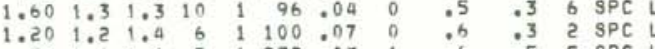

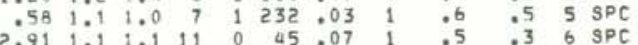
$\begin{array}{lllllllllllll}7.18 & 1.2 & .7 & 13 & 0 & 175 & .09 & 1 & 1.3 & 1.3 & 10 & \text { MER }\end{array}$

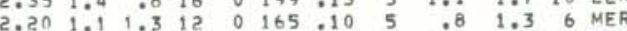

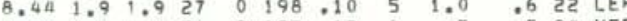
$\begin{array}{llllllllllll}7.14 & 1.8 & 1.8 & 31 & 0 & 127 & .12 & 1 & .7 & .7 & 24 & \text { MER }\end{array}$ $\begin{array}{lllllllllllll}7.5 A & 1.7 & 1.6 & 25 & 1 & 91 & .08 & 5 & .5 & .9 & 22 & \text { UER }\end{array}$ $5.22 \quad 1.311 \quad 0 \quad 146,11 \quad 4 \quad: 8 \quad 2.7909$ MER 


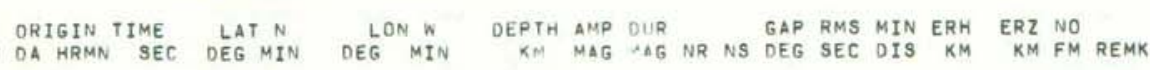
$\begin{array}{lllllll}1979 & \text { SEP } 22 \quad 2027 \quad 28.00 & 19 & 21.57 \quad 155 \quad 6.20\end{array}$

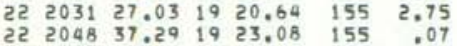

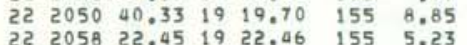
22 $2115 \quad 37.42 \quad 19 \quad 19.55 \quad 155 \quad 10.52$

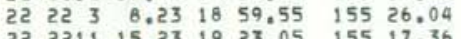

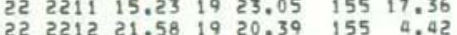
$22221916.891924 .59 \quad 15528.40$ $\begin{array}{llllllr}22 & 2243 & 17.71 & 19 & 21.23 & 155 & 1.50 \\ 22 & 2252 & 11.59 & 19 & 28.44 & 154 & 51.04\end{array}$

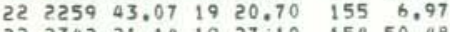

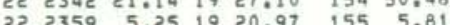

$\begin{array}{lllllll}23 & 020 & 59.90 & 19 & 20,48 & 155 & 6.35 \\ 23 & 029 & 4.917 & 19 & 19.82 & 155 & 6.86\end{array}$ $\begin{array}{rrrrrrr}23 & 029 & 4.17 & 19 & 19.82 & 155 & 6.86 \\ 23 & 128 & 19.88 & 19 & 23.02 & 155 & 4.23\end{array}$

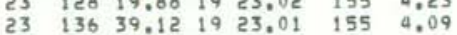

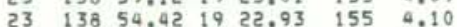

$\begin{array}{lllllll}23 & 215 & 44.90 & 19 & 21.81 & 155 & 1.78 \\ 23 & 255 & 54.26 & 19 & 21.77 & 155 & 18.36\end{array}$ $23 \quad 338$ 44.91 $1922,46 \quad 155 \quad 2.02$

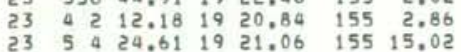
$\begin{array}{lllllll}23 & 537 & 36.98 & 19 & 21.58 & 155 & 3.53\end{array}$

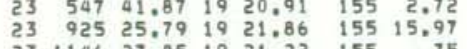

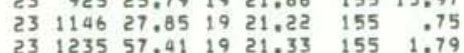
$\begin{array}{llllllll}23 & 13 & 25.02 & 19 & 20.63 \quad 155 \quad 1.88\end{array}$

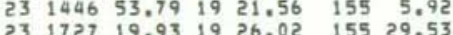
$23 \quad 2026 \quad 44.65 \quad 1921.61 \quad 155 \quad 5,92$ $232123 \quad 28,43 \quad 1921,46 \quad 155 \quad 5,73$

$\begin{array}{lllllll}24 & 052 & 3.97 & 19 & 24.98 & 155 & 28.18\end{array}$ 24 7 1 32.03 19 $20.69 \quad 155 \quad 4.39$ $\begin{array}{rrrrrrr}24 & 1031 & 46.13 & 19 & 21.11 & 155 & 6.61 \\ 24 & 1251 & 6.07 & 19 & 21.32 & 155 & 5.26\end{array}$ $\begin{array}{lllllll}24 & 1324 & 17.98 & 19 & 23.79 & 155 & 15.21\end{array}$

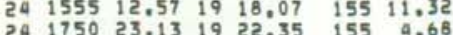
$\begin{array}{lllllll}24 & 1933 & 53.17 & 1921.28 & 155 & 2.79 \\ 24 & 2116 & 24.23 & 19 & 21.59 & 155 & 5.24\end{array}$ $\begin{array}{llllllll}24 & 22 & 7 & 50.29 & 19 & 19.24 & 155 & 11.08\end{array}$

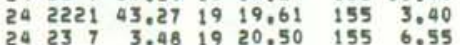

$\begin{array}{lllllllllll}7.40 & 1.3 & 1.3 & 27 & 1 & 84 & .10 & 3 & .5 & .9 & 22 \\ & \text { UER }\end{array}$

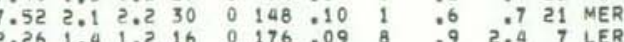

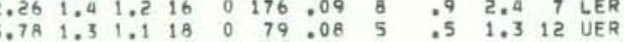

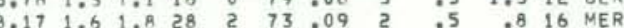
$\begin{array}{rrrrrrrrrrrr}9.05 & 1.9 & 1.1 & 15 & 0 & 118 & .04 & 5 & 1.0 & 2.1 & 12 & \text { UER } \\ 4.41 & 2.3 & 1.5 & 23 & 1 & 234 & .07 & 19 & 1.1 & 1.9 & 17 & \text { DIS }\end{array}$

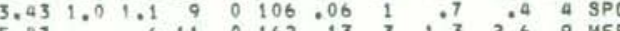

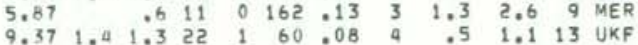

$\begin{array}{lllllllllllll}7.17 & 1.3 & 1.2 & 17 & 0 & 183 & 09 & 4 & 1.0 & .8 & 9 & \text { MER } \\ 6.74 & 1.5 & 0.2 & 16 & 1 & 282 & 09 & 4 & 1.6 & .6 & 4 \\ 7 & 4 E R\end{array}$

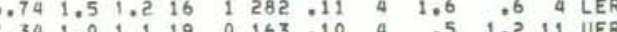
.921 .711 .419 a 290.13 6 $1.6 \quad .510$ LER $\begin{array}{lllllllllllll}8.36 & 1.9 & 1.7 & 27 & 1 & 107 & .08 & 5 & .5 & .7 & 15 & \text { UER }\end{array}$

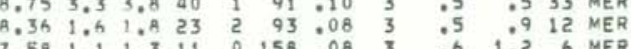
$\begin{array}{lllllllllll}7.75 & 1.5 & 1.3 & 16 & 0 & 164 & .09 & 4 & .9 & .9 & 8 \\ 3 & \text { MER }\end{array}$

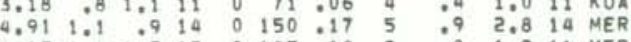
$\begin{array}{rrrrrrrrrrrr}8.15 & 1.6 & 1.5 & 17 & 0 & 143 & 10 & 2 & 98 & 1.2 & 16 & \text { MER } \\ 9.74 & 1.2 & 0 & 16 & 0 & 81 & .05 & 3 & .7 & 1.4 & 16 & \text { KOA }\end{array}$

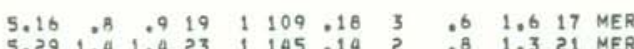
$\begin{array}{lllllllllll}7.45 & 2.3 & 2.9 & 27 & 0 & 196 & .10 & 5 & 99 & .6 & 26 \\ 6.42 & \text { LER }\end{array}$ $\begin{array}{llllllllllll}6.77 & 1.6 & 16 & 0 & 192 & .11 & 2 & 1.2 & 1.3 & 16 & \text { MER }\end{array}$

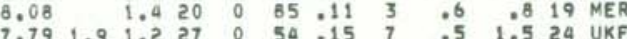

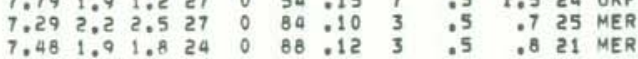
$\begin{array}{rrrrrrrrrrrr}0.18 & 1.9 & 1.6 & 27 & 0 & 55 & .11 & 5 & .5 & 1.0 & 22 & \text { UKF }\end{array}$ $6.74 \quad 1.524 \quad 0107,12 \quad 3 \quad .6 \quad 024$ MER

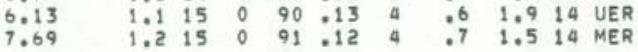
$\begin{array}{llllllllll}3.17 & 1.6 & 9 & 0 & 94 & .05 & 2 & .5 & .5 & 9 \mathrm{SPC}\end{array}$

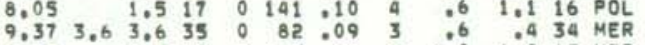

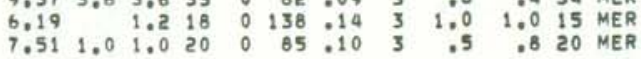

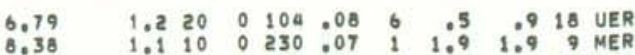

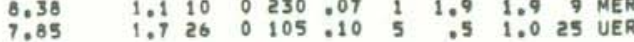

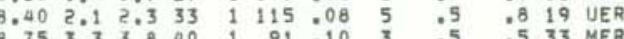

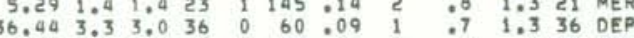

ORIGIN TIME LATN LON N

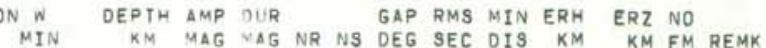

1979 SEP $25 \quad 1 \quad 1 \quad 29.97 \quad 1920.74 \quad 155 \quad 4.85$

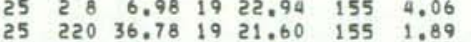
$\begin{array}{llll}25 & 240 & 8.63 & 19 \\ 25 & 0.22 & 155 & 12.60\end{array}$

$\begin{array}{lllllll}25 & 550 & 45.10 & 19 & 19.80 & 155 & 3.82\end{array}$ $\begin{array}{lllllll}25 & 856 & 7.11 & 19 & 41.93 & 155 & 46.68\end{array}$

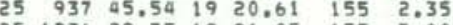
25123609.751921 .05 155 7.00

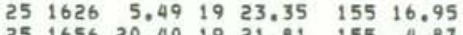
$\begin{array}{lllllll}25 & 1656 & 20.40 & 19 & 21.81 & 155 & 4.87 \\ 25 & 1820 & 34.95 & 19 & 20.59 & 154 & 59.11\end{array}$ $251924 \quad 52.17$ 19 $20.06 \quad 154 \quad 58.89$ $25194229,341921,60 \quad 155 \quad 6,23$ $\begin{array}{lllllll}25 & 2017 & 4.54 & 19 & 22.18 & 155 & 1.07 \\ 25 & 2031 & 33.79 & 19 & 21.33 & 155 & 5.65\end{array}$ $\begin{array}{lllllll}25 & 2137 & 35.49 & 19 & 19.97 & 155 & 6.28 \\ 25 & 2156 & 32.57 & 19 & 19.54 & 155 & 9.08\end{array}$ $\begin{array}{lllllll}25 & 2311 & 24.01 & 19 & 21.55 & 155 & 2.72 \\ 25 & 2323 & 29.55 & 19 & 31.34 & 155 & 36.37\end{array}$ $2603311.211920 .78 \quad 155 \quad 2.25$

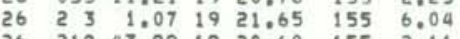

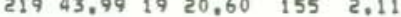
$26 \quad 249 \quad 15.34 \quad 1920.48 \quad 155 \quad 4.42$ $26 \quad 632 \quad 14.601920 .25 \quad 155 \quad 6.33$ $26 \quad 65132,46 \quad 1921.81 \quad 155 \quad 25,64$

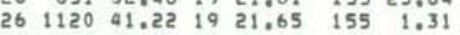
$\begin{array}{lllllll}26 & 1344 & 35.16 & 19 & 17.73 & 155 & 23.58\end{array}$ $2615132.43 \quad 19 \quad 32.46 \quad 155 \quad 55.43$ $\begin{array}{lllllll}26 & 1628 & 31.97 & 19 & 20.83 & 155 & 5.73 \\ 26 & 1715 & 27.22 & 19 & 20.81 & 155 & 4.75\end{array}$

$\begin{array}{lllllllll}26 & 22 & 6 & 24.73 & 20 & 8.71 & 155 & 49.22 & 11\end{array}$ 27233643.771921 .15 155 5.49

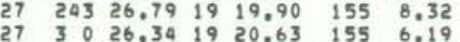

$\begin{array}{lllllll}37 & 352 & 53.76 & 19 & 21.44 & 155 & 3.24\end{array}$ $27 \quad 535 \quad 45.49 \quad 19 \quad 19.76 \quad 155 \quad 7.23$ $\begin{array}{lllllll}27 & 540 & 41.97 & 19 & 19.66 & 155 & 6.24 \\ 27 & 543 & 3.66 & 19 & 19.50 & 155 & 7.09\end{array}$

$\begin{array}{lllllll}27 & 548 & 4.84 & 19 & 21.76 & 155 & 6.35\end{array}$

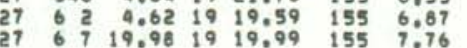
$\begin{array}{lllllllllll}7.41 & 1.4 & 19 & 0 & 107 & .09 & 4 & .6 & 1.1 & 19 & \text { MEP } \\ 8.15 & 3.2 & 22 & 0 & 90 & .09 & 3 & .5 & .8 & 19 & \text { MER }\end{array}$ $\begin{array}{rllllllllll}6.38 & 1.2 & 17 & 0 & 165 & .14 & 4 & 1.0 & 1.2 & 17 & \text { MER } \\ 7.09 & 1.0 & 19 & 0 & 72 & 11 & 5 & .6 & 1.0 & 18 & \text { UEF }\end{array}$

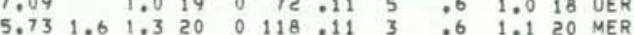
$\begin{array}{llllllllllllll}8.28 & 2.0 & 2.2 & 24 & 0 & 157 & .09 & 2 & .9 & .6 & 23 & \text { MER }\end{array}$

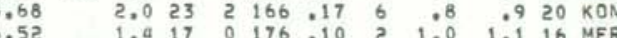

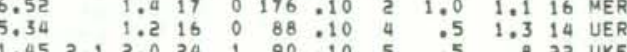
$\begin{array}{llllllllllll}3.19 & 1.6 & 1.8 & 17 & 1 & 62 & .06 & 3 & .3 & .5 & 16 & \mathrm{SPC}\end{array}$

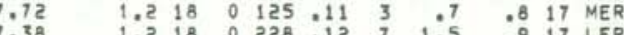

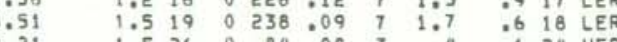
$\begin{array}{llllll}1.52 & 26 & 0 & 84 & 08 & 3\end{array}$ $\begin{array}{lllllllllll}7.83 & 1.3 & 17 & 0 & 172 & 010 & 5 & 1.1 & .8 & 17 & \text { MER } \\ 23 & 1 & 20 & 0 & 91 & 10 & 3 & .5 & 03 & 20 & \text { MER }\end{array}$

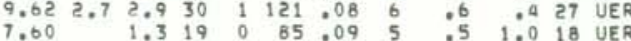

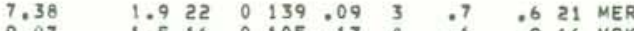

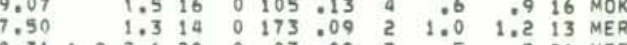

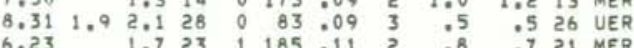
$\begin{array}{lllllllllll}7.52 & 1.0 & 19 & 0 & 116 & .10 & 3 & .6 & .8 & 18 & \text { MER }\end{array}$

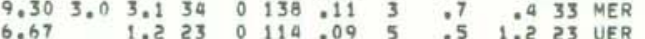

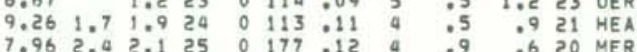
$3.00 \quad 2.0 \quad 1.9 \quad 18 \quad 11113 \quad .10 \quad 5 \quad .6 \quad 1.2 \quad 14$ SWR

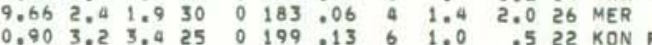
\begin{tabular}{lllllllllll}
.19 & 1.3 & 21 & 0 & 103 & 13 & 4 & .6 & .9 & 16 & MER \\
.56 & 2.1 & 1.9 & 27 & 0 & 105 & .12 & 4 & .6 & .6 & 23 \\
\hline
\end{tabular}

$\begin{array}{llllllllllll}1.17 & 2.9 & 2.2 & 32 & 3 & 291 & .11 & 51 & 3.4 & 5.2 & 28 & \mathrm{KOH}\end{array}$ $\begin{array}{lllllllllllll}7.30 & 1.9 & 1.4 & 17 & 0 & 96 & 11 & 4 & .5 & .7 & 15 & \text { MER } \\ 7.22 & 1.7 & 1.3 & 23 & 0 & 91 & : 11 & 5 & : 5 & : 8 & 19 & \text { UER }\end{array}$ $\begin{array}{rrrrrrrrrrrr}7.81 & 1.3 & 21 & 0 & 83 & .09 & 5 & .5 & .8 & 16 & \text { UER } \\ 8.91 & 2.2 & 2.4 & 30 & 0 & 105 & .09 & 4 & .6 & .4 & 28 & \text { UER }\end{array}$ $\begin{array}{llllllllllll}6.95 & 1.4 & 1.0 & 22 & 0 & 118 & .12 & 3 & .7 & .7 & 18 & \text { MER }\end{array}$

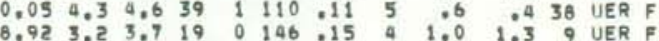
$\begin{array}{llllllllllll}7.02 & & 1.4 & 12 & 0 & 131 & .06 & 5 & .6 & 1.5 & 9 & \text { UER } \\ 7.56 & 1.7 & 1.3 & 18 & 0 & 118 & .07 & 4 & .6 & 1.2 & 13 & \text { UER }\end{array}$

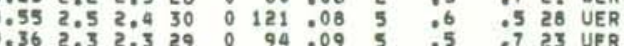

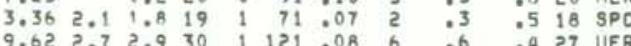
$8.232 .22 .326 \quad 0 \quad 80.0825 \quad .5 \quad .721$ UER 
ORIGIN TIME LATN LON W OEPTH AMP DUR GAP RMS MIN ERH ERZ NO

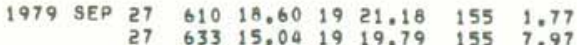
$\begin{array}{lllllll}27 & 633 & 15.04 & 19 & 19.79 & 155 & 7.97 \\ 27 & 734 & 9.95 & 19 & 21.86 & 155 & 5.62\end{array}$

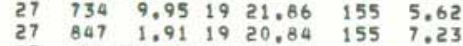
$\begin{array}{lllllll}27 & 848 & 59,65 & 19 & 19,47 & 155 \quad 7,10\end{array}$ $\begin{array}{lccccccc}27 & 9 & 8 & .34 & 19 & 19.33 & 155 & 11.75 \\ 27 & 1035 & 18: 44 & 19 & 19.51 & 155 & 7.46\end{array}$

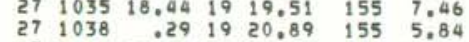

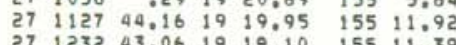

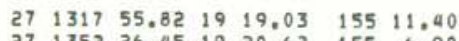
$\begin{array}{lllllll}27 & 1352 & 26.45 & 19 & 20.63 & 155 & 6.90 \\ 27 & 1520 & 56.00 & 19 & 20 & & \end{array}$ $27 \quad 1527 \quad 34.34 \quad 19 \quad 18.76 \quad 155 \quad 23.39$

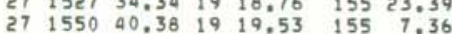

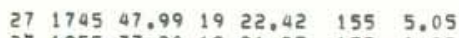
$\begin{array}{lllllll}27 & 1955 & 33.20 & 19 & 21.25 & 155 & 1.82 \\ 27 & 2113 & 30.24 & 19 & 17.25 & 155 & 23.56\end{array}$

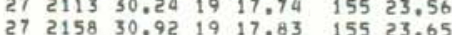

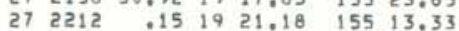
$27 \quad 2249 \quad 51.69 \quad 19 \quad 29.62 \quad 15 a 52.69$ $\begin{array}{lllllllll}28 & 0 & 3 & 18.48 & 19 & 23.09 & 155 & 17.00 \\ 28 & 112 & 23.53 & 10 & 23.40 & 155 & 1.50\end{array}$

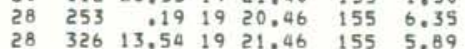
$\begin{array}{lllllll}28 & 345 & 43.59 & 19 & 20.30 & 155 & 12.58\end{array}$ $28 \quad 3405.67 \quad 1920.94 \quad 155 \quad 6.09$ $\begin{array}{lllllll}28 & 450 & 25.06 & 19 & 20.25 & 155 & 9.49 \\ 28 & 733 & 55.77 & 19 & 19.69 & 155 & 7.93\end{array}$

$\begin{array}{lllllll}28 & 1123 & 32.89 & 19 & 20.06 & 155 & 6.49\end{array}$

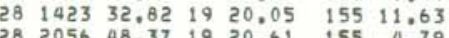
$\begin{array}{rrrrrrr}29 & 112 & 3.01 & 19 & 27.00 & 155 & 28,95 \\ 29 & 123 & 39.55 & 19 & 19.56 & 155 & 10,38\end{array}$ $\begin{array}{lllllll}29 & 624 & 26.16 & 19 & 23.72 & 155 & 16.71\end{array}$ $29 \quad \begin{array}{llllll}4 & 17.88 & 19 & 23.79 & 155 & 16.82\end{array}$

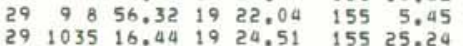
$\begin{array}{lllllll}29 & 1050 & 40.08 & 19 & 18.45 & 155 & 13.57\end{array}$

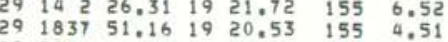

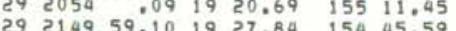

$\begin{array}{lllllll}29 & 2157 & 25.64 & 19 & 27.49 & 154 & 44.66\end{array}$

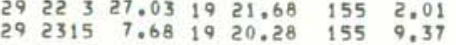
$\begin{array}{llllllllllll}6.99 & 2.5 & 2.3 & 23 & 0 & 177 & 10 & 3 & .7 & .7 & 18 & \text { MER } \\ 8.91 & 2.4 & 2.3 & 31 & 0 & 91 & 0.08 & 5 & .4 & .7 & 27 & \text { UER }\end{array}$

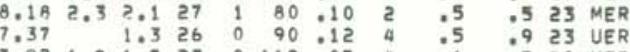
$\begin{array}{lllllllllll}4.77 & 1.1 & 18 & 0 & 98 & -10 & 5 & .5 & 2.2 & 16 & \text { UER }\end{array}$

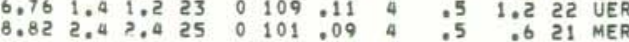
$8.182 .02 .2 \quad 27 \quad 0 \quad 83.08 \quad 5 \quad .450 .626$ UER $\begin{array}{lllllllllll}5.21 & 1.0 & 20 & 0 & 108 & -10 & 5 & .6 & 1.8 & 18 & \text { UER }\end{array}$

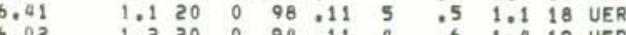

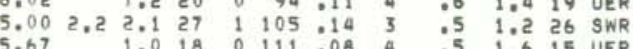

$\begin{array}{lllllllllll}7.72 & 1.9 & 1.8 & 27 & 0 & 76 & .10 & 2 & .5 & .6 & 25 \\ 0.55 & \text { MER }\end{array}$

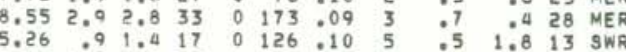

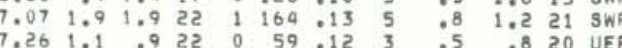

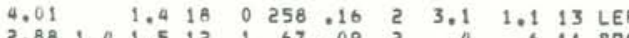

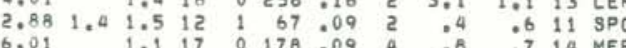

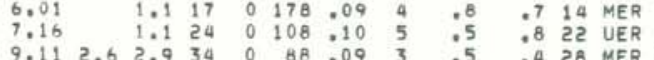

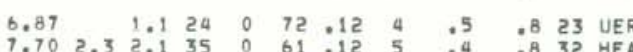

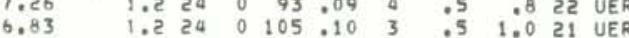
\begin{tabular}{lllllllllll}
8.68 & 1.9 & 2.2 & 27 & 0 & 94 & .09 & 4 & .6 & .6 & 25 \\
\hline & $5 E R$
\end{tabular} $\begin{array}{llllllllllll}7.65 & 2.2 & 1.9 & 32 & 1 & 116 & .09 & 5 & .4 & .8 & 22 & \text { UER }\end{array}$

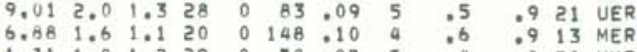

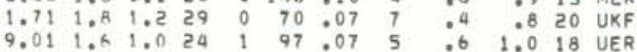
$\begin{array}{lllllllllllll}2.65 & 1.1 & 1.1 & 14 & 1 & 52.07 & 0 & .4 & .3 & 8 & \mathrm{SPC}\end{array}$

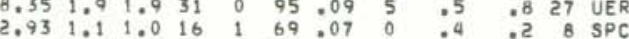

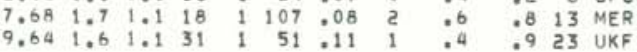

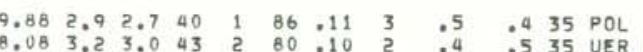

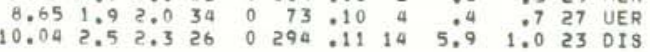
$\begin{array}{llllllllllll}9.06 & 2.1 & 1.7 & 21 & 1 & 301 & .09 & 16 & 2.5 & .6 & 11 & \text { DIS }\end{array}$

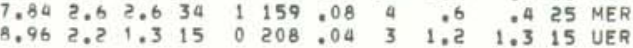
7.26
7.3 $8.20 \quad 2,1 \quad 2.133 \quad 0 \quad 114 \quad .08 \quad 3 \quad$ is 5 :5 29 MER

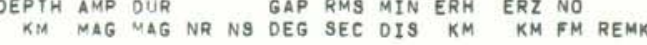
YEAR MON ORA HRMN TIME LEAT N DEG LON W

1979 SEP $30 \quad 110 \quad 19.87 \quad 1920.42 \quad 155 \quad 11.62$

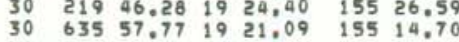
$\begin{array}{lllllll}30 & 1021 & 4.33 & 19 & 19.69 & 155 & 11.43 \\ 30 & 1410 & 24.29 & 19 & 19.55 & 155 & 14.02\end{array}$

$\begin{array}{lllllll}30 & 1513 & 44.69 & 19 & 19.85 & 155 & 6.23\end{array}$

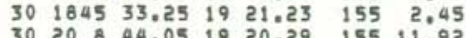

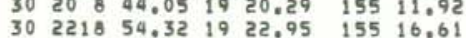

OCT

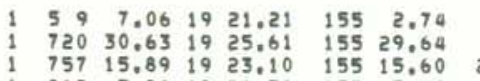
$812 \quad 3.281921 .51 \quad 155 \quad 3.10$ $0.65 \quad 155 \quad 3.91$

$\begin{array}{rrrrrr}844 & 16.57 & 19 & 19.82 & 155 & 6.17 \\ 1852 & 14.02 & 19 & 19.19 & 155 & 9.73\end{array}$

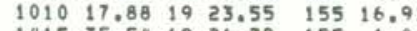

$142353.18 \quad 19 \quad 19.55 \quad 155 \quad 1.04$

$\begin{array}{rrrrrrr}1 & 1427 & 2.41 & 19 & 22.12 & 155 & 5.74 \\ 1 & 1511 & 44.80 & 19 & 18.19 & 155 & 13.43\end{array}$

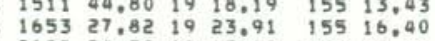

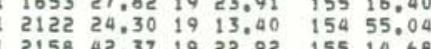
$\begin{array}{lllllll}1 & 2159 & 11.71 & 19 & 22.94 & 155 & 14.47\end{array}$

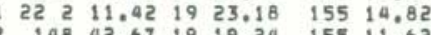
\begin{tabular}{l}
$152 \quad 51.95 \quad 19 \quad 19.36 \quad 155 \quad 11.38$ \\
\hline
\end{tabular} $226 \quad 58.75 \quad 1920,77 \quad 155 \quad 12.65$

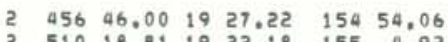

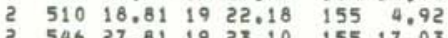
$2610 \quad 17.62 \quad 1929.38 \quad 154 \quad 55.68$ $39.00 \quad 1921.64 \quad 155 \quad 6.40$ $\begin{array}{rrrrrrr}2 & 951 & 28.82 & 19 & 24.49 & 155 & 25.33 \\ 2 & 1416 & 20.04 & 19 & 11.50 & 155 & 37.74\end{array}$

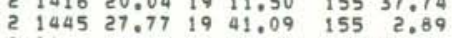

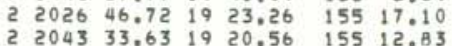

$\begin{array}{llllll}2 & 2357 \quad 52.76 \quad 19 & 21.17 \quad 155 \quad 7.68\end{array}$ $305922.22 \quad 19 \quad 23.54 \quad 155 \quad 16.90$

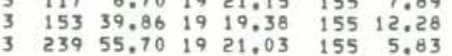

$\begin{array}{lllllll}3 & 459 & 35.14 & 19 & 23.06 & 155 & 2.41\end{array}$ $1229 \quad 0.73 \quad 19 \quad 19.61 \quad 155 \quad 11.90$

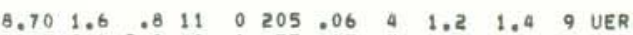

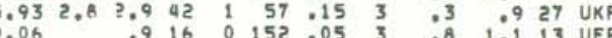

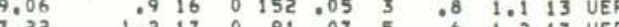

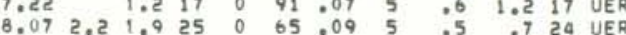
$\begin{array}{lllllllllllll}7.60 & 1.4 & 1.2 & 16 & 0 & 126 & .09 & 6 & .7 & 1.5 & 16 & \text { UER }\end{array}$ \begin{tabular}{lllllllllll}
.82 & 2.1 & 1.7 & 18 & 0 & 152 & 09 & 3 & .7 & .7 & 16 \\
\hline
\end{tabular}

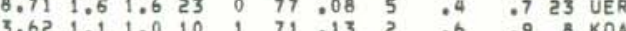

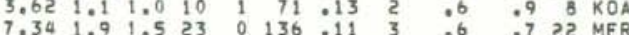

$\begin{array}{lllllllllllllll}7.37 & 2.1 & 2.0 & 28 & 0 & 141 & .10 & 2 & .6 & .5 & 26 & \text { MER }\end{array}$

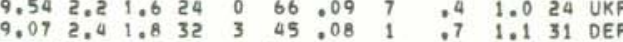

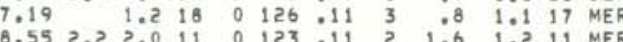
$\begin{array}{lllllllllllll}7.19 & 2.0 & 1.6 & 18 & 0 & 127 & .09 & 6 & .6 & 1.5 & 18 & \text { UER } \\ 6.96 & & 1.3 & 18 & 0 & 102 & 09 & 5 & .5 & 1.4 & 18 & \text { UER }\end{array}$ $\begin{array}{llllllllllll}3.21 & 2.6 & 3.0 & 36 & 1 & 44 & .09 & 3 & .3 & .5 & 25 & \text { SPC }\end{array}$

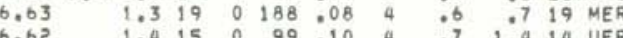

$\begin{array}{llllllllllll}7.50 & 1.9 & 1.6 & 23 & 1 & 99 & .10 & 2 & .5 & 7 & 21 & \text { MER } \\ 8.03 & 9 & 1.0 & 13 & 0 & 86 & : 10 & 2 & : 7 & 9 & 13 & \text { POL }\end{array}$

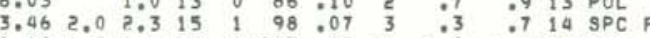

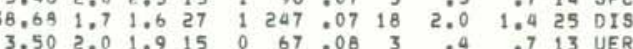
$\begin{array}{llllllllllll}4.83 & 2.6 & 2.9 & 14 & 1 & 58 & .09 & 3 & .4 & 2.0 & 13 & \text { UER F }\end{array}$ $\begin{array}{lllllllll}4.7 & 13 & 6 L N\end{array}$

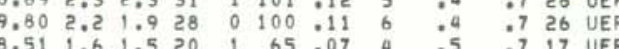

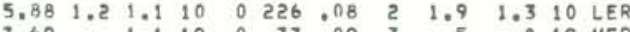

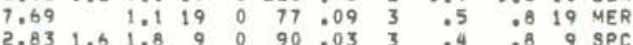

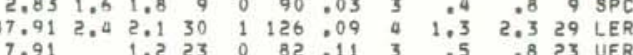
$\begin{array}{llllllllllll}0.05 & 2.3 & 2.1 & 25 & 0 & 54 & .11 & 5 & .4 & .8 & 22 & \text { UKF }\end{array}$

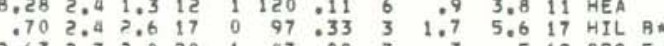

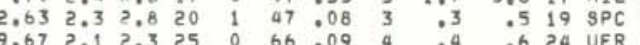
$\begin{array}{lllllllllll}8.02 & 1.0 & 21 & 0 & 79 & .10 & 4 & .8 & .7 & 21 & \text { UER }\end{array}$

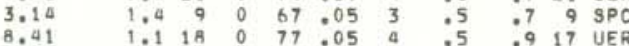
\begin{tabular}{lllllllllll}
9.28 & 1.0 & 14 & 0 & 90 & 06 & 5 & .06 & 1.95 & 14 & UER \\
9.44 & 2.7 & 2.5 & 26 & 0 & 97 & .10 & 4 & .6 & .5 & 26 \\
\hline
\end{tabular} $6.751 .3 \quad 1.11900131 \quad 12 \quad 4 \quad .6 \quad 1.117$ MER

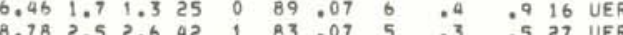


HVO EARTHQUAKE SUMMARY LIST

PAGE 75

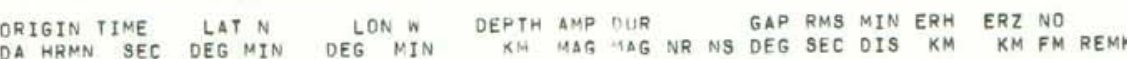

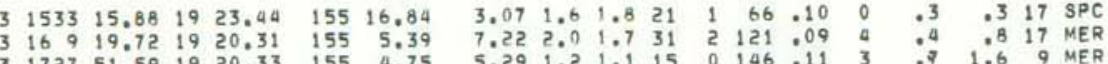
$\begin{array}{llllll}1727 & 51.59 & 19 & 20.33 & 155 & 4.75\end{array}$ 3 (1743 46.421920 .901556 .86

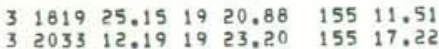
32040
3 $2.001923 .06 \quad 15516.83$ 33204331.301923 .19 155 17.10

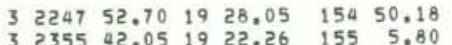

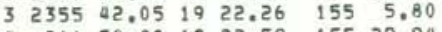
$4 \quad 21650.081922 .59 \quad 15529.94$ $\begin{array}{llllll}750 & 5.68 & 19 & 19.84 & 155 & 12.03\end{array}$

$\begin{array}{lllllll}4 & 837 & 35.91 & 19 & 23.81 & 155 & 15.37 \\ 4 & 845 & 14.60 & 19 & 24.44 & 155 & 16.23\end{array}$ $912 \quad 35.791923 .13 \quad 15514,82$ $4 \quad 1059 \quad 26.72 \quad 1923.03 \quad 155 \quad 14.75$

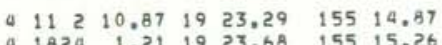

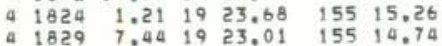

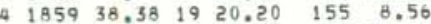
195737.9819
$16.83 \quad 15521.60$ $1957 \quad 53.46 \quad 19 \quad 22.86 \quad 155 \quad 14.99$

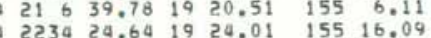
$\begin{array}{rrrrrrr}5 & 015 & 4.84 & 19 & 21.22 & 155 & 2.42 \\ 5 & 1 & 1.49 .35 & 19 & 23.48 & 155 & 16.78\end{array}$

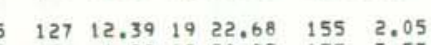

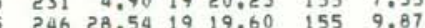

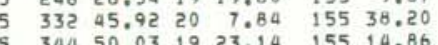

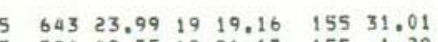

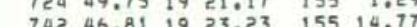
5
5

$\begin{array}{lllllll}6 & 046 & 12.24 & 19 & 20.15 & 155 & 12.90\end{array}$

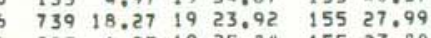

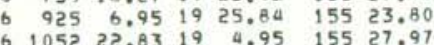

$1159 \quad .06 \quad 19 \quad 13.09 \quad 155 \quad 32.64$

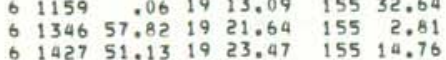
$\begin{array}{lllllllllllll}7.14 & 1.1 & 1.3 & 25 & 1 & 93 & .11 & 4 & .5 & 1.1 & 15 & \text { UER } \\ 8.04 & 2.3 & 2.3 & 35 & 1 & 134 & .09 & 2 & .5 & .5 & 26 & \text { MER }\end{array}$ $\begin{array}{lllllllllllll}9.91 & 1.8 & 1.0 & 13 & 0 & 70 & .03 & 4 & .5 & 1.1 & 10 & \text { UER }\end{array}$

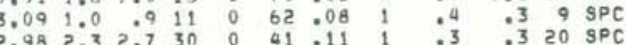

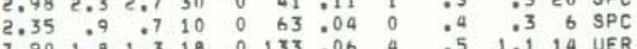

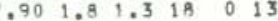
$\begin{array}{llllllllllll}.01 & 2.4 & 2.3 & 21 & 2 & 273 & .23 & 6 & 1.2 & .5 & 13 & \text { LER }\end{array}$

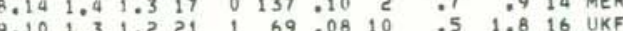

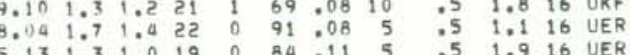
$5.131 .31 .019 \quad 0 \quad 80$ $\begin{array}{rrrrrrrrrrr}3.00 & 1.0 & 1.3 & 11 & 1 & 99 & .06 & 2 & .4 & .5 & 9 \mathrm{SPC}\end{array}$

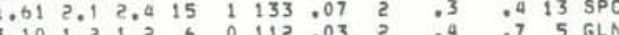

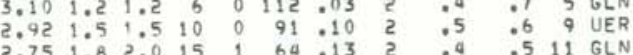

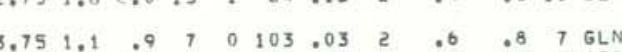
$\begin{array}{rrrrrrrrrrrr}3.75 & 1.1 & .9 & 7 & 0 & 103 & 03 & 2 & .6 & .8 & 7 & G L N \\ 2.95 & 1.0 & 1.2 & 6 & 0 & 97 & .04 & 2 & .5 & 09 & 5 & 5 P C\end{array}$

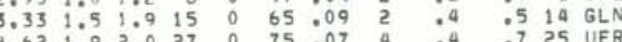

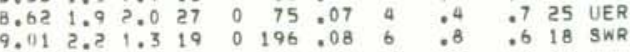
$\begin{array}{lllllllllllll}3.82 & 1.5 & 1.8 & 9 & 0 & 117 & .13 & 2 & .6 & .9 & 8 & \text { UER }\end{array}$ $\begin{array}{rllllll:lllll}6.24 & 1.6 & 1.4 & 23 & 0 & 154 & 11 & 3 & .7 & .8 & 19 & \text { MER } \\ 2.52 & 1.9 & 2.2 & 22 & 2 & 52 & .13 & 3 & .3 & .04 & 20 & \text { SPC }\end{array}$ $\begin{array}{llllllllllll}7.23 & 1.3 & 1.4 & 22 & 1 & 144 & .12 & 5 & .8 & .6 & 19 & \text { MER }\end{array}$

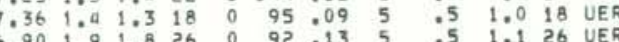

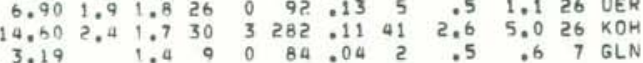

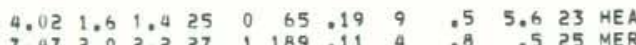
$\begin{array}{lllllllllll}3.22 & 1.1 & 9 & 0 & 8 B & 04 & 3 & .4 & .6 & 8 & G L N\end{array}$

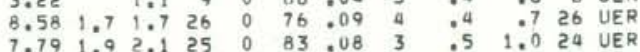

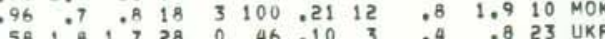
$\begin{array}{llllllllllll}0.45 & 2.0 & 1.8 & 24 & 1 & 217 & .13 & 6 & .7 & .9 & 9 & \text { LSW }\end{array}$

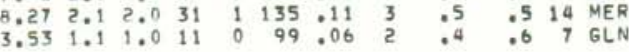

HYO EARTHQUAKE SUMMARY LIST

PAGE 76

ORIGIN TIME LAT N
YEAR MON DA HRMN SEC DEG MIN DEG MIN DEPTH AMP RIR N MAP RMS MIN ERH ERZ NO

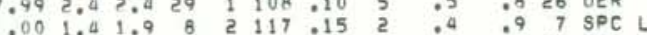

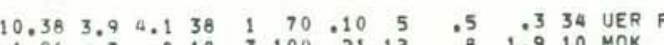

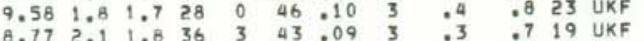

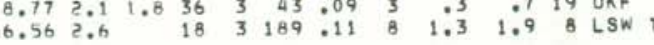

1979 OCT $6 \quad 143932.58 \quad 1923.23 \quad 155 \quad 14.90$

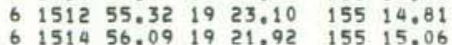
$6153145.651924 .53 \quad 15526.02$ $\begin{array}{llllll}6 & 16 \quad 8 \quad 21.41 & 19 & 23.07 \quad 155 & 14.78\end{array}$

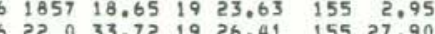

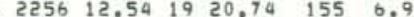

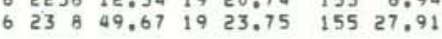

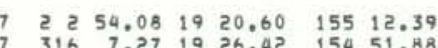

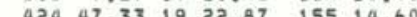
$\begin{array}{llllll}527 & 1.79 & 19 & 23,39 & 155 & 14.90\end{array}$ $\begin{array}{llllll}536 & 42.51 & 19 & 22.11 & 155 & 1.23\end{array}$

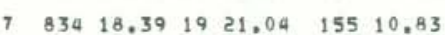

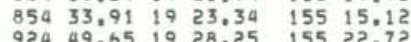
$\begin{array}{llllll}7833 & 57.89 & 19 & 26.30 & 155 & 25.03 \\ 72241 & 30.79 & 19 & 13.51 & 155 & 37.66\end{array}$

$\begin{array}{lllllll}8 & 322 & 21.73 \quad 19 & 19.25 & 155 & 11.23\end{array}$ $64137.401920 .68 \quad 155 \quad 5.90$

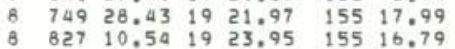
$\begin{array}{llllll}8 & 1138 & 26.93 & 19 & 22.12 \quad 155 & 2.18\end{array}$ B $1321 \quad 43.491920 .57 \quad 155 \quad 5.84$ $8 \quad 135736.73 \quad 1920.33 \quad 155 \quad 9.60$
8 $\begin{array}{lllllr}8 & 1424 & 56.85 & 1926.20 & 154 & 55.31 \\ 8 & 1553 & 54.32 & 1921.50 & 155 & 5.59\end{array}$

$\begin{array}{lllllll}8 & 1640 & 19.80 & 19 & 19.20 & 155 & 11.35\end{array}$

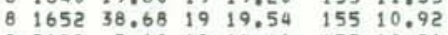
$\begin{array}{lllllll}8 & 2122 & 2.10 & 19 & 19.41 & 155 & 10.81 \\ 8 & 2231 & 34.71 & 19 & 13.46 & 155 & 39.78\end{array}$

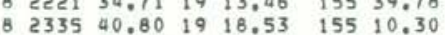
$\begin{array}{rrrrrrr}8 & 2345 & 42.71 & 19 & 21.17 & 155 & 5.68 \\ 9 & 425 & 9.32 & 20 & 27.14 & 155 & 15.37\end{array}$ $9 \quad 43452.391921 .50 \quad 155 \quad 15.28$ 9. $72923.471920 .02 \quad 15515.79$ $9 \begin{array}{llllll}1140 & 23.26 \quad 19 & 22.16 \quad 155 \quad 5.87\end{array}$ $9 \begin{aligned} & 114352.12 \quad 1922.33 \quad 155 \quad 5.72 \\ & 9 \\ & 125454.59\end{aligned}$ $9125454.591920 .69 \quad 155 \quad 5.65$

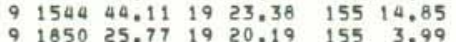

$92341 \quad 55.36 \quad 1923.19 \quad 155 \quad 14.79$

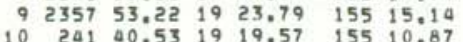

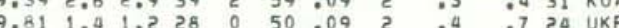
$\begin{array}{lllllllllllll}3.45 & 1.1 & 1.0 & 10 & 0 & 111 & .05 & 2 & .5 & .6 & 8 & \text { GLN }\end{array}$

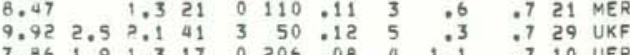

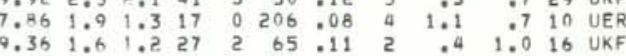

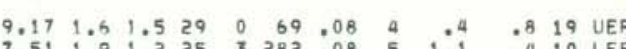

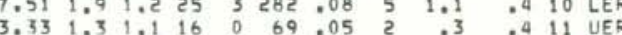

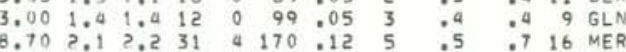

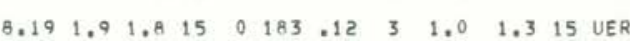

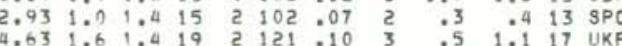
$\begin{array}{rrrrrrrrrrrr}6.55 & 1.8 & 1.2 & 19 & 1 & 63 & 08 & 2 & .5 & 1.1 & 18 & \text { UKF } \\ 8.39 & 2.4 & 2.3 & 32 & 2 & 199 & .18 & 3 & .7 & .7 & 28 & \text { HEA }\end{array}$

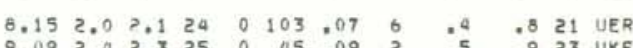

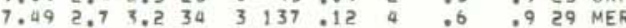
$\begin{array}{lllllllllllll}2.89 & 1.2 & 1.2 & 13 & 1 & 88 & 09 & 3 & .4 & .7 & 11 & K O A \\ 3.16 & & 1.7 & 9 & 0 & 84 & .06 & 2 & .5 & .6 & 8 & \text { SPC }\end{array}$ $\begin{array}{lllllllllll}7.00 & 1.7 & 1.6 & 19 & 0 & 148 & .11 & 4 & .8 & .5 & 19\end{array}$ MER

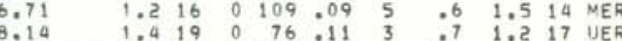

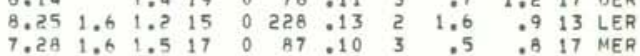
$\begin{array}{llllllllllll}10.14 & 3.7 & 3.7 & 29 & 0 & 104 & .09 & 5 & .5 & .4 & 29 & \text { UER F }\end{array}$

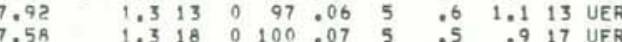

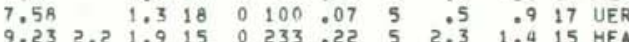
$\begin{array}{llllllllllll}7.23 & 2.2 & 1.9 & 15 & 0 & 233 & .22 & 5 & 2.3 & 1.4 & 15 & \text { HEA } \\ 7.1 & 15 & 0 & 127 & .11 & 5 & 1.0 & 1.4 & 14 & \text { POL }\end{array}$

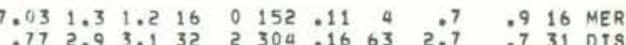

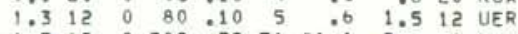
$\begin{array}{lllllllllll}8.57 & 1.0 & 15 & 0 & 74 & .07 & 2 & .5 & 1.1 & 15 & \text { MER }\end{array}$

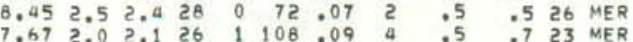
$3.66 \quad 1.81 .912$ O $80.05 \quad 3 \quad: 4 \quad .6$ 12 GLN

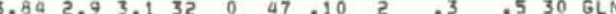

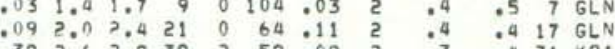
$\begin{array}{llllllllllllll}2.69 & 1.1 & 1.3 & 17 & 1 & 61 & .12 & 1 & .3 & .3 & 12 & \text { SPC }\end{array}$

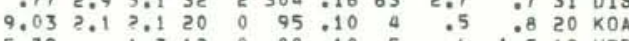

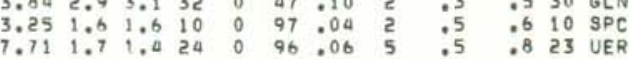


ORIGIN TIME LATN LON $K$ DEPIH AMP OIIR N GAP RMS MIN ERH ERZ NO
DA HRMN SEC DEG MIN DEG MIN KM MAG MAG NR NS OEG SEC DIS KM KM FM REMK

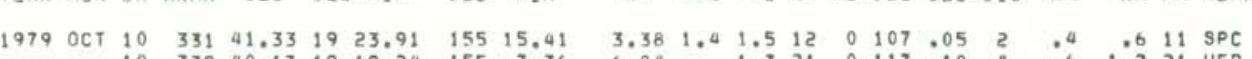

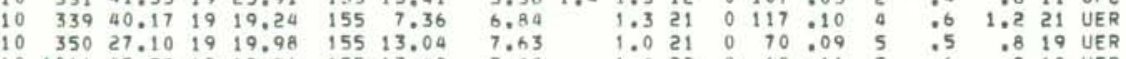

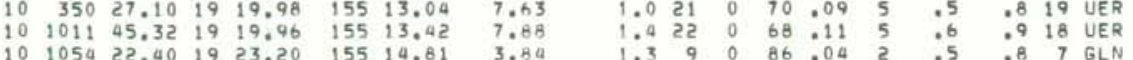
$\begin{array}{llllllllllllllllll}10 & 1059 & 28.19 & 19 & 19.76 & 155 & 11.24 & 8.15 & 2.6 & 3.0 & 30 & 1 & 91 & .10 & 5 & .4 & .6 & 21 \\ 1 & \text { UER }\end{array}$

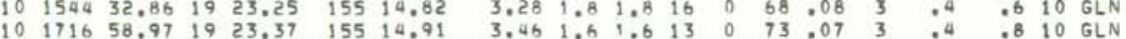

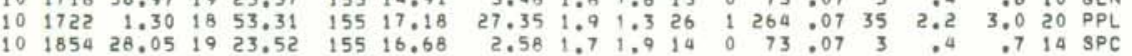
$\begin{array}{lllllll}10 & 1916 \quad 4.54 & 19 & 23.23 \quad 155 & 14.68\end{array}$ $10193720.891922 .91 \quad 15514.79$

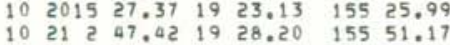

$1022>45.61 \quad 19 \quad 19.70 \quad 15511.10$ 10 $2221 \quad 45.16 \quad 19 \quad 19.31 \quad 15511.80$

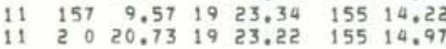
$11 \quad 34129.16 \quad 1926.45 \quad 155 \quad 27.28$ $11 \quad 532 \quad 37.42 \quad 1927.28 \quad 155$ 15.99

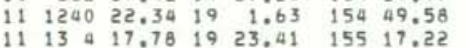
$\begin{array}{llllllll}11 & 13 & 737.96 & 19 & 23.43 & 155 & 16.94\end{array}$ 11 13 $8 \quad 15.42 \quad 1923.44 \quad 155 \quad 17.09$ $111416 \quad 13.22 \quad 1920.59 \quad 155 \quad 5.82$ $11.165159 .691923,20 \quad 15515.15$

$\begin{array}{lllllll}11 & 2033 & 37.27 & 19 & 22.16 & 155 & 1.19\end{array}$ 11 2055 $29.251920 .18 \quad 155 \quad 6.03$

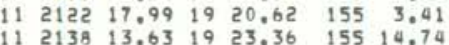

$12 \quad 014 \quad 5.76 \quad 19 \quad 18.96 \quad 155 \quad 16.12$

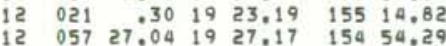
$\begin{array}{lllll}12 & 057 & 27.04 & 19 & 27.17 \\ 12 & 314 & 48,84 & 154 & 54,29 \\ 12 & 23,07 & 155 & 14,86\end{array}$ $12 \quad 4603.91$ i9 $52,49 \quad 15518,93$ $\begin{array}{rrrrrrr}12 & 422 & 5.46 & 19 & 21.76 & 155 & 13.09 \\ 12 & 510 & 13.59 & 19 & 50.20 & 155 & 46.26\end{array}$ $1261415.141926 .43 \quad 15521.66$ $12 \quad 7479.97 \quad 1923.54 \quad 155 \quad 30,38$ $\begin{array}{rrrrrrr}12 & 849 & 53.70 & 19 & 22.00 & 155 & 13.47 \\ 12 & 1110 & 30.95 & 19 & 23.09 & 155 & 14.84\end{array}$

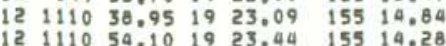

$\begin{array}{lllllllllllll}3.83 & 1.6 & 1.9 & 9 & 0 & 91 & .04 & 3 & .6 & 1.1 & 8 & \text { GLN } \\ 3.29 & .5 & 1.9 & 13 & 0 & 67 & .08 & 3 & 0 & .7 & 10 & \text { UER }\end{array}$

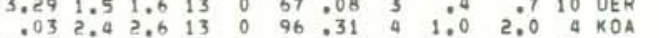
$\begin{array}{llllllllllll}9.48 & 2.2 & 2.1 & 32 & 0 & 51 & .12 & 3 & .4 & .8 & 25 & \mathrm{UKF} \\ 8.55 & 1.4 & 1.4 & 18 & 1 & 152 & .16 & 7 & .9 & .9 & 15 & \mathrm{KON}\end{array}$ $\begin{array}{llllllllllllll}9.07 & 2.1 & 2.2 & 26 & 0 & 92 & .10 & 5 & .5 & .7 & 22 & \text { UER }\end{array}$

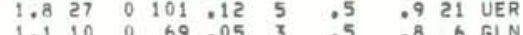

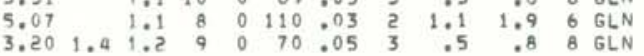

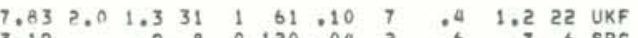

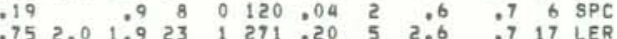

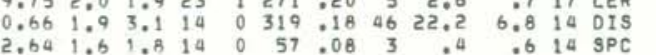
$\begin{array}{llllllllllll}2.78 & 1.3 & 1.5 & 9 & 0 & 114 & .05 & 3 & .4 & .6 & 8 & \mathrm{SPC}\end{array}$ $\begin{array}{llllllllll}.77 & 1.0 & 16 & 0 & 109 & 06 & 5 & .6 & 1.2 & 16 \\ 3 & \text { MER }\end{array}$

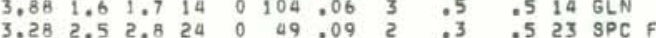

$\begin{array}{lllllllllll}2.91 & 1.3 & 13 & 0 & 171 & .11 & 5 & .6 & 1.5 & 13 & \text { MER }\end{array}$

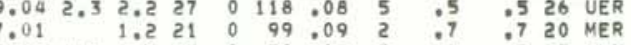

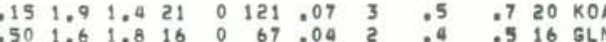
4.781 .21 .215 o $216: 12$ द 1.9 :8 15 LER

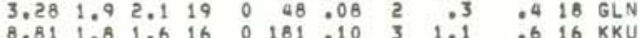
$\begin{array}{llllllllllll}2.91 & & 1.0 & 10 & 0 & 101 & 03 & 2 & .03 & .5 & 10 & \text { UER } \\ 24.99 & 2.6 & 2.0 & 19 & 1 & 155 & .06 & 18 & 0 & 0.1 & 18 & \text { KON }\end{array}$

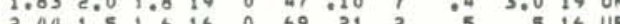

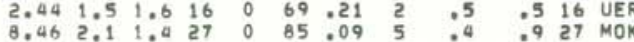
$\begin{array}{lllllllllll}2.85 & .9 & 8 & 0 & 143 & .03 & 1 & .9 & .4 & 5 & 5 E R \\ 0.61 & 1.0 & 6 & 0 & 153 & .02 & 2 & 1.6 & .5 & 5 & 6 L N\end{array}$

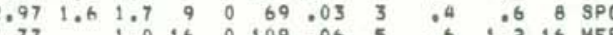
$\begin{array}{llllllllllll}9.03 & 1.6 & 1.5 & 24 & 0 & 68 & .09 & 6 & .5 & 1.0 & 24 & \text { URF }\end{array}$

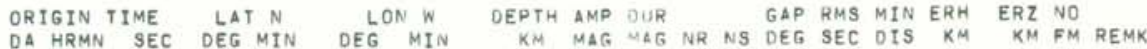

1979 OCT $12 \quad 1113 \quad 7.21 \quad 1923.12 \quad 15514.86$

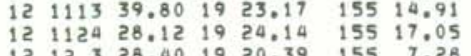

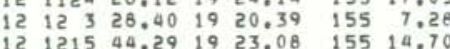
$12 \quad 1216 \quad 14.641923 .97 \quad 155 \quad 15.48$ $12133938,451923,27 \quad 155$ 14 14.89 12155524.801926 .74 155 21.41 $12155925.76 \quad 1923.04 \quad 155 \quad 14.76$ $12 \quad 1616 \quad 32,47 \quad 1923,30 \quad 155 \quad 14,85$

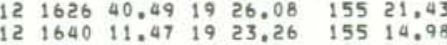
$\begin{array}{lllllll}12 & 1753 & 29.02 & 19 & 23,20 & 155 & 14.90\end{array}$ \begin{tabular}{l}
$1812 \quad 25.97 \quad 1923.11 \quad 155 \quad 16.33$ \\
\hline
\end{tabular} $12181832.441923 .15 \quad 15514.73$ $\begin{array}{llllllll}12 & 19 & 80.25 & 19 & 21.37 & 155 & 4.41\end{array}$ $\begin{array}{llllllll}12 & 2056 & 43.17 & 19 & 17.51 & 155 & 14.1 & 14 \\ 13 & 052 & 48.39 & 19 & 21.74 & 155 & 5.9\end{array}$

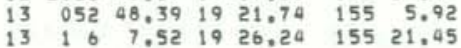
$\begin{array}{lllllll}13 & 116 & 26.04 & 19 & 26.68 & 155 & 20.97 \\ 13 & 151 & 48.24 & 19 & 26.49 & 155 & 21.60\end{array}$ $\begin{array}{lllllll}13 & 224 & 53.75 & 19 & 26.20 & 155 & 21.53 \\ 13 & 258 & 51.02 & 19 & 27.05 & 155 & 21.55\end{array}$ $13 \quad 35141.69 \quad 1922,97 \quad 15514,82$

$\begin{array}{lllllll}13 & 652 & 39.07 & 19 & 20.88 & 155 & 2.62\end{array}$

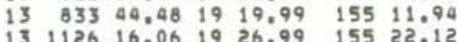

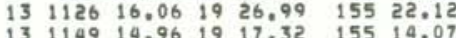
$13120258,101920.96 \quad 155 \quad 5.87$

$\begin{array}{llllllll}13 & 12 & 4 & 57.84 & 19 & 23.30 & 155 & 16,98\end{array}$ $\begin{array}{llllllll}13 & 12 & 5 & 57.36 & 19 & 23.43 & 155 & 16.93 \\ 13 & 1233 & 31.71 & 19 & 17.88 & 155 & 14.19\end{array}$

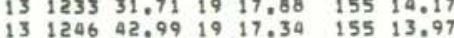
$\begin{array}{lllllll}13 & 1246 & 42.99 & 19 & 17.34 & 155 & 13.97 \\ 13 & 1248 & 33.01 & 19 & 17.39 & 155 & 19.15\end{array}$

$13 \quad 135021.56 \quad 19 \quad 17.45 \quad 155 \quad 14.15$

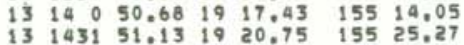
13 1442 $47.401918 .12 \quad 15512.99$ 15512.99 $\begin{array}{rrrrrrr}13 & 1446 & 34.27 & 19 & 17.42 & 155 & 12.99 \\ 13 & 1447 & 9.97 & 19 & 16.97 & 155 & 12.67\end{array}$ $13145148.22 \quad 19 \begin{array}{lll}17.84 & 155 & 12.77\end{array}$

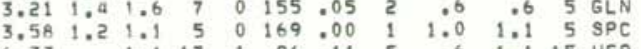
$\begin{array}{rrrrrrrrrrrr}6.33 & & 1.1 & 17 & 1 & 96 & .11 & 5 & .6 & 1.1 & 15 & \text { UER } \\ 3.17 & 1.5 & 1.5 & 12 & 0 & 130 & .06 & 2 & .4 & .4 & 10 & \text { GLN }\end{array}$ $\begin{array}{llllllllllllllll}3.22 & 1.5 & 1.8 & 9 & 0 & 117 & .05 & 3 & .5 & .7 & 8 & \mathrm{SPC}\end{array}$

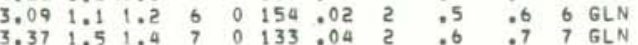

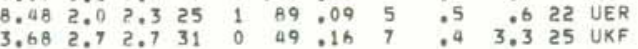

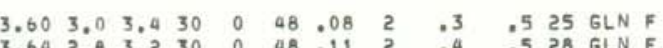

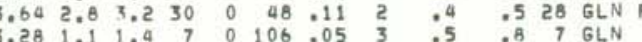
$\begin{array}{lllllllllll}.81 & 2.1 & 2.2 & 22 & 0 & 48 & .15 & 8 & .5 & 3.8 & 20 \\ 3 & \text { UKF }\end{array}$ $\begin{array}{llllllllllll}3.14 & 1.8 & 1.6 & 12 & 0 & 69 & .06 & 2 & .4 & .5 & 11 & \text { GLN }\end{array}$

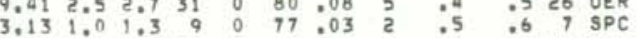

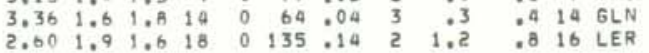
\begin{tabular}{lllllllllllll}
7.67 & 1.9 & 1.3 & 22 & 0 & 84 & .10 & 4 & .5 & .7 & 18 & MER \\
\hline
\end{tabular}

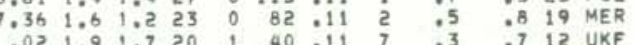

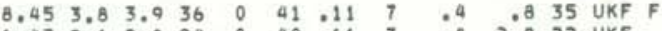

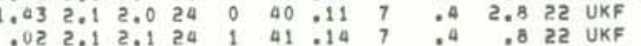

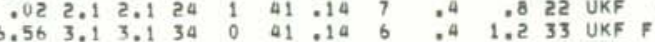

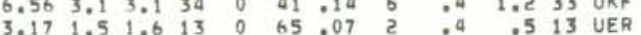

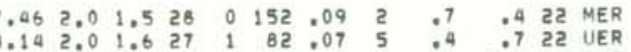

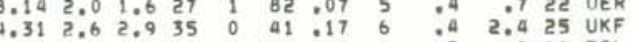

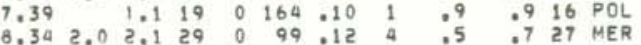

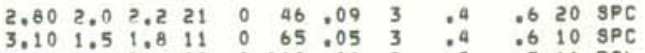

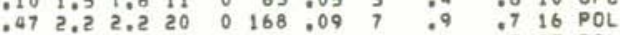
$\begin{array}{lllllllllllll}8.09 & 1.6 & 1.4 & 26 & 0 & 118 & 10 & 1 & .6 & 7 & 23 & \text { POL } \\ 7.94 & 1.7 & 1.7 & 22 & 0 & 146 & 10 & 1 & 9 & 0 & 18 & \text { POL }\end{array}$

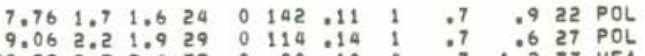

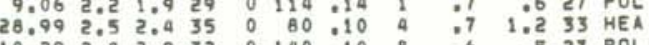

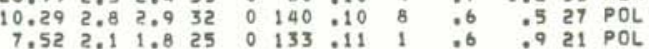

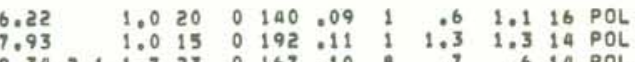

$\begin{array}{llllllllllll}3.09 & 1.1 & .9 & 6 & 0 & 154 & 03 & 2 & .6 & .6 & 6 & 6 L N\end{array}$

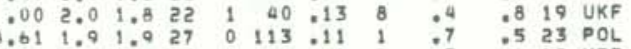


ORIGIN TIME
DA HRMN SEE DEG MIN DEG MIN TON W DEPTH AMP OUR $\begin{array}{llllllll}1979 & \text { OCT } 13 \quad 1647 & 36.27 & 19 & 26.51 & 155 & 21.65\end{array}$

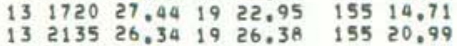

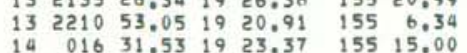

$\begin{array}{lllllll}14 & 148 & 44.07 \quad 19 & 27.09 \quad 155 & 21.65\end{array}$ $14 \quad 157 \quad 1.591923 .57 \quad 15514.93$ $14 \quad 251 \quad 37.601927 .17 \quad 15520.97$

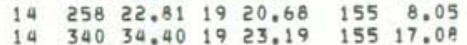

$\begin{array}{lllllllll}14 & 6 & 5 & 10.27 & 19 & 18.47 & 155 & 13.67\end{array}$ $\begin{array}{lllllll}14 & 737 & 16.92 & 19 & 54.38 & 155 & 10.73 \\ 14 & 947 & 50.17 & 19 & 22.13 & 155 & 1\end{array}$ $1410148.691920 .40 \quad 15512.81$

$\begin{array}{lllllll}14 & 1215 & 40.56 & 19 & 22.69 & 155 & 14.36\end{array}$

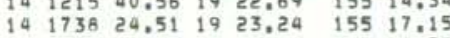

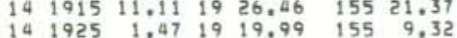
$142046 \quad 9,241921,58 \quad 155 \quad 5,39$

$\begin{array}{lllllll}14 & 2143 & 58.24 & 19 & 18.91 & 155 & 11.74 \\ 14 & 2235 & 7.85 & 19 & 26.59 & 155 & 21.26\end{array}$ $14225434.62 \quad 1926.63 \quad 15521.25$ $\begin{array}{llllll}14 & 2340 & 18.75 \quad 19 & 21.97 \quad 155 & 25.53\end{array}$

$\begin{array}{lllllll}15 & 015 & 53.37 & 19 & 26.49 & 155 & 20.28 \\ 15 & 032 & 56.34 & 19 & 26.54 & 155 & 20.45\end{array}$ $\begin{array}{lllllll}15 & 032 & 56.34 & 19 & 26.54 & 155 & 20.45 \\ 15 & 044 & 12.27 & 19 & 55.75 & 156 & 27.75\end{array}$ $1505756.921923 .27 \quad 155 \quad 15.41$

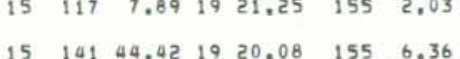

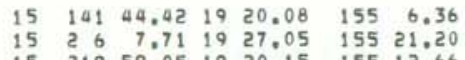
$15 \quad 21959.051920 .15$ 155 12.66

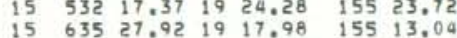

$\begin{array}{rrrrrrr}15 & 744 & 8.73 & 19 & 20.11 & 155 & 6.16 \\ 15 & 943 & 15.76 & 19 & 27.55 & 154 & 52.61\end{array}$

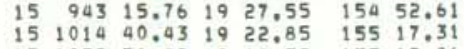
$\begin{array}{lllllll}15 & 1032 & 51.49 & 19 & 19.59 & 155 & 12.86 \\ 15 & 1146 & 46.62 & 19 & 20.68 & 155 & 11.14\end{array}$

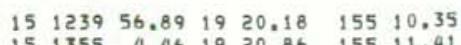
$151355 \quad 4.461920 .86 \quad 15511.41$ $15 \quad 1536 \quad 30.9919 \quad 27.08 \quad 155 \quad 21.36$

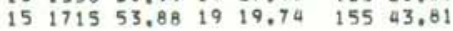

$\begin{array}{lllllll}15 & 2112 & 12.78 & 19 & 20.01 & 155 & 13.46 \\ 15 & 2357 & 55.92 & 19 & 20.04 & 155 & 13.46\end{array}$

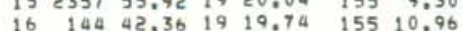

$\begin{array}{llllllllllllllll}.02 & 1.7 & 1.6 & 11 & 0 & 102 & 17 & 7 & .7 & 3.8 & 10 & \text { UKF } & 0\end{array}$

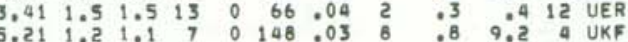
6.951 .91 .2511009 .12 4 51.51 .021 UER

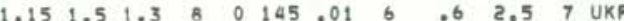

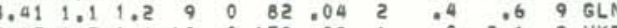

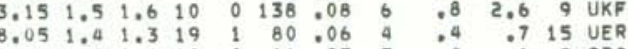

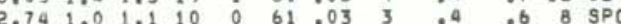
$\begin{array}{rrrrrrrrrrrr}8.60 & 2.2 & 2.0 & 29 & 0 & 89 & 10 & 3 & .6 & .5 & 26 & \text { POL F } \\ 0.57 & 9.0 & 0.3 & 38 & 1 & 213 & .10 & 17 & 1.0 & 1.7 & 37 & \mathrm{KKU} F\end{array}$ $\begin{array}{lllllllllll}0.73 & 2.0 & 1.8 & 25 & 1 & 163 & 0.10 & 5 & .6 & .7 & 22 \\ 0.9 & \text { MER }\end{array}$

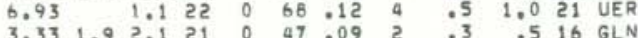

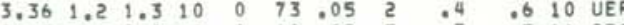

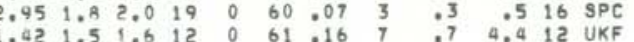

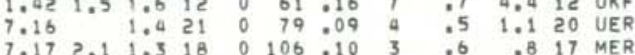
$\begin{array}{llllllllllllll}7.71 & 0 & 1.6 & 19 & 0 & 108 & -12 & 5 & .6 & 1.0 & 19 & \text { POL } \\ 2,31 & 1.6 & 1.6 & 10 & 0 & 111 & 016 & 7 & .8 & 3.5 & 10 & \text { UKF . }\end{array}$ $\begin{array}{rlllllllllll}.07 & 1.4 & 1.4 & 9 & 0 & 112 & .07 & 7 & .6 & 3.3 & 7 & \text { UKF } \\ 7.62 & 1.4 & 1.3 & 20 & 0 & 102 & .08 & 4 & .6 & .8 & 20 & \text { UER } \\ 8.23 & & 1.3 & 1.4 & 0 & 109 & .07 & 4 & .5 & 1.2 & 13 & \text { HEA }\end{array}$ $\begin{array}{lllllllllllll}6.58 & 2.1 & 2.1 & 20 & 0 & 94 & .11 & 6 & .5 & 1.7 & 18 & \text { UKF }\end{array}$

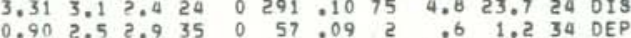

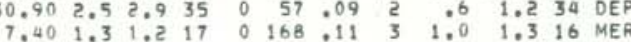
$\begin{array}{rrrrrrrrrrrr}7.17 & 1.4 & 1.4 & 20 & 0 & 117 & .08 & 5 & .5 & 1.1 & 20 & \text { UER } \\ 3.14 & 2.0 & 2.0 & 15 & 0 & 95 & .10 & 6 & .5 & 2.6 & 15 & \text { UKF }\end{array}$

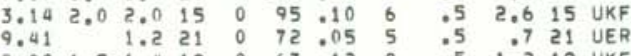

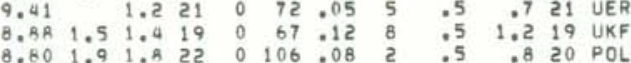
$\begin{array}{lllllllllllll}8.37 & 2.4 & 2.3 & 31 & 1 & 119 & .09 & 5 & .5 & .7 & 30 & \text { UER }\end{array}$

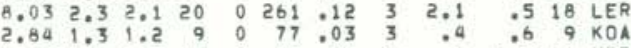
$\begin{array}{llllllllllll}6.75 & 1.6 & 1.3 & 13 & 0 & 78 & .06 & 5 & .6 & 1.2 & 11 & \text { UER } \\ 9.15 & 2.1 & 2.3 & 24 & 0 & 74 & .07 & 3 & .5 & .6 & 23 & \text { UER }\end{array}$ $\begin{array}{rrrrrrrrrrr}7.51 & 1.2 & 17 & 0 & 83 & .09 & 4 & .6 & 1.1 & 16 & \text { UER }\end{array}$

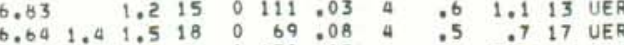

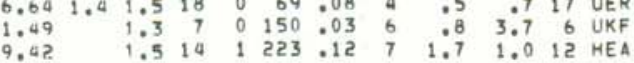
$\begin{array}{lllllllllllll}5.36 & 1.4 & 1.3 & 20 & 1 & 67 & .10 & 5 & .4 & 1.1 & 18 & \text { UER } \\ 7.69 & 18 & 1.4 & 17 & 0 & 79 & .06 & 4 & .4 & .9 & 15 & \text { UER }\end{array}$

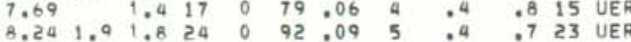

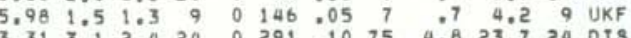

ORIGIN TIME LAT N LON W YEAR MON DA HRMN SEC DEG MIN DEG MIN

$\begin{array}{llllllll}1979 & \text { OCT } 16 \quad 248 & 27.71 & 19 & 19.59 & 155 & 8.55\end{array}$ $\begin{array}{llllllllllll}6.17 & 1.6 & 1.8 & 30 & 1 & 80.09 & 4 & 4 & .8 & 28 & \text { UER }\end{array}$

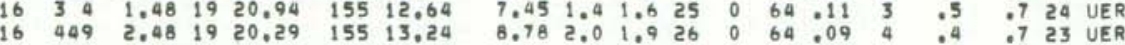

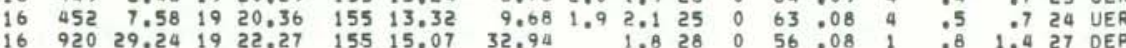
$16 \quad 95329.62 \quad 1926.79 \quad 155238.56$

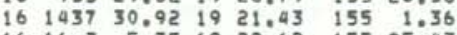
$16 \quad 163 \quad 5.351922 .12 \quad 155 \quad 25.43$

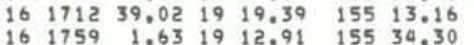
$\begin{array}{lllllll}16 & 1954 & 37.81 & 19 & 19.44 & 155 & 8.45 \\ 16 & 20 & 52.30 & 19 & 21.09 & 155 & 5.55\end{array}$

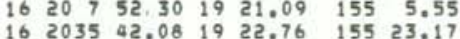

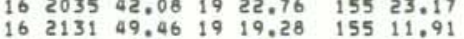

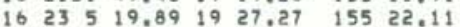

$\begin{array}{lrlllll}16 & 2332 & 35.49 & 19 & 19.74 & 155 & 10.19 \\ 17 & 026 & 17.85 & 19 & 20.79 & 155 & 1.82\end{array}$

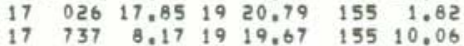

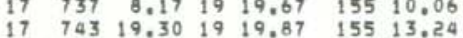
$\begin{array}{lllllll}17 & 1245 & 37.74 & 19 & 19.22 & 155 & 11.08\end{array}$

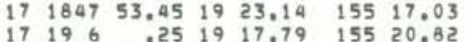

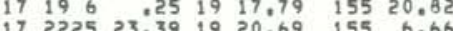
18 1 $1997.281926 .73 \quad 15526.49$ $\begin{array}{lllllll}18 & 1017 & 26.86 & 19 & 23.36 & 155 & 14.93\end{array}$ 18 1219 $36.131921 .09 \quad 155 \quad 7.11$ $18 \quad 1414 \quad 59.67 \quad 1927.47 \quad 15518.49$ $\begin{array}{lllllll}18 & 1728 & 4.73 & 19 & 19,83 & 155 & 13.19\end{array}$ $\begin{array}{lllllll}18 & 1833 & 2.11 & 19 & 20.49 & 155 & 11.74\end{array}$

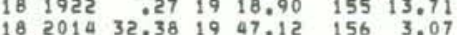
$\begin{array}{llllllll}18 & 2122 & 26.50 & 19 & 21.37 & 155 & 5.55\end{array}$ $\begin{array}{llll}18 & 2319 & 16.76 \quad 19 & 20.15 \quad 155 \quad 13.55\end{array}$

$\begin{array}{lllllll}19 & 036 & 11.28 & 19 & 23.21 & 155 & 15.03\end{array}$ $19 \quad 13254.641923 .06 \quad 155 \quad 14.82$ $19 \quad 2 \quad 8 \quad 12.91 \quad 1922.97 \quad 155 \quad 14.96$

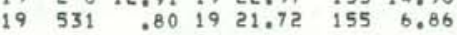
$19 \quad 1025 \quad 59.19 \quad 1923.41 \quad 155 \quad 16.29$ $19163539.011923 .56 \quad 15516.99$ 19172051.291923 .45 155 16.99

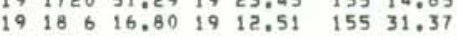

$\begin{array}{llllllll}19 & 2026 & 7.32 & 19 & 20.70 \quad 155 & 13.27\end{array}$

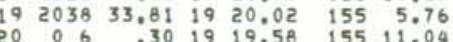

$\begin{array}{llllllllllll}9.80 & 1.5 & 17 & 0 & 68 & .08 & 8 & .6 & 1.7 & 16 & \text { UKF }\end{array}$ $\begin{array}{llllllllllll}6.52 & 1.3 & 19 & 0 & 180 & 10 & 4 & .8 & .7 & 19 & \text { MER } \\ 8.78 & 2.0 & 2.1 & 19 & 0 & 104 & : 06 & 4 & .6 & 1.1 & 19 & \text { UKF }\end{array}$

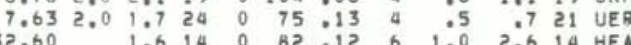

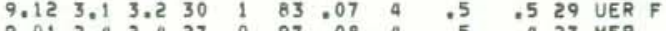

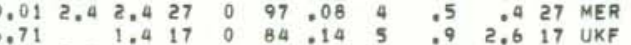

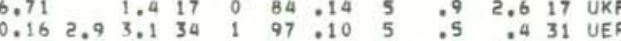

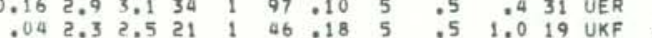
$\begin{array}{lllllllllllll}7.90 & 1.9 & 1.6 & 21 & 0 & 91 & .06 & 4 & .5 & .7 & 20 & \text { UER } \\ 6.49 & 0.7 & 0 & 12 & 0 & 189 & 011 & 3 & 0.0 & 1.4 & 12 & \text { MER }\end{array}$

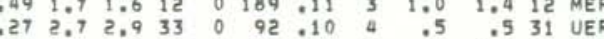
$\begin{array}{llllllllll}9.10 & 915 & 0 & 69 & : 11 & 5 & .5 & 2.0 & 15 & \text { UER }\end{array}$ $\begin{array}{llllllllllll}2.90 & 1.1 & 9 & 0 & 68 & .04 & 2 & .4 & .6 & 6 & \text { SPC }\end{array}$

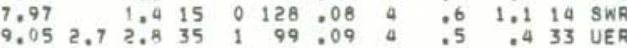
$9.672 .62 .44550046 .11 \quad 8 \quad 040.632$ UKF $\begin{array}{llllllllllllll}3.60 & 1.2 & 1.5 & 10 & 1 & 100 & .04 & 2 & .4 & .7 & 9 & 6 L N\end{array}$

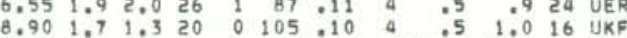

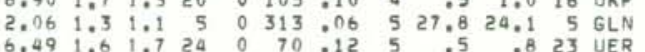

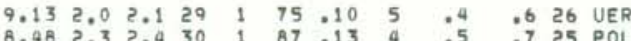

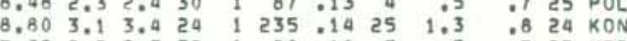

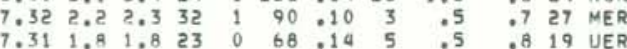
$\begin{array}{llllllllllll}3.16 & 1.5 & 1.9 & 13 & 0 & 73 & .09 & 2 & .4 & .5 & 11 & \text { SPC }\end{array}$ $3.02 \quad 1.6 \quad 1.7720072 .05 \quad 3 \quad 0 \quad .626 \mathrm{GLN}$

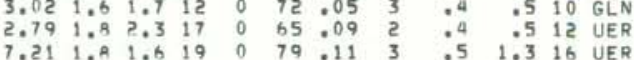
$\begin{array}{llllllllllll}3.65 & 1.4 & 1.3 & 8 & 1 & 86 & .05 & 2 & .4 & .7 & 3 & \text { SPC }\end{array}$

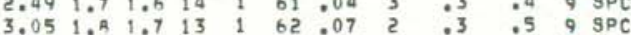
$3.592,52.524 \quad 0 \quad 45,09$ 2 $0.4 \quad .523$ GLN $\begin{array}{lllllllllll}8.25 & 1.3 & 25 & 2 & 80 & : 13 & 8 & : 4 & 1.0 & 18 & \text { L9N }\end{array}$

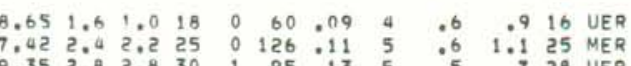




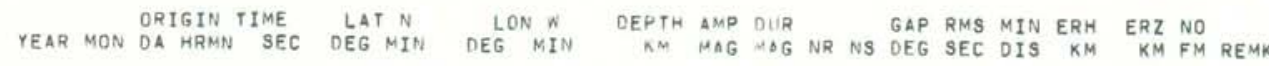

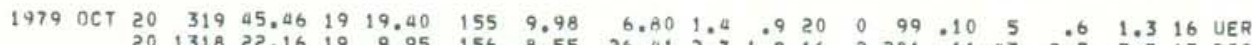

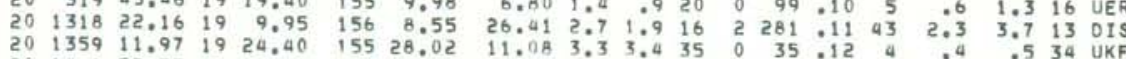

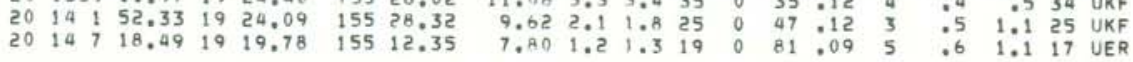

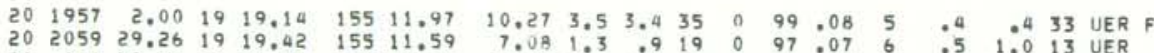
$21 \quad 115 \quad 32.94 \quad 19 \quad 24.30 \quad 155 \quad 25.04$ 21 $3241.681923 .37 \quad 15514.69$

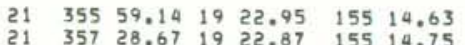
$21 \quad 4 \quad 4 \quad 19.27 \quad 1923.13$ is5 14.77

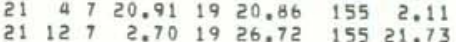
$\begin{array}{lllllll}21 & 1315 & 39.65 & 19 & 19,36 & 155 & 11.58 \\ 21 & 149 & 27.48 & 19 & 23.27 & 155 & 17.06\end{array}$ $\begin{array}{llllllll}21 & 14 & 9 & 27.48 & 19 & 23.27 & 155 & 17.06 \\ 21 & 15 & 8 & 49.63 & 19 & 23.60 & 155 & 15.01\end{array}$ $21173336.591923 .17 \quad 15515.79$

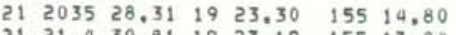
$\begin{array}{llllllll}21 & 21 & 4 & 30.81 & 19 & 23.19 & 155 & 17.04\end{array}$ $21224044.16 \quad 1920.01 \quad 15511.66$ $22021 \quad 51.321923 .21 \quad 15516.80$

$\begin{array}{lllllll}22 & 148 & 1.88 & 19 & 20.73 & 155 & 12.97\end{array}$

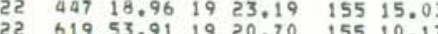
$\begin{array}{lllllll}22 & 740 & 45.41 & 19 & 22.96 & 155 & 17.33\end{array}$ $22996 \quad 34.8519 \quad 20.60 \quad 155 \quad 6.41$

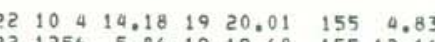

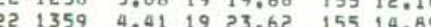
$\begin{array}{llll} & \end{array}$ $\begin{array}{llllll}1652 & 11.58 & 19 & 20.04 & 155 & 10,90\end{array}$

$22 \begin{array}{lllll}1853 & 44.12 \quad 19 & 19.69 \quad 155 \quad 10.46\end{array}$

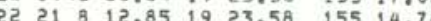
$22 \quad 3344 \quad 42.18 \quad 1921.34 \quad 155$ $22 \quad 2355 \quad 16.741922 .89 \quad 155 \quad 16.97$

$22 \quad 235926.99 \quad 1923.44 \quad 155 \quad 14.83$ $\begin{array}{llllllll}23 & 0 & 5 & 50.76 & 18 & 50.61 & 155 & 11.85 \\ 23 & 0 & 9 & 37.83 & 19 & 19.39 & 155 & 7.83\end{array}$ $\begin{array}{llllllll}23 & 1 & 3 & 52.29 & 19 & 13.82 & 155 & 1.40 \\ 23 & 1 & 9 & 32.12 & 19 & 23.53 & 155 & 14.87\end{array}$ $\begin{array}{lllllll}23 & 251 & 30.83 & 19 & 23.40 & 155 & 17.07\end{array}$ $\begin{array}{lllllll}23 & 348 & 55.15 & 19 & 20.75 & 155 & 6.66 \\ 23 & 919 & 47.52 & 19 & 19.36 & 155 & 12,95\end{array}$

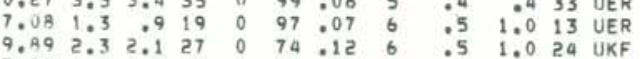

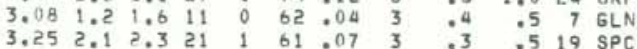

$\begin{array}{lllllllllll}3.69 & 2.4 & 2.3 & 22 & 0 & 49 & .09 & 2 & .4 & .5 & 20 \\ 3 & \text { UER }\end{array}$

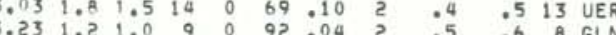

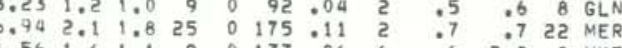

9.84
3.40

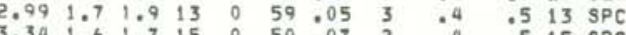

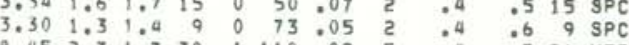

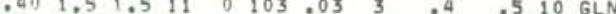
$\begin{array}{llllllllllllll}3.36 & 1.8 & 0 & 17 & 0 & 61 & 05 & 3 & .05 & .3 & .6 & 17 & \text { SPC }\end{array}$

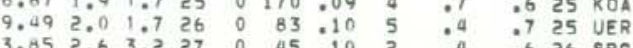

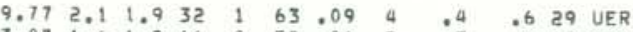

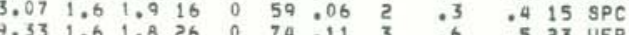

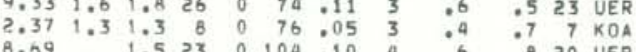
$\begin{array}{llllll}.69 & 1.5 & 23 & 0 & 104 & .10\end{array}$ $\begin{array}{rrrrrrrrrrrr}6.4 A & 2.1 & 1.7 & 19 & 1 & 179 & .10 & 6 & .7 & 1.3 & 17 & \text { MER } \\ 8.44 & 2.3 & 1.9 & 25 & 0 & 85 & .12 & 5 & .5 & .8 & \text { 22 } & \text { UER }\end{array}$

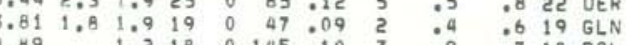

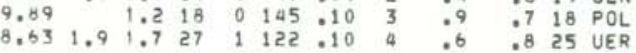
$\begin{array}{llllllllll}8.80 & 1.5 & 25 & 0 & 93 & .12 & 5 & .6 & .7 & 25 \\ 3 & \text { UER }\end{array}$

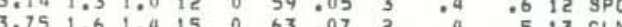

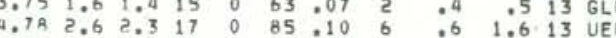

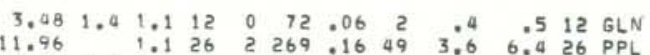

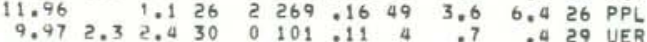

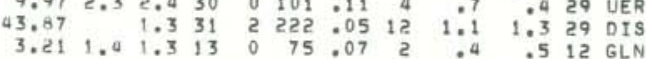
$\begin{array}{llllllllllll}2.79 & 1.1 & 1.1 & 11 & 0 & 59 & .05 & 3 & .4 & .6 & 11 & \text { SPC }\end{array}$

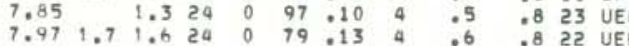

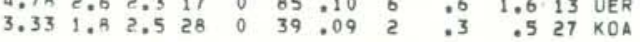

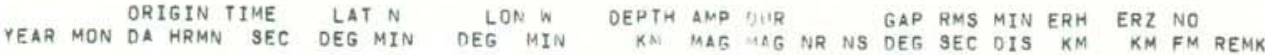

1979 OCT $23 \quad 923 \quad 15.73 \quad 1922.76 \quad 155 \quad 14.40 \quad 3$

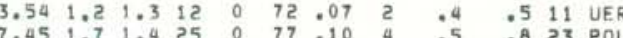

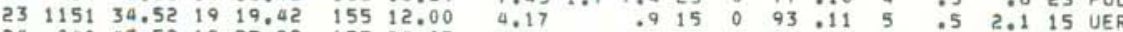

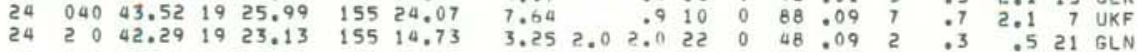
$\begin{array}{llllllllllllllllll}24 & 514 & 6.57 & 19 & 11.50 & 155 & 21.32 & 45.58 & 1.2 & 18 & 0 & 170 & .09 & 13 & 1.1 & 3.4 & 17 \quad \mathrm{LSW} T\end{array}$

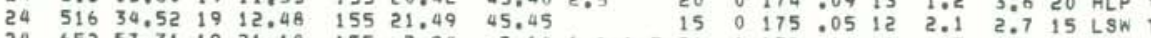

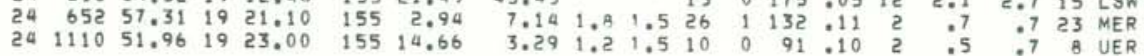

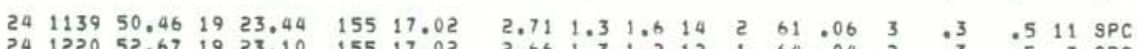

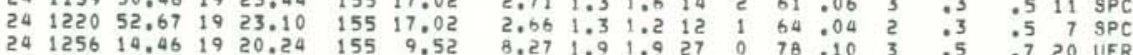

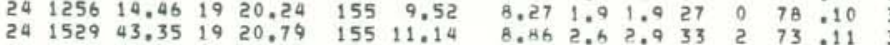
241531.07 .351920 .7915511 .14

$24 \quad 2233 \quad 5.441921 .66 \quad 155 \quad 18.37$

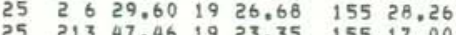

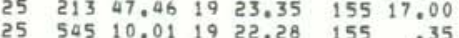
$25 \quad 74017.02 \quad 1920,12 \quad 155 \quad 8.53$

$\begin{array}{lllll}25 & 735 & 42.65 \quad 19 & 26.12 \quad 154 \quad 56.76\end{array}$ $25181757.701925 .40 \quad 15520.98$ $252018 \quad 45.941924 .33 \quad 15516.33$ (5) 21.823 .291923 .96 155 15.11

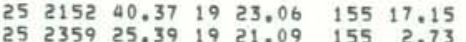

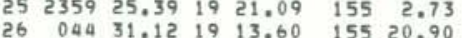

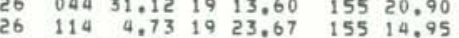
26 $\quad \begin{array}{llllll}133 & 15,01 & 19 & 23,12 & 155 & 17,01\end{array}$

$\begin{array}{llrrrrr}26 & 239 & 6.17 & 19 & 19.94 & 155 & 9.72 \\ 26 & 244 & 51.48 & 19 & 24.77 & 155 & 25.13\end{array}$ $\begin{array}{lllllll}26 & 244 & 51.48 & 19 & 24.77 & 155 & 25.13 \\ 26 & 535 & 21.40 & 19 & 22.13 & 155 & 17.31\end{array}$

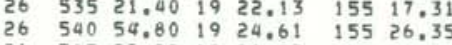

$\begin{array}{llllllll}26 & 8 & 1 & 35.25 & 19 & 23.01 & 155 & 17.10\end{array}$

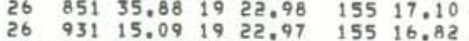

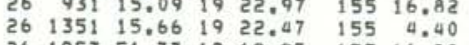
195351.771919 .97 155 11.20 $\begin{array}{lllllll}26 & 2227 & 30.87 & 19 & 18.70 & 155 & 23.54 \\ 26 & 2328 & 24.75 & 19 & 21.26 & 155 & 1.96\end{array}$

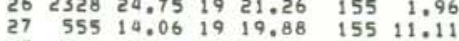

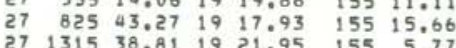

$\begin{array}{llllllll}27 & 14 & 0 & 2.28 & 19 & 20.50 & 155 & 10.3\end{array}$

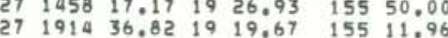

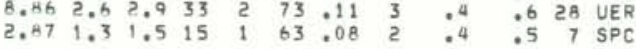

$\begin{array}{llllllllllll}3.00 & 1.2 & 1.1 & 8 & 0 & 77 & .06 & 3 & .8 & 1.6 & 7 & \text { KOA }\end{array}$

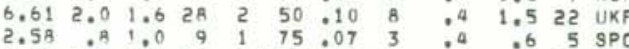

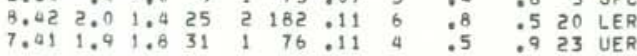
$\begin{array}{llllllllllll}4.94 & 2.2 & 1.4 & 24 & 2 & 176 & .13 & 4 & .8 & 1.1 & 18 & \text { LER }\end{array}$ $9.671 .9 \quad 1.3 \quad 12 \quad 0 \quad 115: 09 \quad 6 \quad: 7 \quad 2.511$ UKF $\begin{array}{lllllllllllllll}2.79 & 1.5 & 1.5 & 9 & 1 & 126 & 08 & 3 & .4 & .5 & 6 & \mathrm{SPC}\end{array}$

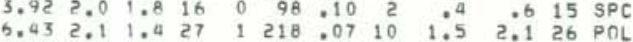

$\begin{array}{lllllllllllll}2.56 & 1.0 & 1.2 & 12 & 1 & 66 & .06 & 3 & .3 & .6 & 10 & \mathrm{SPC}\end{array}$

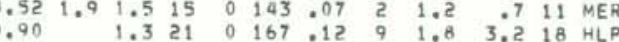

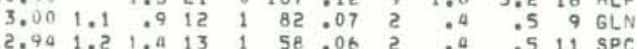

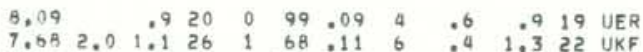
$\begin{array}{llllllllllllll}3.16 & 1.2 & 1.2 & 11 & 1 & 101 & 09 & 3 & .4 & .8 & 10 & \text { KOA }\end{array}$

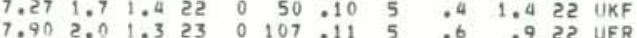

$\begin{array}{llllllllllllll}2.89 & 1.6 & 1.6 & 15 & 1 & 63 & .06 & 2 & .3 & .6 & 12 & \mathrm{SPC}\end{array}$

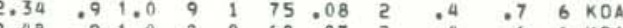

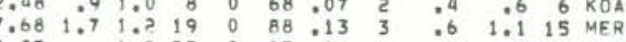

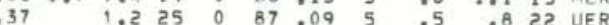

$\begin{array}{lllllllllllll}4.06 & 1.4 & 1.7 & 19 & 0 & 120 & .10 & 3 & .5 & 1.1 & 14 & \text { SWR } \\ 8.30 & 3.5 & 0.0 & 28 & 1 & 109 & .08 & 3 & .7 & .6 & 26 & \text { WER }\end{array}$

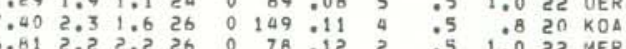
$\begin{array}{llllllllllll}8.27 & 2.1 & 2.2 & 21 & 0 & 88 & .13 & 3 & .7 & 1.0 & 19 & \text { UER }\end{array}$

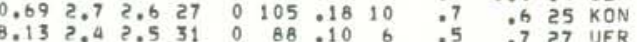

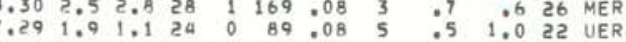




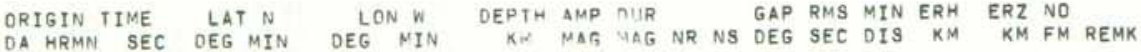
979 OCT $28 \quad 011 \quad 30.20 \quad 19 \quad 19.40 \quad 155 \quad 15.10$

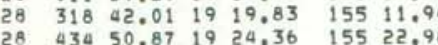

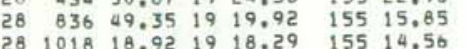
$\begin{array}{lllllll}28 & 1254 & 6.03 & 19 & 21.11 & 155 & 6.80\end{array}$ $\begin{array}{lllllll}28 & 1327 & 54.75 & 19 & 23.11 & 155 & 17.00\end{array}$

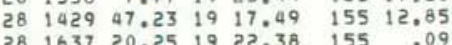
$\begin{array}{lllllll}28 & 1853 \quad 33.20 & 19 & 24.92 \quad 155 & 26.38\end{array}$ $\begin{array}{lllllllll}28 & 20 & 8 & 45.85 & 19 & 16.00 & 155 & 32.53\end{array}$

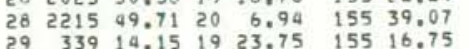

$\begin{array}{llllllll}29 & 8 & 0 & 43.93 & 19 & 24.20 & 155 & 28.81\end{array}$

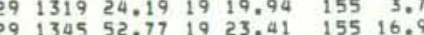
29 $1357 \quad 44.5919 \quad 31.31 \quad 155 \quad 37.49$ $\begin{array}{lllllll}29 & 1625 & 22.29 & 19 & 21.78 & 155 & .77 \\ 29 & 1643 & 32.57 & 19 & 21.78 & 155 & 21.03\end{array}$ $291816 \quad 40.67 \quad 1926.90 \quad 15521.52$

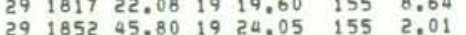
$292126 \quad 15.17 \quad 1920.08 \quad 155 \quad 11.50$

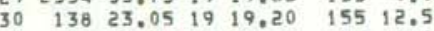
$30 \quad 14159.22 \quad 1923.17 \quad 155 \quad 14.95$

$\begin{array}{lllllll}30 & 410 & 59.54 & 19 & 23.08 & 155 & 3.79\end{array}$ $\begin{array}{lllllll}30 & 715 & 17.42 & 19 & 19.65 & 155 & 10.82\end{array}$

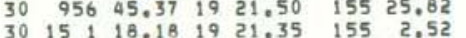
$301521 \quad 32.551921 .83 \quad 15518.11$

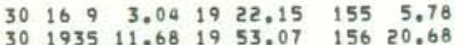

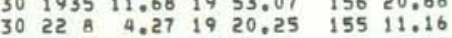
$\begin{array}{rrrrrrr}30 & 2315 & 38.72 & 19 & 21.28 & 155 & 4.68 \\ 31 & 020 & 43.88 & 19 & 26.80 & 155 & 27.30\end{array}$

$\begin{array}{llllllr}31 & 253 & 1.79 & 19 & 22.04 & 155 & 2.21 \\ 31 & 523 & 30.09 & 19 & 58.43 & 155 & 47.87\end{array}$ 31526111.791921 .56 155 2.02

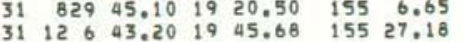

$\begin{array}{lllllll}31 & 1229 & 15.12 & 19 & 19.84 & 155 & 8.03 \\ 31 & 1248 & 53.57 & 19 & 20.19 & 155 & 12.20\end{array}$ $31 \quad 1248 \quad 53.57 \quad 1920.19 \quad 155 \quad 12.20$

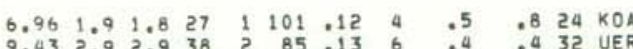
$B, 9 B 2,3,332 \quad 1 \quad 51.10 \quad 8 \quad 4 \quad 738$ UK

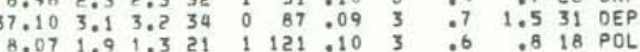
$\begin{array}{llllllllllllll}7.70 & 2.1 & 1.9 & 27 & 2 & 89 & .08 & 4 & .9 & .8 & 24 & \text { UER }\end{array}$

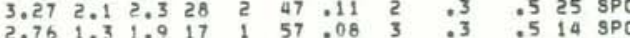

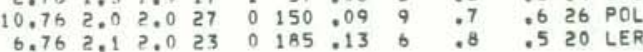
$\begin{array}{llllllllllll}10.44 & 2.4 & 2.1 & 30 & 0 & 59 & .17 & 10 & .6 & .8 & 27 & \text { UKF }\end{array}$ $\begin{array}{llllllllllll}8.58 & 1.3 & 24 & 0 & 117 & 115 & 8 & .7 & 1.1 & 19 & \text { HEA } \\ 4.59 & 1.6 & 1.9 & 19 & 1 & 178 & 09 & 3 & : 7 & 1.2 & 17 & \text { SWR }\end{array}$

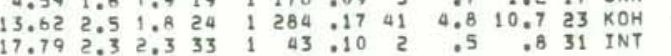

$\begin{array}{llllllllllll}9.33 & 1.9 & 1.7 & 24 & 1 & 75 & .11 & 4 & .5 & 1.2 & 20 & \text { UKF }\end{array}$

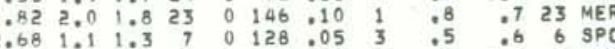

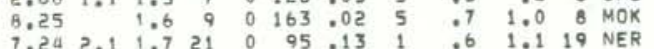

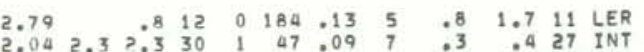

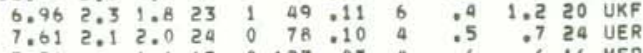
7.36 16 1700123.07 4 0 .6 16 MER $\begin{array}{rllllllllllll}7.82 & 1.7 & 1.3 & 20 & 0 & 91 & .08 & 5 & .5 & .7 & 20 & \text { UER } \\ 7.30 & & 1.2 & 15 & 0 & 140 & .09 & 4 & .7 & 1.1 & 14 & \text { UER }\end{array}$ $\begin{array}{lllllllllll}7.95 & 2.1 & 2.0 & 34 & 0 & 89.13 & 4 & .4 & .6 & 30 & \\ 30 & \text { UER }\end{array}$

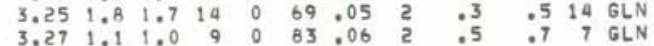
$\begin{array}{llllllllllll}8.11 & 2.6 & 2.6 & 32 & 1 & 99 & .11 & 3 & .5 & .5 & 27 & \text { MER } \\ 7.45 & & .5 & 21 & 0 & 94 & 010 & 5 & 5 & 1.1 & 20 & \text { UER }\end{array}$

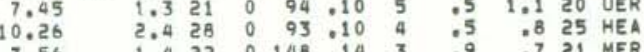

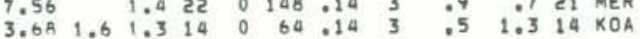

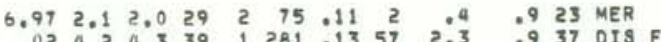

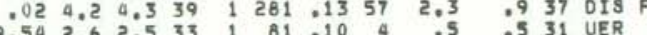

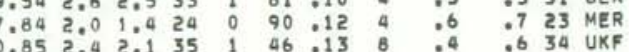

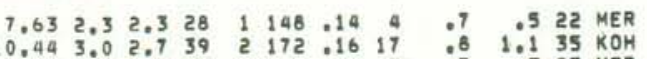

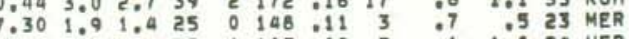

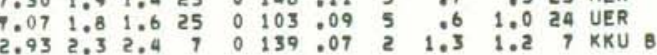
$\begin{array}{llllllllllll}8.51 & 1.9 & 2.0 & 27 & 0 & 69 & .08 & 5 & .6 & .6 & 27 & \text { UER } \\ 6.41 & 1.6 & 1.5 & 19 & 0 & 76 & 09 & 5 & .6 & 1.3 & 18 & \text { UER } \\ 4.26 & 2.5 & 1.9 & 17 & 0 & 173 & .09 & 4 & 17 & 3.8 & 17 & \text { HEA }\end{array}$
ORIGIN TIME LATN LON W YEAR MON DA HRMN SEC DEG MIN DEG MIN $\begin{array}{llllllll}1979 & \text { OCT } 31 \quad 1549 \quad 34.96 & 20 & .30 & 155 & 48.68\end{array}$

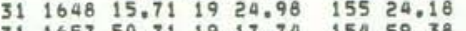
$\begin{array}{lllllll}31 & 1657 & 50.71 & 19 & 17.74 & 154 & 59.38 \\ 31 & 17 & 34.28 & 19 & 20.88 & 155 & 5.82\end{array}$ $\begin{array}{lllllll}31 & 1720 & 21.08 & 18 & 55.37 & 155 & 10.61\end{array}$ $\begin{array}{llllll}31 & 1734 & 26.75 \quad 19 & 20.15 \quad 155 & 11.00\end{array}$

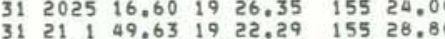

Nov $1023 \quad 6.25 \quad 1919.10 \quad 15513.20$

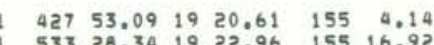

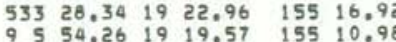
$\begin{array}{llllll}95 & 54.26 & 19 & 19.57 & 155 & 10.9 \\ 921 & 45.26 & 19 & 20.32 & 155 & 12.5\end{array}$ $124131.411921 .11 \quad 15514.5$ $\begin{array}{llllll}1411 & 38.69 & 19 & 23.13 & 155 & 16.95 \\ 1423 & 22.60 & 19 & 19.89 & 155 & 11.63\end{array}$ $\begin{array}{llllll}1750 & 21.45 & 19 & 17.89 & 155 & 14.86 \\ 1752 & 21.57 & 19 & 21.71 & 155 & 2.00\end{array}$

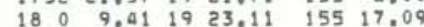

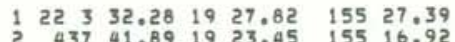

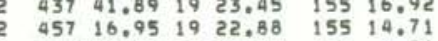
$9557.01 \quad 1920.50 \quad 155 \quad 4.20$

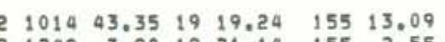

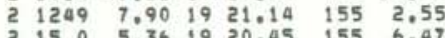

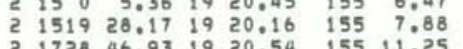
$\begin{array}{lllllll}2 & 1747 & 19.27 & 19 & 25.21 & 155 & 26.23\end{array}$ $\begin{array}{lllllll}2 & 1758 & 8.10 & 19 & 26.51 & 154 & 55.93 \\ 2 & 2110 & 11.87 & 19 & 20.65 & 155 & 12.80\end{array}$ 2 $111011.871920 .61 \quad 15512.88$ $3 \quad 13490.651920 .16 \quad 155 \quad 25.17$

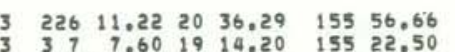

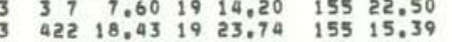

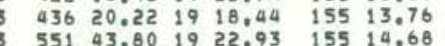
$\begin{array}{lllllll}3 & 610 & 42.17 & 19 & 23.21 & 155 & 14.79\end{array}$ $62516.751923 .35 \quad 15514.78$ 355
3 $6.121917 .62 \quad 15516.11$

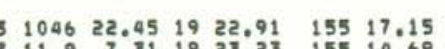
$31210 \quad 0.511923,05 \quad 15514.60$
DEPTH AMP NUR GAP RMS MIN ERH ERZ NO
KM MAG MAG NR NS DEG SEC DIS KM KMM FM REMK $\begin{array}{lllllllllllll}14.09 & 2.5 & 1.9 & 29 & 2 & 299 & 08 & 35 & 2.5 & 2.5 & 27 & \mathrm{KOH}\end{array}$

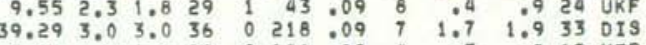

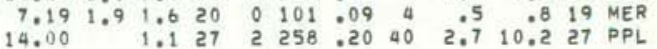

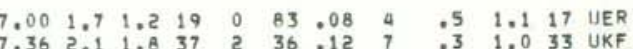

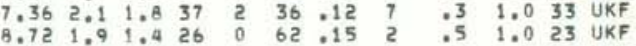

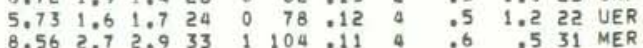

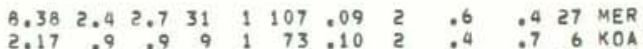

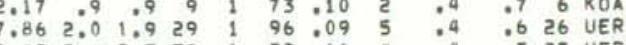

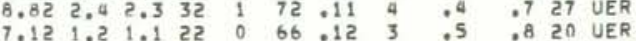

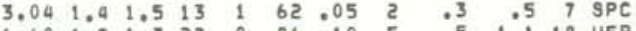

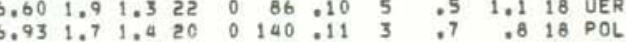

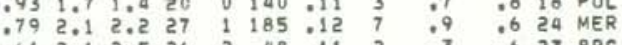

$\begin{array}{llllllll}9.07 & 2.1 & 1.7 & 20 & 1 & 48 & 0.10 & 8\end{array}$

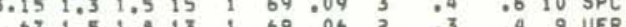

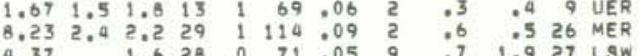
$\begin{array}{lllllllllllll}0.21 & 1.9 & 1.3 & 22 & 1 & 79 & .11 & 4 & .5 & 1.2 & 21 & 1 & \text { UER }\end{array}$ $\begin{array}{lllllllllllll}6.14 & 1.9 & 1.5 & 21 & 1 & 150 & : 13 & 2 & : 6 & 0 & 18 & \text { MER } \\ 7.19 & 2.1 & 1.7 & 25 & 0 & 106 & : 09 & 5 & : 5 & 7 & 25 & \text { UER }\end{array}$

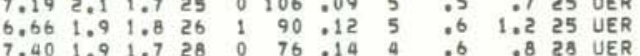
$\begin{array}{llllllllllll}0.13 & 1.6 & 1.3 & 20 & 0 & 57 & .09 & 6 & .5 & 1.4 & 19 & \text { UKF }\end{array}$ $\begin{array}{rrrrrrrrrrrr}4.82 & 1.5 & 1.2 & 14 & 0 & 197 & 16 & 2 & 1.8 & 1.7 & 13 & \text { UER } \\ 7.08 & 1.8 & 1.5 & 29 & 1 & 65 & 1.2 & 4 & .5 & .7 & 28 & \text { UER }\end{array}$

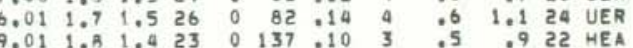
$\begin{array}{lllllllllllll}8.13 & 2.8 & 2.8 & 17 & 3 & 196 & .10 & 37 & 1.6 & 1.8 & 15 & 018\end{array}$

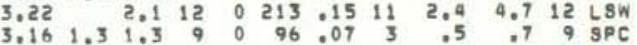
$\begin{array}{llllllllllll}8.01 & 1.6 & 1.6 & 26 & 1 & 93 & 012 & 3 & .5 & .7 & 25 & \text { POL } \\ 3.39 & 1.7 & 1.7 & 17 & 1 & 57 & .08 & 2 & .4 & .5 & 16 & \text { UER }\end{array}$ $\begin{array}{lllllllllllll}3.69 & 1.2 & 1.1 & 8 & 0 & 105 & .03 & 2 & .5 & .7 & \text { \& GLN }\end{array}$ .

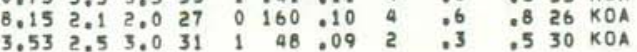
$\begin{array}{llllllllllllll}2.68 & 1.3 & 1.0 & 12 & 1 & 72 & .09 & 2 & .3 & .6 & 10 & \mathrm{KOA}\end{array}$

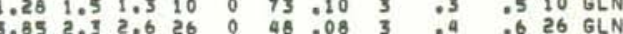




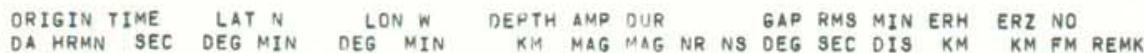

1979 NOV $3 \quad 1310 \quad 24.88 \quad 19 \quad 17.97 \quad 155 \quad 15.88$ $\begin{array}{lllllll}3 & 1412 & 17.57 & 19 & 19.37 & 155 & 11.44 \\ 3 & 1517 & 24.60 & 19 & 20.26 & 155 & 12.48\end{array}$

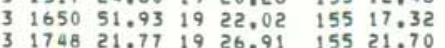

$3 \begin{array}{llllll}18 & 51.64 & 20 & 3.51 \quad 155 & 38.74\end{array}$ $\begin{array}{lllllll}3 & 2030 & 8.68 & 19 & 20.64 & 155 & 6.78 \\ 3 & 2045 & 21.20 & 19 & 22.95 & 155 & 16.72\end{array}$

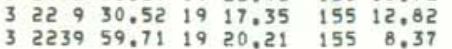

$\begin{array}{lllllll}3 & 2254 & 53.29 & 19 & 19.17 & 155 & 1.22\end{array}$ - $11831.971923 .82 \quad 15528.56$

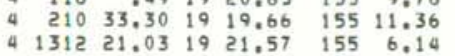
4 $1328 \quad 39.82 \quad 1922.30 \quad 155 \quad 19.51$ $4182152.52 \quad 1919.27 \quad 15511.86$

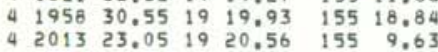

$\begin{array}{lllllll}4 & 2147 & 28.56 & 19 & 20.17 & 155 & 11.53\end{array}$

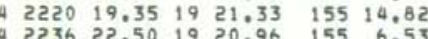

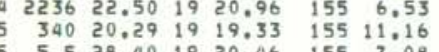

$\begin{array}{llllllll}5 & 543 & 9.20 & 19 & 17.77 & 155 & 12,81\end{array}$

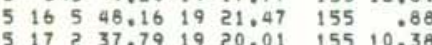

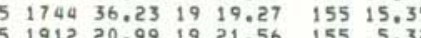

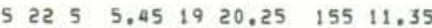

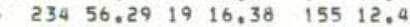
$\begin{array}{lllllll}6 & 310 & 38.37 & 19 & 19.39 & 155 & 13.22\end{array}$

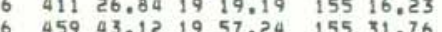

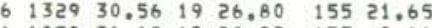
6
6 \begin{tabular}{l}
$605128.191920 .68 \quad 155 \quad 7.15$ \\
\hline
\end{tabular} $31438.821918 .10 \quad 15523.94$

$\begin{array}{lllllll}7 & 1459 & 49.29 & 19 & 20.26 & 155 & 11.46 \\ 7 & 1533 & 23.27 & 19 & 21.73 & 155 & 18.31\end{array}$ $\begin{array}{llllll}1717 & 13.59 & 19 & 21.53 & 155 & 5.25\end{array}$ $\begin{array}{llllll}20 & 3 & 13.99 & 19 & 22.51 \quad 155 & 29.78\end{array}$

$\begin{array}{rlllll}2110 & 44.31 & 19 & 19.71 & 155 & 8.65\end{array}$ $\begin{array}{lllllll}8 & 113 & 39.43 & 19 & 20.48 & 155 & 18.72 \\ 8 & 232 & 10.79 & 19 & 20.57 & 155 & 9.05\end{array}$ $\begin{array}{llllllllllll}7.54 & 2.2 & 2.1 & 25 & 0 & 150 & .09 & 5 & .5 & .7 & 24 & K O A\end{array}$

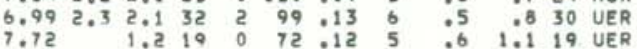

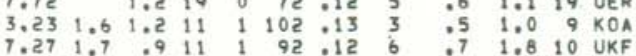

$\begin{array}{lllllllllll}3.10 & 3.0 & 2.4 & 29 & 1 & 181 & .10 & 16 & 1.2 & .9 & 25 \\ \mathrm{KOH} F\end{array}$

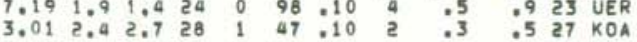

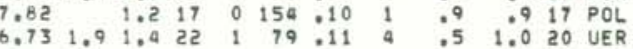

$\begin{array}{llllllllllll}37.60 & 2.3 & 1.6 & 30 & 1 & 213 & .08 & 3 & .8 & 1.1 & 29 & \text { MER }\end{array}$

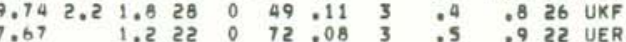

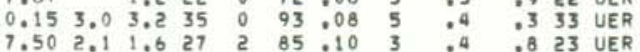
$\begin{array}{llllllllllll}4.09 & 1.3 & 1.0 & 8 & 1 & 221 & .16 & 6 & 1.9 & 4.0 & 5 & \text { UKF }\end{array}$

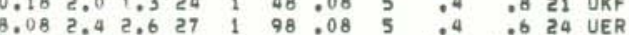

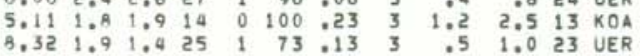
$\begin{array}{llllllllllll}6.81 & 1.9 & 1.3 & 21 & 0 & 82 & .12 & 5 & .5 & 1.0 & 19 & \text { UER }\end{array}$

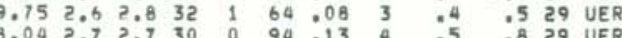

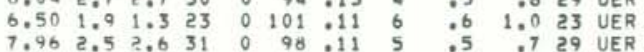
$\begin{array}{llllllllllllll}7.80 & 1.7 & 1.3 & 19 & 0 & 125 & .12 & 2 & .9 & 1.0 & 16 & \mathrm{POL}\end{array}$

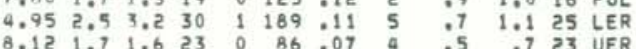

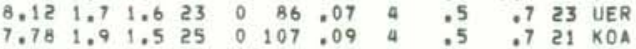

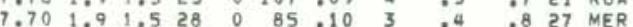
$\begin{array}{llllllllllll}.57 & 2.1 & 1.8 & 27 & 0 & 80 & .11 & 4 & .4 & .7 & 25 & \text { UER }\end{array}$

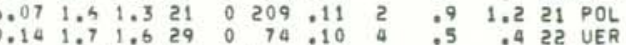
$9.602 .25 .029 \quad 1113.09 \quad 3 \quad .5 \quad .621 \quad \mathrm{KOA}$ $\begin{array}{lllllllllllll}8.98 & 2.0 & 1.2 & 18 & 1 & 76 & .13 & 6 & .6 & 1.0 & 16 & \text { UKF }\end{array}$

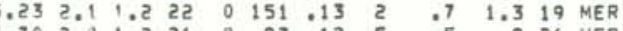

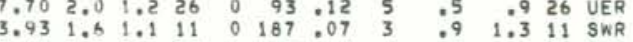

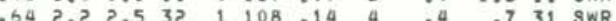
$\begin{array}{lllllllllllll}7.51 & 1.8 & 1.1 & 22 & 1 & 80 & 12 & 4 & .5 & 1.1 & 18 & \text { UER } \\ 2.55 & 1.6 & 1.5 & 20 & 1 & 56 & 08 & 3 & .03 & .8 & 18 & \text { KOA }\end{array}$

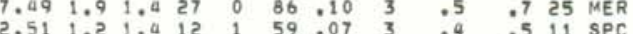

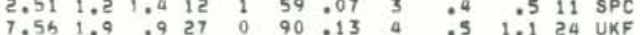
$\begin{array}{llllllllllll}7.05 & 1.8 & 1.1 & 28 & 0 & 77 & .11 & 5 & .5 & .8 & 25 & \text { UER }\end{array}$ $\begin{array}{lllllllllllll}31.60 & 2.6 & 2.2 & 38 & 2 & 53 & 110 & 2 & .6 & 1.1 & 34 & \text { OEP } \\ 6.44 & 1.6 & 1.1 & 26 & 0 & 68 & 12 & 3 & : 5 & 1.0 & 26 & \text { UER }\end{array}$

ORIGIN TIME
YEAR MON DA HRMN SEC DEG MIN DEG MIN DEPTH AMP DUR

1979 NOV $\begin{array}{llrllll}8 & 635 & .54 & 19 & 21.48 & 155 & 6.08 \\ 8 & 920 & 55.98 & 19 & 20.05 & 155 & 8.63 \\ 8 & 1359 & 40.53 & 19 & 21.51 & 155 & 5.85 \\ 8 & 1734 & 44.72 & 19 & 21.25 & 155 & 6.90 \\ 8 & 1712 & 34.78 & 19 & 17.90 & 155 & 16.55\end{array}$ $\begin{array}{ll}8226 & 58.651921 .75 \quad 15512.72\end{array}$ $0.017351918 .05 \quad 155 \quad 4.10$

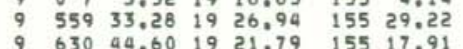

9 $1317 \quad 36.17 \quad 19 \quad 20.44 \quad 155 \quad 13.01$ $\begin{array}{llllll}173 & 16 & 19 & 23.12 & 155 & 17.36\end{array}$

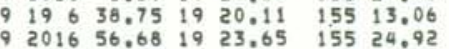

$9 \begin{aligned} & 2022 \quad 48.35 \quad 19 \quad 20.29 \quad 155 \quad 8.59\end{aligned}$ $\begin{array}{lllllll}10 & 311 & 7.99 & 19 & 22.48 & 155 & 16.64\end{array}$ $10 \quad 347 \quad 47.65 \quad 19 \quad 31,37 \quad 15541.17$

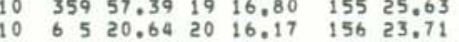

$\begin{array}{lllllll}10 & 648 & 18.33 & 19 & 19.48 & 155 & 8.98\end{array}$

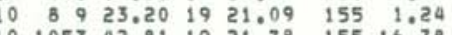
$\begin{array}{lllllll}10 & 1053 & 42.81 & 19 & 21.78 & 155 & 16.78 \\ 10 & 132 & 12.64 & 19 & 20.54 & 155 & 12.84\end{array}$ $10 \quad 1643 \quad 56.43 \quad 19 \quad 11.77 \quad 155 \quad 40.58$ 1028 8 $16.111923 .89 \quad 155$ $10216 \quad 30.93 \quad 1920.90 \quad 15512.67$ $10213124.711920 .42 \quad 155 \quad 6.39$

$\begin{array}{lllllll}3 & 5 & 51.23 & 19 & 23.37 & 155 & 14.99\end{array}$ $11313 \quad 26.641923 .10 \quad 155 \quad 16.90$

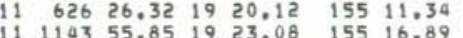
$11.23029 .23 \quad 1924.45 \quad 155 \quad 16.57$

$11131928.32 \quad 1920.41 \quad 15512.76$

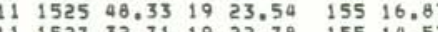
1152732.71 19 22.78 155 14.87 $\begin{array}{lllllll}11 & 2116 & 7.93 & 19 & 19.86 & 155 & 7.97\end{array}$

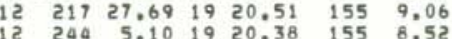
$12 \quad 4941.031920 .15 \quad 15510.53$

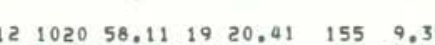

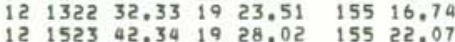

$\begin{array}{lllllllllllll}8.12 & 2.4 & 2.5 & 32 & 0 & 87 & 110 & 3 & .5 & .7 & 29 & \text { UER }\end{array}$

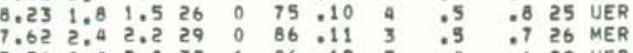

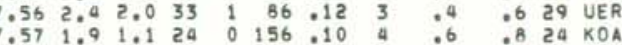
$\begin{array}{llllllllllll}2.34 & 1.9 & 1.6 & 16 & 0 & 65 & .15 & 2 & .5 & .7 & 15 & \text { UER }\end{array}$

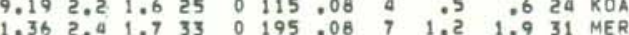

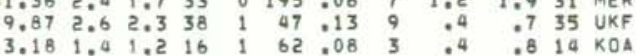

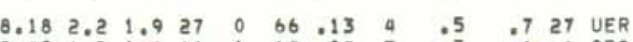

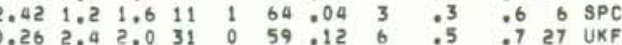

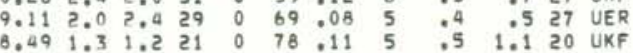

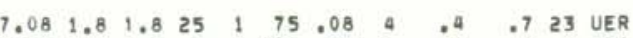

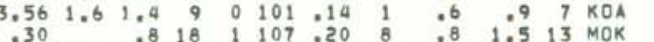
$\begin{array}{llllllllllll}7.00 & 1.9 & 1.7 & 26 & 1 & 109 & .12 & 8 & 0.4 & .9 & 20 & \text { HEA } \\ 2.28 & 2.3 & 2.0 & 30 & 4 & 222 & .21 & 57 & 1.7 & 3.7 & 21 & \text { DIS }\end{array}$

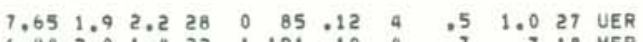

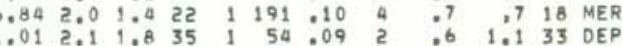

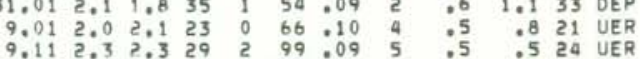
$\begin{array}{lllllllllll}3.18 & 2.5 & 2.6 & 25 & 1 & 191 & .24 & 8 & 1.5 & 3.4 & 21 \\ \text { HEA }\end{array}$

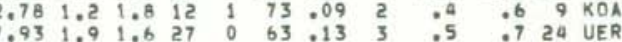

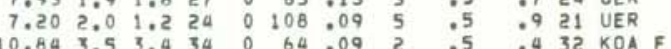
$\begin{array}{llllllllllll}2.93 & 1.1 & 1.2 & 14 & 1 & 75 & 12 & 2 & .4 & .6 & 11 & \mathrm{GLN}\end{array}$

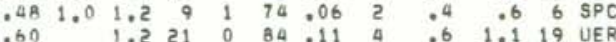

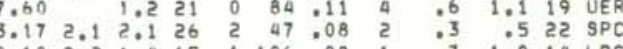
9.512 .42 .725 1 69.09 4 40.624 UER

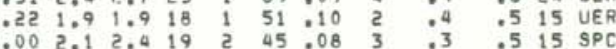

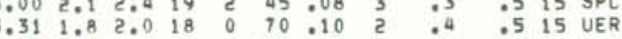

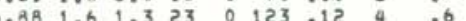
$\begin{array}{llllllllllll}.04 & 1.9 & 1.6 & 27 & 0 & 90 & 010 & 5 & .6 & .7 & 26 & \text { UER } \\ 36 & 1.1 & 24 & 0 & 70 & : 13 & 3 & .6 & 1.0 & 19 & \text { UER }\end{array}$

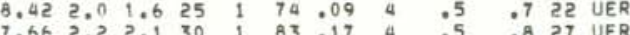

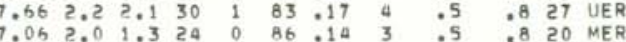
$\begin{array}{llllllllllll}7.66 & 1.9 & 1.6 & 27 & 0 & 73 & .09 & 3 & .5 & .8 & 27 & \text { UER } \\ 3.72 & 1.3 & 1.2 & 12 & 0 & 70 & 09 & 3 & .0 & .5 & 12 & \text { SPC }\end{array}$

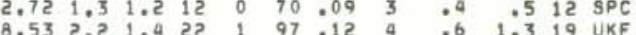


HVO EARTHQUAKE SUMMARY LIST

PAGE 87 ORIGIN TIME LAT N L LN W OEPTH AMP DUR N GAP RMS MIN ERH ERZ NO
DA HRMN SEC DEG MIN DEG MIN OKM MAG NAG NR NS DEG SEC OIS KM KM FM REMK YEAR MON DA HRMN SEC DEG MIN DEG MIN KM MAG "AG NR NS DEG SEC OIS KM KM FM REMK $12 \quad 2135 \quad 1.75 \quad 1923.65 \quad 15516.72$

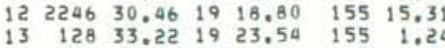
$\begin{array}{lllllll}13 & 520 & 37.42 & 19 & 19.40 & 155 & 21.87\end{array}$ $\begin{array}{lllllll}13 & 917 & 7.95 & 19 & 23.27 & 155 & 17.65 \\ 13 & 919 & 9.59 & 19 & 23.03 & 155 & 16.9\end{array}$

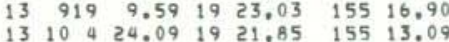

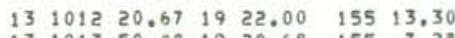

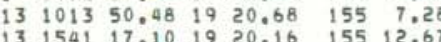

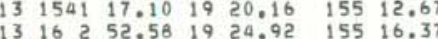
$13165356.03 \quad 1926.73 \quad 15520.93$

$\begin{array}{lllllll}13 & 1749 & 31.66 & 19 & 22.73 & 155 & 14.33 \\ 13 & 1759 & 18.22 & 19 & 23.24 & 155 & 17.22\end{array}$ $\begin{array}{lllllll}13 & 18 & 69.25 & 19 & 22.89 & 155 & 17.03\end{array}$ $132138 \quad 57.13 \quad 1920.70 \quad 155 \quad 18.40$

$\begin{array}{llllllll}14 & 1156 & 37.11 & 19 & 20.27 & 155 & 13.06\end{array}$

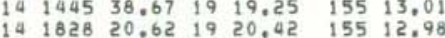
$142118 \quad 36.591919 .90 \quad 155 \quad 13.28$

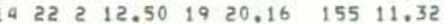

$\begin{array}{llllllll}15 & 0 & 6 & 33.73 & 19 & 23.31 & 155 & 14.93\end{array}$

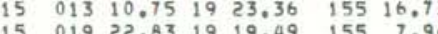
$15 \quad 1320.011923 .23 \quad 15515.01$

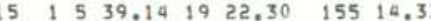

$\begin{array}{lllllll}15 & 130 & 5.47 & 19 & 23.27 & 155 & 14.84\end{array}$

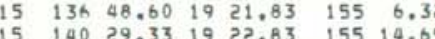
$\begin{array}{lllllll}15 & 158 & 7.55 & 19 & 23.13 & 155 & 14.79\end{array}$ $\begin{array}{lllllll}15 & 159 & 36.16 & 19 & 23.15 & 155 & 14.82\end{array}$

$\begin{array}{lllllll}15 \quad 212 & 51.61 & 19 & 23.27 & 155 & 15.00\end{array}$ $\begin{array}{lllllll}15 & 233 & 17.07 & 19 & 29.34 & 155 & 23.9\end{array}$

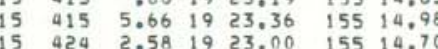

$15 \quad 512 \quad 20.02 \quad 1923.53 \quad 155 \quad 14.95$ $\begin{array}{lllllll}15 & 610 & 3.28 & 19 & 18.99 & 155 & 15.8\end{array}$

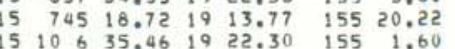

$15 \quad 1044 \quad 15.89 \quad 1920.52 \quad 15511.60$

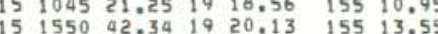

$\begin{array}{rrrrrrrrrrrr}5.42 & 1.9 & 1.0 & 21 & 0 & 78 & .09 & 4 & .5 & 1.8 & 20 & \text { UKF } \\ 3.0 A & 2.0 & 0.0 & 21 & 0 & 72 & .08 & 2 & .0 & .5 & 21 & \text { SPC }\end{array}$

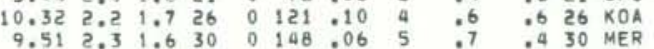

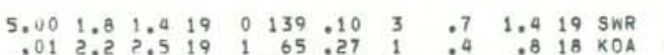

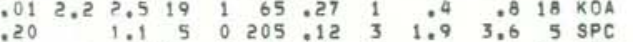

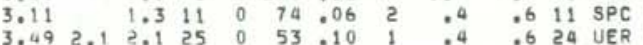
$\begin{array}{lllllllllllll}3.73 & 1.4 & 1.0 & 13 & 0 & 92 & .05 & 5 & .4 & 1.2 & 13 & \text { UER } \\ 0 & 2.7 & 2.3 & 2.3 & 28 & 0 & 91 & 07 & 5 & .5 & .5 & 26 & \text { UER }\end{array}$

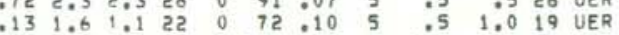
$\begin{array}{llllllllllll}3.44 & 1.6 & 1.1 & 15 & 0 & 72 & .06 & 2 & .4 & .5 & 14 & \text { UER } \\ 3.29 & 1.4 & 1.4 & 12 & 1 & 69 & .09 & 3 & .4 & .6 & 11 & \text { SPC }\end{array}$

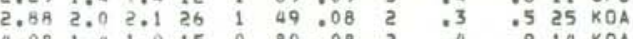
$\begin{array}{llllllllllll}4.08 & 1.4 & 1.0 & 15 & 0 & 80 & 08 & 2 & .4 & .9 & 14 & \text { KOA } \\ 8.19 & 1.9 & 1.4 & 28 & 2 & 86 & 10 & 5 & : 4 & .6 & 26 & \text { UER }\end{array}$

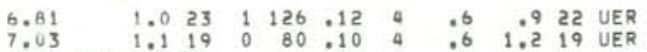

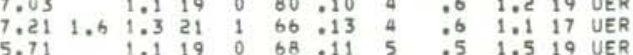

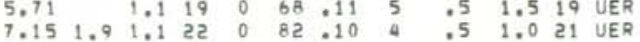
$\begin{array}{lllllllllllllll}3.55 & 1.2 & 1.0 & 7 & 0 & 103 & .02 & 2 & .7 & .6 & 6 & \text { GLN } \\ 3.95 & 2.4 & 2.6 & 21 & 0 & 101 & 07 & 2 & .4 & .7 & 20 & \text { SPC }\end{array}$

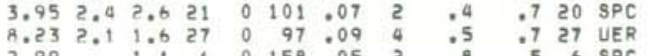

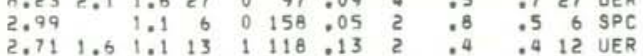
$\begin{array}{llllllllllll}3.57 & 1.6 & 1.5 & 14 & 0 & 69 & .08 & 2 & .4 & .5 & 14 & \mathrm{GLN}\end{array}$

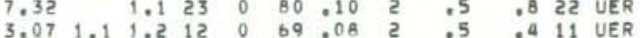

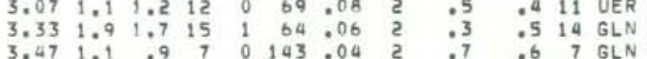
$\begin{array}{llllllllllll}2.78 & 1.0 & 1.0 & 4 & 0 & 159 & .01 & 2 & .7 & 1.0 & 4 & \text { GLN }\end{array}$

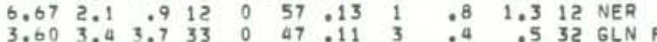

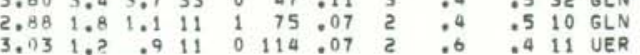

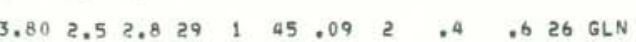

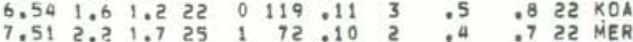

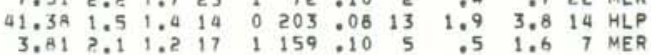
$\begin{array}{lllllllllllll}7.36 & 2.1 & .8 & 17 & 0 & 134 & .06 & 4 & .6 & .8 & 8 & \text { UER }\end{array}$

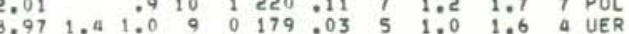

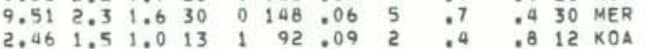
$\begin{array}{llllllllllll}2.53 & 1.3 & 1.1 & 6 & 0 & 149 & 13 & 1 & 1.0 & .7 & 5 & \text { SPC } \\ 4.41 & 1.8 & 1.1 & 17 & 0 & 77 & 11 & 7 & .5 & 6.4 & 17 & \text { UKF }\end{array}$
HVO EARTHQUAKE SUMMARY LIS

PAGE 88

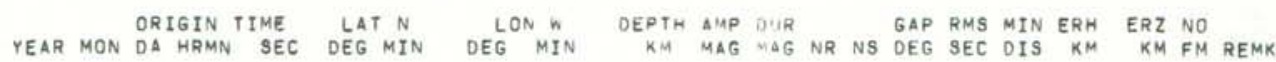

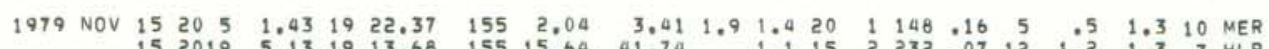

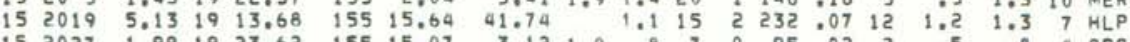

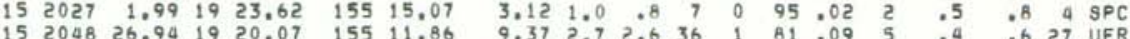

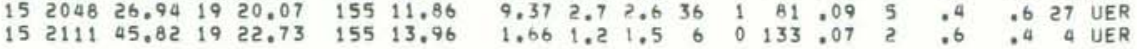

$\begin{array}{lllll}15 & 2119 \quad 7.21 & 19 & 22.09 \quad 155 \quad 13.62\end{array}$

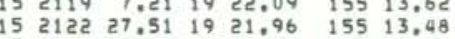

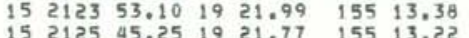

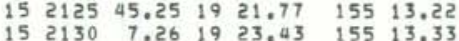

$\begin{array}{lllllll}15 & 2132 & 3.96 & 19 & 21.89 & 155 & 13.08 \\ 15 & 2137 & 6.02 & 19 & 21.65 & 155 & 13.37\end{array}$ $15213941.061922 .11-15513.50$

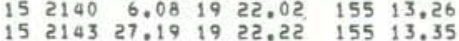

$\begin{array}{lllllll}15 & 2153 & 17.08 & 19 & 21.79 & 155 & 13.10\end{array}$

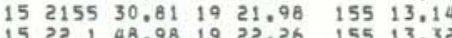

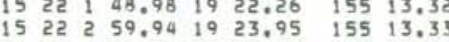
15 22 $556,701921.92 \quad 15513,06$

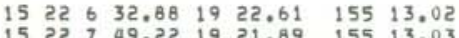
$15221035.22 \quad 1922.14 \quad 15513.19$

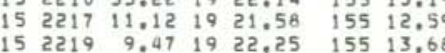

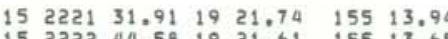
15 2236 18.51 19 $21.61 \quad 155 \quad 13.65$ $152237 \quad 8.32 \quad 19222.17 \quad 15513.04$

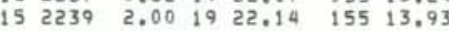
$\begin{array}{llllll}15 & 2241 & 32.57 \quad 19 & 22.04 \quad 155 \quad 13.90\end{array}$

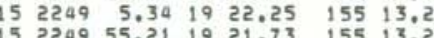
$\begin{array}{ll}15 & 225232.92 \quad 1922.95 \quad 155 \quad 14.49\end{array}$ $152257 \quad 39.16 \quad 1922.60 \quad 155 \quad 13,95$

$\begin{array}{lllllll}15 & 2258 & 42.28 & 19 & 21.75 & 155 & 13.65\end{array}$

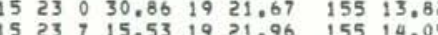

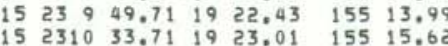

$\begin{array}{lllllll}15 & 2312 & 1.72 & 19 & 21.72 \quad 155 & 13.55\end{array}$ 15.231711 .071923 .2215513 .09

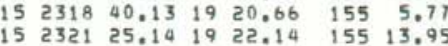
$\begin{array}{llllll}15 & 2325 \quad .38 & 19 & 22.46 & 155 & 14.23\end{array}$

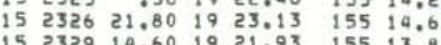

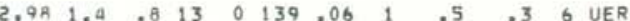

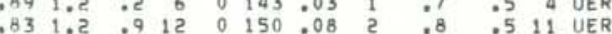

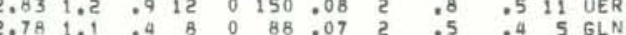

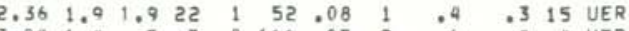
\begin{tabular}{llllllllllll}
3.08 & 1.4 & .7 & 7 & 0 & 166 & .07 & 2 & .6 & .4 & 4 & 4 \\
\hline
\end{tabular}

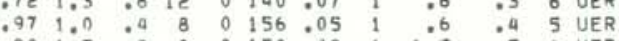

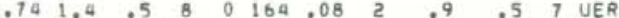

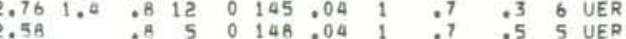
$\begin{array}{llllllllllllll}1.12 & 1.2 & .9 & 6 & 0 & 149 & .06 & 2 & .6 & .6 & 3 & 6 L N\end{array}$ $\begin{array}{llllllllllll}2.22 & 1.3 & 1.0 & 11 & 0 & 129 & .11 & 0 & .6 & .4 & 5 & \text { UER }\end{array}$

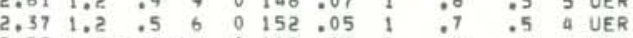

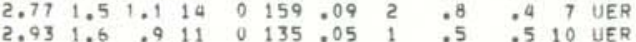

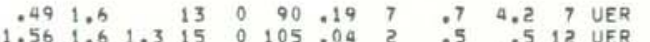

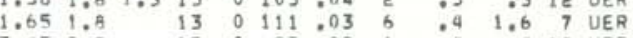

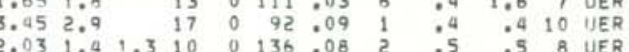
$\begin{array}{llllllllllll}1.42 & 2.7 & 3.2 & 19 & 0 & 85 & .09 & 2 & .3 & .5 & 18 & \text { UER }\end{array}$

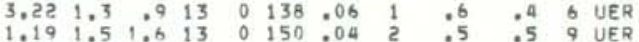

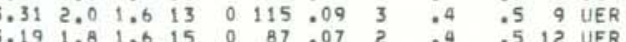
$\begin{array}{lllllllllll}1.49 & 1.8 & 2.2 & 17 & 0 & 55.08 & 2 & .4 & .5 & 14 & \text { UER }\end{array}$

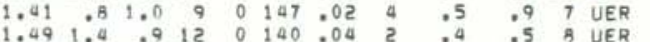

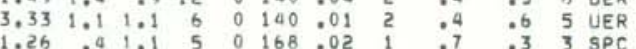
$\begin{array}{lllllllllllllll}1.61 & 1.6 & 1.5 & 10 & 0 & 148 & .07 & 2 & .5 & .5 & 8 & \text { UER }\end{array}$

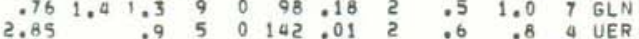

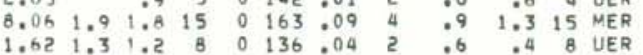
$\begin{array}{lllllllllllllll}1.23 & 1.2 & 1.3 & 6 & 0 & 151 & .03 & 2 & .7 & .5 & \text { a UER }\end{array}$

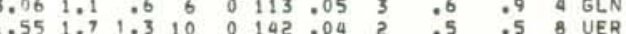

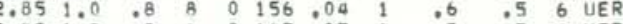




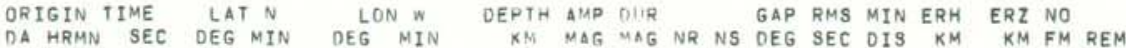
1979 NOV $152332 \quad 22.541922 .20 \quad 155 \quad 13.98$

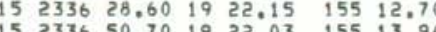

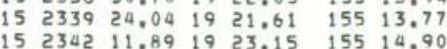

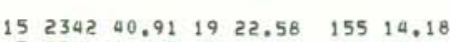
$\begin{array}{lllllll}15 & 2347 & 11.73 & 19 & 22.34 & 155 & 13.87\end{array}$ $\begin{array}{lllllll}15 & 2352 & 35.77 & 19 & 22.11 & 155 & 13.11 \\ 15 & 2354 & 29.53 & 19 & 21.18 & 155 & 14.01\end{array}$

$\begin{array}{lllllll}15 & 2358 & 19.58 & 19 & 22.89 & 155 & 14.53\end{array}$ $\begin{array}{llllllll}16 & 0 & 4 & 46.15 & 19 & 22.27 & 155 & 13.3\end{array}$ 16 010 14.731921 .80 155 14.03 $\begin{array}{lllllll}16 & 017 & 59.21 & 19 & 21.88 & 155 & 13,79\end{array}$ $\begin{array}{lllllll}16 & 021 & 54.75 & 19 & 22.99 & 155 & 14.37\end{array}$ $160023 \quad 54,93 \quad 1921.74 \quad 155 \quad 14,0$ $\begin{array}{lllllll}16 & 025 & 12.23 & 19 & 22.01 & 155 & 13.97\end{array}$ $16037 \quad 86.34 \quad 1922.20 \quad 155 \quad 14.09$ $\begin{array}{rrrrrrr}16 & 044 & 2.62 & 19 & 22.70 & 155 & 14,57 \\ 16 & 045 & 47.17 & 19 & 21.78 & 155 & 14.05\end{array}$ $\begin{array}{lllllll}16 & 045 & 47.17 & 19 & 21.78 & 155 & 14.05 \\ 16 & 047 & 58.86 & 19 & 21.48 & 155 & 14.22\end{array}$

$\begin{array}{lllllll}16 & 049 & 27.24 & 19 & 22.39 & 155 & 13.96\end{array}$

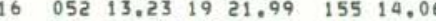
$16 \quad 05224.06 \quad 1921.64 \quad 15514.13$ $\begin{array}{lllllll}16 & 053 & 15.59 & 19 & 21.76 & 155 & 14.25 \\ 16 & 053 & 52.85 & 19 & 22.09 & 155 & 13.15\end{array}$

$\begin{array}{llllllllll}16 & 1 & 1 & 17.77 & 19 & 21.68 & 155 & 14.07 \\ 16 & 1 & 4 & 27.35 & 19 & 21.82 & 155 & 13.96\end{array}$ $\begin{array}{llllllll}16 & 1 & 4 & 27.35 & 19 & 21.82 & 155 & 13.96 \\ 16 & 1 & 8 & 16.84 & 19 & 22.82 & 155 & 14.28\end{array}$ $16 \quad 1 \quad 851.181921 .86 \quad 15514.10$

$\begin{array}{lllllll}16 & 126 & 39.34 & 19 & 22.60 & 155 & 14.11\end{array}$ $\begin{array}{lllllll}16 & 129 & 3.48 & 19 & 22.12 & 155 & 13.97 \\ 16 & 130 & 33.24 & 19 & 21.84 & 155 & 14.24\end{array}$ 1613033.241921 .84 155 14.20 $16 \quad 136 \quad 11,101922.05 \quad 15513.92$

$\begin{array}{lllllll}16 & 140 & 35.43 & 19 & 21.34 & 155 & 14.37 \\ 16 & 144 & 16.75 & 19 & 21.98 & 155 & 13.98\end{array}$ $16 \quad 14730.641921 .76 \quad 15514.23$

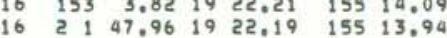
$\begin{array}{llllllll}16 & 2 & 4 & 58.69 & 19 & 22.10 & 155 & 12.99 \\ 16 & 2 & 5 & 33.65 & 19 & 22.27 & 155 & 13.86\end{array}$ $\begin{array}{llllllll}16 & 2 & 5 & 33.65 & 19 & 22.27 & 155 & 13.86 \\ 16 & 2 & 6 & 49.38 & 19 & 21.99 & 155 & 14.20\end{array}$ $\begin{array}{rrrrrrrrrrrr}.89 & 2.3 & 2.9 & 18 & 0 & 82 & .06 & 2 & .3 & .4 & 13 & \text { UER } \\ 2.70 & & .8 & 7 & 0 & 155 & .05 & 1 & .8 & .5 & 4 & \text { UER }\end{array}$

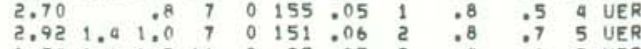

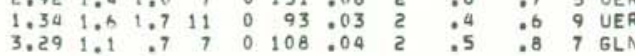

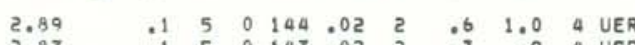

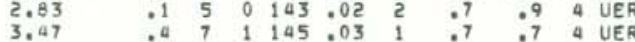
$\begin{array}{llllllllllllll}3.44 & 1.4 & .9 & 10 & 0 & 141 & 02 & 1 & .6 & .4 & 6 & \text { UER } \\ 1.50 & 3.4 & 3.8 & 25 & 0 & 62 & .09 & 3 & .2 & 06 & 18 & \text { UER }\end{array}$ $\begin{array}{llllllllllll}3.62 & 2.6 & 2.5 & 22 & 0 & 65 & .08 & 3 & .4 & .5 & 21 & \text { UER }\end{array}$

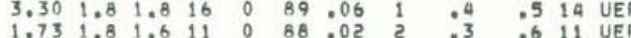

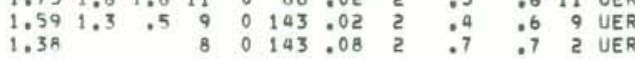
$\begin{array}{llllllllllll}1.61 & 1.4 & 1.3 & 9 & 0 & 119 & .07 & 2 & .4 & .4 & 6 & \text { UER } \\ 1.76 & 1.9 & 1.1 & 8 & 0 & 142 & .05 & 2 & .5 & 1.0 & 7 & \text { UER }\end{array}$

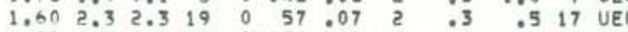

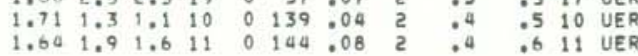
$\begin{array}{lllllllllllll}1.67 & 1.6 & 1.6 & 11 & 0 & 135 & 04 & 2 & .3 & .4 & 8 & \text { UER } \\ 1.70 & & 1.1 & 11 & 1 & 136 & 05 & 2 & .3 & .5 & 7 & \text { UER }\end{array}$ $\begin{array}{llllllllllll}1.47 & 1.8 & 2.1 & 15 & 0 & 71 & .06 & 2 & .3 & .4 & 12 & \text { UER } \\ 1.69 & 1.9 & 2.2 & 12 & 0 & 88 & .03 & 2 & .4 & 0 & 10 & \text { UER }\end{array}$

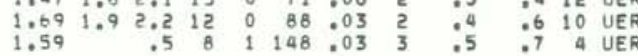
$\begin{array}{lllllllllllllll}3.17 & 2.2 & 2.0 & 17 & 0 & 80 & .04 & 2 & .3 & .4 & 16 & \text { UER }\end{array}$

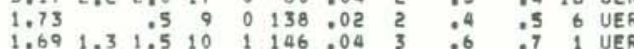

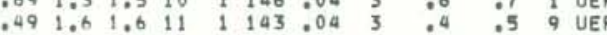

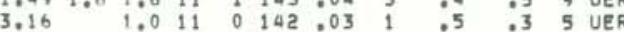
$\begin{array}{llllllllllllll}1.69 & 1.7 & 1.7 & 12 & 0 & 104 & 04 & 2 & .3 & .6 & 11 & \text { UER }\end{array}$

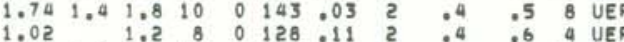

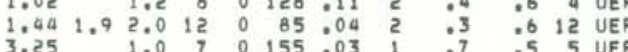
$\begin{array}{llllllllllllll}3.14 & 1.3 & 1.7 & 6 & 1 & 135 & .04 & 2 & .5 & .7 & 4 & \text { UER } \\ 1.52 & 2.1 & 2.5 & 14 & 0 & 84 & .06 & 2 & .3 & 05 & 13 & \text { UER }\end{array}$ 1,41 1.8 2.2 12 $00141: 03 \quad 3 \quad 04 \quad .611$ UER

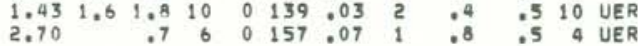
\begin{tabular}{llllllllllll}
0.97 & 2.1 & 1.8 & 14 & 0 & 108 & 08 & 3 & .4 & .7 & 3 & UER \\
1.64 & & 1.2 & 9 & 0 & 140 & 03 & 2 & 04 & .5 & 7 & 7 \\
\hline
\end{tabular}

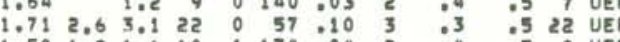

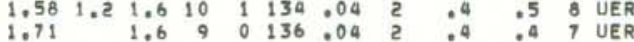

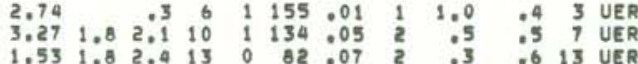

ORIGIN TIME LATN LON W DEPTH AMP OUR GAP RMS MIN ERH ERZ NO

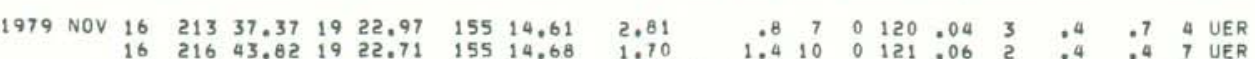

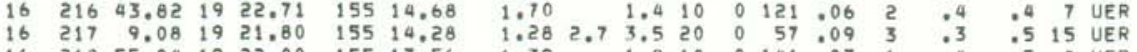

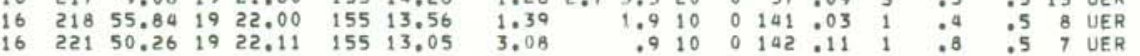

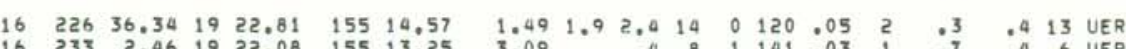

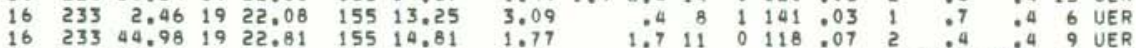

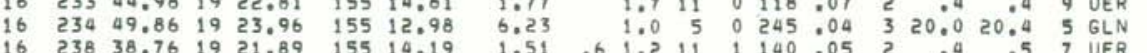
$\begin{array}{lllllll}16 & 245 & 15.53 \quad 19 & 22.67 \quad 155 & 14.38\end{array}$ $16 \quad 253 \quad 13.431922 .49 \quad 15513.8$

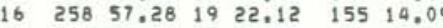

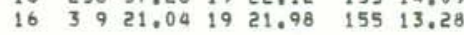

$\begin{array}{lllllll}16 & 310 & 14.29 & 19 & 21.09 & 155 & 14.22\end{array}$ $16 \quad 323 \quad 17.23 \quad 1922.09 \quad 15514.47$

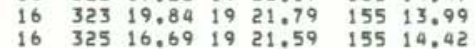

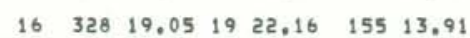

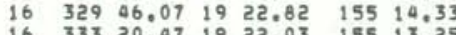

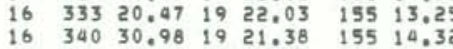
$16 \quad 352 \quad 26.081922 .90 \quad 15515.10$

$\begin{array}{lllllll}16 & 356 & 15.41 & 19 & 22.85 & 155 & 14.92\end{array}$

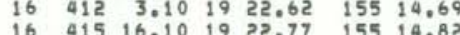
16 416 59.76 19 $23.09 \quad 15514.69$

$\begin{array}{lllllll}16 & 434 & 2.92 & 19 & 20.39 & 155 & 13.79\end{array}$

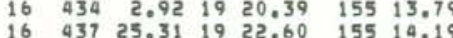

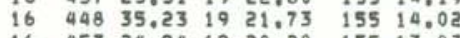
$\begin{array}{lllllll}16 & 453 & 24.94 & 19 & 20,20 & 155 & 13.03 \\ 16 & 456 & 50,55 & 19 & 21.70 & 155 & 14.37\end{array}$ $\begin{array}{llllllll}16 & 5 & 1 & 58.41 & 19 & 22.66 & 155 & 14.49 \\ 16 & 5 & 4 & 33.77 & 19 & 22.19 & 155 & 13.38\end{array}$ $\begin{array}{llllllll}16 & 5 & 0 & 46.57 & 19 & 21.58 & 155 & 14.18 \\ 16 & 5 & 5 & 42.13 & 19 & 22.23 & 155 & 14.37\end{array}$

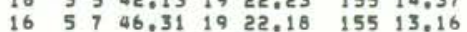

$\begin{array}{lllllll}16 & 519 & 13.41 & 19 & 21,75 & 155 & 13.78 \\ 16 & 519 & 42.92 & 19 & 22,08 & 155 & 13.94\end{array}$

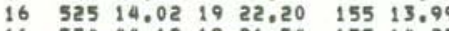
i6 $53729.4919 \quad 23.09 \quad 15514.25$

$\begin{array}{lllllll}16 & 541 & 59.56 & 19 & e 1.97 & 155 & 13.90 \\ 16 & 542 & 25.32 & 19 & 22.85 & 155 & 14.54\end{array}$

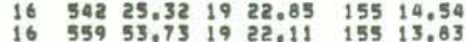

$\begin{array}{lllllllllllll}1.05 & .3 & 1.0 & 9 & 0 & 129 & 08 & 2 & .6 & .5 & 4 & 4 & \text { UER }\end{array}$

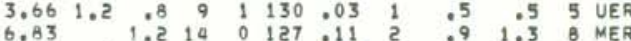

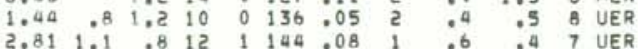
$\begin{array}{lllllllllllll}1.11 & 1.3 & 1.0 & 10 & 1 & 158 & .05 & 3 & .4 & .9 & 6 & \text { UER }\end{array}$

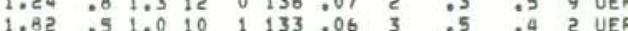
$\begin{array}{llllllllllll}2.14 & 2.1 & 2.5 & 21 & 0 & 56 & .14 & 2 & .3 & .6 & 14 & \text { UER } \\ 1.83 & .8 & .8 & 7 & 1 & 211 & .03 & 3 & .9 & .5 & 2 & \text { UER }\end{array}$ $\begin{array}{llllllllllllll}2.84 & 1.1 & 1.0 & 13 & 1 & 136 & .07 & 2 & .4 & .4 & 9 \\ 3 & \text { UER }\end{array}$

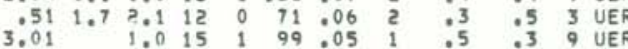

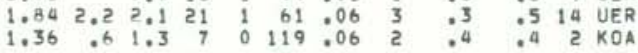
$\begin{array}{llllllllllll}1.29 & 1.4 & 1.8 & 13 & 1 & 80 & .10 & 2 & .4 & .4 & 9 \text { UER }\end{array}$

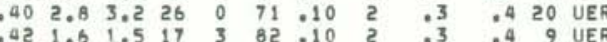

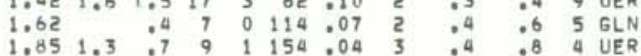
$\begin{array}{llllllllllll}.24 & 1.9 & 1.8 & 9 & 0 & 178 & .10 & 4 & .5 & 1.9 & 2 \\ 3 & \text { UER }\end{array}$

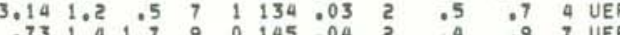

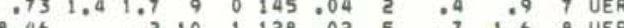

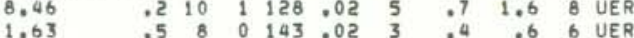
$\begin{array}{lllllllllllllll}1.21 & .5 & 5 & 0 & 137 & .02 & 2 & .4 & .5 & 2 & \text { UER }\end{array}$

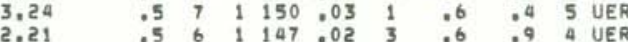

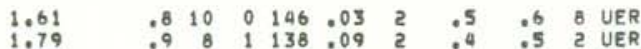

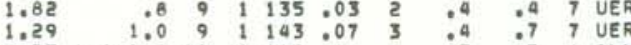
$2.731 .21 .210+107.06 \quad 2 \quad .5 \quad .766 \mathrm{GLN}$

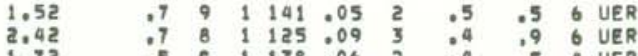
$\because 7 \quad 6 \quad 0185.04$ a 1.0 .5 4 UER 


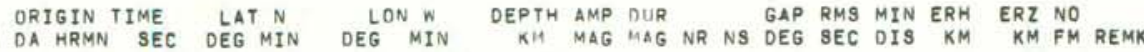

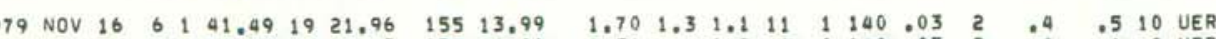

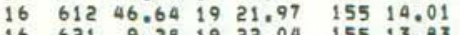
$16 \quad 653 \quad 3.11$ 19 $21.63 \quad 15513.83$ $16 \quad 656 \quad 48,13 \quad 1922.64 \quad 155 \quad 14,56$ $\begin{array}{llllllll}16 & 720 & 4.18 & 19 & 21.80 & 155 & 14.06 \\ 16 & 727 & 23.86 & 19 & 21.63 & 155 & 14.01\end{array}$

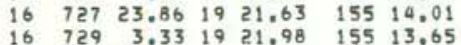

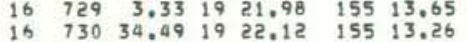
$16 \quad 740 \quad 24.13 \quad 1921.76 \quad 155 \quad 14.59$ $\begin{array}{lllllll}16 & 743 & 46.87 & 19 & 21.47 & 155 & 14.02\end{array}$ $16 \quad 74653.981923 .37-15515.19$ $\begin{array}{lllllll}16 & 759 & 7.86 & 19 & 21.59 & 155 & 14.030\end{array}$

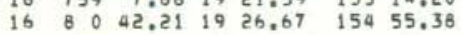
$\begin{array}{llllllll}16 & 8 & 5 & 37.28 & 19 & 21.50 & 155 & 14.43\end{array}$

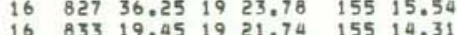
$16 \quad 84901,78$ 19 $21,57 \quad 15519,59$ $\begin{array}{lllllll}16 & 852 & 46.24 & 19 & 18.38 & 155 & 14.92\end{array}$

$\begin{array}{llllllll}16 & 853 & .19 & 19 & 21.57 & 155 & 14.25\end{array}$

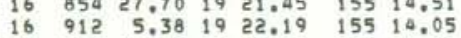
$\begin{array}{lllllll}16 & 912 & 41.79 & 19 & 22.57 & 155 & 14.30 \\ 16 & 931 & 40.57 & 19 & 21.19 & 155 & 14.08\end{array}$

$\begin{array}{lllllll}16 & 935 & 16.35 & 19 & 22.03 & 155 & 14.05\end{array}$ $\begin{array}{lllllll}16 & 936 & 18.74 & 19 & 22.07 & 155 & 14.04\end{array}$

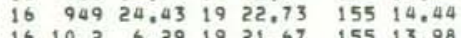

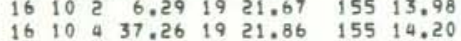
$\begin{array}{lll}16 & 10 & 4 \\ 53.90 & 19 & 21.66 \quad 155 \quad 14.08\end{array}$ $16 \quad 106 \quad 3.90 \quad 1922.15 \quad 155 \quad 14.18$ $16 \quad 1015 \quad 47.87 \quad 1922.68 \quad 155 \quad 14.51$

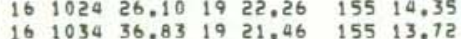

$\begin{array}{lllllll}16 & 1044 & 27.81 & 19 & 22.13 & 155 & 13.70\end{array}$

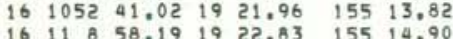
$\begin{array}{lllllll}16 & 11 & 58.19 & 19 & 22,83 & 155 & 14,90 \\ 16 & 1112 & 21.20 & 19 & 22,06 & 155 & 14,25\end{array}$ $\begin{array}{llll}16 & 1115 & 8.67 & 19 \\ 21.96 & 15513.80\end{array}$ $\begin{array}{lllllll}16 & 1123 & 57.70 & 19 & 22.36 & 155 & 14.44\end{array}$

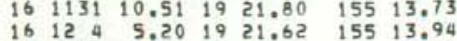

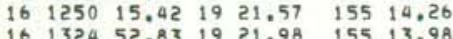

$\begin{array}{lllllll}16 & 1325 & 43.68 & 19 & 23.79 & 155 & 15.69\end{array}$

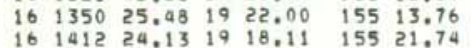

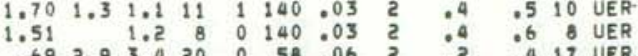

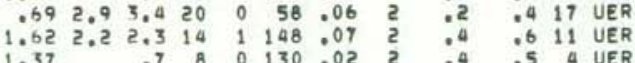

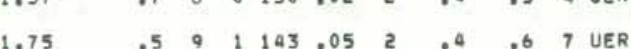

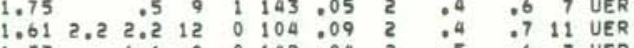

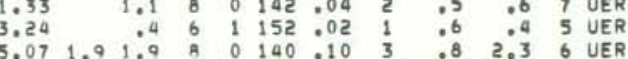
$\begin{array}{lllllllllllll}1.19 & 1.0 & 7 & 0 & 151.02 & 4 & .4 & 1.3 & 6 & \text { UER }\end{array}$

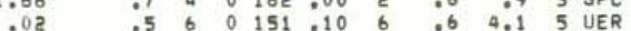
$\begin{array}{lllllllllll}2.45 & .6 & 6 & 0 & 147 & .03 & 6 & .5 & 2.2 & 4 & \text { UER }\end{array}$

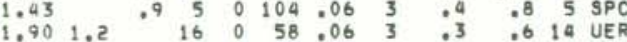

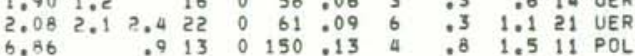
$\begin{array}{llllllllllll}2.03 & 1.6 & 1.2 & 9 & 0 & 84 & .05 & 3 & .4 & 1.1 & 7 & 7 \text { UER }\end{array}$

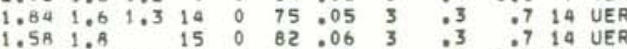

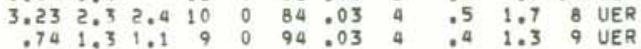
$\begin{array}{llllllllllll}1.26 & 1.3 & 1.8 & 9 & 0 & 87 & .04 & 3 & .4 & 1.1 & 8 & \text { UER }\end{array}$

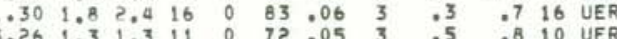

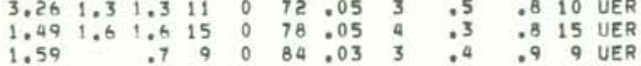
$\begin{array}{llllllllllllll}1.99 & 1.7 & 2.1 & 13 & 0 & 76 & .06 & 3 & .3 & 1.1 & 12 & \text { UER }\end{array}$

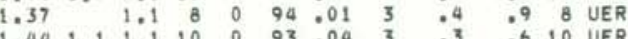

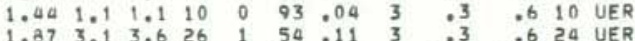

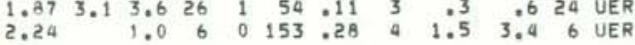
$\begin{array}{rrrrrrrrrrrr}.79 & 1.3 & 1.0 & 9 & 0 & 103 & 04 & 4 & .4 & 1.1 & 8 & \text { UER } \\ 86 & & 1.2 & 8 & 0 & 93 & 02 & 4 & .5 & 1.1 & 8 & \text { UER }\end{array}$

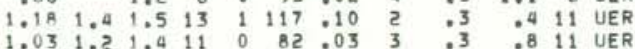
$\begin{array}{llllllllllll}1.03 & 1.2 & 1.4 & 11 & 0 & 82.03 & 3 & .3 & .8 & 11 & \text { UER } \\ .21 & 2.1 & 2.7 & 13 & 1 & 88 & .15 & 4 & .9 & .8 & 11 & \text { UER }\end{array}$ $\begin{array}{llllllllllll}1.43 & 1.9 & 2.6 & 18 & 0 & 77 & .08 & 3 & .3 & .5 & 17 & \text { UER }\end{array}$

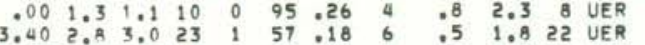

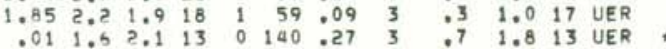

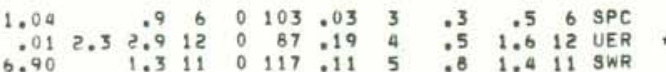
$\begin{array}{lllllllllll}1.14 & 1.0 & 11 & 0 & 147 & 04 & 3 & .3 & : 9 & 7 \text { UER } \\ 5.56 & 1.1 & 15 & 0 & 238 & 011 & 2 & 2.6 & 1.9 & 12 & \text { LER }\end{array}$
ORIGIN TIME LAT N LON W DEPTH AMP DUR GAP RMS MIN ERH ERZ NO YEAR MON OA HRMN SEC DEG MIN DEG MIN KM MAG MAG NR NS DEG SEC DIS KM KM FM REMK

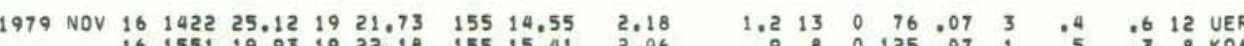

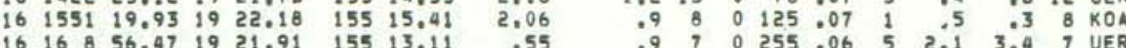

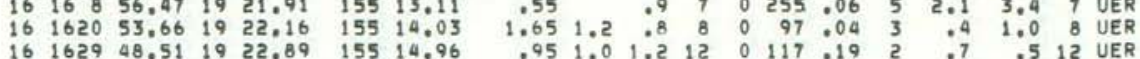
$\begin{array}{lllllll}16 & 1923 \quad 43.30 \quad 19 \quad 21.28 \quad 155 \quad 13.67\end{array}$ 16 $201356.421923 .06 \quad 15515.73$ 16 21 403,01 19 $23.14 \quad 15514,35$

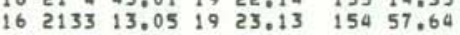

$\begin{array}{lllllll}16 & 2151 \quad 19.44 \quad 19 & 22.03 \quad 155 & 13.31\end{array}$

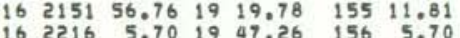
$\begin{array}{rrrrrrr}17 & 138 & 32.70 & 19 & 21.36 & 155 & 14.89 \\ 17 & 442 & 5.77 & 19 & 21.42 & 155 & 14.20\end{array}$

$\begin{array}{llllll}17 & 611 & 59.55 & 19 & 20.92 \quad 155 \quad 13.29\end{array}$ $\begin{array}{lllllll}17 & 647 & 40.21 & 19 & 22.44 & 155 & 29.87 \\ 17 & 756 & 34.31 & 19 & 23.85 & 155 & 15.41\end{array}$

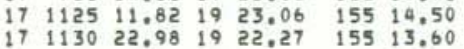

$17 \quad 1456 \quad 44.74 \quad 1927.42 \quad 155 \quad 21.24$

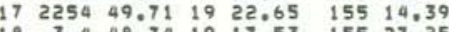
18 3 $3408.7401917 .53 \quad 15527.25$ $\begin{array}{lllllll}18 & 337 & 24.11 & 19 & 20.95 & 155 & 6.54 \\ 18 & 518 & 16.62 & 19 & 21.88 & 155 & 1.61\end{array}$

$\begin{array}{lllllll}18 & 520 & 43.61 & 19 & 22.92 & 155 & 14.31\end{array}$ $18 \quad 545 \quad 49.931922 .88$ 155 14.48

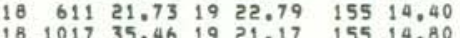
$\begin{array}{lllllll}18 & 1017 & 35.46 & 19 & 21.17 & 155 & 14.80 \\ 18 & 1312 & 18.12 & 19 & 23,10 & 155 & 14.86\end{array}$

$18 \quad 1459 \quad 49.02 \quad 19 \quad 10.58 \quad 155 \quad 26.19$ $18 \quad 1618 \quad 11.91$ i9 $23.13 \quad 155 \quad 14.63$ $\begin{array}{llllllll}18 & 2153 & 41.34 & 19 & 14.04 & 155 & 16.38 \\ 18 & 2332 & 42.83 & 19 & 23.23 & 155 & 14.76\end{array}$ $\begin{array}{lllllll}18 & 2332 & 42.83 & 19 & 23.23 & 155 & 14.76 \\ 18 & 2342 & 28.69 & 19 & 22.99 & 155 & 14.89\end{array}$

$\begin{array}{lllllll}18 & 2343 & 49.12 & 19 & 23.13 & 155 & 14.91\end{array}$

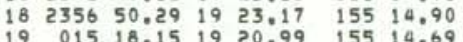

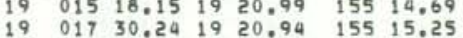

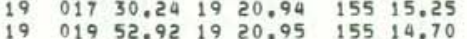

$\begin{array}{lllllll}19 & 020 & 47.88 & 19 & 21.01 & 155 & 14.60\end{array}$ $\begin{array}{llllllll}19 & 056 & 10.79 & 19 & 19.96 & 155 & 13.04 \\ 19 & 217 & 1.31 & 19 & 16.10 & 155 & 11.87\end{array}$

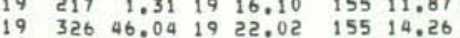

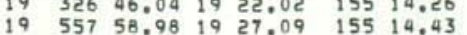

$\begin{array}{lllllll}19 & 648 & 37.22 & 19 & 22.59 & 155 & 13.54 \\ 19 & 930 & 41.82 & 19 & 23.20 & 155 & 14.77\end{array}$ 19 $931 \quad 53.46 \quad 19 \quad 23.74 \quad 155 \quad 5.56$

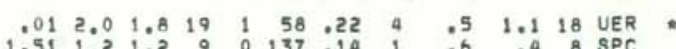

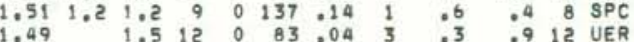

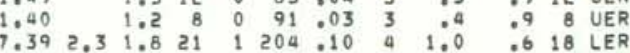
$\begin{array}{llllllllll}3.46 & .8 & 4 & 0 & 155.00 & 5 & .9 & 3.6 & 3 & \text { UER }\end{array}$

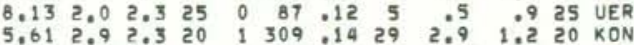
\begin{tabular}{llllllllllll}
1.44 & 1.5 & 1.7 & 14 & 0 & 65 & 12 & 3 & 4 & 8 & 14 & UER \\
.01 & 1.3 & 1.1 & 12 & 0 & 89 & 12 & 3 & $: 4$ & 9 & 12 & 12 \\
\hline
\end{tabular} $\begin{array}{lllllllllllll}9.60 & 3.3 & 3.5 & 34 & 1 & 58 & .08 & 3 & .3 & .4 & 31 & \text { UER F }\end{array}$

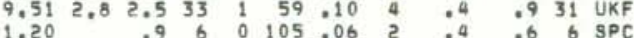

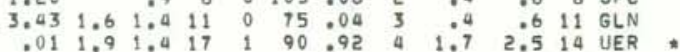

$\begin{array}{lllllllllllll}8.61 & 2.0 & 1.5 & 19 & 1 & 96 & .11 & 6 & .6 & 1.0 & 18 & \text { UKF }\end{array}$

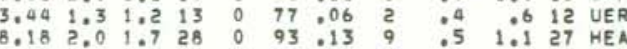

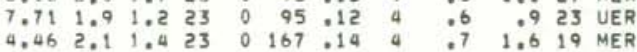
$\begin{array}{llllllllllll}3.43 & 1.8 & 1.6 & 18 & 1 & 68 & .11 & 2 & .4 & .6 & 17 & \text { UER }\end{array}$ $\begin{array}{llllllllllll}3.08 & 1.7 & 1.3 & 16 & 0 & 69 & .10 & 3 & .4 & .5 & 15 & 15 \\ .06 R\end{array}$

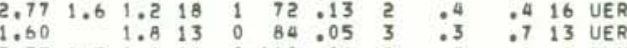
$3331,51,010$ 0 110.04204 .6 8 GLN

$\begin{array}{llllllllllll}9.17 & 2.2 & 1.3 & 15 & 0 & 168 & .14 & 4 & 2.9 & 5.5 & 8 & \text { LSW }\end{array}$

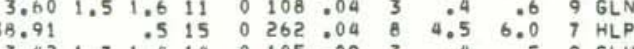
$\begin{array}{llllllllllll}3.42 & 1.7 & 1.4 & 14 & 0 & 105 & 09 & 3 & .4 & .5 & 9 & \text { GLN } \\ 3.11 & 1.6 & 1.3 & 10 & 0 & 114 & .06 & 2 & .5 & .6 & 8 & \text { UER }\end{array}$ $\begin{array}{llllllllllll}2.67 & 1.1 & .7 & 7 & 0 & 109 & .03 & 2 & .4 & .6 & 5 & 5 L N\end{array}$

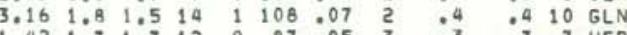

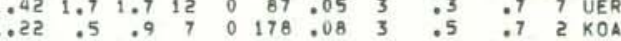

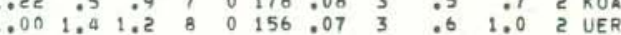

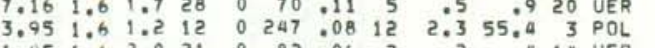
$\begin{array}{llllllllllll}1.05 & 1.6 & 2.0 & 21 & 0 & 82 & 06 & 2 & .2 & .04 & 14 & \text { UER } \\ 33.01 & 2.5 & 2.3 & 40 & 2 & 50 & .09 & 5 & .6 & 1.1 & 32 & \text { DEP }\end{array}$

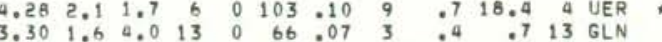
$\begin{array}{llllllllllll}1.66 & 2.2 & 2.2 & 18 & 0 & 68 & .06 & 3 & .3 & .6 & 12 & \text { UER } \\ 7.16 & 1.6 & 1.7 & 28 & 0 & 70 & .11 & 5 & 5 & .9 & 20 & \text { UER }\end{array}$ 
HVO EARTHLUAKE SUMMARY LIST

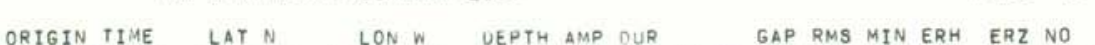
YEAR MON DA HRMN SEC DEG MIN DEG NIN UEPTH AMP OUR MR GAP RMS MIN ERH ERZ NO 1979 Nov $19124512.801922 .78 \quad 155 \quad 14.45$ $\begin{array}{lllllll}19 & 1658 & 13.86 & 19 & 19.88 & 155 & 8.07\end{array}$

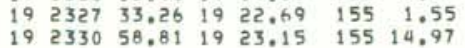

$\begin{array}{lllllll}20 & 127 & 9.45 & 19 & 23.01 & 155 & 14.44\end{array}$

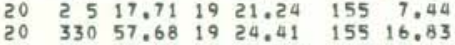
$20 \quad 34658.021923 .05 \quad 155 \quad 14.62$

$\begin{array}{lllllll}20 & 937 & 30.74 & 19 & 23.24 & 155 & 14.95\end{array}$ $\begin{array}{llrllll}20 & 1035 & 4.40 & 19 & 23.13 & 155 & 14.76 \\ 20 & 1035 & 25.60 & 19 & 23.13 & 155 & 14.72\end{array}$ 20103525.601923 .13 155 14,72 $201144 \quad 16.741922 .49 \quad 155 \quad 13.96$

$\begin{array}{lllllll}20 & 1357 & 57.93 & 19 & 23.27 & 155 & 15.10 \\ 20 & 1437 & 25.75 & 19 & 23.43 & 155 & 24.02\end{array}$ $20 \quad 1532 \quad 40.71 \quad 1920.29 \quad 155 \quad 8.62$ 20 17939.79 , 19.61 155 10.93

$\begin{array}{lllllll}20 & 1950 & 46.24 & 19 & 28.93 & 154 & 54.93 \\ 20 & 2019 & 31.68 & 19 & 22.05 & 155 & 13.96\end{array}$

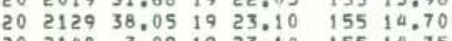
$2133018.36 \quad 1926,10 \quad 155 \quad 23,10$

$\begin{array}{lllllll}21 & 318 & 27.17 & 19 & 22.83 & 155 & 14.52 \\ 21 & 319 & 54.00 & 19 & 19.88 & 155 & 10.42\end{array}$

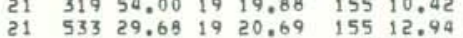
$\begin{array}{lllllll}21 & 533 & 29.668 & 19 & 20.69 & 155 & 12.44 \\ 21 & 558 & 48.82 & 19 & 23.17 & 155 & 14.84\end{array}$ 216 8 57.171921019 155 6.70 $\begin{array}{llllllll}21 & 9 & 7 & 27.57 & 19 & 16.27 & 155 & 46.60 \\ 21 & 943 & 39.22 & 19 & 22.67 & 155 & 14.43\end{array}$ 21 $943 \quad 39.23 \quad 1922.70 \quad 15514.43$ $21 \quad 13241.051923 .66 \quad 155 \quad 15,04$

$\begin{array}{llllllll}21 & 14 & 6 & 40.24 & 19 & 23.08 & 155 & 14.84\end{array}$

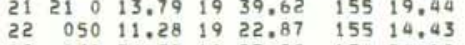
$22 \quad 15954.57 \quad 19 \quad 27.90 \quad 15454.10$

$\begin{array}{lllllll}22 & 550 & 36.92 & 19 & 22.01 & 155 & 6.32\end{array}$ $22 \quad 63814.151923 .33 \quad 15514.93$ $\begin{array}{lllllll}22 & 1328 & 25.55 & 19 & 23.09 & 155 & 14.81 \\ 22 & 1437 & 28.90 & 19 & 23.41 & 155 & 15.05\end{array}$ $\begin{array}{lllllll}22 & 1742 \quad 30.75 & 19 & 18.64 & 155 & 13.45\end{array}$ $22 \quad 2111 \quad 7.91 \quad 1922.76 \quad 155 \quad 14.49$

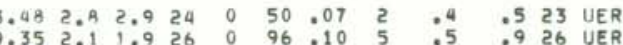

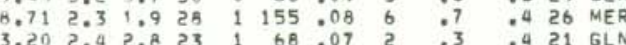
$\begin{array}{rlllllllllll}3.53 & 1.2 & 1.4 & 13 & 0 & 112 & .07 & 3 & .5 & .6 & 13 & \text { GLN } \\ 3.26 & 2.5 & 0.6 & 32 & 0 & 81 & : 11 & 4 & .5 & .6 & 31 & \text { UER }\end{array}$ $\begin{array}{llllllllllllll}1.34 & 1.0 & 1.3 & 6 & 0 & 100 & .04 & 1 & .5 & .5 & 4 & S P C\end{array}$ $\begin{array}{rrrrrrrrrrrr}3.41 & 1.8 & 1.6 & 18 & 0 & 71 & .08 & 2 & .3 & .4 & 11 & \mathrm{GLN} \\ 3.18 & 1.7 & 1.4 & 15 & 0 & 109 & .08 & 2 & 04 & .4 & 9 & G L N\end{array}$ $\begin{array}{llllllllllll}3.26 & 1.7 & .0 & 13 & 1 & 108 & .04 & 2 & .4 & .5 & 7 & 6 L N\end{array}$

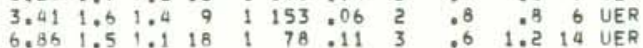
$\begin{array}{llllllllllll}3.09 & 2.1 & .9 & 16 & 0 & 74 & .08 & 2 & .4 & .4 & 12 & \text { SPC } \\ 9.58 & 1.6 & 4 & 21 & 1 & 69 & 06 & 4 & 04 & .8 & 14 & \text { UKF }\end{array}$

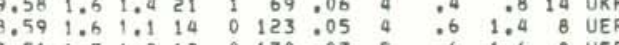

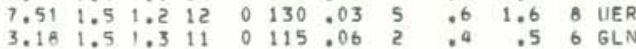
$\begin{array}{rrrrrrrrrrrr}3.04 & 2.0 & .9 & 10 & 0 & 116 & .06 & 3 & 1.3 & 1.3 & 5 & \text { LER } \\ 1.38 & 1.3 & 1.3 & 11 & 0 & 139 & .05 & 2 & .4 & .4 & 7 \text { UER }\end{array}$ 4.141 .01 .116 $\begin{array}{llllllllllllll}3.25 & 1.3 & 1.2 & 11 & 0 & 121 & 03 & 3 & .4 & .5 & 4 & \text { UER } \\ 8.96 & 1.9 & 1.7 & 17 & 1 & 120 & 05 & 4 & 0 & 1.2 & 13 & \text { UER }\end{array}$

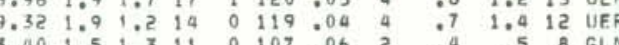
$\begin{array}{lllllllllllll}3.40 & 1.5 & 1.3 & 11 & 0 & 107 & 06 & 2 & .4 & .5 & 8 & \text { GLN } \\ 8.33 & 1.6 & 1.2 & 9 & 0 & 161 & .04 & 3 & .8 & 1.8 & 8 & \text { UER }\end{array}$ $\begin{array}{llllllllllll}10.30 & 3.1 & 2.7 & 33 & 1 & 108 & .13 & 10 & .5 & .6 & 17 & \text { KON } \\ 3.22 & 1.5 & 1.3 & 14 & 0 & 123 & .04 & 2 & .5 & .4 & 10 & \text { UER }\end{array}$

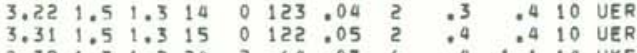

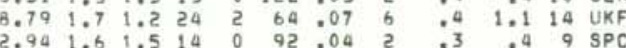
$\begin{array}{lllllllllll}3.09 & 1.4 & 1.1 & 12 & 0 & 110 & .04 & 2 & .3 & .5 & 9\end{array}$

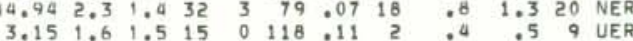

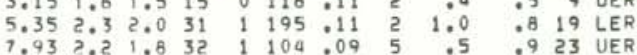
$\begin{array}{llllllllllll}7.92 & 1.7 & 1.4 & 16 & 0 & 111 & .08 & 2 & .5 & .9 & 9 & 9 \\ 3 & 0 E R\end{array}$

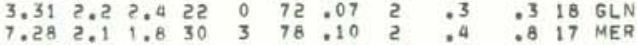
$\begin{array}{llllllllllll}3.29 & 2.6 & 2.8 & 32 & 1 & 48 & .07 & 2 & .3 & .3 & 21 & \text { GLN } \\ 3.26 & 1.0 & 1.0 & 10 & 0 & 99 & 04 & 0 & .3 & .0 & 8 & \text { SPC }\end{array}$ $\begin{array}{llllllllllll}9.18 & 3.1 & 3.0 & 43 & 0 & 78 & .10 & 3 & .4 & .4 & 34 & \text { POL }\end{array}$

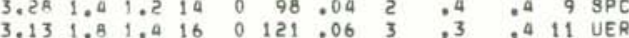

HVO EARTHQUAKE SUMMARY LIST

PAGE 94

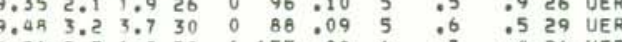
$\begin{array}{llllllllllllll}3.35 & 1.7 & : 3 & 12 & 0 & 111 & 05 & 3 & 0 & 4 & .4 & 12 & \mathrm{GLN} \\ 3.30 & 2.4 & 1.3 & 18 & 0 & 180 & : 12 & 9 & 9 & 5.2 & 15 & \mathrm{kON}\end{array}$

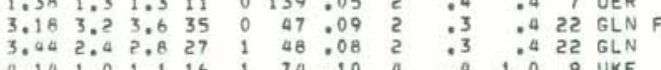

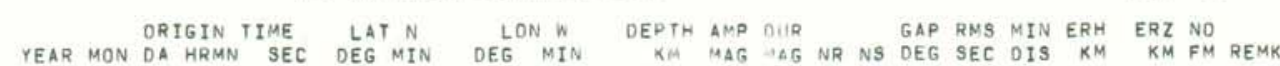

1979 NOV $22 \quad 2131 \quad 53.36 \quad 19 \quad 19.18 \quad 155 \quad 8.69$

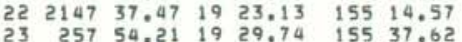

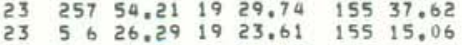
$2350 \quad 4.48 \quad 1923,03 \quad 155 \quad 14.79$

$\begin{array}{lllllll}23 & 541 & 15.75 & 19 & 22.06 & 155 & 14.22\end{array}$

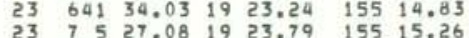

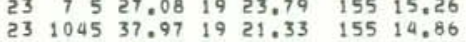

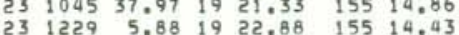

$\begin{array}{lllllll}23 & 1250 & 14.24 & 19 & 23.07 & 155 & 14.60\end{array}$ 23184026.701910 .6515517 .033 $\begin{array}{llllllll}23 & 1848 & 27.67 & 19 & 26.79 & 155 & 23.68\end{array}$

$\begin{array}{lllllll}24 & 414 & 34.88 & 19 & 23.67 & 155 & 15.13\end{array}$ $24 \quad 91908.391923 .01 \quad 15514.57$ 24 $95731.971920 .45 \quad 15512.84$ $\begin{array}{llllllll}24 & 2048 & 13.50 & 19 & 19.87 & 155 & 8.43\end{array}$ $242051 \quad 30.62 \quad 1922.79 \quad 155 \quad 14.40$ 24 $2131 \quad 6.641924 .001551927 .16$

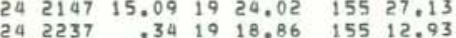
$25 \quad 050 \quad 1.94 \quad 19 \quad 18.95 \quad 155 \quad 11.31$

$\begin{array}{lllllll}25 & 523 & 29.74 & 19 & 23.02 & 155 & 14.45\end{array}$

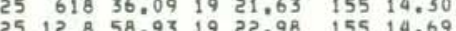
$\begin{array}{lllllll}25 & 1327 & 31.61 & 19 & 17.94 & 155 & 21.11 \\ 25 & 1531 & 47.10 & 19 & 23.13 & 155 & 14.97\end{array}$

$\begin{array}{llllllll}25 & 16 & 3 & 27.64 & 19 & 23.84 & 155 & 15.40\end{array}$

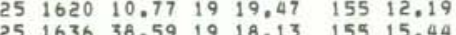
$\begin{array}{lllllll}25 & 1751 & 25.03 & 19 & 19.89 & 155 & 19.03\end{array}$

$\begin{array}{lllllll}25 & 1911 & 46.79 & 19 & 12.46 & 155 & 40.47\end{array}$

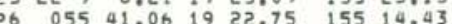

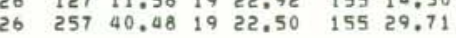
$\begin{array}{llllllll}26 & 9 & 2 & 29.35 & 19 & 17.64 & 155 & 13.06\end{array}$

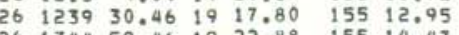

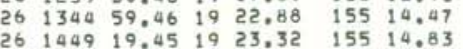

$\begin{array}{lllllll}26 & 1520 & 42.27 & 19 & 23.33 & 155 & 14.84\end{array}$

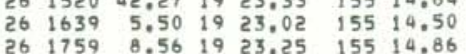

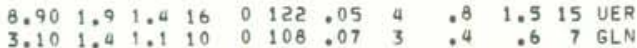

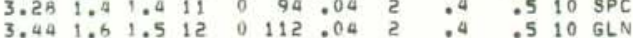

$\begin{array}{llllllllllll}1.44 & 1.6 & 2.3 & 18 & 0 & 81 & 06 & 2 & .3 & .5 & 16 & \text { UER } \\ 3.53 & 3.4 & 3.4 & 37 & 0 & 47 & .09 & 2 & .3 & .0 & 25 & \text { GLN F }\end{array}$

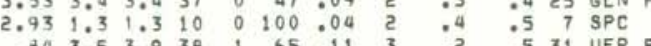

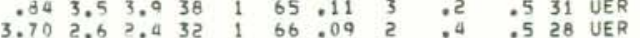

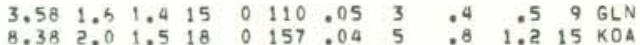

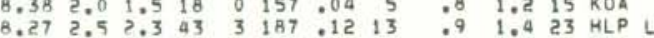

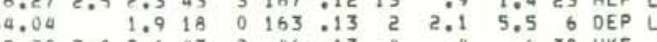

$\begin{array}{llllllllllll}3.24 & 1.8 & 1.9 & 19 & 0 & 88 & .05 & 2 & .3 & .3 & 17 & \mathrm{SPC}\end{array}$

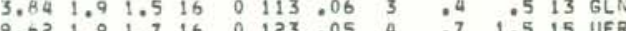

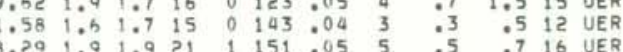

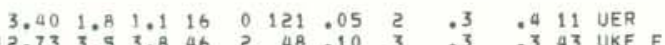

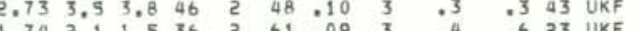

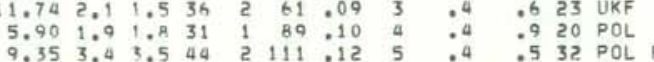
$\begin{array}{llllllllllll}3.72 & 1.9 & 1.6 & 22 & 0 & 64 & .08 & 3 & .3 & .5 .14 & 6 L N\end{array}$

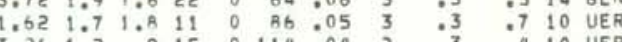

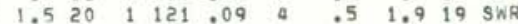

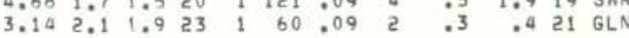
$\begin{array}{llllllllllllllll}3.02 & 1.0 & 1.0 & 9 & 0 & 105 & .05 & 2 & .5 & .6 & 7 & \mathrm{SPC}\end{array}$

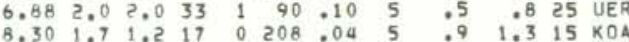
$\begin{array}{llllllllllll}31.62 & 3.1 & 3.0 & 46 & 3 & 51 & .10 & 3 & : 6 & 09 & 43 & \text { OEP } \\ 3.16 & 1.9 & 1.7 & 20 & 1 & 73 & .06 & 3 & .3 & .3 & 13 & \text { GLN }\end{array}$ $\begin{array}{llllllllllll}6.22 & 2.4 & 1.9 & 30 & 3 & 185 & .24 & 7 & 1.1 & 1.8 & 16 & \text { HEA }\end{array}$

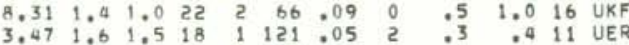
$\begin{array}{llllllllllll}3.34 & 1.6 & 1.7 & 16 & 0 & 117 & 07 & 3 & .4 & .4 & 14 & \text { UER } \\ 9.75 & 1.0 & 1.2 & 19 & 3 & 70 & : 08 & 4 & .4 & 1.0 & 15 & \text { UKF }\end{array}$ $\begin{array}{llllllllllll}6.90 & 2.0 & 1.4 & 31 & 1 & 119 & .11 & 1 & .5 & 1.0 & 23 & \text { POL }\end{array}$ $\begin{array}{llllllllllll}5.40 & 2.1 & 1.5 & 34 & 3 & 118 & .10 & 2 & .4 & 1,0 & 23 & \text { POL }\end{array}$ $\begin{array}{lllllllllllll}3.43 & 1.8 & : .3 & 18 & 0 & 118 & 05 & 3 & .3 & .4 & 11 & \text { UER } \\ 3.20 & 1.6 & 1.1 & 12 & 0 & 106 & .04 & 3 & 04 & .5 & 10 & 6 L N\end{array}$ $\begin{array}{lllllllllll}3.45 & 1.2 & .8 & 11 & 0 & 101 & .03 & 3 & .4 & .5 & 8 \\ 3 & 6 L N\end{array}$

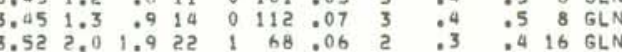

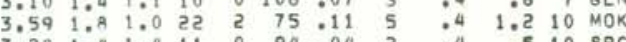
$23171626.9719 \quad 16.74 \quad 15515.13$ 


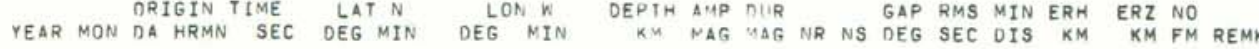
1979 NOV $26 \quad 1922 \quad 18.56 \quad 1926.88 \quad 15521.35$ $\begin{array}{lllllll}26 & 2235 & 1.48 & 19 & 13.05 & 155 & 32.04\end{array}$

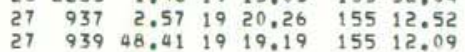

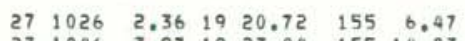
$\begin{array}{lllllll}27 & 1046 & 3.97 & 19 & 23.04 & 155 & 14.83\end{array}$ $\begin{array}{lllllll}27 & 1440 & 45.95 & 19 & 16.22 & 155 & 12.01\end{array}$

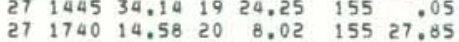
27 $2020 \quad 46.51 \quad 19 \quad 19.67 \quad 155 \quad 11.59$ 27. $2330 \quad 17.91 \quad 19 \quad 18.02 \quad 155 \quad 17.07$ $\begin{array}{lllllll}27 & 2330 & 33.54 & 19 & 18.15 & 155 & 16.80\end{array}$ $\begin{array}{lllllll}28 & 245 & 38.28 & 19 & 22.33 & 155 & 1.33\end{array}$

$\begin{array}{lllllll}28 & 348 & 21.61 & 19 & 20.17 & 155 & 3.84\end{array}$ 28 449 32.46 i9 $20.04 \quad 15512.16$

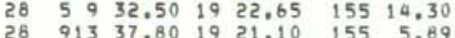

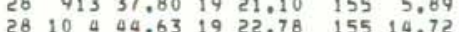

$\begin{array}{llllllll}28 & 12 & 8 & 13.27 & 19 & 18.11 & 155 & 23.10\end{array}$

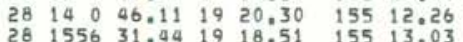
$\begin{array}{lllllll}28 & 1556 & 31.44 & 19 & 18.51 & 155 & 13.03 \\ 28 & 1853 & 16.88 & 19 & 20.51 & 155 & 12.59\end{array}$

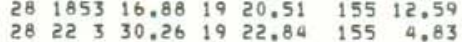

$\begin{array}{lllllll}29 & 415 & 35.60 & 19 & 18.92 & 155 & 13.72 \\ 29 & 612 & 45.59 & 19 & 19.41 & 155 & 13.78\end{array}$

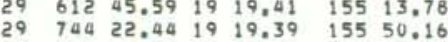
$\begin{array}{lllllll}29 & 8 & 8.56 & 19 & 20.94 & 155 & 5.07\end{array}$ $\begin{array}{lllllll}29 & 1150 & 59.64 & 19 & 21.65 & 155 & 1.93 \\ 29 & 1427 & 2.65 & 19 & 20.69 & 155 & 13.53\end{array}$ $29 \begin{array}{llllll}1542 & 36.46 & 19 & 31.03 & 155 & 55.53\end{array}$ $291645 \quad 19,49 \quad 1920.24 \quad 155 \quad 11.11$

$\begin{array}{llllllll}29 & 23 & 7 & 38.06 & 19 & 20.80 & 155 & 5.36 \\ 30 & 055 & 47.92 & 19 & 34.84 & 155 & 59.21\end{array}$ $\begin{array}{llll}30 & 425 & 32.85 & 19 \\ 30 & 26.49 & 155 & 14.44\end{array}$

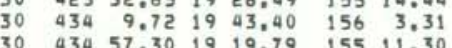
$\begin{array}{rrrrrrr}30 & 1015 & 41.80 & 19 & 23.67 & 155 & 15.07 \\ 30 & 1141 & 3.82 & 19 & 30.47 & 155 & 51.11\end{array}$

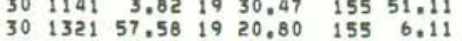

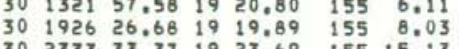
DEC $1 \quad 1612 \quad 30.90 \quad 19 \quad 22.37 \quad 155 \quad 49.34$

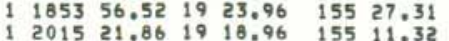

$\begin{array}{llllllllllllll}4.76 & 1.9 & 1.4 & 18 & 1 & 77 & .12 & 6 & .6 & 3.8 & 16 & \text { UKF }\end{array}$ $6.45 \quad 2.0 \quad 1.024 \quad 3 \quad 77.14 \quad 5 \quad .4 \quad 1.1 \quad 10 \quad 5 W$

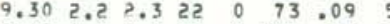

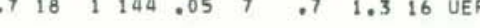
$\begin{array}{llllllllllll}7.85 & 1.8 & 1.3 & 15 & 0 & 138 & .06 & 4 & .6 & 1.1 & 10 & \text { UER }\end{array}$

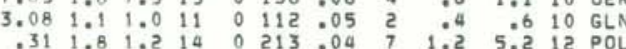

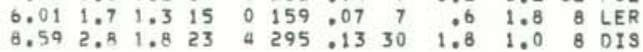
$\begin{array}{lllllllllllll}9.21 & 1.7 & 1.3 & 18 & 0 & 132 & .04 & 5 & .7 & 1.3 & 16 & \text { UER }\end{array}$ $\begin{array}{lllllllllllll}.01 & 0.0 & 13 & 0 & 175 & .06 & 3 & .7 & 1.0 & 7 & \mathrm{KOA}\end{array}$

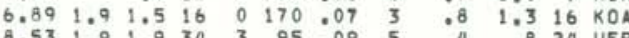

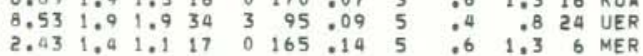
$\begin{array}{lllllllllllll}8.55 & 2.8 & 2.7 & 39 & 2 & 129 & .12 & 2 & .5 & .5 & 28 & \text { MER }\end{array}$ $\begin{array}{llllllllllll}.19 & 1.6 & 1.3 & 19 & 0 & 128 & 05 & 5 & .7 & 1.2 & 17 & \\ 3 & 0 E R\end{array}$ $\begin{array}{llllllllllll}3.26 & 1.2 & 1.0 & 14 & 1 & 124 & .06 & 2 & .4 & .5 & 10 & \text { UER } \\ 8.32 & 2.1 & 1.9 & 27 & 0 & 96 & .06 & 4 & .5 & 0 & 25 & \text { MER }\end{array}$ $\begin{array}{lllllllllllll}4.21 & 1.7 & 1.6 & 18 & 1 & 150.08 & 4 & .6 & 1.3 & 14 & \text { SWR }\end{array}$

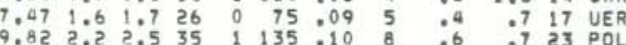

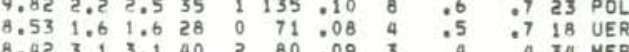

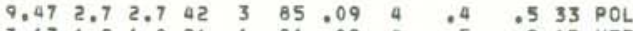

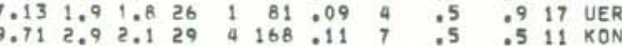
$9.43 \quad 1.199 \quad 00211: 03$ a $2.6 \quad 1.799$ MER $\begin{array}{lllllllllll}7.51 & 1.2 & 1.2 & 13 & 0 & 162 & 08 & 4 & 9 & 1.2 & \text { O MER }\end{array}$

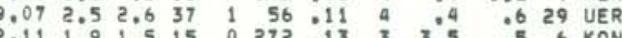
2.75 2.2 1.5 10 00197.10 a 1.1 1.:. 1.2 9 MOK

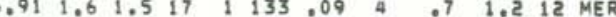

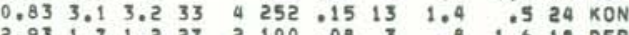
$\begin{array}{llllllllllll}32.93 & 1.7 & 1.2 & 27 & 2 & 100 & 08 & 3 & 0.8 & 1.6 & 18 & \text { OEP } \\ 37.82 & & 1.8 & 27 & 2 & 233 & : 12 & 23 & 1.0 & 2.1 & 19 & K O N\end{array}$ $3.493 .03 .028 \quad 1 \quad 78.11 \quad 2 \quad 4 \quad, 527 \mathrm{SPC}$

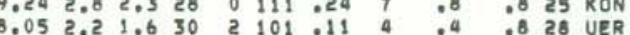

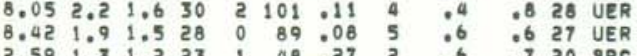
$9.012 .3 \quad 1.525 \quad 2 \quad 118.1213 \quad .5 \quad .818$ KON

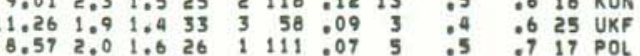

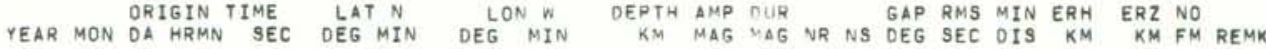
$\begin{array}{rrrrrrr}2 & 245 & 9.65 & 19 & 20.84 & 155 & 10.03 \\ 2 & 435 & 2.10 & 19 & 23.98 & 155 & 27.51 \\ 2 & 452 & 7.48 & 19 & 17.89 & 155 & 14.20 \\ 3 & 625 & 47.32 & 19 & 19.64 & 155 & 12.04 \\ 3 & 759 & 31.83 & 19 & 21.20 & 155 & 7.07\end{array}$ $\begin{array}{llllllllllll}8.64 & 1.7 & 1.4 & 30 & 2 & 71.08 & 2 & .4 & .6 & 12 & 2 & \text { UER }\end{array}$

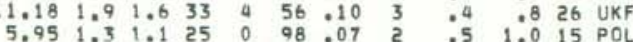

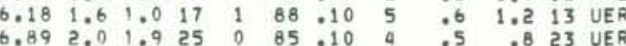

$\begin{array}{lllllllllllllllllll}3 & 1122 & 34.23 & 19 & 25.64 & 155 & 30.09 & 7.94 & 2.0 & 1.8 & 27 & 1 & 54 & .11 & 7 & .4 & 1.2 & 23 & \text { MOK }\end{array}$

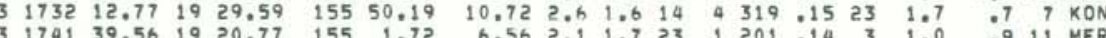

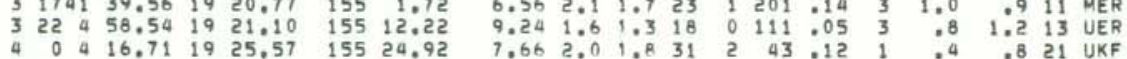

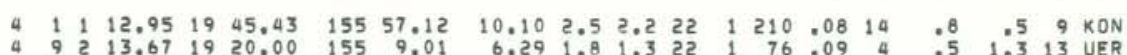

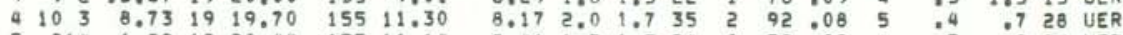

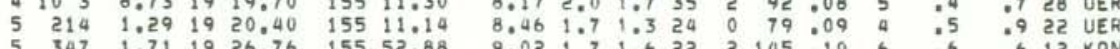
$.6 .612 \mathrm{KON}$

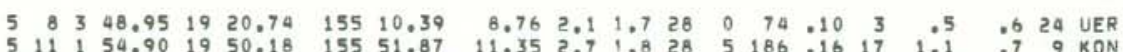

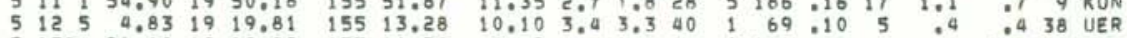

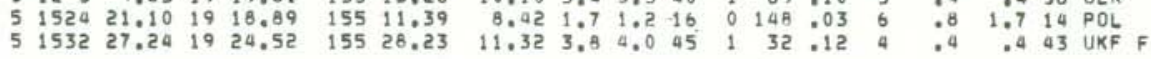
$\begin{array}{llllllllllllllllll}5 & 1539 & .81 & 19 & 25.64 & 155 & 28.27 & 9.34 & 1.8 & 1.1 & 34 & 2 & 49 & .10 & 6 & .4 & .8 & 24 \\ \text { UKF }\end{array}$

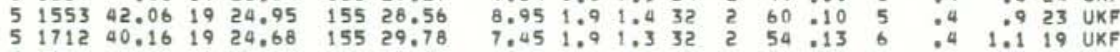

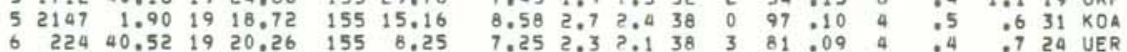

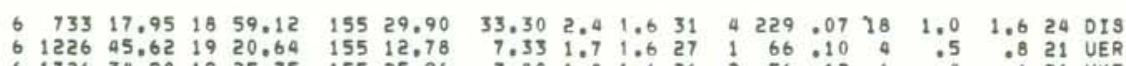

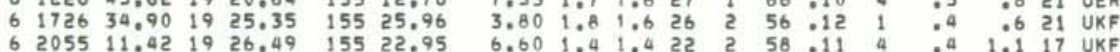

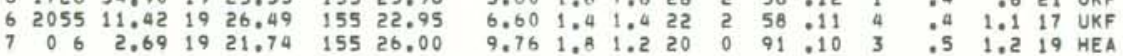

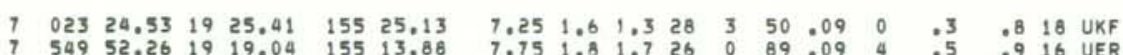

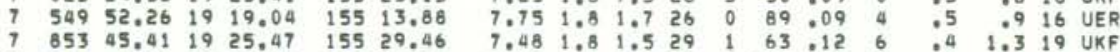

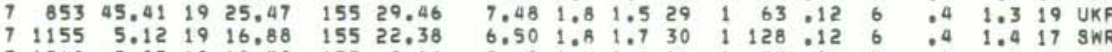

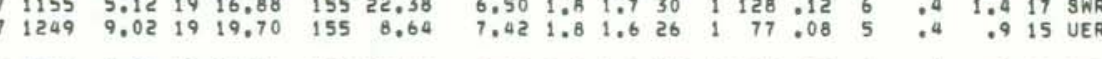
$\begin{array}{llllllllllllllllllllll}7 & 1319 & 5.94 & 19 & 25.61 & 155 & 25.19 & 4.68 & 1.6 & 1.4 & 17 & 0 & 123 & 08 & 1 & .4 & .8 & 14 & \text { UKF } \\ 716 & 57.13 & 19 & 20.41 & 155 & 8.76 & 7.52 & 1.4 & 1.3 & 22 & 0 & 71 & .07 & 4 & .5 & .0 & 15 & \text { UER }\end{array}$

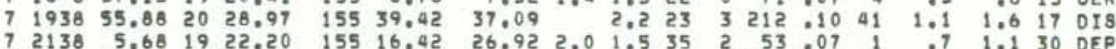

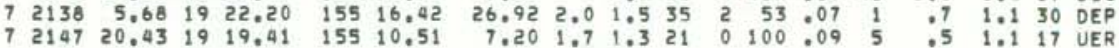

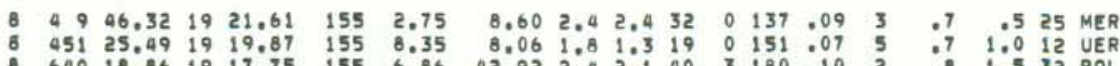

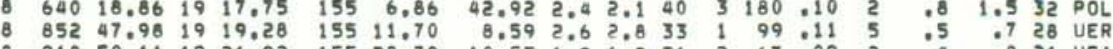

C.

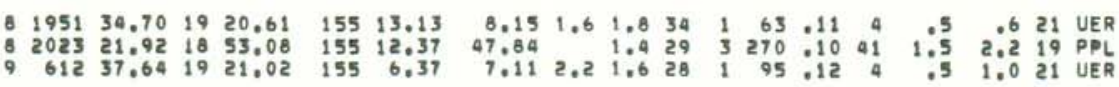


ORIGIN TIME LAT N LON W DEPTH AMP OUR N GAP RMS MIN ERH ERZ NO
DA HRMN SEE DEG MIN DEG MIN

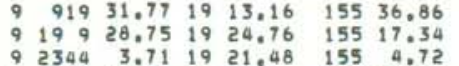
$\begin{array}{rrrrrrr}9 & 2344 & 3.71 & 19 & 21.48 & 155 & 4.72 \\ 10 & 259 & 45.63 & 19 & 24.76 & 155 & 32.35\end{array}$

$10 \quad 921 \quad 14,54 \quad 19 \quad 24,62 \quad 155 \quad 24,76$ $\begin{array}{lllllll}10 & 1657 & 37.25 & 19 & 27.30 & 155 & 24.00 \\ 10 & 23 & 51.30 & 19 & 24.44 & 155 & 24.89\end{array}$ $115014.621920 .42 \quad 1557.23$ $\begin{array}{lllllll}11 & 1058 & 45.24 & 19 & 12.30 & 155 & 10.63\end{array}$ $\begin{array}{rrrrrrr}11 & 1442 & 54.63 & 19 & 27.39 & 155 & 20.76 \\ 11 & 1517 & .36 & 19 & 27.42 & 155 & 20.93\end{array}$ $\begin{array}{lllllll}11 & 1517 & .36 & 19 & 27.42 & 155 & 20.93\end{array}$ $11184735.70 \quad 1920.31 \quad 155 \quad 8.83$

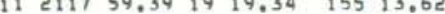
$\begin{array}{lllllll}11 & 2125 & 18.14 & 19 & 26.88 & 155 & 21.16\end{array}$

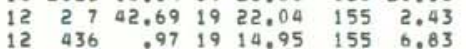

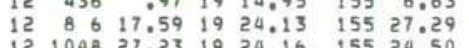

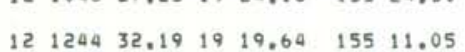
$12135918.03 \quad 1923.31 \quad 155 \quad 15.11$ $\begin{array}{lllllll}12 & 1643 & 13.15 & 19 & 20.33 & 155 & 10.83 \\ 12 & 1740 & 45.06 & 19 & 19.81 & 155 & 12.60\end{array}$

$\begin{array}{llllll}12 & 1745 & 47.61 & 19 & 19.34 \quad 155 \quad 11.33\end{array}$ $12 \quad 1824 \quad 56.24 \quad 19 \quad 19.32 \quad 155 \quad 11.72$ $\begin{array}{llllllll}13 & 2 & 4 & 23.24 & 19 & 22.20 & 155 & 2.78\end{array}$ $\begin{array}{llllllr}13 & 436 & 30.55 & 19 & 15.66 & 155 & 7.32 \\ 13 & 555 & 52.06 & 19 & 23.18 & 155 & 14.81\end{array}$ $\begin{array}{lrrrrrr}13 & 844 & 5.80 & 19 & 20.80 & 155 & 2.61\end{array}$

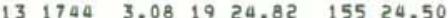
$13205121.211910,33 \quad 15526.97$

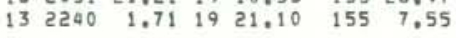
$13232258.42 \quad 1921.71 \quad 15515.15$ $14 \quad 35829,901924,28 \quad 15524.82$

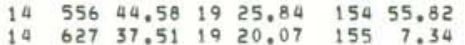
$\begin{array}{lllllll}14 & 638 & 41.17 & 19 & 27.35 & 155 & 20.99\end{array}$

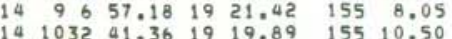
$14 \quad 1251 \quad 24.18 \quad 1956.43 \quad 15529.10$

$\begin{array}{lllllll}15 & 212 & 45.89 & 19 & 26.62 & 154 & 55.93\end{array}$

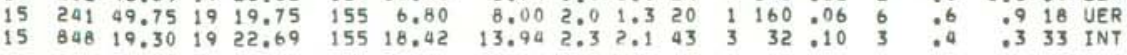

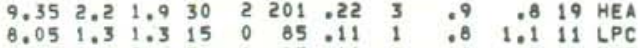

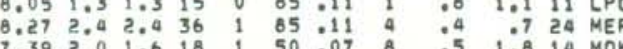
$\begin{array}{llllllllllll}7.39 & 2.0 & 1.6 & 18 & 1 & 50 & 07 & 8 & .5 & 1.8 & 14 & \text { MOK } \\ 7.70 & 1.5 & 1.2 & 24 & 2 & 46 & .12 & 1 & .4 & 9 & 19 & \text { UKF }\end{array}$ $\begin{array}{lllllllllllll}3.46 & 1.9 & 1.6 & 24 & 3 & 66 & : 12 & 4 & .3 & 0 & 17 & 17 & \text { UKF } \\ 0.91 & 2.1 & 2.2 & 28 & 2 & 88 & : 09 & 2 & : 5 & : 8 & 19 & \text { UKF }\end{array}$

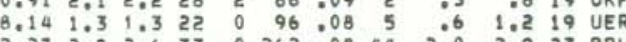

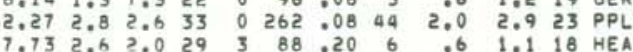
$\begin{array}{lllllllllllll}8.17 & 1.8 & 1.2 & 18 & 1 & 103 & 10 & 0 & .6 & 1.2 & 14 & \text { UKF }\end{array}$

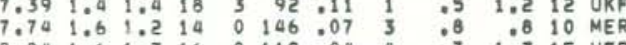

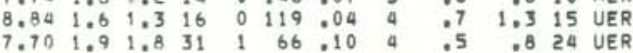
$6.84 \quad 1.92 .024 \quad 1 \quad 46.1025 \quad .4 \quad .815$ UKF $\begin{array}{lllllllllllll}0.47 & 2.1 & 1.6 & 29 & 1 & 142 & .08 & 4 & .5 & .6 & 21 & \text { MER } \\ 4.71 & 1.9 & 1.7 & 21 & 0 & 201 & 06 & 5 & 1.6 & 20 & 18 & \text { PO }\end{array}$ $\begin{array}{lllllllllllll}9.78 & 1.9 & 1.3 & 23 & 1 & 56 & .08 & 3 & .4 & .8 & 19 & \text { UKF } \\ 9.79 & 2.1 & 1 & 3 & 32 & 0 & 48 & 11 & 2 & .4 & .6 & 28 & \text { UKF }\end{array}$ $\begin{array}{lllllllllll}8.35 & 2.6 & 2.7 & 37 & 1 & 94 & .09 & 5 & .4 & .6 & 29 \\ 0 & 4 E R\end{array}$

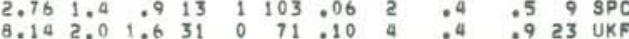

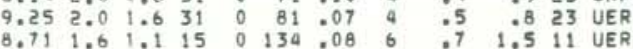
$\begin{array}{lllllllllllllll}8.65 & 1.6 & 1.2 & 17 & 0 & 138 & .04 & 6 & .7 & 1.5 & 13 & \text { UER }\end{array}$

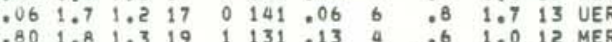

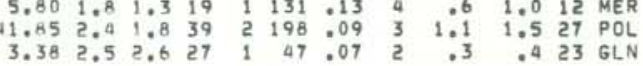
$\begin{array}{lllllllllllllllll}6.48 & 2.0 & 1.5 & 25 & 0 & 155 & .13 & 2 & .7 & 1.0 & 17 & \text { MER }\end{array}$

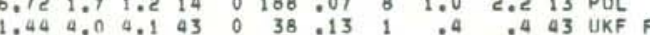

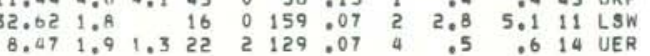
$\begin{array}{llllllllllll}9.19 & 2.0 & 1.6 & 23 & 0 & 92 & .08 & 2 & .5 & .8 & 21 & \text { KOA }\end{array}$

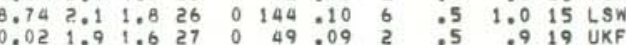

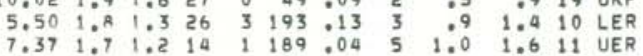
$\begin{array}{lllllllllllll}7.35 & 1.9 & 1.8 & 23 & 1 & 65 & .10 & 1 & .5 & 1.0 & 19 & \text { UKF }\end{array}$

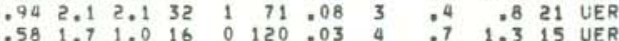

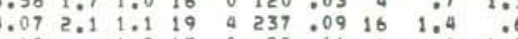

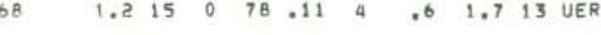
ORIGIN TIME
YEAR MON N

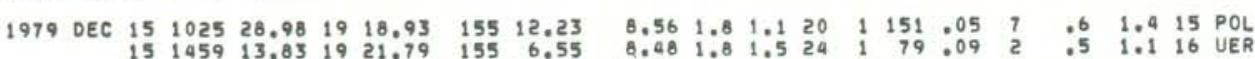

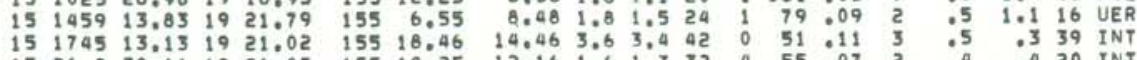

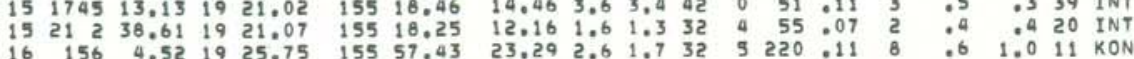
$\begin{array}{lllllllllllllllllll}16 & 214 & 50.34 & 19 & 27.01 & 155 & 14.72 & 32.41 & 2.1 & 1.5 & 38 & 5 & 83 & .09 & 4 & .7 & .9 & 30 & \text { DEP }\end{array}$

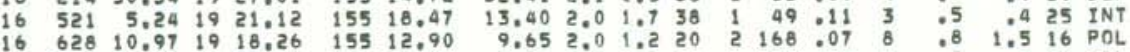

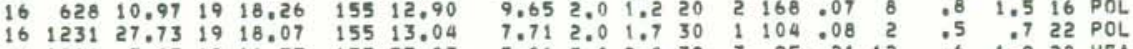

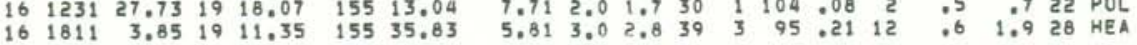
$\begin{array}{lllllllllllllllllll}16 & 1922 & 55.52 & 19 & 19.49 & 155 & 12.03 & 7.36 & 2.4 & 2.7 & 37 & 0 & 91 & .12 & 5 & .4 & .7 & 33 & \text { UER }\end{array}$

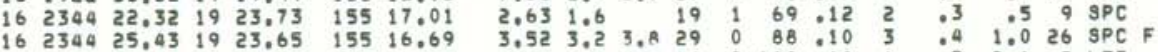

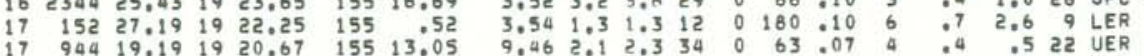

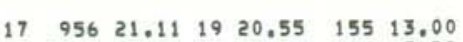

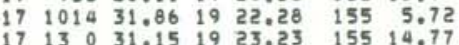
17192913.191922 .26 155 5.67 $\begin{array}{lllllll}18 & 147 & 9.89 & 19 & 21.18 & 155 & 5.85\end{array}$

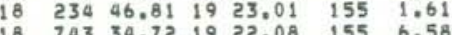

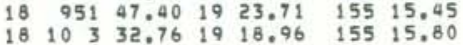

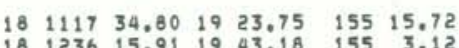
$18 \quad 143622.65 \quad 1920.48 \quad 15513.00$ $\begin{array}{lllllll}18 & 1648 & 25.06 & 19 & 17.29 & 155 & 29.24 \\ 18 & 1810 & 1.43 & 19 & 23.47 & 155 & 14.94\end{array}$

$\begin{array}{llllllll}18 & 19 & 4 & 47.10 & 19 & 25.03 \quad 155 \quad 25.42\end{array}$

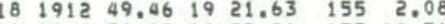

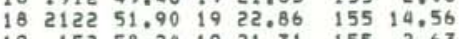

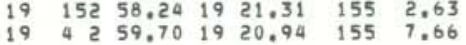
$\begin{array}{llllllll}19 & 4 & 6 & 29.94 & 19 & 22.01 & 155 & 3.30\end{array}$

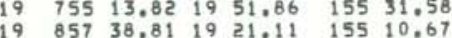

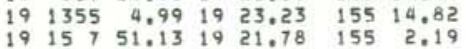

$19 \begin{array}{llllll}1723 & 21.17 \quad 19 & 16.51 \quad 155 \quad 23.43\end{array}$ $191825 \quad 42.941923 .94 \quad 15515.41$ $19194750.5119230 .67 \quad 15513.23$ $202325 \quad 6.601923 .22 \quad 15514.90$

$\begin{array}{lllllll}20 & 228 & 13.35 & 19 & 19.97 & 155 & 6.68\end{array}$ $\begin{array}{lllllll}20 & 343 & 4.66 & 19 & 23.80 & 155 & 15.25 \\ 20 & 345 & 30.04 & 19 & 20.31 & 155 & 11.60\end{array}$ $9.111 .41 .315 \quad 0 \quad 122.05 \quad 4 \quad .6 \quad 1.611$ UER

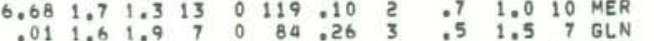
$\begin{array}{llllllllllll}7.74 & 1.9 & 1.8 & 22 & 0 & 72 & 10 & 2 & .4 & .9 & 16 & \text { MER } \\ 8.84 & 1.7 & 1.4 & 15 & 1 & 117 & .06 & 2 & 9 & 1.2 & 11 & \text { POL }\end{array}$ $\begin{array}{llllllllllll}8.53 & 1.2 & 1.4 & 15 & 0 & 127 & .04 & 3 & .6 & 1.0 & 11 & \text { MER }\end{array}$

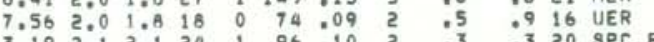

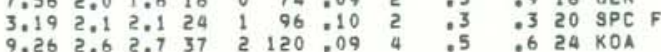
$\begin{array}{lllllllllllll}2.83 & 1.4 & 1.6 & 16 & 1 & 100 & .09 & 2 & .4 & .2 & 13 & \mathrm{SPC}\end{array}$

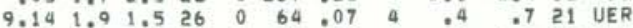

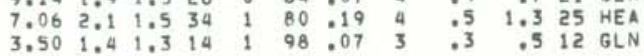
$\begin{array}{lllllllllllll}7.75 & 1.7 & 1.5 & 27 & 1 & 50 & 0 & 10 & 1 & .4 & .9 & 22 & \text { UKF }\end{array}$

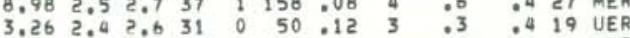

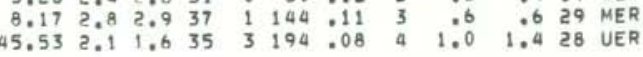

$\begin{array}{lllllllllllll}7.14 & 2.0 & 1.4 & 28 & 0 & 116 & .15 & 4 & .5 & .920 & 20 & \text { MER }\end{array}$

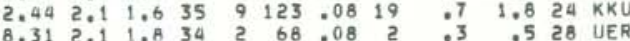

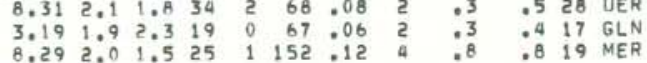
$\begin{array}{lllllllllllllll}6.91 & 1.8 & 1.6 & 21 & 2 & 172 & .11 & 7 & .6 & 1.9 & 19 & \text { SWR }\end{array}$

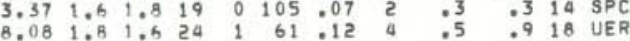
$\begin{array}{lllllllllllll}3.21 & 1.5 & .6 & 16 & 0 & 69 & .05 & 2 & .3 & .4 & 14 & 6 L N \\ 2.40 & 1.6 & 1.6 & 17 & 0 & 63 & .07 & 2 & .3 & .4 & 14 & 6 L N\end{array}$ $\begin{array}{rrrrrrrrrrrr}8.25 & 2.0 & 1.6 & 27 & 0 & 115 & 10 & 5 & .6 & 1.0 & 26 & \text { UER } \\ 3.22 & 1.0 & 1.4 & 27 & 0 & 97 & 0.06 & 2 & 04 & 0.4 & 13 & 3 P C\end{array}$ $\begin{array}{llllllllllll}3.22 & 1.6 & .4 & 15 & 0 & 97 & 06 & 2 & .4 & .4 & 13 & \text { SPC } \\ 9.51 & 2.2 & 1.8 & 31 & 0 & 78 & .07 & 5 & .4 & .8 & 23 & \text { UER }\end{array}$ 


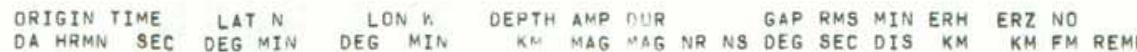

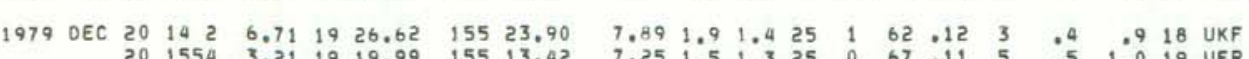

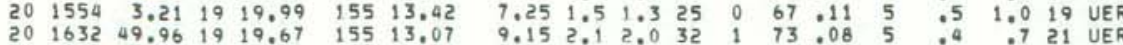

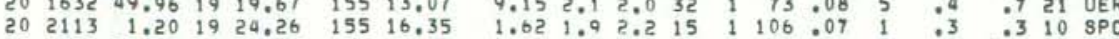

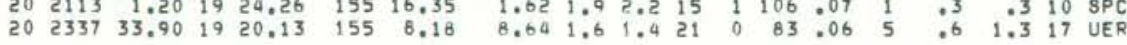

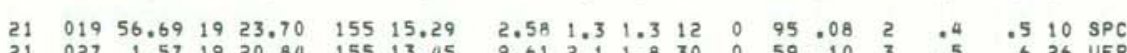

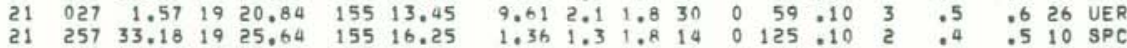

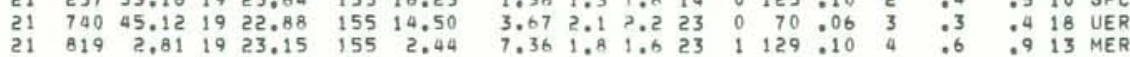

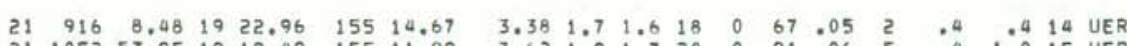

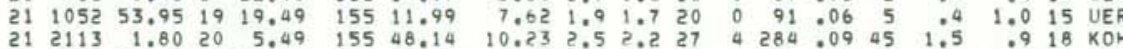

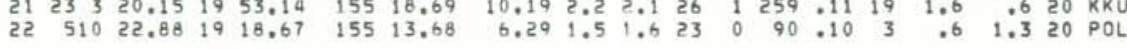

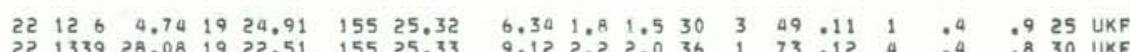

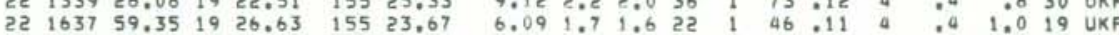

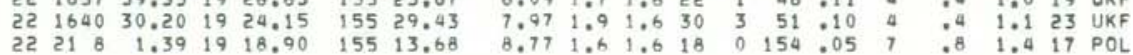

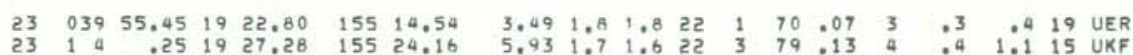

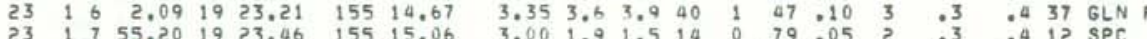

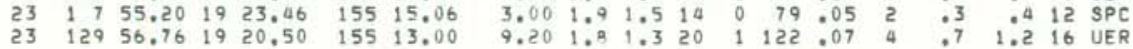
$\begin{array}{lllllllllllllllllll}23 & 131 & 12.78 & 19 & 23.45 & 155 & 15.04 & 3.13 & 2.0 & 2.3 & 26 & 1 & 69 & .06 & 2 & .3 & .3 & 22 & \text { SPC F }\end{array}$

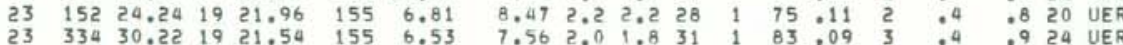

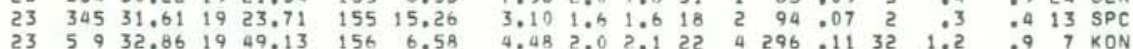

$\begin{array}{lllllllllllllllllll}23 & 511 & 40.04 & 19 & 22.33 & 156 & 21.99 & 36.50 & 2.4 & 2.4 & 20 & 4 & 319 & .09 & 49 & 1.7 & 2.1 & 13 & 019\end{array}$

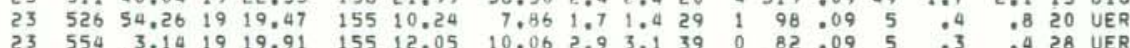

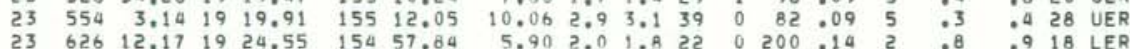

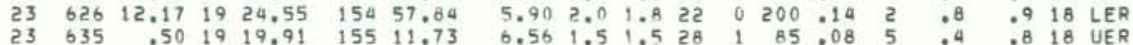

$\begin{array}{lllllllllllllllllll}23 & 718 & 57.18 & 19 & 24.15 & 155 & 25.38 & 9.84 & 2.0 & 1.6 & 34 & 2 & 37 & 11 & 2 & .4 & .7 & 24 & \text { UKF }\end{array}$

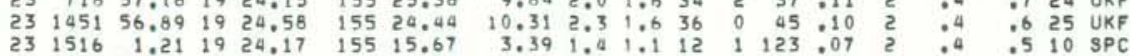

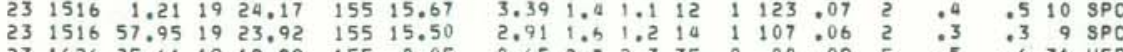

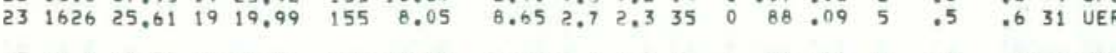
$\begin{array}{llllllllllllllllllll}23 & 21 & 8 & 26.64 & 19 & 26.81 & 155 & 23.70 & 8.23 & 2.1 & 1.5 & 33 & 0 & 46 & .11 & 4 & .4 & .8 & 26 & \text { UKF } \\ 24 & 144 & 8.17 & 19 & 22.53 & 155 & 6.02 & 8.32 & 3.1 & 3.1 & 40 & 0 & 69 & .10 & 1 & .4 & .6 & 31 & \text { UER }\end{array}$

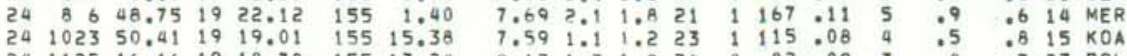

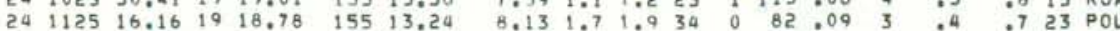

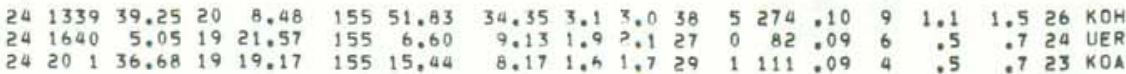

ORIGIN TIME LAT N LON W DEPTH AMP PUR GAP RMS MIN ERH ERZ NO YEAR MON DA HRMN SEC DEG MIN DEG MIN KIA MAG WAG NR NS OEG SEC DIS KM KM FM REMK

1979 DEC $24 \quad 2013 \quad 5.18 \quad 1920.21 \quad 155 \quad 11.93$

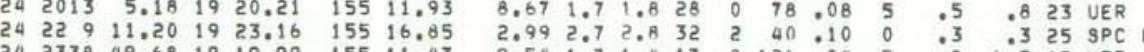

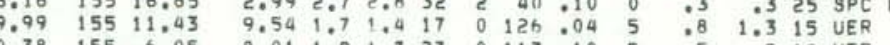

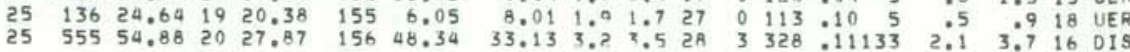

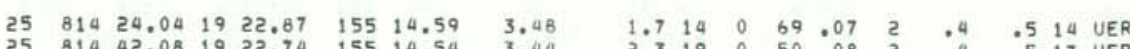

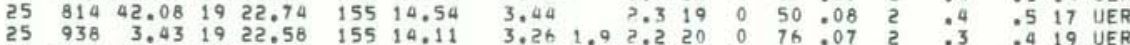

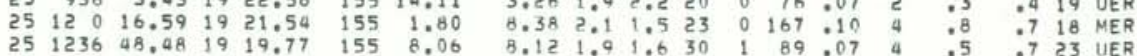
$\begin{array}{llllllllllllllllll}25 & 1611 & 52.07 & 19 & 20.18 & 155 & 13.31 & 8.73 & 1.9 & 2.2 & 32 & 0 & 64 & .09 & 5 & .4 & .7 & 22 \\ \text { UER }\end{array}$

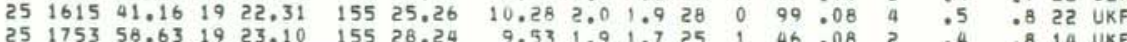

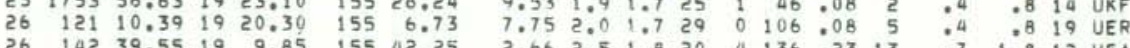

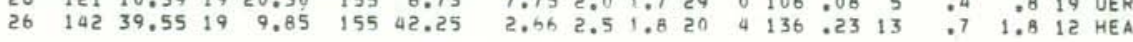

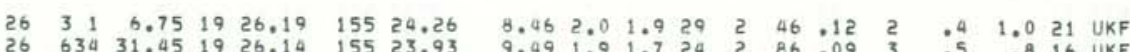

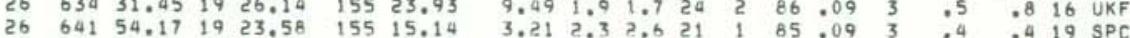

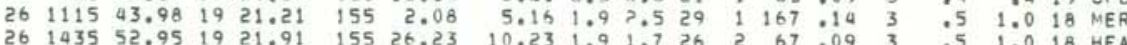
$\begin{array}{llll} & \end{array}$ $\begin{array}{llllllllllllllllllll}26 & 1535 & 17.77 & 19 & 23.59 & 155 & 15.22 & 2.80 & 1.4 & 1.7 & 19 & 2 & 87 & .09 & 3 & .3 & .4 & 13 & \text { SPC } \\ 26 & 17 & 43.43 & 19 & 21.71 & 155 & 6.60 & 8.83 & 2.0 & 2.2 & 25 & 0 & 80 & 09 & 2 & : 4 & : 9 & 22 & \text { UER }\end{array}$

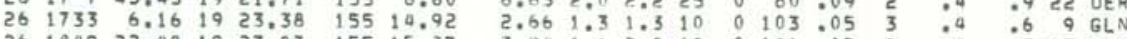

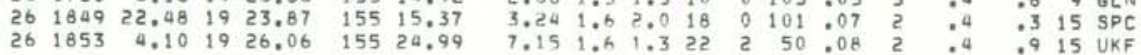
$\begin{array}{lllllll}26 & 2017 & 19.64 & 19 & 20.74 \quad 155 \quad 4.50\end{array}$ $26 \quad 2044 \quad 5.391924 .02 \quad 155 \quad 15.50$

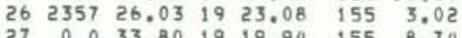

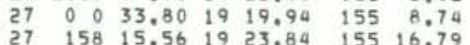

$\begin{array}{llllllll}27 & 4 & 5 & 27.05 & 19 & 22.90 & 155 & 14.49\end{array}$ $27 \quad 1011 \quad 10.77 \quad 19 \quad 19.57 \quad 155 \quad 13.04$ $\begin{array}{lllllll}27 & 1319 & 1.19 & 19 & 23.09 & 155 & 3.14 \\ 27 & 1539 & 7.35 & 19 & 21.25 & 155 & 2.85\end{array}$

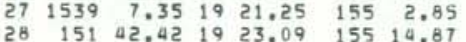

$\begin{array}{lllllll}28 & 412 & 39.41 & 19 & 26.99 & 155 & 24.58\end{array}$

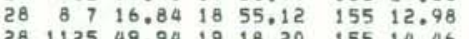
$28 \quad 1125 \quad 49.941918 .20 \quad 15514.46$

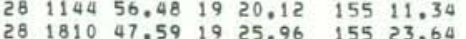

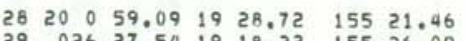

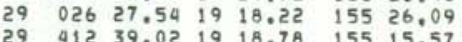
$\begin{array}{lllllll}29 & 412 & 39.02 & 19 & 18.78 & 155 & 15.57\end{array}$

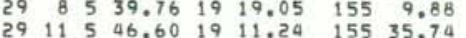

$\begin{array}{llllll}29 & 1730 & 17.82 & 19 & 22.03 \quad 155 & 6.45\end{array}$

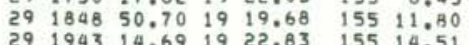

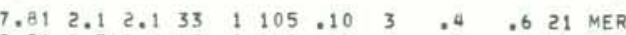
$\begin{array}{lllllllllllll}2.76 & 1.3 & 1.4 & 13 & 1 & 113 & .05 & 2 & .3 & .3 & 11 & \text { SPC } \\ 8.45 & 2.3 & 0.1 & 29 & 0 & 115 & .09 & 3 & : 5 & : 5 & 20 & \text { MEP }\end{array}$

$\begin{array}{llllllllllll}3.31 & 1.6 & .9 & 15 & 1 & 117 & .07 & 3 & .4 & .4 & 12 & \text { UER }\end{array}$

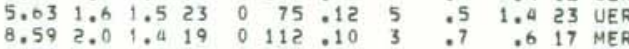

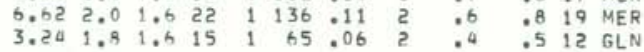
$\begin{array}{llllllllllllll}\text { B.19 } & 2.1 & 1.7 & 27 & 0 & 47 & .14 & 6 & .5 & 1.3 & 24 & \text { UKF }\end{array}$

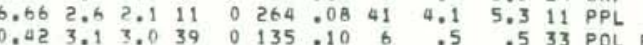

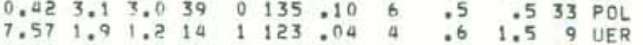
$\begin{array}{llllllllllll}5.70 & 2.4 & 2.1 & 32 & 2 & 53 & .14 & 3 & .4 & 1.0 & 26 & \text { UKF }\end{array}$

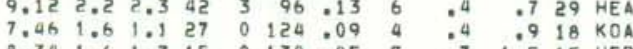

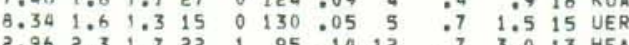
$\begin{array}{llllllllllllllll}8.51 & 2.4 & 7.4 & 37 & 1 & 75 & .07 & 2 & 3 & 5 & 24 & 4 E R\end{array}$

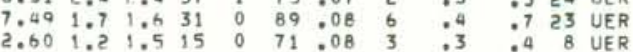
$\begin{array}{rlllllllllll}8.93 & 1.1 & 1.1 & 10 & 0 & 122 & .03 & 5 & 1.0 & 3.2 & 6 & \text { UER } \\ 2.87 & 1.4 & 1.9 & 19 & 1 & 80 & .07 & 0 & .0 & .2 & 13 & \text { SPC }\end{array}$ 


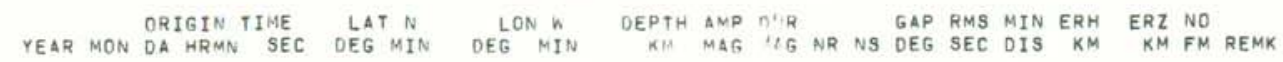

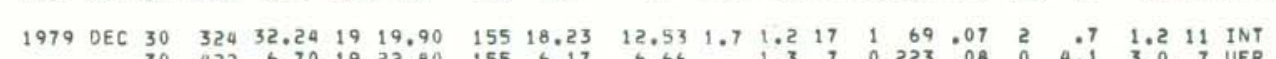

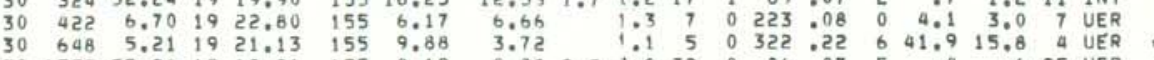

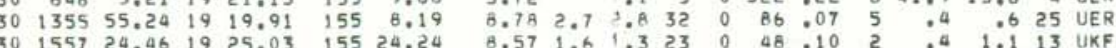

$\begin{array}{llllllllllllllllllllll}30 & 16 & 4 & 9.97 & 19 & 56.33 & 155 & 43.14 & 10.42 & 3.0 & 2.6 & 21 & 2 & 151 & .08 & 22 & .7 & .7 & 10 & K O H\end{array}$

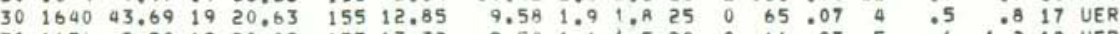

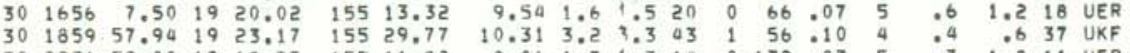

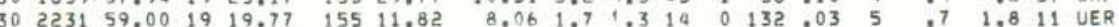

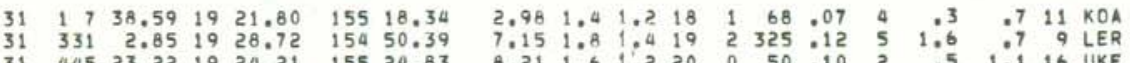

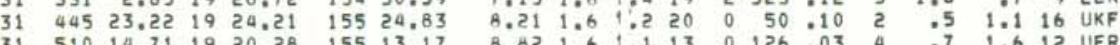

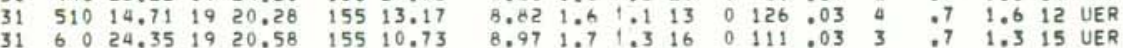

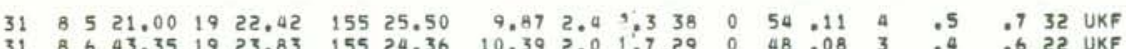

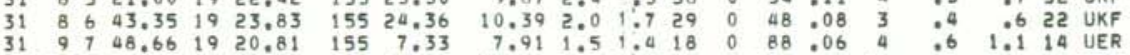

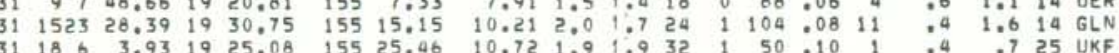


ORIG IN TIME 1979 JAN $11821 \quad 36.01 \quad 1921.18$ $\begin{array}{lllll}12031 & 25.20 & 20 & 57.37\end{array}$ $\begin{array}{llllll}2 & 5 & 3 & 39.79 & 19 & 21.12\end{array}$

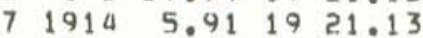

$9230044.73 \quad 1923.09$

$11 \quad 224 \quad 45.59 \quad 19 \quad 22.11$ $\begin{array}{lllll}11 & 524 & 52.57 & 19 & 23.34\end{array}$ $\begin{array}{llllll}13 & 755 & 26.99 & 19 & 20.06\end{array}$ $142112 \quad 36.55 \quad 19 \quad 22.32$ $\begin{array}{llllll}16 & 1947 & 13.78 \quad 20 & 22.36\end{array}$

$\begin{array}{lllll}17 & 236 & 45.58 & 19 & 18.10\end{array}$ $19 \quad 1419 \quad 15.41 \quad 1920.12$ $\begin{array}{llllll}24 & 1322 & 45.87 & 19 & 15.74\end{array}$ $\begin{array}{llllll}25 & 1821 & 5.83 & 19 & 19.69\end{array}$ $\begin{array}{lllll}26 & 622 & 47.36 & 19 & 22.32\end{array}$

$\begin{array}{llllll}28 & 1922 & 53.48 & 19 & 22.64\end{array}$ FEB $11425 \quad 32.2120 \quad 30.58$ $\begin{array}{lllll}3 & 249 & 4.28 & 19 & 20.40\end{array}$ $\begin{array}{lllll}3 & 528 & 45.52 & 19 & 20.06\end{array}$

$\begin{array}{lllll}4 & 837 & 8.64 & 19 & 19.57\end{array}$

$\begin{array}{llllll}4 & 911 & 3.00 & 19 & .22\end{array}$ $\begin{array}{llllll}5 & 22 & 39.97 & 19 & 20.45\end{array}$ $\begin{array}{llllll}6 & 1651 & 18.16 & 19 & 22.76\end{array}$

$\begin{array}{llllll}8 & 217 & 35.69 & 19 & 19.37\end{array}$

13165251.051920 .60

$\begin{array}{lllll}14 & 1349 & 39.87 & 19 & 20.01\end{array}$ $\begin{array}{llllll}18 & 445 & 44.07 & 19 & 26.97\end{array}$ $\begin{array}{lllll}20 & 1914 & 20.28 & 19 & 21.82\end{array}$ $\begin{array}{llllll}22 & 1715 & 6.29 & 19 & 22.41\end{array}$ $\begin{array}{lllll}23 & 252 & 21.39 & 19 & 23.92\end{array}$

$\begin{array}{lllll}24 & 139 & 53.92 & 19 & 19.73\end{array}$ $\begin{array}{lllll}27 & 052 & 53.05 & 19 & 11.80\end{array}$ $\begin{array}{llllll}\text { MAR } 2 & 227 & 18.22 & 19 & 20.21\end{array}$ $\begin{array}{lllll}2 & 2148 & 11.81 & 19 & 24.58\end{array}$ $\begin{array}{lllll}5 & 2041 & 58.58 & 19 & 20.88\end{array}$

$\begin{array}{lllll}6 & 259 & 50.14 & 19 & 19.97\end{array}$ $\begin{array}{llllll}6 & 5 & 7 & 58.54 & 19 & 31.21\end{array}$ $\begin{array}{llllll}6 & 1254 & 43.52 & 19 & 23.59\end{array}$ $\begin{array}{lllll}6 & 1957 & 20.28 & 19 & 20.89\end{array}$

$\begin{array}{lllllll}7 & 5 & 8 & 7.91 & 19 & 19.97\end{array}$

$\begin{array}{lllll}8 & 1411 \quad 36.68 \quad 19 \quad 23.02\end{array}$ $\begin{array}{llllll}8 & 1442 & 37.15 & 19 & 24.54\end{array}$

$10 \quad 355 \quad 14.65 \quad 19 \quad 20.05$

$10 \quad 454 \quad 49.29 \quad 19 \quad 12.30$

$10949 \quad 33.311918 .03$

$\begin{array}{llllll}11 & 014 & 56.50 & 19 & 17.59\end{array}$ $\begin{array}{llllll}11 & 1728 & 5.17 & 19 & 30.84\end{array}$

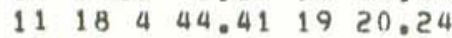

LON W DEG MIN

$155 \quad 4.19$

$156 \quad 3.60$

$155 \quad 2.63$

15513.30

$155 \quad 5.37$

$155 \quad 5.68$

$155 \quad 5.42$

1557.98

$155 \quad 4.57$

15459.98

15514.14

$155 \quad 12.38$

$155 \quad 26.94$

15511.29

$155 \quad 28.82$

15530.13

$155 \quad 35.75$

15512.08

$155 \quad 11.96$

1557.85

$156 \quad 27.12$

$155 \quad 6.76$

$155 \quad 6.46$

$155 \quad 7.07$

1554.25

1558.24

15529.27

$155 \quad 15.78$

$155 \quad 5.71$

$155 \quad 15.95$

$155 \quad 8.38$

15540.09

15511.18

15528.12

$155 \quad 6.10$

$155 \quad 6.99$

15516.18

1551.68

1552.06

1557.45

$155 \quad 5.52$

$155 \quad 14.72$

1556.68

$155 \quad 41.07$

1557.03

$155 \quad 5.97$

$155 \quad 16.84$

1558.05

DEPTH AMP DIIR GAP RMS MIN ERH

ERZ NO KiH MAG $A G$ NR NS DEG SEC DIS KM KM FM REMK

$6.58 \quad 2.3 \quad 3.034$ $11.73 \quad 3.5 \quad .237$ $\begin{array}{lllll}7.93 & 2.7 & 3.2 & 33\end{array}$ $10.21 \quad 2.8 \quad 3.2 \quad 34$ $\begin{array}{lllll}1.42 & 2.7 & 3.1 & 23\end{array}$

$2.53 \quad 3.0 \quad 3.4 \quad 27$ $.86 \quad 3.8 \quad 4.0 \quad 18$ $8.60 \quad 2.8 \quad 3.133$ $9.42 \quad 3.1 \times .333$ .093 .45 .132

$10.792 .9 \quad 3.136$ $9.97 \quad 3.0 \quad 3.236$ $9.00 \quad$ 2.A $3.0 \quad 35$ $9.73 \quad 2.7 \quad 3.0 \quad 31$ $10.25 \quad 2.8 \quad 3.232$

9.073 .02 .833 $\begin{array}{lllll}17.00 & 2.3 & 3.1 & 21\end{array}$ $9.24 \quad 3.5 \quad 3.6 \quad 34$ $10.06 \quad 2.9 \quad 3.0 \quad 34$

$9.00 \quad 3.5 \quad 3.9 \quad 29$

$\begin{array}{lllll}36.31 & 3.6 & 3.7 & 23\end{array}$ $8.833 .2 \quad 3.134$ .812 .73 .321 $9.40 \quad$ 2.ค 3.133 $8.58 \quad 3.9 \quad 4.3 \quad 36$

$8.19 \quad 2.8 \quad 3.3 \quad 30$

$10.58 \quad 3.2 \quad 3.5 \quad 37$

$26.89 \quad 3.1 \quad 3.1 \quad 31$

$8.823 .2 \quad 3.429$

$3.172 .5 \quad 3.0 \quad 29$

$9.21 \quad 2.7 \quad 3.1 \quad 33$

$\begin{array}{llll}5.87 & 3.2 & 2.9 & 24\end{array}$

$9.91 \quad 3.6 \quad 4.1 \quad 37$

11.133 .03 .140

$9.443 .3 \quad 3.536$

$9.80 \quad 3.7 \quad 4.0 \quad 37$

27.434 .74 .938

$8.42 \quad 2.8 \quad 3.2 \quad 28$

$\begin{array}{llll}7.81 & 2.8 & 3.3 & 29\end{array}$

$9.162 .6 \quad 3.229$

$\begin{array}{llll}1.23 & 2.7 & 3.3 & 23\end{array}$

$\begin{array}{lllll}2.81 & 2.7 & 3.1 & 20\end{array}$

$9.57 \quad 4.5 \quad 4.4 \quad 37$

$\begin{array}{lllll}7.19 & 3.3 & 3.5 & 29\end{array}$

10.133 .13 .331

$10.893 .4 \quad 3.9 \quad 30$ $24.25 \quad 3.4 \quad 3.236$ $8.27 \quad 2.6 \quad 3.2 \quad 30$
$187.14 \quad 3$

$3278: 1229$

1146.09 ?

157.093

$0 \quad 78.16 \quad 3$

$\begin{array}{ll}75 & .15 \\ 79 & .08 \\ 88 & .10 \\ 84 & .08\end{array}$

$0308.14 \quad 6512.1$

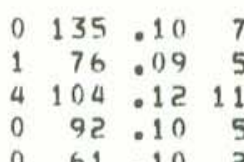

$0 \quad 61.10$ 2

$\begin{array}{llll}0 & 57.11 & 4\end{array}$

3303.1147

$\begin{array}{llll}0 & 74.08 \quad 4\end{array}$

0
01.115

$0204.10 \quad 6$

$\begin{array}{lllll}0 & 309.10 \quad 68 \quad 13.2\end{array}$

0143.106

184.07

1121.10

$0 \quad 109.10$

$0 \quad 83.10 \quad 5$

146.11

160.09

070.095

$\begin{array}{llll}0 & 37 \quad .12 \quad 3\end{array}$

$183.08 \quad 4$
19

$\begin{array}{lll}0 & 190 & .20\end{array}$

182.08

2 34.12

ट 100.10

1110.105

$0 \quad 50.0911$

$\begin{array}{llll}1 & 137 & .10 \quad 5\end{array}$

$1176.10 \quad 2$

1101.095

$\begin{array}{llll}0 & 70 & .18 & 4\end{array}$

$044.15 \quad 1$

$\begin{array}{llll}0 & 113.10 \quad 5\end{array}$

$0 \quad 115.20$

$0 \quad 184.11$

0199.117

$0 \quad 59.0910$

0
$85.07 \quad 5$
.5 .931 MER

$3.3 \quad 35$ DIS

.428 MER

.430 UER

1.123 MER

.5 2.6 27 MER

.4 .817 MER

.5 .628 UER

.5 .532 MER

6.732 DIS

$.6 \quad .536 \mathrm{POL}$ .4 .432 UER

$.3 .920 \mathrm{LSW}$

.5 .530 UER

.4 .731 UKF

.4 .832 MOK

$4.498 .9 \quad 17$ DIS

.4 .333 UER

.5 .431 UER

1.1 .628 UER

$\begin{array}{llll}3.2 & 3.321 & \text { DIS }\end{array}$

.6 .533 UER

.3 .811 UER

.6 .429 UER

.6 .436 MER F

$.5 \quad .725$ UER

.4 .636 UKF F

.7 .926 DEP

$.5 \quad .727$ MER

.3 .525 SPC

.5 .430 UER

1.21 .522 HEA

.3 .433 UER

.4 .537 UKF

.5 .433 UER

.5 .436 UER F

$.51 .3 \quad 34$ NER

$.6 \quad .625$ MER

.8 .623 MER

$\begin{array}{lll}.6 & .5 & 2.7 \text { UER }\end{array}$

.5 1.4 23 MER

.4 .819 GLN F

.6 .436 UER F

1.01 .326 HEA F

$.9 .531 \mathrm{POL}$

$1.0 \quad .529$ MER

.61 .226 GLN F

.5

.728 UER 
ORIGIN TIME LAT N YEAR MON DA HRMN SEC DEG MIN

1979 MAR $13 \quad 821 \quad 39.42 \quad 1920.08$ $\begin{array}{llllll}13 & 957 & 8.81 & 19 & 21.10\end{array}$ $\begin{array}{llllll}15 & 855 & 1.06 & 19 & 22.12\end{array}$ $\begin{array}{llllll}15 & 1010 & 14.66 & 19 & 22.67\end{array}$ $\begin{array}{llllll}20 & 13 & 3 & 9.91 & 19 & 21.06\end{array}$

$\begin{array}{llllll}20 & 16 & 8 & 35.18 & 19 & 40.60\end{array}$ $\begin{array}{lllll}21 & 2046 & 59.79 & 20 & 5.99\end{array}$ $\begin{array}{llllll}23 & 2136 & 30.93 & 19 & 20.73\end{array}$ $\begin{array}{llllll}25 & 650 & 17.87 & 19 & 21.25\end{array}$ $\begin{array}{llll}26 & 1341 & 25.47 \quad 19 & 20.80\end{array}$

$\begin{array}{lllll}27 & 2130 & 9.80 & 20 & 5.42\end{array}$ $27 \quad 2134 \quad 44.9220 \quad 3.82$ $\begin{array}{llllll}28 & 554 & 50.56 & 19 & 21.38\end{array}$ $\begin{array}{lllll}29 & 056 & 2.32 & 20 & 8.61\end{array}$ $\begin{array}{llllll}29 & 1324 & 46.69 & 18 & 47.67\end{array}$

$\begin{array}{lllllll}29 & 23 & 6 & 44.78 & 20 & 48.35\end{array}$ $\begin{array}{lllll}30 & 1256 & 21.12 & 20 & 3.63\end{array}$ $A P R \quad 2 \quad 1923 \quad 24.55 \quad 19 \quad 22.13$ $\begin{array}{lllll}4 & 2014 & 58.71 & 19 & 20.94\end{array}$

$\begin{array}{llllll}8 & 18 & 8 & 57.88 & 19 & 26.34\end{array}$

$\begin{array}{llllll}12 & 839 & 46.44 \quad 19 & 20.15\end{array}$ $\begin{array}{llllll}14 & 331 & 12.71 & 19 & 23.97\end{array}$ $\begin{array}{lllll}14 & 1524 & .29 & 19 & 20.94\end{array}$ $\begin{array}{llllll}15 & 115 & 18.55 & 19 & 23.70\end{array}$ $\begin{array}{llllll}16 & 2029 & 53.87 & 19 & 15.34\end{array}$

$21 \quad 1214 \quad 52.33 \quad 19 \quad 20.12$ $\begin{array}{llllll}21 & 1857 & 6.78 & 19 & 18.69\end{array}$ $\begin{array}{llllll}24 & 112 & 38.11 & 20 & 6.79\end{array}$ $\begin{array}{llllll}26 & 2114 & 45.57 & 20 & 19.50\end{array}$ $27 \quad 1426 \quad 55.26 \quad 18 \quad 57.73$

$\begin{array}{lllllll}30 & 22 & 3 & 32.53 & 18 & 42.45\end{array}$ $\begin{array}{llllllll}\text { MAY } 2 & 21 & 8 & 47.95 & 19 & 24.71\end{array}$

$\begin{array}{lllll}5 & 1851 & 7.32 & 19 & 13.30\end{array}$

$\begin{array}{llllll}5 & 2122 & 47.41 & 19 & 4.66\end{array}$

$\begin{array}{llllll}10 & 1037 & 45.76 \quad 19 & 26.28\end{array}$

$\begin{array}{llllll}11 & 1345 & 26.94 & 19 & 20.14\end{array}$ $111359 \quad 37.331920 .41$

$11 \quad 1455 \quad 50.61 \quad 1920.44$

$\begin{array}{llllll}12 & 1226 & 17.33 & 19 & 23.26\end{array}$

$13 \quad 1138 \quad 39.85 \quad 19 \quad 23.05$

$\begin{array}{llll}14 & 1057 & 31.42 \quad 19 \quad 16.80\end{array}$

$\begin{array}{lllll}14 & 1838 & 45.11 & 19 & 2.42\end{array}$

$\begin{array}{llllll}17 & 530 & 52.27 & 19 & 27.90\end{array}$

$\begin{array}{llllll}21 & 2142 & 26.24 & 19 & 19.83\end{array}$

$\begin{array}{llllll}23 & 39 & 44.27 & 19 & 23.88\end{array}$

$23 \quad 1651 \quad 14.06 \quad 19 \quad 57.66$ $\begin{array}{llllll}24 & 1626 \quad 6.09 \quad 19 & 23.05\end{array}$ $\begin{array}{lllll}28 & 644 & 58.32 & 18 & 50.07\end{array}$
LON W DEG MIN

$155 \quad 12.09$

15526.16

1556.30

$155 \quad 5.91$

1557.66

1553.82

15550.44

1557.50

$155 \quad 7.88$

1558.48

15550.08

15548.83

$155 \quad 4.71$

$155 \quad 51.49$

15517.58

$158 \quad 41.18$

$155 \quad 49.97$

15459.91

$155 \quad 1.33$

$155 \quad 27.23$

15513.29

15515.76

15513.31

15515.28

15524.17

15529.04

$155,3.25$

15554.62

15532.39

15528.07

$155 \quad 11.13$

15529.21

15535.88

15531.46

$155 \quad 24.47$

$\begin{array}{ll}155 & 3.87\end{array}$

$155 \quad 3.91$

$155 \quad 3.81$

$155 \quad 3.36$

$155 \quad 3.04$

$155 \quad 47.21$ 1552.28

15524.29

15512.00

15526.82

$155 \quad 37.08$

15514.98

15421.42
DEPTH AMP NIIR

GAP RMS MIN ERH

ERZ NO KM MAG NAG NR NS DEG SEC DIS KM

KM FM REMK

$\begin{array}{rrrrrrrrrrrr}9.21 & 2.8 & 3.3 & 31 & 0 & 79 & .10 & 5 & .4 & .6 & 28 & \text { UER } \\ 11.28 & 3.5 & 3.7 & 38 & 1 & 75 & .12 & 4 & .4 & .5 & 36 & \text { HEA F } \\ 1.43 & 3.4 & 3.7 & 27 & 0 & 75 & .18 & 6 & .6 & 2.6 & 25 & \text { UER } \\ .11 & 3.4 & 1.0 & 21 & 0 & 76 & .11 & 5 & .4 & 1.3 & 20 & \text { MER } \\ 8.94 & 3.3 & 3.8 & 31 & 1 & 81 & .09 & 4 & .4 & .5 & 25 \text { UER F }\end{array}$

$10.00 \quad 2.6 \quad 3.1 \quad 15$ $16.22 \quad 4.5 \quad 4.5 \quad 37$

$9.08 \quad 2.7 \quad 3.1 \quad 31$

$9.993 .13 .6 \quad 35$

$\begin{array}{lllll}7.09 & 3.2 & 3.9 & 28\end{array}$

0212.1730 1144.1146

$188.07 \quad 5$

$1.5 \quad 2.114 \mathrm{HIL} \mathrm{B}$ $.717 .632 \mathrm{KOH} \mathrm{F*}$ .5 .529 UER .5 .332 UER .5 .926 UER F

$12.314 .94 .6 \quad 36$ $10.34 \quad 3.1 \quad 3.4 \quad 30$

$9.13 \quad 3.0 \quad 3.0 \quad 32$

$13.14 \quad 3.13 .1 \quad 34$

$12.93 \quad 2.7 \quad 3.025$

$.117 \quad 5.5 \quad 5.7 \quad 37$

$\begin{array}{lllll}21.79 & 3.1 & 3.3 & 33\end{array}$

$6.17 \quad 2.6 \quad 3.0 \quad 31$

$6.87 \quad 3.3 \quad 3.6 \quad 32$

$\begin{array}{lllll}9.78 & 3.1 & 7.7 & 34\end{array}$

$9.443 .0 \quad 3.133$

5.373 .43 .836

$9.542 .8 \quad 3.0 \quad 33$

$\begin{array}{lllll}3.41 & 2.6 & 3.0 & 30\end{array}$

$47.44 \quad 3.6 \quad 3.8 \quad 33$

$8.23 \quad 3.0 \quad 3.226$ 10.853 .03 .431 31.053 .13 .133 $\begin{array}{lllll}1.70 & 2.9 & 3.3 & 29\end{array}$ $36.70 \quad 3.4 \quad 3.6 \quad 36$ $1281.10 \quad 49$

$3.25 .932 \mathrm{KOH} \mathrm{F}$ .6 .423 MER $\begin{array}{llllllll}1 & 294 & 12 & 51 & 3.6 & 6.3 & 28 & \mathrm{KOH}\end{array}$ $\begin{array}{llllllll}0 & 276 & .06 & 44 & 3.5 & 23.7 & 21 & \mathrm{PPL}\end{array}$

$\begin{array}{rrrrrrrr}1 & 285 & .18 & 98 & 13.2 & 2.3 & 34 & \text { DIS * } \\ 1 & 208 & .10 & 9 & 3.1 & 4.6 & 23 & \text { KOH } \\ 0 & 191 & .11 & 6 & .9 & .6 & 30 & \text { LER F } \\ 0 & 194 & .09 & 3 & .7 & .5 & 28 & \text { MER } \\ 2 & 47 & .11 & 7 & .4 & .8 & 29 & \text { UKF }\end{array}$

$164.08 \quad 5$ $044: 13 \quad 3$ 158.093 168.09 $0 \quad 129.09$

$.4 \quad .628$ UER $.4 \quad .733$ SPC F .4 .529 UER $.3 \quad .520 \mathrm{SPC}$ .92 .231 LSW F

$\begin{array}{rrrr}0 & 71 & .11 & 5 \\ 1 & 133 & .08 & 7\end{array}$

$1262.10 \quad 14$

$\begin{array}{lllll}0 & 277 & .14 & 33\end{array}$

.41 .026 HEA $.6 .428 \mathrm{POL}$ $2.5 \quad 2.829 \mathrm{KOH}$ $\begin{array}{llll}3.6 & 3.0 & 29 & \text { DIS }\end{array}$

7.003 .02 .534 $10.56 \quad 3.23 .239$

$8.16 \quad 3.12 .9 \quad 30$

$56.563 .0 \quad 26$

$10.96 \quad 3.02 .6 \quad 33$

1231.0521

$1.0 \quad 1.7 \quad 33$ DIS

$0292.1364 \quad 5.4 \quad 85.134 \mathrm{PPL}$. $132.10 \quad 5 \quad .4 \quad .532$ UKF $\begin{array}{lllllll}0 & 85 & .24 & 4 & .8 & 1.3 & 24 \\ \end{array}$ $\begin{array}{lllllllll}0 & 175 & 19 & 11 & 2.0 & 4.6 & 11 & \text { LSW T }\end{array}$ $\begin{array}{llllllll}0 & 36 & .13 & 7 & .4 & .7 & 32 & \text { UKF }\end{array}$

$9.31 \quad 2.8 \quad 3.1 \quad 35$

1132.12

$9.26 \quad 3.3 \quad 3.7 \quad 35$

$8.97 \quad 2.9 \quad 3.132$

8.823 .13 .224

$\begin{array}{lllll}0 & 114 & 111 & 2\end{array}$

$1110.10 \quad 2$

9.613 .03 .225

1.106 .103

9.453 .52 .933 51.073 .02 .937 $10.01 \quad 3.4 \quad 3.3 \quad 35$

9.233 .23 .235

$8.983 .3 \times .535$

0103.11 2. 248.0729

. B .5 30 MER

$.8 \quad .433$ MER F

.7 .428 MER

.6 .519 MER $.5 \quad .423$ MER

$\begin{array}{rrrrrrrrrrrr}11.35 & 3.2 & 2.7 & 26 & 1 & 247 & .06 & 26 & 1.2 & .6 & 16 & \mathrm{KOH} \\ 3.41 & 3.2 & 3.5 & 36 & 1 & 49 & .09 & 2 & .3 & .5 & 32 & \mathrm{GLN} F \\ 7.01 & 3.1 & 1.6 & 21 & 0 & 337 & .52 & 91 & 99.0 & 99.0 & 14 & \text { DIS }\end{array}$ 
ORIGIN TIME LAT N LON W DEPTH AMP DUR GAP RMS MIN ERH YEAR MON DA HRMN SEC DEG MIN DEG NIN

1979 MAY $28 \quad 1850 \quad 33.82 \quad 1922.39$ $\begin{array}{llllll}29 & 1743 & 55.40 \quad 19 & 21.86\end{array}$ $\begin{array}{llllll}29 & 1744 & 54.97 & 19 & 22.33\end{array}$ $\begin{array}{llllll}29 & 1748 & 7.55 & 19 & 22.25\end{array}$ $29 \quad 1752 \quad 17.05 \quad 1921.42$

JUN $1016 \quad 11.64 \quad 19 \quad 20.36$ 105458.241922 .20 $\begin{array}{lllll}5 & 1216 & 36.49 & 19 & 22.32\end{array}$ $\begin{array}{llllll}7 & 5 & 2 & 54.34 & 19 & 23.14\end{array}$ $\begin{array}{lllllll}9 & 0 & 2 & 17.88 & 19 & 49.82\end{array}$

$99753.30 \quad 1924.70$ $\begin{array}{llll}12 & 036 & 51.07 \quad 19 & 20.71\end{array}$ $\begin{array}{lllll}12 & 1655 & 48.83 & 19 & 24.35\end{array}$ $19 \quad 1517 \quad 38.34 \quad 19 \quad 20.03$ $21622 \quad 31.61 \quad 19 \quad 19.58$

$\begin{array}{lllll}25 & 1835 & 50.26 \quad 19 & 21.81\end{array}$ $\begin{array}{lllllll}26 & 2147 & 59.77 & 19 & 28.54\end{array}$

JUL ट $1842 \quad 44.83 \quad 1923.83$

$\begin{array}{llllll}4 & 1727 & 15.89 & 19 & 20.77\end{array}$

$5 \quad 233 \quad 46.11 \quad 19 \quad 28.09$

$8 \quad 546 \quad 34.42 \quad 19 \quad 23.74$

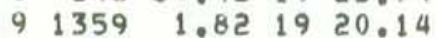
$\begin{array}{llllll}14 & 515 & 34.31 & 19 & 43.36\end{array}$ $\begin{array}{llllll}15 & 1642 & 7.27 & 19 & 22.86\end{array}$ $\begin{array}{llllll}15 & 22 & 3 & 40.51 & 19 & 29.37\end{array}$

$\begin{array}{llllll}16 & 248 & 49.72 & 19 & 23.31\end{array}$

$\begin{array}{llllll}16 & 413 & 15.73 & 19 & 24.00\end{array}$

$\begin{array}{lllllll}18 & 0 & 1 & 50.19 & 19 & 23.32\end{array}$

$\begin{array}{llllll}20 & 2322 & 30.25 & 19 & 24.56\end{array}$

$\begin{array}{lllllll}24 & 18 & 7 & 38.33 & 19 & 19.68\end{array}$

$\begin{array}{lllll}26 & 950 & 41.65 & 19 & 45.52\end{array}$

$\begin{array}{lllll}27 & 856 & 33.62 & 19 & 19.65\end{array}$

$\begin{array}{lllll}29 & 839 & 24.67 & 19 & 25.00\end{array}$

$\begin{array}{lllll}31 & 330 & 51.28 & 19 & 27.98\end{array}$

$\begin{array}{llllll}31 & 2237 & 36.45 & 19 & 20.54\end{array}$

AUG

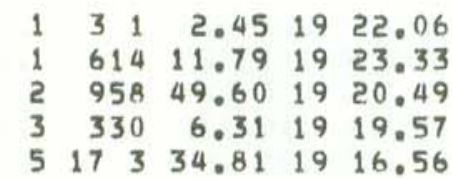

$\begin{array}{lllll}10 & 1757 & 53.10 & 19 & 18.37\end{array}$

$\begin{array}{lllll}12 & 2032 & 1.32 & 19 & 19.87\end{array}$

$136340.6119 \quad 17.80$

$\begin{array}{llllll}14 & 251 & 42.19 & 20 & 48.87\end{array}$

$\begin{array}{llllll}15 & 0 & 0 & 8.12 & 19 & 24.35\end{array}$

$\begin{array}{lllllll}16 & 13 & 4 & 19.38 & 19 & 22.66\end{array}$ $\begin{array}{lllllll}25 & 21 & 8 & 14.65 & 19 & 20.80\end{array}$ $\begin{array}{llllll}28 & 521 & 59.08 & 19 & 18.56\end{array}$
$155 \quad 4.77$

$155 \quad 12.64$

$155 \quad 12.47$

$155 \quad 12.89$

15512.92

$155 \quad 13.78$

$155 \quad 4.96$

1554.53

1554.04

$155 \quad 45.37$

15528.23

1553.05

15524.70

15510.70

1558.56

$155 \quad 1.92$

$155 \quad 52.01$

15526.68

$154 \quad 49.45$

$\begin{array}{lll}155 & 15.71\end{array}$

15511.69

156.04

$155 \quad 5.65$

15555.21

$155 \quad 5.24$

$155 \quad 1.73$

$155 \quad 15.25$

15527.60

1558.30

15558.42

1558.22

15525.28

15525.89

15513.02

$155 \quad 4.49$

15516.93

$155 \quad 12.87$

$155 \quad 12.30$

15533.54

$155 \quad 13.17$ 1557.13 $155 \quad 15.59$ $156 \quad 17.42$ 15525.00

15527.22 15513.32 15513.21
1557.86

GAP RMS MIN ERH
$9.05 \quad 3.0 \quad 3.0 \quad 36$

6.793 .02 .932

4.743 .13 .316 .223 .13 .120

$6.72 \quad 3.22 .820$

$9.83 \quad 3.0 \quad 2.7 \quad 37$

$9.20 \quad 3.0 \quad 2.8 \quad 34$

$\begin{array}{llll}8.82 & 3.1 & 2.8 \quad 37\end{array}$

$8.312 .8 \quad 3.129$

13.92 2.B 3.133

$\begin{array}{lllll}10.87 & 3.1 & 3.3 & 38\end{array}$

$\begin{array}{lllll}7.71 & 2.9 & 3.2 & 32\end{array}$

$\begin{array}{llll}12.22 & 3.1 & 3.0 & 38\end{array}$

$9.98 \quad 3.2 \quad 3.536$

$\begin{array}{lllll}8.88 & 2.8 & 3.1 & 27\end{array}$

$\begin{array}{lllll}10.11 & 3.4 & 3.3 \quad 32\end{array}$

$10.74 \quad 3.3 \quad 3.6 \quad 40$

$9.42 \quad 3.4 \quad 3.7 \quad 35$

$11.613 .13 .5 \quad 34$

$\begin{array}{llll}2.89 & 2.9 & 3.1 \quad 27\end{array}$

$9.41 \quad 2.7 \quad 3.1 \quad 38$

$33.87 \quad 3.0 \quad 3.2 \quad 38$

$1.47 \quad 3.6 \quad 4.0 \quad 34$

$13.47 \quad 3.0 \quad 2.6 \quad 19$

$8.92 \quad 3.5 \quad 3.936$ $10.833 .6 \quad 3.9 \quad 39$

$\begin{array}{rrr}1 & 81 & .09 \\ 1 & 55 & .11 \\ 0 & 89 & .15 \\ 1 & 117 & .15 \\ 0 & 99 & .11\end{array}$

256.10

177.11

086.093

094.093

$0 \quad 147.0918$

2125.08

187.12

$0 \quad 79.10$

0159.09

3103.14

$0-195010$

151.112

2 81.105

$\begin{array}{llll}2 & 223 & .12 & 17\end{array}$

068.11,

1245.0925

$20.30 \quad 3.6 \quad 4.138$

$9.27 \quad 3.5 \quad 4.0 \quad 32$

$9.132 .8 \quad 3.0 \quad 34$

$11.65 \quad 4.3 \quad 4.242$

$10.072 .93 .0 \quad 34$

$9.39 \quad 2.9 \quad 3.1 \quad 33$

$3.433 .0 \quad 3.530$

$9.30 \quad 2.7 \quad 3.2 .37$

$10.30 \quad 3.3 \quad 3.7 \quad 42$

9.833 .53 .429

$10.27 \quad 3.0 \quad 3.238$

$8.26 \quad 3.0 \quad 3.3 \quad 34$

$9.713 .4 \quad 3.4 \quad 36$

$24.48 \quad 4.5 \quad 4.9 \quad 44$

$10.59 \quad 3.0 \quad 2.7 \quad 33$

$10.76 \quad 3.9 \quad 4.0 \quad 43$

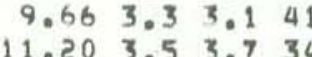

9.542 .93 .229

$\begin{array}{llll}1.97 & 3.1 & 3.7 & 28\end{array}$

$\begin{array}{lllll}3.41 & 2.7 & 3.2 & 23\end{array}$

$9.84 \quad 3.53 .7 \quad 34$

132.104

2 40.10

2 47.11

$1.82 \cdot 10$
277

ERZ NO

KM FM REMK

$.5 \quad .530$ MER

.4 .825 UER

$.6 \quad 2.212$ UER

.6 .912 UER

$.6 \quad .711$ UER

.4 .432 UER

.5 .429 MER

.5

. 5 31 MER

.528 MER

- $830 \mathrm{KON}$

.4

.535 UKF

.728 MER

.326 UKF

.428 UER F

.422 UER

.427 MER

$.525 \mathrm{KON}$

.530 UKF $F$

. 435 UER F

.530 LER

.321 SPC

.531 UER

$1.21 .531 \mathrm{KON}$

.3 .227 MER

$\begin{array}{lll}1.5 & .7 & 7 \mathrm{KON}\end{array}$

.5

. 318 MER

.532 MER

.718 SPC

.529 UKF F

. 5 28 Uer F

1.1

$2.132 \mathrm{KON} F$ .427 UER F

1.028 UKF

. 4 40 UKF

. 428 UER F

. 429 MER

. 525 SPC F

.526 UER

.437 UER

.825 HEA F

$.532 \mathrm{POL}$

.728 UER

.635 KOA

.843 DIS F

.630 UKF

.5 41 UKF

. 433 UER F

.431 INT 

ORIGIN TIME LAT N YEAR MON DA HRMN SEC DEG MIN 1979 AUG $28 \quad 547 \quad 24.79 \quad 19 \quad 18.98$ $\begin{array}{llllll}28 & 655 & 13.20 & 19 & 18.58\end{array}$ SEP $11216 \quad 33.48 \quad 19 \quad 21.74$ $\begin{array}{lllll}3 & 127 & 12.58 & 19 & 19.50\end{array}$ $\begin{array}{lllll}4 & 130 & 9.18 \quad 19 & 44.61\end{array}$

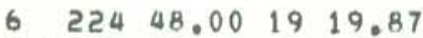

$\begin{array}{lllllll}8 & 1334 & 42.18 & 19 & 18.75\end{array}$

$\begin{array}{lllll}11 & 1455 & 25.20 & 19 & 23.17\end{array}$

$\begin{array}{llllll}14 & 432 & 17.38 & 19 & 23.56\end{array}$

$14 \quad 73518.711920 .09$

$\begin{array}{lllll}14 & 1532 & 48.01 \quad 19 & 21.10\end{array}$

$\begin{array}{llllll}16 & 951 & 36.66 & 19 & 23.81\end{array}$

$\begin{array}{lllll}20 & 918 & 32.85 & 19 & 23.57\end{array}$

$\begin{array}{llllll}21 & 129 & 24.09 & 19 & 20.23\end{array}$

$\begin{array}{lllll}21 & 2159 & 37.62 & 19 & 20.81\end{array}$

$\begin{array}{llllll}21 & 2329 & 12.34 & 19 & 21.18\end{array}$ $\begin{array}{llllll}21 & 2336 & 17.34 & 19 & 20.71\end{array}$ $\begin{array}{llllll}22 & 151 & 2.41 & 19 & 20.54\end{array}$ $\begin{array}{llllll}23 & 128 & 19.88 & 19 & 23.02\end{array}$ $\begin{array}{llllll}23 & 925 & 25.79 & 19 & 21.86\end{array}$

$\begin{array}{llllll}24 & 1750 & 23.13 & 19 & 22.35\end{array}$ $\begin{array}{llllll}26 & 621 & 22.85 & 19 & 21.68\end{array}$ $\begin{array}{lllll}26 & 151 & 32.43 \quad 19 & 32.46\end{array}$ $\begin{array}{llllll}27 & 535 & 45.49 & 19 & 19.76\end{array}$ $\begin{array}{lllll}27 & 538 & 31.20 & 19 & 20.33\end{array}$

$\begin{array}{llllllll}29 & 14 & 2 & 26.31 & 19 & 21.72\end{array}$ $\begin{array}{llllll}1 & 1010 & 17.88 & 19 & 23.55\end{array}$

$\begin{array}{lllll}6 & 046 & 12.24 & 19 & 20.15\end{array}$

$\begin{array}{llllll}8 & 641 & 37.40 & 19 & 20.68\end{array}$

8164019.801919 .20

$9425 \quad 9.32 \quad 20 \quad 27.14$

$9234155.36 \quad 1923.19$ $\begin{array}{llllll}10 & 1059 & 28.19 & 19 & 19.76\end{array}$ $\begin{array}{lllll}11 & 1240 & 22.34 & 19 & 1.63\end{array}$ $\begin{array}{lllll}12 & 1559 & 25.76 & 19 & 23.04\end{array}$

$12 \quad 16 \quad 6 \quad 51.09 \quad 19 \quad 23.06$ $\begin{array}{lllll}13 & 116 & 26.04 & 19 & 26.68\end{array}$ $\begin{array}{lllll}13 & 258 & 51.02 & 19 & 27.05\end{array}$ $\begin{array}{llllll}14 & 737 & 16.92 & 19 & 54.38\end{array}$ $\begin{array}{llllll}15 & 044 & 12.27 & 19 & 55.75\end{array}$

$\begin{array}{lllll}16 & 1954 & 37.81 & 19 & 19.44\end{array}$ $\begin{array}{llllll}16 & 2131 & 49.46 \quad 19 & 19.28\end{array}$ $\begin{array}{llllll}18 & 2014 & 32.38 & 19 & 47.12\end{array}$ $19 \quad 132 \quad 54.64 \quad 19 \quad 23.06$ $201359 \quad 11.97 \quad 19 \quad 24.40$

$\begin{array}{llllll}20 & 1957 & 2.00 & 19 & 19.14\end{array}$

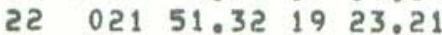
$\begin{array}{lllll}28 & 836 & 49.35 & 19 & 19.92\end{array}$

LON W DEPTH AMP DUR DEG MIN

KN MAG $M A G$

DEG SEC DIS KM

ERZ NO

$\begin{array}{llllll}155 & 13.08 & 10.88 & 3.4 & 3.7 & 35\end{array}$ $\begin{array}{lll}155 & 13.17\end{array}$ $155 \quad 4.92$

$155 \quad 10.43$ $10.98 \quad 3.8 \quad 3.7 \quad 39$ $9.54 \quad 3.8 \quad 4.0 \quad 43$ $9.46 \quad 2.9 \quad 3.139$ $9.063 .2 \quad 3.3 \quad 36$

$0 \quad 128 \cdot 10$ $0 \quad 130.08$ 180.11 198.08 1225.1330

$9.55 \quad 3.4 \quad 3.5 \quad 37$ $10.48 \quad 3.4 \quad 3.7 \quad 41$ $\begin{array}{llll}3.41 & 2.9 & 3.1 & 30\end{array}$ $3.00 \quad 3.0 \quad 3.5 \quad 37$ $10.40 \quad 3.2 \quad 3.3 \quad 38$

$10.96 \quad 3.8 \quad 3.8 \quad 43$ $9.24 \quad 3.2 \quad 3.6 \quad 42$ 3.362 .93 .231 $10.06 \quad 3.4 \quad 3.6 \quad 41$

$9.195 .5 \quad 5.4 \quad 43$

9.214 .34 .343 $8.343 .2 \quad 3.6 \quad 38$ 8.47 2.8 3.140 $8.753 .3 \quad 3.8 \quad 40$

36.443 .33 .036

$9.37 \quad 3.6 \quad 3.6 \quad 35$ $9.30 \quad 3.0 \quad 3.134$ $10.90 \quad 3.2 \quad 3.425$ $10.05 \quad 4.3 \quad 4.6 \quad 39$ $8.92 \quad 3.2 \quad 3.7 \quad 19$

$8.08 \quad 3.2 \quad 3.0 \quad 43$ $\begin{array}{lllll}3.21 & 2.6 & 3.0 & 26\end{array}$ $\begin{array}{lllll}10.38 & 3.9 & 4.1 & 38\end{array}$ $\begin{array}{lllll}7.49 & 2.7 & 3.2 & 34\end{array}$ $10.14 \quad 3.7 \quad 3.7 \quad 29$

$155 \quad 15.37$

$155 \quad 14.79$

15511.24

$154 \quad 49.58$

$155 \quad 14.76$

$155 \quad 14.74$

15520.97

$155 \quad 21.55$

$155 \quad 10.73$

$156 \quad 27.75$

$155 \quad 8.45$

15511.91

$156 \quad 3.07$

15514.82

15528.02

$155 \quad 11.97$

15516.80

15515.85
.772 .93 .132 $\begin{array}{llll}3.84 & 2.9 & 3.1 & 32\end{array}$ $8.152 .6 \quad 3.0 \quad 30$ $30.66 \quad 1.9 \quad 3.1 \quad 14$ $3.60 \quad 3.0 \quad 3.4 \quad 30$

$\begin{array}{llll}3.64 & 2.8 \quad 3.2 \quad 30\end{array}$ $8.45 \quad 3.8 \quad 3.936$ $\begin{array}{lllll}6.56 & 3.1 & 3.1 & 34\end{array}$ $40.57 \quad 4.0 \quad 4.3 \quad 38$ $\begin{array}{lllll}3.31 & 3.1 & 2.4 & 24\end{array}$

$9.12 \quad 3.13 .230$ $\begin{array}{lllll}10.16 & 2.9 & 3.1 & 34\end{array}$ $8.80 \quad 3.1 \quad 3.4 \quad 2.4$ $\begin{array}{lllll}3.72 & 2.8 & 3.0 & 27\end{array}$ 11.083 .33 .435

$10.27 \quad 3.5 \quad 3.4 \quad 35$ $\begin{array}{llll}3.85 & 2.6 & 3.2 & 27\end{array}$ $\begin{array}{lllll}37.10 & 3.1 & 3.2 & 34\end{array}$

$\begin{array}{rrr}1 & 114 & .09 \\ 1 & 73 & .11 \\ 1 & 48 & .09 \\ 0 & 35 & .09 \\ 1 & 81 & .09\end{array}$

1113.1211 1119.10 145.10 $0 \quad 79.09$

0101.11

1160.11

1158.09

2 114.10

191.10

60.09

$0 \quad 82.09$

$0 \quad 138.11$

$0 \quad 199.13$

1110.11

0146.15

280.10

144.09

170.10

$3 \quad 137.12$

0104.09

$304.16 \quad 63$ $47.10 \quad$ ?

$191.10 \quad 5$

$0 \quad 319.18 \quad 46 \quad 22$. 48.08

\subsection{1}

41.117 41.146 $\begin{array}{llll}213 & .10 & 17\end{array}$ $0 \quad 291.1075$

$\begin{array}{rrl}1 & 83 & .07 \\ 1 & 97 & .10 \\ 1 & 235 & .14 \\ 0 & 49 & .10 \\ 0 & 35 & .12 \\ & & \\ 0 & 99 & .08 \\ 0 & 45 & .10\end{array}$

.2

$\because 6$

.5

.7

.6

.

.7

1.0

1.0
.5

.6

.5

.4

1.3

.5

.4

.3

.0

.5

.6

.5

.6

.7

.6

.4 .535 UER F .3 .525 SPC F .5 .334 UER F

.6 .929 MER

.5 .429 UER F

$2.7 \quad .731$ DIS

$.3 \quad .530$ GLN

.4 .621 UER

.36 .814 DIS

.525 GLN F

.4 .528 GLN F .4 .835 UKF F .41 .233 UKF F $1.0 \quad 1.7 \quad 37 \quad \mathrm{KKU} F$ 4.823 .724 DIS *
.5 .529 UER F
.5 .431 UER
$1.3 .824 \mathrm{KON}$
.4 .626 GLN
.4 .534 UKF
$\begin{array}{rrrr}.4 & .4 & 33 & \text { UER F } \\ .4 & .6 & 26 & \text { SPC } \\ .7 & 1.5 & 31 & \text { DEP }\end{array}$

87.09 
ORIGIN TIME LAT N YEAR MON DA HRMN SEC DEG MIN

$\begin{array}{lllllll}1979 & \text { OCT } 30 & 1935 & 11.68 & 19 & 53.07\end{array}$

$\begin{array}{llllll}31 & 523 & 30.09 & 19 & 58.43\end{array}$

$\begin{array}{llllll}31 & 1657 & 50.71 & 19 & 17.74\end{array}$

NOV $3 \quad 658 \quad 3.13 \quad 19 \quad 17.08$

$\begin{array}{lllllll}3 & 10 & 8 & 31.88 & 19 & 22.96\end{array}$

$\begin{array}{lllll}318951.64 & 20 \quad 3.51\end{array}$

$\begin{array}{llllll}4 & 210 & 33.30 & 19 & 19.66\end{array}$

$\begin{array}{llllll}5 & 16 & 5 & 48.16 & 19 & 21.47\end{array}$

$11 \quad 025 \quad 33.60 \quad 1921.46$

$\begin{array}{lllll}15 & 413 \quad .88 & 19 & 23.19\end{array}$

$\begin{array}{lllll}15 & 2241 & 32.57 & 19 & 22.04\end{array}$

$\begin{array}{lllll}15 & 2354 & 29.53 & 19 & 21.18\end{array}$

$\begin{array}{llll}16 & 147 \quad 30.64 \quad 19 \quad 21.76\end{array}$

$\begin{array}{llllll}16 & 217 & 9.08 & 19 & 21.80\end{array}$

$\begin{array}{lllll}16 & 412 \quad 3.10 & 19 & 22.62\end{array}$

$\begin{array}{lllll}16 & 6219.28 \quad 19 & 22.04\end{array}$

$\begin{array}{llllll}16 & 1024 & 26.10 & 19 & 22.26\end{array}$

$\begin{array}{llllll}16 & 12 & 4 & 5.20 & 19 & 21.62\end{array}$

$\begin{array}{lllll}17 & 611 & 59.55 & 19 & 20.92\end{array}$

$\begin{array}{lllll}19 & 930 & 41.82 & 19 & 23.20\end{array}$

$\begin{array}{llllll}19 & 1658 & 13.86 & 19 & 19.88\end{array}$ $\begin{array}{lllll}20 & 2129 & 38.05 & 19 & 23.10\end{array}$ $\begin{array}{llllll}21 & 9 & 7 & 27.57 & 19 & 16.27\end{array}$ 2. $1742 \quad 30.75 \quad 19 \quad 18.64$ 2364134.031923 .24

$\begin{array}{lllll}23 & 1045 & 37.97 & 19 & 21.33\end{array}$ $\begin{array}{llllll}23 & 1848 & 27.67 & 19 & 26.79\end{array}$ $\begin{array}{llllll}24 & 2131 & 6.84 & 19 & 24.00\end{array}$ $\begin{array}{llllll}25 & 050 & 1.94 & 19 & 18.95\end{array}$ $\begin{array}{lllll}25 & 1751 & 25.03 & 19 & 19.89\end{array}$

$\begin{array}{lllllll}28 & 22 & 3 & 30.26 & 19 & 22.84\end{array}$ $\begin{array}{llllll}30 & 055 & 47.92 & 19 & 34.84\end{array}$ $\begin{array}{llllll}30 & 1015 & 41.80 & 19 & 23.67\end{array}$

DEC

$\begin{array}{rlrrrr}5 & 12 & 5 & 4.83 & 19 & 19.81 \\ 5 & 1532 & 27.24 & 19 & 24.52\end{array}$

$\begin{array}{lllll}13 & 1744 & 3.08 & 19 & 24.82\end{array}$ $\begin{array}{llllll}15 & 1745 & 13.13 & 19 & 21.02\end{array}$ $\begin{array}{llllll}16 & 1811 & 3.85 & 19 & 11.35\end{array}$ $\begin{array}{lllll}16 & 2344 & 25.43 & 19 & 23.65\end{array}$ $\begin{array}{llllll}23 & 16 & 2.09 & 19 & 23.21\end{array}$

$\begin{array}{lllll}23 & 554 & 3.14 & 19 & 19.91\end{array}$ $\begin{array}{llllll}24 & 144 & 8.17 & 19 & 22.53\end{array}$ $\begin{array}{llll}24 & 1339 & 39.25 \quad 20 \quad 8.48\end{array}$ $\begin{array}{lllll}25 & 555 & 54.88 & 20 & 27.87\end{array}$ $\begin{array}{lllll}28 & 1125 & 49.94 & 19 & 18.20\end{array}$

$\begin{array}{llllll}30 & 16 & 4 & 9.97 & 19 & 56.33\end{array}$ $\begin{array}{lllll}30 & 1859 & 57.94 & 19 & 23.17\end{array}$
LON W DEG MIN

$156 \quad 20.68$

$155 \quad 47.87$

15459.38

$155 \quad 15.92$

15516.93

$155 \quad 38.74$

$155 \quad 11.36$

$155 \quad .88$

$155 \quad 15.05$

15514.63

$155 \quad 13.98$

15514.01

15514.23

15514.28

15514.69

$155 \quad 13.83$

$155 \quad 14.35$

15513.94

$155 \quad 13.29$

15514.77

$155 \quad 8.07$

15514.70

15546.60

$155 \quad 13.45$

15514.83

$155 \quad 14.86$ 15523.68 $155 \quad 27.16$ 15511.31 15519.03

$155 \quad 4.83$

15559.21

15515.07

15513.28

15528.23

$155 \quad 24.50$ $155 \quad 18.46$ 15535.83 15516.69 15514.67

$155 \quad 12.05$ $155 \quad 6.02$ 15551.83 $156 \quad 48.34$ 15514.46

$155 \quad 43.14$ $155 \quad 29.77$

DEPTH AMP DUR

GAP RMS MIN ERH KM MAG NAG NR NS DEG SEC DIS KM

ERZ NO

$.024 .24 .3 \quad 39$

$10.44 \quad 3.0 \quad 2.7 \quad 39$

39.293 .03 .036

$10.73 \quad 3.3 \quad 3.3 \quad 35$

$\begin{array}{llll}3.53 & 2.5 & 3.0 & 31\end{array}$

$13.10 \quad 3.0 \quad 2.4 \quad 29$

$10.153 .0 \quad 3.235$

$4.95 \quad 2.5 \quad 3.2 \quad 30$

$10.84 \quad 3.5 \quad 3.4 \quad 34$

$3.603 .4 \quad 3.7 \quad 33$

$1.42 \quad 2.7 \quad 3.219$

$1.50 \quad 3.4 \quad 3.825$

$\begin{array}{llll}1.71 & 2.6 & 3.1 & 22\end{array}$

$1.28 \quad 2.7 \quad 3.5 \quad 20$

1.402 .83 .226

$\begin{array}{llll}.69 & 2.9 \quad 3.4 \quad 20\end{array}$

$\begin{array}{llll}1.87 & 3.1 \quad 3.6 \quad 26\end{array}$

3.402 .83 .023

$9.60 \quad 3.3 \quad 3.5 \quad 34$

$3.301 .6 \quad 4.013$

$9.48 \quad 3.2 \quad 3.7 \quad 30$

$3.18 \quad 3.2 \quad 3.6 \quad 35$

$10.30 \quad 3.12 .7 \quad 33$

$9.18 \quad 3.1 \quad 3.0 \quad 43$

$3.53 \quad 3.4 \quad 3.4 \quad 37$

$\begin{array}{rrr}.84 & 3.5 \quad 3.9 \quad 38\end{array}$

$9.20 \quad 3.02 .6 \quad 43$

$\begin{array}{lllll}12.73 & 3.5 & 3.8 & 46\end{array}$

$9.35 \quad 3.4 \quad 3.5 \quad 44$

31.6 ? 3.13 .046

$8.42 \quad 3.13 .140$

$10.833 .13 .2 \quad 33$

$3.49 \quad 3.0 \quad 3.0 \quad 28$

$10.10 \quad 3.4 \quad 3.3 \quad 40$

$11.32 \quad 3.8 \quad 0.0 \quad 45$

$11.44 \quad 4.04 .143$

$14.46 \quad 3.6 \quad 3.442$

$\begin{array}{llll}5.81 & 3.0 & 2.8 & 39\end{array}$

$\begin{array}{llll}3.52 & 3.2 & 3.8 & 29\end{array}$

$\begin{array}{lllll}3.35 & 3.6 & 3.9 & 40\end{array}$

$10.062 .9 \quad 3.139$

$\begin{array}{llll}8.32 & 3.1 \quad 3.140\end{array}$

$34.35 \quad 3.13 .0 \quad 38$

$33.13 \quad 3.2 \quad 3.5 \quad 28$

10.423 .13 .039

10.423 .02 .621

10.313 .23 .343
1281.1357

$\begin{array}{lllll}172 & 172 & 16 & 17\end{array}$

$\begin{array}{llll}0 & 218 & .097\end{array}$

1141.11

148.09

$1 \quad 181.10 \quad 16$

093.08

1189.11

$0 \quad 64.09$

$0 \quad 47.11$

$0 \quad 85.09$

$0 \quad 62.09$

$0 \quad 57.10$

$0 \quad 57.09$

071.10

$0 \quad 58.06$

154.11

157.18

158.08

$0 \quad 66.07$

0
$08.09 \quad 5$

$0 \quad 47.09 \quad 2$

1108.1310

078.103

$0 \quad 47.09 \quad 2$

165.113

2 46.13

248.10

2111.12

351.10

2 $80.09 \quad 3$

$452 \quad .15 \quad 13$
13

78.11 ?

69.105

32.12

038.131

$0 \quad 51.113$

395.2112

088.103

47.103

$0 \quad 82.09 \quad 5$

$0 \quad 69.10 \quad 1$

$5274.10 \quad 9$

3328.11133

$0 \quad 135.106$

2. 151.0822

$156.10 \quad 4$
2.3 .937 DIS F*

$.8 \quad 1.135 \mathrm{KOH}$

1.71 .933 DIS

$.6 .533 \mathrm{KOA}$

$.3 .530 \mathrm{KOA}$

$1.2 .925 \mathrm{KOH} \mathrm{F}$

.4 .333 UER

.71 .125 LER

.5 .432 KOA F

.4 .532 GLN F

.3 .518 UER

.2 .618 UER

.3 .522 UER

.3 .515 UER

.3 .420 UER

$.2 \quad .417$ UER

.3 .624 UER

$.51 .8 \quad 22$ UER

.3 .431 UER F

$.4 \quad .713 \mathrm{GLN}$

.6 .529 UER

.3 .422 GLN F

$.5 .617 \mathrm{KON}$

$.4 .434 \mathrm{POL}$

.3 .425 GLN F

.2 .5 31 UER F

.4 .638 UKF

.343 UKF F

.532 POL F

.943 DEP

.4
1.4
$: 4$
$: 4$

.434 MER

$.524 \mathrm{KON}$

.527 SPC F

. 438 UER

.443 UKF F

.4 .443 UKF F

.5 .339 INT

.61 .928 HEA

.41 .026 SPC F

.3 .437 GLN F

.3 .428 UER

$.4 \quad .631$ UER

$1.11 .526 \mathrm{KOH}$

$2.13 .7 \quad 16$ DIS .533 POL F

$.7 \quad .710 \mathrm{KOH}$ .637 UKF 